\title{
Design and Development of New Carbon-based Sorbent Systems for an Effective Containment of Hydrogen
}

\author{
DOE Award No. DE-FC36-04G014006
}

\section{Final Report}

Revised April 2012

Project Period: 1 February $2004-31$ March 2009

Recipient: Air Products and Chemicals, Inc.

7201 Hamilton Blvd.

Allentown, PA 18195

Contact: $\quad$ Alan C. Cooper, Air Products Program Manager

$610-481-2607$

cooperac@airproducts.com 


\section{Hydrogen Storage by Reversible Hydrogenation of Solid- and Liquid- phase Hydrogen Carriers}

\section{Executive Summary}

This is a summary of the research and development activities performed under cooperative agreement DE-FC36-04G014006 ("Design and Development of New Carbon-based Sorbent Systems for an Effective Containment of Hydrogen"). The project was directed to discover new solid and liquid materials that use reversible catalytic hydrogenation as the mechanism for hydrogen capture and storage. After a short period of investigation of solid materials, the inherent advantages of storing and transporting hydrogen using liquid-phase materials focused our attention exclusively on organic liquid hydrogen carriers ("liquid carriers").

While liquid carriers such as decalin and methylcyclohexane were known in the literature, these carriers suffer from practical disadvantages such as the need for very high temperatures to release hydrogen from the carriers and difficult separation of the dehydrogenated carriers from the hydrogen. In this project, we were successful in using the prediction of reaction thermodynamics to discover liquid carriers that operate at temperatures up to $150{ }^{\circ} \mathrm{C}$ lower than the previously known carriers. The means for modifying the thermodynamics of liquid carriers involved the use of certain molecular structures and incorporation of elements other than carbon into the carrier structure. The temperature decrease due to the more favorable reaction thermodynamics results in less energy input to release hydrogen from the carriers. For the first time, the catalytic reaction required to release hydrogen from the carriers could be conducted with the carrier remaining in the liquid phase. This has the beneficial effect of providing a simple means to separate the hydrogen from the carrier.

In order to utilize the liquid carrier prototypes discovered in the project, efficient catalysts that operate at relatively mild temperatures were required. Thus, we pursued a catalyst discovery task that designed, synthesized, and tested catalysts for the most promising liquid carrier prototypes. The best liquid carrier/catalyst combinations that were developed in the project resulted in the reversible storage of hydrogen at densities of 5.3-6.7 wt. \% $\mathrm{H}_{2}$ and $50-70 \mathrm{~g}$ $\mathrm{H}_{2} /$ liter (on a material basis) with hydrogen purities in excess of $99.9 \%$ from the 
dehydrogenation of the liquid carriers. The dehydrogenation temperatures required for hydrogen release range from $180-235^{\circ} \mathrm{C}$, depending on the specific liquid carrier and other operating factors such as hydrogen pressure.

Recognizing that operating temperatures of $>180{ }^{\circ} \mathrm{C}$ will not allow the use of waste heat from PEM fuel cells to liberate hydrogen from the carriers, we developed a liquid carrier hydrogen storage technology that does not require external heat input for hydrogen release. Our "autothermal" hydrogen storage technology uses a set of chemical reactions involving liquid carriers to provide hydrogen to the power source. The endothermic (heat consuming) hydrogen release is balanced by an exothermic (heat generating) reaction that uses air to partially oxidize the carrier liquid. The oxidized carrier is not consumed and no carbon dioxide is generated during the autothermal hydrogen release. The autothermal hydrogen carriers can be fully regenerated with hydrogen in a similar process to the conventional liquid carriers.

The project was successful in realizing the original goals of discovering a set of promising hydrogen storage materials and demonstrating efficient hydrogen storage at levels that could enable hydrogen storage systems that meet many of the criteria specified in the DOE targets at the time of the project. However, additional development is required to bring this hydrogen storage technology to a stage where it can be used for consumer applications. One necessary research and development activity is the design and demonstration of equipment that can efficiently conduct the catalytic reaction that releases the hydrogen from the liquid carrier. This goal was pursued in a separate DOE-funded project for hydrogen delivery (DE-FC36-

05G015015). Additional development of liquid carriers should be considered for inclusion in future solicitations by DOE. This could include testing of liquid carrier stability under repeated cycling and continued development of the autothermal hydrogen storage technology. Due to the decoupling of heat transfer from the fuel cell, autothermal hydrogen storage could be an enabling technology for applications that utilize PEM fuel cells. Additional development of dehydrogenation catalysts with the goals of increasing the reaction rate and increasing the reaction selectivity would also assist with the economics of hydrogen storage and delivery using both conventional and autothermal hydrogen carriers. 
Final Report for Contract DE-FC36-04G014006: Hydrogen Storage by Reversible Hydrogenation of Solid- and Liquid-phase Hydrogen Carriers

\begin{tabular}{|c|c|c|}
\hline Executive Summary & Pages i-ii & \\
\hline Chapter List & Page iii & \\
\hline Chapter 1 & Pages 1-23 & Introduction \\
\hline Chapter 2 & Pages 24-45 & $\begin{array}{l}\text { Reversible Hydrogenation of Large Polyaromatic } \\
\text { Hydrocarbons }\end{array}$ \\
\hline Chapter 3 & Pages 46-76 & Carbazole-based Hydrogen Carriers \\
\hline Chapter 4 & Pages 77-132 & Alkylated Indole Hydrogen Carriers \\
\hline Chapter 5 & Pages $133-150$ & Hydrogen Carriers with Multiple Nitrogen Atoms \\
\hline Chapter 6 & Pages 151-185 & $\begin{array}{l}\text { Hydrogen Carriers Incorporating Aromatic Ring } \\
\text { Systems with 6-membered Rings }\end{array}$ \\
\hline Chapter 7 & Pages 186-198 & Oxygen-containing Hydrogen Carriers \\
\hline Chapter 8 & Pages 199-210 & Amine/Nitrile Couples as Potential Carriers \\
\hline Chapter 9 & Pages 211-238 & $\begin{array}{l}\text { Autothermal Hydrogen Storage and Delivery } \\
\text { Employing Liquid Carriers }\end{array}$ \\
\hline Appendix A & Pages 239-256 & $\begin{array}{l}\text { Computational Modeling Methods and Calculation } \\
\text { of Hydrogenation Thermodynamics }\end{array}$ \\
\hline Appendix B & Pages 257-261 & General Experimental Details \\
\hline Append & Pages 262-263 & Abbreviations \\
\hline
\end{tabular}




\section{Chapter 1: Introduction}

\section{Project Background}

This is the final report for cooperative agreement DE-FC36-04G014006, "Design and Development of New Carbon-based Sorbent Systems for an Effective Containment of Hydrogen." The award from DOE was made under solicitation DE-PS36-02G092004 for the following summarized statement of work (SOW):

Task 1: Chemical sorbents for hydrogen storage. This task encompasses studies of the reversible catalytic hydrogenation of large polyaromatic hydrocarbons and graphitic carbon materials.

Task 2: Hydrogen storage by the adsorption of $\mathrm{H}_{2}$ on small-diameter, chiral, single-walled carbon nanotubes.

Task 3: Adsorption of hydrogen on "naked" fluoride salts.

As a result of our preliminary experimental findings, the cooperative agreement was amended in October 2003 to eliminate Task 2 and focus project resources on Task 1. Within Task 1, the types of chemical sorbents under consideration were narrowed by a down-selection process to place the primary project focus on liquid polyaromatic compounds ${ }^{1 \mathrm{a}}$. As a result of the successful pursuit of liquid polyaromatic hydrogen carriers, including oxygen and nitrogen heterocyclic systems, Task 3 was removed from the cooperative agreement and was transferred in July 2007 to a more suitable program within the Hydrogen Sorption Center of Excellence called "Designing Microporous Carbons for Hydrogen Storage Systems" (DE-FC3605G015074).

This final report thus comprises substantially the work within Task 1 as modified in October 2003. We report briefly on the reversible hydrogenation of solid polyaromatic hydrocarbons, but the primary content is the discovery and testing of various classes of heterocyclic aromatic molecules as hydrogen-reversible liquid carriers and associated hydrogenation and dehydrogenation catalysts. 


\section{Introduction and Objectives}

Hydrogen is a large-scale chemical commodity ${ }^{1}$ that is an important feedstock for ammonia and methanol production. Hydrogen is consumed in increasing volumes in refining operations such as hydro-treating transportation fuels for sulfur removal and upgrading heavier crudes. Much of the hydrogen used at refineries is captured from the refinery operations, but gradually more and more merchant hydrogen delivered by pipeline from remote plants is employed. A smaller segment of merchant hydrogen is delivered in the form of a cryogenic liquid or compressed gas to chemical and metallurgical industry consumers.

Currently envisaged is the larger-scale use of hydrogen as an energy carrier to replace our traditional hydrocarbon fuels. This is the much-touted hydrogen economy ${ }^{2,3}$. The principal drivers for a hydrogen economy are the option of obtaining hydrogen from a variety of sources, preferably beyond reforming of fossil fuels, and the relatively higher efficiency with which the gas can be converted to electrical energy and motive power using electrochemical fuel cells and advanced internal combustion engines. The electrochemical or thermal combustion of hydrogen to produce power yields only water as a byproduct. These attractive features of a hydrogen fuel infrastructure are offset by the societal cost of such a massive investment, and the safety and environmental concerns of a widespread use of hydrogen at the level of typical consumers. From a technology point of view, however, the "Grand Challenge" toward this hydrogen economy as articulated by the U.S. Department of Energy is in developing technologies for an effective production, distribution, and vehicular storage of hydrogen. Specifically, the goals for vehicular storage are related to containing hydrogen (or an equivalent $\mathrm{H}_{2}$ source) in an onboard system with gravimetric and volumetric storage densities and operational technical targets as defined in the DOE's Hydrogen and Fuel Cells R\&D Plan ${ }^{4}$.

Current prototypes of fuel cell vehicles generally store hydrogen in lightweight, high-pressure carbon-fiber composite tanks. While the approximate target of a 300 mile driving range for a fuel cell vehicle has now been met by at least two manufacturers ${ }^{5}$, the volumetric $\mathrm{H}_{2}$ storage density is far from optimal, and there are remaining concerns regarding the safety of extremely high storage pressures of gas (up to 700 bar) in a consumer's environment. Considerable research has been directed at finding solid-state, reversible hydrogen sorbent materials that can contain hydrogen in a more compact form without the need for high pressures, while meeting 
DOE system targets. The materials that have been widely studied include traditional metal alloy hydrides $^{6}$, complex metal hydrides which have the promise of higher gravimetric capacities ${ }^{7}$, high-surface-area materials including activated carbons, single-wall carbon nanotubes ${ }^{8}$, and metal-organic framework compositions ${ }^{9}$, sometimes in combination with catalytic metal additives $^{10}$. This is a broad and rapidly evolving area of knowledge and, at the time of this writing, there is no independently validated hydrogen sorbent/storage system that can meet the DOE targets for vehicular hydrogen storage.

We began the present work with the notion of potentially storing hydrogen via a catalytic chemical hydrogenation/dehydrogenation of pi-conjugated molecules, such as large polyaromatic hydrocarbons, as solid-state reaction media. Some solid-state, reversible hydrogen-incorporating systems were found (Chapter 2), but were not considered viable principally because of the very slow, diffusion-limited $\mathrm{H}_{2}$ sorption/desorption chemical reaction kinetics. This led to the consideration of more conventional liquid-phase hydrogenation/ dehydrogenation substrates as hydrogen carriers in the context of a "liquids" hydrogen infrastructure.

\section{Hydrogen Storage Carriers and Delivery Infrastructure}

The major focus of our hydrogen storage research was dedicated to the concept of chemically storing hydrogen in organic liquids that are easily transportable carriers for the gas. A liquidsbased hydrogen infrastructure for the storage and delivery of hydrogen to fixed sites or mobile systems is illustrated schematically in Figure 1-1. 

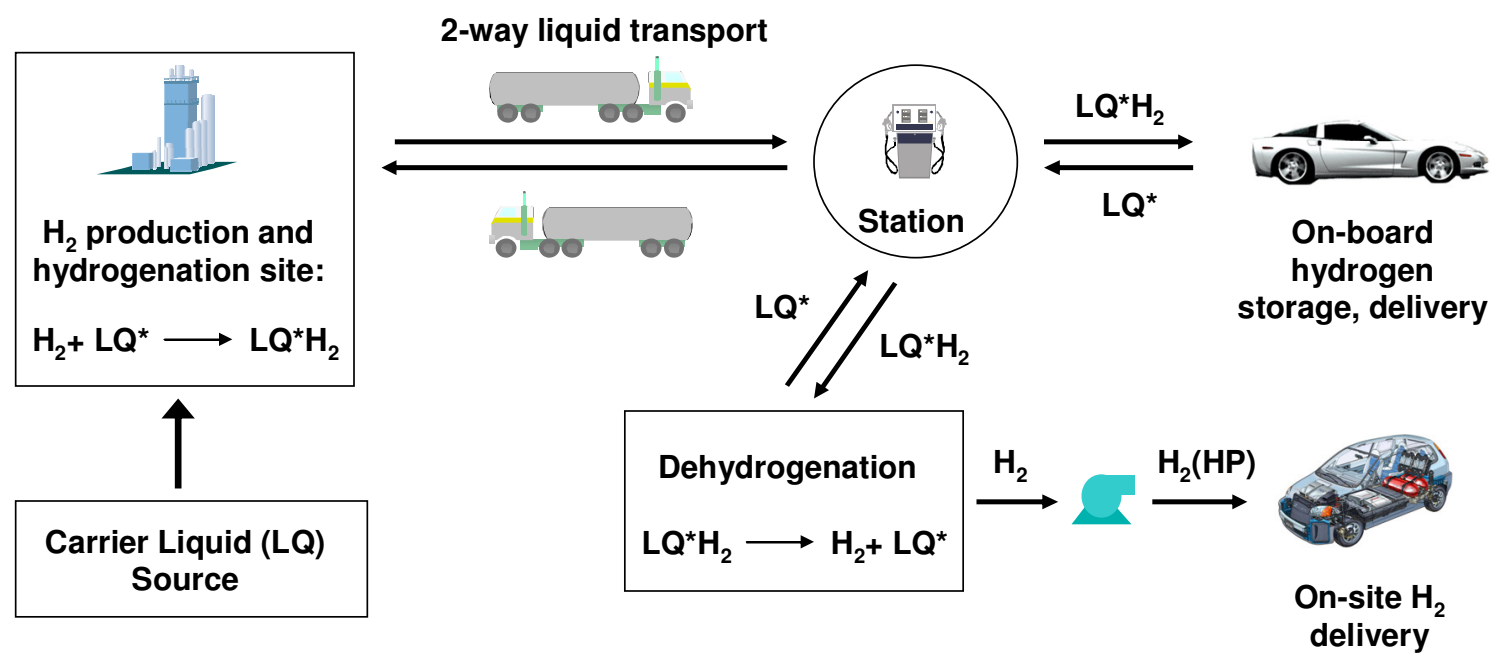

Figure 1-1. Illustration of liquids-based hydrogen storage and delivery infrastructure.

Hydrogen from sources such as conventional steam-methane reforming, ambient or hightemperature water/steam electrolysis, and "futuristic" processes such as photoelectrolysis and solar or nuclear-based water splitting using thermo-chemical cycles can be used to catalytically hydrogenate a liquid carrier molecule (LQ), where $L Q^{*} \mathrm{H}_{2}$ represents the hydrogen-loaded carrier:

$$
\mathrm{LQ}+\mathrm{H}_{2} \stackrel{\text { catalyst }}{\longrightarrow} \mathrm{LQ}^{*} \mathrm{H}_{2}+\text { heat }
$$

The hydrogenated carrier can be conveyed by pipeline, rail, or over-the-road transport to local distribution systems. At local distribution sites, such as automobile fueling stations, there are two options for the use of the hydrogenated carrier. The carrier can be catalytically dehydrogenated (the reverse of Equation 1) with an input of heat. The produced hydrogen gas can be compressed and stored for vehicular fueling as needed. The spent carrier, $L Q$, is then returned to the hydrogenation site. In the second option, the liquid carrier can be used to both store and deliver hydrogen on-board vehicles. The fueling with liquid $L Q^{*} \mathrm{H}_{2}$ is performed in a similar manner to conventional hydrocarbon fueling, except that a dual-nozzle filling device (Figure 1-2) would be used which permits a simultaneous dispensing of the hydrogenated carrier $\left(L Q^{*} H_{2}\right)$ and a withdrawal of the spent carrier (LQ). 


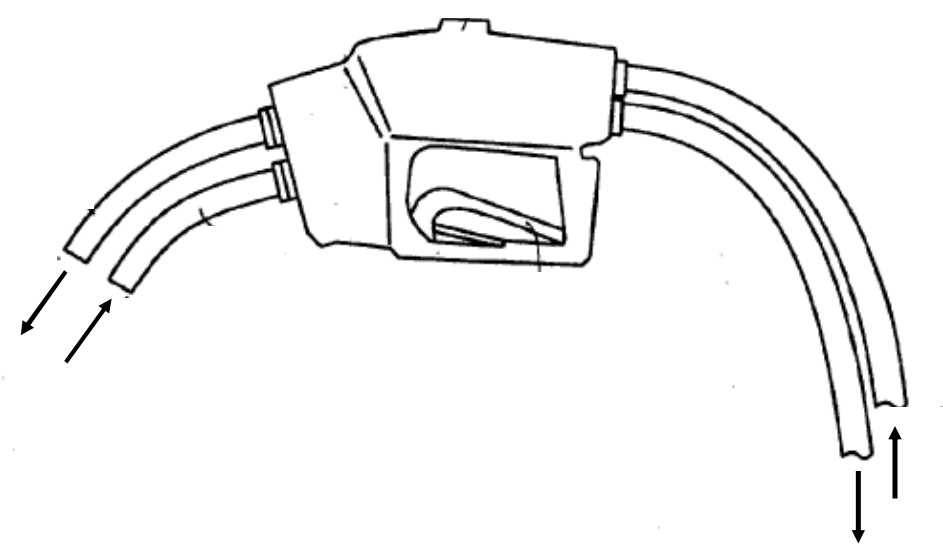

Figure 1-2. Dual nozzle device for a simultaneous fueling and recovery of liquid carrier.

In the vehicle (Figure 1-3), a tank containing a moveable partition or membrane is used to accommodate the two carrier liquids without mixing of the liquids. The total volume of the two liquids will be roughly constant throughout use, due to the similar density of hydrogenated and dehydrogenated carriers. Onboard the vehicle, the $L Q^{*} \mathrm{H}_{2}$ liquid is passed through a catalytic dehydrogenation reactor, releasing hydrogen. The hydrogen is fed to the power generation unit, which may be a fuel cell or $\mathrm{H}_{2}$ internal combustion engine. Heat is required to extract hydrogen from the carrier at the required dehydrogenation temperature. Even with a PEM fuel cell at $60 \%$ efficiency, there should be about $96 \mathrm{~kJ} /$ mole $\mathrm{H}_{2}$ of waste heat available from the power generation unit (based on the LHV for $\mathrm{H}_{2}$ ). The estimated heats of dehydrogenation, $\Delta \mathrm{H}_{d}$, of our liquid carriers range from 40-60 $\mathrm{kJ} / \mathrm{mole}_{2}$. In principle, this thermal load could be supplied from the fuel cell waste heat after accounting for the efficiency of the heat transfer, requirements for cabin heating, and other on-board energy needs. In any case, in the absence of other heat sources the dehydrogenation temperature for the carrier would be limited to that of the rejected heat from the fuel cell. If the power generation unit is a PEM fuel cell, the waste heat will be available at a temperature somewhat less than the fuel cell operating temperature (currently $\left.\sim 80^{\circ} \mathrm{C}\right)$. Higher temperature waste heat $\left(\sim 120^{\circ} \mathrm{C}\right)$ may be available from advanced PEM fuel cells that are currently in development. Very high waste heat temperatures should be available from hydrogen IC engines. 


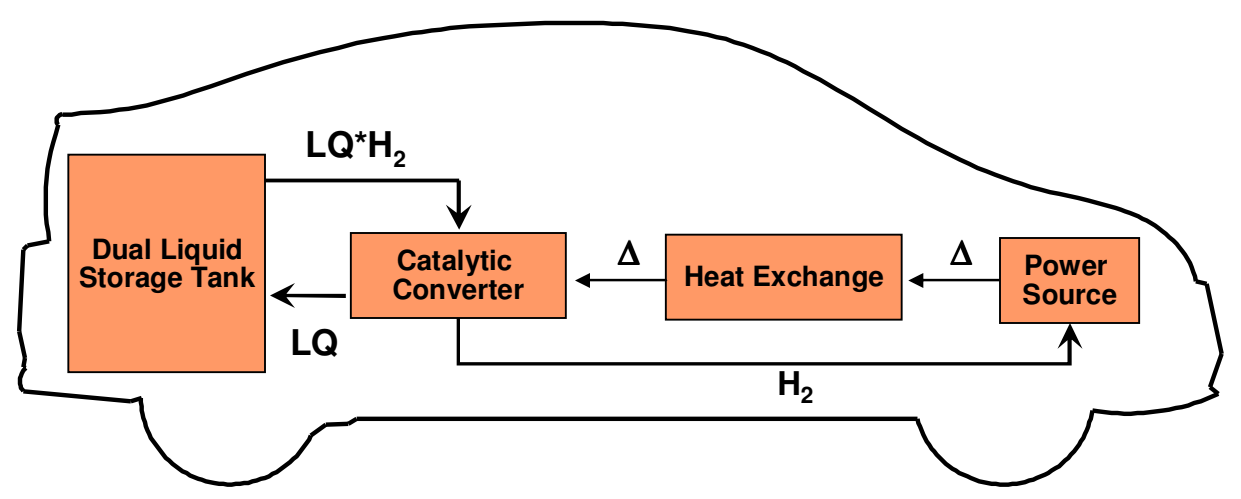

Figure 1-3. Schematic of vehicle with dual tank, catalytic reactor, heat exchange unit, and power source.

Overall, the most energy-efficient use of the carrier would be realized when the hydrogenation reaction exotherm (Equation 1) can be exploited as useful process heat (e.g., for low-pressure steam generation), and the dehydrogenation is driven by rejected waste heat from the power source.

The research detailed in this report was aimed at the discovery of $\mathrm{H}_{2}$-reversible liquid carriers and the operative catalytic hydrogenation/dehydrogenation conditions that would meet the DOE hydrogen storage and delivery targets, in the context of a "non- $\mathrm{H}_{2}$-gas" hydrogen infrastructure (Figure 1-1). We searched for unsaturated organic molecules that could undergo a very selective catalytic hydrogenation with the addition of at least 1 hydrogen atom per carbon or light $(\mathrm{O}, \mathrm{N}, \mathrm{B})$ element atom. The desired molecules would undergo a corresponding catalytic dehydrogenation, with $\mathrm{H}_{2}$ release occurring at modest temperatures, preferably $<80^{\circ} \mathrm{C}$. Other important discerning factors were the carriers' melting point, vapor pressure, and EH\&S profile.

\section{Literature Background}

The general concept of storing hydrogen via a reversible hydrogenation of organic compounds (in a liquid or gaseous phase) has been known for several decades. ${ }^{11}$ The concept has been developed using benzene, toluene, and naphthalene as hydrogenation substrates. The corresponding saturated molecules, cyclohexane, methylcyclohexane, and decalin (which have covalent $\mathrm{C}-\mathrm{H}$ linkages) have been described in the literature as "organic hydrides" or "liquid hydrides." In contrast to the classical hydrogen-reactive metal-alloy hydrides (such as FeTi, 
$\mathrm{LaNi}_{5}$, etc. $)^{6}$, the organic compounds can display a reversible reactivity with hydrogen only in the presence of suitable metal catalysts. The hydrogenation of aromatic molecules is an exothermic and thermodynamically spontaneous process which allows high conversions to the cycloalkanes under relatively moderate conditions $\left(100-200^{\circ} \mathrm{C}, 10-50 \mathrm{~atm} . \mathrm{H}_{2}\right)$. However, the reverse process, selective dehydrogenation of cycloalkanes, is correspondingly unfavorable and is only possible with an input of heat (dehydrogenation enthalpy, $\Delta \mathrm{H}_{\mathrm{D}}$ ) at higher temperatures (generally $>300^{\circ} \mathrm{C}$ ), where the process is largely entropy-driven by the release of hydrogen gas. The most recent investigations of hydrogen storage with organic liquids have mainly employed the naphthalene/decalin system with an emphasis on devising reactor systems ${ }^{12}$ which can minimize the effect of the thermodynamic barrier, thus permitting a partial release of the stored hydrogen at lower temperatures $\left(<300^{\circ} \mathrm{C}\right)$. There has been additional focus on developing more active dehydrogenation catalysts for enhanced dehydrogenation rates while maintaining a high reaction selectivity. ${ }^{13}$

Our approach toward more practical, lower operating temperatures has been to search for new organic liquid dehydrogenation/hydrogenation "couples" that inherently have a lower $\Delta H_{D}$ barrier. The novel concepts are the subject of three US patents and two pending patent applications. ${ }^{14}$ Recently, several publications ${ }^{15-19}$ on organic liquid carriers have appeared which have extended the field by building on our contributions, as documented in the patent disclosures, with due acknowledgement.

In parallel with the discovery work on liquid carriers, we have pursued a complementary DOE-funded hydrogen delivery project ${ }^{20}$ with the goal of designing and developing a prototype dehydrogenation reactor which will provide a 0.1 to $0.5 \mathrm{~kW}$ supply of hydrogen.

\section{Technical Approaches}

\section{Thermodynamic Considerations}

Having recognized the need for high temperature conditions and reaction catalyst requirements for a practical selective dehydrogenation of the cyclic alkanes, $\mathrm{C}_{6} \mathrm{H}_{12}, \mathrm{C}_{6} \mathrm{H}_{11} \mathrm{CH}_{3}$, and $\mathrm{C}_{10} \mathrm{H}_{18}$, we sought alternative reaction systems where the release of hydrogen could occur at much lower temperatures and close to ambient pressures. Fundamentally, this search was directed at identifying perhydrogenated aromatic/aromatic molecule couples, or in more general terms, 
hydrogenated/dehydrogenated $\pi$-conjugated systems. These systems should have a Gibbsfree energy for dehydrogenation, $\Delta G_{D}$, appreciably less than that for the cyclic alkanes under the same conditions.

In Equation 2, $\Delta S_{D}$ represents the increase in entropy of the system from dehydrogenation of hydrogenated carrier and is mostly reflective of the change of state from bound hydrogen (as $\mathrm{C}-\mathrm{H}$ bonds) to free, gas-phase hydrogen. Thus, the $\Delta \mathrm{S}_{\mathrm{D}}$ is close to the standard entropy for $\mathrm{H}_{2}$, which is $130 \mathrm{~J} / \mathrm{deg}$ mole, varying only slightly with respect to the other system components.

$$
\Delta G_{D}=\Delta H_{D}-T \Delta S_{D}
$$

The quantity that most determines the dehydrogenation temperature is the reaction dehydrogenation enthalpy, $H_{D}$, which needs to be at least lower than those for cyclohexane, methylcyclohexane, and decalin, the principal liquid carriers of the prior art. However, $\Delta \mathrm{H}_{\mathrm{D}}$ must not be so low as to preclude a substantial re-hydrogenation of the "spent" carrier (not less than ca. $\left.40 \mathrm{~kJ} / \mathrm{mole}_{2}\right){ }^{21}$ The experimentally determined average $\Delta \mathrm{H}_{\mathrm{D}}$ value for the cyclohexane/ benzene couple, $69 \mathrm{~kJ} / \mathrm{mole}_{2}$, is far lower than that for a dehydrogenation of cyclohexene to cyclohexane, $118 \mathrm{~kJ} /$ mole $_{2}$. This is a well understood consequence of the very significant aromatic stabilization energy of benzene $\left(3 \times 118-3 \times 69=149 \mathrm{~kJ} / \mathrm{mole}_{2}\right)$. We therefore investigated by computational modeling a broad spectrum of saturated/unsaturated aromatic molecule pairs with the notion that the use of aromatic or, in more general terms, highly conjugated systems to stabilize the dehydrogenated state could lead to the desirably lower reaction enthalpy.

\section{a. Polyaromatic Hydrocarbons}

We first examined polyaromatic hydrocarbon systems, calculating $\Delta \mathrm{H}_{\mathrm{D}}{ }^{\circ}$ for a wide range of perhydroaromatic/aromatic molecule pairs. The results of this initial computational survey using the PM3 semi-empirical computational method are shown in Figure 1-4 where $\Delta H_{D}{ }^{\circ}$ is plotted as a function of the number of rings in the respective molecules. For the poly(1,2-acene) series of Curve I, $\Delta \mathrm{H}_{\mathrm{D}}{ }^{\circ}$ becomes more favorable in going from benzene to naphthalene, and then increases as more rings are added. In contrast, for phenanthrene-based systems (Curve II), $\Delta \mathrm{H}_{\mathrm{D}}{ }^{\circ}$ is not only more favorable, but there is a continuing improvement with increasing molecular size. Starting with pyrene $\left(\mathrm{C}_{16} \mathrm{H}_{10}\right)$, coronene $\left(\mathrm{C}_{24} \mathrm{H}_{12}\right)$, and ultimately towards 
graphene, fused polyaromatic compounds having a roughly circular shape (Curve III) are observed to have the lowest heats of dehydrogenation in our calculations.

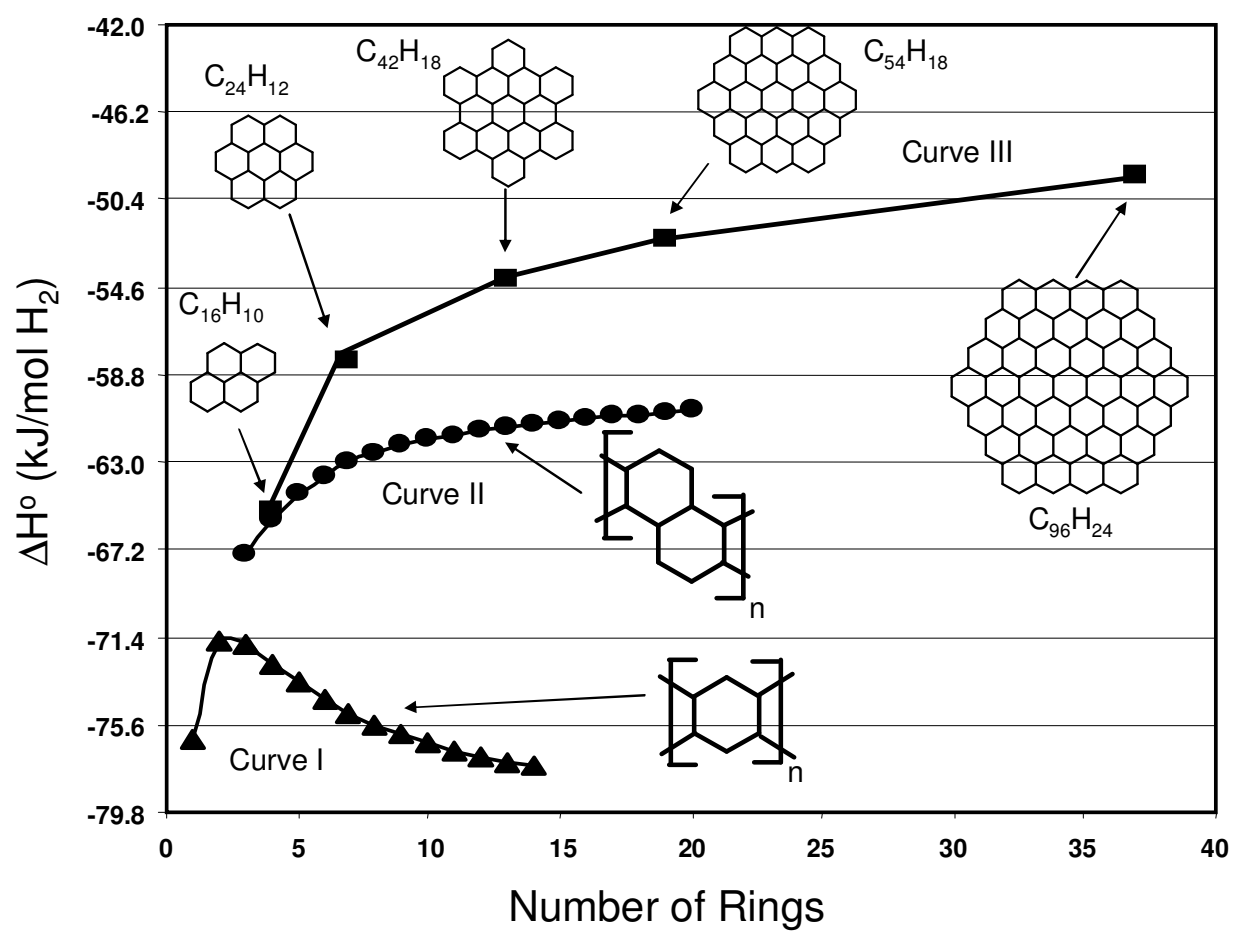

Figure 1-4. Calculated dehydrogenation enthalpy, $\Delta \mathrm{H}_{\mathrm{D}}{ }^{\circ}\left(\mathrm{kJ} / \mathrm{mole}_{2}\right)$, for three series of polyaromatic hydrocarbons as a function of the number of rings. The PM3 semi-empirical method was used for the calculations.

Clar first proposed that the aromatic stabilization energy of polyaromatic hydrocarbons is determined by the relative number of aromatic sextet structures in the molecules. ${ }^{21}$ Most of the data in Figure 1-4 can be explained on this basis. Molecules for which the maximum number of aromatic sextets can be drawn (Curve III) are expected to have the most favorable heat of dehydrogenation. For the naphthalene, anthracene, tetracene, etc. series (Figure 1-5), for which the aromatic sextet is shared among all of the rings, $\Delta \mathrm{H}_{\mathrm{D}}{ }^{\circ}$ is, by Clar's rule, ${ }^{22}$ expected to be increasingly unfavorable, as shown in Curve I of Figure 1-4.<smiles>c1ccc2ccccc2c1</smiles><smiles>c1ccc2cc3ccccc3cc2c1</smiles><smiles>c1ccc2cc3cc4ccccc4cc3cc2c1</smiles>

Figure 1-5. Molecular structures of naphthalene, anthracene, and tetracene. 
However, the latter point begs the question as to why $\Delta \mathrm{H}_{\mathrm{D}}{ }^{\circ}$ should be more favorable for naphthalene than benzene, the "ideal sextet". ${ }^{22}$ Our hypothesis is that in decahydronaphthalene there is in conjunction with the fusion of the two rings a small amount of strain which leads to an increase in the standard enthalpy of the molecule and hence a lower $\Delta \mathrm{H}^{\circ}$ for the dehydrogenation. This was a valuable hint of the possibility of diminishing $\Delta \mathrm{H}_{D}{ }^{\circ}$ by altering the energetics of the perhydrogenated molecule (vide infra).

\section{b. Molecules with Nitrogen Heteroatoms}

In view of the lower average $\Delta \mathrm{H}_{\mathrm{D}}{ }^{\circ}$ for the dehydrogenation of piperidine to pyridine $(63 \mathrm{~kJ} / \mathrm{mole}$ $\mathrm{H}_{2}$ ) as compared to $\Delta \mathrm{H}_{\mathrm{D}}{ }^{\circ}$ for cyclohexane to benzene $\left(69 \mathrm{~kJ} / \mathrm{mole}_{2}\right)$, we decided to broadly scope the effect of nitrogen atom substitution for a series of polycyclic molecules using the PM3 computational method. Figure 1-6 shows the variation of $\Delta \mathrm{H}_{\mathrm{D}}{ }^{\circ}$ with molecular size for fused $\mathrm{N}$ heterocycles vis-a-vis their hydrocarbon counterparts.

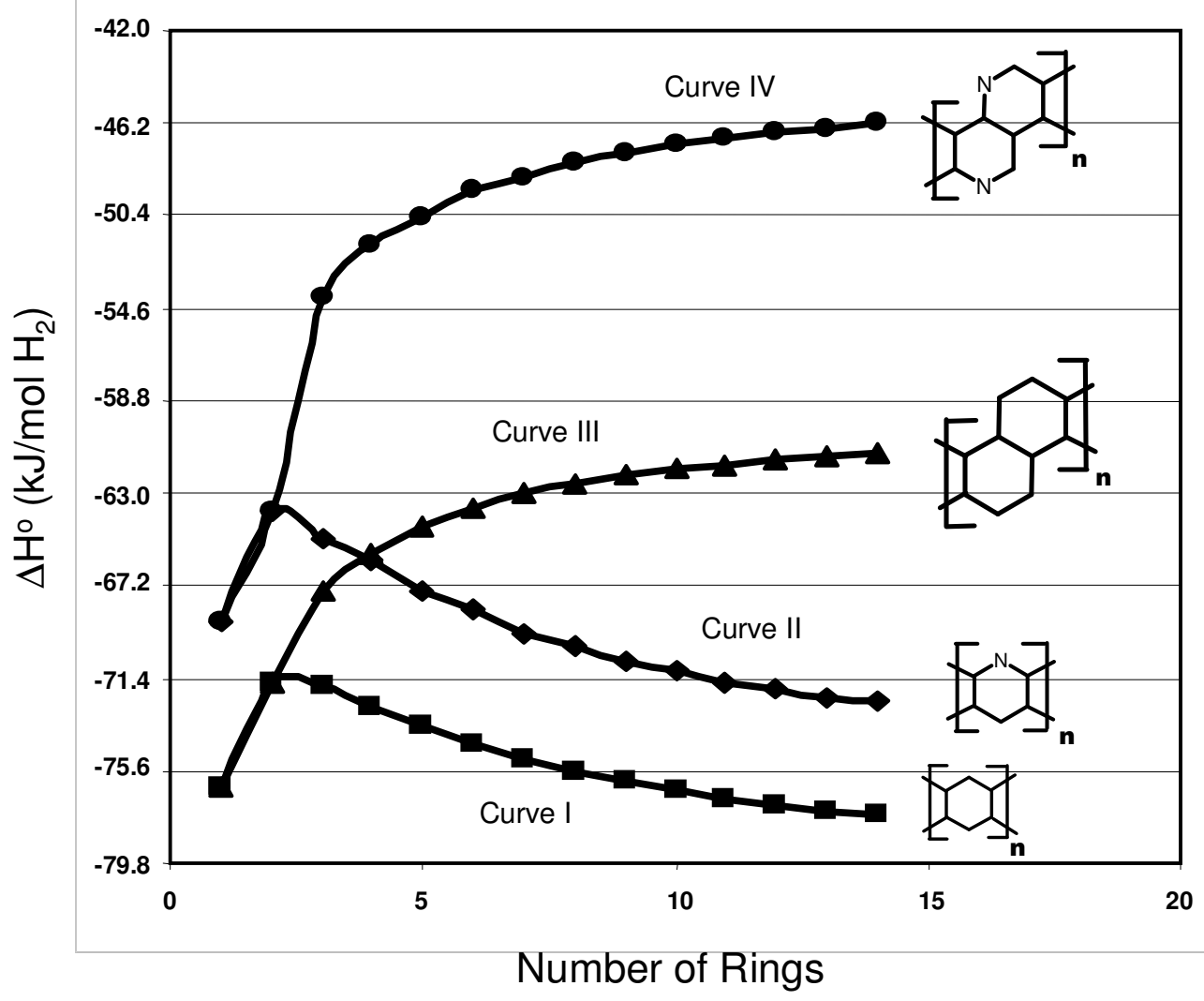

Figure 1-6. Calculated dehydrogenation enthalpy, $\Delta \mathrm{H}_{\mathrm{D}}{ }^{\circ}\left(\mathrm{kJ} / \mathrm{mole}_{2}\right)$ for $\mathrm{N}$-heterocycles and the corresponding polyaromatic hydrocarbons (PM3 method). 
There is a clear and significant lowering of $\Delta \mathrm{H}_{\mathrm{D}}{ }^{\circ}$ with substitutional incorporation of nitrogen, which once again depends on the overall "shape" of the molecule. The generally beneficial effect of nitrogen substitution in polycyclic aromatics with retention of the aromatic sextet structure was validated using the more precise DFT calculation method (vide infra) for a large number of hydrogenated/dehydrogenated $\mathrm{N}$-heterocyclic molecules.

\section{c. Polyaromatic Hydrocarbons Comprising 5-Membered Rings}

We found that, surprisingly, the $\Delta \mathrm{H}_{\mathrm{D}}{ }^{\circ}$ of fused polycyclic hydrocarbons is significantly lower for structures that incorporate 5 -membered rings. This is clearly seen in a comparison of the calculated average $\Delta H_{D}{ }^{\circ}$ for anthracene $\left(-66 \mathrm{~kJ} / \mathrm{mole}_{2}\right)$ and fluorene $\left(-62 \mathrm{~kJ} / \mathrm{mole}_{2}\right)$ using the DFT method. Both molecules have three rings and two ring junctions, but the influence of the central 5-membered ring of fluorene on the $\Delta \mathrm{H}_{\mathrm{D}}{ }^{\circ}$ is substantial.

\section{d. Oxygen Containing Molecules}

The relatively facile catalytic dehydrogenation of isopropanol to acetone is well known. This system has been proposed for hydrogen storage, but its theoretical gravimetric capacity (3.3 wt. \%) is relatively low. In our survey of liquid carrier candidates, we considered polyaromatic ketones and ethers. A computational survey is provided in Table 1a, Appendix A.

\section{e. N-Heterocyclics Comprising Structures with 5-Membered Rings}

This subset of liquid carriers combine features from categories $a, b$ and $c$ in the current list. Aromatic molecules comprising of five and six-membered rings with one or more $\mathrm{N}$-heteroatoms provided the most fruitful source of potentially usefully lower $\Delta \mathrm{H}_{D}{ }^{\circ}$ hydrogenation "redox" pairs (Table 1b, Appendix A).

\section{f. Amines to Nitriles: Candidates for a $>1: 1 \mathrm{H}: \mathrm{C}$ Hydrogen Storage}

The catalytic hydrogenation of alkyl and aromatic nitriles to primary amines is well known. However, there is only scant literature relating to the reverse process, the selective 
dehydrogenation of the amines. This reaction could in principle allow a greater than 1:1 H: C hydrogen storage capacity for the liquid carrier. The thermodynamic barrier for catalytically releasing hydrogen from amines is greater than that for cyclohexane to benzene $\left(\Delta \mathrm{H}_{\mathrm{D}}>67 \mathrm{~kJ} / \mathrm{mole} \mathrm{H}_{2}\right.$, see Table $1 \mathrm{~d}$, Appendix A). Nevertheless, we investigated primary amine/nitrile pair molecules for hydrogen storage with the promise of higher capacities, while recognizing the need for generally high dehydrogenation temperatures.

\section{Effect of Isomers}

The $\Delta H_{D}{ }^{\circ}$ values are routinely estimated using the minimum energy configuration of the respective molecule pairs, considering all possible stereoisomers. For the dehydrogenated aromatic molecules, which are overridingly planar, finding the most stable configuration is usually relatively straightforward. On the other hand, with perhydrogenated, fused cyclic molecules, there can be the possibility of various combinations of cis and trans isomers, depending on the number of ring junctions. For amines, there are $\mathrm{N}$ atom "in and out" isomers, as well as potential conformers. Cis-trans isomerism in fused ring systems, exemplified in Figure 1-7 by the respective isomers of decahydronaphthalene (decalin), is in our context a potentially very useful molecular property. If the higher energy $\left(\mathrm{H}_{\mathrm{f}}{ }^{\circ}\right)$ isomer can be selectively prepared by a catalytic hydrogenation of the corresponding aromatic compound, then effectively, a lowering in $\Delta \mathrm{H}_{\mathrm{D}}{ }^{\circ}$ is realized. Thus, for the more energetic cis-decalin isomer $(13 \mathrm{~kJ} / \mathrm{mole})$, which can be prepared by a shape-selective catalytic hydrogenation of naphthalene ${ }^{23}$, there is a $4 \%$ decrease in $\Delta H_{D}{ }^{\circ}$ which corresponds to a significant ca. $20^{\circ} \mathrm{C}$ reduction in the dehydrogenation temperature (Table 1a in Appendix $A$ ). In the course of our work we have advantageously exploited the use of cis-trans isomerism associated with fused ring systems for a more facile dehydrogenation of liquid carriers. 


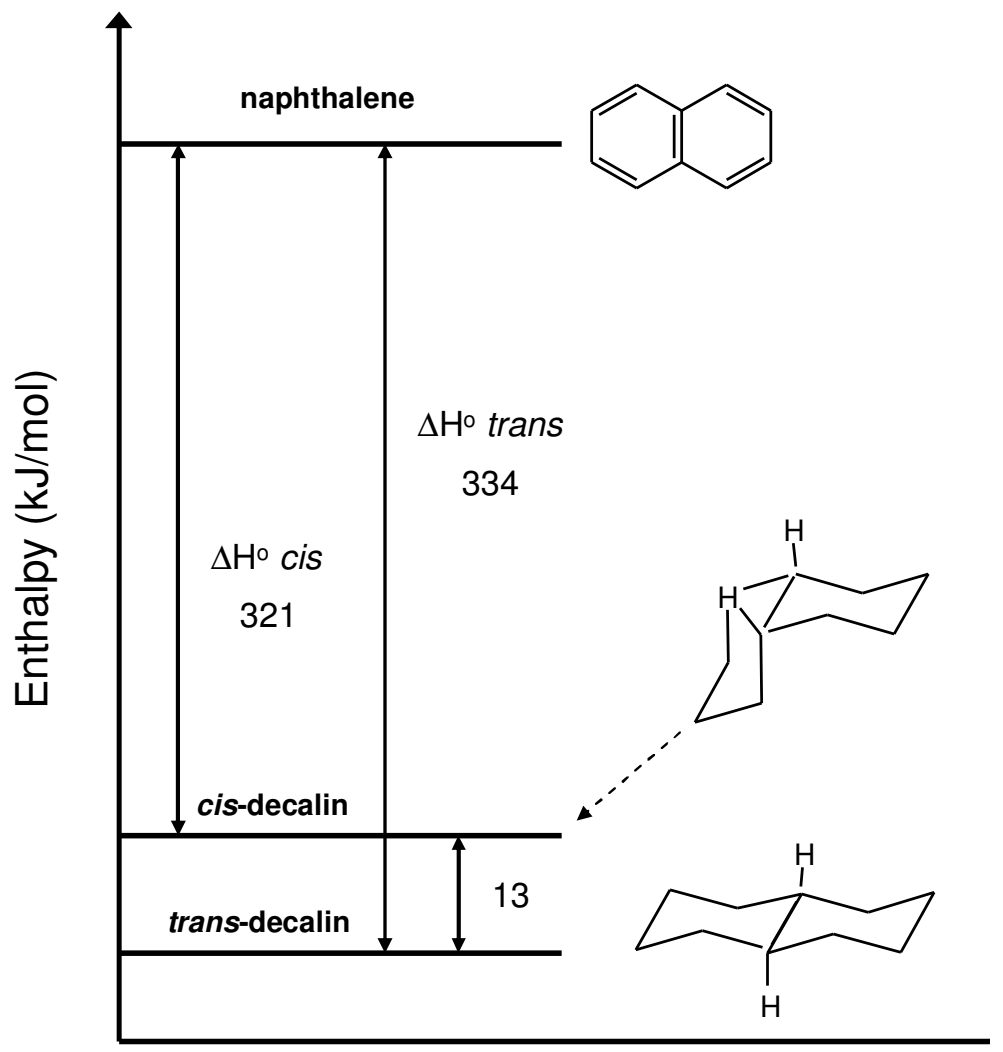

Figure 1-7. Cis-trans isomerism in naphthalene and the relative energetics.

\section{Genesis of Lower Carrier Dehydrogenation Enthalpies $\left(\Delta H_{D}\right)$}

As discussed, the utility of previously investigated liquid carriers, of which cyclohexane/benzene is a base case, is principally limited by the elevated temperatures required for dehydrogenation. The most temperature-determining factor is the endothermic dehydrogenation enthalpy, $\Delta \mathrm{H}_{\mathrm{D}}{ }^{\circ}$. Since $\Delta H_{D}{ }^{\circ}$ is the difference between the respective standard heats of formation, $\mathrm{H}_{\mathrm{f}}{ }^{\circ}$, of the hydrogenated/dehydrogenated molecule pairs, we searched for systems where the hydrogenated carrier is rendered more energetic (higher $\mathrm{H}_{\mathrm{f}}^{\circ}$ ), while the corresponding unsaturated molecule is relatively stabilized (lower $\mathrm{H}_{\mathrm{f}}{ }^{\circ}$ ). This required a study of the potential energetics of the individual hydrogenated and dehydrogenated forms of the carrier. 


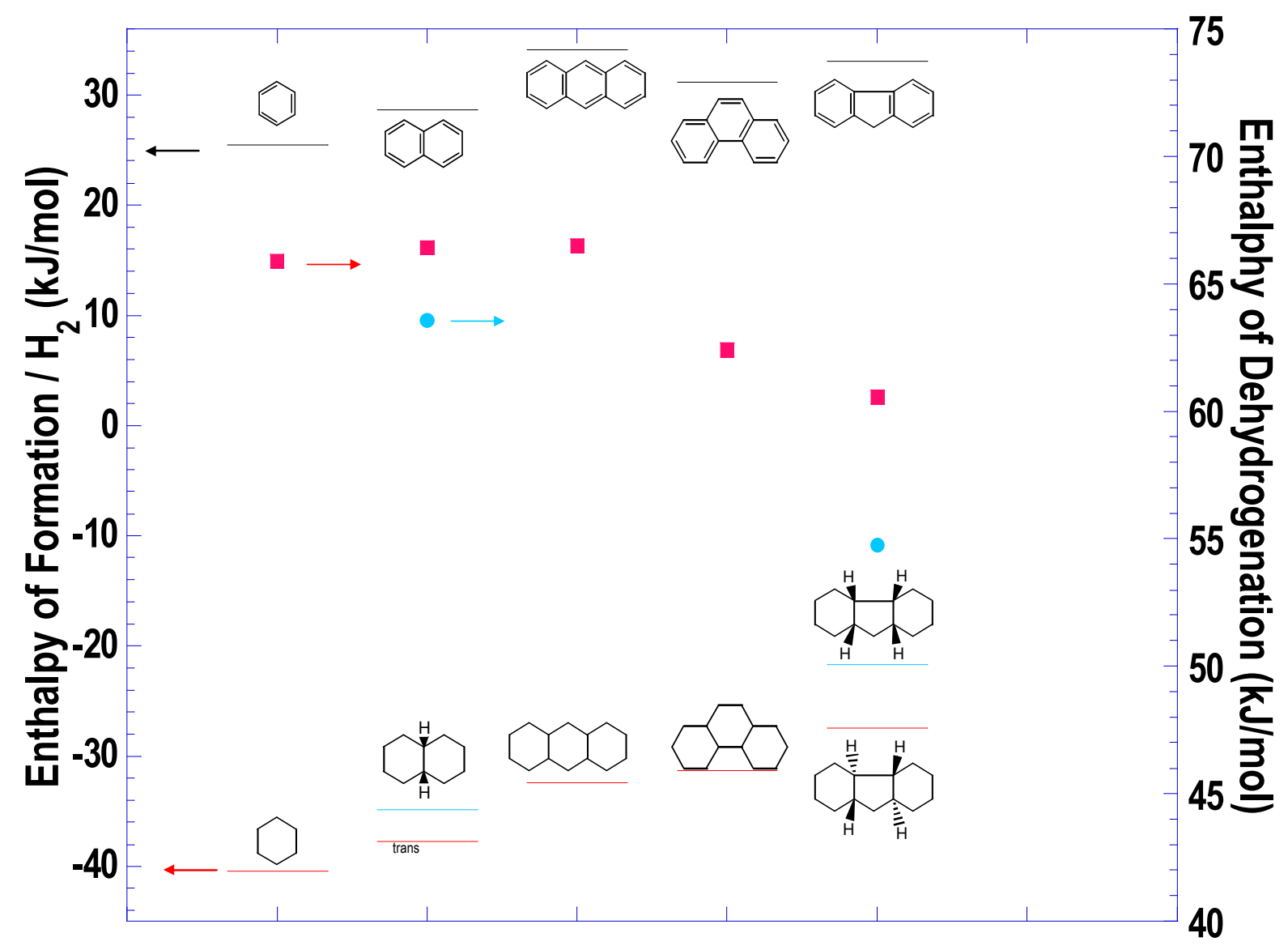

Figure 1-8. Standard enthalpies of formation, $\mathrm{H}_{\mathrm{D}}{ }^{\circ}$ and standard dehydrogenation enthalpies, $\Delta \mathrm{H}_{\mathrm{D}}{ }^{\circ}$, of aromatic and cycloaliphatic molecule pairs.

In Figures 1-8 and 1-9 our approach is quantitatively illustrated for a series of aromatic hydrocarbons and the corresponding nitrogen-containing heterocyclic molecules. All enthalpies are gas-phase values (calculated as described in Appendix A) and are expressed as $\mathrm{kJ} / \mathrm{mole}$ $\mathrm{H}_{2}$, assuming one $\mathrm{H}_{2}$ molecule per double bond of the aromatic moiety. For the cyclic hydrocarbons (Figure 1-8), there is a gradual lowering of $\Delta H_{D}{ }^{\circ}$ for the series of cyclohexane, cis-decalin, perhydrophenanthrene and perhydrofluorene. The underlying contributions to this trend are revealed by the respective $\mathrm{H}_{\mathrm{f}}{ }^{\circ}$ values of the hydrogenated/dehydrogenated molecule pairs. For the series of aromatics, benzene, with its ideal sextet, has the lowest $\mathrm{H}_{f}{ }^{\circ}$. The enthalpy of formation is higher for the naphthalene and anthracene polyene structures for which, as noted by Clar, ${ }^{22}$ the aromatic sextet is "diluted" among several "linear" polyene structures. 
Also apparent in Figure 1-8, the aromaticity is greater for multiply fused ring systems, and thus phenanthrene (two aromatic sextets for 3 rings) is more stable than anthracene (one aromatic sextet for 3 rings).

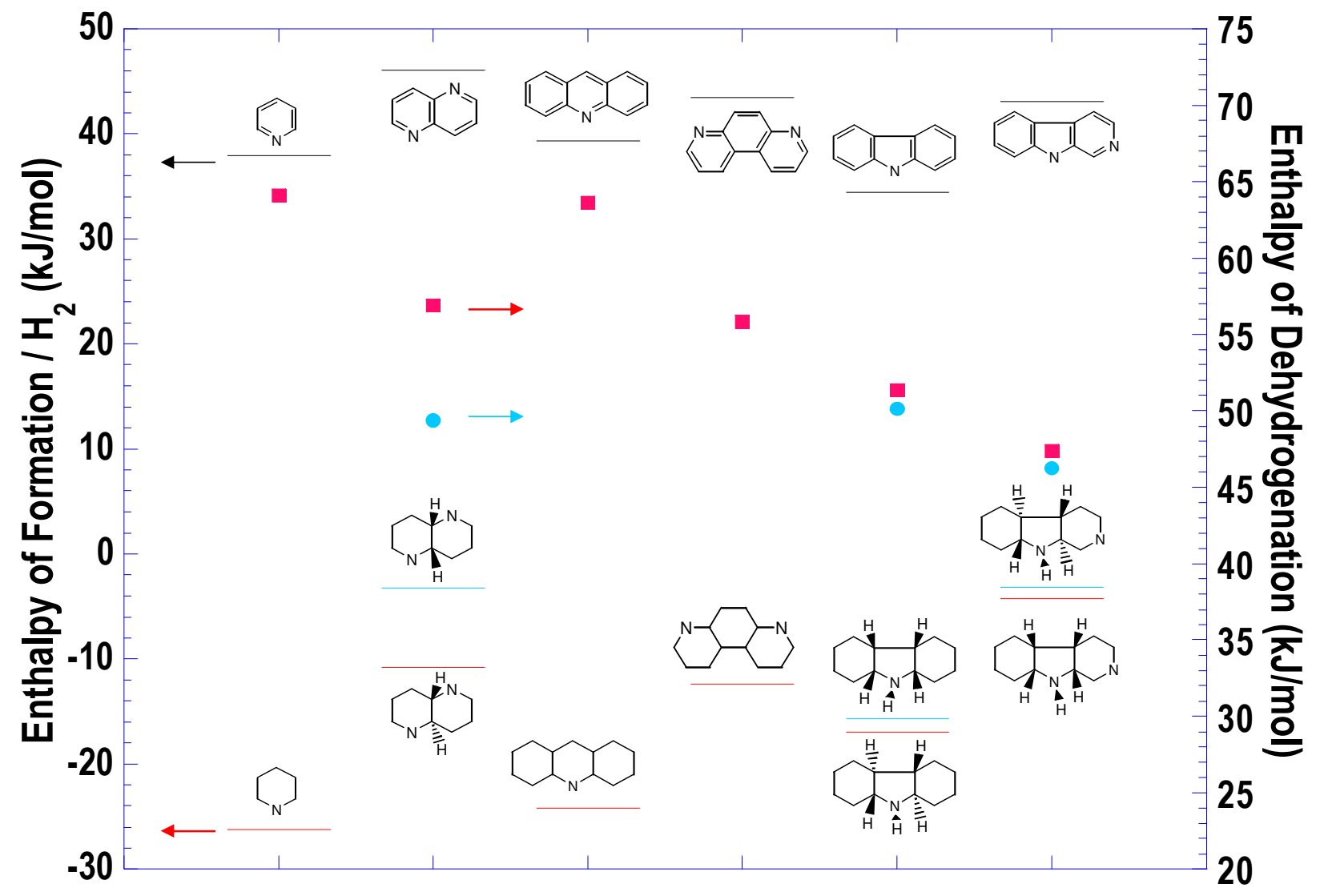

Figure 1-9. Standard enthalpies of formation, $\mathrm{H}_{\mathrm{f}}{ }^{\circ}$ and standard dehydrogenation enthalpies, $\Delta \mathrm{H}_{\mathrm{D}}{ }^{\circ},\left(\mathrm{kcal} / \mathrm{mole}_{2}\right)$ of $\mathrm{N}$-heterocyclic aromatic and cycloaliphatic molecule pairs.

For the series of saturated cyclic hydrocarbons, there is an apparent rise in $\mathrm{H}_{\mathrm{f}}{ }^{\circ}$ with the formation of multiple ring systems, presumably due to strain, and this is particularly evident for molecules containing 5-membered rings ( $\mathrm{cf} . \mathrm{H}_{\mathrm{f}}{ }^{\circ}$ for perhydroanthracene and perhydrofluorene). Also evident is the above-discussed advantage of cis-structural isomers for lowering $\Delta \mathrm{H}_{\mathrm{D}}{ }^{\circ}$. It is noteworthy that these trends and variations in the standard enthalpy of formation of the saturated cyclic hydrocarbons represent the dominating contributions to a favorable lowering $\Delta \mathrm{H}_{\mathrm{D}}{ }^{\circ}$ for some of the hydrogenated/dehydrogenated molecule pairs. 
Figure 1-9 displays the heat of formation, $H_{f}^{\circ}$, and dehydrogenation enthalpy, $\Delta H_{D}^{\circ}$, for the corresponding nitrogen-containing compounds (pyridine, polyaromatic $\mathrm{N}$-heterocycles and their saturated counterparts). For the series of aromatic $\mathrm{N}$-heterocycles, there is no clear trend in $\mathrm{H}_{\mathrm{f}}{ }^{\circ}$ except to note that (in this limited sample) the values are a little higher for the molecules that contain two nitrogen atoms. On the other hand, $\mathrm{H}_{\mathrm{f}}{ }^{\circ}$ varies greatly over the series of corresponding cyclic aliphatic amines and is, once again, the dominant factor (as illustrated in Figure 1-9) in the observed favorable trend toward decreasing dehydrogenation enthalpies.

From a comparison of $\Delta \mathrm{H}_{\mathrm{D}}{ }^{\circ}$ data for the hydrocarbons (Figure 1-8) and corresponding $\mathrm{N}$-heterocyclic molecules (Figure 1-9), it is evident that substitution of nitrogen for carbon consistently results in lower values of the dehydrogenation enthalpy (as was also seen from the PM3 calculations in Figure 1-6). While it is tempting to propose that this is due to an increased aromatic stabilization energy (e.g., pyridine vs. benzene), such a contrast is not supported from a review of the relevant literature. ${ }^{24}$ As suggested by Crabtree et al. ${ }^{16}$ the favorable effect of incorporating nitrogen more likely stems from the well-known weakening of the $\mathrm{C}-\mathrm{H}$ bonds of carbon atoms which are adjacent to nitrogen in the organic amines ${ }^{25-27}$, thus facilitating the overall dehydrogenation of the molecule. This reduction in $\mathrm{C}-\mathrm{H}$ bond dissociation energy (BDE) is explained by Wayner et al. ${ }^{25}$ by reference to the structures of methylamine and the methylamine radical (Figure 1-10).

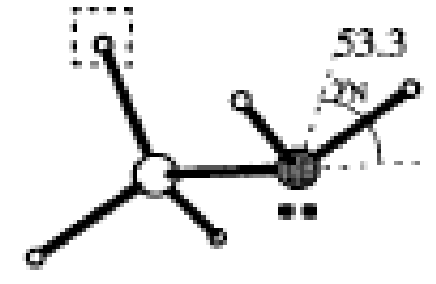

$\mathrm{CH}_{1}=\mathrm{NH}_{2}$

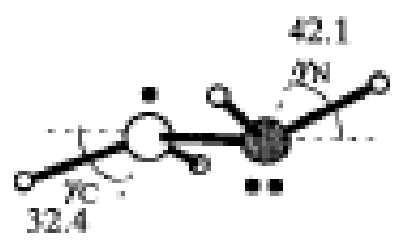

$\mathrm{CH}_{3}-\mathrm{NH}_{2}^{*}$

Figure 1-10. Structures of methylamine and the methylamine radical (reproduced from Reference 25).

For the methylamine radical, $\mathrm{CH}_{2} \mathrm{NH}_{2}{ }^{\circ}$, an interaction of the semi-occupied orbital on the carbon center with the orbital containing the lone pair on nitrogen results in a stabilization of the radical and, hence, a lowering of the homolytic $\mathrm{C}-\mathrm{H}$ bond dissociation energy. This effect is seen 
quantitatively in the calculated representative $\mathrm{C}-\mathrm{H}$ bond dissociation energies of perhydrogenated carrier molecules of our most current interest (Figure 1-11).
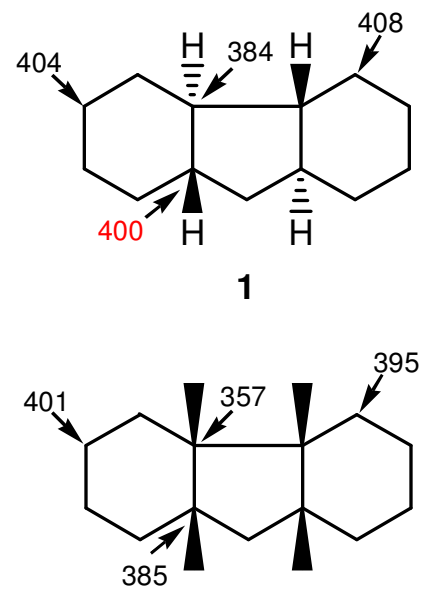

2

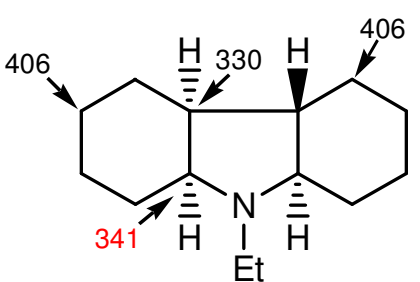

3

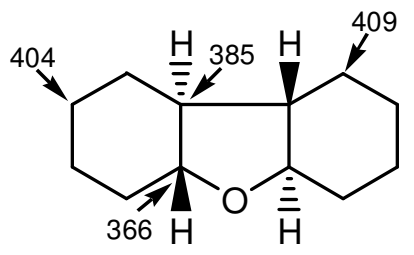

4

Figure 1-11. Calculated $\mathrm{C}-\mathrm{H}$ bond dissociation energies $(\mathrm{kJ} / \mathrm{mole})$ for trans, trans perhydrofluorene (1), cis-syn-cis-perhydrofluorene (2), perhydro-N-ethylcarbazole (3) and perhydrodibenzofuran (4). See Appendix A for details of the computational methods.

The $\mathrm{C}-\mathrm{H}$ bond dissociation energy for the carbon atom that is adjacent to the bridging methylene in perhydrofluorene (1) is dramatically lowered from $400 \mathrm{~kJ} / \mathrm{mole}$ to $341 \mathrm{~kJ} / \mathrm{mole}$ for the $\mathrm{C}-\mathrm{H}$ bond at the same site of perhydro-N-ethylcarbazole. A smaller but significant reduction from 400 to $366 \mathrm{~kJ} /$ mole for perhydrodibenzofuran is seen as a result of a similar radical stabilizing effect by the oxygen heteroatom. ${ }^{25,28}$ The other $\mathrm{C}-\mathrm{H}$ bonds at equivalent positions in molecules 1, 3 and 4 are quite comparable with $\mathrm{C}-\mathrm{H}$ bonds at bridgehead carbons displaying relatively lower BDEs as a result of ring strain. ${ }^{29}$ This effect is accentuated in the cis-syn-cis isomer of perhydrofluorene (2), for which $\mathrm{H}_{\mathrm{f}}^{\circ}$ is $5.8 \mathrm{~kJ} / \mathrm{mole} \mathrm{H}_{2}$ (or $34.8 \mathrm{~kJ} /$ molecule) higher than that for the more stable all trans perhydrofluorene (Figure 1-8).

\section{Thermodynamics of Intermediate Hydrogenation/Dehydrogenation Products}

The thermodynamic arguments thus far have only considered the relative energetics of the fully hydrogenated and dehydrogenated molecules. Catalytic hydrogenation and dehydrogenation reactions will in most cases involve a series of intermediates. The relative heats of formation of the intermediates will thus be central to facile reversibility. This is particularly important for the 
more thermodynamically demanding dehydrogenation process chemistry, as illustrated by the two sequences in Figure 1-12. Ideally, the dehydrogenation of molecule $L Q * H_{2}$ to $L Q+H_{2}$ proceeds along Path I, which involves a series of fairly regular steps of enthalpy change. In the alternate pathway, Path II, the irregular steps will lead to incomplete conversion under moderate reaction conditions, and at best only a small equilibrium amount will be evident as product $L Q$, even though the overall $\Delta H_{D}$, the sum of all step energies, is the same. The overall transformation may actually have several possible pathways; the one largely travelled will depend on the catalyst employed and reaction conditions.

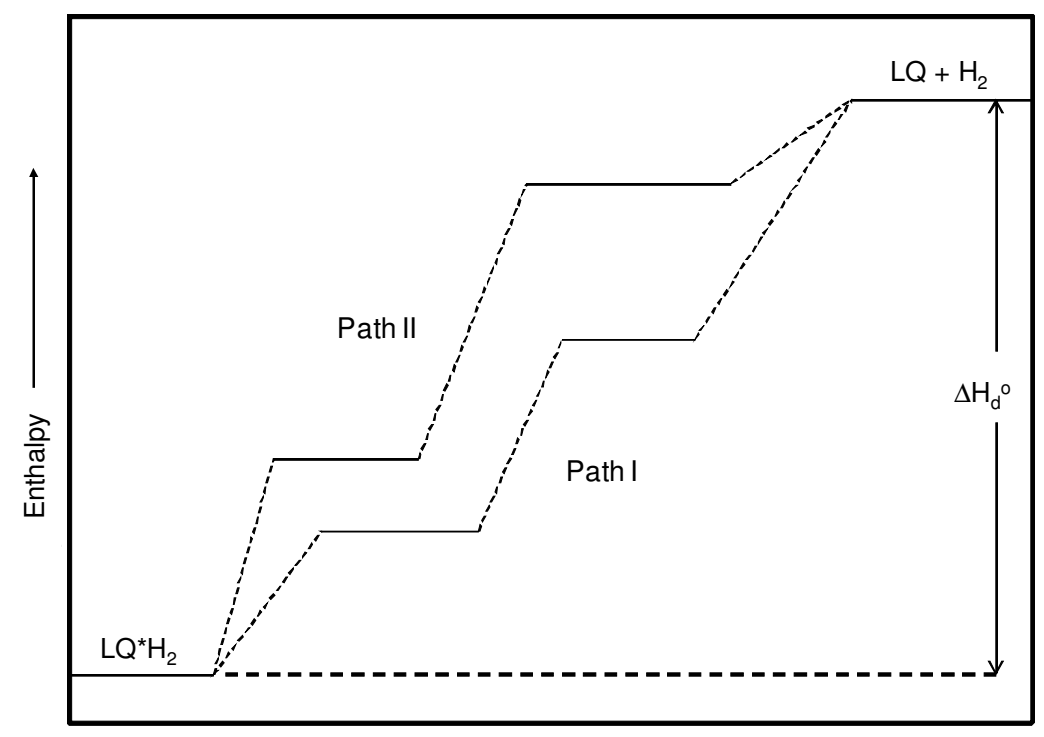

Figure 1-12. An illustration of the relative energetics of two dehydrogenation pathways.

\section{Conclusions Regarding Thermodynamics}

In conclusion, the molecular design features to consider for an optimal ( 40-55 kJ/mole $\mathrm{H}_{2}$ ) dehydrogenation enthalpy for the hydrogenated/aromatic molecular pair are:

(1) Fused ring systems containing a maximum of aromatic sextets

(2) Rings incorporating nitrogen and/or oxygen heteroatoms

(3) Structures comprising 5-membered rings

(4) Less stable structural isomers of the hydrogenated carrier

(5) The thermochemistry of the intermediate carrier hydrogenation/dehydrogenation species 


\section{Carrier Physical Properties}

Vapor pressure: b.p. $>100^{\circ} \mathrm{C}$ less than dehydrogenation temperature.

Liquidity range: Preferably down to $-30^{\circ} \mathrm{C}$.

For designing carriers having an as low as possible freezing point, we used (a) the concept of having multi-component mixtures (including having residual dehydrogenation intermediates) and (b) following Wei, ${ }^{25}$ molecules that display the lowest order of rotational symmetry. Generally, the hydrogenated form of the carrier will have the greatest liquidity (lower m.p.) because the efficient solid-state packing of aromatic molecules usually leads to higher melting temperatures. While for transient vehicular use it would seem necessary to have the dehydrogenated carrier in a liquid form, for fixed site delivery applications it should be practical to return the dehydrogenated carrier in large heated containers (as is commonly done with some hydrocarbon fuels).

\section{EH\&S and Societal Requirements}

Ideally, the liquid carrier should have properties that would make it acceptable as a chemical for introduction in society at a large scale. It should largely be insoluble in water, to avoid the ground water contamination problems as occurred with methyl tert-butyl ether (MTBE), it should not have an objectionable odor, should be thermally stable, and should be at least less toxic than gasoline.

Protocols for an evaluation of toxicity involve testing for skin irritation, and oral toxicity using animal subjects and an Ames test for mutagenity. A desirable level of thermal stability is gauged by a high flash point and only a low level exotherm for any self-heating substance. ${ }^{31}$

\section{References and Notes}

1. (a) Letter from P. A. Alba (Air Products) to D. W. Hooker, DOE Golden Field Office: Supplemental Documentation for the project "Design and Development of New Carbon Based Sorbent Systems for an Effective Containment of Hydrogen" under solicitation DE-PS36-02G092004.

(b) Letter from R. Mazakas (Air Products) to Jesse Adams, DOE Golden Field Office: Remove Scope from the project "Design and Development of New Carbon-Based 
Sorbent Systems for an Effective Containment of Hydrogen" - DOE Instrument \# DE-FC36-04G014006.

(c) The world consumption of on-purpose $\mathrm{H}_{2}$ estimates for 2006, according to the SRI Consulting Chemical Economics Handbook, was about 22.1 trillion standard cubic feet or about 58 million metric tons. Source: SRI Consulting PEP Report 32C, "Hydrogen Production“ by Syed Naqvi, September 2007.

2. "The Hydrogen Economy: Opportunities, Costs, Barriers and R\&D Needs (2004)," National Academy of Engineering (NAE). The National Academies Press, Washington, D.C. (www.nap.edu).

3. "A National Vision of America's Transition to a Hydrogen Economy - to 2003 and Beyond," U.S. Department of Energy, February 2002

(http://www1.eere.energy.gov/hydrogenandfuelcells/pdfs/vision_doc.pdf).

4. Hydrogen, Fuel Cells \& Infrastructure Technologies Program Multi-Year Research, Development, and Demonstration Plan: Section 3.3, Hydrogen Storage, October 2007 (http://www1.eere.energy.gov/hydrogenandfuelcells/mypp/pdfs/storage.pdf).

5. Honda's 08 FCX Clarity hydrogen fuel cell powered car is claimed to go 280 miles before refueling (http://automobiles.honda.com/fcx-clarity). Toyota has announced the development of a fuel cell hybrid vehicle with a range of about 516 miles; the $\mathrm{H}_{2}$ is stored in a $70 \mathrm{MPa}(\sim 10,000 \mathrm{psi})$ tank (http://www.greencarcongress.com/2008/06/toyotaunveils.html).

6. Mueller, W.M.; Blackledge, J.P.; Libowitz, G.G., in "Metal Hydrides", Academic Press, 1968.

7. Orimo, S.; Nakamori, Y.; Eliseo, J. R.; Züttel, A.; Jensen, C. M. Chem. Rev. 2007, 107, 4111.

8. Dillon, A.C.; Heben, M.J. Applied Physics A. (Materials and Processing) 2001, A72, 133.

9. Collins, D.J.; Zhou, H.-C. J. Mater. Chem. 2007, 17, 3154.

10. Li, Y.; Yang, R.T J. Am. Chem. Soc. 2006, 128, 8136.

11. (a) The concept of a mobile storage of hydrogen, "A system of hydrogen powered vehicles using organic liquid hydrides," was described in 1981 by $M$. Taube of the Swiss Institute of Reactor Research: EIR-Ber (1981), (436), CAN 96:38328; CAN 98:75373. A toluene/methylcyclohexane system was employed; with a dehydrogenation of the latter performed at $>400^{\circ} \mathrm{C}, 20$ bar $\mathrm{H}_{2}$ pressure. The work culminated in the development and demonstration of a prototype truck powered by a $150 \mathrm{~kW}$ (mech) hydrogen burning engine; with the $\mathrm{H}_{2}$ fuel being supplied by a continuous dehydrogenation of methyl cyclohexane aboard the truck: Taube, M.; Rippin, D.; Knecht, W.; Hakimifarod, B.; Milisavljevic, B.; Gruenenfelder, N. Int. J. Hydrogen Energy 1985, 10, 595.

(b) At about the same time, G. Belanger of the Inst. Rech. D'Hydra-Quebec prepared a $\mathrm{H}_{2}$ storage scheme using the $\mathrm{C}_{6} \mathrm{H}_{6} / \mathrm{C}_{6} \mathrm{H}_{12}$ and $\mathrm{C}_{6} \mathrm{H}_{5} \mathrm{CH}_{3} / \mathrm{C}_{6} \mathrm{H}_{11} \mathrm{CH}_{3}$ "liquid hydride" 
hydrogenation/dehydrogenation couples: Advances in Hydrogen Energy (1982), 3 (Hydrogen Energy Prog. 4, Vol. 3), 1335-43, CAN 97:200747. This was followed by the design of a dehydrogenation reactor: Tauzani, A.; Klavana, D.; Belanger, B., Advances in Hydrogen Energy (1986), 5 (Hydrogen Energy Prog. 6, Vol. 2), 858-67, CAN 105:229915.

(c) The methylcyclohexane and decalin "liquid hydrogen carriers" were conceptually employed in several studies by Newson, et al. for a seasonal storage of electricity: Newson, E.; Truong, T.B.; Hottinger, P.; Von Roth, F.; Schacan, Th. H., Hydrogen Energy Progress x11, Proceedings of the World Hydrogen Energy Conference, $12^{\text {th }}$, Buenos Aires (1998), CAN 133:20066. See also: Scherer, G.W.H.; Newson, E.; Wohaun, A. Int. J. Hydrogen Energy 1999, 24, 1157.

(d) Recently, a hydrogen storage and delivery method employing organic thiols as liquid carriers has been proposed: Ratner, B.D., U.S. Pat. No. 7,186,396 B2 (2007, assigned to Asemblon, Inc., Seattle, WA, USA). The organic thiol is dehydrogenated in the presence of a gold powder catalyst; however, no data is provided on a re-hydrogenation of the resultant product with $\mathrm{H}_{2}$.

12. (a) Saito, Hodoshima, et al. at the Tokyo University of Science and Ichikawa, et al. at Hokkaido University have employed the catalytic reactor concepts of, respectively, "superheated liquid-film-type catalysis" and catalysis under "wet-dry multiphase conditions" for practically conducting a dehydrogenation of decalin to naphthalene at lower temperatures (below $300^{\circ} \mathrm{C}$ ). As noted by Kariya, et al. (see ref. 13), fundamentally the two approaches are the same. A film of decalin on a superheated catalytic surface undergoes both dehydrogenation and some evaporation with the byproduct naphthalene upon refluxing. The higher temperature at the liquid/catalyst interface may be expected to improve the equilibrium conversion and reaction kinetics and it is also postulated that by having a high coverage of reactant (decalin) at the surface the rehydrogenation reaction rate is minimized. See: Hodoshima, S.; Shono, A.; Saito, Y. Energy and Fuels 2008, 22, 2559 and references therein; Saito, Y.; Aramaki, K.; Hodoshima, S.; Saito, M.; Shono, A.; Kuwano, J.; Otake, K. Chemical Engineering Science 2008, 63, 4935.

(b) For use of the "wet-dry" concept in a pulse mode spray reactor, see: Kariya, N.; Fukuoka, A.; Utagowa, T.; Sakunomoto, M.; Goto, Y. and Ichikawa, M. Applied Catalysis A 2003, 247, 247.

(c) Work by Ichikawa, et al. has, it appears, led to a formation of the Hrein Energy Company that recently announced a successful test-drive of a "cleaner" gasoline/hydrogen bi-fuel vehicle for which the $\mathrm{H}_{2}$ is derived from an onboard dehydrogenation of a liquid hydride (www.hrein.jp/english/pressrelease/index.htm).

13. (a) Kariya, N.; Fukuoka, A.; Ichikawa M. Applied Catalysis A 2002, 233, 91.

(b) Sebastian, D.; Bordeje, E.G.; Calvillo, L.; Lazaro, M.J.; Moliner, R. Int. J. Hydrogen Energy 2008, 33, 1329.

(c) Wang, B.; Goodman, D.W.; Froment, G.F. J. Catal. 2008, 253, 229. 
14. (a) Pez, G.P.; Scott, A.R.; Cooper, A.C.; Cheng, H., "Hydrogen storage by reversible hydrogenation of pi-conjugated substrates," U.S. Pat. No. 7,101,530 (2006).

(b) Pez, G.P.; Scott, A.R.; Cooper, A.C.; Cheng, H.; Wilhelm, F.C.; Abdourazak, A.H., "Hydrogen storage by reversible hydrogenation of pi-conjugated substrates," U.S. Pat. No. 7,351,395 (2008).

(c) Pez, G.P.; Scott, A.R.; Cooper, A.C.; Cheng, H., "Hydrogen storage by reversible hydrogenation of pi-conjugated substrates," U.S. Pat. No. 7,429,372 (2008).

(d) Bagzis, L.D.; Appleby, J.B.; Pez, G.P.; Cooper, A.C., "Method of delivering a reversible hydrogen storage fuel to a mobile or stationary fuel source," U.S. Pat. Appl. 2004/833467 (2005).

15. Moores, A.; Poyatos, Y.L.; Crabtree, R.H. New J. Chem. 2006, 30, 1675.

16. Clot, E.; Eisenstein, O.; Crabtree, R.H. Chem. Commun. 2007, 2231.

17. Sung, J.S.; Choo, K.Y.; Kim, T.H.; Tarasov, A.L.; Tkachenoko, O.P.; Kustov, L.M. Int. J. Hydrogen Energy 2008, 33, 2721.

18. Cui, Y.; Kwok, S.; Bucholtz, A.; Boyd, D.; Whitney, R.A.; Jessop, P.G. New J. Chem. 2008, 32, 1027.

19. (a) Wechsler, D.; Cui, Y.; Dean, D.; Davis, B.; Jessop, P.G. J. Am. Chem. Soc. 2008, 130, 17203.

(b) Biniwale, R.R.; Rayalu, S.; Devotta, S.; Ichikawa, M. Int. J. Hydrogen Energy 2008, 33, 360. This is a review of "Chemical hydrides - A solution to high capacity hydrogen storage supply" which cites our work referring to U.S. Pat. Appl. 2005/0002857, which unfortunately is "played down" incorrectly quoting low hydrogen capacities.

20. "Reversible Liquid Carriers for an Integrated Production, Storage and Delivery of Hydrogen," Contract No. DE-FG36-05G015015. This project is informally referred to as the " $\mathrm{H}_{2}$ Delivery" project.

21. Estimated for a model hydrogenation reaction:

$\mathrm{A}+3 \mathrm{H}_{2}=\mathrm{AH}_{6}$ where $\mathrm{K}_{\mathrm{eq}}=\left[\mathrm{AH}_{6}\right] /[\mathrm{A}] \times \mathrm{P}\left(\mathrm{H}_{2}\right)^{3} \mathrm{~atm}^{-3}$

For $\mathrm{P}\left(\mathrm{H}_{2}\right)=100$ atm. and $\mathrm{T}=423 \mathrm{~K}$ (reasonable catalytic hydrogenation conditions), and assuming $\Delta S=-30 \mathrm{cal} / \mathrm{deg} \cdot \mathrm{mol} \mathrm{H} \mathrm{H}_{2}$; a hydrogenation of $\mathrm{A}$ to $\left[\mathrm{AH}_{6}\right]:[\mathrm{A}]=50: 1$ at equilibrium, $\Delta \mathrm{H}$ would be approximately $9.3 \mathrm{kcal} / \mathrm{mol} \mathrm{H}_{2}\left(40 \mathrm{~kJ} / \mathrm{mol} \mathrm{H}_{2}\right)$.

22. Clar, E. in "Polycyclic Hydrocarbons", Academic Press, NY, 1964, Chapter 16.

23. Schmitz, A.D.; Bowers, G.; Song, C. Catalysis Today, 1996, 31, 45.

24. Katritzky, A.R.; Karelson, M.; Malkotna, N. Heterocycles 1991, 32, 127. 
25. Wayner, D.D.M.; Clark, K.B.; Rauk, A.; Yu, D.; Armstrong, P.A. J. Am. Chem. Soc. 1997, $119,8295$.

26. Dombrowski, G.W.; Dinnocenzo, J.P.; Farid, S.; Goodman, J.L.; Gould, I.R. J. Org. Chem. 1999, 64, 427.

27. Laleve, J.; Allonas, X.; Foussier, J.-P. J. Am. Chem. Soc. 2002, 124, 9613.

28. Tumanov, V.E.; Kromkin, E.A.; Denisov E.T. Russian Chemical Bulletin (Int. Ed.), 2002, $51,1641$.

29. Feng, Y.; Lei, L.; Wang, J.-T.; Zhao, S.-W.; Guo, Q.-X. J. Org. Chem. 2004, 69, 3192.

30. Wei, J. Ind. \& Eng. Chem. Res. 1999, 38, 5019.

31. Recommendations on the Transport of Dangerous Goods, Seventh Ed., United Nations Publ., New York, 1991. 


\section{Chapter 2: Reversible Hydrogenation of Large Polyaromatic Hydrocarbons}

\section{Introduction}

Polyaromatic hydrocarbons (PAH) are a class of hydrocarbon molecules that contain carbon atoms in the form of fused five- and six-membered rings. The smallest PAH is naphthalene, which contains two fused rings. The upper limit for the size of PAH is somewhat arbitrary because very large PAH have physical properties (e.g., melting point, vapor pressure) that resemble materials rather than molecules. In some respects, graphene (a single layer of graphite) can be considered an almost-infinitely large $\mathrm{PAH}$.

The reversible hydrogenation of naphthalene for the storage of hydrogen is well known in the literature (see Chapter 1). As demonstrated herein by quantum mechanics-based thermodynamics calculations and supporting experimental data, a reversible hydrogenation of larger and more extended PAH is generally thermodynamically more favorable; it can be carried out at a lower temperature than is possible with the commonly used one, two, or three six-membered ring aromatic substrates of the prior art. Essentially, the modulus of the heat or enthalpy of the (exothermic) hydrogenation reaction and of the (endothermic) dehydrogenation step is reduced, thus resulting in a hydrogenation/dehydrogenation system which is more easily reversible at modest and practical temperatures. For this discussion, large polyaromatic hydrocarbons (LPAH) are defined to be a polycyclic aromatic hydrocarbon comprising a fused ring system having at least four rings wherein at least two rings of the fused ring system are represented as six-membered aromatic sextet structures. Examples of PAH with four aromatic rings are triphenylene (9,10-benzophenanthrene), chrysene (1,2-benzophenanthrene), and pyrene.

An added potential advantage of using LPAH for hydrogen storage is that they are relatively involatile, thus precluding the need for a separate unit operation for totally separating the LPAH from the product hydrogen, which greatly simplifies the overall hydrogen storage equipment and the process of operation. 
While, on a thermodynamic basis, generally preferred are the larger molecules (i.e., those with considerably more than four rings), the value of the standard enthalpy change of hydrogenation, $\Delta \mathrm{H}^{\circ}$, and thus the ease of reversible hydrogenation can be very dependent on the "external" shape or structure of the LPAH molecule.

Fundamentally, the LPAH molecules that have the highest aromatic resonance stabilization energy will have the lowest modulus (absolute value) of the standard enthalpy of hydrogenation, $\Delta \mathrm{H}^{\circ}$. It is a general principle that the stability of isomers of fused ring substrates increases with the number of aromatic sextets. ${ }^{1}$ For instance, anthracene (Figure 2-1, left) has one aromatic sextet (conventionally represented by three alternating single and double bonds in a single ring or by an internal circle), while phenanthrene (Figure 2-1, right) has two aromatic sextets, with the result that phenanthrene is more stable by $18.5 \mathrm{~kJ} / \mathrm{mol}$ (based on the molecules' relative heats of formation).<smiles>c1ccc2cc3ccccc3cc2c1</smiles>

anthracene

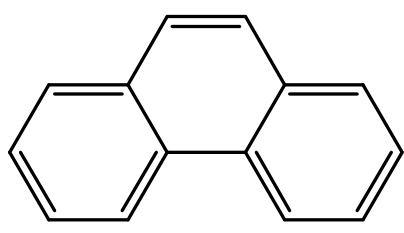

phenanthrene

Figure 2-1. Molecular structures of anthracene and phenanthrene.

Thus, for a LPAH of a given number of fused rings, the structural isomer that is represented by the largest number of aromatic sextets will be preferred on a thermodynamic basis as a hydrogen carrier. These two criteria provide a useful but only qualitative indication of the relative $\Delta \mathrm{H}^{\circ}$ of hydrogenation of a series of LPAH isomers and LPAH molecules of varying size.

Quantum mechanics calculations utilizing the PM3 methodology provide a more useful and quantitative prediction of the $\Delta \mathrm{H}^{\circ}$ values for hydrogenation, as summarized in Figure 2-2. 


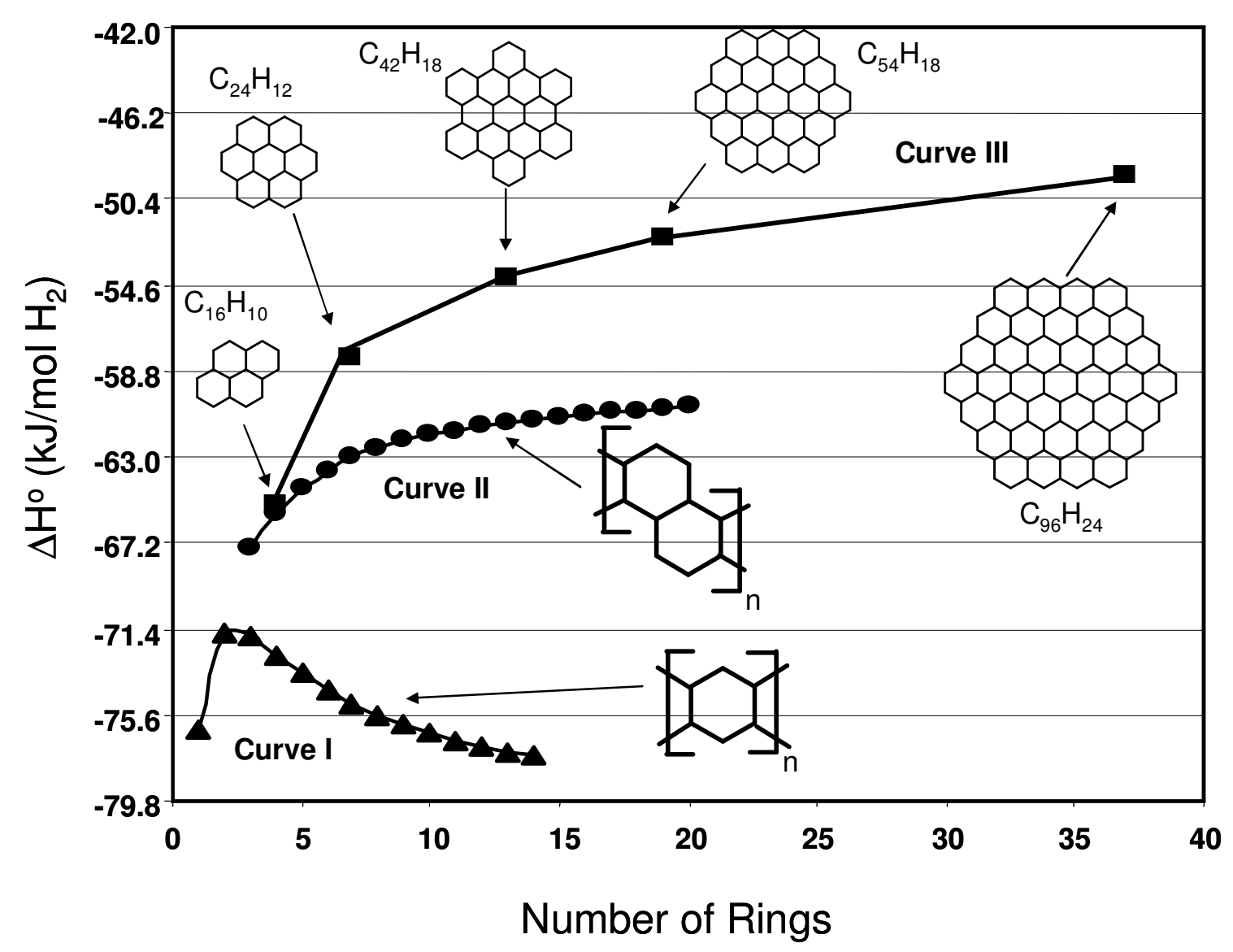

Figure 2-2. Plot of the calculated standard enthalpies of hydrogenation $\left(\Delta \mathrm{H}^{\circ}\right)$ for three structural classes of polyaromatic hydrocarbons as a function of the number of fused aromatic rings (the representations of double bonds, or aromatic sextets, have been omitted for simplicity).

In Figure 2-2, Curve 1 shows the variation of $\Delta \mathrm{H}^{\circ}$ of hydrogenation for a series of linear polyacenes for which the first three members are benzene, naphthalene, and anthracene. The heat, or enthalpy, of hydrogenation reaches its least negative value at naphthalene (2 rings) and becomes increasingly more negative with an increasing number of aromatic rings. We have discovered that fusing the aromatic rings in a staggered ("armchair") linear arrangement results in a less negative $\Delta H^{\circ}$ of hydrogenation as the number of rings increases (Figure 2-2, Curve II). We have also observed a more pronounced effect of fused ring number on $\Delta \mathrm{H}^{\circ}$ of hydrogenation for a series of roughly circular polyaromatic hydrocarbons (Figure 2-2, Curve III). Increasing 
the number of fused rings from pyrene (4 rings) to coronene (7 rings) results in a decrease of $7.2 \mathrm{~kJ} / \mathrm{mol} \mathrm{H}_{2}$ in the $\Delta \mathrm{H}^{\circ}$ of hydrogenation. This curve portraying larger and still larger polyaromatic hydrocarbons suggests that the $\Delta \mathrm{H}^{\circ}$ of hydrogenation of a single sheet of graphite will be of the order of about -34 to about $-46 \mathrm{~kJ} / \mathrm{mol} \mathrm{H}_{2}$, representing the ultimate polyaromatic hydrocarbon for reversible hydrogenation. The large effect of polyaromatic hydrocarbon shape on the $\Delta \mathrm{H}^{\circ}$ of hydrogenation can also be illustrated by comparing the $\Delta \mathrm{H}^{\circ}$ of hydrogenation values for the three 13-ring polyaromatic hydrocarbons in Figure 2-2. There is a span of $23 \mathrm{~kJ} / \mathrm{mol} \mathrm{H}_{2}$ between the $\Delta \mathrm{H}^{\circ}$ of hydrogenation of hexabenzocoronene (Figure 2-3; $\mathrm{C}_{42} \mathrm{H}_{18},-54 \mathrm{kcal} / \mathrm{mol} \mathrm{H}_{2}$ ) and the also 13-ring linear polyacene of Curve 1 of Figure 2-2, for which the $\Delta \mathrm{H}^{\circ}$ of hydrogenation is $77 \mathrm{~kJ} / \mathrm{mol} \mathrm{H}_{2}$.

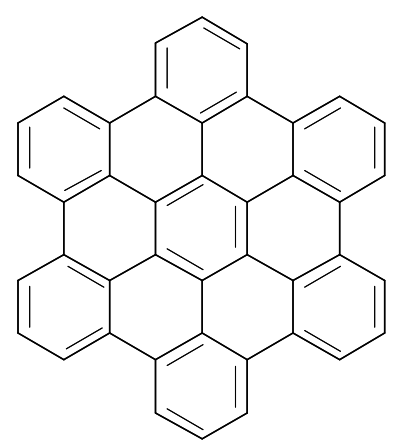

Figure 2-3. Molecular structure of hexabenzocoronene.

While classical examples of hydrogenation and dehydrogenation always invoke reactions in the liquid phase or gas phase, there have been a small number of reports of the solid-state hydrogenation of organic compounds that appear to implicate hydrogen spillover as the mechanism for hydrogenation. In these experiments, there are no solvents employed and no physical means of moving catalyst particles throughout the solid organic compound (e.g., no grinding of the mixture under hydrogen). In the absence of means to transport the organic compound to the heterogeneous hydrogenation catalyst (e.g., dissolving the organic compound in a solvent or sublimation of the organic compound) or a means of transporting the hydrogenation catalyst to the 
solid organic compound (e.g., solvent-dissolved homogeneous catalyst), it has to be considered that the mobile species is atomic hydrogen.

The reversible chemisorption (hydrogenation) of aromatic molecules on silica-supported platinum has been demonstrated using IR spectroscopy. In these experiments, the aromatics are adsorbed on the surface of the metal catalyst and remain adsorbed throughout the sequence of hydrogenation and dehydrogenation. ${ }^{2}$ In a more remarkable report, Lamartine and Parrin have described a series of experiments using platinum or palladium on carbon catalysts to hydrogenate bulk crystalline thymol and other thymol derivatives in the solid state. ${ }^{3}$ Their evidence for solid-state reactions included the hydrogenation of parathymol (3-methyl-4-isopropylphenol) in the form of chiral crystals which remarkably retained the rotatory power after hydrogenation.

\section{Results and Discussion}

In our survey of LPAH, we performed experimental testing with readily available hydrogen carrier candidates. Pyrene is a PAH that is formed from four fused rings (first point on Curve 3 of Figure 2-2). The quantum mechanics calculations utilizing the PM3 methodology yield an average $\Delta \mathrm{H}^{\circ}$ value for hydrogenation for pyrene of about $-65 \mathrm{~kJ} / \mathrm{mol} \mathrm{H}_{2}$. Coronene is a larger $\mathrm{PAH}$ that is formed from seven fused rings (second point on Curve 3 of Figure 2-2). The quantum mechanics calculations utilizing the PM3 methodology yield an average $\Delta \mathrm{H}^{\circ}$ value for hydrogenation for coronene of about $-58 \mathrm{~kJ} / \mathrm{mol} \mathrm{H}_{2}$. Hexabenzocoronene is the largest PAH that was evaluated in our study. This 13-ring $\mathrm{PAH}$ also has the lowest calculated average $\Delta \mathrm{H}^{\circ}$ value for hydrogenation $\left(-54 \mathrm{~kJ} / \mathrm{mol} \mathrm{H}_{2}\right)$ of any LPAH that was evaluated experimentally.

\section{A. Pyrene}

Our experimental testing confirmed that the reversible hydrogenation of pyrene $\left(\mathrm{C}_{16} \mathrm{H}_{10}\right)$ could be achieved under mild conditions and short reaction times using solid pyrene and a solid admixed catalyst. Under 1000 psia (69 bar) of hydrogen gas pressure at $95^{\circ} \mathrm{C}$ with moderate mechanical grinding, the conversion of pyrene to hydrogenated pyrene compounds $\left(\mathrm{C}_{16} \mathrm{H}_{12}-\mathrm{C}_{16} \mathrm{H}_{26}\right)$ is $99.5 \%$ in 1.5 hours (Table 2-1). This is a 2.3 wt. \% 
increase in the gravimetric hydrogen capacity on a total sample weight basis (pyrene + catalyst). The temperature at which the hydrogenation is performed is well below the melting point of pyrene $\left(149^{\circ} \mathrm{C}\right)$. The hydrogenated pyrene can be isolated as a solid material at room temperature that shows a melting point onset of approximately $110^{\circ} \mathrm{C}$. Thus, it is likely that pyrene, a solid at the onset of the hydrogenation, remains a solid during the hydrogenation reaction performed at $95^{\circ} \mathrm{C}$.

Table 2-1. Products from the hydrogenation of pyrene at $95^{\circ} \mathrm{C}$.

\begin{tabular}{|c|c|c|}
\hline $\begin{array}{c}\text { Hydrogenated Pyrene } \\
\text { Sample Components }\end{array}$ & Molecular Formula & $\begin{array}{c}\text { Percentage of Sample } \\
\text { Mixture }\end{array}$ \\
\hline Pyrene & $\mathrm{C}_{16} \mathrm{H}_{10}$ & 0.5 \\
\hline Dihydropyrene & $\mathrm{C}_{16} \mathrm{H}_{12}$ & 6.0 \\
\hline Tetrahydropyrene & $\mathrm{C}_{16} \mathrm{H}_{14}$ & 25 \\
\hline Hexahydropyrene & $\mathrm{C}_{16} \mathrm{H}_{16}$ & 36 \\
\hline Decahydropyrene & $\mathrm{C}_{16} \mathrm{H}_{20}$ & 2.6 \\
\hline Hexadecahydropyrene & $\mathrm{C}_{16} \mathrm{H}_{26}$ & \\
\hline
\end{tabular}

The mixture of hydrogenated pyrene compounds can be dehydrogenated, under 15 psia ( 1 bar) hydrogen gas pressure, at $95^{\circ} \mathrm{C}$ with moderate mechanical grinding. After three hours under these conditions, $25 \%$ of the sample was converted back to pyrene, and the abundance of dihydropyrene $\left(\mathrm{C}_{16} \mathrm{H}_{12}\right)$ was increased relative to the more deeply hydrogenated species (Table 2-2). 
Table 2-2. Dehydrogenation of the mixture from Table $2-1$ at $95^{\circ} \mathrm{C}$.

\begin{tabular}{|c|c|c|}
\hline $\begin{array}{c}\text { Dehydrogenated Pyrene } \\
\text { Sample Components }\end{array}$ & Molecular Formula & $\begin{array}{c}\text { Percentage of Sample } \\
\text { Mixture }\end{array}$ \\
\hline Pyrene & $\mathrm{C}_{16} \mathrm{H}_{10}$ & 25 \\
\hline Dihydropyrene & $\mathrm{C}_{16} \mathrm{H}_{12}$ & 8 \\
\hline Tetrahydropyrene & $\mathrm{C}_{16} \mathrm{H}_{14}$ & 30 \\
\hline Hexahydropyrene & $\mathrm{C}_{16} \mathrm{H}_{16}$ & 23 \\
\hline Decahydropyrene & $\mathrm{C}_{16} \mathrm{H}_{20}$ & \\
\hline
\end{tabular}

When the pyrene is hydrogenated to higher conversion than the mixture detailed in Table 2-1, the mixture of products is liquid at room temperature. The dehydrogenation of the liquid mixture was conducted using mechanical grinding, rather than conventional stirring, in case the dehydrogenated products solidified during the dehydrogenation.

Table 2-3. Mixture of hydrogenated pyrene.

\begin{tabular}{|c|c|c|}
\hline $\begin{array}{c}\text { Hydrogenated Pyrene } \\
\text { Sample Components }\end{array}$ & Molecular Formula & $\begin{array}{c}\text { Percentage of Sample } \\
\text { Mixture }\end{array}$ \\
\hline Pyrene & $\mathrm{C}_{16} \mathrm{H}_{10}$ & 0 \\
\hline Decahydropyrene & $\mathrm{C}_{16} \mathrm{H}_{20}$ & 35 \\
\hline Hexadecahydropyrene & & 65 \\
\hline
\end{tabular}

Starting from a liquid hydrogenated pyrene and a solid admixed catalyst under 23 psia (1.7 bar) of hydrogen gas pressure at $150^{\circ} \mathrm{C}$ with moderate mechanical grinding, the conversion of hydrogenated pyrene (Table 2-3; $\mathrm{C}_{16} \mathrm{H}_{20}-\mathrm{C}_{16} \mathrm{H}_{26}$ ) to dehydrogenated pyrene compounds $\left(\mathrm{C}_{16} \mathrm{H}_{10}-\mathrm{C}_{16} \mathrm{H}_{26}\right)$ resulted in liberation of $25 \%$ of the stored hydrogen (Table 2-4). After the dehydrogenation was ended, the mixture of dehydrogenated pyrene compounds $\left(\mathrm{C}_{16} \mathrm{H}_{10}-\mathrm{C}_{16} \mathrm{H}_{26}\right)$ was still found to be liquid at $25^{\circ} \mathrm{C}$. 
Table 2-4. Dehydrogenation of the mixture from Table $2-3$ at $150^{\circ} \mathrm{C}$.

\begin{tabular}{|c|c|c|}
\hline $\begin{array}{c}\text { Dehydrogenated Pyrene } \\
\text { Sample Components }\end{array}$ & Molecular Formula & $\begin{array}{c}\text { Percentage of Sample } \\
\text { Mixture }\end{array}$ \\
\hline Pyrene & $\mathrm{C}_{16} \mathrm{H}_{10}$ & 9.1 \\
\hline Dihydropyrene & $\mathrm{C}_{16} \mathrm{H}_{12}$ & 0.4 \\
\hline Tetrahydropyrene & $\mathrm{C}_{16} \mathrm{H}_{14}$ & 20.3 \\
\hline Hexahydropyrene & $\mathrm{C}_{16} \mathrm{H}_{16}$ & 0.3 \\
\hline Octahydropyrene & $\mathrm{C}_{16} \mathrm{H}_{18}$ & 19.4 \\
\hline Decahydropyrene & $\mathrm{C}_{16} \mathrm{H}_{20}$ & 46 \\
\hline Hexadecahydropyrene & $\mathrm{C}_{16} \mathrm{H}_{26}$ & \\
\hline
\end{tabular}

\section{B. Coronene}

This highly symmetrical molecule has the maximum possible number of aromatic sextets for a 7-ring PAH due to the circular shape, which leads to greater aromatic stabilization for coronene than other $\mathrm{PAH}$ with the same number of rings (Figure 2-4, inset). Coronene is one of the largest PAH that is commercially available and amenable to analytical characterization by Nuclear Magnetic Resonance (NMR) and Gas Chromatography-Mass Spectroscopy (GC-MS). In our experimental studies, we have observed the hydrogenation of coronene in the solid state at temperatures substantially below the melting point. Intimate mixtures of coronene (melting point $428^{\circ} \mathrm{C}$ ) and heterogeneous hydrogenation catalysts were exposed to moderate pressures of hydrogen at $150^{\circ} \mathrm{C}$, resulting in the formation of partially hydrogenated derivatives of coronene.

A sample of coronene was impregnated with palladium metal particles by RF sputtering. Subsequent thermal gravimetric combustion analysis demonstrated a 3\% loading of palladium metal in the coronene solid. The sample was then placed in a differential pressure adsorption unit ${ }^{4}$, consisting of two identical pressure cells which are spanned by a differential pressure gauge. Under 995 psia of hydrogen gas pressure at $150^{\circ} \mathrm{C}$, a 
$4.9 \mathrm{wt}$ \% increase in the gravimetric hydrogen capacity on a total sample weight basis (coronene + catalyst) was observed over a period of 63 hours (Figure 2-4, Cycle 1). The mixture of hydrogenated coronene compounds was dehydrogenated, under 20 psia hydrogen gas pressure, at temperatures of 150 and $200^{\circ} \mathrm{C}$ in the absence of any mechanical agitation or grinding (Figure 2-5, Cycle \#1). After 24 hours at $150^{\circ} \mathrm{C}$ and 14 hours at $200^{\circ} \mathrm{C}$, the sample desorbed 4.5 wt. \% hydrogen $(92 \%$ of the sorbed hydrogen).

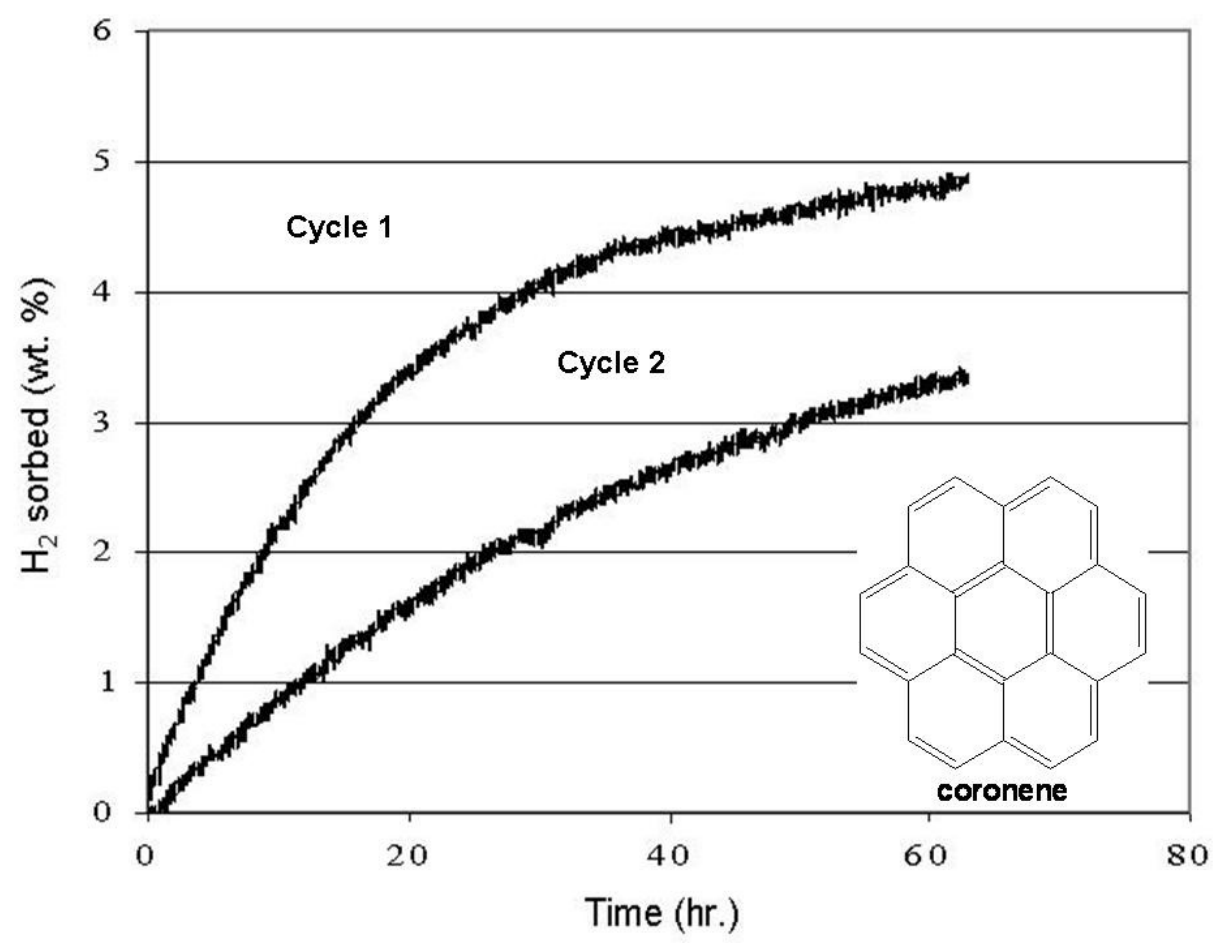

Figure 2-4. The reversible catalytic solid-state hydrogenation of coronene with palladium nanoparticles over two cycles. The inset shows the molecular structure of coronene.

The sample was hydrogenated a second time; under 1005 psia of hydrogen gas pressure at $150^{\circ} \mathrm{C}$, a $3.9 \mathrm{wt}$ \% increase of the gravimetric hydrogen capacity on a total sample weight basis (coronene + catalyst) was observed over a period of 91 hours (Figure 2-4, Cycle 2, data shown only up to 60 hours). The mixture of hydrogenated coronene compounds was again dehydrogenated, under 20 psia hydrogen gas 
pressure, at $200^{\circ} \mathrm{C}$ (Figure 2-5, Cycle \#2). After 9 hours at $200^{\circ} \mathrm{C}$, the sample desorbed $3.5 \mathrm{wt}$ \% hydrogen ( $90 \%$ of the sorbed hydrogen). These results appear to demonstrate gas/solid hydrogenation and dehydrogenation of a two-component solid system (hydrogenated and dehydrogenated forms of the solid substrate) in that the hydrogenation and dehydrogenation of the solid can easily and effectively go to completion under equilibrium conditions.
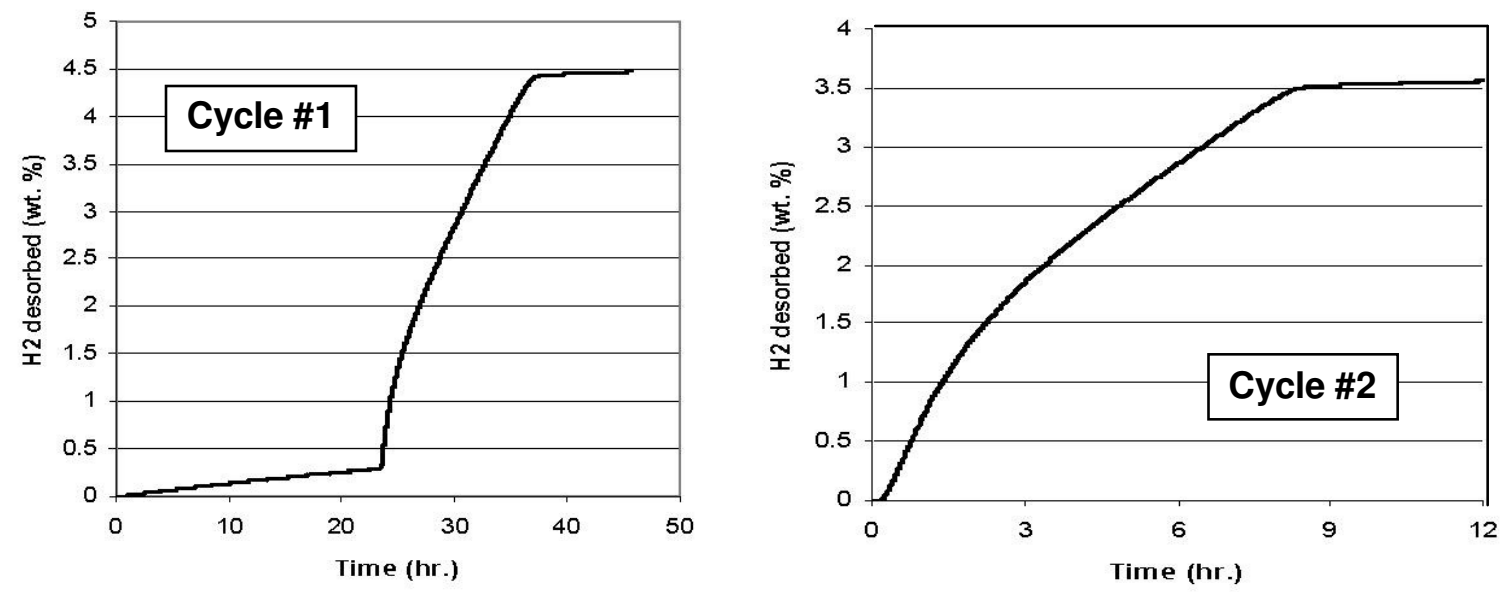

Figure 2-5. Desorption of hydrogen from hydrogenated coronene over two cycles via solid-state catalytic dehydrogenation with palladium nanoparticles.

Other metal catalysts were also found to be effective for the hydrogenation and dehydrogenation of coronene in the solid state. In one representative experiment, a $2: 1$ mixture of coronene and rhodium on carbon catalyst ( $5 \%$ rhodium) was ground with an agate mortar and pestle for 15 minutes until a uniform mixture was formed. The sample was degassed at ambient temperature for 30 minutes under vacuum. Both the sample cell and reference cells were placed under 970 psia hydrogen and heated to $150^{\circ} \mathrm{C}$. The hydrogen pressure in the sample cell dropped, relative to the reference cell, for a period of 17 hours, indicating adsorption of $3.2 \mathrm{wt}$. \% hydrogen by the sample. After 17 hours, the cells were cooled to ambient temperature, and the pressure in both cells was reduced to 20 psia. Upon heating both cells to $150^{\circ} \mathrm{C}$, there was an increase in the pressure of the sample cell relative to the reference cell, indicating desorption of hydrogen from the sample. After 70 hours, the sample had desorbed $1.0 \mathrm{wt}$ \% 
hydrogen (31\% of the sorbed hydrogen). Analysis of the sample after hydrogenation/dehydrogenation by GC-MS confirmed that partially hydrogenated intermediates of coronene were present in the sample.

While the preceding experiments have shown that coronene can be reversibly hydrogenated in the solid state without physical mixing or agitation, the reaction rates are very slow. The reversible hydrogenation of coronene can be achieved under mild conditions and much shorter reaction times using solid coronene and a solid admixed catalyst and mechanical grinding. Under 1045 psia (72 bar) of hydrogen gas pressure at $150^{\circ} \mathrm{C}$ with moderate mechanical grinding, the conversion of coronene to hydrogenated coronene compounds $\left(\mathrm{C}_{24} \mathrm{H}_{22}-\mathrm{C}_{24} \mathrm{H}_{30}\right)$ using a rhodium on carbon catalyst is $99+\%$ in 4 hours (Table 2-5). This is a $3.5 \mathrm{wt}$ \% increase in the gravimetric hydrogen capacity on a total sample weight basis (coronene + catalyst).

Table 2-5. Hydrogenation of coronene with Rh/C catalyst.

\begin{tabular}{|c|c|c|}
\hline $\begin{array}{c}\text { Hydrogenated Coronene } \\
\text { Sample Components }\end{array}$ & Molecular Formula & $\begin{array}{c}\text { Percentage of Sample } \\
\text { Mixture }\end{array}$ \\
\hline Coronene & $\mathrm{C}_{24} \mathrm{H}_{12}$ & 2 \\
\hline Decahydrocoronene & $\mathrm{C}_{24} \mathrm{H}_{22}$ & 27 \\
\hline Tetradecahydrocoronene & $\mathrm{C}_{24} \mathrm{H}_{26}$ & 70 \\
\hline Octadecahydrocoronene & $\mathrm{C}_{24} \mathrm{H}_{30}$ & $<1$ \\
\hline
\end{tabular}

The hydrogenated coronene was isolated as a solid material that shows a melting point onset at approximately $280^{\circ} \mathrm{C}$. Thus, it is likely that coronene, a solid at the onset of hydrogenation, remains a solid during the hydrogenation reaction performed at $150^{\circ} \mathrm{C}$. The mixture of hydrogenated coronene compounds with the $\mathrm{Rh} / \mathrm{C}$ catalyst can be dehydrogenated, under 15 psia ( 1 bar) hydrogen gas pressure, at $150^{\circ} \mathrm{C}$ with moderate mechanical grinding. After 16 hours under these conditions, $91 \%$ of the sample was converted back to coronene (Table 2-6). 
Table 2-6. Dehydrogenation of the mixture from Table 2-5.

\begin{tabular}{|c|c|c|}
\hline $\begin{array}{c}\text { Dehydrogenated } \\
\text { Coronene Sample } \\
\text { Components }\end{array}$ & Molecular Formula & $\begin{array}{c}\text { Percentage of Sample } \\
\text { Mixture }\end{array}$ \\
\hline Coronene & $\mathrm{C}_{24} \mathrm{H}_{12}$ & 92 \\
\hline Octahydrocoronene & $\mathrm{C}_{24} \mathrm{H}_{20}$ & 0.82 \\
\hline Tetradecahydrocoronene & $\mathrm{C}_{24} \mathrm{H}_{26}$ & 0.54 \\
\hline Octadecahydrocoronene & $\mathrm{C}_{24} \mathrm{H}_{30}$ & 6.7 \\
\hline
\end{tabular}

We observed the reversible hydrogenation of coronene under mild conditions and short reaction times starting from solid coronene and a solid admixed catalyst from the group of early transition metals ( $\mathrm{Sc}, \mathrm{Y}, \mathrm{Ti}, \mathrm{Zr}, \mathrm{Hf}, \mathrm{V}, \mathrm{Nb}, \mathrm{Ta}$ ). This is notable in that metals and metal alloys of the late transition metals ( $\mathrm{Fe}, \mathrm{Ru}, \mathrm{Os}, \mathrm{Co}, \mathrm{Rh}, \mathrm{Ir}, \mathrm{Ni}, \mathrm{Pd}, \mathrm{Pt}$ ) are typically employed as catalysts for both hydrogenation and/or dehydrogenation. In addition, our experiments suggest that a catalyst in the form of a stable metal hydride $\left(\mathrm{MH}_{\mathrm{x}}\right)$ can be utilized for the reversible hydrogenation of $\mathrm{PAH}$. Using a titanium hydride catalyst, under 1185 psia (82 bar) of hydrogen gas pressure at $200^{\circ} \mathrm{C}$ with moderate mechanical grinding, the conversion of coronene to hydrogenated coronene compounds (hcoronene) is $44 \%$ in only 2 hours. The mixture of hydrogenated coronene compounds can be dehydrogenated with the same titanium catalyst, under 15 psia (1 bar) hydrogen gas pressure, at $150^{\circ} \mathrm{C}$ with moderate mechanical grinding. After 7 hours under these conditions, $90 \%$ of the hydrogenated coronene mixture was converted back to coronene.

\section{Hexabenzocoronene}

The reversible hydrogenation of hexabenzocoronene $\left(\mathrm{HBC}, \mathrm{C}_{42} \mathrm{H}_{18}\right)$ can be achieved starting from solid hexabenzocoronene and admixed $5 \%$ rhodium on carbon catalyst. Under 1130 psia (78 bar) of hydrogen gas pressure at $200^{\circ} \mathrm{C}$ with moderate mechanical grinding, the conversion of hexabenzocoronene to hydrogenated hexabenzocoronene 
compounds $\left(\mathrm{C}_{42} \mathrm{H}_{24}-\mathrm{C}_{42} \mathrm{H}_{36}\right)$ is $72 \%$ in 8 hours (Table 2-7). This represents a 1.65 wt. \% increase in the gravimetric hydrogen capacity on a total sample weight basis (hexabenzocoronene + catalyst).

Table 2-7. Hydrogenation of hexabenzocoronene.

\begin{tabular}{|c|c|c|c|}
\hline $\begin{array}{c}\text { Hydrogenated HBC } \\
\text { Sample Components }\end{array}$ & $\begin{array}{c}\text { Molecular } \\
\text { Formula }\end{array}$ & $\begin{array}{c}\text { Molecular } \\
\text { Weight }\end{array}$ & $\begin{array}{c}\text { Percentage of } \\
\text { Sample Mixture }\end{array}$ \\
\hline HBC & $\mathrm{C}_{42} \mathrm{H}_{18}$ & 522 & 28 \\
\hline Hexahydro-HBC & $\mathrm{C}_{42} \mathrm{H}_{24}$ & 528 & 71 \\
\hline Octadecahydro-HBC & $\mathrm{C}_{42} \mathrm{H}_{36}$ & 540 & 1 \\
\hline
\end{tabular}

The temperature at which the hydrogenation is performed is $>500^{\circ} \mathrm{C}$ below the melting point of hexabenzocoronene $\left(>700^{\circ} \mathrm{C}\right)$. Thus, it is extremely likely that hexabenzocoronene, a solid at the onset of hydrogenation, remains a solid during the hydrogenation reaction performed at $200^{\circ} \mathrm{C}$. The mixture of hydrogenated hexabenzocoronene compounds can be dehydrogenated, under 15 psia (1 bar) hydrogen gas pressure, at $200^{\circ} \mathrm{C}$ with moderate mechanical grinding. After 16 hours under these conditions, $58 \%$ of the hydrogenated hexabenzocoronene was converted back to hexabenzocoronene (Table 2-8)

Table 2-8. Dehydrogenation of mixture in Table 2-7.

\begin{tabular}{|c|c|c|c|}
\hline $\begin{array}{c}\text { Dehydrogenated HBC } \\
\text { Sample Components }\end{array}$ & $\begin{array}{c}\text { Molecular } \\
\text { Formula }\end{array}$ & $\begin{array}{c}\text { Molecular } \\
\text { Weight }\end{array}$ & $\begin{array}{c}\text { Percentage of } \\
\text { Sample Mixture }\end{array}$ \\
\hline HBC & $\mathrm{C}_{42} \mathrm{H}_{18}$ & 522 & 86 \\
\hline Hexahydro-HBC & $\mathrm{C}_{42} \mathrm{H}_{24}$ & 528 & 5.5 \\
\hline Dodecahydro-HBC & $\mathrm{C}_{42} \mathrm{H}_{30}$ & 534 & 8.65 \\
\hline Octadecahydro-HBC & $\mathrm{C}_{42} \mathrm{H}_{36}$ & 540 & \\
\hline
\end{tabular}




\section{Pentacene (comparative example)}

The $\Delta \mathrm{H}^{\circ}$ for the hydrogenation of pentacene $\left(-74 \mathrm{~kJ} / \mathrm{mol} \mathrm{H}_{2}\right)$ is substantially larger than the $\Delta \mathrm{H}^{\circ}$ for the hydrogenation of coronene $\left(-58 \mathrm{~kJ} / \mathrm{mol} \mathrm{H}_{2}\right)$. This should disfavor the dehydrogenation of hydrogenated pentacene at moderate temperatures.

Pentacene and rhodium on carbon catalyst (5\% rhodium) were ground with an agate mortar and pestle for 15 minutes until a uniform mixture was formed. The sample was then placed in the differential pressure adsorption unit for hydrogenation and dehydrogenation testing. Under 980 psia (67.6 bar) of hydrogen gas pressure at $150^{\circ} \mathrm{C}$, a $5.5 \mathrm{wt}$ \% increase in the gravimetric hydrogen capacity on a total sample weight basis (pentacene + catalyst) was observed over a period of 10 hours (Figure 2-6). The temperature at which the hydrogenation was performed was far below the melting point of pentacene (m.p. $>300^{\circ} \mathrm{C}$ ). Thus, it is likely that pentacene, a solid at the onset of hydrogenation, remains a solid during the hydrogenation and dehydrogenation reactions performed at $150^{\circ} \mathrm{C}$.

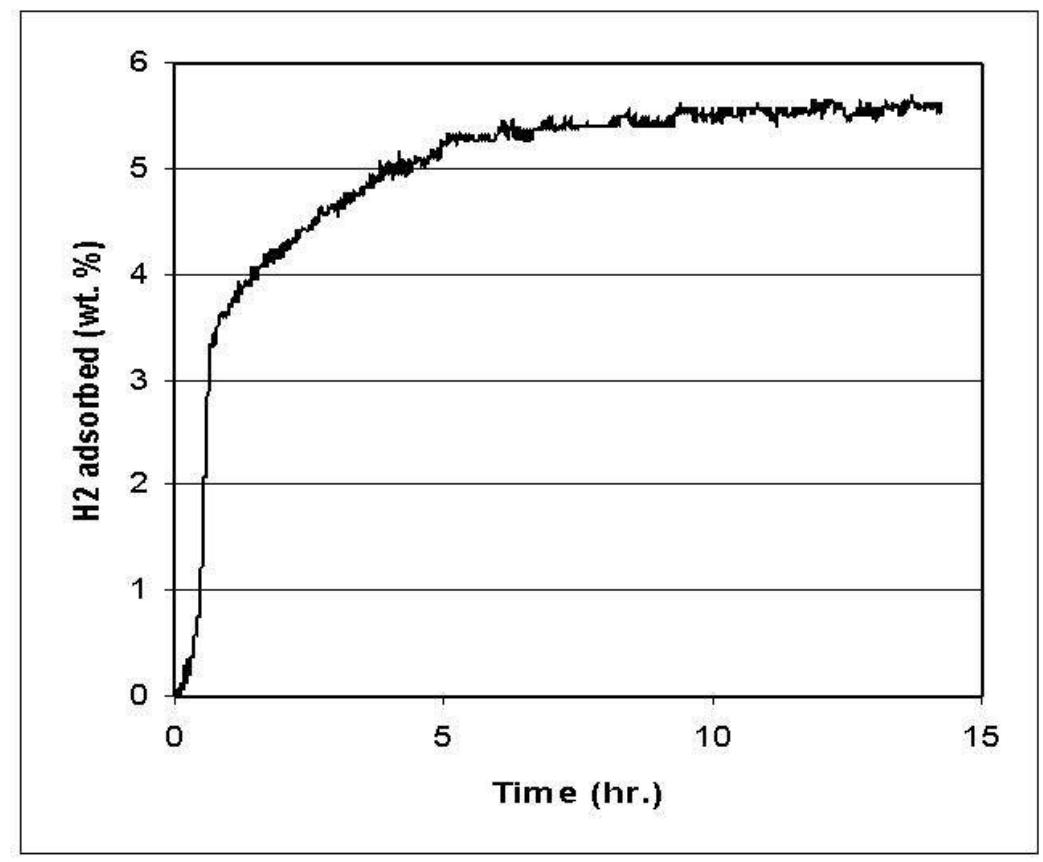

Figure 2-6. Hydrogenation of pentacene. 
The mixture of hydrogenated pentacene compounds was not dehydrogenated to any appreciable conversion under $18 \mathrm{psia}(1.25 \mathrm{bar})$ hydrogen gas pressure at $150^{\circ} \mathrm{C}$. After 70 hours under these conditions, the sample desorbed only $0.15 \mathrm{wt}$. \% hydrogen (only $2.7 \%$ of the sorbed hydrogen). This can be compared to the dehydrogenation of coronene, where after 70 hours at these same conditions the hydrogenated coronene sample desorbed $1.0 \mathrm{wt}$. \% hydrogen (Figure $2-5,31 \%$ of the initially sorbed hydrogen). The $1150 \%$ decrease in the percentage of desorbed hydrogen for pentacene compared to coronene under identical process conditions again illustrates the substantial difference in $\Delta \mathrm{H}^{\circ}$ for hydrogenation between these two polyaromatic hydrocarbon molecules and the importance of $\Delta \mathrm{H}^{\circ}$ of hydrogenation in a hydrogen storage system based upon reversible hydrogenation of polyaromatic hydrocarbons.

\section{Conclusions}

The reversible hydrogenation of PAH has been demonstrated in both solid- and liquidstate reactions. The solid-state reactions can be performed rapidly if mechanical grinding of the hydrogen carrier and the hydrogenation/dehydrogenation catalyst is used. Remarkably, we observed the reversible hydrogenation of coronene, even in the absence of grinding or other mechanical mixing/agitation. Intimate mixtures of coronene and heterogeneous transition metal catalysts were used to demonstrate reversible hydrogenation of coronene at reaction temperatures several hundred degrees Celsius below the melting point of the solid, favoring our interpretation of solid-state hydrogenation. These experimental studies of the solid-state hydrogenation of a graphene-like large polyaromatic hydrocarbon add experimental evidence for the migration of hydrogen atoms in a solid-state material and the ability of hydrogen atoms to hydrogenate an aromatic carbon-based material in the absence of direct metalmediated catalytic hydrogenation. 


\section{Experimental Details}

\section{General Details}

The apparatus for hydrogenation and dehydrogenation testing while grinding the substrate and catalyst was constructed from a $50 \mathrm{cc}$ high-pressure reactor (Parr instruments) equipped with a customized grinding apparatus. The grinding apparatus consisted of an elongated stirrer shaft with an arc-shaped paddle. The bottom of the reactor contained a stainless steel insert with a concave bottom, which allows the paddle of the stirrer shaft to sweep the bottom of the reactor with 1/8" clearance. Mechanical agitation of the sample mixture was performed by adding 5-8 stainless steel ball bearings of varying size (1/16" - 1/4" diameter). The stirrer motor was programmed such that rotational direction of the stirrer would alternate between clockwise and counterclockwise directions during the course of the reaction in order to ensure that all of the sample mixture would contact the grinding balls.

The differential adsorption measurement apparatus consists of two identical pressure cells which are spanned by a differential pressure gauge. The absolute pressures of the two cells are measured independently by pressure transducers. Adsorption of hydrogen by the sample is characterized by a decrease in the pressure in the sample cell relative to the reference cell while an identical temperature is maintained between the two cells. ${ }^{4}$

\section{Reversible hydrogenation of pyrene with $5 \% \mathrm{Rh}$ on carbon catalyst and mechanical grinding}

A $0.2 \mathrm{~g}$ sample of pyrene (>99\%, Fluka) and $0.1 \mathrm{~g}$ of rhodium on carbon catalyst ( $5 \% \mathrm{Rh}$, Acros Organics) were ground by hand with an agate mortar and pestle until a uniform color mixture was formed. The mixture was then placed in the customized grinding apparatus. The system was pressurized with helium to 1000 psia (69 bar) and vented. Pressurization and venting with helium was repeated three times. The reactor system was then twice pressurized with 1000 psia (69 bar) hydrogen and vented. Once purging was completed, the sample mixture was hydrogenated by grinding the mixture at $95^{\circ} \mathrm{C}$ under 1000 psia (69 bar) hydrogen pressure for 1.5 hours. After the reaction time, the reactor was then quickly cooled to room temperature and vented. Half of the sample 
mixture was removed from the reactor, and the remaining material was left in the reactor for dehydrogenation testing. The material to be dehydrogenated was purged with helium and hydrogen, as described above, and ground at $95^{\circ} \mathrm{C}$ under $15 \mathrm{psia}$ ( 1 bar) hydrogen for three hours before the reactor was cooled to room temperature. Both of the hydrogenated pyrene and dehydrogenated $\mathrm{h}$-pyrene samples were separated from the catalyst by extraction with chloroform (HPLC grade, Fisher) and filtering of the insoluble catalyst. The chloroform was then removed under vacuum to obtain the pure products. GC-MS and proton NMR spectroscopy analyses were performed on the hydrogenated and dehydrogenated pyrene. GC-MS indicated that $>99 \%$ of the pyrene starting material was converted to a mixture of hydrogenated products, having a gravimetric storage capacity for hydrogen of $2.3 \mathrm{wt}$. \% on a total sample weight basis (pyrene + catalyst). Upon dehydrogenation, GC-MS showed that approximately $25 \%$ of the hydrogenated material was converted back to pyrene. Proton NMR was used to verify the hydrogenation/dehydrogenation of pyrene. Although the proton NMR spectra were too complex for peak assignment of individual hydrogenated pyrene products, integration of pyrene peak intensity versus new resonances in the spectrum indicated substantial conversion during hydrogenation and partial regeneration of pyrene after dehydrogenation.

\section{Dehydrogenation of liquid pyrene under 1 bar hydrogen pressure}

A $0.4 \mathrm{~g}$ sample of substantially hydrogenated pyrene (colorless liquid at $25^{\circ} \mathrm{C}$ ) and $0.2 \mathrm{~g}$ rhodium on carbon catalyst (5\% Rh, Acros Organics) was placed in the customized grinding apparatus. The system was evacuated for 5 minutes and pressurized with helium to 1000 psia (69 bar) and vented. Pressurization and venting with helium was repeated three times. The system was filled with 14.5 psia hydrogen ( 1 bar). The sample was dehydrogenated by grinding at $150^{\circ} \mathrm{C}$ under 23 psia (1.6 bar) hydrogen pressure for 16 hours. After the reaction time, the reactor was quickly cooled to room temperature and vented. The still-liquid sample mixture was removed from the reactor and separated from the catalyst by extraction with chloroform (HPLC grade, Fisher) and filtering of the insoluble catalyst. The chloroform was then removed under vacuum to obtain the pure products. GC-MS was used to analyze the hydrogenated and dehydrogenated pyrene. The gravimetric hydrogen storage capacity of the hydrogenated pyrene + catalyst was $4.7 \mathrm{wt}$ \%, and after dehydrogenation, the capacity 
was reduced to $3.5 \mathrm{wt}$. \% hydrogen. This corresponded to approximately $25 \%$ of the stored hydrogen being released during dehydrogenation of hydrogenated pyrene.

\section{Reversible hydrogenation of coronene with $5 \% \mathrm{Rh}$ on carbon catalyst and mechanical grinding}

A $0.125 \mathrm{~g}$ sample of coronene (95\%, Acros Organics) and $0.065 \mathrm{~g}$ of rhodium on carbon catalyst (5\% Rh, Acros Organics) were ground by hand with an agate mortar and pestle until a uniform dark green mixture was formed. The mixture was then placed in the customized grinding apparatus. The system was pressurized with helium to 1000 psia (69 bar) and vented. Pressurization and venting with helium was repeated three times. The reactor system was then twice pressurized to 1000 psia (69 bar) hydrogen and vented. The coronene was hydrogenated by heating the sample mixture to $150^{\circ} \mathrm{C}$ under 1045 psia (72 bar) hydrogen while the mixture was continuously ground for four hours. The reactor was then quickly cooled to room temperature and vented to atmospheric pressure. The mixture was removed from the reactor, weighed, and half of the material was returned to the reactor for dehydrogenation. The hydrogenated coronene was removed from the mixture by extracting with chloroform, filtering of the insoluble catalyst, and drying under vacuum. Dehydrogenation was performed by continuous grinding at $150^{\circ} \mathrm{C}$ under 15 psia ( 1 bar) hydrogen for 16 hours. After the reactor was cooled to room temperature, the sample mixture was removed from the reactor, and the dehydrogenated coronene was isolated by extraction with chloroform, filtering of the insoluble catalyst, and drying under vacuum. Both of the hydrogenated and dehydrogenated coronene samples were analyzed by proton NMR and DEP Probe CI (isobutane) mass spectrometry. The coronene parent ion (molecular weight 300) was not present in the mass spectrum of the hydrogenated coronene. The mass spectrum mainly consisted of masses 318,314 and 310 . The mass spectrum of dehydrogenated coronene showed that the three masses at 310,314, and 318 decreased in intensity, and a new peak was formed at $300 \mathrm{~m} / \mathrm{z}$, the molecular weight of coronene. With the assumption that the response factors for each product were similar, a particular ion contribution to the spectrum and the weight increase of coronene upon hydrogenation were calculated. Upon hydrogenation, the coronene added $3.5 \mathrm{wt}$ \% hydrogen, and 80 percent of those hydrogenated products were converted back to coronene upon dehydrogenation. The irreversibly hydrogenated product mainly consisted of the mass 
318 isomer. Proton NMR spectroscopy was in good agreement with the mass spectroscopy results. The coronene resonance (singlet at $9 \mathrm{ppm}$ ) diminished significantly after hydrogenation, while new upfield resonances, assigned to methylene hydrogens, appeared. The intensity of the coronene resonance was regained after dehydrogenation, while the methylene resonances diminished.

\section{Reversible hydrogenation of coronene with $5 \%$ Rh on carbon catalyst}

A $0.066 \mathrm{~g}$ sample of coronene (95\%, Acros Organics) and $0.033 \mathrm{~g}$ of rhodium on carbon catalyst (5\% Rh, Acros Organics) were ground with an agate mortar and pestle for 15 minutes until a uniform dark green mixture was formed. The sample was then placed in the differential pressure adsorption unit. The sample was degassed at ambient temperature for 30 minutes under vacuum. Both the sample cell and reference cells were placed under $970 \mathrm{psia}$ (67 bar) hydrogen and heated to $150^{\circ} \mathrm{C}$. The hydrogen pressure in the sample cell dropped, relative to the reference cell, for a period of 17 hours, indicating adsorption of $3.2 \mathrm{wt}$ \% hydrogen by the sample. After 17 hours, the cells were cooled to ambient temperature, and the pressure in both cells was reduced to 20 psia (1.4 bar). Upon heating both cells to $150^{\circ} \mathrm{C}$, there was a increase in the pressure of the sample cell relative to the reference cell, indicating desorption of hydrogen from the sample. After 70 hours, the sample had desorbed $1.0 \mathrm{wt}$. \% hydrogen ( $31 \%$ of the sorbed hydrogen).

\section{Reversible hydrogenation of coronene with sputtered palladium}

In an argon glovebox, a $0.1 \mathrm{~g}$ sample of coronene (95\%, Acros Organics) was impregnated with palladium metal particles by RF sputtering. Subsequent TGA combustion analysis demonstrated a $3 \%$ loading of palladium metal in the coronene solid. In the glovebox, the sample was then placed in the differential pressure adsorption unit cell. The sample was degassed at ambient temperature for 20 minutes under vacuum. Both the sample cell and reference cells were placed under $995 \mathrm{psia}$ (69 bar) hydrogen and heated to $150^{\circ} \mathrm{C}$. The hydrogen pressure in the sample cell dropped, relative to the reference cell, for a period of 63 hours, indicating adsorption of 4.9 wt. \% hydrogen by the sample. After 63 hours, the pressure in both cells was reduced to 20 psia (1.4 bar). After about 24 hours at $150^{\circ} \mathrm{C}$, both cells were heated to 
$200^{\circ} \mathrm{C}$. Throughout the period of heating, up to about 40 hours, there was a increase in the pressure of the sample cell relative to the reference cell, indicating desorption of hydrogen from the sample. After 40 hours, the sample had desorbed $4.5 \mathrm{wt}$. \% hydrogen (92\% of the sorbed hydrogen). The cells were then cooled to $150^{\circ} \mathrm{C}$, and the hydrogen pressure in both cells was raised to $1005 \mathrm{psia}$ (69 bar). The hydrogen pressure in the sample cell dropped, relative to the reference cell, for a period of 91 hours, indicating adsorption of $3.9 \mathrm{wt}$ \% hydrogen by the sample. The pressure was dropped to 20 psia (1.4 bar) and the temperature raised to $200^{\circ} \mathrm{C}$ in both cells. Throughout the period of heating, up to about 9 hours, there was a increase in the pressure of the sample cell relative to the reference cell, indicating desorption of $3.5 \mathrm{wt}$. $\%$ hydrogen from the sample ( $90 \%$ of the sorbed hydrogen).

\section{Reversible hydrogenation of coronene with titanium hydride and mechanical grinding}

In an argon glovebox, a $0.1 \mathrm{~g}$ sample of coronene and $0.047 \mathrm{~g}$ of titanium hydride ( $\mathrm{TiH}_{2}$, Alfa Aesar) were ground by hand with an agate mortar and pestle until a uniform mixture was formed. In the glovebox, the mixture was then placed in the customized grinding apparatus. The system was pressurized to $1000 \mathrm{psia}$ (69 bar) hydrogen and vented. The coronene was hydrogenated by heating the sample mixture to $200^{\circ} \mathrm{C}$ under 1185 psia (82 bar) hydrogen while the mixture was continuously ground for two hours. The reactor was then quickly cooled to room temperature and vented to atmospheric pressure. The mixture was removed from the reactor and the hydrogenated coronene was removed from the mixture by extracting with chloroform, filtering of the insoluble catalyst, and drying under vacuum. Proton NMR spectroscopy showed that the intensity of the coronene resonance (singlet at $9 \mathrm{ppm}$ ) diminished significantly after hydrogenation while new upfield resonances, assigned to methylene hydrogens, appeared. The integration of these methylene resonances vs. the unhydrogenated coronene suggested a $44 \%$ conversion of coronene to hydrogenated coronene products. To study the dehydrogenation of hydrogenated coronene using the titanium hydride catalyst, $0.03 \mathrm{~g}$ of a hydrogenated coronene mixture containing $52 \%$ coronene and $48 \%$ hydrogenated coronene was ground by hand with $0.03 \mathrm{~g}$ of titanium hydride $\left(\mathrm{TiH}_{2}\right.$, Alfa Aesar) in an argon glovebox with an agate mortar and pestle until a uniform mixture was formed. In the glovebox, the mixture was then placed in the customized grinding 
apparatus. The system was pressurized to 1000 psia (69 bar) hydrogen and vented to 15 psia (1 bar). The hydrogenated coronene was dehydrogenated by heating the sample mixture to $150^{\circ} \mathrm{C}$ under 15 psia (1 bar) hydrogen while the mixture was continuously ground for seven hours. The reactor was then quickly cooled to room temperature. The mixture was removed from the reactor, and the dehydrogenated coronene was removed from the mixture by extracting with chloroform, filtering of the insoluble catalyst, and drying under vacuum. GC-MS analysis was performed on the dehydrogenated coronene, and indicated that approximately $90 \%$ of the hydrogenated coronene was converted to coronene upon dehydrogenation with titanium hydride.

\section{Reversible hydrogenation of hexabenzocoronene with $5 \% \mathrm{Rh}$ on carbon and mechanical grinding}

Hexabenzocoronene (HBC) was prepared according to a literature procedure. ${ }^{5}$ A $0.06 \mathrm{~g}$ sample of $\mathrm{HBC}$ and $0.03 \mathrm{~g}$ of rhodium on carbon catalyst ( $5 \% \mathrm{Rh}$, Acros Organics) were ground by hand with an agate mortar and pestle until a uniform dark green mixture was formed. The mixture was then placed in the customized grinding apparatus. The system was pressurized with helium to 1000 psia (69 bar) and vented. Pressurization and venting with helium was repeated three times. The reactor system was then twice pressurized to 1000 psia (69 bar) hydrogen and vented. The HBC was hydrogenated by heating the sample mixture to $200^{\circ} \mathrm{C}$ under 1130 psia (78 bar) hydrogen while the mixture was continuously ground for eight hours. The reactor was then quickly cooled to room temperature and vented to atmospheric pressure. Half of the sample mixture was removed from the reactor, and the remaining material was left in the reactor for dehydrogenation. The material to be dehydrogenated was purged as described above and ground at $200^{\circ} \mathrm{C}$ and 15 psia (1 bar) hydrogen for 16 hours before the reactor was cooled to room temperature. Both of the hydrogenated HBC and dehydrogenated HBC samples were separated from the catalyst by extraction with chloroform and filtering of the insoluble catalyst. The chloroform was then removed under vacuum to obtain the pure products, which were analyzed by MALDI mass spectrometry using tetracyanoquinodimethane as the matrix. Upon hydrogenation of HBC (molecular weight 522), a new mass arose at $540 \mathrm{~m} / \mathrm{z}$. This mass increase would account for the addition of nine hydrogen molecules. The mass spectrum of the dehydrogenated HBC showed the regeneration of $\mathrm{HBC}$ and diminished intensity for the hydrogenated product at 
$540 \mathrm{~m} / \mathrm{z}$. The conversion of HBC during hydrogenation was approximately $70 \%$, whereas during dehydrogenation the conversion back to $\mathrm{HBC}$ from hydrogenated $\mathrm{HBC}$ was approximately $63 \%$. Thus, the hydrogen storage capacity of HBC during hydrogenation was approximately $2.4 \mathrm{wt}$ \%.

\section{Hydrogenation of pentacene with $5 \%$ Rh on carbon catalyst}

A $0.10 \mathrm{~g}$ sample of pentacene (Aldrich) and $0.050 \mathrm{~g}$ of rhodium on carbon catalyst ( $5 \% \mathrm{Rh}$, Acros Organics) were ground with an agate mortar and pestle for 15 minutes until a uniform mixture was formed. The sample was then placed in the differential pressure adsorption unit. The sample was degassed at ambient temperature for 20 minutes under vacuum. Both the sample cell and reference cells were placed under 980 psia (67.6 bar) hydrogen and heated to $150^{\circ} \mathrm{C}$. The hydrogen pressure in the sample cell dropped, relative to the reference cell, for a period of about 8 hours, indicating adsorption of $5.5 \mathrm{wt}$. \% hydrogen by the sample (Figure 2-9). After 14 hours, the cells were cooled to ambient temperature, and the pressure in both cells reduced to 18 psia (1.25 bar). Upon heating both cells to $150^{\circ} \mathrm{C}$, there was a very small increase in the pressure of the sample cell relative to the reference cell, indicating desorption of hydrogen from the sample (Figure 2-10). After 70 hours, the sample had desorbed only 0.15 wt. \% hydrogen ( $2.7 \%$ of the sorbed hydrogen).

\section{$\underline{\text { References }}$}

1. Clar, E. in "Polycyclic Hydrocarbons," Academic Press, 1984, Chapter 6.

2. Davydov, V. Y.; Sheppard, N.; Osawa, E. Int. J. Hydrogen Energy 2004, 29, 1157.

3. Lamartine, R.; Perrin, R. in "Spillover of adsorbed species: proceedings of the international symposium, Lyon-Villeurbanne," September 12-16, 1983, Elsevier: Amsterdam; New York, 1983.

4. Zielinski, J. M.; Coe, C. G.; Nickel, R. J.; Romeo, A. M.; Cooper, A. C.; Pez, G. P. Adsorption 2007, 13, 1.

5. Dotz, F.; Brand, J.D.; Ito, S.; Gherghel, L.; Müllen, K. J. Am. Chem. Soc. 2000, 122, 7707. 


\section{Chapter 3: Carbazole-based Hydrogen Carriers}

\section{Introduction}

According to the molecular structure-based trends affecting the average heat of hydrogenation for multi-ring organic compounds (Chapter 1), carbazole or a derivative of carbazole should present an attractive target for organic liquid carriers. The carbazole framework incorporates both a five-membered ring and a nitrogen atom in the center ring, both beneficial for lowering the heat of hydrogenation. While carbazole has an attractive hydrogen capacity $\left(6.7 \mathrm{wt} . \% \mathrm{H}_{2}\right)$, a melting point of $250^{\circ} \mathrm{C}$ precludes its use as a near-ambient temperature liquid carrier. The well-known and commercially available $\mathrm{N}$-alkylated derivatives of carbazole, $\mathrm{N}$-methylcarbazole (m.p. $90^{\circ} \mathrm{C}$ ) and $\mathrm{N}$-ethylcarbazole (m.p. $70^{\circ} \mathrm{C}$ ), offer substantially lower melting points with modest reductions in the reversible hydrogen capacity of the perhydrogenated compounds (6.2 and 5.7 wt. $\% \mathrm{H}_{2}$, respectively). N-ethylcarbazole (NEC) is used as a raw material for a number of consumer products and is commercially available on a ton scale.

A number of $\mathrm{N}$-alkylcarbazoles and $\mathrm{N}$-arylcarbazoles were evaluated for selectivity and rate for reversible hydrogenation at temperatures $<200^{\circ} \mathrm{C}$. In general, the $\mathrm{N}$-alkylcarbazoles performed well, demonstrating high selectivity for both the hydrogenation and dehydrogenation reactions. While $\mathrm{N}$-arylcarbazole liquid carrier candidates offered the potential for higher hydrogen capacity, assuming a reversible hydrogenation of the $\mathrm{N}$-aryl ring, insurmountable problems with hydrogenation selectivity made their use as hydrogen carriers impractical.

Using perhydro-N-ethylcarbazole as an example, a number of general concepts related to reversible hydrogenation were explored in great detail. These concepts include the thermodynamics of intermediate transformation during dehydrogenation, the effect of isomer distribution on the temperature of dehydrogenation, the variability of rate and selectivity as a function of metal and support in the dehydrogenation catalyst, and the purity of the hydrogen evolved from the carrier during various stages of the dehydrogenation reaction. 


\section{Results and Discussion}

A. Perhydro-N-ethylcarbazole (ph-NEC)

\section{Hydrogenation}

The catalytic hydrogenation of NEC to produce perhydro-N-ethylcarbazole (ph-NEC), can be achieved with very high selectivity and yield, either in solution (e.g., using THF as a solvent) or in the melt using a number of known aromatic hydrogenation catalysts (supported ruthenium and rhodium, Raney nickel). Moderate pressures of hydrogen (e.g., 500-1000 psia $\mathrm{H}_{2}$ ) and moderate temperatures (e.g., 120-190 ${ }^{\circ} \mathrm{C}$ ), conditions which are amenable to large-scale hydrogenation, are used with little variation in the selectivity of the hydrogenation process.

In a typical hydrogenation reaction, a $100 \mathrm{cc}$ stainless steel pressure reactor was loaded with $50 \mathrm{~g} \mathrm{~N}$-ethylcarbazole and $2.0 \mathrm{~g}$ of $5 \%$ ruthenium on lithium aluminate. After the headspace was purged with hydrogen, the hydrogen pressure was increased to $800 \mathrm{psia}$. The reactor was heated to $160^{\circ} \mathrm{C}$, and the hydrogen pressure increased to $1000 \mathrm{psia}$ and stirring to $800 \mathrm{rpm}$. After 2.5 hours, the reactor was cooled to $25^{\circ} \mathrm{C}$, and the contents filtered to remove catalyst. The ph-NEC product is a colorless, free-flowing liquid with a viscosity of 13.5 centipoise and a boiling point of ca. $295^{\circ} \mathrm{C}$ at atmospheric pressure. If desired, ph-NEC can be distilled for purification at $130^{\circ} \mathrm{C}$ at $7 \times 10^{-2}$ torr. GC-MS analysis of ph-NEC samples hydrogenated with a variety of catalysts typically showed $>99 \%$ selectivity for the hydrogenation reaction. Trace impurities, typically found at ppm levels, include perhydrocarbazole and dicyclohexyl.

\section{Simultaneous hydrogenation and alkylation of carbazole}

Carbazole is a readily available, inexpensive precursor to $\mathrm{N}$-alkylcarbazoles. Therefore, it was desirable to develop a one-step, easily scalable route to convert carbazole to the hydrogenated, $\mathrm{N}$-alkylcarbazoles that can be used as liquid carriers.

The hydrogenation of carbazole in alcohols using Raney nickel afforded hydrogenated $\mathrm{N}$-alkylcarbazoles in moderate to high yields, using an inexpensive source of the alkyl 
group (Me, Et, " $\mathrm{Pr}, \mathrm{I}^{\mathrm{Pr}}$ ) as the alcohols (methanol, ethanol, 1-propanol, isopropanol;

Scheme 3-1).
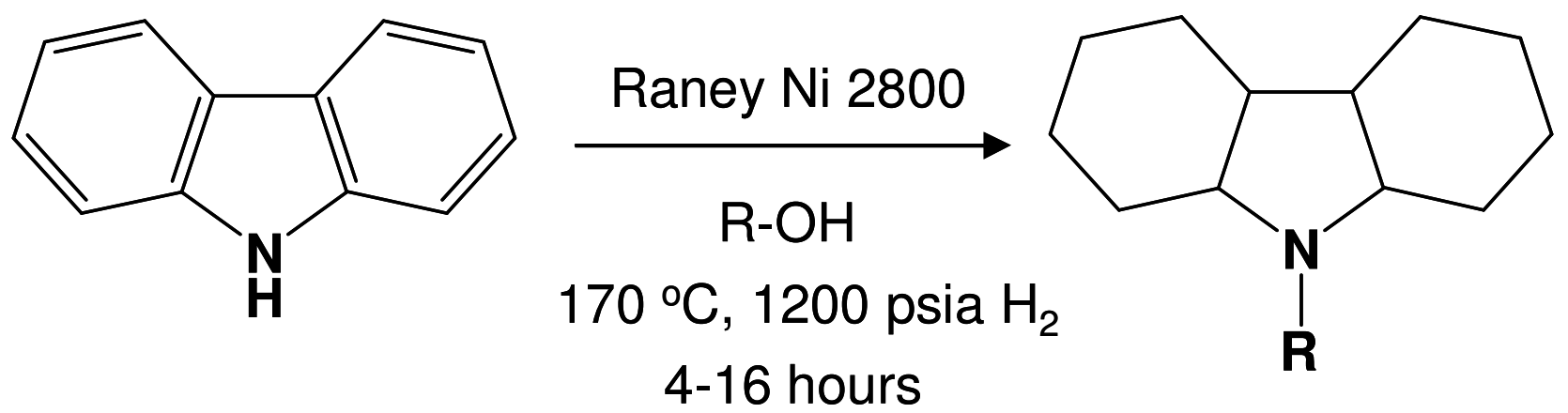

Scheme 3-1. Nickel-catalyzed hydrogenation and N-alkylation of carbazole.

The hydrogenation of carbazole as a 10-33 wt.\% solution in anhydrous alcohols using ca. 5 wt. \% Raney 2800 nickel catalyst produced the desired perhydro-N-alkylcarbazole in high yield with methanol, ethanol, and 1-propanol (Table 3-1). The hydrogenation in isopropanol yielded only $66 \%$ alkylated carbazoles, with the balance present as perhydrogenated carbazole. This is likely due to the much slower reaction rate for catalytic alkylation of carbazole using a secondary alcohol vs. primary alcohols.

Table 3-1. Yields of nickel-catalyzed hydrogenation and N-alkylation of carbazole.

\begin{tabular}{|c|c|c|c|}
\hline Alkyl group & $\begin{array}{c}\text { Yield of perhydro- } \\
\text { N-alkylcarbazole }\end{array}$ & $\begin{array}{c}\text { Yield of partially hydrogenated } \\
\text { N-alkylcarbazole }\end{array}$ & $\begin{array}{c}\text { Hydrogenated } \\
\text { carbazole }\end{array}$ \\
\hline Methyl & $98 \%$ & $2 \%$ & $0 \%$ \\
\hline Ethyl & $97 \%$ & $3 \%$ & $0 \%$ \\
\hline n-propyl & $97.5 \%$ & $2.5 \%$ & $34 \%$ \\
\hline iso-propyl & $59 \%$ & $7 \%$ & \\
\hline
\end{tabular}




\section{Formation of ph-NEC isomers from catalytic hydrogenation}

In general, the hydrogenation of liquid carriers may yield more than one product of the same overall chemical composition. There may be found isomers of the product molecule that differ only in the relative disposition of its carbon-hydrogen bonds or of other atoms or groups of atoms in the molecule. The isomers will each have different energies (standard heats of formation, $\Delta \mathrm{H}_{\mathrm{f}}^{\circ}$ ), the thermodynamically most stable isomer having the lowest $\Delta \mathrm{H}^{\circ}$. This occurs in the hydrogenation of naphthalene, which can result in the formation of the two isomers, both saturated molecules, cis-decalin and trans-decalin, which differ in the relative disposition of the two $\mathrm{C}-\mathrm{H}$ linkages, along the common carbon-carbon bond. Commercial decalin contains a 67:33 distribution of cis and trans isomers. The trans isomer, where the $\mathrm{C}-\mathrm{H}$ bonds are on opposite sides of the common C-C linkage, is more stable by ca. $12.5 \mathrm{~kJ} / \mathrm{mol}$. Larger, fully hydrogenated piconjugated molecules, and particularly those containing nitrogen heteroatoms, can potentially have a myriad of isomers which may differ in energy $\left(\Delta \mathrm{H}^{\circ}\right)$ by several $\mathrm{kcal} / \mathrm{mol}$ vis-à-vis the most stable configuration.

Under our typical hydrogenation conditions, ph-NEC was produced as a mixture of 3 major isomers, as observed by GC-MS, with occasional trace amounts of other isomers. As shown in Figure 3-1, ph-NEC can exist as a number of isomers with different cis or trans configurations of the hydrogen atoms on the 5-membered ring. DFT calculations of the relative energies of the possible isomers show that the most stable isomer has a trans,trans configuration of the hydrogen atoms on the 5-membered ring (Figure 3-2). The least stable isomer, cis-syn-cis-perhydro-N-ethylcarbazole, has a calculated electronic energy that is $61 \mathrm{~kJ} / \mathrm{mole}$ higher than the trans, trans configuration. 

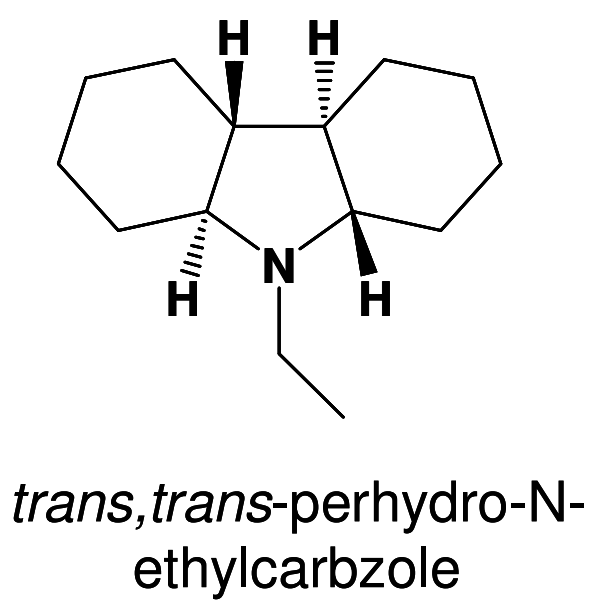
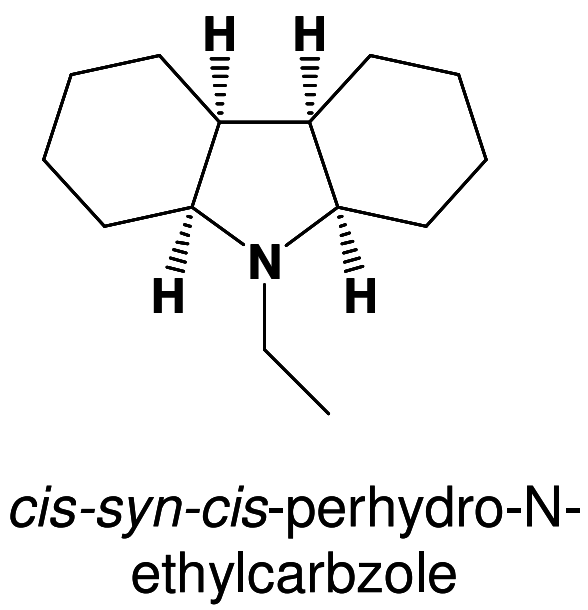

Figure 3-1. Two of the possible isomers of perhydro-N-ethylcarbazole.

In practice, the formation of isomers will depend on the conditions under which the catalytic hydrogenation of the liquid carrier candidate is conducted; lower hydrogenation reaction temperatures favor formation of the less stable (i.e., more energetic), conformer molecules. Conversely, a hydrogenation of the pi-conjugated substrate at higher temperatures results in an equilibrium (i.e., a lower energy distribution of conformers). The formation of such non-equilibrium conformers provides a means of desirably lowering the hydrogenation enthalpy $\Delta \mathrm{H}_{H_{2}}^{o}$ of the pi-conjugated unsaturated molecule by making its hydrogenation product less stable, thus enabling the dehydrogenation process to occur at a lower temperature. In some cases, depending on the catalytic dehydrogenation mechanism, there may be the additional advantage of a kinetically more facile dehydrogenation of the more energetic non-equilibrium conformers. 


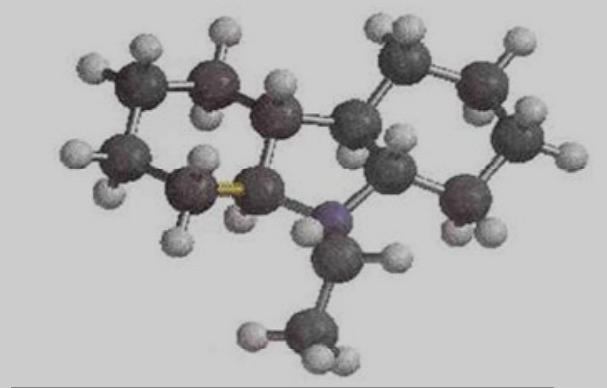

$\Delta \mathrm{E}=0 \mathrm{~kJ} / \mathrm{mole}$

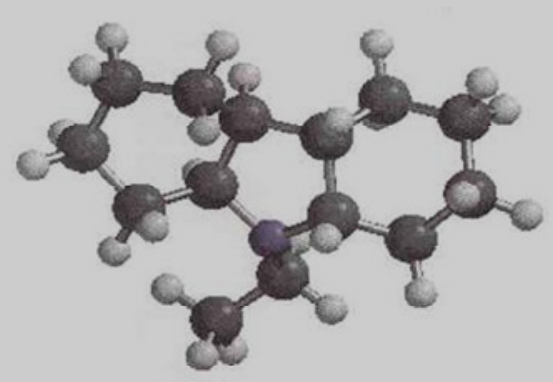

$\Delta \mathrm{E}=36 \mathrm{~kJ} / \mathrm{mole}$

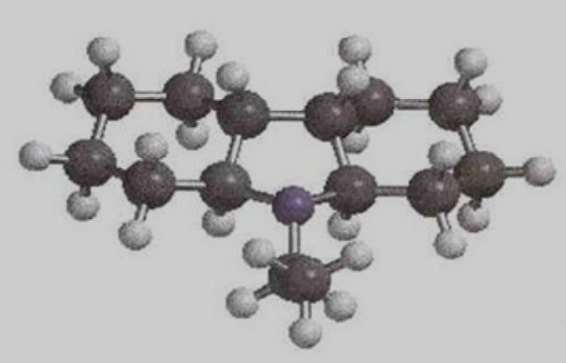

$\Delta \mathrm{E}=11 \mathrm{~kJ} / \mathrm{mole}$

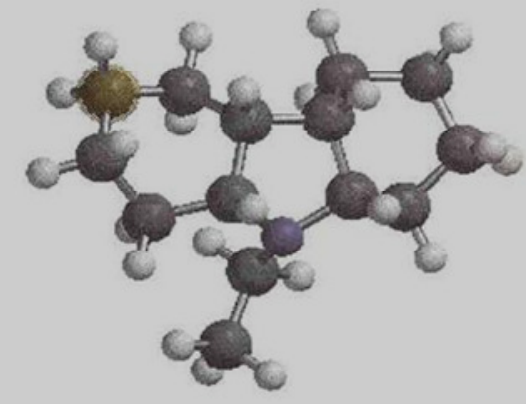

$\Delta \mathrm{E}=61 \mathrm{~kJ} / \mathrm{mole}$

Figure 3-2. Relative electronic energies of ph-NEC isomers.

\section{Dehydrogenation of perhydro-N-ethylcarbazole}

In a typical dehydrogenation experiment, a slurry of the dehydrogenation catalyst (15 wt. \% catalyst) in liquid ph-NEC was ramped at a controlled rate to a temperature of $150-200^{\circ} \mathrm{C}$ under $1 \mathrm{~atm}$. hydrogen while the slurry was stirred at a rate of 500-800 rpm. The rate of hydrogen release from the reactor was measured by a set of flow meters (see experimental details).

Dehydrogenation experiments that were suspended after partial conversion yielded information on the stepwise conversion of ph-NEC. GC-MS analysis of intermediates found during various stages of conversion for the dehydrogenation of ph-NEC suggest that the dehydrogenation proceeds in steps liberating two molecules of hydrogen. The 
stepwise thermodynamics for conversion through these intermediates were calculated using ab initio methods (Scheme 3-2).

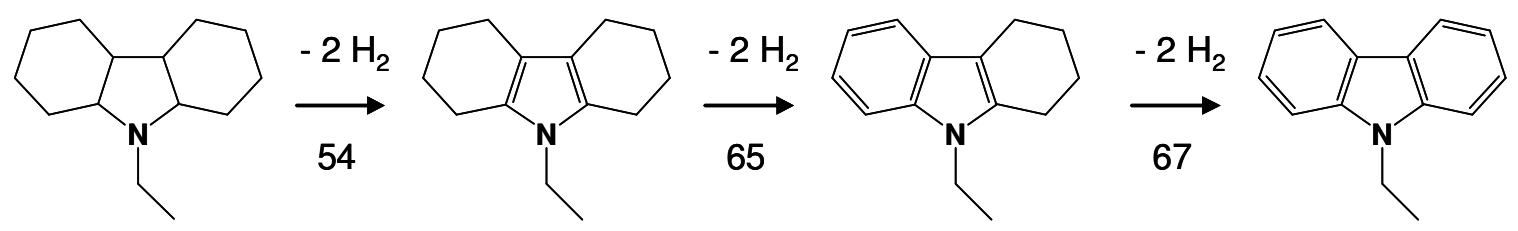

Scheme 3-2. Calculated electronic energy change $\left(\Delta \mathrm{E} ; \mathrm{kJ} / \mathrm{mol} \mathrm{H}_{2}\right)$ for the stepwise dehydrogenation of ph-NEC (computational details in Appendix A). The trans,trans isomer was used to calculate the electronic energy of ph-NEC.

Our initial dehydrogenation studies involved repeated hydrogenation and dehydrogenation of NEC in the same pressure-capable stirred tank reactor system. These cycling studies, which were designed to test the stability of the NEC and catalyst, yielded promising results regarding liquid carrier and catalyst lifetime. During five complete cycles of hydrogenation and dehydrogenation at hydrogen storage levels of $>5.5 \mathrm{wt}$. \% for each cycle, there was no detectable chemical degradation of the NEC and no systematic loss in the rates of the hydrogenation catalyst $\left(\mathrm{Ru} / \mathrm{Al}_{2} \mathrm{O}_{3}\right)$ or dehydrogenation catalyst $\left(\mathrm{Pd} / \mathrm{Al}_{2} \mathrm{O}_{3}\right)$. The very low volatility of $\mathrm{N}$-ethylcarbazole and the hydrogenated intermediates allowed us to perform the cycling experiment in a single small stirred tank reactor system, with no significant loss of liquid due to evaporation during the extended periods of heating and hydrogen evolution. In the cycling experiment, the reactor was loaded with N-ethylcarbazole $(5 \mathrm{~g})$, $\mathrm{Ru} / \mathrm{Al}_{2} \mathrm{O}_{3}$ (200 mg, hydrogenation catalyst), and $\mathrm{Pd} / \mathrm{Al}_{2} \mathrm{O}_{3}$ (200 mg, dehydrogenation catalyst). Hydrogenation was accomplished by stirring the slurry of catalyst in neat $\mathrm{N}$ ethylcarbazole at $170^{\circ} \mathrm{C}$ under 1000 psia hydrogen for several hours. After cooling to ambient temperature, the reactor was vented down to 15 psia hydrogen pressure, and the temperature ramped from ambient to $197^{\circ} \mathrm{C}$. Hydrogen evolution under 15 psia hydrogen was recorded using calibrated flow meters and started during the temperature ramp, continuing for a period of $2-3$ hours while holding at $197^{\circ} \mathrm{C}$. This hydrogenation/ dehydrogenation cycle was repeated a total of five times, each time yielding over $5.5 \mathrm{wt}$ \% according to the measured hydrogen flow (Figure 3-3). 


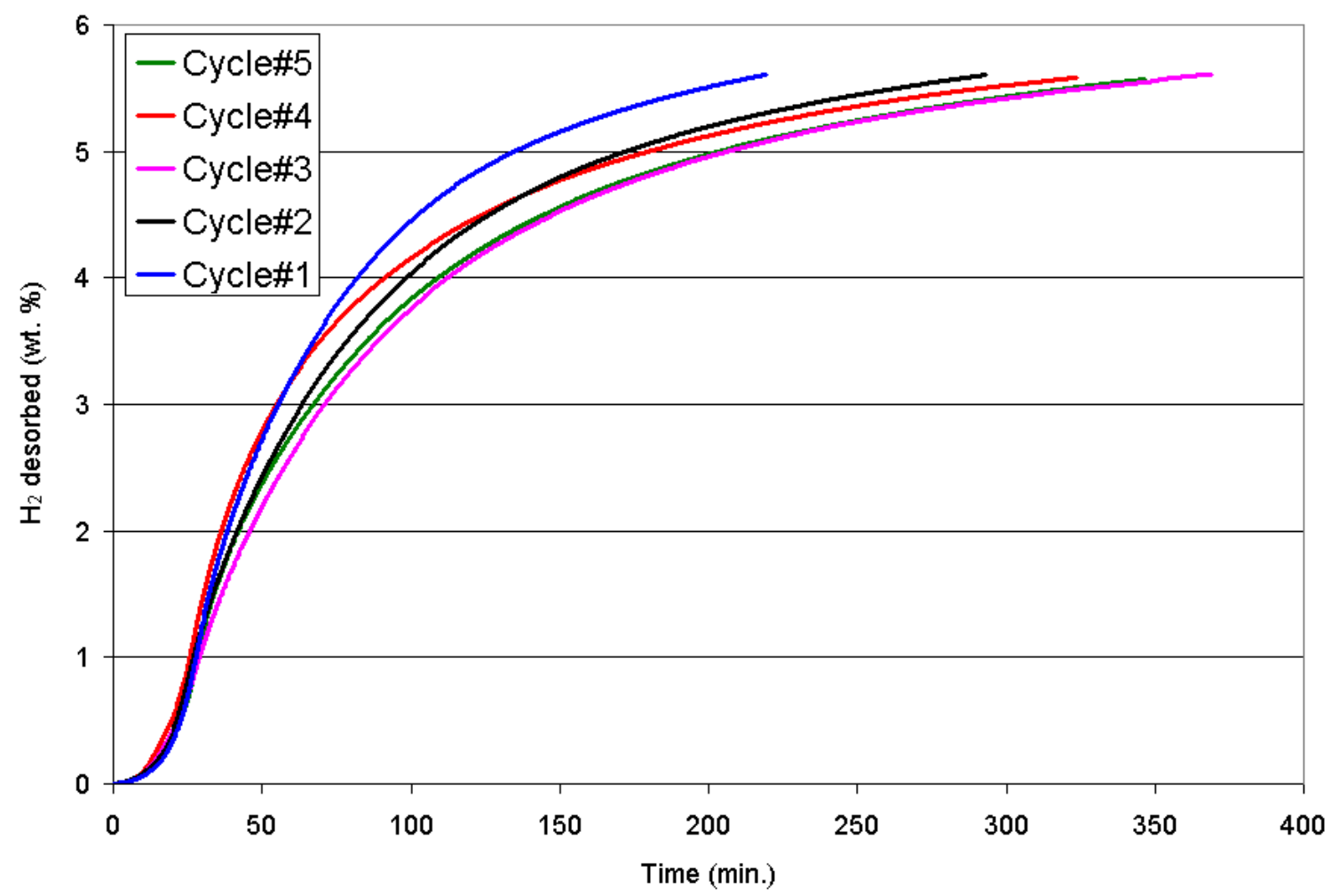

Figure 3-3. Five sequential dehydrogenation steps of hydrogenation/dehydrogenation cycling experiments of NEC.

\section{Effect of isomer distribution on NEC catalytic dehydrogenation}

Two hydrogenated $\mathrm{N}$-ethylcarbazole mixtures with varying perhydro-N-ethylcarbazole isomer distributions were prepared by altering the temperature of hydrogenation. Mixture A (prepared by hydrogenating $\mathrm{N}$-ethylcarbazole at $120^{\circ} \mathrm{C}$ ) contained $90 \%$ perhydro-N-ethylcarbazole (molecular weight 207) and 10\% octahydro-N-ethylcarbazole (molecular weight 203). The perhydro-N-ethylcarbazole fraction of mixture A consisted of $90 \%$ of two less stable isomers and $10 \%$ of the more stable isomer. Mixture $B$ (obtained from a hydrogenation of $\mathrm{N}$-ethylcarbazole conducted at $170^{\circ} \mathrm{C}$ ), contained 100\% perhydro-N-ethylcarbazole (molecular weight 207). The perhydro-Nethylcarbazole fraction of mixture B consisted of $19 \%$ of two less stable isomers and $81 \%$ of the more stable isomer. The mixtures were dehydrogenated by temperature ramping of a slurry of dehydrogenation catalyst (4 wt. \%; $\mathrm{Pt} / \mathrm{Re} / \mathrm{Al}_{2} \mathrm{O}_{3}$ ) to $150-200^{\circ} \mathrm{C}$ 
under 1 atm. hydrogen while they were stirred at a rate of $700 \mathrm{rpm}$. The hydrogen flow data shows a clear difference between the two mixtures (Figure 3-4).

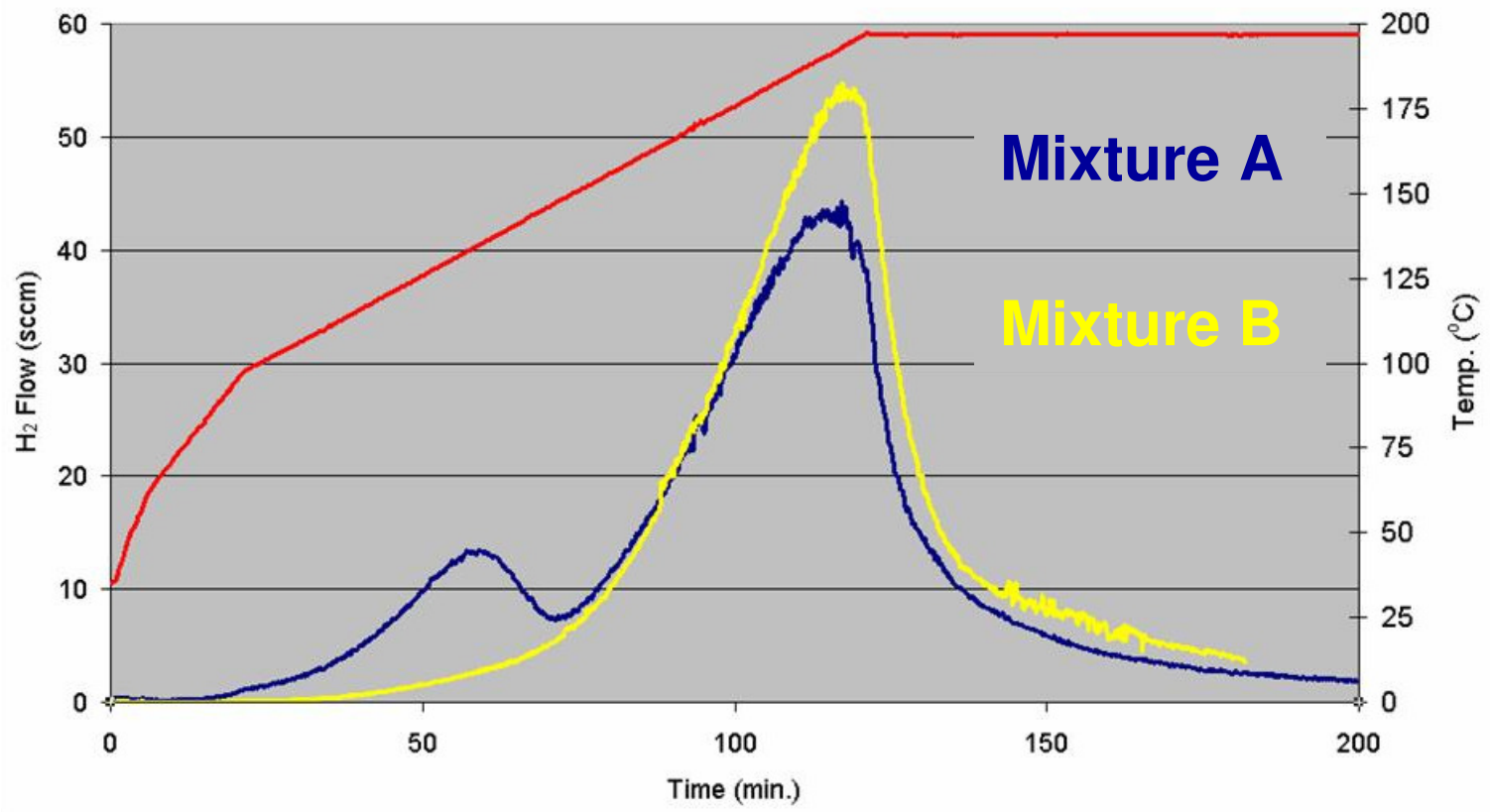

Figure 3-4. Hydrogen flow data for dehydrogenation of two mixtures of ph-NEC isomers using $\mathrm{Pt} / \mathrm{Re} / \mathrm{Al}_{2} \mathrm{O}_{3}$ catalyst.

Mixture A, mostly comprising less stable ph-NEC isomers, demonstrated a much lower temperature onset of hydrogen flow and showed two peaks in the hydrogen flow profile during temperature ramping. The origin of the low temperature peak is likely due to the dehydrogenation of ph-NEC to the octahydro-N-ethylcarbazole intermediate shown in Scheme 3-2. The temperature of this peak is greatly influenced by the energy of the ph-NEC isomer. Since the isomers are "lost" after the dehydrogenation of the fivemembered ring, the remaining hydrogen release for all isomers follows an identical profile, as observed in the large hydrogen flow peak at in the end of the temperature ramp (Figure 3-4).

Mixture B, mainly comprising stable ph-NEC isomers (e.g., trans, trans-perhydro-Nethylcarbazole), demonstrated a higher temperature onset of hydrogen flow and showed 
only a single higher temperature peak in the hydrogen flow profile during temperature ramping. The same trends were observed while using a palladium on alumina dehydrogenation catalyst (Table 3-2). Mixture A consistently showed a lower temperature onset of dehydrogenation (i.e., temperature for $1 \%$ and $5 \%$ conversion).

Table 3-2. Dehydrogenation of ph-NEC mixtures with different isomer distributions. Temp. (1\%) and Temp. (5\%) are the temperatures at which there are $1 \%$ and $5 \%$ conversions from ph-NEC to NEC. Conversion at $150^{\circ} \mathrm{C}$ is the percentage of conversion from ph-NEC to NEC at the point when the temperature ramp reached $150^{\circ} \mathrm{C}$.

\begin{tabular}{|c|c|c|c|c|}
\hline Catalyst & Mixture & $\begin{array}{c}\text { Temp. } \\
(1 \% \text { conversion })\end{array}$ & $\begin{array}{c}\text { Temp. } \\
(5 \% \text { conversion })\end{array}$ & $\begin{array}{c}\text { Conversion } \\
\text { at } 150^{\circ} \mathrm{C}\end{array}$ \\
\hline $5 \% \mathrm{Pd} / \mathrm{Al}_{2} \mathrm{O}_{3}$ & $\mathrm{~A}$ & $109^{\circ} \mathrm{C}$ & $131^{\circ} \mathrm{C}$ & $8.8 \%$ \\
\hline $5 \% \mathrm{Pd} / \mathrm{Al}_{2} \mathrm{O}_{3}$ & $\mathrm{~B}$ & $131^{\circ} \mathrm{C}$ & $160{ }^{\circ} \mathrm{C}$ & $2.9 \%$ \\
\hline $\begin{array}{c}2.9 \% \mathrm{Pt} / 0.3 \% \mathrm{Re} / \\
\mathrm{Al}_{2} \mathrm{O}_{3}\end{array}$ & $\mathrm{~A}$ & $108^{\circ} \mathrm{C}$ & $126^{\circ} \mathrm{C}$ & $14.8 \%$ \\
\hline $\begin{array}{c}2.9 \% \mathrm{Pt} / \mathrm{O}_{3} \% \mathrm{Re} / \\
\mathrm{Al}_{2} \mathrm{O}_{3}\end{array}$ & $\mathrm{~B}$ & $129^{\circ} \mathrm{C}$ & $153^{\circ} \mathrm{C}$ & $4.0 \%$ \\
\hline
\end{tabular}

\section{Dehydrogenation catalyst testing}

A large variety and number of potential dehydrogenation catalysts were tested for use with ph-NEC (Figure 3-5 and Table 3-3). Hydrogen flow data from experiments with identical temperature ramp and hold conditions was used to determine the relative rates of hydrogen release. GC-MS analysis of the dehydrogenated NEC was utilized to verify the hydrogen flow data (by calculating the theoretical amount of hydrogen released as a function of the distribution of products and hydrogen content of each product). The GCMS analysis also was used to determine the selectivity of the dehydrogenation reaction (i.e., screening for the presence of byproducts).

The selection of potential heterogeneous dehydrogenation catalysts was based upon systematic variation of several variables in the catalyst formulation, namely, the 
supported metal(s), the identity of the support material, and the amount of metal present in the catalyst.

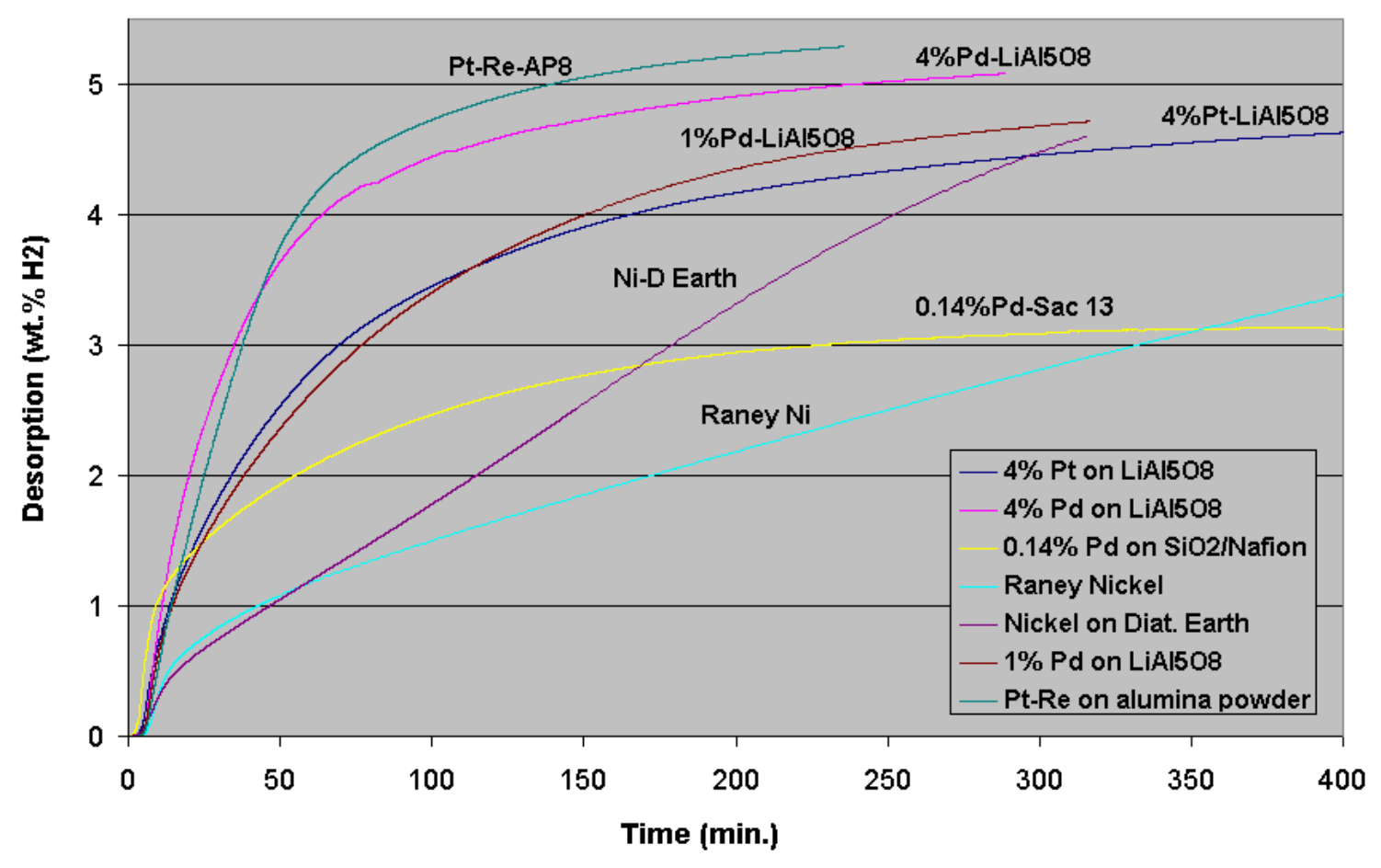

Figure 3-5. An overlay of ph-NEC dehydrogenation data from a range of catalysts.

In general, the most effective catalysts for the dehydrogenation of ph-NEC contained platinum-group metals, specifically platinum and palladium. A large number of bimetallic catalysts (e.g., platinum/rhenium) were also evaluated. It is noteworthy that base metals such as nickel did show some dehydrogenation activity, albeit at reduced levels relative to the platinum group metals. 
Table 3-3. Assay of catalysts for dehydrogenation of perhydro-N-ethylcarbazole.

\begin{tabular}{|c|c|c|c|c|c|}
\hline Catalyst & $\begin{array}{c}\text { Maximum } \\
\mathrm{H}_{2} \text { Flow } \\
\text { Rate (cc } \\
\mathrm{H}_{2} / \mathrm{min} . / \\
\text { g catalyst) }\end{array}$ & $\begin{array}{c}\text { Flow Rate } \\
\text { at } 50 \% \\
\text { conversion } \\
\text { (cc } \mathrm{H}_{2} / \mathrm{min} . / \\
\text { g catalyst) }\end{array}$ & $\begin{array}{c}\text { Flow Rate } \\
\text { at } 70 \% \\
\text { conversion } \\
\text { (ccH }{ }_{2} / \text { min./ } \\
\text { g catalyst) }\end{array}$ & $\begin{array}{l}\text { Time }(\min .) \\
\text { required to } \\
\text { reach } 50 \% \\
\text { conversion }\end{array}$ & $\begin{array}{c}\text { Final } \mathrm{H}_{2} \\
\text { storage } \\
\text { capacity, } \\
\text { GC-MS } \\
\text { data } \\
\left(\text { wt. } \% \mathrm{H}_{2}\right)\end{array}$ \\
\hline $\begin{array}{l}3.6 \% \mathrm{Pt} / 1.7 \% \mathrm{Sn} \\
\text { on } \mathrm{Al}_{2} \mathrm{O}_{3}\end{array}$ & 497 & 9 & 4 & 233 & 3.9 \\
\hline $\begin{array}{l}2.0 \% \mathrm{Pt} / 1.6 \% \mathrm{Pd} \\
\text { on } \mathrm{Al}_{2} \mathrm{O}_{3}\end{array}$ & 457 & 51 & 9 & 122 & 5.0 \\
\hline $\begin{array}{l}2.83 \% \text { Pt on } \\
\mathrm{Al}_{2} \mathrm{O}_{3}\end{array}$ & 212 & 80 & 10 & 102 & 5.0 \\
\hline $\begin{array}{l}\text { 2.91\%Pton } \\
\mathrm{LiAl}_{5} \mathrm{O}_{8}\end{array}$ & 766 & 96 & 22 & 64 & 5.3 \\
\hline $\begin{array}{l}2.56 \% \text { Pt on } \\
\mathrm{Li}-\mathrm{Al}_{2} \mathrm{O}_{3}\end{array}$ & 175 & 87 & 13 & 122 & 5.0 \\
\hline $\begin{array}{l}3.96 \% \mathrm{Pt} \text { on } \\
\text { Carbon- } \mathrm{Al}_{2} \mathrm{O}_{3}\end{array}$ & 57 & $<15$ & $<10$ & $>400$ & 3.2 \\
\hline $\begin{array}{l}1.34 \% \mathrm{Pt} / \mathrm{Re} \text { on } \\
\mathrm{Al}_{2} \mathrm{O}_{3}\end{array}$ & 680 & 111 & 19 & 65 & 4.9 \\
\hline $\begin{array}{l}2.13 \% \mathrm{Pt} \text { on } \\
\mathrm{Nafion} \text { coated } \\
\mathrm{SiO}_{2}\end{array}$ & 570 & 18 & $<10$ & 231 & 3.6 \\
\hline $\begin{array}{l}5.5 \% \mathrm{Pd} \text { on } \\
\mathrm{Al}_{2} \mathrm{O}_{3}\end{array}$ & 475 & 87 & 14 & 66 & 5.2 \\
\hline $\begin{array}{l}2.67 \% \mathrm{Pd} \text { on } \\
\text { Carbon- } \mathrm{Al}_{2} \mathrm{O}_{3}\end{array}$ & 197 & $<21$ & $<10$ & $>300$ & 3.8 \\
\hline $\begin{array}{l}3.36 \% \mathrm{Pd} \text { on Li- } \\
\mathrm{Al}_{2} \mathrm{O}_{3}\end{array}$ & 403 & 89 & 48 & 77 & 5.6 \\
\hline $\begin{array}{l}\text { 3.89\% } \% \text { d } \\
\text { on } \mathrm{LiAl}_{5} \mathrm{O}_{8}\end{array}$ & 1038 & 254 & 86 & 35 & 5.7 \\
\hline $\begin{array}{l}0.14 \% \mathrm{Pd} \text { on } \\
\mathrm{Nafion} \text { coated } \\
\mathrm{SiO}_{2}\end{array}$ & 1040 & 15 & $<10$ & 187 & 4.3 \\
\hline
\end{tabular}


The relative activity of three large sets of ph-NEC dehydrogenation catalysts is shown in Figures 3-6 and 3-7.

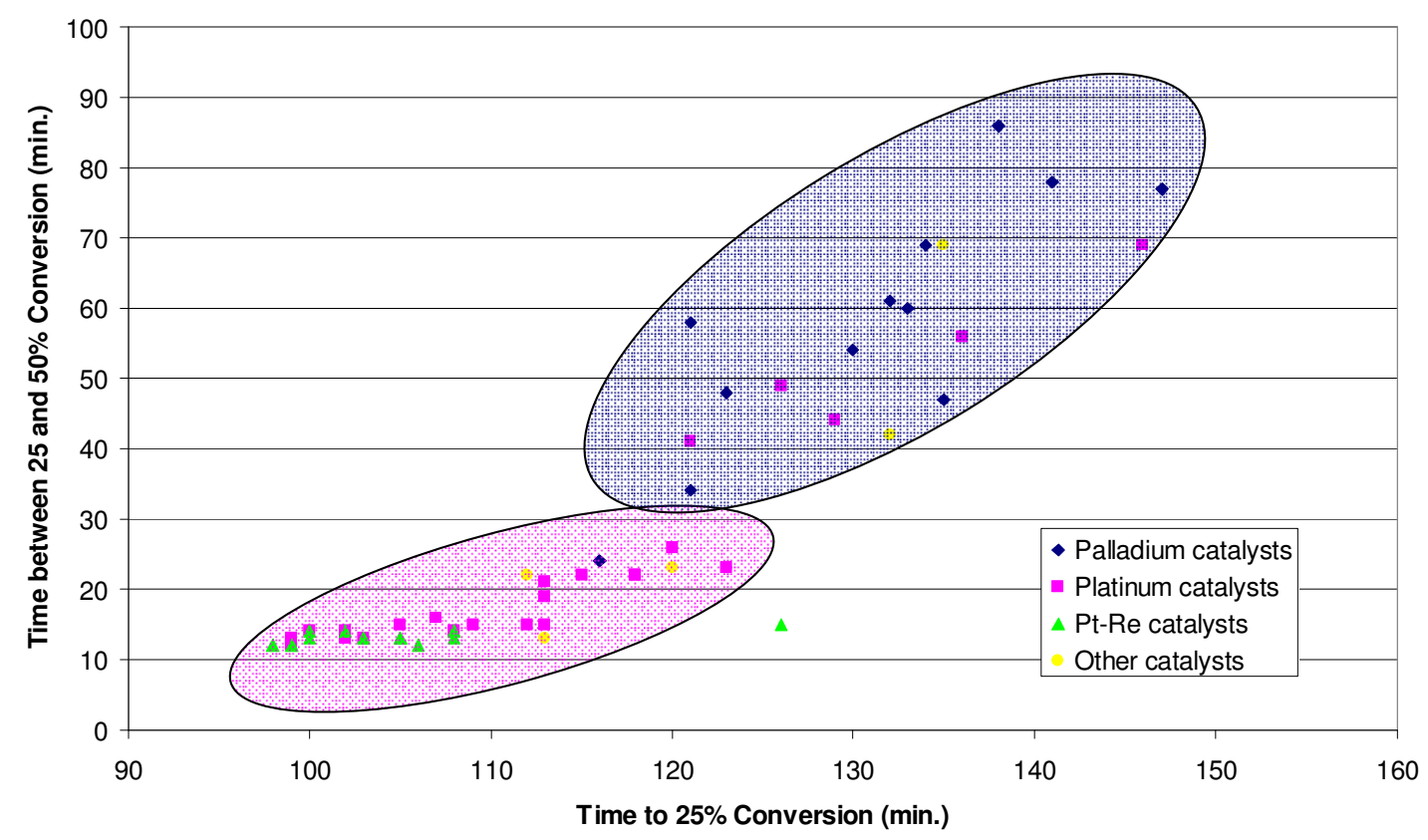

Figure 3-6. Plot of time required to reach $25 \%$ conversion vs. the time required for conversion from $25 \%$ to $50 \%$ for ph-NEC. See the experimental details for information on the temperature ramping profile.

From Figure 3-6, it is apparent that the first several steps of the dehydrogenation (i.e., ph-NEC converting to octahydro-NEC with additional conversion to tetrahydroNEC) proceeds most rapidly with platinum-based catalysts, including Pt/Re bimetallic catalysts. Palladium-based catalysts are slower than the platinum-based catalysts, particularly for the $25-50 \%$ conversion. Catalysts that do not contain either Pt or Pd that show some dehydrogenation activity include pure iridium and nickel-based catalysts. 


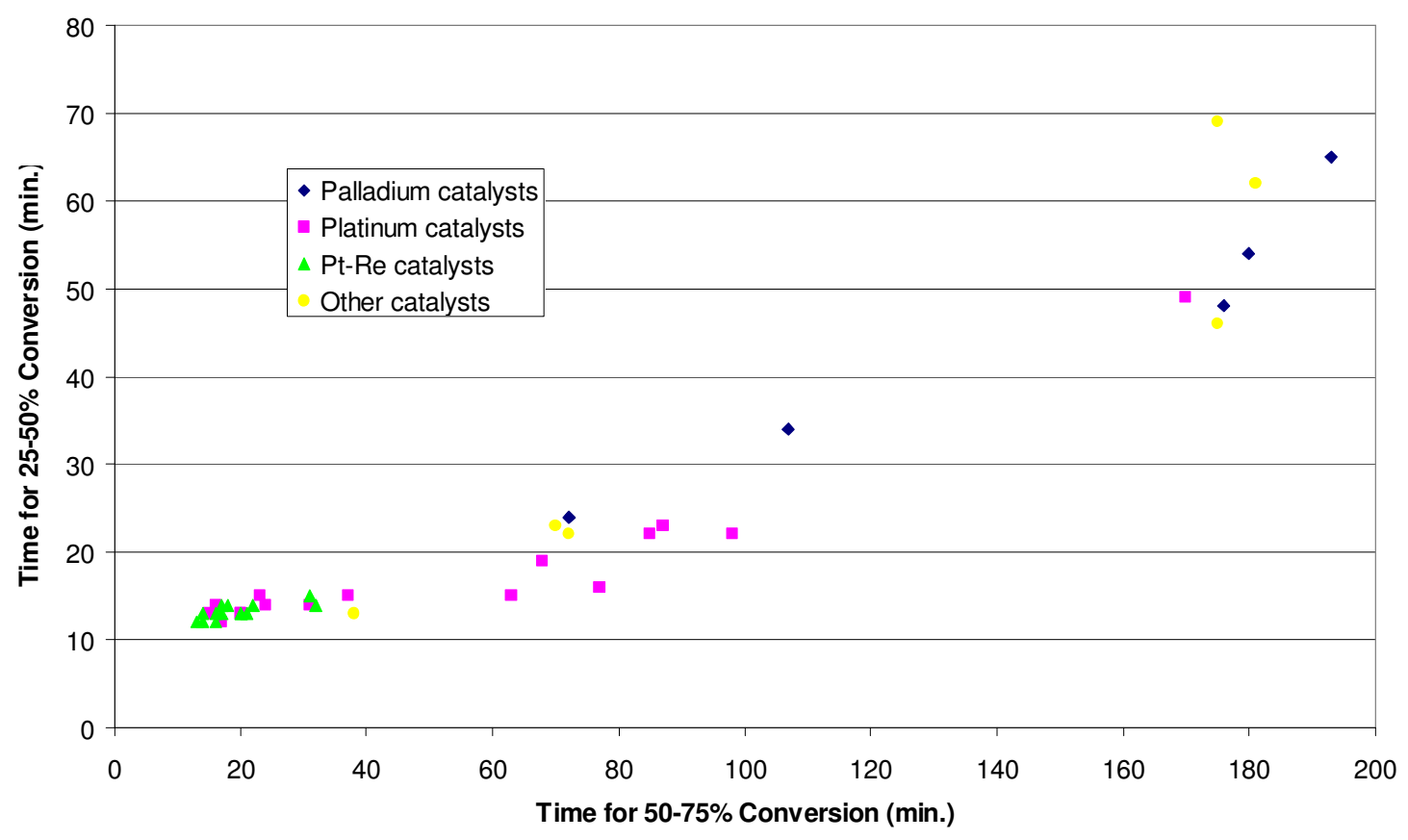

Figure 3-7. Plot of time required for conversion from $25 \%$ to $50 \%$ vs. the time required for conversion from $50 \%$ to $75 \%$ for ph-NEC. See the experimental details for information on the temperature ramping profile.

From the data presented in Figure 3-7, it is observed that the intermediate and final steps of the dehydrogenation (i.e., octahydro-NEC converting to tetrahydro-NEC with additional conversion to NEC) proceeds most rapidly with platinum-based catalysts, especially $\mathrm{Pt} / \mathrm{Re}$ bimetallic catalysts. Again, palladium-based catalysts are slower than the platinum-based catalysts, particularly for the $50-75 \%$ conversion.

In an effort to extend our understanding of the low temperature dehydrogenation catalysis, we attempted to modify the catalytic dehydrogenation of ph-NEC with various $\mathrm{acid} / \mathrm{base} / \mathrm{solvent}$ additives. The dehydrogenation of ph-NEC with a $5 \% \mathrm{Pd}$ on carbon catalyst was performed with $3 \mathrm{~mol}$. \% additive (unless otherwise noted), which was added to the liquid before the reactor was charged. Figure 8 summarizes the results of this study. 


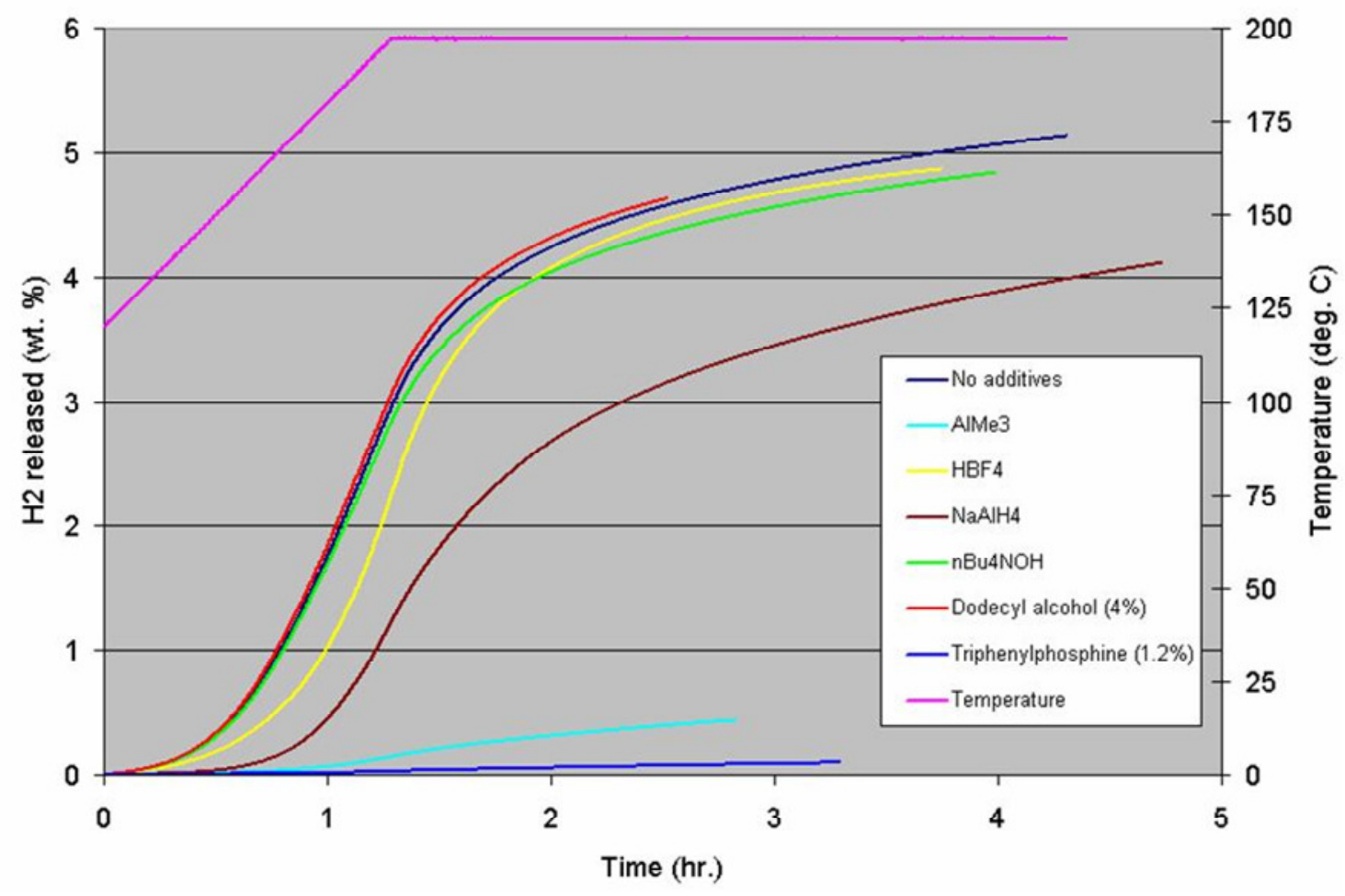

Figure 3-8. Dehydrogenation of ph-NEC in the presence of various additives.

The results show that added alcohol solvent and strong Bronsted acids or bases have very little effect on the dehydrogenation rate. With the addition of $\mathrm{HBF}_{4}$, there was formation of white solid in the liquid (the ammonium salt of perhydro- $\mathrm{N}$-ethylcarbazole). The addition of an insoluble hydride $\left(\mathrm{NaAlH}_{4}\right)$, which can also be considered a Bronsted base, lowered the dehydrogenation rate.

Lewis acids and bases had a larger negative effect on the dehydrogenation rate. The addition of $1.2 \mathrm{~mol}$ \% triphenylphosphine (a strong Lewis base donor) severely affected the dehydrogenation, presumably due to competitive binding of the dehydrogenation catalyst. The addition of a Lewis acid (trimethylaluminum, $3 \mathrm{~mol}$. \%) to perhydro-Nethylcarbazole did not result in precipitate, but did lower the dehydrogenation rate. In this case, it is less clear why the dehydrogenation rate is lowered, except that an acidbase adduct of $\mathrm{N}$-ethylcarbazole and trimethylaluminum would be expected, which may 
interfere with the reversible adsorption of the N-ethylcarbazole with the heterogeneous catalyst surfaces.

\section{Measurements of hydrogen purity}

The dehydrogenation catalyst testing equipment was reconfigured during the project so that the hydrogen produced during dehydrogenation catalyst testing could be analyzed in real time via gas chromatography. The system was calibrated using a gas standard comprising methane, ethane and propane in helium. Several catalysts were chosen from our dehydrogenation catalyst library and tested using our standard heating protocols. The hydrogen effluent was sampled at multiple points during the standard heating profile for dehydrogenation. The flow data and corresponding GC data are shown in Tables 3-4a-e. The data in blue was sampled at a $\mathrm{H}_{2}$ flow rate of $5 \mathrm{sccm}$ during the temperature ramp (low conversion). The data in red was collected at the peak $\mathrm{H}_{2}$ flow rate, typically at the conclusion of the temperature ramp (moderate conversion). The data in black was sampled at a $\mathrm{H}_{2}$ flow rate of $5 \mathrm{sccm}$ near the end of the isothermal period at $200^{\circ} \mathrm{C}$ (high conversion). These sampling points were chosen in order to assay intermediates at low conversion (ph-NEC and octahydro-NEC), moderate conversion (primarily octahydro-NEC and tetrahydro-NEC), and high conversion (primarily tetrahydro-NEC and NEC).

In general, it is clear that the palladium catalysts produce fewer total hydrocarbon impurities than the platinum-based catalysts. However, there is little difference between activated carbon and alumina supports for the same metal. The total impurity analysis in Table 3-4 is based on methane, ethane and propane only. Other unidentified peaks (<50 ppm) were not calibrated. 
Table 3-4a. Dehydrogenation of ph-NEC with palladium on alumina. The table shows the temperature and hydrogen flow rate at the time when the samples were analyzed.

\begin{tabular}{|c|c|c|c|}
\hline Temp $\left.\mathbf{~}^{\mathbf{}} \mathbf{C}\right)$ & $\begin{array}{c}\mathbf{H}_{\mathbf{2}} \text { Flow } \\
\text { (sccm) }\end{array}$ & $\begin{array}{c}\text { Methane } \\
\text { Conc. }(\mathbf{p p m})\end{array}$ & $\begin{array}{c}\text { Ethane } \\
\text { Conc. }(\mathbf{p p m})\end{array}$ \\
\hline 144 & 5.0 & 177 & 2 \\
147 & 6.4 & 164 & 10 \\
149 & 7.3 & 137 & 19 \\
152 & 8.1 & 99 & 31 \\
154 & 9.0 & 80 & 34 \\
197 & 45.0 & & 1 \\
197 & 39.0 & & 13 \\
197 & 35.0 & & 23 \\
197 & 30.0 & & 23 \\
197 & 26.0 & & 25 \\
197 & 5.0 & & 44 \\
197 & 4.6 & & 47 \\
197 & 4.4 & & 48 \\
197 & 4.1 & & 48 \\
197 & 3.9 & & \\
\hline
\end{tabular}

Table 3-4b. Dehydrogenation of ph-NEC with platinum on alumina. The table shows the temperature and hydrogen flow rate at the time when thesamples were analyzed.

\begin{tabular}{|c|c|c|c|}
\hline $\begin{array}{c}\text { Temp } \\
\left({ }^{\circ} \mathbf{C}\right)\end{array}$ & $\begin{array}{c}\mathbf{H}_{2} \text { Flow } \\
(\mathbf{s c c m})\end{array}$ & $\begin{array}{c}\text { Methane } \\
\text { Conc. }(\mathbf{p p m})\end{array}$ & $\begin{array}{c}\text { Ethane } \\
\text { Conc. }(\mathbf{p p m})\end{array}$ \\
\hline 144 & 5 & 196 & 44 \\
147 & 6.4 & 194 & 163 \\
153 & 7.3 & 188 & 374 \\
155 & 8.1 & 178 & 631 \\
157 & 9 & 169 & 991 \\
197 & 58 & & 2723 \\
197 & 45 & & 2865 \\
197 & 32.9 & & 3327 \\
197 & 22.8 & & 4146 \\
197 & 17.5 & & 5093 \\
197 & 5 & & 7244 \\
197 & 4.8 & & 7350 \\
197 & 5.5 & & 7398 \\
197 & 4.9 & & 7355 \\
197 & 4 & & 7343 \\
\hline
\end{tabular}


Table 3-4c. Dehydrogenation of ph-NEC with platinum/rhenium on alumina.

The table shows the temperature and hydrogen flow rate at the time when the samples were analyzed.

\begin{tabular}{|c|c|c|c|}
\hline $\begin{array}{c}\text { Temp } \\
\left({ }^{\circ} \mathbf{C}\right)\end{array}$ & $\begin{array}{c}\mathbf{H}_{2} \text { Flow } \\
\text { (sccm) }\end{array}$ & $\begin{array}{c}\text { Methane } \\
\text { Conc. (ppm) }\end{array}$ & $\begin{array}{c}\text { Ethane } \\
\text { Conc. (ppm) }\end{array}$ \\
\hline 149 & 5 & 163 & 71 \\
151 & 5.7 & 136 & 312 \\
154 & 6.5 & 158 & 716 \\
157 & 7.6 & 141 & 1142 \\
159 & 9 & & 1702 \\
197 & 62 & & 2180 \\
197 & 45.6 & & 2079 \\
197 & 33 & & 2229 \\
197 & 24 & & 2604 \\
197 & 17.3 & & 3154 \\
197 & 5 & & 6073 \\
197 & 4.6 & & 6238 \\
197 & 4.3 & & 6349 \\
197 & 4.1 & & 6425 \\
197 & 3.7 & & 6448 \\
\hline
\end{tabular}

Table 3-4d. Dehydrogenation of ph-NEC with platinum on carbon. The table shows the temperature and hydrogen flow rate at the time when the samples were analyzed.

\begin{tabular}{|c|c|c|c|}
\hline $\begin{array}{c}\text { Temp } \\
\left({ }^{\circ} \mathbf{C}\right)\end{array}$ & $\begin{array}{c}\mathbf{H}_{\mathbf{2}} \text { Flow } \\
(\mathbf{s c c m})\end{array}$ & $\begin{array}{c}\text { Methane } \\
\text { Conc. (ppm) }\end{array}$ & $\begin{array}{c}\text { Ethane } \\
\text { Conc. (ppm) }\end{array}$ \\
\hline 154 & 5 & 171 & 15 \\
157 & 6.7 & 214 & 25 \\
160 & 8.2 & 335 & 591 \\
163 & 10.3 & 319 & 1594 \\
165 & 12 & 229 & 2140 \\
197 & 38 & 183 & 3052 \\
197 & 35 & 190 & 3171 \\
197 & 30 & 193 & 3218 \\
197 & 27.7 & 194 & 3236 \\
197 & 25 & 188 & 3283 \\
197 & 5 & 180 & 6333 \\
197 & 4.8 & 176 & 6484 \\
197 & 4.6 & 176 & 6600 \\
197 & 4.5 & 161 & 6590 \\
197 & 4.3 & 161 & 6665 \\
\hline
\end{tabular}


Table 3-4e. Dehydrogenation of ph-NEC with palladium on carbon. The table shows the temperature and hydrogen flow rate at the time when the samples were analyzed.

\begin{tabular}{|c|c|c|c|}
\hline $\begin{array}{c}\text { Temp } \\
\text { (') }\end{array}$ & $\begin{array}{c}\mathbf{H}_{2} \text { Flow } \\
\text { (sccm) }\end{array}$ & $\begin{array}{c}\text { Methane } \\
\text { Conc. (ppm) }\end{array}$ & $\begin{array}{c}\text { Ethane } \\
\text { Conc. (ppm) }\end{array}$ \\
\hline 145 & 5.3 & 315 & 16 \\
147 & 6.5 & 591 & 7 \\
149 & 7.8 & 593 & 7 \\
152 & 9.4 & 437 & 16 \\
154 & 11 & 298 & 16 \\
197 & 45 & 187 & 26 \\
197 & 38 & 190 & 26 \\
197 & 32 & 190 & 26 \\
197 & 27 & 194 & 26 \\
197 & 24 & 191 & 21 \\
197 & 5 & 205 & 17 \\
197 & 4.7 & 206 & 21 \\
197 & 4.4 & 208 & 20 \\
197 & 4.2 & 208 & 22 \\
197 & 3.9 & 209 & 22 \\
\hline
\end{tabular}

B. Mixed N-alkylcarbazoles

While perhydro-N-ethylcarbazole and mixtures of partially hydrogenated $\mathrm{N}$ -

ethylcarbazole are liquids at ambient temperature, pure $\mathrm{N}$-ethylcarbazole has a melting point of ca. $67^{\circ} \mathrm{C}$. This represents a practical obstacle to the use of pure $\mathrm{N}$ ethylcarbazole as a hydrogen storing "liquid." Blending of various $\mathrm{N}$-alkyl carbazoles has yielded mixtures that are liquid at room temperature in all stages of hydrogenation and dehydrogenation. A nominal 2:1 mixture of perhydro-N-propylcarbazole and perhydro-N-ethylcarbazole remained liquid at room temperature for at least a period of several days after near-complete dehydrogenation. This mixture has a slightly depressed $\mathrm{H}_{2}$ capacity (ca. 5.5 wt. \%) relative to pure perhydro-N-ethylcarbazole (ca. $5.7 \mathrm{wt}$ \%). The cycling stability of the mixture appears to be similar to the pure perhydro-N-ethylcarbazole (Figure 3-9). 


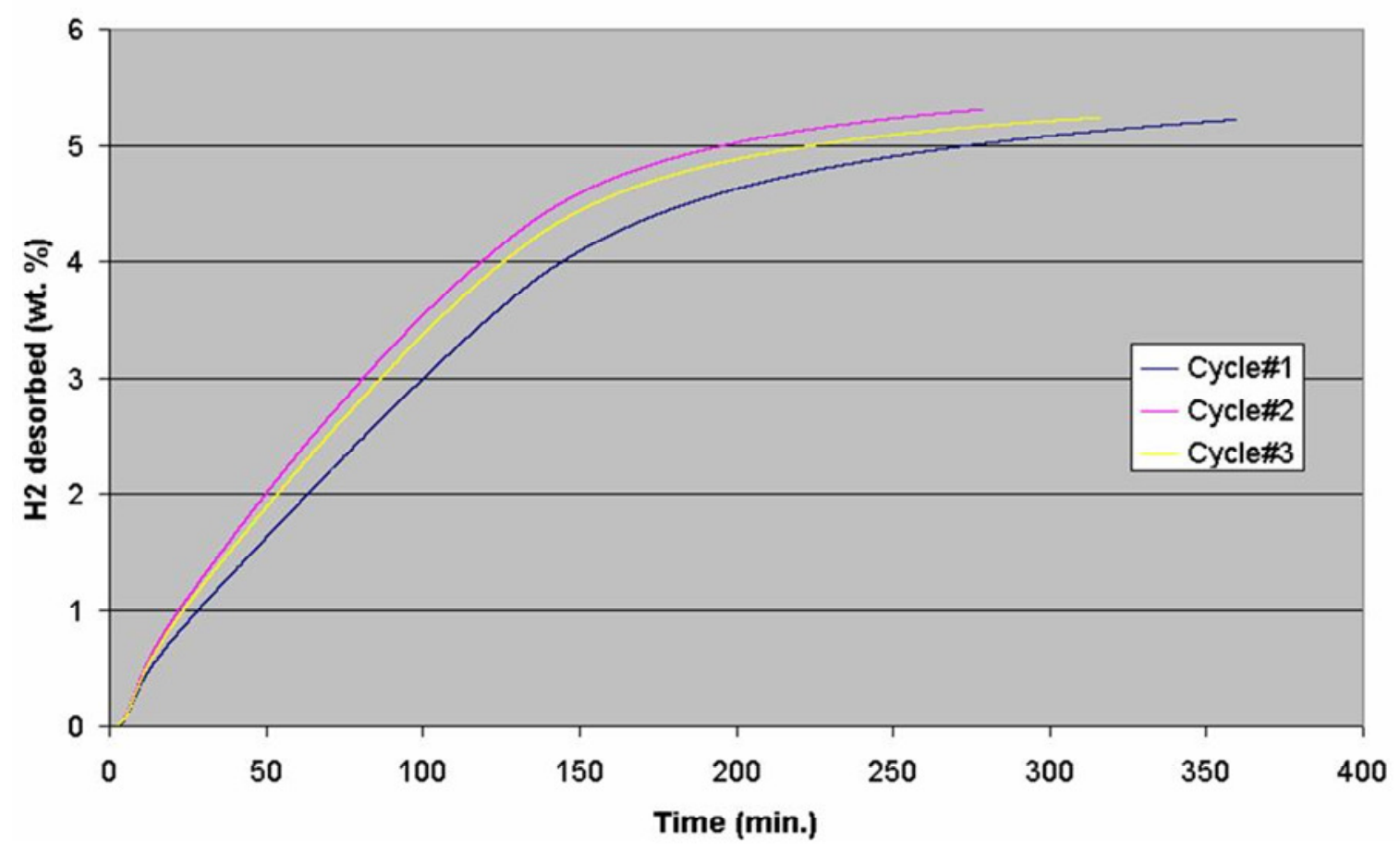

Figure 3-9. Dehydrogenation data for three consecutive cycles of hydrogenation and dehydrogenation for a 2:1 mixture of perhydro-N-propylcarbazole and perhydro- $\mathrm{N}$ ethylcarbazole.

\section{Perhydro-3-methyl-N-ethylcarbazole.}

One general strategy for lowering the melting point of aromatics and aromatic heterocycles is alkylation of the aromatic ring. We synthesized a ring-methylated derivative of $\mathrm{N}$-methylcarbazole (m.p. $90 \mathrm{oC}$ ), but, unfortunately, found that the melting point was only slightly depressed. Pure samples of 3-methyl-N-methylcarbazole demonstrated a melting point of $850 \mathrm{C}$. 


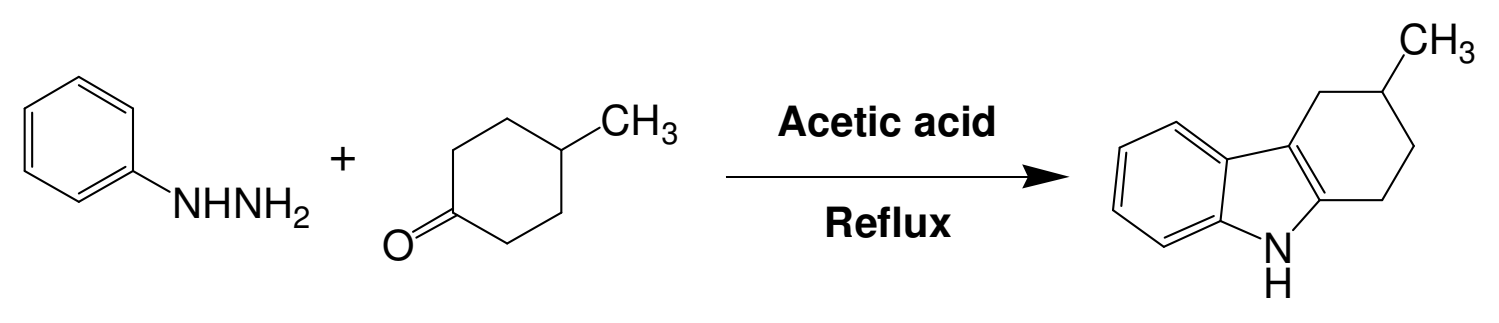

Yield: 92\%

Scheme 3-3. Synthesis of tetrahydro-3-methylcarbazole

Perhydro-3-methyl-N-methylcarbazole was obtained by Fischer indole synthesis using phenylhydrazine and 4-methylcyclohexanone (Scheme 3-3), followed by hydrogenation and methylation under hydrogen with methanol and Raney nickel (Scheme 3-4).<smiles>Cc1ccc2c(c1)c1ccc(NC(=O)OCc3ccccc3)cc1n2C</smiles>

Scheme 3-4. Synthesis and dehydrogenation of perhydro-3-methyl-N-ethylcarbazole.

Dehydrogenation with $5 \% \mathrm{Pd} / \mathrm{C}$ at $200^{\circ} \mathrm{C}$ yielded a high conversion to the fully dehydrogenated 3-methyl-N-ethylcarbazole with dehydrogenation rates very similar to those for perhydro-N-ethylcarbazole (Figure 3-10). However, due to the limited depression of the melting point by ring methylation, no additional dehydrogenation studies were performed. 


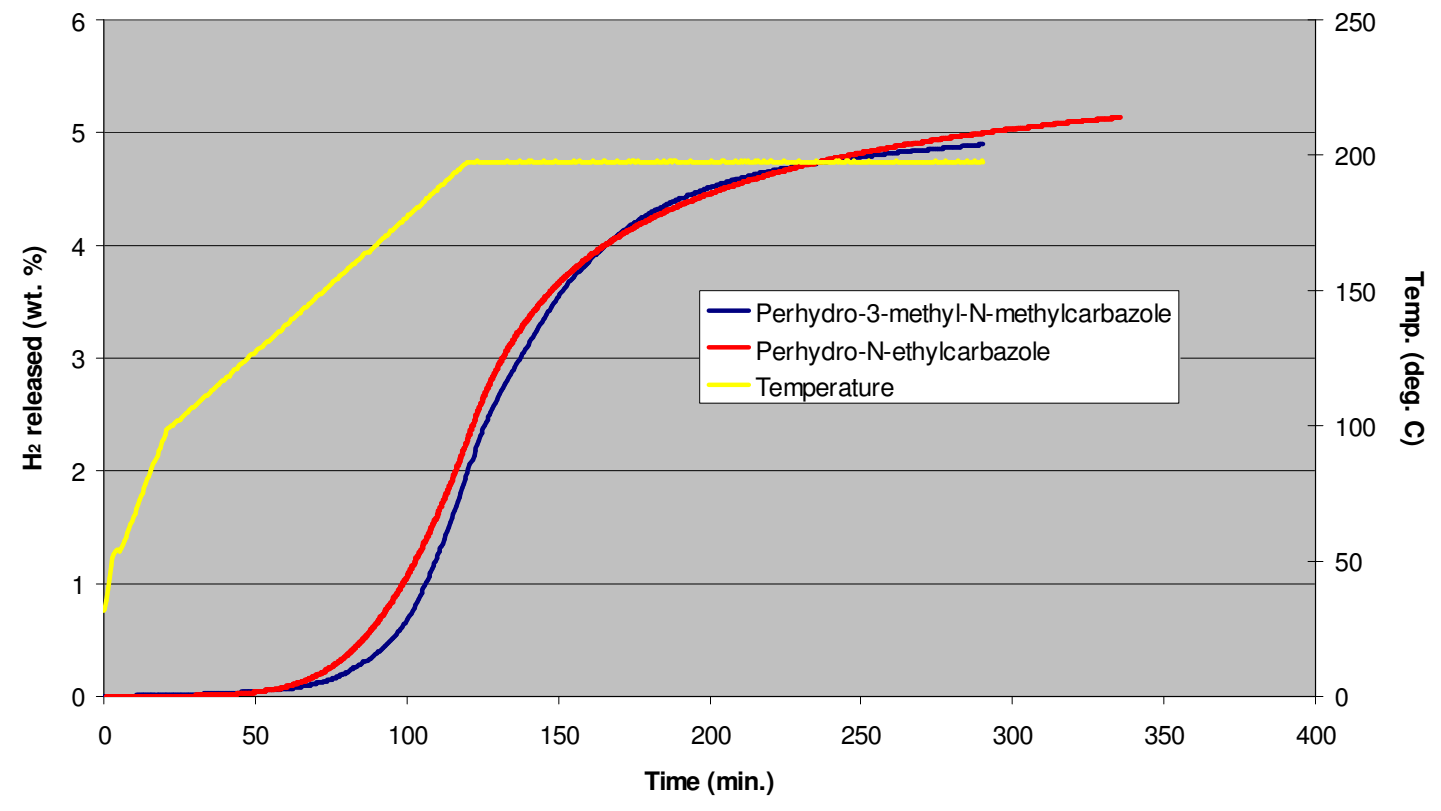

Figure 3-10. Dehydrogenation of perhydro-3-methyl-N-ethylcarbazole and perhydro-Nethylcarbazole with $5 \%$ palladium on carbon.

D. 3-methyl-N-ethylcarbazole

Tetrahydro-3-methylcarbazole was prepared according to the procedure in Scheme 3-3. The N-ethylation was performed using iodoethane and potassium hydroxide in dimethylformamide at room temperature (Scheme 3-5).
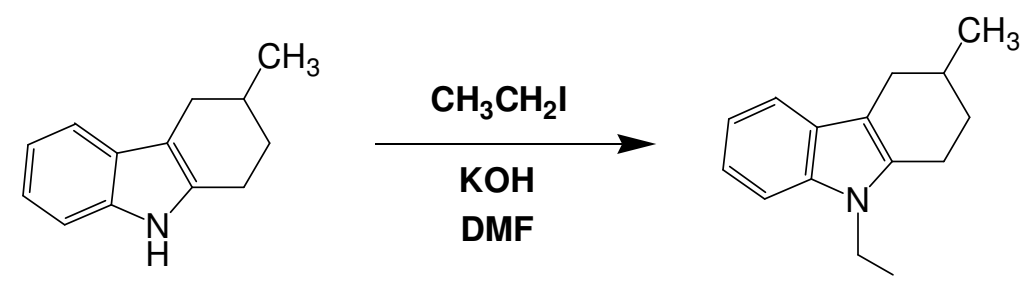

Scheme 3-5. Synthesis of tetrahydro-3-methyl-N-ethylcarbazole.

Complete hydrogenation of the tetrahydro-3-methyl-N-ethylcarbazole was accomplished with a ruthenium on lithium aluminate hydrogenation catalyst at $150^{\circ} \mathrm{C}$ under $800 \mathrm{psia}$ 
hydrogen pressure. Dehydrogenation of perhydro-3-methyl-N-ethylcarbazole at $200^{\circ} \mathrm{C}$ using palladium on carbon yielded only a moderate conversion to 3-methyl-Nethylcarbazole. A comparative dehydrogenation experiment with $\mathrm{N}$-ethylcarbazole demonstrated a slower dehydrogenation rate for perhydro-3-methyl-N-ethylcarbazole.

\section{E. 9-ethanolcarbazole}

In an effort to explore methods for decreasing the melting point of carbazole-based liquid carrier candidates while having a minimal effect on the reversible hydrogen storage capacity, we have investigated low melting, low volatility polyether oligomers as additives. With addition of $10 \mathrm{wt}$. \% or less of several different types of oligomers, there are substantial effects on the melting point. It is clear from Table 3-5 that liquid carriers with polar functional groups (e.g., 9-ethanolcarbazole) show more substantial melting point depression in the presence of polyether oligomers. There are also differences in the performance of the polyether additives, depending on the functionalization (or lack thereof) of the oligomer termini.

Table 3-5. Melting point and theoretical hydrogen capacity of $\mathrm{N}$-alkylcarbazole/ polyether oligomer mixtures.

\begin{tabular}{|c|c|c|c|}
\hline Mixture & Ratio & M.P. (deg. C) & H2 Cap. (wt. \%) \\
\hline NMC & pure & 90 & 6.2 \\
\hline ECE & pure & 82 & 5.4 \\
\hline NEC & pure & 70 & 5.8 \\
\hline NEC/PPGDB & $10: 1$ & 55 & 5.2 \\
\hline NEC/PPGMB & $10: 1$ & 40 & 5.2 \\
\hline NEC/PEG & $10: 1$ & 40 & 5.2 \\
\hline NEC/PEGEH & $10: 1$ & 40 & 5.2 \\
\hline NMC/ECE/PPGMB & $10: 5: 1$ & 36 & 5.3 \\
\hline NMC/ECE/PEGEH & $10: 5: 1$ & 35 & 5.3 \\
\hline NMC/ECE/PEG & $10: 5: 1$ & 35 & 5.3 \\
\hline NMC/ECE/PPGDB & $10: 5: 1$ & 35 & 5.3 \\
\hline NEC/ECE/PPGMB & $10: 5: 1$ & 33 & 5.0 \\
\hline NEC/ECE/PEG & $10: 5: 1$ & 32 & 5.0 \\
\hline NEC/ECE/PPGMB & $10: 5: 1$ & 25 & 5.0 \\
\hline NEC/ECE/PPGDB & $10: 5: 1$ & 25 & 5.0 \\
\hline ECE/PEG & $10: 1$ & $<25$ & 4.9 \\
\hline ECE/PPGMB & $10: 1$ & $<25$ & 4.9 \\
\hline ECE/PPGDB & $10: 1$ & $<25$ & 4.9 \\
\hline
\end{tabular}


NMC: $\quad \mathrm{N}$-methylcarbazole

NEC: N-ethylcarbazole

ECE: 9-ethanol carbazole

PEG: $\quad$ Poly(ethylene glycol), avg. $\mathrm{M}_{\mathrm{n}} 200$, m.p. $-65^{\circ} \mathrm{C}$, b.p. $>200^{\circ} \mathrm{C}$

PPGDB: Poly(propylene glycol)dibenzoate, avg. $\mathrm{M}_{\mathrm{n}} 410$, m.p. $<20^{\circ} \mathrm{C}$, b.p. $217^{\circ} \mathrm{C} / 1 \mathrm{~mm} \mathrm{Hg}$

PEGEH: Poly(ethylene glycol) bis(2-ethylhexanoate)

Avg. $\mathrm{M}_{\mathrm{n}} 650$, m.p. $-48^{\circ} \mathrm{C}$, b.p. $>200^{\circ} \mathrm{C}$

PPGMB: Poly(propylene glycol)monobutylether

Avg. $\mathrm{M}_{\mathrm{n}} 340$, m.p. $-57^{\circ} \mathrm{C}$, b.p. $>200^{\circ} \mathrm{C}$

The selective hydrogenation of 9-ethanolcarbazole proved to be very difficult.

Hydrogenation under our typical conditions (e.g., supported ruthenium or rhodium catalyst, temperature of $150-170^{\circ} \mathrm{C}$, hydrogen pressure of $500-1200 \mathrm{psia}$ ) resulted in extensive cleavage of the carbon-oxygen bond, yielding perhydro-N-ethylcarbazole as the primary product. We found that only a very slow hydrogenation under moderate temperature conditions using rhodium on carbon resulted in a high yield and high conversion to perhydro-9-ethanolcarbazole (Scheme 3-6).
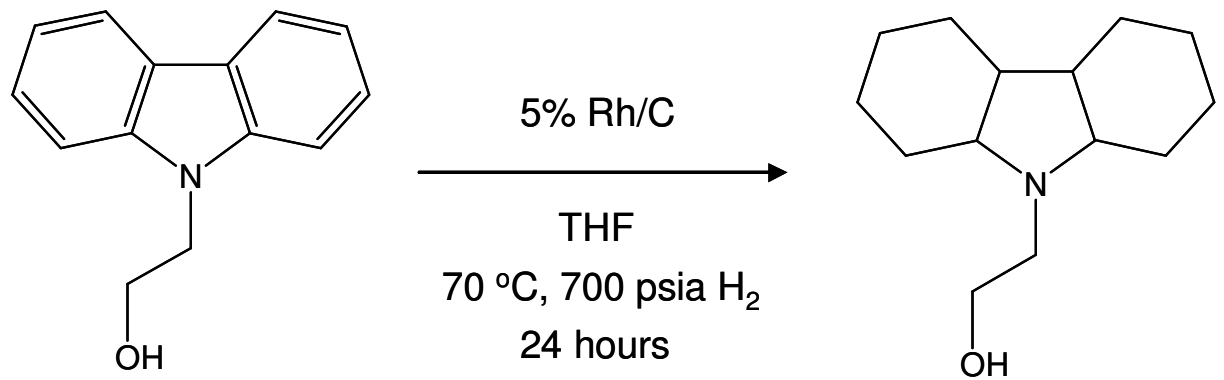

Scheme 3-6. Hydrogenation of 9-ethanolcarbazole. 
Unfortunately, the dehydrogenation of perhydro-9-ethanolcarbazole invariably resulted in the cleavage of the carbon-oxygen bond with either palladium or platinum-based catalysts under our typical dehydrogenation conditions (e.g., temperature ramp to $200^{\circ} \mathrm{C}$, 4 wt. \% catalyst, 1 atm. hydrogen pressure). While hydrogen, and presumably water, was evolved, the major products of the dehydrogenation reactions were $\mathrm{N}$-ethylcarbazole and partially hydrogenated $\mathrm{N}$-ethylcarbazole. Due to the low selectivity, no additional research was performed using 9-ethanolcarbazole.

\section{F. Azaphenylcarbazoles}

As part of the development of new carbazole-based liquid carrier candidates, we investigated the hydrogenation and dehydrogenation of azaphenylcarbazoles ( $\mathrm{N}$-pyridine, $\mathrm{N}$-pyrimidine and $\mathrm{N}$-pyrazine). An efficient method for the preparation of these compounds was needed, since these molecules are not commercially available and no practical method of making them has been reported in the literature. A one-step $\mathrm{N}$-arylation synthesis starting from carbazole and the halogenated $\mathrm{N}$-heterocycles was developed using cesium carbonate base in dimethylformamide solvent. While the reactions were performed with quantitative yields using chloropyrimidine and chloropyrazine at $130-150^{\circ} \mathrm{C}$ (Scheme 3-7), no reaction was observed with chloropyridine.

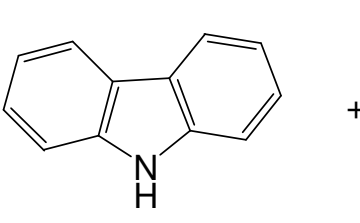<smiles>Clc1ncccn1</smiles>
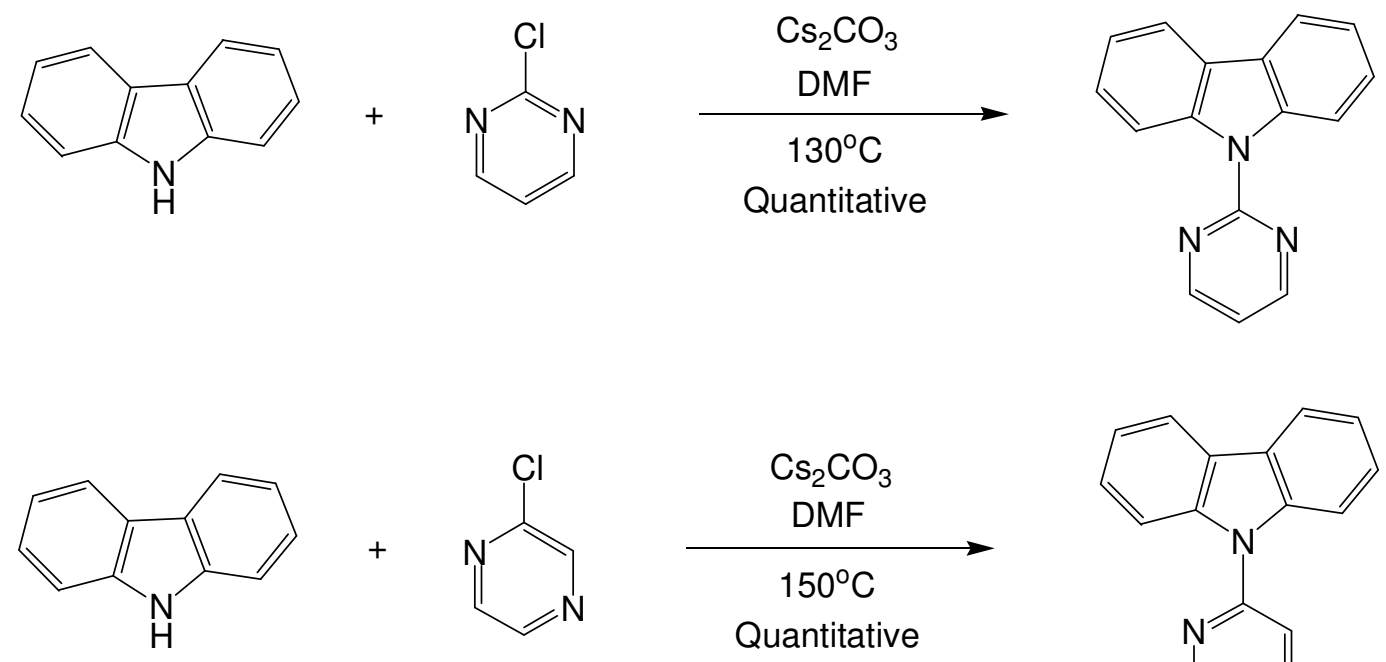<smiles>Clc1cnccn1</smiles>
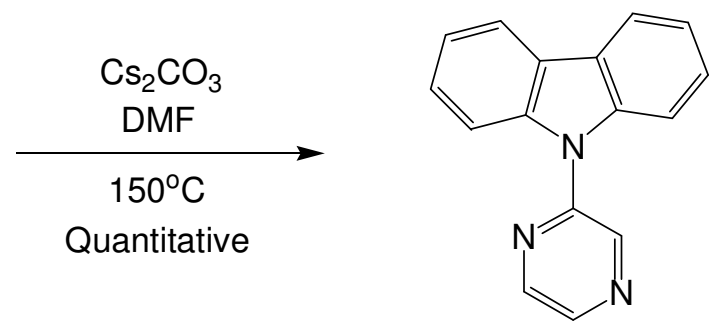

Scheme 3-7. Synthesis of N-pyrimidylcarbazole (top) and N-pyrazylcarbazole (bottom). 
$\mathrm{N}$-pyridylcarbazole was obtained quantitatively from fluoropyridine and carbazole at a slightly higher temperature of $170^{\circ} \mathrm{C}$ (Scheme 3-8).

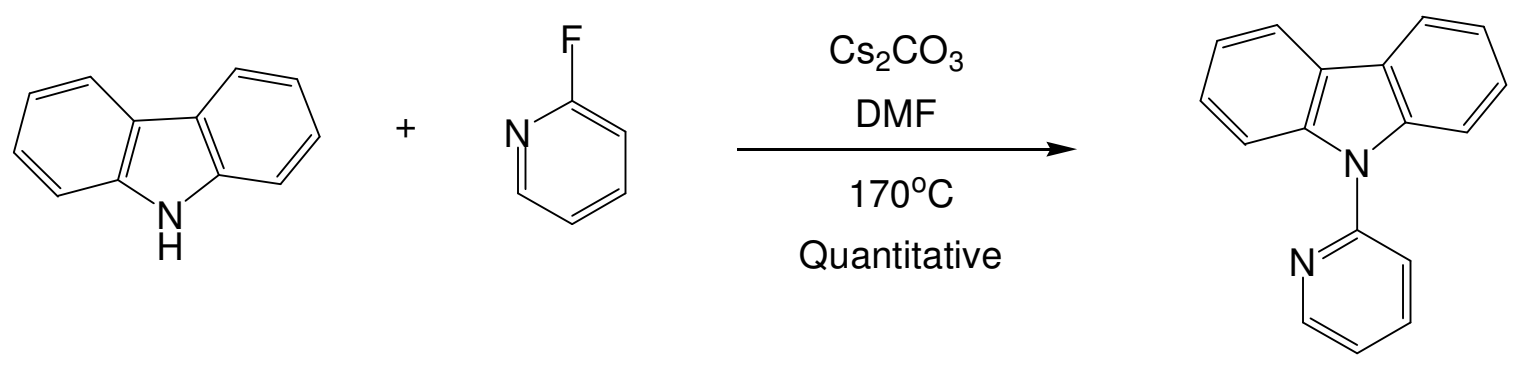

Scheme 3-8. Synthesis of N-pyridylcarbazole.

Under our standard hydrogenation conditions (e.g., $160^{\circ} \mathrm{C}, 800 \mathrm{psi}$ hydrogen pressure, neat or in a solvent) with various catalysts (ruthenium on lithium aluminate, rhodium on bohemite or rhodium on lithium aluminate), N-pyridylcarbazole undergoes hydrogenolysis with cleavage at the carbon-nitrogen bond, yielding perhydrocarbazole and piperidine. At lower temperatures $\left(100^{\circ} \mathrm{C}\right), \mathrm{N}$-pyridylcarbazole solutions and neat $\mathrm{N}$-pyridylcarbazole were not hydrogenated in the presence of ruthenium on lithium aluminate or rhodium on lithium aluminate. With rhodium on bohemite as the catalyst, only two moles of hydrogen were added to the $\mathrm{N}$-pyridylcarbazole starting material (Scheme 3-9).

The reactivity of $\mathrm{N}$-pyrimidylcarbazole and $\mathrm{N}$-pyrazylcarbazole under hydrogenation conditions generally mirrored the reactivity of $\mathrm{N}$-pyridylcarbazole. For example, hydrogenation of $\mathrm{N}$-pyrimidylcarbazole with ruthenium on lithium aluminate at $160^{\circ} \mathrm{C}$ and 800 psi of hydrogen pressure, either neat or in tetrahydrofuran solvent, stopped after addition of only one mole of hydrogen. A sample of this dihydro-derivative was submitted for ${ }^{1} \mathrm{H}$ NMR analysis, and the results suggested that addition of the hydrogen occurred at the carbons in the 4,5 positions. 


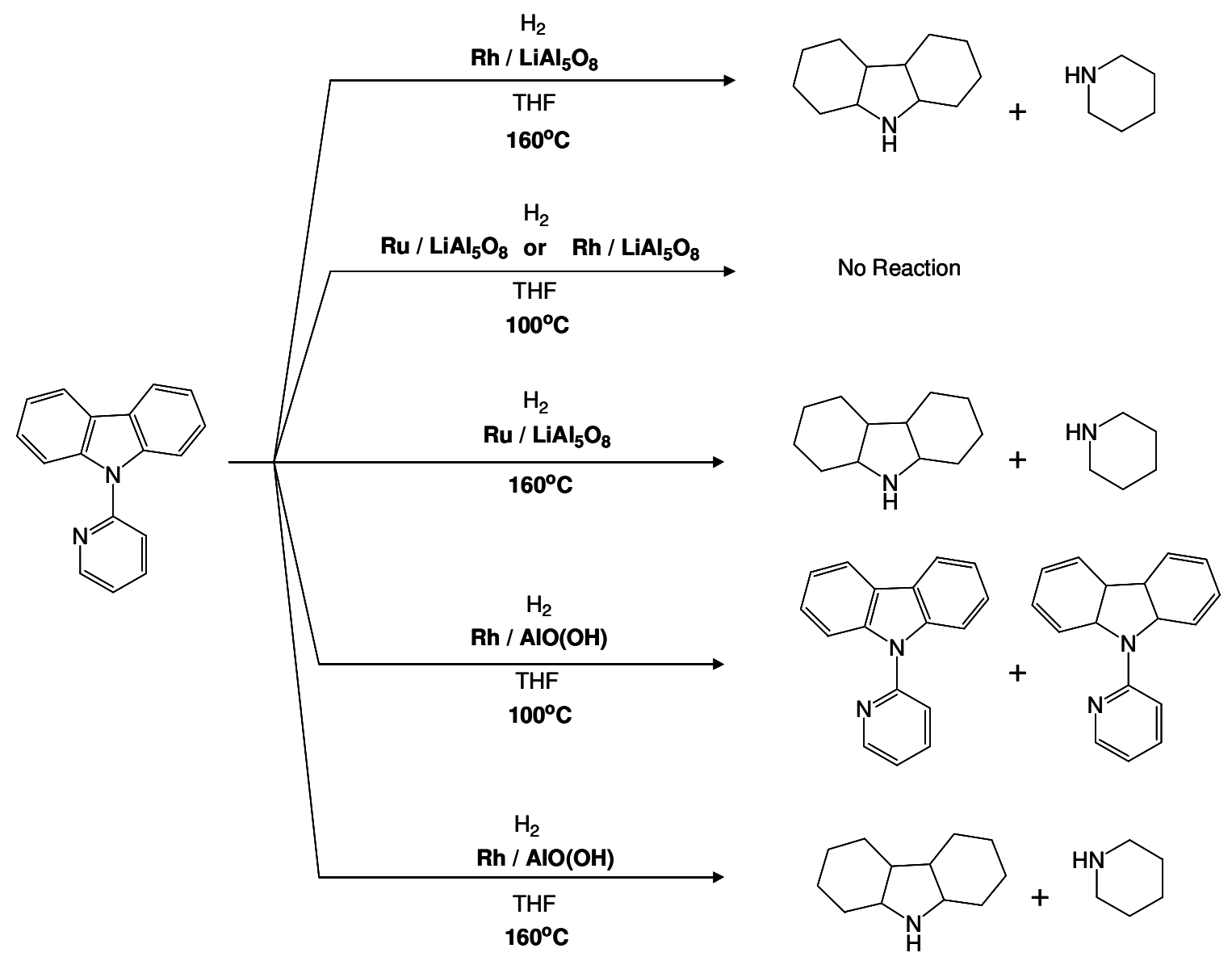

Scheme 3-9. Hydrogenation reactions of N-pyridylcarbazole.

\section{Experimental Details}

See Appendix B for general experimental details.

\section{Dehydrogenation catalyst testing}

For comparison of dehydrogenation catalyst activity, two $20 \mathrm{cc}$ stirred tank reactors were used in the same positions in the dehydrogenation testing manifold for every catalyst screening reaction. The performance of these reactors was periodically checked by performing a dehydrogenation with a reference catalyst $(5 \%$ palladium on alumina, Engelhard catalyst 99021). Efforts were undertaken to use the same batch of perhydro$\mathrm{N}$-ethylcarbazole for screening a set of dehydrogenation catalysts. When batches of 
perhydro-N-ethylcarbazole were changed, a set of dehydrogenation experiments with the reference catalyst was used to check for significant differences in the dehydrogenation rate and selectivity.

In a typical dehydrogenation test, $4.0 \mathrm{~g}$ ph-NEC and $0.1 \mathrm{~g}$ dehydrogenation catalyst ( 2.5 wt. \% catalyst) were placed in a 20 cc stirred tank reactor in an argon glove box. The reactor was sealed inside the glove box and connected to a manifold containing a vacuum source, hydrogen source, and a flow measurement system consisting of calibrated 10 and $100 \mathrm{sccm}$ flow meters in series. After evacuation of the connecting lines, the reactor was pressurized with hydrogen to ca. 200 psia and vented to atmospheric pressure three times. With stirring (500-800 rpm), the reactor was quickly heated from room temperature to $100^{\circ} \mathrm{C}$. The temperature of the reactor was then ramped from 100 to $200^{\circ} \mathrm{C}$ at a rate of $1^{\circ} \mathrm{C}$ per minute and held at $200^{\circ} \mathrm{C}$ until the conclusion of the experiment. The data recorded from the 10 and $100 \mathrm{sccm}$ flow meters was used to calculate the conversion as a function of time. At the conclusion of the dehydrogenation testing, the reactor contents were analyzed by GC-MS.

\section{Dehydrogenation of perhydro- $\mathrm{N}$-ethylcarbazole isomers}

Two hydrogenated N-ethylcarbazole samples with varying levels of perhydro-Nethylcarbazole isomers were degassed by evacuation $\left(1.0 \times 10^{-3}\right.$ torr $)$ at $20^{\circ} \mathrm{C}$ for 20 minutes. Mixture A (prepared by hydrogenating $\mathrm{N}$-ethylcarbazole at $120^{\circ} \mathrm{C}$ with $5 \%$ ruthenium on lithium aluminate) contained $90 \%$ perhydro- $\mathrm{N}$-ethylcarbazole (molecular weight 207) and 10\% octahydro-N-ethylcarbazole (molecular weight 203). The perhydro-N-ethylcarbazole fraction of mixture A consisted of $90 \%$ of two less stable isomers and $10 \%$ of the more stable isomer. Mixture $B$ (obtained from a hydrogenation of $\mathrm{N}$-ethylcarbazole with $5 \%$ ruthenium on lithium aluminate conducted at $170^{\circ} \mathrm{C}$ ), contained $100 \%$ perhydro-N-ethylcarbazole (molecular weight 207). The perhydro-Nethylcarbazole fraction of mixture B consisted of $19 \%$ of two less stable isomers and $81 \%$ of the more stable isomer. Under inert atmosphere, $4.0 \mathrm{~g}$ of Mixture A or B of hydrogenated $\mathrm{N}$-ethylcarbazole and $0.1 \mathrm{~g}$ of either $5 \%$ palladium on alumina or $2.9 \%$ platinum $/ 0.3 \%$ rhenium on alumina (dehydrogenation catalysts) were placed in a $20 \mathrm{cc}$ stirred tank reactor. The reactor was connected to the dehydrogenation testing manifold. After evacuation of residual air from the manifold lines, hydrogen was purged 
through the reactor to displace the argon from the reactor headspace. The reactor was heated to $100^{\circ} \mathrm{C}$ with stirring $(500 \mathrm{rpm})$ under $1 \mathrm{~atm}$. hydrogen. The temperature was then ramped from 100 to $200^{\circ} \mathrm{C}$ at $1^{\circ} \mathrm{C} /$ minute and held at $200^{\circ} \mathrm{C}$ until $>75 \%$ of the available hydrogen was desorbed.

\section{Preparation of 3-methyl-1,2,3,4-tetrahydrocarbazole}

A solution containing $11.8 \mathrm{~g}$ of 4-methylcyclohexanone and $40 \mathrm{ml}$ acetic acid was prepared, and phenylhydrazine was added by drop under a nitrogen purge. The solution was then refluxed for one hour. The solution was cooled to room temperature, poured into $100 \mathrm{ml}$ water, and extracted twice with $100 \mathrm{ml}$ portions of ethyl acetate. The organic was then washed twice with $100 \mathrm{ml}$ portions of $20 \%$ aq. sodium bicarbonate, dried over magnesium sulfate, and the solvent was removed under vacuum. The product was recovered as $17.4 \mathrm{~g}$ of a white solid (yield 94\%).

\section{Preparation of 3-methyl-9-ethyl-1,2,3,4-tetrahydrocarbazole}

A mixture of 3-methyl-1,2,3,4-tetrahydro-carbazole $(5 \mathrm{~g}, 27.0 \mathrm{mmol})$, iodoethane $(5.05 \mathrm{~g}$, $32.4 \mathrm{mmol})$ and potassium hydroxide $(1.8 \mathrm{~g}, 32.4 \mathrm{mmol})$ in $100 \mathrm{~mL}$ of dimethylformamide was stirred at room temperature for one hour. The reaction mixture was then poured onto a dilute solution of $\mathrm{HCl}$ ( $\mathrm{pH}$ slightly acidic) and extracted with ethyl acetate. The organic phase was washed with water, dried with magnesium sulfate, and filtered. After evaporation of the solvent and drying under vacuum, $5.7 \mathrm{~g}$ of product was obtained (yield 99\%).

\section{Hydrogenation of 3-methyl-9-ethyl-1,2,3,4-tetrahydrocarbazole}

A 30 cc stainless steel pressure reactor was loaded with $5.5 \mathrm{~g}$ of 3-methyl-9-ethyl$1,2,3,4$-tetrahydro-carbazole and $0.6 \mathrm{~g}$ of $5 \%$ ruthenium on lithium aluminate. After the headspace was purged with hydrogen, the hydrogen pressure was increased to 950 psia. The reactor was heated to $180^{\circ} \mathrm{C}$, and the hydrogen pressure increased to 1000 psia. After 7 hours under these conditions with stirring, the reactor was cooled to $25^{\circ} \mathrm{C}$, and the contents were filtered to remove catalyst. GC-MS analysis showed complete conversion to a mixture of isomers of perhydro-3-methyl-9-ethylcarbazole. 
The perhydro-3-methyl-9-ethylcarbazole was then degassed by evacuation to 100 millitorr at $23^{\circ} \mathrm{C}$ for 20 minutes and purged with argon for 5 minutes.

\section{Dehydrogenation of perhydro-3-methyl-9-ethylcarbazole}

Under an argon atmosphere, $4 \mathrm{~g}$ of the perhydro-3-methyl-9-ethylcarbazole and $0.1 \mathrm{~g}$ of $5 \%$ palladium on carbon (dehydrogenation catalyst) were placed in a $30 \mathrm{cc}$ stirred tank reactor and the reactor was sealed. The reactor was connected to the dehydrogenation testing manifold. After evacuation of residual air from the manifold lines, hydrogen was purged through the reactor to displace the argon from the reactor headspace. The reactor temperature was ramped to $180^{\circ} \mathrm{C}$ with stirring $(500 \mathrm{rpm})$ under 1 psia hydrogen. After 5 hours at $180^{\circ} \mathrm{C}$, the measured hydrogen flow corresponded to the desorption of $4.2 \mathrm{wt}$ \% hydrogen (theoretical maximum 5.4 wt. \% hydrogen).

\section{Dehydrogenation of perhydro-3,9-dimethyl-1,2,3,4-carbazole}

An alternative method for preparing perhydro-3,9-dimethyl-carbazole was developed by hydrogenation and N-methylation of 3-methyl-1,2,3,4-tetrahydrocarbazole using Raney Nickel as a catalyst and methanol both as an alkylating agent and a solvent in the same reactor. Dehydrogenation under the same conditions as for perhydro-3-methyl-9-ethyl carbazole yielded a $4.9 \mathrm{wt} \%$ hydrogen desorption.

\section{Preparation of 3-methylcarbazole}

A solution containing $2 \mathrm{~g}$ of 3-methyl-1,2,3,4-tetrahydrocarbazole in $20 \mathrm{ml}$ tertbutylbenzene was prepared. The solution was then inerted under a nitrogen sparge and brought into a glove box where $200 \mathrm{mg}$ of pre-reduced $5 \% \mathrm{Pd} / \mathrm{C}$ was added. The solution was then heated at $170^{\circ} \mathrm{C}$ under a nitrogen atmosphere for 24 hours. The solution was cooled, and the precipitate and catalyst were filtered off and washed with tert-butylbenzene followed by cold hexane. The filtrate was then re-dissolved in acetone, filtered and the solvent removed under vacuum yielding $1.9 \mathrm{~g}$ of a white crystalline solid (yield 99\%). 


\section{General procedure for the preparation of azaphenylcarbazoles}

\section{Example: Preparation of $\mathrm{N}$-pyridylcarbazole}

A mixture of carbazole (17 g, $0.1 \mathrm{~mol})$, 2-fluoropyridine $(10 \mathrm{~g}, 0.1 \mathrm{~mol})$ and cesium carbonate $(40 \mathrm{~g}, 0.12 \mathrm{~mol})$ in dimethylformamide $(200 \mathrm{~mL})$ was heated at $130^{\circ} \mathrm{C}$ for one day. Sampling the reaction and analysis by GC-MS showed only $30 \%$ conversion. The mixture was heated to $170^{\circ} \mathrm{C}$ overnight. After cooling, the reaction mixture was poured onto water $(1 \mathrm{~L})$ and extracted with ethyl acetate. The organic phase was washed with water, dried over magnesium sulfate, filtered, and the solvent was evaporated. The oily residue was chromatographed on silica gel with ethyl acetate: hexanes (3:1). The product was recrystallized from the solvents by dissolving the solid in ethyl acetate, and then adding excess hexanes. With 2-chloropyridine, no reaction was observed. Using this general procedure, $\mathrm{N}$-pyrimidinocarbazole and $\mathrm{N}$-pyrazinocarbazole were prepared using the starting materials 2-chloropyrimidine and 2-chloropyrazine, respectively. 


\section{Chapter 4: Alkylated Indole Hydrogen Carriers}

\section{Introduction}

As a result of our early efforts in predictive computational modeling, molecules based upon indole were identified as a promising class of potential hydrogen carriers. While indole is a solid at ambient temperature (m.p. $51^{\circ} \mathrm{C}$ ), certain alkylated derivatives, such as 1-ethyl-2-methylindole, are liquids at $20^{\circ} \mathrm{C}$, both in the hydrogenated and fully dehydrogenated forms. The vapor pressure of the indole derivatives is higher than the analogous carbazole-based carriers (e.g., the boiling point of 1-ethyl-2-methylindole is $266^{\circ} \mathrm{C}$ at 760 torr), but likely still suitable for hydrogen storage applications. The average heat of hydrogenation of 1-ethyl-2-methylindole is $-52 \mathrm{~kJ} / \mathrm{mol}$ (cf. $-51 \mathrm{~kJ} / \mathrm{mol}$ for $\mathrm{N}$-ethylcarbazole), which should allow for relatively low-temperature dehydrogenation. Therefore, several commercially available indole derivatives were evaluated for reversible catalytic hydrogenation. Other derivatives of indole, such as indole dimers, bis-indolylmethanes, and various indolocarbazole isomers, offered potential for lower average heats of hydrogenation and/or lower vapor pressures relative to the commercially available alkylated indoles. We identified synthetic routes to a number of carrier prototypes and successfully prepared samples in sufficient quantities for preliminary testing. In general, these indole-based carriers suffered from significantly higher melting points than indole and alkylated indoles.

The hydrogenation of a large variety of indole-based compounds has been reported ${ }^{1}$, including hydrogenation under acidic conditions ${ }^{2,3}$. However, relatively few examples of the catalytic dehydrogenation of perhydroindoles or partially saturated indoles exist in the literature. ${ }^{4}$ One exception is the very favorable dehydrogenation of indolines (2,3-dihydroindoles), which has been reported to occur under very mild conditions in several publications. ${ }^{5,6}$

Our hydrogenation and dehydrogenation testing on the indole-based carriers revealed that catalytic selectivity would be a substantial challenge to the success of these potential hydrogen storage materials. 


\section{Results and Discussion}

\section{A. Alkylated Indoles}

The catalytic hydrogenation and dehydrogenation of three alkyl-substituted indoles, shown in Figure 4-1, was studied. While the temperature necessary for high dehydrogenation conversion for these indole compounds was similar to that observed for $\mathrm{N}$-ethylcarbazole, and while they have slightly lower $\mathrm{H}_{2}$ storage capacities (4.8-5.0 wt. \%), they are generally free-flowing liquids at room temperature. Our best liquid carrier composition from this class was a 1,2-dimethylindole/1-ethyl-2-methylindole mixture that, at $185^{\circ} \mathrm{C}$, was $99 \%$ reversible and had a deliverable hydrogen storage capacity of $5.02 \mathrm{wt} . \% \mathrm{H} 2$.

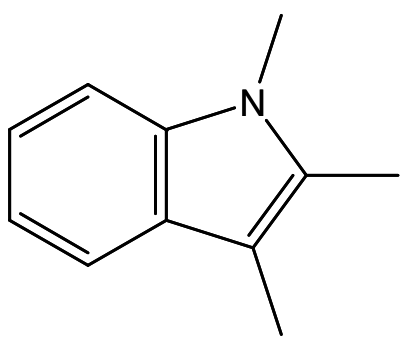

1,2,3-trimethylindole

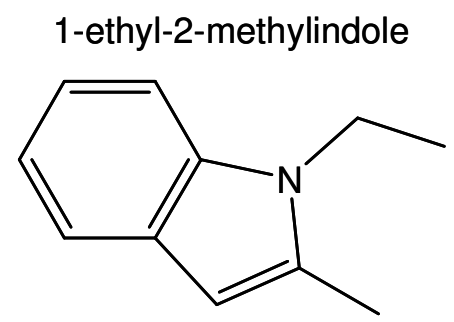

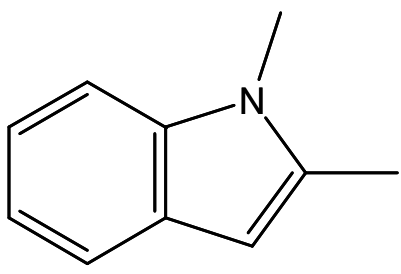

1,2-dimethylindole

Figure 4-1. Alkylated indoles.

\section{a. 1-ethyl-2-methylindole}

A sample of 1-ethyl-2-methylindole (Aldrich, 97\%) was hydrogenated using a 5\% ruthenium on lithium aluminate catalyst at $160^{\circ} \mathrm{C}$ and 1000 psia hydrogen pressure. GC-MS analysis of the products after 2 hours of hydrogenation indicated that the hydrogenated liquid contained 95\% perhydro-1-ethyl-2-methylindole, 3\% partially hydrogenated 1-ethyl-2-methylindole, and 2\% unconverted 1-ethyl-2-methylindole. Without any additional purification, the perhydro-1-ethyl-2-methylindole mixture was dehydrogenated using a $5 \% \mathrm{Pd} / \mathrm{Al}_{2} \mathrm{O}_{3}$ catalyst at $185^{\circ} \mathrm{C}$ under one atmosphere hydrogen pressure (Figure 4-2). 


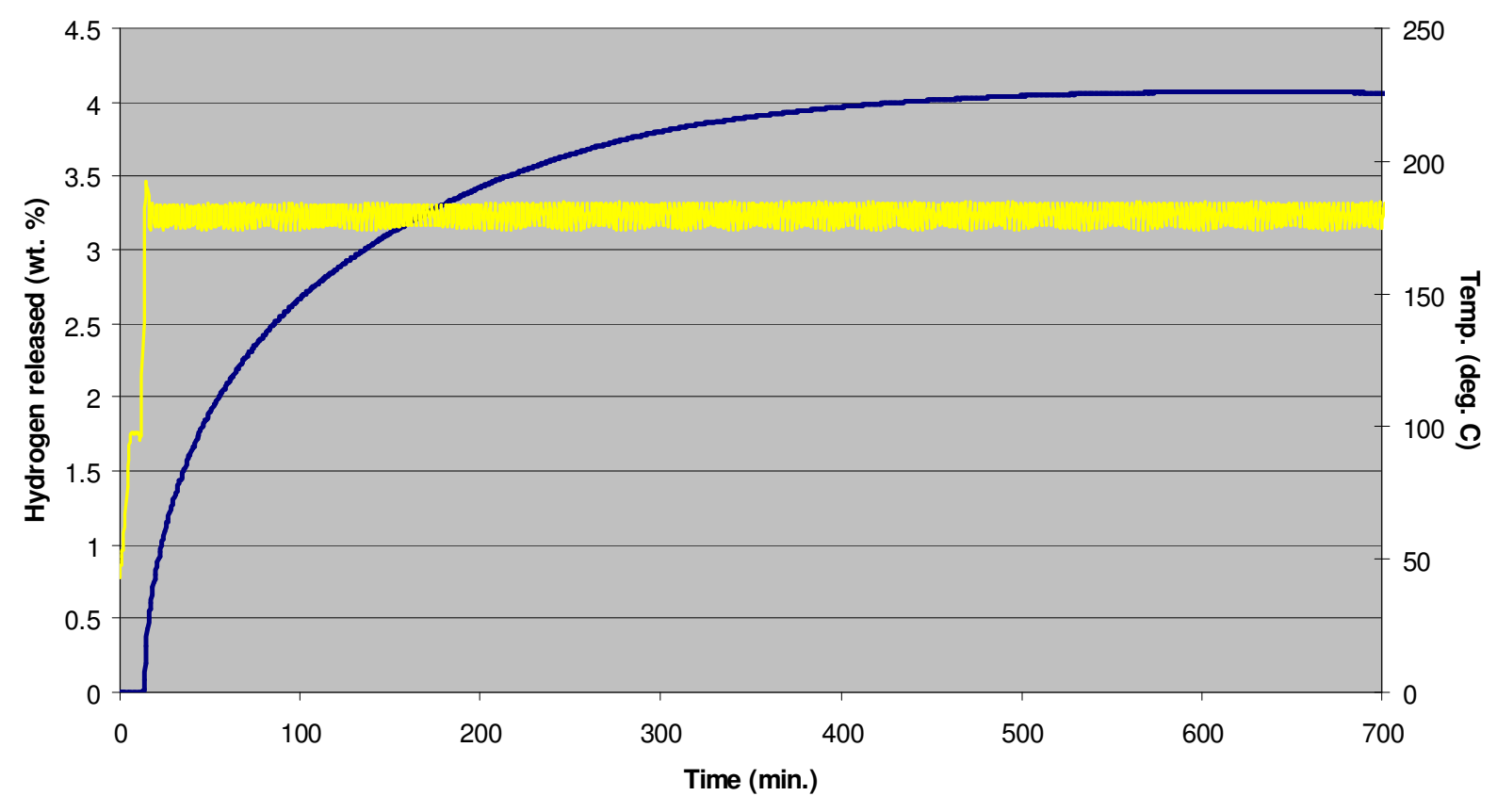

Figure 4-2. Dehydrogenation of perhydro-1-ethyl-2-methylindole.

The results of the dehydrogenation experiment indicated that $85 \%$ of the stored hydrogen was released, giving the material an effective hydrogen storage capacity of approximately 4.1 wt. \% hydrogen under these conditions. GC-MS analysis of the products showed that the dehydrogenation reaction sequence occurs in two steps, each producing 2 hydrogen molecules to reform the unsaturated indole (Scheme 4-1).

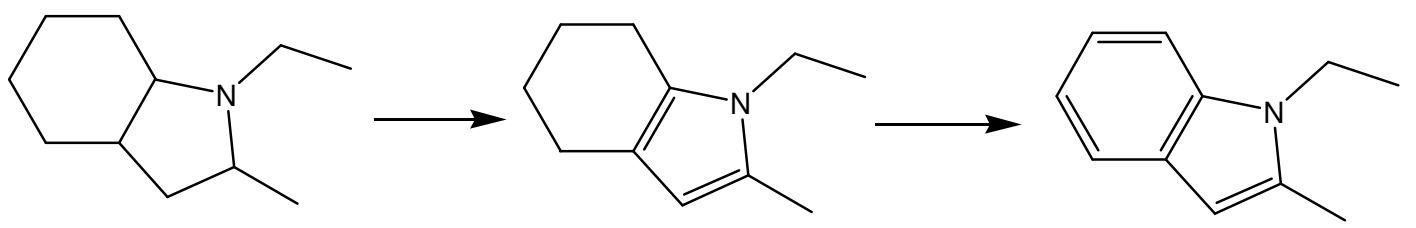

Scheme 4-1. Stepwise dehydrogenation of perhydro-1-ethyl-2-methylindole. The tetrahydro-1-ethyl-2-methylindole structure (MW 163, Table 1) is assumed based upon thermodynamics calculations. 


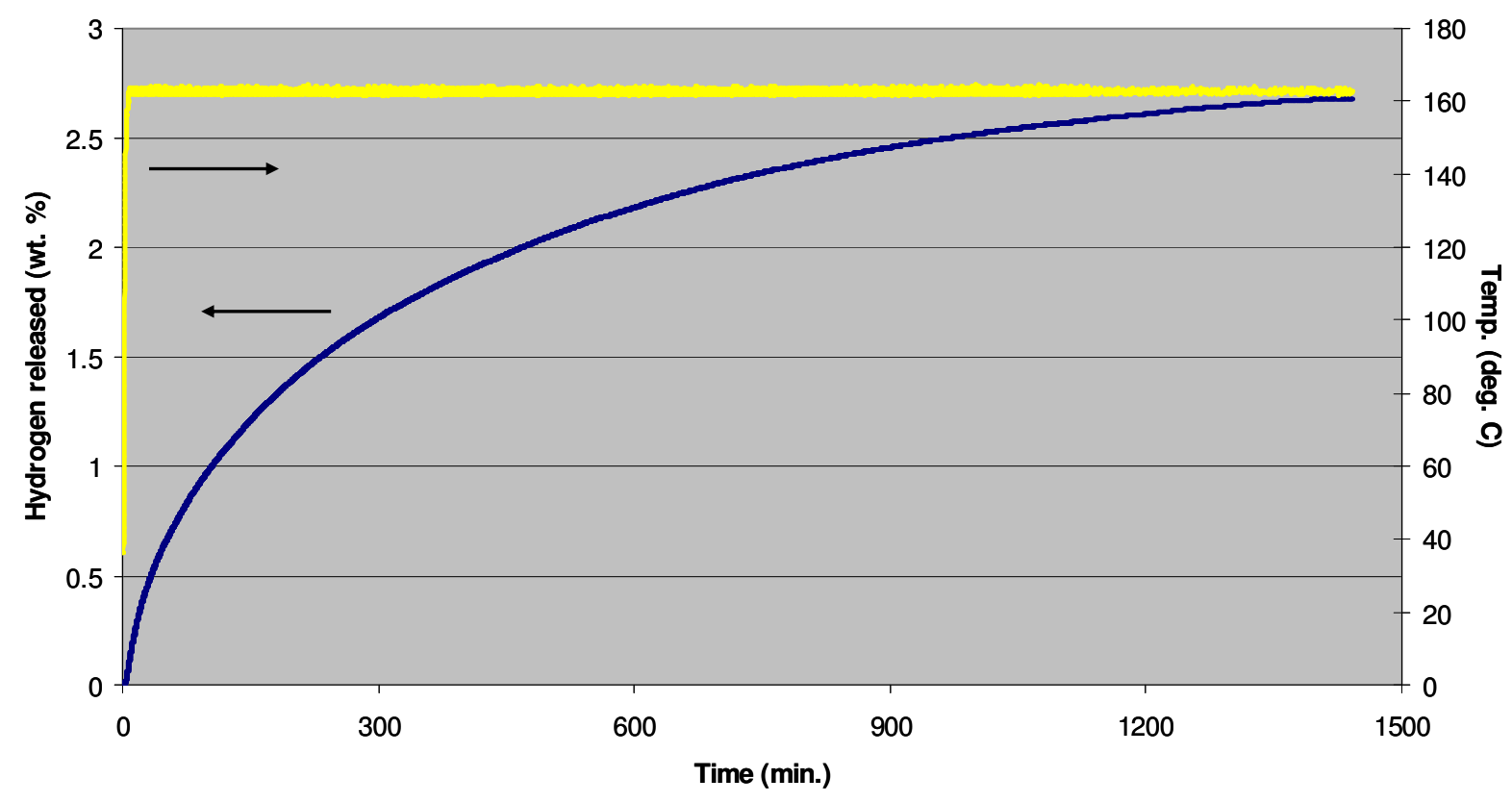

Figure 4-3. Dehydrogenation of perhydro-1-ethyl-2-methylindole at $160^{\circ} \mathrm{C}$.

Dehydrogenation testing of the perhydro-1-ethyl-2-methylindole mixture was repeated at a lower temperature of $160^{\circ} \mathrm{C}$. Under these conditions, the perhydro-1-ethyl-2methylindole conversion over ca. 1400 minutes was approximately $56 \%$, yielding a deliverable hydrogen storage capacity of 2.7 wt. \% hydrogen (Figure 4-3 and Table 4-1).

Table 4-1. GC-MS analysis of dehydrogenated perhydro-1-ethyl-2-methylindole.

\begin{tabular}{|c|c|}
\hline Molecular Weight (Compound) & \% of Total Composition \\
\hline 159 (1-ethyl-2-methylindole) & 56.7 \\
\hline 163 (tetrahydro-1-ethyl-2-methylindole) & 35.6 \\
\hline 167 (perhydro-1-ethyl-2-methylindole) & 7.6 \\
\hline
\end{tabular}

\section{b. 1,2,3-trimethylindole}

A sample of 1,2,3-trimethylindole was synthesized from commercially available 2,3dimethylindole by alkylation with iodomethane in dimethylformamide, using potassium 
hydroxide as the base. After purification by column chromatography, GC-MS analysis showed that the purified product contained 83\% 1,2,3-trimethylindole and 17\% 2,3dimethylindole. Since the product and starting material have very similar silica retention times and boiling points, further attempts at purification of the 1,2,3-trimethylindole were unsuccessful.

The 1,2,3-trimethylindole/2,3-dimethylindole mixture was hydrogenated for 30 hours at $170^{\circ} \mathrm{C}$ under 700 psig hydrogen pressure using $5 \%$ ruthenium on alumina as the catalyst. After hydrogenation, GC-MS analysis showed that the 1,2,3-trimethylindole was converted to approximately $60 \%$ perhydro-1,2,3-trimethylindole and $40 \%$ tetrahydro1,2,3-trimethylindole (Table 4-2). It is interesting to note that for some unknown reason we did not directly observe the hydrogenated 2,3-dimethylindole by GC-MS.

Table 4-2. GC-MS analysis of hydrogenated mixture containing 1,2,3-trimethylindole.

\begin{tabular}{|c|c|}
\hline Molecular Weight (compound) & \% of Total Composition \\
\hline $159(1,2,3-$ trimethylindole) & 39 \\
\hline 163 (tetrahydro-1,2,3-trimethylindole) & 60 \\
\hline 167 (perhydro-1,2,3-trimethylindole) & 1 \\
\hline
\end{tabular}

Dehydrogenation testing of the hydrogenated mixture shown in Table 4-2 was performed by heating a 10:1 (wt./wt.) slurry of the hydrogenated product and $5 \%$ palladium on alumina catalyst to $185^{\circ} \mathrm{C}$ under one atmosphere hydrogen for a total of 16 hours (Figure 4-4). GC-MS analysis of the dehydrogenated mixture indicated that all of the perhydro1,2,3-trimethylindole was converted back to 1,2,3-trimethylindole (Table 4-3). We also were able to observe the 2,3-dimethylindole in the mixture after dehydrogenation. Based upon GC-MS analysis alone, it is not possible to determine if the 2,3dimethylindole was reversibly hydrogenated and additive to the overall hydrogen capacity of the mixture. The reversible hydrogen storage capacity, based solely on the conversion of 1,2,3-trimethylindole, was $3.8 \mathrm{wt}$. \%. 
Table 4-3. GC-MS analysis of the dehydrogenated mixture of perhydro-1,2,3-trimethylindole.

\begin{tabular}{|c|c|}
\hline Molecular Weight (Compound) & \% of Total Composition \\
\hline $145(2,3$-dimethylindole) & 17 \\
\hline $159(1,2,3$-trimethylindole) & 83 \\
\hline
\end{tabular}

One observation during these experiments is the extremely slow kinetics for hydrogenation and dehydrogenation. For example, the plot of hydrogen desorption shown in Figure 4-4 is cut short at 400 minutes; however, dehydrogenation conversion was only $85 \%$ after the full experiment time of 13 hours. The slow reactions could be the result of steric hindrance on the five membered ring due to the multiplicity of methyl groups.

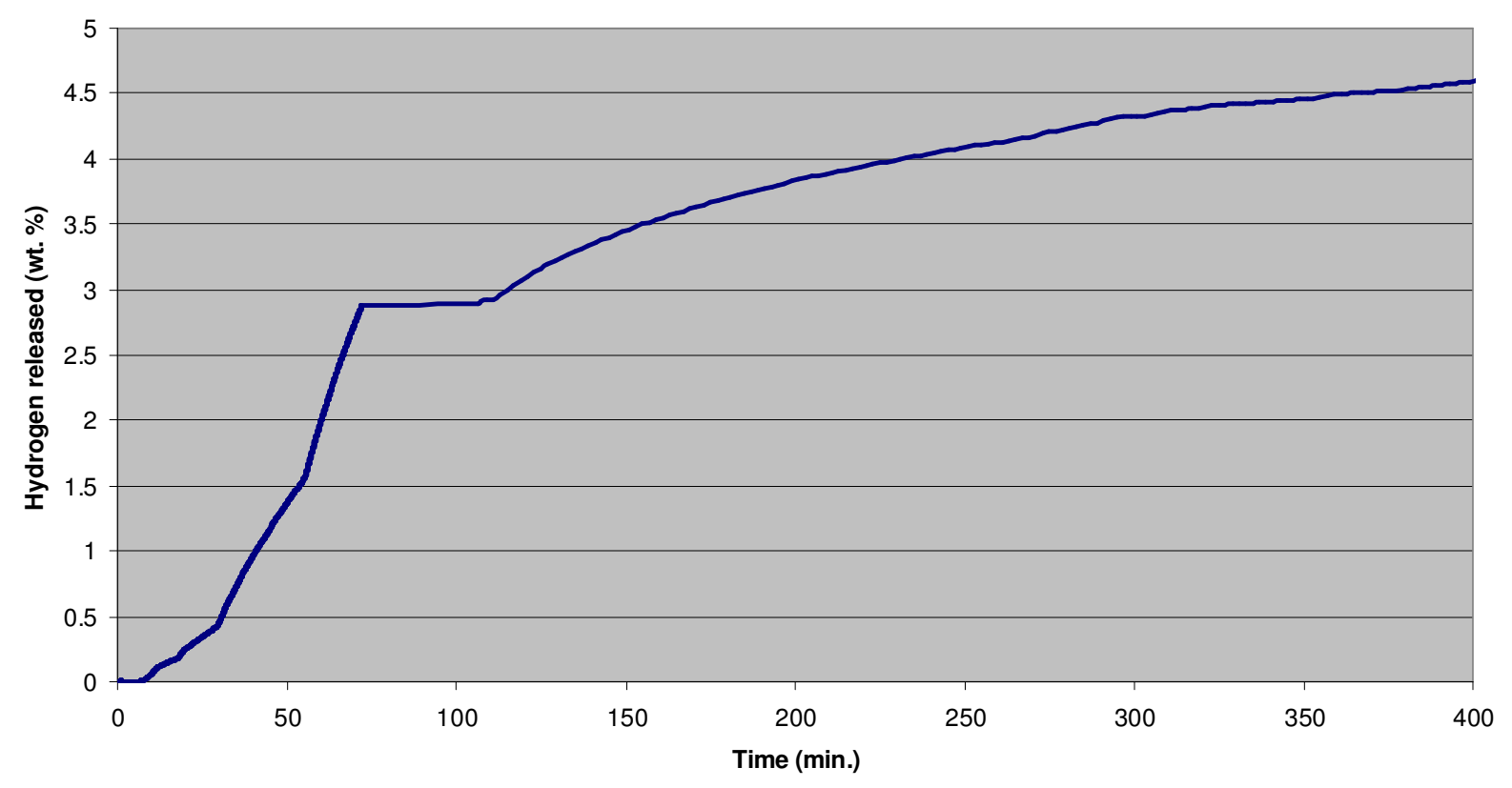

Figure 4-4. Dehydrogenation of hydrogenated 1,2,3-trimethyl indole mixture. 


\section{c. 1,2-dimethylindole/1-ethyl-2-methylindole mixture}

In an effort to increase the deliverable hydrogen capacity of an alkylated indole mixture to $>5$ wt. \%, 1,2-dimethylindole was investigated as a potential liquid carrier. Since 1,2-dimethylindole is a solid at ambient temperature (m.p. $50^{\circ} \mathrm{C}$ ), it was dissolved in a minimal amount of 1-ethyl-2-methylindole to form a liquid mixture (ca. 2:1 by weight 1,2-dimethylindole to 1-ethyl-2-methylindole). The mixture was hydrogenated at $170^{\circ} \mathrm{C}$ and 700 psig hydrogen pressure for 3 hours using a 5\% ruthenium on lithium aluminate catalyst. GC-MS analysis of the hydrogenated mixture indicated full conversion to perhydro-1,2-dimethylindole and perhydro-1-ethyl-2-methylindole (Table 4-4).

Table 4-4. GC-MS analysis of perhydro-1-ethyl-2-methylindole/ 1,2-dimethylindole mixture.

\begin{tabular}{|c|c|c|}
\hline Molecular Weight & $\begin{array}{c}\text { \% of Total } \\
\text { Compositon }\end{array}$ & $\begin{array}{c}\text { Theor. wt. \% } \\
\text { Hydrogen }\end{array}$ \\
\hline 153 (perhydro-1,2-dimethylindole) & 67 & 3.5 \\
\hline 167 (perhydro-1-ethyl-2-methylindole) & 33 & 1.6 \\
\hline
\end{tabular}

The hydrogenated mixture was dehydrogenated using 5\% palladium on alumina catalyst by first ramping the temperature to $150^{\circ} \mathrm{C}$ under one atmosphere hydrogen with stirring. After 1.5 hours, the temperature was increased to $175^{\circ} \mathrm{C}$ and ultimately to $185^{\circ} \mathrm{C}$ (Figure 4-5). After 13 hours total reaction time, the reactor was cooled to room temperature, and GC-MS analysis was performed on the dehydrogenated products (Table 4-5).

Table 4-5. GC-MS analysis of the 1-ethyl-2-methylindole/1,2-dimethylindole mixture after dehydrogenation.

\begin{tabular}{|c|c|c|}
\hline Molecular Weight & $\begin{array}{c}\text { \% of Total } \\
\text { Composition }\end{array}$ & $\begin{array}{c}\text { Wt. \% Unrecovered } \\
\text { Hydrogen }\end{array}$ \\
\hline 153 (perhydrogenated 1,2-dimethylindole) & 6.8 & 0.35 \\
\hline 149 (tetrahydro-1,2-dimethylindole) & 4.7 & 0.13 \\
\hline 145 (1,2-dimethylindole) & 53 & 0 \\
\hline 163 (tetrahydro-1-ethyl-2-methylindole) & 2.9 & 0.07 \\
\hline 159 (1-ethyl-2-methylindole) & 33 & 0 \\
\hline
\end{tabular}


The GC-MS results show that at $175^{\circ} \mathrm{C}$, approximately $90 \%$ of the hydrogen was desorbed, yielding a total deliverable capacity of 4.55 wt.\% hydrogen. When the dehydrogenation temperature was increased to $185^{\circ} \mathrm{C}$, the majority of the remaining hydrogen was desorbed, making the sample $99 \%$ reversible with a deliverable hydrogen capacity of 5.03 wt. \% hydrogen.

Table 4-6. GC-MS analysis of the 1-ethyl-2-methylindole/ 1,2-dimethylindole mixture from dehydrogenation at $185^{\circ} \mathrm{C}$.

\begin{tabular}{|c|c|c|}
\hline Molecular Weight & $\begin{array}{c}\text { \% of Total } \\
\text { Composition }\end{array}$ & $\begin{array}{c}\text { Wt. \% Unrecovered } \\
\text { Hydrogen }\end{array}$ \\
\hline 149 (tetrahydro-1,2-dimethylindole) & 2 & 0.05 \\
\hline 163 (tetrahydro-1-ethyl-2-methylindole) & 1 & 0.02 \\
\hline 159 (1-ethyl-2-methylindole) & 60 & 0 \\
\hline $145(1,2-$ dimethylindole) & 37 & 0 \\
\hline
\end{tabular}

In general, alkylated indole compounds are effective liquid carriers for hydrogen. Various compounds were discovered that showed very good selectivity for both hydrogenation and dehydrogenation. The positive attributes also include liquidity at near-ambient temperatures and at all stages of hydrogenation for carriers such as 1-ethyl-2-methylindole and a mixture of 1,2-dimethylindole and 1-ethyl-2-methylindole. On the negative side, the hydrogen capacity of the alkylated indoles is less than other classes of liquid carriers (e.g., all six-member ring compounds with nitrogen heteroatoms in Chapter 4-6). In addition, the impact of moderate vapor pressures for the alkylated indoles on the use of these carriers is yet to be determined. 


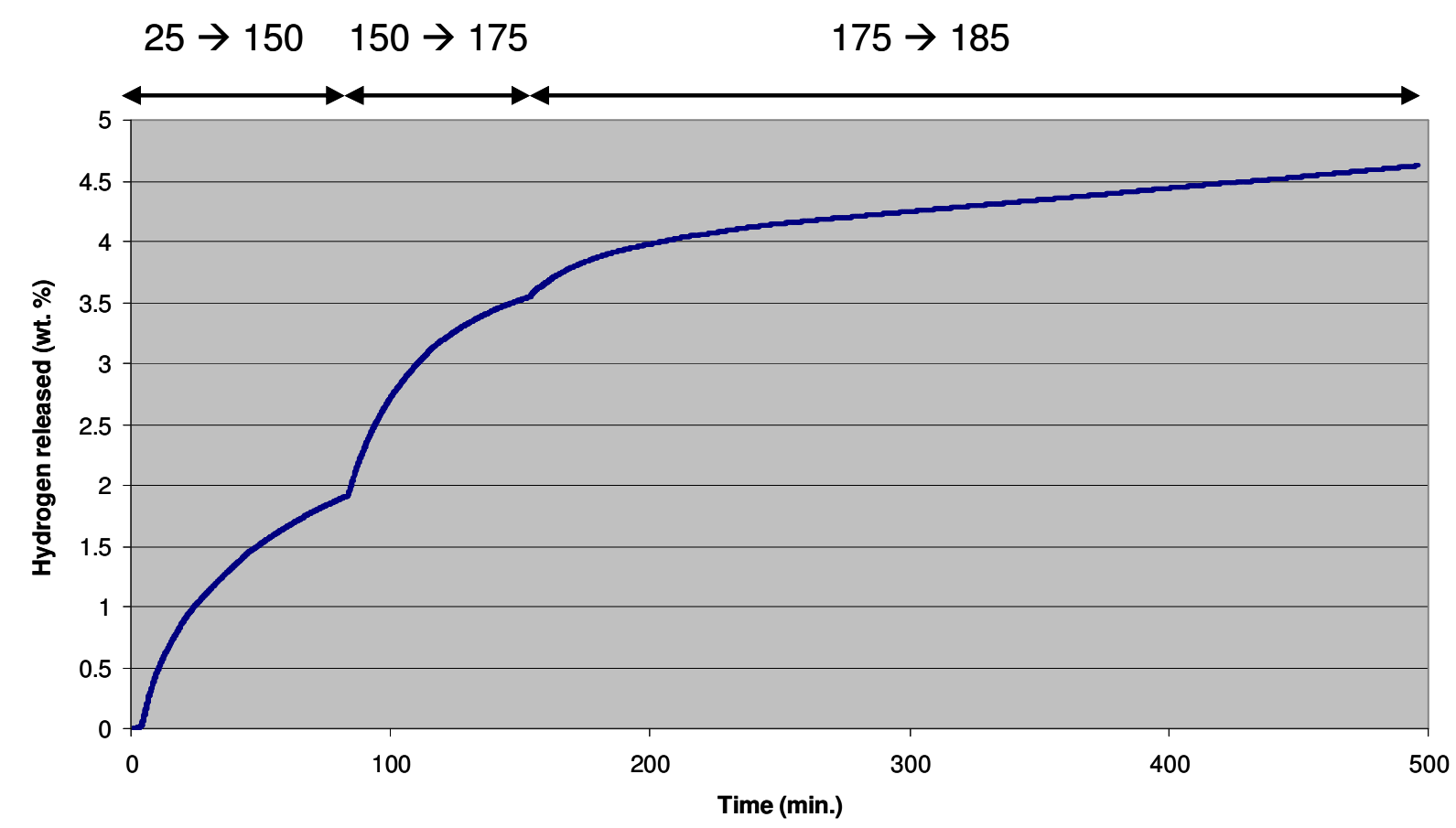

Figure 4-5. Dehydrogenation of hydrogenated 1,2-dimethylindole and 1-ethyl-2methylindole. Temperature ramping is indicated in degrees $\mathrm{C}$.

\section{A. Indole Dimers}

While alkylated indoles have been successfully tested as liquid carriers, repeatedly demonstrating selective hydrogenation and dehydrogenation during our testing, the higher volatility of this general class of compounds may limit their ultimate utility. Therefore, we explored the use of indole dimers as hydrogen carriers, with the notion that the high selectivity of alkylated indoles for reversible hydrogenation would be retained in a compound with substantially lower volatility.

The dimerization of indole occurs in the presence of strong acids in organic solvents. In a typical reaction, a solution of indole in diethyl ether was treated with an excess of $\mathrm{HCl}$ in ether. Upon neutralization with $2 \mathrm{M} \mathrm{NaOH}$, a mixture of two indole dimer compounds, [2-3']-biindolyl and a partially saturated derivative (at C2 and C3), was observed by GC/MS (Scheme 4-2). 

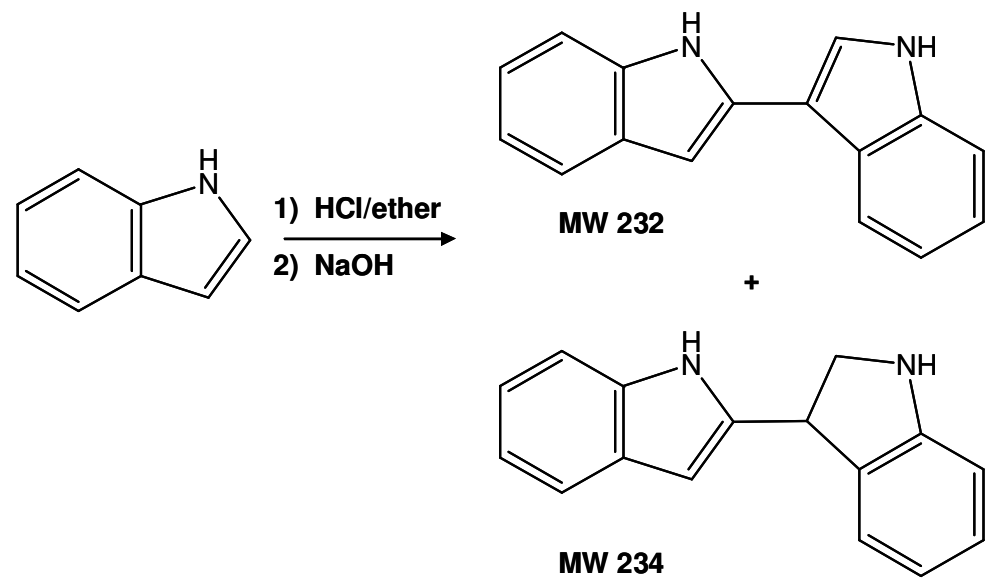

Scheme 4-2. Synthesis of indole dimers.

The two indole dimers shown in Scheme 4-2 are formed in ca. $80 \%$ yield. We found that the MW 234 product where the carbon atoms in the 2 and 3 positions of the 5 -membered ring are saturated slowly oxidizes in air to the fully unsaturated product (MW 232). Our attempts at the catalytic hydrogenation of the MW 232 indole dimer at $100^{\circ} \mathrm{C}$ surprisingly resulted in ring opening due to cleavage of the nitrogen-carbon bonds. In an attempt to prevent the nitrogen-carbon bond cleavage during hydrogenation, N-methylated indole was dimerized (Scheme 4-3).

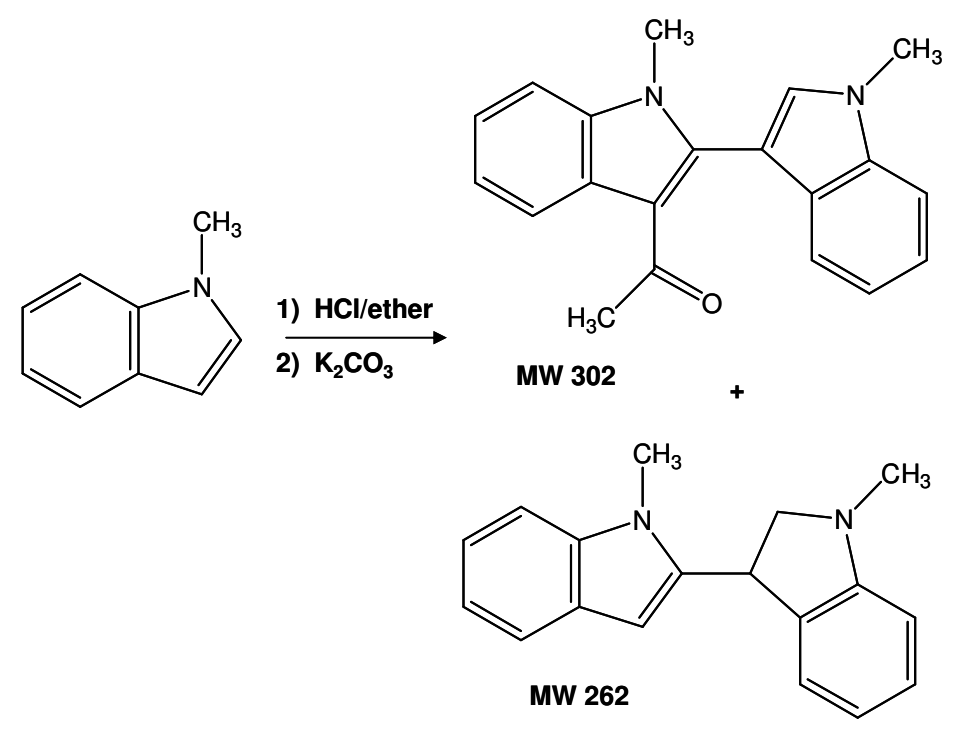

Scheme 4-3. The dimerization of N-methylindole. 
The reaction proceeds in a similar fashion to the indole dimerization, but it appears that the 3 position of the dimerized indole is highly reactive because this position was acylated on the fully unsaturated dimer. It is likely that this position is more reactive relative to indole because of the electron donation of the methyl groups. The acylation may have occurred by attack of partially protonated diethyl ether and subsequent Oxy-Cope rearrangement to the acetal during the basic reaction workup.

The mixture of the MW 262 and 302 dimers shown in Scheme 4-3 was hydrogenated using a $5 \% \mathrm{Rh} / \mathrm{Al}_{2} \mathrm{O}_{3}$ catalyst at $105^{\circ} \mathrm{C}$ and 1000 psig hydrogen pressure. The results of the hydrogenation are shown in Table 4-7.

Table 4-7. Hydrogenation of N-methylated indole dimer products.

\begin{tabular}{|c|c|c|c|}
\hline $\begin{array}{c}\text { Mass of Hydrogenated } \\
\text { Product of MW 262 }\end{array}$ & $\begin{array}{c}\text { \% Composition } \\
\text { in Mixture }\end{array}$ & $\begin{array}{c}\text { Mass of Hydrogenated } \\
\text { Product of MW 302 }\end{array}$ & $\begin{array}{c}\text { \% Composition } \\
\text { in Mixture }\end{array}$ \\
\hline 276 (perhydrogenated) & 12.6 & 318 (perhydrogenated) & 21.1 \\
\hline 272 & 8.1 & 310 & 12.6 \\
\hline 268 & 2.6 & 306 & 13.5 \\
\hline 278 & 12.3 & 304 & 0.5 \\
\hline & & 302 (fully unsaturated) & 16.2 \\
\hline
\end{tabular}

Approximately $30 \%$ of the MW 262 product undergoes ring-opening hydrogenolysis, forming a product with a MW of 278. However, no hydrogenolysis was observed for the substituted MW 302 product, indicating that a substituent at the three position may be beneficial for performing hydrogenation in the absence of ring-opening hydrogenolysis. The hydrogenated mixture shown in Table 4-7 was dehydrogenated using a 5\% $\mathrm{Pd} / \mathrm{Al}_{2} \mathrm{O}_{3}$ catalyst at $145^{\circ} \mathrm{C}$ under 15 psia hydrogen pressure (Table 4-8).

Table 4-8. Dehydrogenation of hydrogenated N-methylated indole dimer products.

\begin{tabular}{|c|c|c|c|}
\hline $\begin{array}{c}\text { Mass of } \\
\text { Dehydrogenated } \\
\text { Product of MW 262 }\end{array}$ & $\begin{array}{c}\text { \% Composition } \\
\text { in Mixture }\end{array}$ & $\begin{array}{c}\text { Mass of } \\
\text { Dehydrogenated } \\
\text { Product of MW 302 }\end{array}$ & $\begin{array}{c}\text { \% Composition in } \\
\text { Mixture }\end{array}$ \\
\hline 247 & 0.3 & 318 & 0.6 \\
\hline 244 & 2.9 & 314 & 10.8 \\
\hline 260 & 0.7 & 310 & 18.5 \\
\hline 239 & 19.3 & 306 & 26.9 \\
\hline 235 & 8.9 & 302 & 7.4 \\
\hline
\end{tabular}


The conditions employed for dehydrogenation appeared to destroy the unsubstituted dimer through loss of mostly primary and secondary amines, whereas the stability of the substituted dimer was remarkably higher under these conditions, and this compound did undergo the desired catalytic dehydrogenation. We did not detect any ring opening or other bond cleavage of the hydrogenated MW 302 material. This result suggests that having substituents on the nitrogen (1-position) and 3-position have a profound effect on the stability of the dimer. To this end, the alkylated dimer shown in Figure 4-6 was an attractive target molecule for dehydrogenation testing.

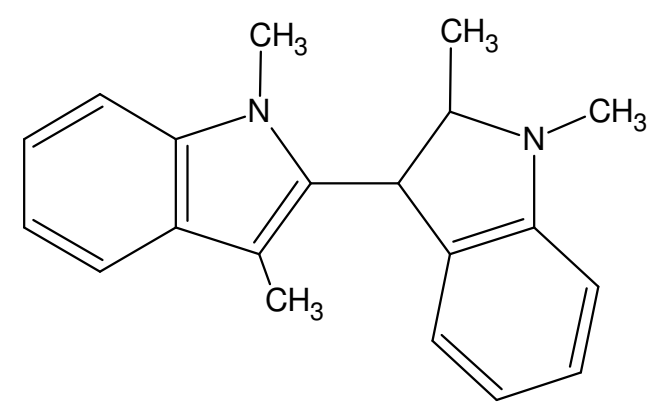

Figure 4-6. Structure of [2-3']-biindolyl with methyl groups in the 1,3- and 1',2'- positions.

To synthesize this molecule, 2-methylindole and 3-methylindole were $\mathrm{N}$-alkylated prior to dimerization. The $\mathrm{N}$-methylation of 3-methylindole was performed with an $80 \%$ yield (balance unreacted 3-methylindole) according to the reaction shown in Scheme 4-4. Unfortunately, our attempted synthesis of the liquid carrier prototype shown in Figure 4-6 via the acid-catalyzed coupling of 1,2-dimethylindole with 1,3-dimethylindole using anhydrous $\mathrm{HCl}$ was unsuccessful. A separate attempt to synthesize a [2-2']-biindolyl by acid-catalyzed coupling of the crude 1,3-dimethylindole mixture from the reaction shown in Scheme 4-4 did not exceed 10\% conversion with the same conditions used in the synthesis of [2-3']-biindolyl or under much more acidic conditions using anhydrous HF and $\mathrm{BF}_{3}$. 


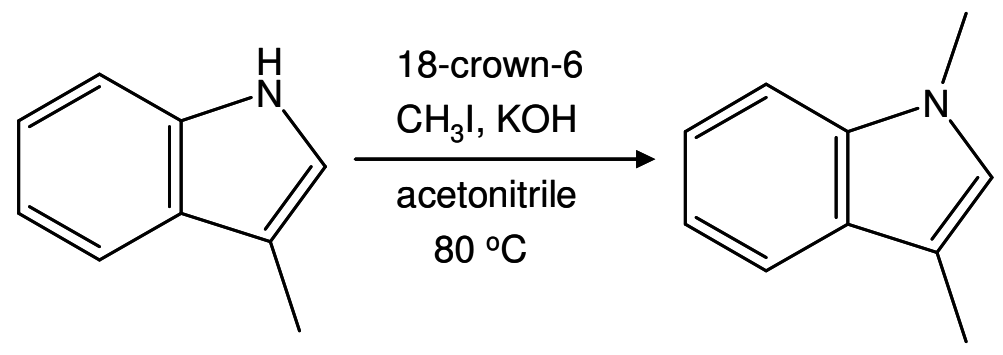

Scheme 4-4. N-alkylation of 3-methylindole.

Since dimerization of 1,3-dimethylindole did not proceed beyond $10 \%$ conversion, we attempted to access this type of indole dimer via a separate route where ring alkylated indole was dimerized and subsequently $\mathrm{N}$-alkylated. The dimer of 3-methylindole was prepared with $95 \%$ conversion (5\% remaining monomer) using acid-catalyzed coupling with anhydrous HF. Alkylation of the resulting mixture with $\mathrm{KOH}$ and methyl iodide yielded a mixture of alkylated products of which only $30 \%$ was the desired 1,3-dimethylindole dimer. This product mixture was extremely difficult to purify, and we did not continue to study the hydrogenation /dehydrogenation cycle of this dimer.

In summary, indole dimers with $\mathrm{N}$-methyl substituents and indole dimers without $\mathrm{N}$-methyl substituents were accessible via acid-catalyzed coupling reactions. However, these dimers are far less stable towards undesired ring-opening hydrogenolysis under conditions where di- and tri-alkylated indole monomers show high hydrogenation selectivity. While it may be possible that more highly alkylated indole dimers would show comparable selectivity to the di- and tri-alkylated indole monomers, the synthesis of these compounds was not successful via acid-catalyzed coupling. Therefore, no additional hydrogenation or dehydrogenation testing was performed.

\section{B. Indolocarbazoles}

Indolocarbazoles are a class of heterocyclic compounds formed by the ring fusion of indole and carbazole, creating an extended pi-conjugated molecule. Based upon the regiochemistry of ring fusion, a wide variety of isomers is possible (Figure 4-7). The extensive conjugation and presence of nitrogen-containing, five-membered rings are 
expected to yield low overall heats of hydrogenation (e.g., average $\Delta \mathrm{H}$ for hydrogenation of indolo[2,3-b]carbazole is $10.7 \mathrm{kcal} / \mathrm{mol} \mathrm{H}_{2}$ ). The melting points of the non-alkylated indolocarbazoles are very high, $>300^{\circ} \mathrm{C}$ for many isomers. Alkylation of these compounds and formation of mixtures of the alkylated indolocarbazoles to depress melting points are necessary for feasibility as liquid carriers.<smiles>c1ccc2c(c1)[nH]c1cc3c(cc12)[nH]c1ccccc13</smiles>

Indolo[2,3-b]carbazole<smiles>c1ccc2c(c1)[nH]c1c2ccc2[nH]c3ccccc3c21</smiles>

Indolo[3,2-a]carbazole<smiles>c1ccc2c(c1)[nH]c1c2ccc2c3ccccc3[nH]c21</smiles>

Indolo[2,3-a]carbazole

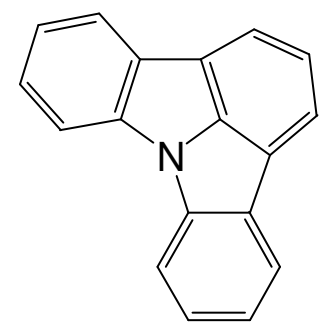

Indolo[3,2,1-jk]carbazole (phenylenecarbazole)<smiles>c1ccc2c(c1)[nH]c1ccc3[nH]c4ccccc4c3c12</smiles>

Indolo[2,3-c]carbazole

Figure 4-7. Structures of various indolocarbazole isomers.

\section{a. Indolo[2,3-b]carbazole}

Two synthetic routes were investigated for the preparation of indolo[2,3-b]carbazole.

The first route consisted of a step to prepare bis-indolylmethane from indole and formaldehyde, followed by reaction with triethyl orthoformate using para-toluenesulfonic acid as a catalyst (Scheme 4-5). 
Unfortunately, the second step of this route had a very poor yield of the desired product, and the separation of the desired product from impurities was very difficult.
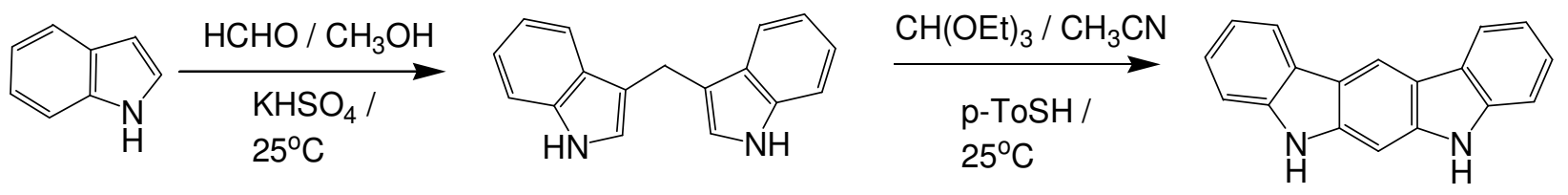

Scheme 4-5. Preparation of indolo[2,3-b]carbazole.

Our second approach to the preparation of indolo[2,3-b]carbazole was a two-step synthesis starting from commercially available 1,3-dichloro-4,6-dinitrobenzene. This starting material was subjected to a Suzuki palladium-catalyzed coupling with phenylboronic acid using 1,3-bis(diphenylphosphino)propane. This was followed by a reductive ring closure of the dinitroterphenyl intermediate using triethylphosphite in refluxing tert-butylbenzene (Scheme $4-6){ }^{7}$

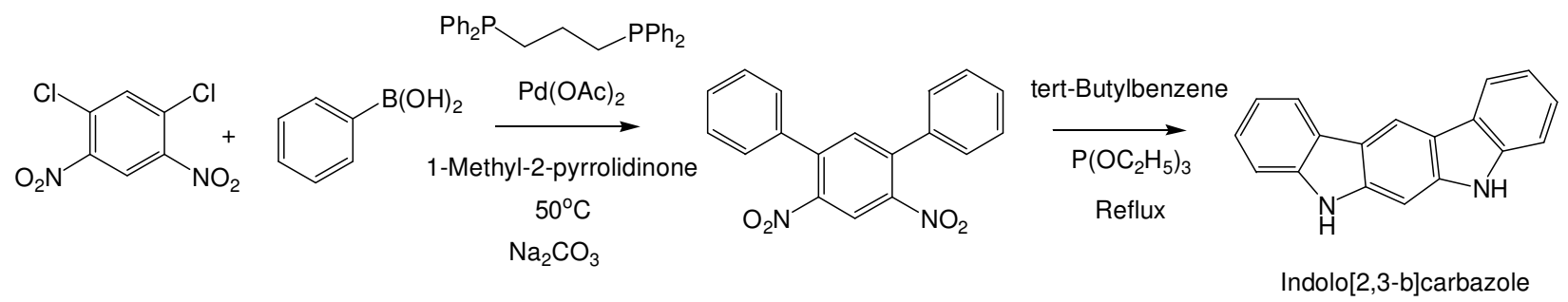

Scheme 4-6. Alternate scheme for the preparation of indolo[2,3-b]carbazole.

While the first step of the synthetic procedure shown in Scheme 4-6 was eventually accomplished with high conversion (Table 4-9), we found that the second step of this sequence worked poorly and resulted in low overall yields. Nonetheless, a small amount of indolo[2,3-b]carbazole was made available for alkylation and melting point studies. 
Table 4-9. Optimization of the Suzuki coupling of 1,3-dichloro-4,6-dinitrobenzene and phenylboronic acid.

\begin{tabular}{|c|c|c|c|c|c|c|c|}
\hline Solvent & Catalyst & Temperature & \multicolumn{5}{|c|}{ Conversion (GC/MS) } \\
\hline Wet NMP & Wet CsF & $100^{\circ} \mathrm{C}$ & $25 \%$ & $4 \%$ & $57 \%$ & $1 \%$ & $13 \%$ \\
\hline Wet NMP & Wet CsF & $25^{\circ} \mathrm{C}$ & $4 \%$ & $2 \%$ & $68 \%$ & - & $26 \%$ \\
\hline Dry NMP & Dry CsF & $25^{\circ} \mathrm{C}$ & - & - & $100 \%$ & - & - \\
\hline Dry NMP & Wet KF & $25^{\circ} \mathrm{C}$ & - & - & $100 \%$ & - & - \\
\hline Dry NMP & Wet $\mathrm{Na}_{2} \mathrm{CO}_{3}$ & $50^{\circ} \mathrm{C}$ & - & - & $100 \%$ & - & - \\
\hline
\end{tabular}

Literature reports detailing the preparation of indolo[2,3-b]carbazole suggested a melting point of $>300^{\circ} \mathrm{C}$, but the exact melting point was not known. We measured the melting point of indolo[2,3-b]carbazole at ca. $420^{\circ} \mathrm{C}$. N-alkylation of indolo[2,3-b]carbazole was achieved by the use of $\mathrm{NaH}$ in THF, followed by the addition of 1-Bromopropane or 1lodobutane to give the previously unreported di-N-propyl and di-N-butyl derivatives. Unfortunately, these compounds have melting points of 162 and $148^{\circ} \mathrm{C}$, respectively, which are not suitable for liquid carriers. Therefore, no additional work on the hydrogenation or dehydrogenation of these alkylated indolo[2,3-b]carbazole was performed.

\section{b. Indolo[3,2-a]carbazole}

The synthesis of an N,N'-dialkylated indolo[3,2-a]carbazole has been reported via the coupling of 3-bromo-N-ethylindole with $\mathrm{N}$-ethylindole and reaction of the resulting dimer with (dimethylamino)acetaldehyde diethyl acetal in the presence of acetic acid (Scheme 4-7). ${ }^{8}$ The liquid carrier prototype di-N-ethylindolo[3,2-a]carbazole has a very low overall calculated heat of hydrogenation of ca. $10.5 \mathrm{kcal} / \mathrm{mol} \mathrm{H}_{2}$ (cf. carbazole at $12.2 \mathrm{kcal} / \mathrm{mol}_{2}$ ). The melting point of the unalkylated compound was reported to be 
ca. $290^{\circ} \mathrm{C}$, thus offering some possibility for synthesizing an $\mathrm{N}$-alkylated derivative or mixture of alkylated derivatives that is liquid at near-ambient temperatures.

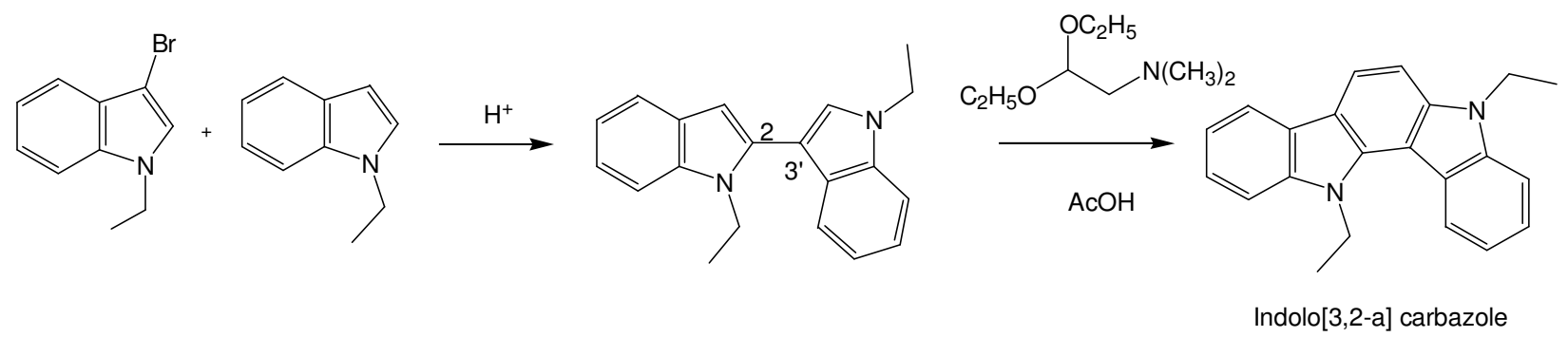

Scheme 4-7. Synthetic approach to N-N'-diethylindolo[3,2-a]carbazole.

Coupling of non-alkylated bromoindole and indole resulted in a mixture of 2,2' and 2,3'-indole dimers, which upon reaction with (dimethylamino)acetaldehyde diethyl acetal in the presence of acetic acid gave a mixture of indolo[3,2-a] carbazole and indolo[2,3-a]carbazole, as was determined by ${ }^{1} \mathrm{H}$ NMR after $\mathrm{N}$-ethylation and separation of the mixture by successive recrystallizations from ethyl acetate and hexanes (Scheme 4-8).<smiles>Brc1c[nH]c2ccccc12</smiles><smiles></smiles><smiles>c1ccc2[nH]c(-c3c[nH]c4ccccc34)cc2c1</smiles><smiles>c1ccc2c(c1)CC(c1cc3ccccc3[nH]1)N2</smiles><smiles>COC(CCN(C)C)OC</smiles><smiles>c1ccc2c(c1)ccc1c3ccccc3[nH]c21</smiles>

Scheme 4-8. A synthetic approach to the synthesis of indolo[3,2-a]carbazole. 
In support of the development of an approach for the production of alkylated derivatives of indolo[3,2-a]carbazole, the synthesis of several alkylated indole dimers was performed. Our procedure using neat anhydrous HF for the dimerization of alkylated indoles was utilized to provide the intermediate for synthesis of indolo[3,2-a]carbazole (Scheme 4-9).

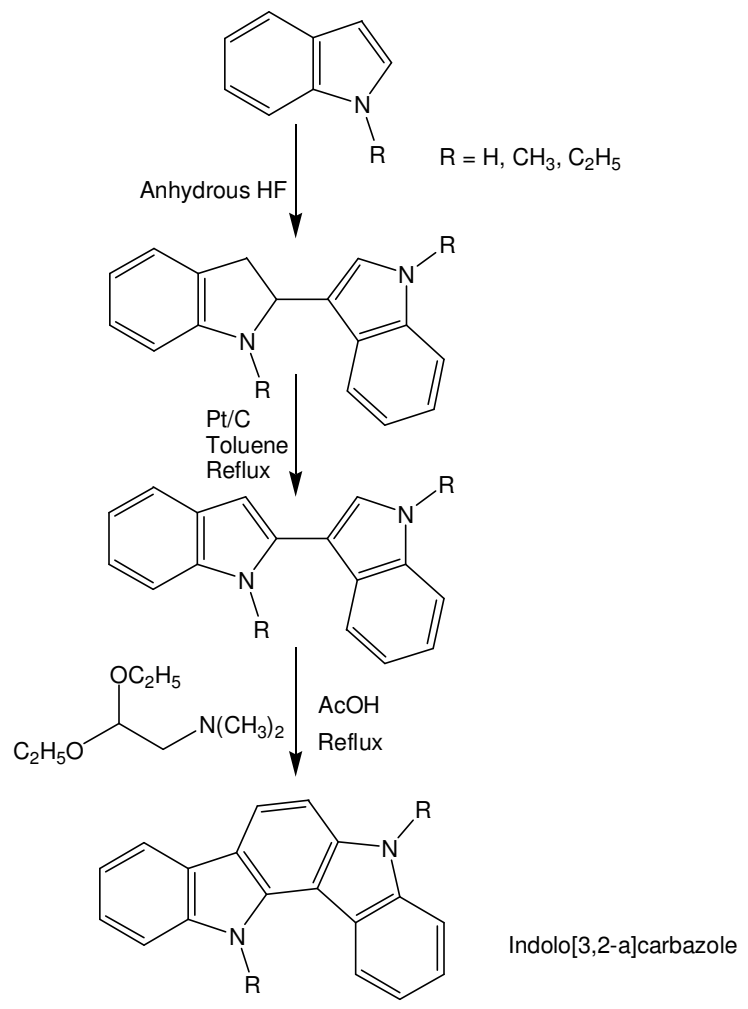

Scheme 4-9. The dimerization of alkylated indole and conversion to indolo[3,2a]carbazole.

Our initial attempts at the hydrogenation of di-N-ethylindolo[3,2-a]carbazole yielded mixed results. The first attempt was conducted using a $5 \% \mathrm{Rh} / \mathrm{Al}_{2} \mathrm{O}_{3}$ catalyst at $140^{\circ} \mathrm{C}$ under 1200 psia hydrogen pressure. The $5 \%$ Rh on alumina catalyst was effective for perhydrogenation of the substrate, but also catalyzed the hydrogenolysis of one of the $\mathrm{C}-\mathrm{N}$ bonds in the molecule, resulting in the opening of a five-membered ring. The resulting perhydrogenated, ring-opened molecule was tested for dehydrogenation. This material was cleanly dehydrogenated, but the dehydrogenation temperature was found to be similar to $\mathrm{N}$-ethylcarbazole (i.e., $200^{\circ} \mathrm{C}$ ) and not reflective of what might be 
expected for an intact perhydroindolo[3,2-a]carbazole. This is not surprising; the ringopened indolocarbazole is essentially a ring-substituted carbazole and should show a similar dehydrogenation temperature.

Neat hydrogenation of di-N-ethylindolo[3,2-a]carbazole at $170^{\circ} \mathrm{C}$ using various catalysts $(\mathrm{Rh}, \mathrm{Ru}, \mathrm{Ni})$ also resulted in hydrogenolysis of the perhydrogenated products.

Ruthenium on lithium aluminate was found to be the mildest catalyst (i.e., most resistant to hydrogenolysis). However, unfortunately, hydrogenation using ruthenium on lithium aluminate with solvents such THF, isopropanol and hexafluoroisopropanol yielded only products of incomplete hydrogenation (e.g., addition of 4 moles of $\mathrm{H}_{2}$ to form an octahydro derivative with a molecular weight of 320). The concentration of the substrate (ratio of substrate to solvent) did not seem to play a role in the extent of conversion during the hydrogenation (Table 4-10). Hydrogenation under either acidic conditions $\left(\mathrm{PtO}_{2}\right.$ with $\left.\mathrm{HBF}_{4}\right)$ or basic conditions (Rh/Ru with $\left.\mathrm{LiOH}\right)$ also did not prevent hydrogenolysis of the $\mathrm{C}-\mathrm{N}$ bond and did not result in high conversion to the desired product.

Table 4-10. Solvent: substrate ratio effects during hydrogenation of di-Nethylindolo[3,2-a]carbazole.

Solvent/ ratio
Isopropanol/ 1:80
Isopropanol/1:4 MW 312
Isopropanol/ $1: 2$




\section{c. Indolo[3,2,1-jk]carbazole}

Indolo[3,2,1-jk]carbazole ("phenylenecarbazole") is an attractive liquid carrier candidate because of its high capacity (6.93 wt. \%). This is the same theoretical capacity as 9 phenylcarbazole, but phenylenecarbazole differs from 9-phenylcarbazole in that all of the rings are fused, lowering the overall average heat of hydrogenation. Thus, one might expect the complete dehydrogenation of all the rings at moderate temperatures $\left(<200^{\circ} \mathrm{C}\right)$. Several approaches were evaluated for the synthesis of indolo[3,2,1jk]carbazole. A potential one-step approach, shown in Scheme 4-10, consists of an internal cyclization of 9-phenylcarbazole using copper(II)-trifluoromethanesulfonate and aluminum chloride. This type of ring-closing chemistry has been proven to be effective for the synthesis of other large polyaromatic hydrocarbons, but failed to produce any of the desired indolo[3,2,1-jk]carbazole.
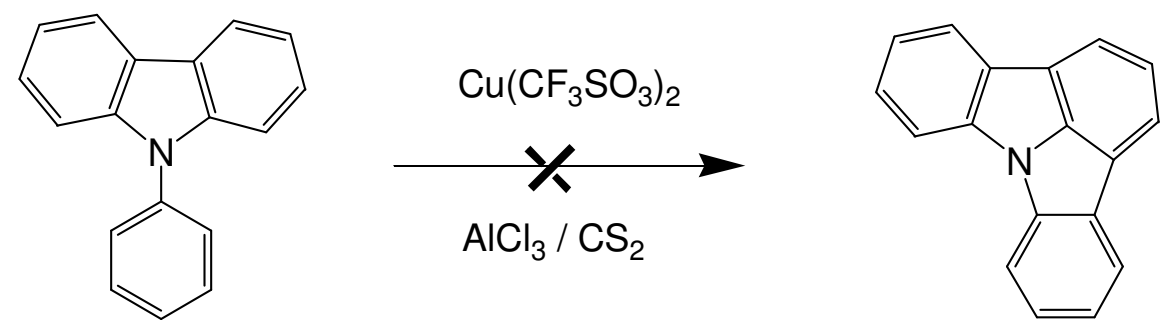

Scheme 4-10. Proposed one-step approach for the synthesis of indolo[3,2,1jk]carbazole.

A different synthetic approach has been reported in the literature and this approach consists of a three-step synthesis starting with an Ullman condensation ( $\mathrm{N}$-arylation) of carbazole with o-chloronitrobenzene to give 9-0-nitrophenylcarbazole, followed by reduction of the nitro group to the amine and, finally, diazotization with ring closure (Scheme 4-11). ${ }^{9}$ 


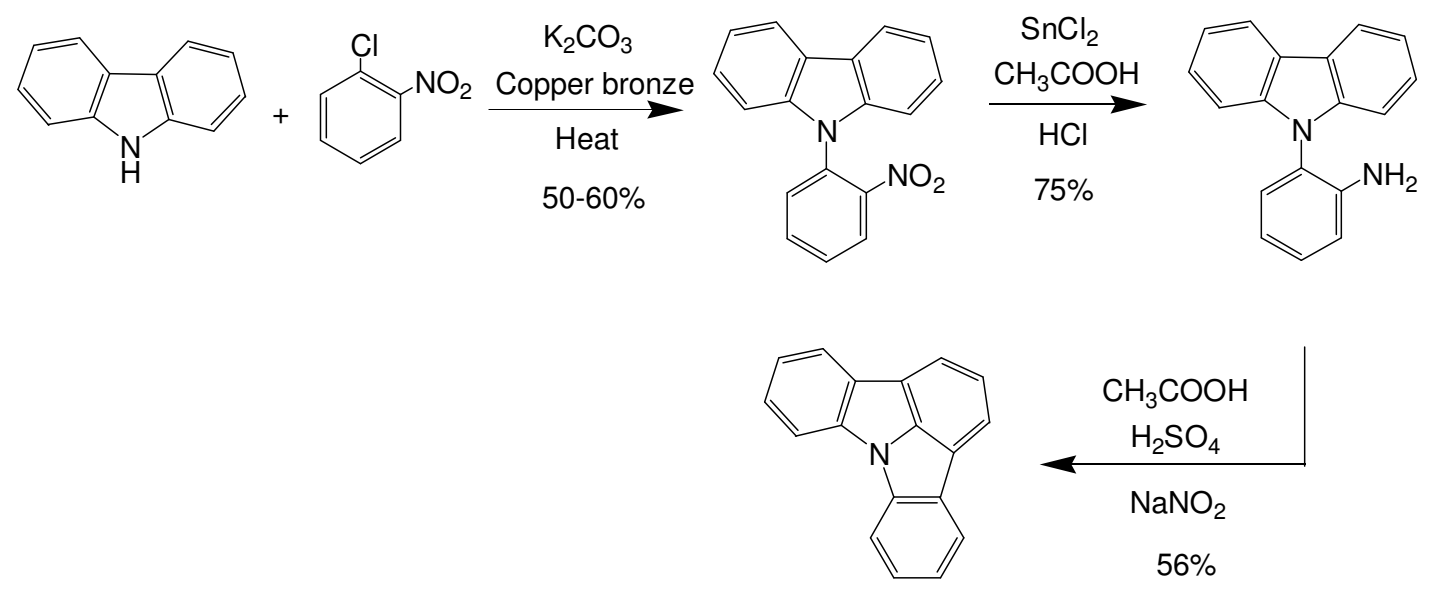

Scheme 4-11. Approach for the synthesis of indolo[3,2,1-jk]carbazole.

Despite the literature report, we found that the Ullman condensation using ochloronitrobenzene and palladium catalyzed $\mathrm{N}$-arylation of carbazole with either the bromo or chloronitrobenzene did not yield 9-o-nitrophenylcarbazole (Scheme 4-12). Therefore, 9-o-nitrophenylcarbazole was initially prepared by Ullman condensation of carbazole and o-bromonitrobenzene in pyridine (Scheme 4-13, top).

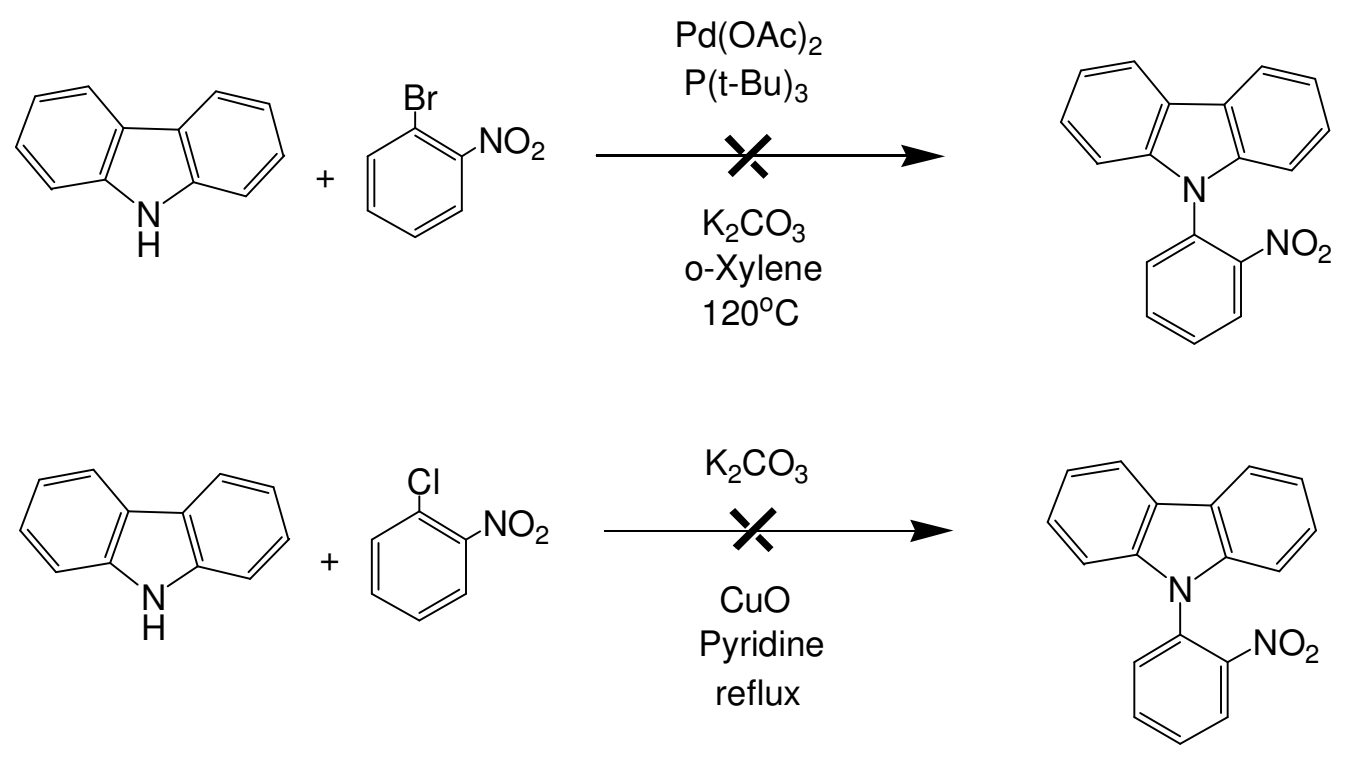

Scheme 4-12. Failed Ullman condensation using o-chloronitrobenzene and failed palladium catalyzed $\mathrm{N}$-arylation of carbazole. 
Reduction of the nitro group in 9-o-nitrophenylcarbazole to the amine was achieved using platinum oxide catalyst in THF or isopropanol at room temperature. The reaction can also be conducted using ruthenium on lithium aluminate, a more cost-effective catalyst than platinum oxide, using THF at $60^{\circ} \mathrm{C}$ (Scheme $4-13$, middle). While the resulting amine can be diazotized with sodium nitrite in a glacial acetic acid/sulfuric acid mixture, the work-up of the reaction mixture was cumbersome and resulted in low yields. Various solvents and nitrites were investigated and the final step of diazotization was ultimately achieved with isoamyl nitrite in acetonitrile in 60-70\% yield (Scheme 4-13, bottom).

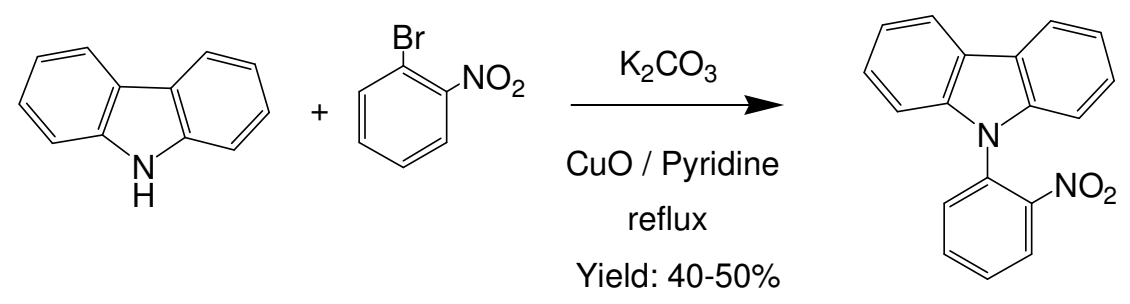<smiles>O=[N+]([O-])c1ccccc1-n1c2ccccc2c2ccccc21</smiles><smiles>Nc1ccccc1-n1c2ccccc2c2ccccc21</smiles>

Yield: Quantitative
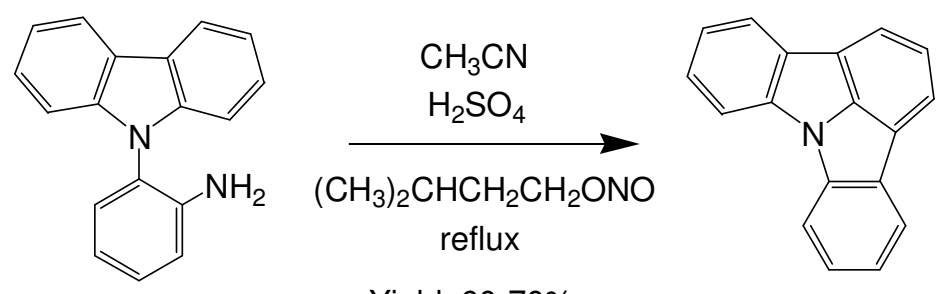

Yield: $60-70 \%$

Scheme 4-13. Preparation of 9-o-nitrophenylcarbazole by Ullman condensation of carbazole and o-bromonitrobenzene in pyridine and subsequent steps to form indolo[3,2,1-jk]carbazole. 
While the yield and effort required for product purification were acceptable for the last two steps shown in Scheme 4-13, the purification of the nitrophenylcarbazole in the first step was found to be cumbersome. An easier and almost quantitative method of preparing nitrophenylcarbazole was developed by the reaction of 2-fluoronitrobenzene with carbazole in dimethylformamide using cesium carbonate as a base (Scheme 4-14).
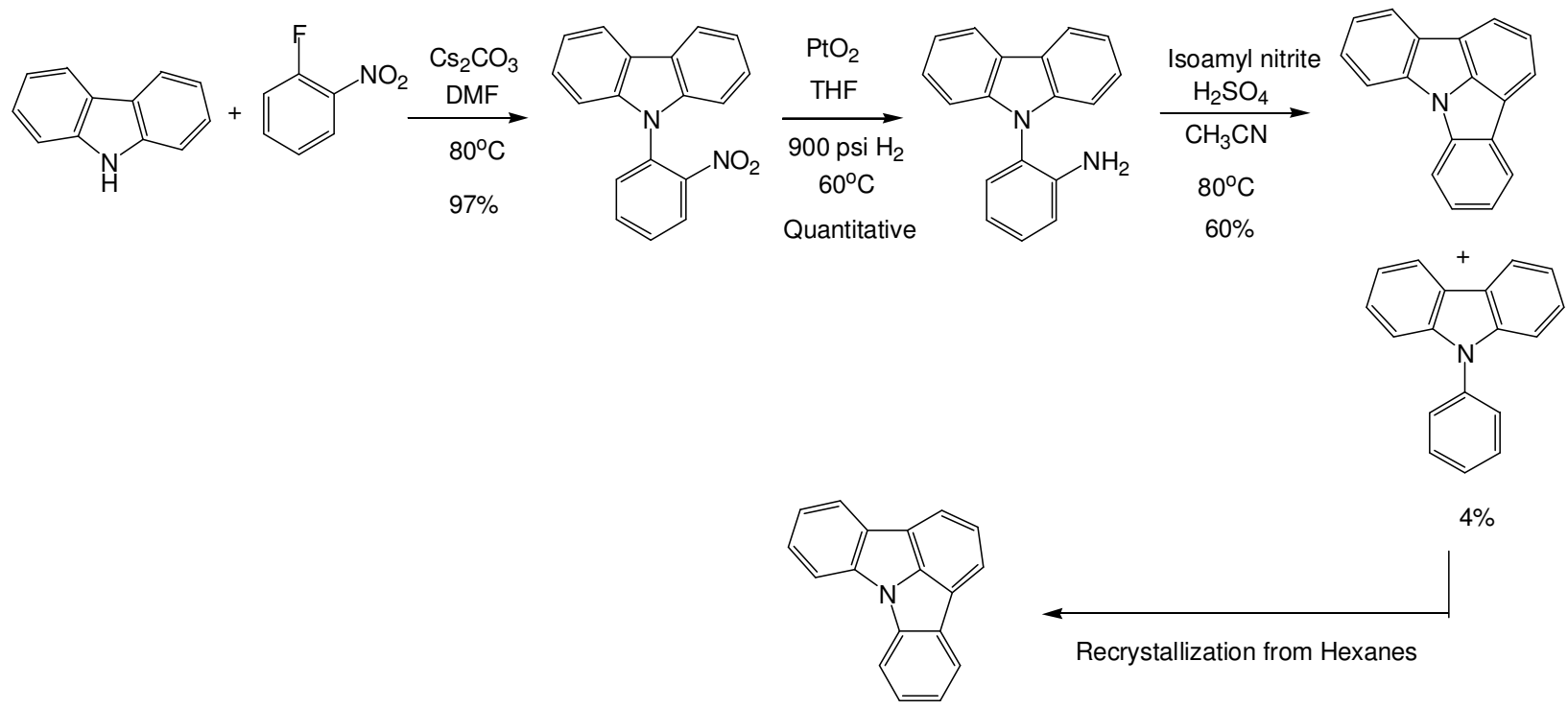

Scheme 4-14. Optimized synthetic scheme for the preparation of indolo[3,2,1jk]carbazole.

Alkylation of indolo[3,2,1-jk]carbazole might be expected to suppress the melting point of this compound. Therefore, 3-methyltetrahydrocarbazole was synthesized, and the optimized synthetic scheme used to produce indolo[3,2,1-jk]carbazole was attempted using 3-methyltetrahydrocarbazole as a starting material (Scheme 4-15). 


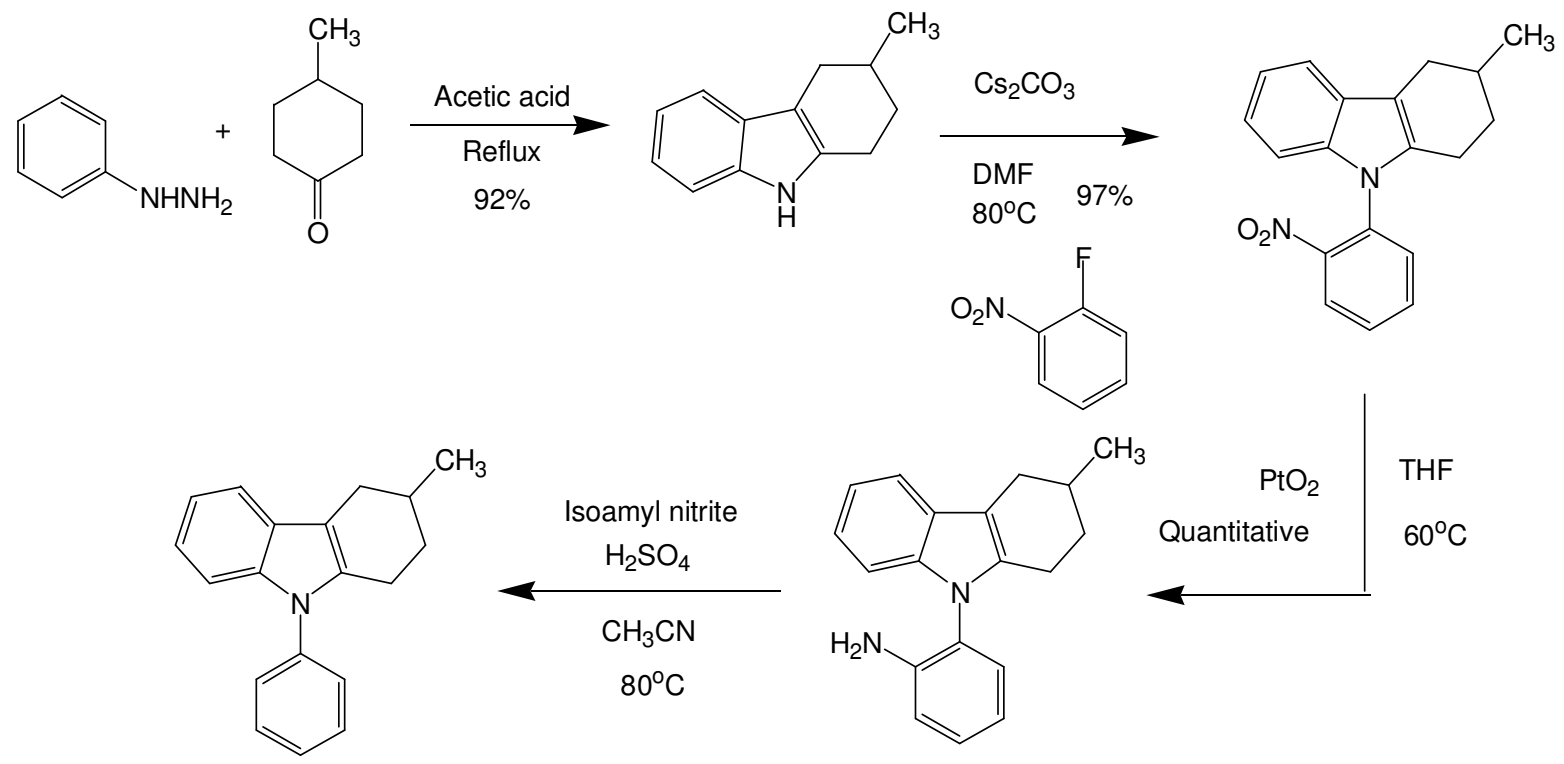

Scheme 4-15. Attempted synthesis of a methylated derivative of indolo[3,2,1jk]carbazole.

3-methyltetrahydrocarbazole was prepared in good yields from phenylhydrazine and 4-methylcyclohexanone. N-arylation was achieved in high yield using 1-fluoro-2nitrobenzene and cesium carbonate as the base in dimethylformamide. Subsequent reduction of the nitro group to a primary amine was performed in quantitative yield. Unfortunately, the final ring closure step of the synthesis did not proceed as expected. Instead of yielding the desired methyl indolo[3,2,1-jk]carbazole, the only isolated product was 3-methyl-9-phenyl-2,3,4,9-tetrahydrocarbazole.

Operating under the hypothesis that the partial saturation of the carbazole moiety was responsible for the failed ring closure step, we added a step to the synthetic scheme in which the tetrahydrocarbazole was subjected to catalytic dehydrogenation to form the fully unsaturated compound. The aromatization of 2,3,4,9-tetrahydrocarbazole was easily accomplished using $5 \%$ palladium on carbon in refluxing tert-butyl benzene under an inert atmosphere to obtain 3-methylcarbazole (Scheme 4-16). 


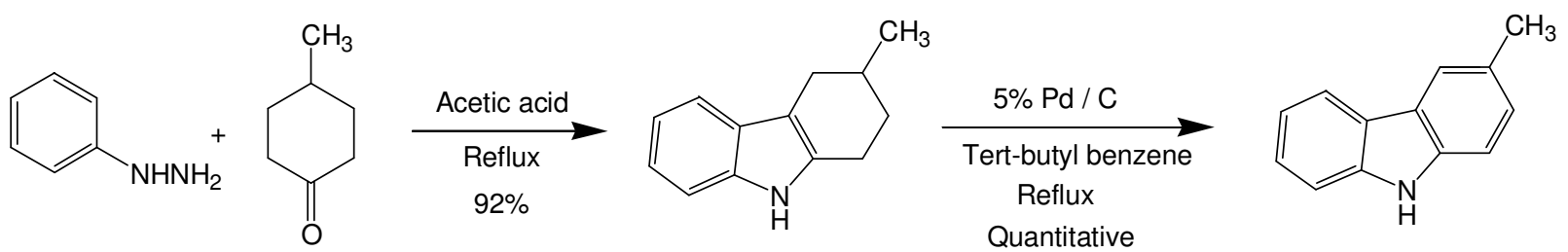

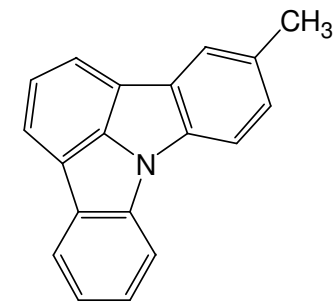

$3: 1$

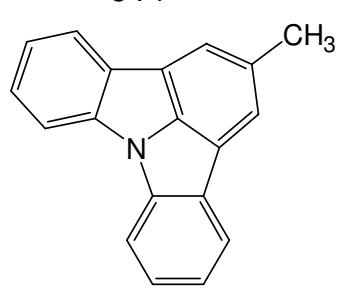

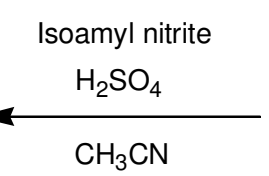

$80^{\circ} \mathrm{C}$
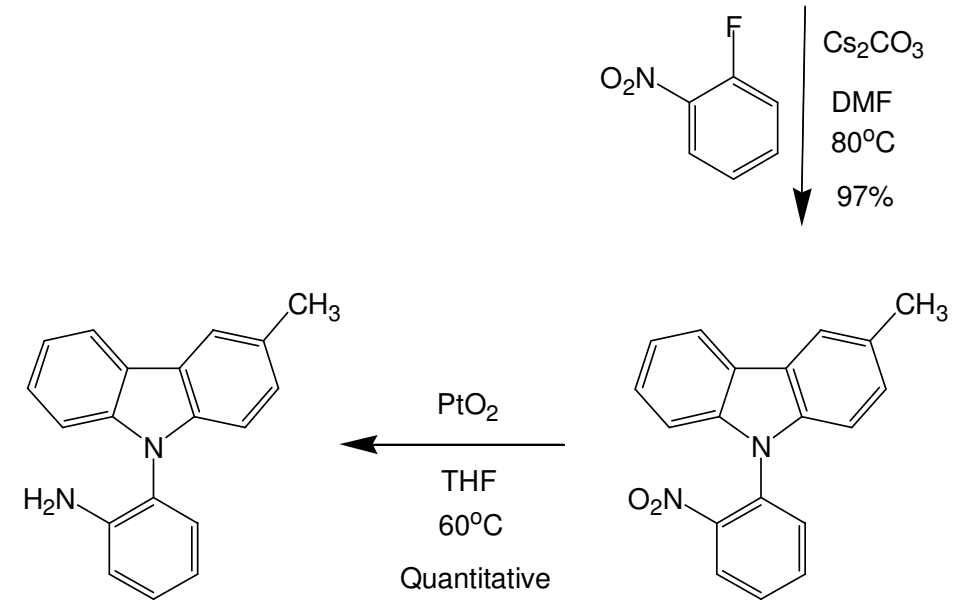

Scheme 4-16. Synthesis of a methylated derivatives of indolo[3,2,1-jk]carbazole.

Using the fully unsaturated N-nitrophenylcarbazole intermediate, the final synthetic step of diazotization and ring closure proceeded to yield two different isomers of methylated indolo[3,2,1-jk]carbazole. The position of the methyl group in the final product is determined by the position on the asymmetric methylcarbazole moiety where ring closure occurs. Under the conditions shown in Scheme 4-16, a 3:1 ratio of 5-methylindolo[3,2,1-jk]carbazole to 2-methyl-indolo[3,2,1-jk]carbazole was produced. Upon performing this ring-closure reaction multiple times, we found that the ratio of isomers was highly variable, and some reactions produced only 5-methyl-indolo[3,2,1jk]carbazole.

Catalytic hydrogenation of phenylenecarbazole was studied using a number of 'standard' hydrogenation catalysts (e.g., rhodium on carbon, ruthenium on lithium aluminate). In a 
typical experiment, a sample of phenylenecarbazole (containing ca. $4 \% \mathrm{~N}$ -

phenylcarbazole as an impurity) was perhydrogenated using $5 \% \mathrm{Rh} / \mathrm{C}$ at $180^{\circ} \mathrm{C}$ under 1000 psig hydrogen. The perhydrogenated material was isolated as a viscous, oily liquid. GC-MS analysis of the reaction product showed high conversion and did not show any traces of products of hydrogenolysis or ring-opening reactions. Depending on the temperature used during the catalytic hydrogenation, one to five fully hydrogenated isomers were observed. Similar to the isomer distribution observed for other liquid carriers, such as $\mathrm{N}$-ethylcarbazole, a low reaction temperature favors the formation of fewer, less thermodynamically stable isomers. At a low temperature (e.g., $\left.140^{\circ} \mathrm{C}\right)$, only a single isomer was observed, and at higher reaction temperatures (e.g., $180^{\circ} \mathrm{C}$ ), five isomers are observed by GC-MS.

The initial testing of hydrogenation/dehydrogenation cycling stability for indolo[3,2,1$\mathrm{jk}$ carbazole was performed as a 1:1 mixture with $\mathrm{N}$-ethylcarbazole. A mixture of partially hydrogenated phenylenecarbazole and $\mathrm{N}$-ethylcarbazole was placed in a reactor with a hydrogenation catalyst ( $5 \%$ ruthenium on lithium aluminate) and dehydrogenation catalyst ( $5 \%$ palladium on carbon). The mixture was initially hydrogenated (to complete the hydrogenation of the phenylenecarbazole).

The cycling experiments, shown in Figure 4-8, revealed that at a maximum temperature of $225^{\circ} \mathrm{C}$, the mixture can be dehydrogenated to near $100 \%$ conversion. The $1: 1$ mixture has a theoretical capacity of ca. $6.3 \mathrm{wt}$ \%. Although the first dehydrogenation run was stopped prematurely, the second run yielded $6.15 \mathrm{wt} . \% \mathrm{H}_{2}$ after 5 hours. The rate of dehydrogenation dropped substantially in the third and fourth cycles. The fourth dehydrogenation cycle was extended to 20 hours in an attempt to maximize conversion before characterization. GC-MS analysis of the mixture after the fourth cycle showed that the $\mathrm{N}$-ethylcarbazole present in the mixture was completely dehydrogenated. However, a surprising finding was that the phenylene carbazole in the dehydrogenated mixture was not completely dehydrogenated, being present almost exclusively in the mixture as an octahydro intermediate with a molecular weight of 249 . 


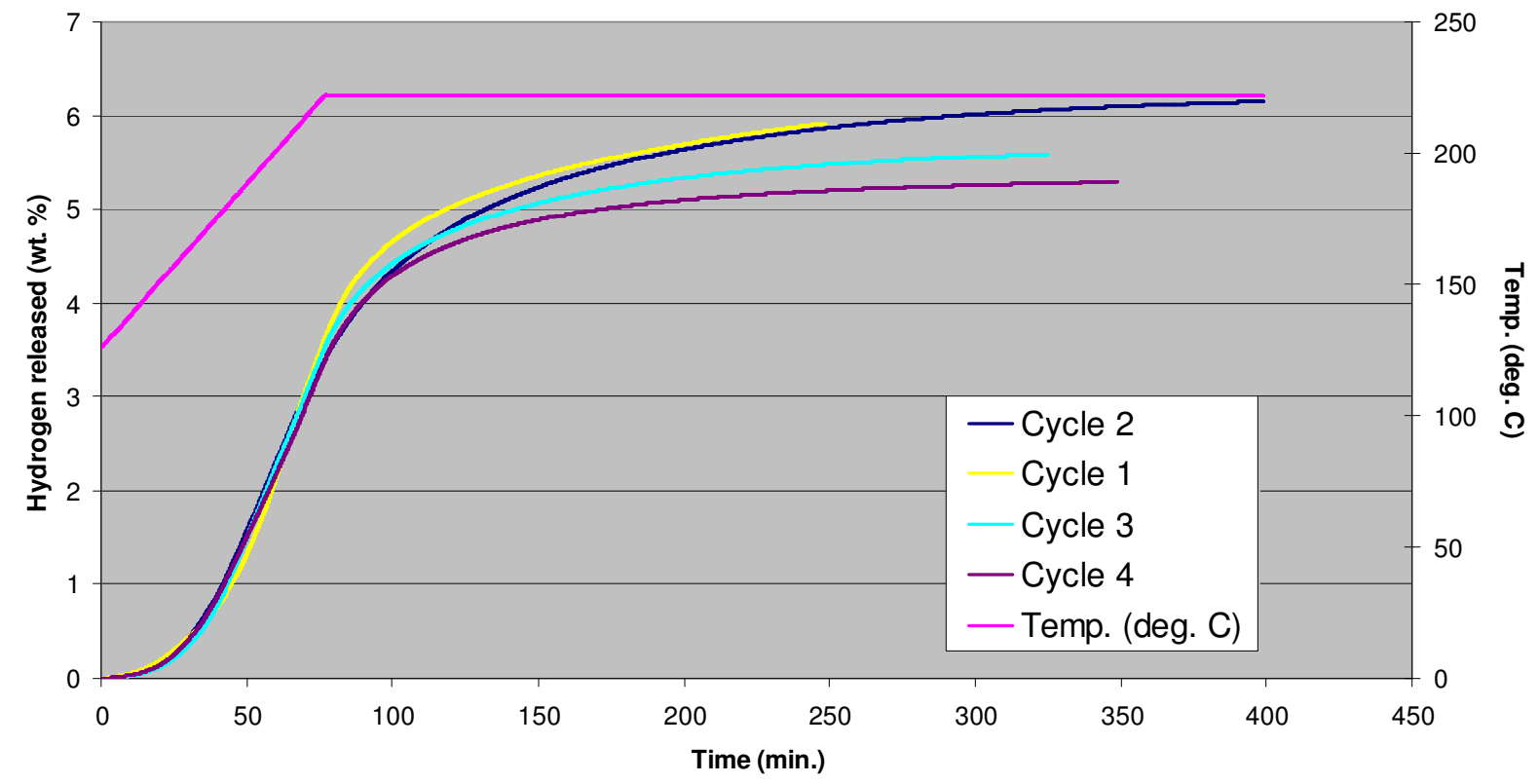

Figure 4-8. Dehydrogenation cycling stability of indolo[3,2,1-jk]carbazole in a 1:1 mixture with $\mathrm{N}$-ethylcarbazole.

The initial dehydrogenation testing of neat phenylenecarbazole was performed using palladium on carbon as the catalyst (Figure 4-9). The sample temperature during the dehydrogenation experiment was slowly ramped to $225^{\circ} \mathrm{C}$. The hydrogen flow rate began to level off when the sample had desorbed approximately $3.5 \mathrm{wt}$. \%, half of the theoretical amount of deliverable hydrogen. At this point, the sample temperature was raised to $255^{\circ} \mathrm{C}$ to desorb the remaining hydrogen. In the end, the sample demonstrated a working capacity of 6 wt. \%. 


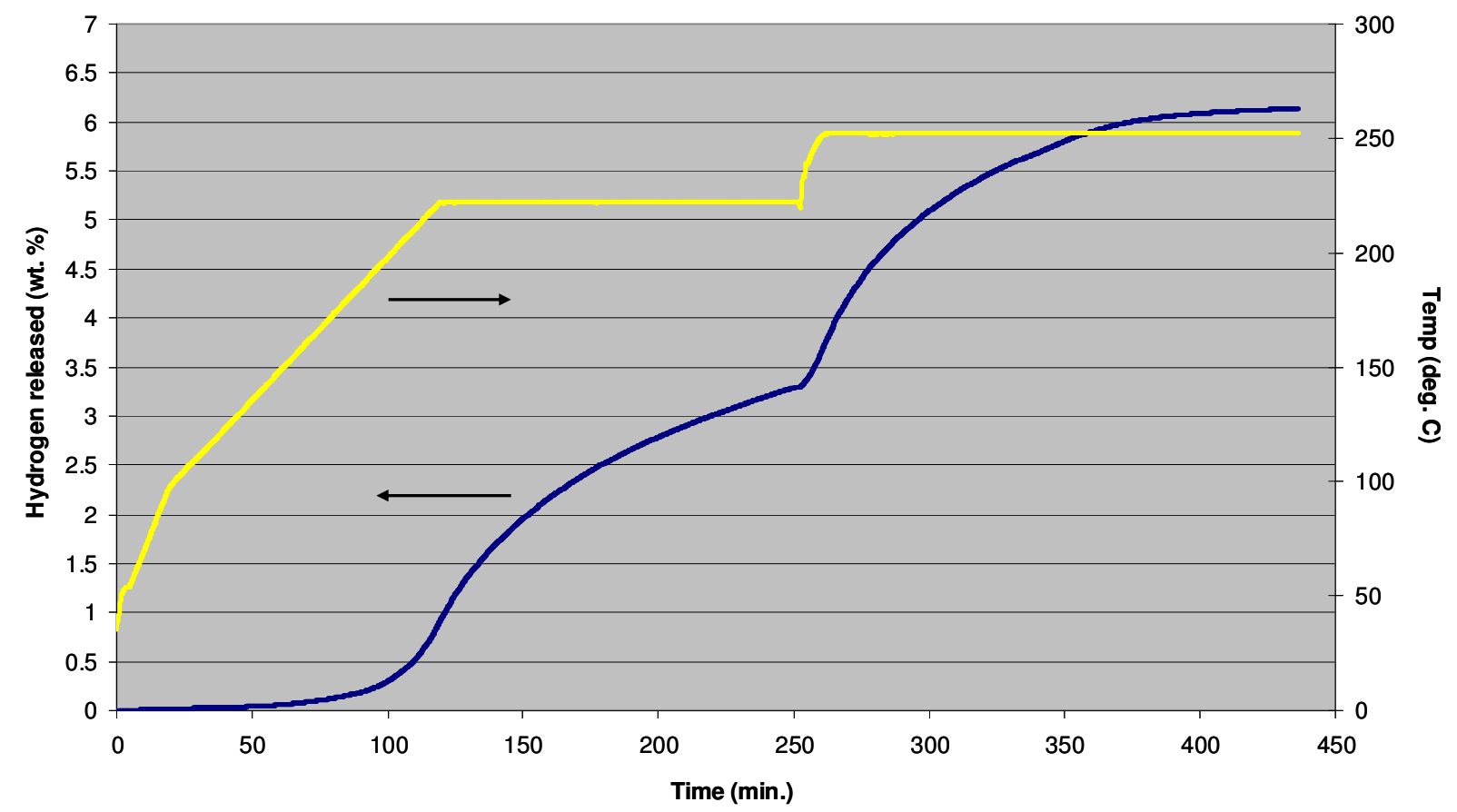

Figure 4-9. Dehydrogenation of neat phenylenecarbazole.

The temperature required for high dehydrogenation conversion was higher than expected based on the earlier cycling experiments in which the phenylenecarbazole was mixed with N-ethylcarbazole. In the cycling experiment shown in Figure 4-8, the dehydrogenation catalyst is exposed to hydrogenation conditions (high temperature under high pressure hydrogen) possibly making the dehydrogenation catalyst more active. Another possibility for the lower dehydrogenation conversion at moderate temperatures for the neat phenylenecarbazole is an impurity (e.g., N-phenylcarbazole) which interfered with the dehydrogenation. Another dehydrogenation experiment was performed using a highly pure sample of phenylenecarbazole containing both hydrogenation and dehydrogenation catalysts. The $5 \% \mathrm{Pd} / \mathrm{C}$ dehydrogenation catalyst was present during an initial hydrogenation step, and in this experiment the working capacity of the sample was $5.8 \mathrm{wt}$ \% hydrogen at a maximum temperature of $220^{\circ} \mathrm{C}$ (Figure 4-10). 


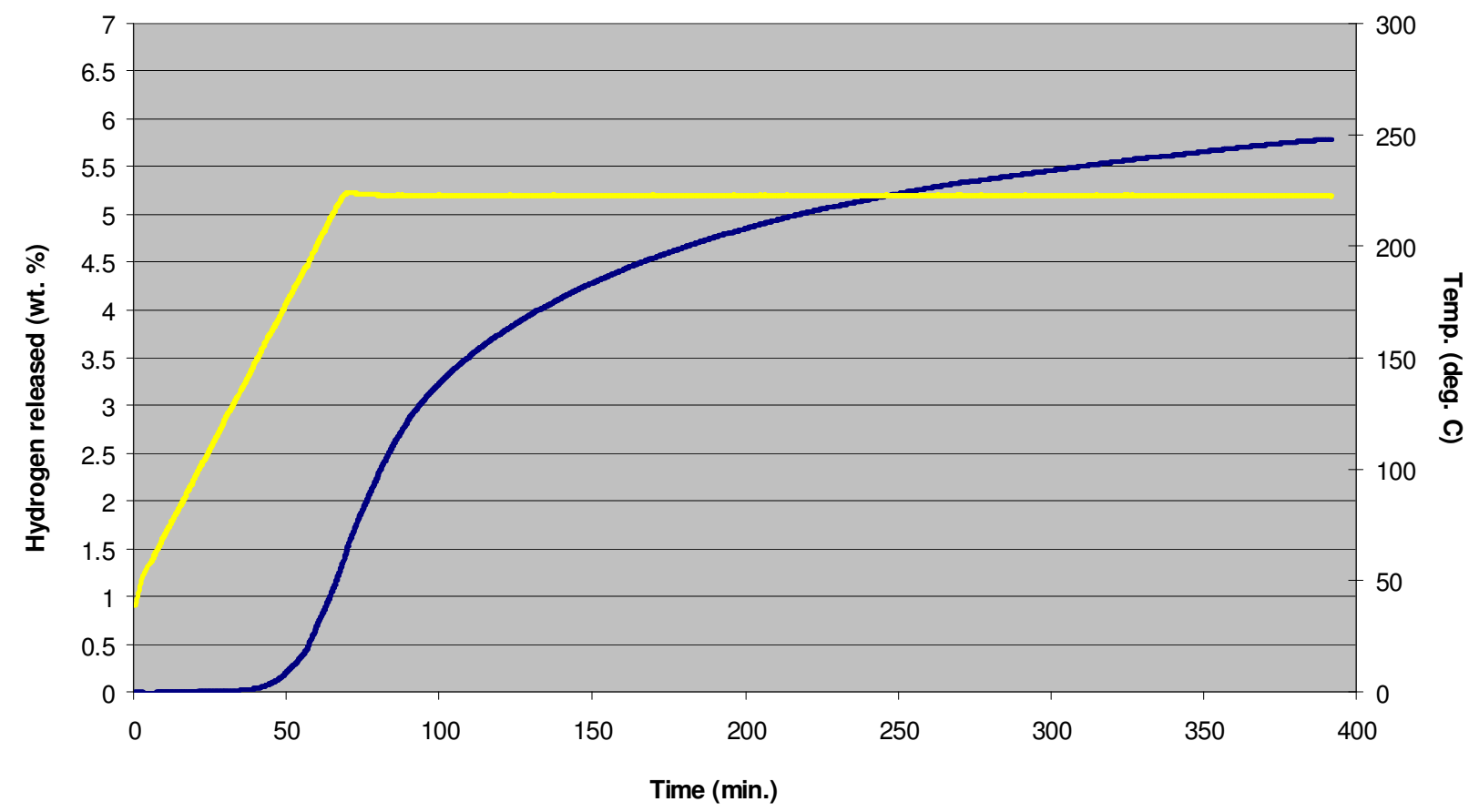

Figure 4-10. Dehydrogenation of phenylenecarbazole.

It may be concluded from the results of this experiment that activation of the dehydrogenation catalyst at higher temperatures and hydrogen pressure may be necessary to substantially dehydrogenate phenylenecarbazole at moderate temperatures. It seems unlikely that the N-phenylcarbazole impurity in the initial dehydrogenation experiment shown in Figure 4-9 caused the poor performance, as it is a tertiary amine, and was present during the earlier cycling experiments with an $\mathrm{N}$ ethylcarbazole/phenylenecarbazole mixture where the capacity was slightly above $6 \mathrm{wt}$. $\%$ at $220^{\circ} \mathrm{C}$. An alternate explanation is that the inability to realize the full $6.9 \mathrm{wt}$. $\%$ of pure phenylenecarbazole is related to the viscosity of the dehydrogenated compounds at high conversion, as the conversion was better in the 1:1 mixture of phenylene carbazole with $\mathrm{N}$-ethylcarbazole.

The distribution of isomers determined by the temperature used in the hydrogenation of phenylenecarbazole was found to have a perceptible influence on the dehydrogenation 
rates of the perhydrogenated compound. Similar to our experience with $\mathrm{N}$-ethylcarbazole, we found that the single isomer formed by hydrogenation at the lower temperatures yielded better initial dehydrogenation kinetics. However, as the perhydrogenated phenylenecarbazole was heated to the minimum practical dehydrogenation temperature of $200^{\circ} \mathrm{C}$, or higher, in the presence of a dehydrogenation catalyst, the perhydrogenated phenylenecarbazole isomerized to a mixture of more stable isomers; thus the benefit of having the less stable kinetic conformer in the mixture was lost. By GC-MS analysis, we found that when a pure sample of the less stable isomer is dehydrogenated at $200^{\circ} \mathrm{C}$, trace amounts of all five fully hydrogenated isomers are observed in the product mixture if the dehydrogenation is stopped at low conversion. Therefore, the dehydrogenation catalysts used in our experiments are also active as isomerization catalysts, and what appears to be a small activation barrier for isomerization is unfortunate, since it is exceeded before dehydrogenation occurs to any appreciable conversion level.

High selectivity in both the hydrogenation and dehydrogenation steps is a requirement for any promising liquid carrier candidate. Unfortunately, careful analysis of the products isolated after our dehydrogenation testing of perhydrogenated phenylenecarbazole revealed that bond cleavage (hydrogenolysis) was occurring during the dehydrogenation. In order to identify the source of the unwanted hydrogenolysis products, we performed experiments to determine the molecular weight and relative amounts of intermediates present during two hydrogenation/dehydrogenation cycles of fully hydrogenated phenylenecarbazole (Figure 4-11 and Table 4-11).

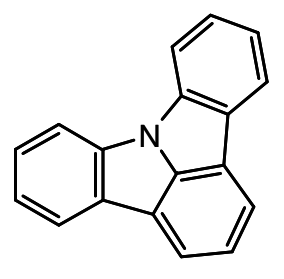

241

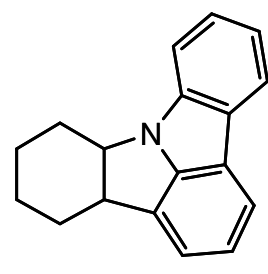

247

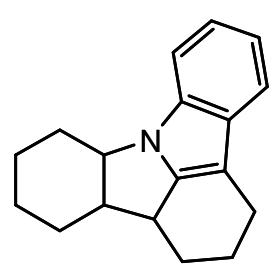

251

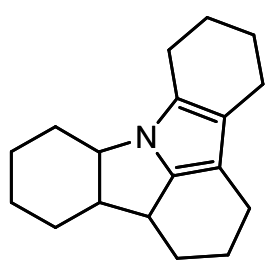

255

Figure 4-11. Proposed structures of intermediates (with molecular weights listed) observed during phenylenecarbazole hydrogenation. 
The structures shown in Figure 4-11 are assumed based on the observed mass numbers in our GC-MS analysis and analogy with N-ethylcarbazole. The intermediate products are formed by the stepwise loss of 2 hydrogen molecules forming aromatic structures similar to substituted pyrrole, indole, and carbazole. The most abundant masses in the dehydrogenation product mixture after dehydrogenation at $200^{\circ} \mathrm{C}$ are MW 247 and MW 251.

Table 4-11. Molecular weight determined by GC/MS after two hydrogenation/ dehydrogenation cycles.

\section{Cycle 1}

Hydrogenation

\begin{tabular}{|c|c|}
\hline Molecular Wt. & \% in Product Mixture \\
\hline 259 & 100 \\
\hline
\end{tabular}

Dehydrogenation

\begin{tabular}{|c|c|}
\hline Molecular Wt. & \% in Product Mixture \\
\hline 241 & 41 \\
\hline 243 & 16 \\
\hline 249 & 23 \\
\hline 251 & 14 \\
\hline 247 & 1 \\
\hline 253 & 7 \\
\hline Observed Capacity & $5.6 \mathrm{wt} . \% \mathrm{H}_{2}$ \\
\hline
\end{tabular}

\section{Cycle 2}

Hydrogenation

\begin{tabular}{|c|c|}
\hline Molecular Wt. & \% in Product Mixture \\
\hline 259 & 68 \\
\hline 261 & 32 \\
\hline
\end{tabular}


Dehydrogenation

\begin{tabular}{|c|c|}
\hline Molecular Wt. & \% in Product Mixture \\
\hline 241 & 18 \\
\hline 243 & 14 \\
\hline 249 & 47 \\
\hline 251 & 5 \\
\hline 253 & 8 \\
\hline Observed Capacity & 5.2 wt. $\% \mathrm{H}_{2}$ \\
\hline
\end{tabular}

While the hydrogenolysis was difficult to observe after the first dehydrogenation cycle due to the multiplicity of products from the dehydrogenation, hydrogenolysis of phenylenecarbazole during dehydrogenation was apparent when the product mixture after the second hydrogenation contained $32 \%$ of a species with a molecular weight of 261, two atomic mass units higher than fully hydrogenated phenylenecarbazole. After a third hydrogenation/dehydrogenation cycle, the amount of this MW 261 byproduct in the final hydrogenated mixture increased to $75 \%$. We were able to determine that the bond cleavage occurred during dehydrogenation rather than hydrogenation, unlike our experience with other potential substrates (e.g., indolo[3,2-jk]carbazole), when hydrogenolysis occurred during hydrogenation. It was found that when the mixture obtained from the second hydrogenation was subjected to our hydrogenation conditions for an additional three hours, no additional hydrogenolysis occurred. It is likely that the higher temperatures $\left(>225^{\circ} \mathrm{C}\right)$ necessary for substantial dehydrogenation are responsible for the hydrogenolysis.

Experiments were performed using various conditions/catalysts for dehydrogenation in an effort to prevent hydrogenolysis during dehydrogenation while still obtaining a high conversion. While we were able to lower the minimum hydrogenation temperature to $140^{\circ} \mathrm{C}$, dehydrogenation did not exceed $60 \%$ conversion until $>200^{\circ} \mathrm{C}$ (Figure 4-12). Based on these experiments, we found that hydrogenolysis occurred with any of the dehydrogenation catalysts developed for other liquid carrier candidates. 


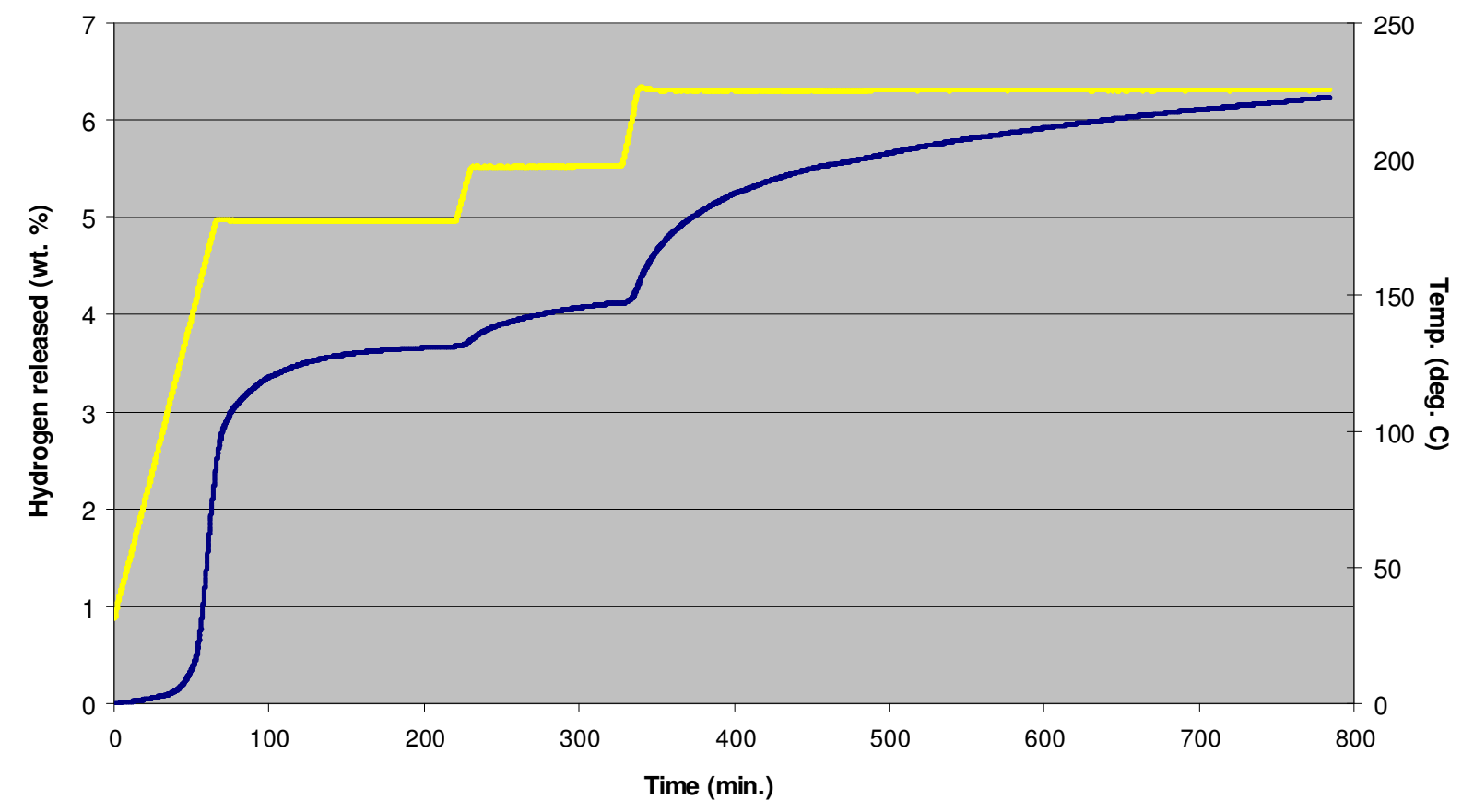

Figure 4-12. Dehydrogenation of phenylenecarbazole.

Our attempts to determine the structure of the products formed by hydrogenolysis using ${ }^{1} \mathrm{H}$ and ${ }^{13} \mathrm{C}$ NMR were unsuccessful due to the complexity of the product mixtures. Two probable bond cleavage pathways exist: 1) cleavage of a $\mathrm{C}-\mathrm{N}$ bond to form 8-phenylcarbazole or 2) cleavage of a C-C bond, yielding N-phenylcarbazole. In our experience, cleavage of the $\mathrm{C}-\mathrm{N}$ bond is the usual pathway, most likely due to ease of metal insertion into the $\mathrm{C}-\mathrm{N}$ bond. To test this hypothesis, product mixtures after hydrogenation and dehydrogenation were methylated via deprotonation, followed by reaction with excess iodomethane. While $\mathrm{N}$-phenylcarbazole would not be expected to undergo methylation, the secondary amines formed by $\mathrm{C}-\mathrm{N}$ bond cleavage should undergo methylation of the nitrogen atom and display a molecular weight increase $(+14$ atomic mass units). The methylation reactions yielded the mass changes shown in Tables 4-12a and 4-12b. 
Table 4-12a. GC-MS analysis of the methylated derivatives of phenylenecarbazole hydrogenation products.

\begin{tabular}{|c|c|}
\hline Molecular Weight Before Reaction & Molecular Weight After Methylation \\
\hline 259 & 259 \\
\hline 261 & 275 \\
\hline
\end{tabular}

Table 4-12b. GC-MS analysis of the methylated derivatives of phenylenecarbazole dehydrogenation products.

\begin{tabular}{|c|c|}
\hline Molecular Weight Before Reaction & Molecular Weight After Methylation \\
\hline 241 & 241 \\
\hline 243 & 275 \\
\hline 249 & 263 \\
\hline 251 & 251 \\
\hline
\end{tabular}

The results suggest that the two dehydrogenation products, MW 243 and MW 249, result from dehydrogenation of a bond cleavage product, and that some of the phenylenecarbazole dehydrogenates completely, along with some intact, partially dehydrogenated material (MW 251). Thus, it appears that two competing reactions are occurring during the dehydrogenation testing: 1) dehydrogenation of phenylenecarbazole and 2) $\mathrm{C}-\mathrm{N}$ bond cleavage and subsequent dehydrogenation of the product of bond cleavage. Many tertiary amines are quite robust towards hydrogenolysis, but this does not appear to be the case for phenylenecarbazole.

It is likely that the C-N bond cleavage occurs to intermediates with MW 247 (hexahydro) and MW 251 (decahydro), since samples taken from dehydrogenation experiments do not show high concentrations of these intermediates relative to the ring-opened byproducts under conditions when hydrogenolysis does occur. The C-N bond cleavage of a partially hydrogenated phenylenecarbazole can occur in only one of two ways:

1) bond cleavage of the 'external' C-N bond to produce a cyclohexylcarbazole or 2) cleavage of the 'internal' C-N bond to form an 8-member ring (Scheme 4-17). 

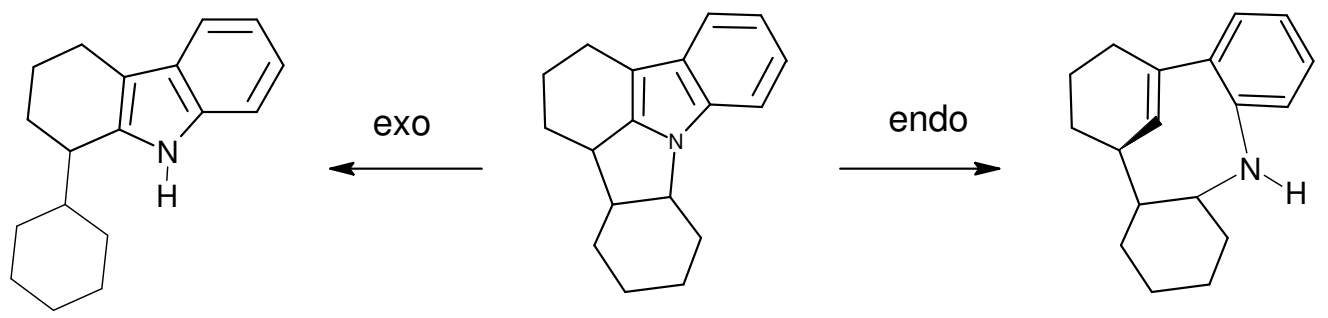

Scheme 4-17. Possible C-N bond cleavage products of decahydrophenylenecarbazole.

The energy values shown in Scheme 4-18 were calculated based on the sequential loss of two hydrogen molecules from perhydrophenylenecarbazole to reach each of the equilibrium structures shown. Since there is a $\mathrm{C} 2$ axis of symmetry in $\mathrm{N}$-phenylenecarbazole, two of the three benzenoid rings are equivalent - labeled as ' 1 ' - and require similar energies to dehydrogenate, whereas with ring 2, more than $18 \mathrm{kcal} / \mathrm{mole}_{2}$ of energy are necessary to arrive at the intermediate labeled with a $\mathrm{B}$. We theorize that in order to dehydrogenate ring 2, the molecule must become planar, which puts strain on the partially saturated five membered ring and, as a consequence, $\mathrm{C}-\mathrm{N}$ bond cleavage occurs to relieve this strain.

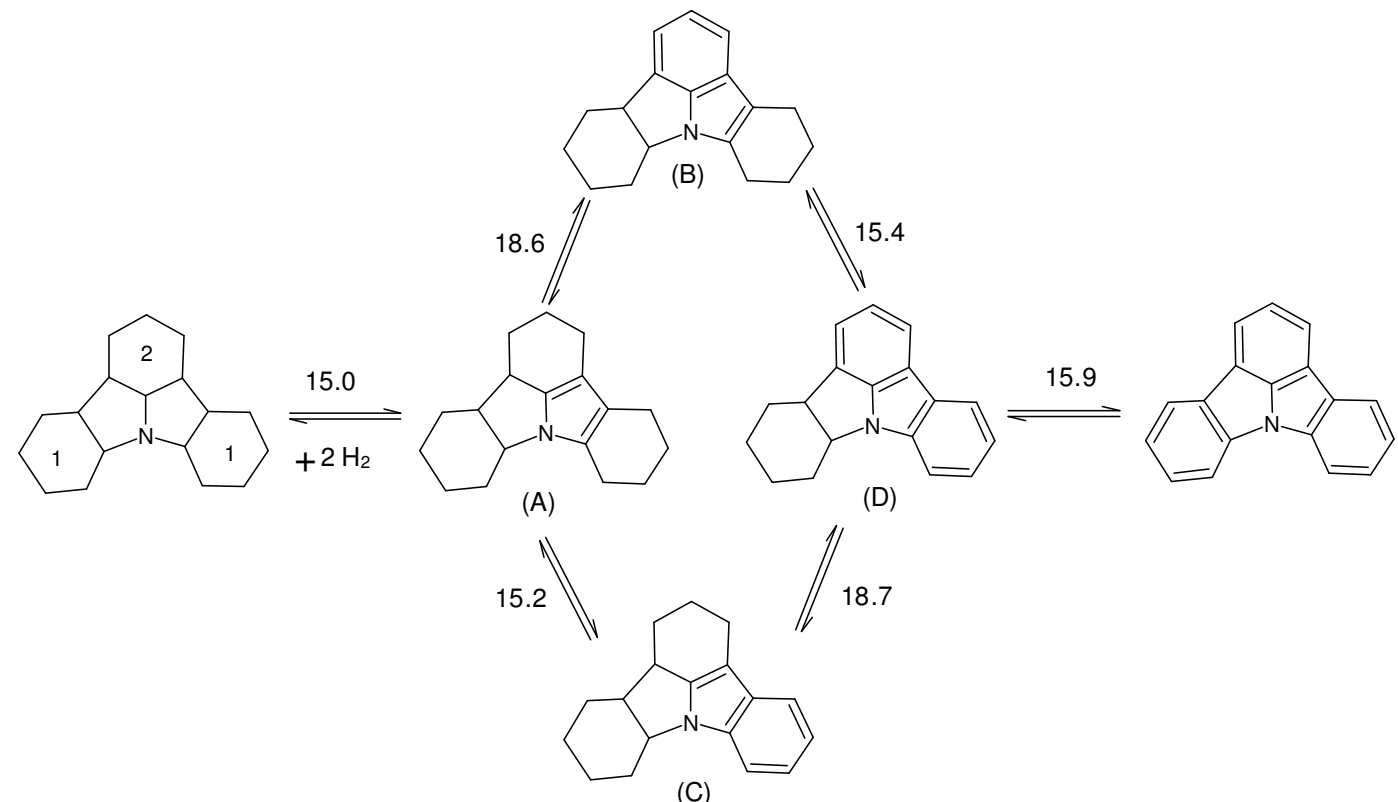

Scheme 4-18. Calculated dehydrogenation pathways for $\mathrm{N}$-phenylenecarbazole, values are electronic energies, $\Delta \mathrm{E}$, calculated in $\mathrm{kcal} / \mathrm{mole}_{2} . \Delta \mathrm{H} \sim \Delta \mathrm{E}-3.5$. 
In a typical dehydrogenation reaction, only ca. $20 \%$ of the dehydrogenation products contain molecules derived from $\mathrm{C}-\mathrm{N}$ bond cleavage. One possible explanation is that, assuming the reaction kinetics are similar throughout, intermediates readily interconvert through equilibria (the dehydrogenation reaction is performed under $1 \mathrm{~atm}$. hydrogen) and when up against a thermodynamically unfavorable transition, some of the intermediates can follow an alternate reaction pathway which leads to $\mathrm{C}-\mathrm{N}$ bond cleavage. Even though pathway $A \rightarrow B$ in Scheme 4-18 is unfavorable compared with the $A \rightarrow C$ pathway, once intermediate $C$ is formed it essentially builds up because dehydrogenation of ring 2 is now even more difficult. Furthermore, as we have learned in the case of dehydrogenating perhydro-N-ethylcarbazole, once some aromatic rings are formed within the molecule, the equilibrium of bound species at the catalyst surface is affected, thus not easily allowing the catalyst to migrate to the saturated ring. The catalyst binding energy coupled with the high energy barrier on going from $C$ to $D$ may make the $A \rightarrow B$ pathway more feasible kinetically and thermodynamically. Any intermediate left at $\mathrm{C}$ is cleaved at the $\mathrm{C}-\mathrm{N}$ bond forming the hydrogenolysis product.

\section{d. 5-methyl-indolo[3,2,1-jk]carbazole}

A batch of 5-methylphenylenecarbazole (containing 5\% of $\mathrm{N}$-phenylcarbazole) was hydrogenated and dehydrogenated under the conditions shown in Scheme 4-19. The melting point of the perhydrogenated compound was $130-145^{\circ} \mathrm{C}$, slightly depressed and broadened relative to the un-methylated phenylenecarbazole (m.p. $138^{\circ} \mathrm{C}$ ).
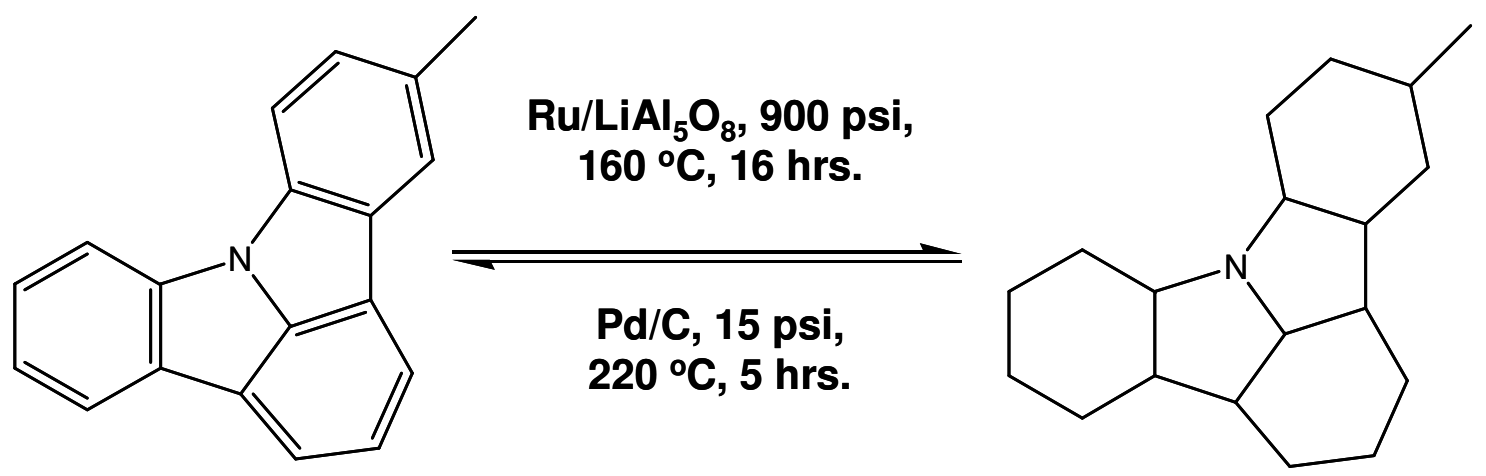

Scheme 4-19. Hydrogenation/dehydrogenation of 5-methylphenylenecarbazole. 
GC-MS analysis of the hydrogenated viscous liquid material showed that the hydrogenation did not go to completion, even after 16 hours. Table 4-13 displays the relative amounts of the hydrogenated products.

Table 4-13. Products of 5-methylphenylenecarbazole hydrogenation.

\begin{tabular}{|c|c|c|}
\hline Molecular Weight & \% in Mixture & Theor. Capacity (wt. \%) \\
\hline 267 & 23.2 & 1.0 \\
\hline 271 & 19.42 & 1.1 \\
\hline 273 & 53.4 & 3.55 \\
\hline 275 (N-phenylcarbazole) & 3.8 & - \\
\hline & & $\mathbf{5 . 6 5}$ \\
\hline
\end{tabular}

The molecular weight of the fully saturated compound is 273 . Other than the fully hydrogenated material, the major products after hydrogenation apparently contain one benzenoid ring (MW 267) and one double bond (MW 271). The resulting theoretical $\mathrm{H}_{2}$ capacity of this mixture was only $5.6 \mathrm{wt}$. \%, less than the theoretical capacity of $6.6 \mathrm{wt}$. $\% \mathrm{H}_{2}$ for the pure perhydrogenated compound.

Dehydrogenation of this hydrogenated mixture using a $\mathrm{Pd} / \mathrm{C}$ catalyst resulted in substantial reversibility at temperatures up to $220^{\circ} \mathrm{C}$ (Figure $4-13$, Table 4-14).

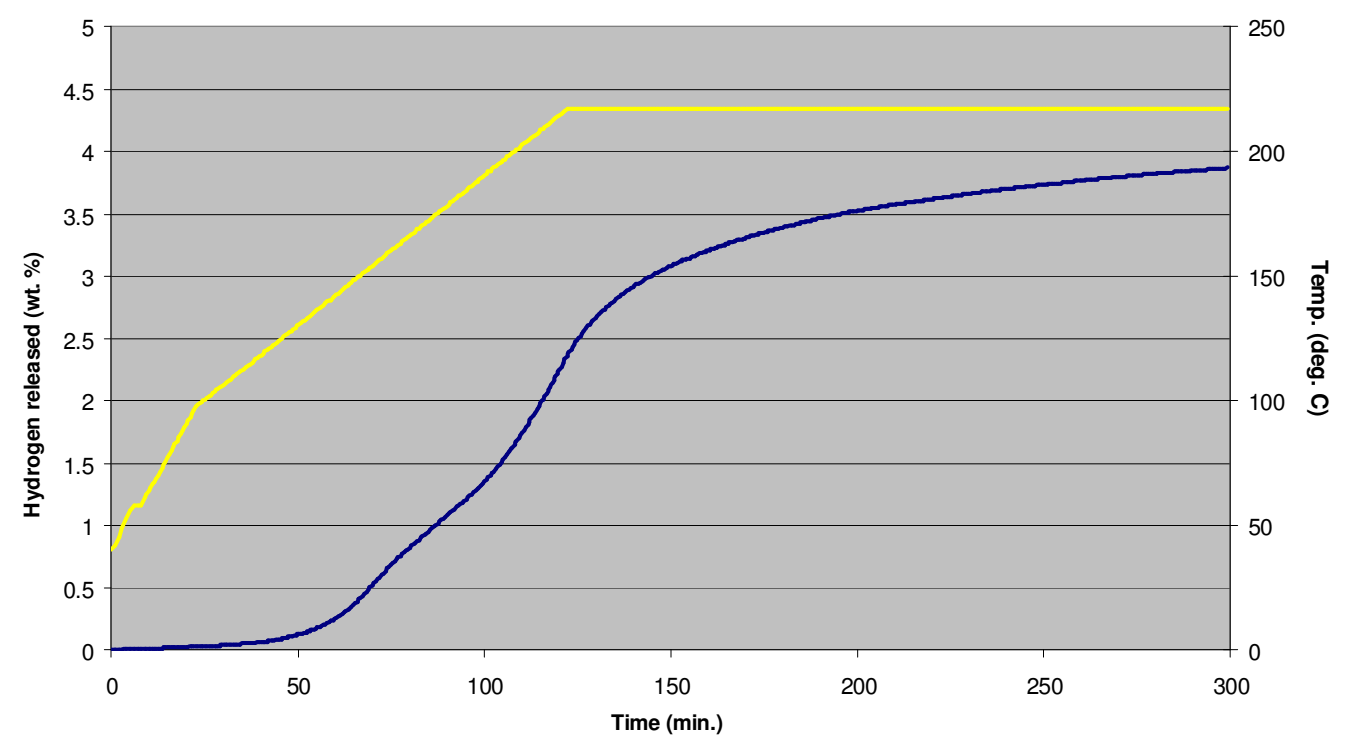

Figure 4-13. Dehydrogenation of 5-methylphenylenecarbazole. 
The raw hydrogen flow rate data from the dehydrogenation experiment showed two maxima at $\sim 150$ and $\sim 220^{\circ} \mathrm{C}$. The non-methylated phenylenecarbazole showed a similar profile; however, the peak maxima were not as distinct, occurring at 180 and $\sim 220^{\circ} \mathrm{C}$. It is possible that the presence of the methyl group somehow helps the initial dehydrogenation steps of 5-methylphenylenecarbazole.

Table 4-14. GC-MS analysis of the products of the dehydrogenation of $\mathrm{N}$-phenylene-7-methylcarbazole and remaining hydrogen capacity of partially hydrogenated intermediates.

\begin{tabular}{|c|c|c|}
\hline Molecular Weight & \% in Mixture & Capacity (wt. \%) \\
\hline 255 & 42.5 & 0 \\
\hline 261 & 2.0 & .04 \\
\hline 263 & 35.9 & 1.1 \\
\hline 265 & 6.6 & .24 \\
\hline 267 & 6.2 & .28 \\
\hline 257 (N-phenylcarbazole) & 6.7 & - \\
\hline & & $\mathbf{1 . 6 6}$ \\
\hline
\end{tabular}

The observed $\mathrm{H}_{2}$ capacity of the material under the stated conditions was 4 wt. \%, or only $70 \%$ reversible. These results compare unfavorably with the un-methylated material with a realized working capacity nearing $6 \mathrm{wt}$. \% hydrogen. The major component of the dehydrogenated mixture, other than the fully aromatized product, is a product with a MW of 263 , which may be an indole-type structure. We were unable to tell from the GC-MS results if the ring containing the methyl group was hydrogenated or dehydrogenated.

It appears that the presence of the methyl group has an overall negative influence, both on hydrogenation and dehydrogenation. The hydrogenation kinetics were very slow, such that after 16 hours under standard conditions, the material was not fully hydrogenated. It should also be noted that the dehydrogenation never reached a very high flow rate, and the hydrogen desorption seemed to be spread over a long period relative to the un-methylated derivative. However, the maximum dehydrogenation rate occurred at a temperature $30^{\circ} \mathrm{C}$ lower with the methylated compound, suggesting that 
whatever is responsible for the low-temperature desorption could be enhanced by the presence of the methyl group. Perhaps these observations are consistent with a combination of the activating effects of the methyl group towards the para ring allowing for low-temperature dehydrogenation, and steric effects slowing the dehydrogenation of the ring containing the methyl group.

In conclusion, the indolocarbazoles yielded mixed results in our testing for use as hydrogen carriers. The expected high melting point issues discouraged the pursuit of certain isomers (e.g., indolo[2,3-b]carbazole). The selectivity for catalytic hydrogenation of N-N'-diethylindolo[3,2-a]carbazole prevented the successful demonstration of this promising liquid carrier candidate. While some perhydro-N-N'-diethylindolo[3,2a]carbazole was obtained, unwanted ring opening due to $\mathrm{C}-\mathrm{N}$ bond hydrogenolysis could not be completely eliminated. The most successful isomer of the group that was tested is indolo[3,2,1-jk]carbazole. This liquid carrier prototype was successfully synthesized and hydrogenated. Unfortunately, the catalytic selectivity of the dehydrogenation reaction likely would preclude the use of this compound as a hydrogen carrier in the current state of development. Our efforts to understand the cause of the poor selectivity revealed that the thermodynamics of intermediate transformations during dehydrogenation demands high dehydrogenation temperatures (e.g., 225-250 ${ }^{\circ} \mathrm{C}$ ). While providing a practical hurdle to the use of this compound as a hydrogen carrier, the high dehydrogenation temperature also contributes to the observed lack of catalytic dehydrogenation selectivity.

\section{Pyrroloindoles}

A class of molecules which should demonstrate low overall heats of hydrogenation based upon their structure are the pyrroloindoles. An example of this class of molecules is 2,3,5,6,8-pentamethyl-1,7-dihydro-pyrrolo[3,2-f]indole ("pentamethylpyrrolo[3,2-

f]indole"), which was synthesized in a one-pot reaction (Scheme 4-20). Two equivalents of 3-chloro-2-butanone and potassium carbonate were stirred and heated in dimethylacetamide (DMAC) to approximately $130^{\circ} \mathrm{C}$. Then a solution of 2,6-diaminotoluene in DMAC was added by drop to the reaction slurry while maintaining 
the temperature at approximately $130^{\circ} \mathrm{C}$. After the addition was complete, the reaction mixture was stirred at $150^{\circ} \mathrm{C}$ for $2-3$ hours. The reaction mixture was cooled and filtered, and the solvent was removed by vacuum distillation. GC-MS and ${ }^{1} \mathrm{H}$ NMR analysis of the products indicated an $85 \%$ yield of the desired product. The product was then purified by column chromatography.

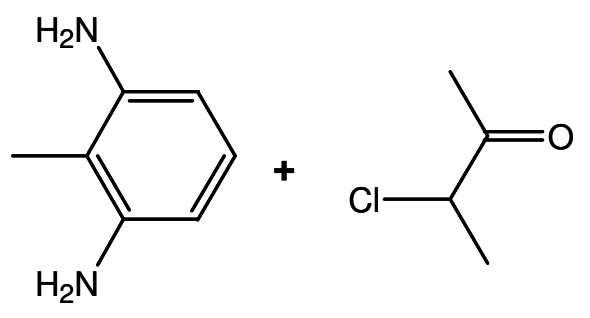<smiles>Cc1[nH]c2c(C)c3[nH]c(C)c(C)c3cc2c1C</smiles>

Scheme 4-20. One-pot synthesis of pentamethylpyrrolo[3,2-f]indole.

A rather simple one-step reaction provided, in high yield, a different pyrroloindole (Scheme 4-21). Alkylation of the nitrogens of this compound, using base and alkyl halides, resulted in a mixture of both the mono- and di-N-alkylated products. The air stability of these compounds was problematic, since the product began to visibly oxidize during purification. The double bond in the middle ring (with expected olefinic character) seemed to be particularly susceptible to oxidation. Hydrogenation of the alkylated pyrroloindole mixture under normal hydrogenation conditions with standard catalysts did not proceed to high conversion. This may have been due to steric congestion (high degree of alkylation), chelation of the catalyst by the nitrogens, or impurities due to oxidation.

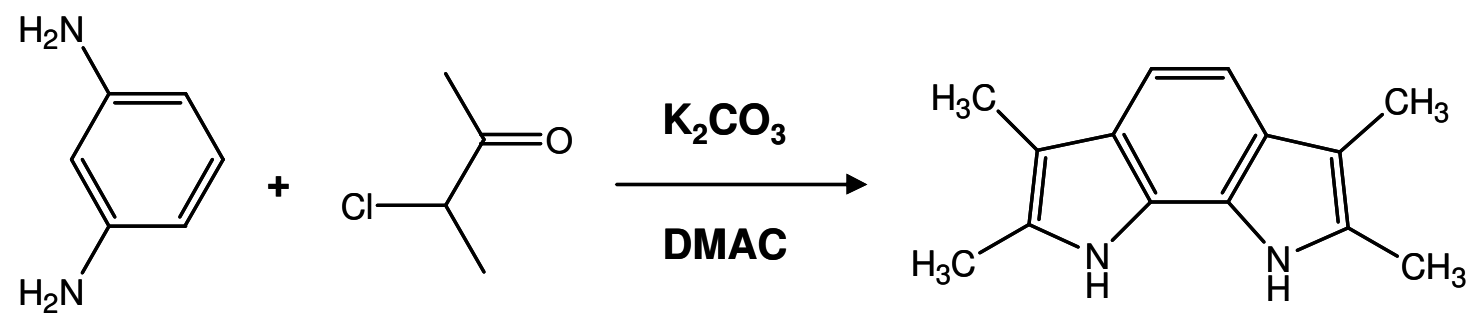

Scheme 4-21. Synthetic procedure for synthesis of tetramethylpyrroloindole. 
In our attempted hydrogenations of the alkylated pyrroloindole, only a single hydrogen molecule was added to the substrate out of the potential five hydrogen molecules. Based on the steric congestion of the five-membered rings, the addition of hydrogen probably occurred on the six-membered ring (note: structurally-related trimethyl indole also showed extremely slow hydrogenation kinetics relative to indole and 1-ethyl-2methylindole).

Since the presence of all the methyl groups on the rings may have blocked catalyst binding during hydrogenation, the synthesis of less substituted derivatives of pyrroloindole was attempted utilizing less substituted aldehydes and ketones as reactants (e.g., protected dimethyl acetal of chloroacetaldehyde). Unfortunately, the products of these reactions yielded black, crystalline solids that by MALDI showed a range of masses greater than MW 500. We could not determine the structure of these products using NMR spectroscopy.

Due to the failure to fully hydrogenate the highly alkylated pyrroloindoles and the inability to use the known synthetic scheme to produce less substituted derivatives, no additional studies were attempted for this class of liquid carrier prototypes.

\section{E. Bis-indolylmethane derivatives}

Two equivalents of indole (or 2-substituted indoles) can be linked through a methylene bridge to form bis-indolylmethanes by a reaction with formaldehyde, using potassium hydrogen sulfate as the catalyst and methanol as the solvent (Scheme 4-22). While the reaction yields were generally good, small amounts of unreacted indole and trimeric byproducts are also formed in the reaction. 


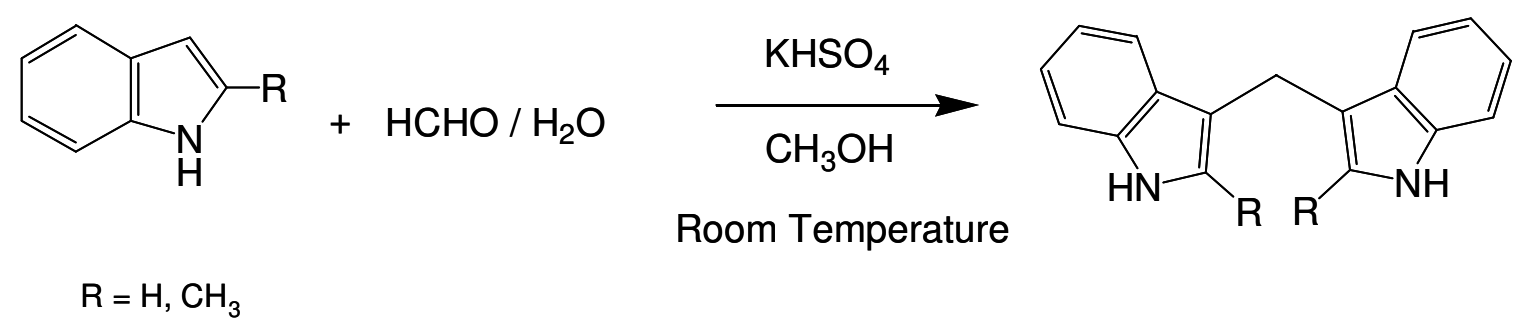

Scheme 4-22. Preparation of bis-indolylmethanes.

The hydrogenation and alkylation of the crude bis-indolylmethanes can be achieved in the same one-pot reaction by hydrogenation of the bis-indolylmethanes in alcohol solvents using a Raney nickel catalyst. For example, N,N'-diethyl-bis-indolylmethane was prepared in high yields using absolute ethanol and Raney 2800 nickel catalyst (Scheme 4-23).
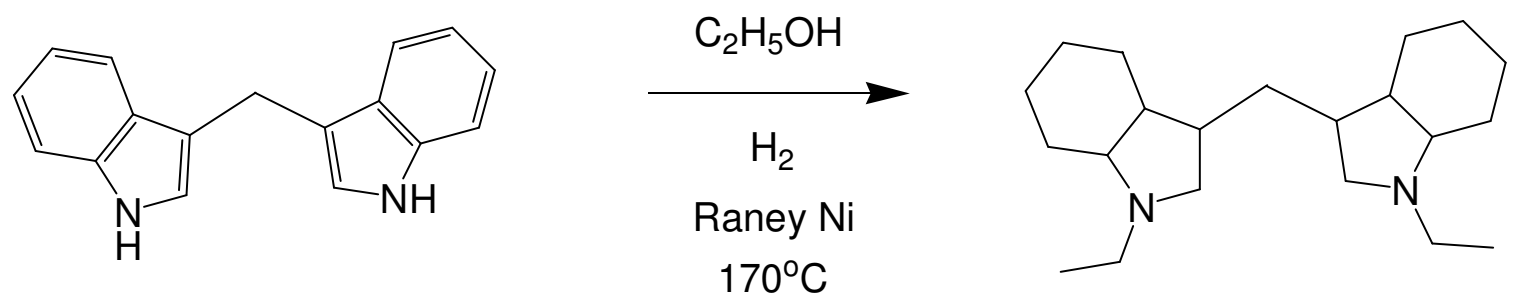

Scheme 4-23. Synthesis of perhydro-N,N'-diethyl-bis-indolylmethane.

The monomer/dimer/trimer mixtures of the hydrogenated, alkylated bis-indolylmethane carriers were separated by a simple vacuum distillation. During the distillation of crude perhydro-N,N'-diethyl-bis-indolylmethane, a small amount of hydrogenated indole was collected at $70^{\circ} \mathrm{C}$ and $8 \times 10^{-1}$ torr. By GC/MS analysis, this monomer is a mixture of MW 153 and 167, which likely corresponds to perhydro-1-ethylindole and perhydro-1ethyl-3-methylindole, respectively. The perhydro- $\mathrm{N}, \mathrm{N}$ '-diethyl-bis-indolylmethane was distilled at $180^{\circ} \mathrm{C}$ and $4 \times 10^{-2}$ torr. A small amount of "trimer" (ca. $5 \%$ ) also was carried 
over. The pure perhydro-N,N'-diethyl-bis-indolylmethane is a colorless liquid with moderate viscosity at ambient temperature.

The crude perhydro-N,N'-diethyl-bis-(2-methyl)indolylmethane was dark brown, qualitatively indicative of oxidation byproducts. During the distillation of crude perhydroN,N'-diethyl-bis-(2-methyl)indolylmethane, a moderate amount of "monomer" was collected at $60^{\circ} \mathrm{C}$ and $3 \times 10^{-1}$ torr. By GC/MS analysis, this monomer is a mixture of MW 167 and 181, which likely corresponds to perhydro-1-ethyl-2-methylindole and perhydro-1-ethyl-2,3-dimethylindole, respectively. The perhydro-N,N'-diethyl-bis(2-methyl)indolylmethane was distilled at $175^{\circ} \mathrm{C}$ and $5 \times 10^{-2}$ torr. The liquid was pure by GC/MS analysis (no monomer, trimer, or unknown byproducts detected). However, a bright yellow coloration of the liquid suggests that a small amount of oxidation byproducts may have co-distilled. The pure perhydro-N,N'-diethyl-bis-(2methyl)indolylmethane has moderate/high viscosity at ambient temperature.

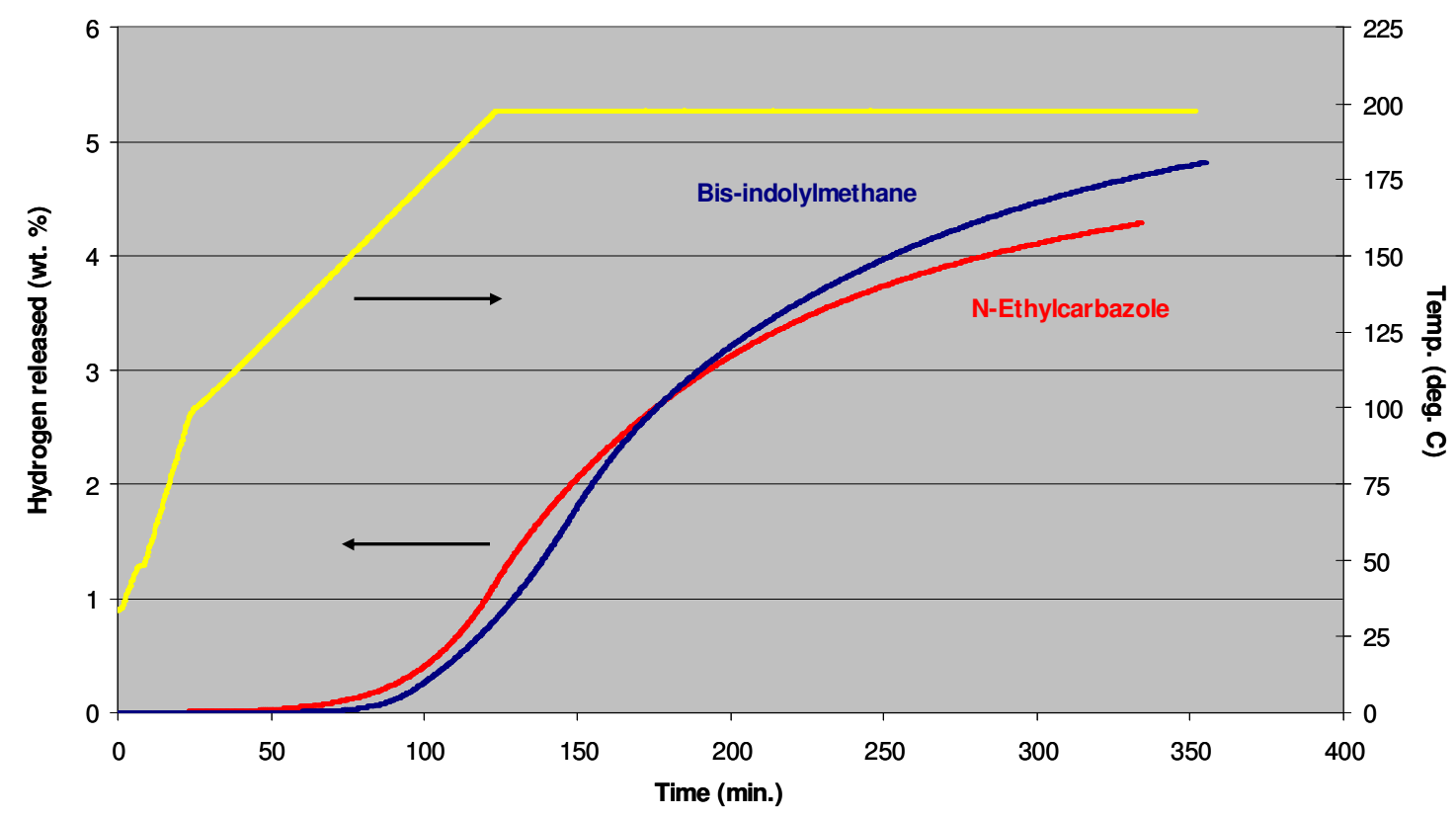

Figure 4-14. Dehydrogenation of perhydro-N,N'-diethyl-bis-indolylmethane. 
The purified perhydro-N,N'-diethyl-bis-indolylmethane was dehydrogenated at $200^{\circ} \mathrm{C}$ using a $5 \% \mathrm{Pd}$ on alumina dehydrogenation catalyst (Figure 4-14). For comparison, we dehydrogenated perhydro- $\mathrm{N}$-ethylcarbazole under the same temperature ramping conditions with the same dehydrogenation catalyst. While the perhydro-Nethylcarbazole demonstrated a faster initial rate of dehydrogenation, the perhydro-N,N'diethyl-bis-indolylmethane liquid carrier evolved more hydrogen by the end of the experiment time (350 minutes). GC-MS analysis of the dehydrogenated perhydro-N,N'diethyl-bis-indolylmethane showed high selectivity for the dehydrogenation reaction.

This is perhaps not surprising given the structural similarity to the alkylated indole liquid carriers (e.g., 1-ethyl-2-methylindole).

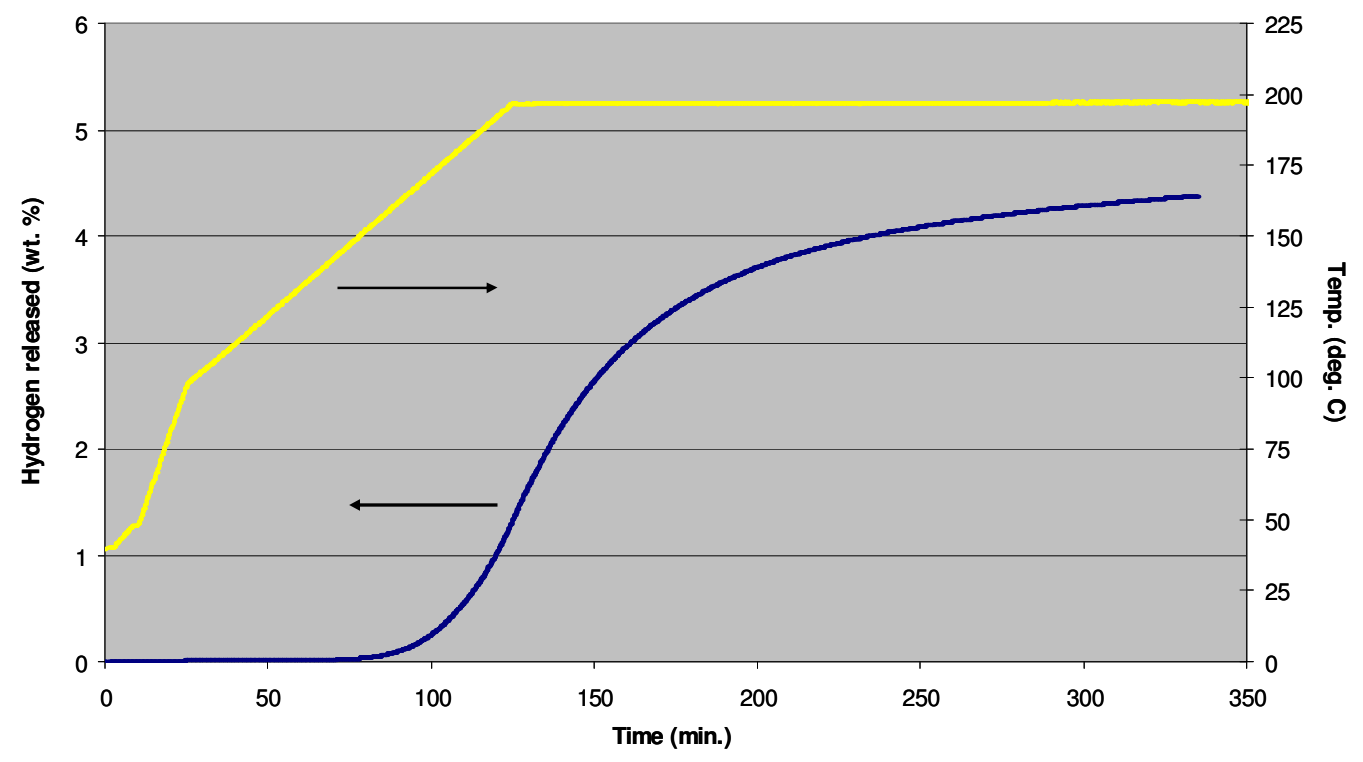

Figure 4-15. Dehydrogenation of perhydro-N,N'-diethyl-bis-(2-methyl)indolylmethane.

The ring-methylated derivative of bis-indolylmethane, perhydro-N,N'-diethyl-bis-(2methyl)indolylmethane, was tested for dehydrogenation activity at $200^{\circ} \mathrm{C}$ using a $5 \% \mathrm{Pd}$ on carbon dehydrogenation catalyst (Figure 4-15). The rate of dehydrogenation was similar to perhydro-N,N'-diethyl-bis-indolylmethane and perhydro-N-ethylcarbazole. This might be surprising given that similar highly alkylated carriers (e.g., 1,2,3-trimethylindole) showed very slow rates of catalytic dehydrogenation. Post-dehydrogenation GC-MS analysis showed high reaction selectivity. 
In summary, bis-indolylmethane derivatives represent a promising class of liquid carriers. Perhydro-N,N'-diethyl-bis-indolylmethane is available in good yields in two catalytic reaction steps using the relatively inexpensive reagents indole, formaldehyde, ethanol, and hydrogen. The dehydrogenation rate using supported palladium catalysts is similar to perhydro-N-ethylcarbazole, even in the case of the methyl-substituted bisindolylmethane. The selectivity of the hydrogenation and dehydrogenation reactions appears to be high, although no cycling studies were performed to confirm long-term stability. The vapor pressure of perhydro-N,N'-diethyl-bis-indolylmethane is lower than that of most of the indole/carbazole-based liquid carriers. The deliverable hydrogen content of these carriers (4.6-5.0 wt. \% hydrogen) is unfortunately lower than that of other types of liquid carriers.

\section{F. Pyrrocoline}

Pyrrocoline can be prepared in one step by heating 2-pyridinepropanol with palladium on carbon (Scheme 4-24). ${ }^{10}$ The reaction produces a mixture consisting of fully unsaturated pyrrocoline and tetrahydropyrrocoline. The reaction mixture was converted to perhydropyrrocoline by hydrogenation at $170^{\circ} \mathrm{C}$ using ruthenium on lithium aluminate.

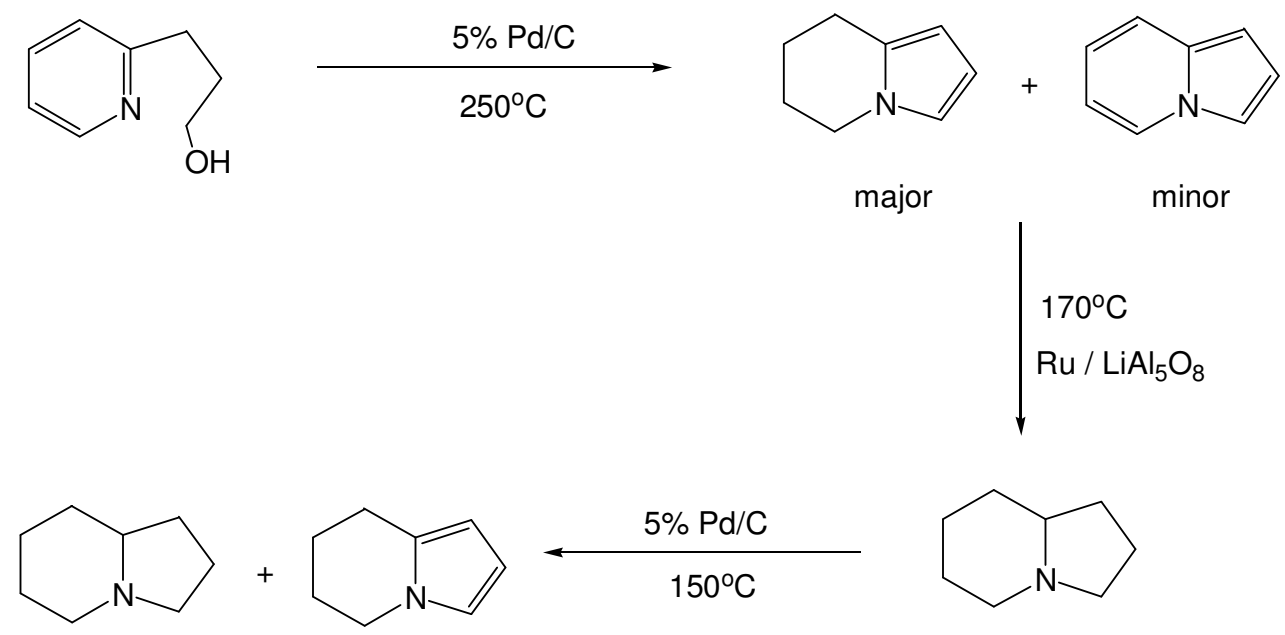

Scheme 4-24. Synthesis, hydrogenation and dehydrogenation of pyrrocoline. 
Dehydrogenation of perhydropyrrocoline with $5 \% \mathrm{Pd} / \mathrm{C}$ at $150^{\circ} \mathrm{C}$ for four hours gave only $6 \%$ conversion to tetrahydropyrrocoline. Analysis of thermodynamics of the stepwise hydrogenation process using calculated energies of hydrogenation shows that while the overall energy change per mole hydrogen for full dehydrogenation is $-19.9 \mathrm{kcal} / \mathrm{mol}(\Delta \mathrm{E})$, the energy change for dehydrogenation of the five membered ring is $-18.6 \mathrm{kcal} / \mathrm{mol} \mathrm{H}_{2}$, and the tetrahydro intermediate (saturation on the six-membered "pyridine" ring) dehydrogenation is $-21 \mathrm{kcal} / \mathrm{mol} \mathrm{H}_{2}$, thus making the dehydrogenation of the sixmembered ring impossible at reasonable temperatures (Figure 4-16).<smiles>c1ccn2cccc2c1</smiles>

$\Delta \mathrm{E}\left(\mathrm{kcal} / \mathrm{mol} \mathrm{H}_{2}\right)$<smiles>C1=CC2CCCN2C=C1</smiles><smiles>c1cc2n(c1)CCCC2</smiles>

$+2 \mathrm{H}_{2}$

$-10.1$

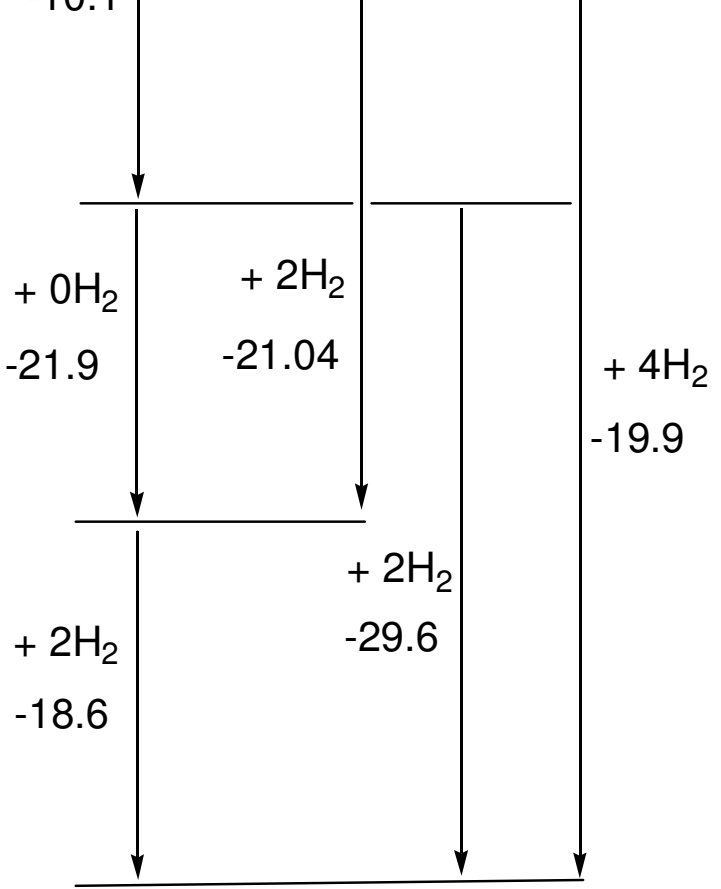

Figure 4-16. Calculated thermodynamics for the stepwise hydrogenation of pyrrocoline. 


\section{Experimental Details}

See Appendix B for general experimental details.

\section{Reversible hydrogenation of 1-ethyl-2-methylindole}

A $100 \mathrm{cc}$ stainless steel pressure reactor was loaded with $55 \mathrm{~g}$ 1-ethyl-2-methylindole and $2.5 \mathrm{~g}$ of $5 \%$ ruthenium on lithium aluminate. After the headspace was purged with hydrogen, the reactor was heated to $160^{\circ} \mathrm{C}$ and the hydrogen pressure increased to 1000 psia with stirring (1000 rpm). After 2 hours the reactor was cooled to ambient temperature. GC-MS analysis of the reactor contents indicated that the hydrogenated product contained 95\% perhydro-1-ethyl-2-methylindole, 2\% 1-ethyl-2-methylindole, and $3 \%$ partially hydrogenated 1-ethyl-2-methylindole. Under inert atmosphere, $4.0 \mathrm{~g}$ of the hydrogenated mixture and $0.1 \mathrm{~g}$ of $5 \%$ palladium on alumina were placed in a $20 \mathrm{cc}$ stirred tank reactor, and the reactor was sealed. The reactor was connected to the dehydrogenation testing manifold. After evacuation of residual air from the manifold lines, hydrogen was purged through the reactor to displace the argon from the reactor headspace. The reactor was heated to $180^{\circ} \mathrm{C}$ with stirring (300 rpm) under $1 \mathrm{~atm}$. hydrogen. After 17.5 hours at $180^{\circ} \mathrm{C}$, the measured hydrogen flow corresponded to the desorption of $4.55 \mathrm{wt}$. \% hydrogen. The first $4 \mathrm{wt}$. \% hydrogen was desorbed after the first 5 hours at $180^{\circ} \mathrm{C}$. The dehydrogenated sample was analyzed by GC-MS.

\section{Reversible hydrogenation of 1-ethyl-2-methylindole/1,2-dimethylindole mixture}

A mixture of $4.2 \mathrm{~g} \mathrm{1,2-dimethylindole} \mathrm{and} 1.8 \mathrm{~g}$ 1-ethyl-2-methylindole was placed in a $20 \mathrm{cc}$ stainless steel reactor. This mixture was a free-flowing liquid at $20^{\circ} \mathrm{C}$. To the liquid mixture was added $1.0 \mathrm{~g}$ of $5 \%$ ruthenium on lithium aluminate. The reactor was sealed and the headspace purged with hydrogen. The mixture was heated to $170^{\circ} \mathrm{C}$ under 700 psia hydrogen with stirring $(500 \mathrm{rpm})$ for 3 hours. The reactor was cooled to ambient temperature and the contents dissolved in $100 \mathrm{cc}$ chloroform. The catalyst was removed by filtration. The hydrogenated 1,2-dimethylindole/1-ethyl-2-methylindole mixture was isolated by removing the chloroform under vacuum. GC/MS analysis of the liquid hydrogenated mixture indicated complete hydrogenation of both 1,2-dimethylindole and 1-ethyl-2-methylindole. 


\section{Dehydrogenation of 1-ethyl-2-methylindole/1,2-dimethylindole mixture}

Under an argon atmosphere, a $20 \mathrm{cc}$ stainless steel reactor was charged with $4.8 \mathrm{~g}$ of the hydrogenated 1,2-dimethylindole/1-ethyl-2-methylindole mixture and $0.5 \mathrm{~g}$ of $5 \%$ palladium on alumina. After evacuation of residual air from the manifold lines, hydrogen was purged through the reactor to displace the argon from the reactor headspace. The mixture was heated to $175^{\circ} \mathrm{C}$ with stirring under 1 atmosphere of hydrogen pressure for 13 hours. After cooling to $25^{\circ} \mathrm{C}$, the reactor was opened under argon and the reactor contents were analyzed by GC/MS.

\section{Preparation of dihydro-N,N'-dimethylindole dimer}

One gram of $\mathrm{N}$-methylindole was added to a $30 \mathrm{cc}$ FEP tube reactor. The tube reactor was then cooled to $-30^{\circ} \mathrm{C}$ and anhydrous hydrogen fluoride was added until a red solution was formed. Excess hydrogen fluoride was removed under vacuum and the solution warmed to room temperature under vacuum, yielding a red, gelatinous material. The residue was taken up in chloroform and neutralized with aqueous potassium carbonate. After separation of the aqueous and organic layers, the solvent was removed under vacuum, giving the desired compound (yield 80\%; $\mathrm{m} / \mathrm{z}=162 \mathrm{amu}$ ).

\section{Preparation of N,N'-dimethylindole dimer}

One gram of dihydro-N,N'-dimethylindole dimer was added to $30 \mathrm{ml}$ toluene and $0.5 \mathrm{~g}$ of $5 \% \mathrm{Pt} / \mathrm{C}$. The solution was then refluxed under a nitrogen purge for $6 \mathrm{hrs}$. The catalyst was then filtered off and solvent removed under vacuum (yield $90 \% ; \mathrm{m} / \mathrm{z}=160 \mathrm{amu}$ ). ${ }^{1} \mathrm{H}$ NMR: 3.75 (s), 3.9 (s), 6.6 (s), 7.05 (t), 7.15 (q), 7.3 (t), 7.4 (d), 7.5 (d), 7.6 (d), 7.7 (d).

\section{Hydrogenation of $\mathbf{N}, \mathrm{N}^{\prime}$-dimethylindole dimer}

A mixture containing $3 \mathrm{~g}$ of $\mathrm{N}, \mathrm{N}^{\prime}$-dimethylindole dimer and $1.5 \mathrm{~g}$ of $5 \% \mathrm{Rh} / \mathrm{C}$ was added to a $50 \mathrm{cc}$ Parr reactor. After the reactor was purged with hydrogen, the mixture was then hydrogenated at $140^{\circ} \mathrm{C}$ for $8 \mathrm{hrs}$ under hydrogen pressure. After filtration of the 
catalyst, only fully hydrogenated monomer was recovered, indicating that dimer cleavage had occurred.

\section{Preparation of 2'4,'-dinitro-1,1'5',1'-terphenyl}

A mixture of 1,3-dichloro-4,6-dintirobenzene (16.2 g, $68.4 \mathrm{mmol})$, phenylboronic acid (18 g, $147.6 \mathrm{mmol})$, palladium acetate $(0.62 \mathrm{~g}, 2.8 \mathrm{mmol})$, sodium carbonate $(29.3 \mathrm{~g}$ dissolved in $200 \mathrm{~mL}$ of water) and diphenylphosphinopropane (1.6 g, $2.8 \mathrm{mmol})$ in $230 \mathrm{~mL}$ of toluene was refluxed for 7 hours ( $\mathrm{N}$-methylpyrrolidine can also be used as solvent). The reaction mixture was cooled to room temperature and the phases were separated. The aqueous layer was extracted with ethyl acetate. The organic phases were combined and the solvent evaporated. Chromatography on silica gel with hexanes:ethyl acetate (7:1) as eluant and recrystallization from ethanol gave 16 grams of the product.

\section{Preparation of indolo[2,3-b]carbazole}

A mixture of 2,4,-dinitro-1,1',5',1"-terphenyl (16 g, $50 \mathrm{mmol})$, triethyl phosphate (35 mL) in tert-butylbenzene was refluxed for two days. The reaction was cooled to room temperature and the solvent distilled under vacuum. Ethanol $(75 \mathrm{~mL})$ and hexanes $(75 \mathrm{~mL})$ were added to the residue, and the resulting mixture was cooled to $5^{\circ} \mathrm{C}$ overnight. Filtration of the suspension yielded 2 grams of the desired product. The filtrate was purified by chromatography on silica gel with hexanes:ethyl acetate (3:1 then $1: 3)$. The red oily product was recrystallized again in $1: 1$ ethanol/hexanes to afford another $2.5 \mathrm{~g}$ of the desired product.

\section{Preparation of 3-bromoindole}

To a solution of N-ethylindole $(5.9 \mathrm{~g}, 40 \mathrm{mmol})$ in $100 \mathrm{~mL}$ of dimethylformamide was slowly added a solution of bromine $(6.5 \mathrm{~g}, 40 \mathrm{mmol})$ in $100 \mathrm{~mL}$ of dimethylformamide. At the end of the addition, the reaction was stirred for one hour, then poured onto ice water with ammonium hydroxide and extracted with ethyl acetate. The organic layer was washed first with a dilute solution of metabisulfite, then with water, dried over 
magnesium sulfate, and the solvent evaporated. A red oil (5.9 grams) was obtained. GC/MS analysis of the product showed the presence of 3-bromoindole, which was contaminated with a dibrominated byproduct.

\section{Preparation of N,N'-diethyl-2,3'-bisindolyl}

A mixture of 3-bromoindole $(5.8 \mathrm{~g}, 26 \mathrm{mmol}), \mathrm{N}$-ethylindole $(3.8 \mathrm{~g}, 26 \mathrm{mmol})$ and trifluoroacetic acid $(1 \mathrm{~mL})$ in $200 \mathrm{~mL}$ of dichloromethane was stirred at room temperature for one hour. The reaction mixture was poured onto an ammonium hydroxide solution. The phases were separated and the solvent evaporated. The organic residue was dissolved in ethyl acetate, washed with water, and dried over magnesium sulfate. After filtration, the solution was concentrated and chromatographed on silica gel with hexanes:ethyl acetate $(7: 1$ then $3: 1)$. The crude oily product was used in a subsequent reaction with no additional purification.

\section{Preparation of N,N'-diethyl-indolo[3,2-a]carbazole}

A mixture of N,N'-diethyl-2,3'-bisindolyl (6 g, $25.9 \mathrm{mmol})$ and dimethylaminoacetaldehyde diethyl acetate $(5.2 \mathrm{~mL}, 28.5 \mathrm{mmol})$ in acetic acid $(250 \mathrm{~mL})$ was refluxed for six hours. After cooling to room temperature, the solvent was evaporated and the residue chromatographed on silica gel with ethyl acetate:hexanes $(1: 4)$.

\section{Hydrogenation of N,N'-diethyl-indolo[3,2-a]carbazole}

Neat hydrogenation of N,N'-diethyl-indolo[3,2-a] carbazole at $170^{\circ} \mathrm{C}$ using various catalysts (Rh, $\mathrm{Ru}$, Raney $\mathrm{Ni}$ ) resulted in hydrogenolysis of the perhydrogenated products. Ruthenium on lithium aluminate was found to be the mildest catalyst for the hydrogenation, yielding the smallest amount of byproducts. Hydrogenation using ruthenium on lithium aluminate with solvents such as THF, isopropanol and hexafluoroisopropanol gave incomplete conversion (addition of 4 moles of $\mathrm{H}_{2}$ instead of 8 moles). Hydrogenation under acidic $\left(\mathrm{PtO}_{2}\right.$ with $\left.\mathrm{HBF}_{4}\right)$ or basic conditions $(\mathrm{Rh} / \mathrm{Ru}$ with 
$\mathrm{LiOH}$ ) did not prevent hydrogenolysis of the $\mathrm{C}-\mathrm{N}$ bond. Concentration (ratio of substrate to solvent) did not seem to play a role in the conversion of the hydrogenation.

\section{Preparation of 9-o-nitrophenylcarbazole}

To a solution containing $5 \mathrm{~g}$ of carbazole in $150 \mathrm{ml}$ dimethylformamide was added $5.07 \mathrm{~g}$ of 1-fluoro-2-nitrobenzene and $19.5 \mathrm{~g}$ cesium carbonate. The solution was stirred at $80^{\circ} \mathrm{C}$ under a nitrogen purge for $12 \mathrm{hrs}$. After cooling to room temperature, the mixture was added to $200 \mathrm{ml}$ water and extracted three times with $150 \mathrm{ml}$ portions of ethyl acetate. The organic was washed again with water and dried over magnesium sulfate. The solvent was removed under vacuum to obtain $8.4 \mathrm{~g}$ of an orange solid.

\section{Preparation of 9-o-aminophenylcarbazole}

A $300 \mathrm{cc}$ stainless steel Parr reactor was charged with $50 \mathrm{~g}$ of 9-0aminophenylcarbazole, $200 \mathrm{ml}$ tetrahydrofuran, and $5 \mathrm{~g}$ of $\mathrm{PtO}_{2}$. The reactor was then purged with hydrogen, pressurized to $1000 \mathrm{psig}$ hydrogen and heated to $60^{\circ} \mathrm{C}$. After 5 hours, the hydrogen uptake stopped. After cooling to room temperature and venting the reactor, the catalyst was filtered and the solvent removed under vacuum. The residue was recrystallized from hexanes to yield $35 \mathrm{~g}$ of 9-0-aminophenylcarbazole.

\section{Preparation of N-phenylenecarbazole}

With stirring, $5 \mathrm{~g}$ of 9-o-aminophenylcarbazole was dissolved in $350 \mathrm{ml}$ acetonitrile. Sulfuric acid $(2.0 \mathrm{~g})$ was added dropwise to the acetonitrile solution followed by the dropwise addition of $2.4 \mathrm{~g}$ of isoamylnitrite. The mixture was stirred at $23^{\circ} \mathrm{C}$ for 30 minutes and then heated to reflux for 2 hours. GC-MS analysis of samples taken during the reflux was used to monitor the conversion. Once the reaction was complete, the solvent was removed under vacuum and the excess acid was neutralized with potassium carbonate. The resulting product was purified by silica gel column chromatography (eluant: $15 \%$ ethyl acetate in hexanes) and recrystallized in hexanes to yield $2.5 \mathrm{~g}$ of a light yellow solid. 


\section{Hydrogenation of $\mathrm{N}$-phenylenecarbazole}

A 30 cc stainless steel pressure reactor was loaded with $4 \mathrm{~g}$ of $\mathrm{N}$-phenylenecarbazole and $0.15 \mathrm{~g}$ of $5 \%$ ruthenium on lithium aluminate. After the headspace was purged with hydrogen, the hydrogen pressure was increased to 920 psia. The reactor was heated to $140^{\circ} \mathrm{C}$, and the hydrogen pressure increased to 950 psia. After 6 hours under these conditions with stirring, the reactor was cooled to $25^{\circ} \mathrm{C}$, and the contents were filtered to remove catalyst. GC-MS analysis showed complete conversion to one isomer of perhydro-N-phenylenecarbazole. The perhydro-N-phenylenecarbazole was then degassed by evacuation to 100 millitorr at $23^{\circ} \mathrm{C}$ for 20 minutes and then purged with argon for 5 minutes.

\section{Dehydrogenation of perhydro- $\mathrm{N}$-phenylenecarbazole}

Under an argon atmosphere, $3.4 \mathrm{~g}$ of the perhydro- $\mathrm{N}$-phenylenecarbazole and $0.2 \mathrm{~g}$ of $5 \%$ palladium on alumina (dehydrogenation catalyst) were placed in a $30 \mathrm{cc}$ stirred tank reactor and the reactor was sealed. The reactor was connected to the dehydrogenation testing manifold. After evacuation of residual air from the manifold lines, hydrogen was purged through the reactor to displace the argon from the reactor headspace. The reactor was heated to $180^{\circ} \mathrm{C}$ with stirring (500 rpm) under 16 psia hydrogen. After 2.5 hours at $180^{\circ} \mathrm{C}$, the measured hydrogen flow corresponded to the desorption of $3.7 \mathrm{wt}$ \% hydrogen. The temperature was then raised to $200^{\circ} \mathrm{C}$ and, after 1.5 hours, the measured hydrogen flow corresponded to desorption of an additional $0.4 \mathrm{wt}$ \% hydrogen. The temperature was then raised again to $225^{\circ} \mathrm{C}$ and, after 7.5 hours, an additional $2.1 \mathrm{wt}$ \% hydrogen was desorbed, yielding a total hydrogen desorption of 6.2 wt. \% hydrogen. The dehydrogenated substrate was analyzed by GC-MS. Hydrogenolysis of approximately $28 \%$ of the phenylenecarbazole was observed under these reaction conditions.

\section{Preparation of N-(2-nitrophenyl)-3-methylcarbazole}

A solution containing $7.7 \mathrm{~g}$ of 3-methylcarbazole, $9.5 \mathrm{~g}$ 1-fluoro-2-nitrobenzene, and $27.3 \mathrm{~g}$ of cesium carbonate in $120 \mathrm{~mL}$ of dimethylformamide was prepared. The solution was then heated to $120^{\circ} \mathrm{C}$ until reaction was completed (determined by GC-MS analysis 
of aliquot). The solution was cooled, filtered, and added to $300 \mathrm{ml}$ water. The mixture was extracted with ethyl acetate. The combined ethyl acetate extracts were dried over magnesium sulfate and filtered, and the solvent was removed under vacuum to obtain $12.5 \mathrm{~g}$ of an orange solid (yield 95\%).

\section{Preparation of $\mathrm{N}$-(2-aminophenyl)-3-methylcarbazole}

To a 50 cc Parr reactor, $13 \mathrm{~g}$ of $\mathrm{N}$-(2-nitrophenyl)-3-methylcarbazole with $1.5 \mathrm{~g}$ platinum (IV) oxide and $25 \mathrm{ml}$ tetrahydrofuran were added. The reactor was then pressurized to 900 psig and heated to $60^{\circ} \mathrm{C}$ and stirred for 4 hours. The catalyst was then filtered off and the solvent removed under vacuum to obtain a viscous yellow oil.

\section{Preparation of 3-methyl-N-phenylenecarbazole}

With stirring, $5.9 \mathrm{~g}$ of $\mathrm{N}$-(2-aminophenyl)-3-methylcarbazole was dissolved in $700 \mathrm{ml}$ acetonitrile. Sulfuric acid ( $2.3 \mathrm{~g})$ was added by drop to the acetonitrile solution and allowed to stir for 5 minutes. Isoamyl nitrite $(2.8 \mathrm{~g})$ was dissolved in $10 \mathrm{ml}$ acetonitrile and was added by drop to the solution containing $\mathrm{N}$-(2-aminophenyl)-3-methylcarbazole and acid. The solution turned red and was heated to $85^{\circ} \mathrm{C}$ for 18 hours. The solvent was then removed under vacuum and the residue taken up in water and extracted with ethyl acetate. The product was further purified by column chromatography $(20 \%$ ethyl acetate in hexanes) and recrystallized in $90 \%$ hexane/10\% ethylacetate (yield $40 \%$ ).

\section{Preparation of bis-indolymethanes}

Potassium hydrogen sulfate $(20 \mathrm{~g}, 0.14 \mathrm{~mol})$ was added to a mixture of indole $(50 \mathrm{~g}$, $0.42 \mathrm{~mol}$ ) and formaldehyde (37\% by weight in water, $22 \mathrm{~g}, 0.27 \mathrm{~mol})$. The mixture was stirred overnight at room temperature. The reaction mixture was poured onto water, filtered, washed with water, and dried under vacuum to give 52 grams of solid which contained a mixture of monomer/dimer/trimer products. 


\section{Hydrogenation of bis-indolylmethanes}

An approximately 100 gram sample of the crude N,N'-diethyl-bis-indolylmethane or $\mathrm{N}, \mathrm{N}$ '-diethyl bis-(2-methyl)indolylmethane was placed in a 1 liter stirred tank reactor with ca. $500 \mathrm{cc}$ of anhydrous ethanol. After the reactor was purged with nitrogen, a charge of Raney nickel $(5 \mathrm{~g})$ suspended in anhydrous ethanol $(100 \mathrm{cc})$ was added to the sealed reactor via cannula. The reactor was pressurized with hydrogen (700 psia) and heated to $170^{\circ} \mathrm{C}$ for 16 hours. After the reactor was cooled and vented to atmospheric pressure, the ethanol solution was filtered to remove catalyst. The crude products were isolated by evaporation of the ethanol.

The monomer/dimer/trimer mixtures were separated by simple vacuum distillation. During the distillation of crude perhydro-N,N'-diethyl bis-indolylmethane, a small amount (ca. $1 \mathrm{cc}$ ) of "hydrogenated monomer" was collected at $70^{\circ} \mathrm{C}$ and $8 \times 10^{-1}$ torr. By GC/MS analysis, this monomer is a mixture of MW 153 and 167, which likely corresponds to perhydro-1-ethylindole and perhydro-1-ethyl-3-methylindole, respectively. The perhydro-N,N'-diethyl bis-indolylmethane was distilled at $180^{\circ} \mathrm{C}$ and $4 \times 10^{-2}$ torr. A small amount of "trimer" (ca. $5 \%$ ) also was carried over. The pure N, $\mathrm{N}$-Diethyl bis-indolylmethane is a colorless liquid with moderate viscosity at ambient temperature.

The crude perhydro-N, N'-diethyl-bis-(2-methyl)indolylmethane was dark brown, qualitatively indicative of oxidation byproducts. During the distillation, a moderate amount (ca. $15 \mathrm{cc}$ ) of "monomer" was collected at $60^{\circ} \mathrm{C}$ and $3 \times 10^{-1}$ torr. By GC/MS analysis, this monomer is a mixture of MW 167 and 181, which likely corresponds to perhydro-1-ethyl-2-methylindole and perhydro-1-ethyl-2,3-dimethylindole, respectively. The perhydro-N, N'-diethyl-bis-(2-methyl)indolylmethane was distilled at $175^{\circ} \mathrm{C}$ and $5 \times 10^{-2}$ torr. The liquid was pure by GC/MS analysis (no monomer, trimer, or unknown byproducts detected). However, a bright yellow coloration of the liquid suggests that a small amount of oxidation byproducts may have co-distilled. The perhydro- $\mathrm{N}, \mathrm{N}^{\prime}$-diethylbis-(2-methyl)indolylmethane has moderate/high viscosity at room temperature. 


\section{Dehydrogenation of perhydro-N,N'-diethyl-bis-indolylmethane}

Perhydro-N,N'-diethyl-bis-indolylmethane was degassed by evacuation ( $1 \times 10^{-3}$ torr) at $20^{\circ} \mathrm{C}$ for 20 minutes. Under inert atmosphere, $4.0 \mathrm{~g}$ perhydro-N,N'-diethyl-bis-

indolylmethane and $0.1 \mathrm{~g}$ of $5 \%$ palladium on carbon (dehydrogenation catalyst) were placed in a $20 \mathrm{cc}$ stirred tank reactor and the reactor was sealed. The reactor was connected to the dehydrogenation testing manifold. After evacuation of residual air from the manifold lines, hydrogen was purged through the reactor to displace the argon from the reactor headspace. The reactor was rapidly heated to $175^{\circ} \mathrm{C}$ with stirring (550 rpm) under 1 atm. hydrogen. The reactor was then heated from 175 to $200^{\circ} \mathrm{C}$ at $0.2^{\circ} \mathrm{C} /$ minute and held at $200^{\circ} \mathrm{C}$ until completion of the dehydrogenation experiment. After completion of the experiment, the reactor was cooled to $25^{\circ} \mathrm{C}$ and the contents filtered to remove catalyst. GC/MS analysis of the chemical composition of the dehydrogenated mixture was used to verify the amount of hydrogen generated by the dehydrogenation reaction and measured by the flow meters.

\section{Preparation, hydrogenation, and dehydrogenation of pyrrocoline}

A mixture of 3-pyridinepropanol $(25 \mathrm{~g}, 0.18 \mathrm{~mol})$ and $1 \mathrm{~g}$ of $5 \% \mathrm{Pd} / \mathrm{C}$ was heated to $250^{\circ} \mathrm{C}$ with stirring for 8 hours. After cooling to room temperature, the mixture was taken up with water and extracted with ethyl acetate. The organic layer was washed with water, dried over magnesium sulfate, and filtered. Evaporation of the solvent and chromatography of the residue on silica gel with ethyl acetate/hexanes (1:1) gave 7 grams of a mixture of pyrrocoline and tetrahydropyrrocoline. The mixture was hydrogenated using the standard hydrogenation procedures. Four grams of this material were dehydrogenated using $5 \% \mathrm{Pd} / \mathrm{C}(100 \mathrm{mg})$ at $150^{\circ} \mathrm{C}$ for 4 hours, giving only $6 \%$ conversion to the tetrahydropyrrocoline (by GC-MS analysis). 


\section{References}

1. Nishimura, S., in "Handbook of Heterogeneous Catalytic Hydrogenation for Organic Synthesis", Wiley-Intescience, 2001.

2. Smith, A.; Utley, J. H. P. Chem. Commun. 1965, 427.

3. Smith, A.; Utley, J. H. P. J. Chem. Soc. (C) 1970, 1.

4. $\quad$ Cui, Y.; Kwok, S.; Bucholtz, A.; Davis, B.; Whitney, R. A.; Jessop, P. G. New J. Chem. 2008, 32, 1027.

5. Hara, T.; Mori, K.; Mizugaki, T.; Ebitani, K.; Kaneda, K. Tetrahedron Letters 2003, 44, 6207.

6. Moores, A.; Poyatos, M.; Luo, Y. Crabtree, R. H. New J. Chem. 2006, 11, 1675.

7. Kistenmacher, A; Müllen, K. J. Heterocyclic Chem. 1992, 29, 1237.

8. Janosik, T.; Bergman, J. Tetrahedron 1999, 55, 2371.

9. Dunlop, H.G.; Tucker, S.H. J. Chem. Soc. 1939, 1945.

10. Boekelheide, V.; Windgassen, Jr., R.J. J. Am Chem. Soc. 1959, 81, 1456. 


\section{Chapter 5: Hydrogen Carriers with Multiple Nitrogen Atoms}

\section{Introduction}

Chapters 3 and 4 of this report have detailed a number of promising liquid carrier candidates that incorporate a nitrogen heteroatom into fused ring structures. As outlined in Chapter 1, the presence of nitrogen has a profound influence on the heat of hydrogenation of these molecules due to the weakening of $\alpha-\mathrm{CH}$ bonds adjacent to the nitrogen atoms. Using this guidance, it is reasonable to assume that liquid carrier analogs that contain multiple nitrogen atoms may result in average $\Delta \mathrm{H}_{d}$ values that are lower than those molecules with only one nitrogen atom. In this chapter, we detail the synthesis and reversible hydrogenation testing of several molecules which are analogs of successful liquid carriers from Chapters 3 and 4 . One example is 9 -ethyl- $\beta$-carboline, which is an analog of N-ethylcarbazole.

\section{Results and Discussion}

\section{A. Carboline derivatives}

Owing to favorable thermodynamics and physical properties, ${ }^{1} \beta$-carboline and its 9 -alkyl derivatives (e.g., 1 in Figure 5-1) were of interest as potential hydrogen carriers. While 9-ethyl- $\beta$-carboline (1; $\mathrm{R}=\mathrm{C}_{2} \mathrm{H}_{5}$ ) can be prepared by alkylation of $\beta$-carboline, dimethylformamide (DMF) is typically used as a cosolvent for alkylation. ${ }^{2}$

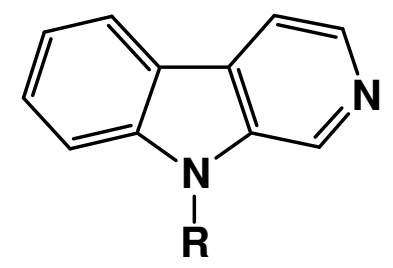

$$
1 ; \mathrm{R}=\mathrm{H}, \mathrm{CH}_{3}, \mathrm{C}_{2} \mathrm{H}_{5}
$$

Figure 5-1. The molecular structure of $\beta$-carboline derivatives. 
Even small quantities of DMF $(<0.5 \%)$ in the product will poison the rhodium catalyst that was used for ring hydrogenation. Complete removal of DMF is very difficult. An alternate route to $\mathbf{1}$ (or to the corresponding tetrahydro analogs) was sought to facilitate evaluation of these liquid carrier candidates.

Inclusion of carbazole among the substrates exemplified in a recent methylation procedure for substituted indoles ${ }^{3}$ suggested a possible route. In that reference, methylation with dimethyl carbonate is favored by electron-withdrawing substituents on the indole nucleus. These observations suggested that $\beta$-carboline might also be selectively alkylated by the same procedure. We found that this procedure could be simplified, and the cosolvent, DMF, could be excluded without detriment. Treatment of $\beta$-carboline with dimethyl carbonate in the absence of DMF provided a single product with the correct molecular weight in quantitative conversion. Regioselective location of the methyl group on the 9 position, the indole nitrogen, was established by ${ }^{13} \mathrm{C} N M R$. This result immediately raised the question of whether commercially available tetrahydro- $\beta$-carboline might also be selectively methylated under the same conditions. If the latter could be done, the initial dehydrogenation of tetrahydro- $\beta$-carboline needed to prepare $\beta$-carboline could be avoided, and the resulting 9-methyl-tetrahydro- $\beta$ carboline would be equally useful for hydrogenation/dehydrogenation studies. However, reaction of tetrahydro- $\beta$-carboline with dimethylcarbonate under the same conditions as those that were successful for methylation of $\beta$-carboline provided a complex mixture (Figure 5-2), which included 9-methyltetrahydro- $\beta$-carboline (2; 35\%), as well as low concentrations $(10-20 \%)$ of both isomeric $\mathrm{N}$-methyl- $\mathrm{N}$-methylcarbamate derivatives (e.g., 3) and both $\mathrm{N}$-methylcarbamates (e.g., 4).

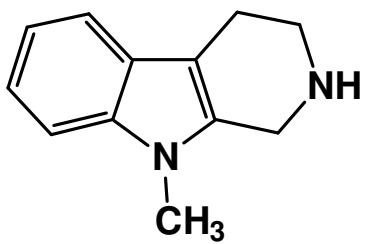

2

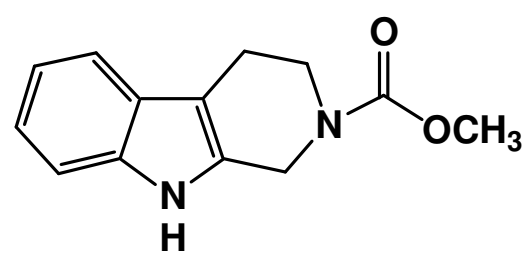

3

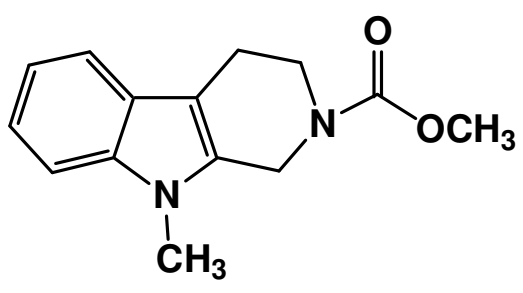

4

Figure 5-2. Products from the attempted alkylation of tetrahydro- $\beta$-carboline. 
Because of the poor selectivity of this reaction and the ready availability of $\mathbf{1}\left(\mathrm{R}=\mathrm{CH}_{3}\right)$, no further work was done on alkylation of tetrahydro- $\beta$-carboline.

We found that while high conversions could be attained by hydrogenation of $\mathrm{N}$ ethylcarboline with in situ-reduced rhodium/lithium aluminate catalyst, formation of significant amounts of $\mathrm{N}$-methyl derivatives, even from DMF-free 9 -ethyl- $\beta$-carboline, was found. This observation was totally unexpected. With the improved route to 9-methyl- $\beta$-carboline reported above, attention shifted to selective hydrogenation of this substrate, and specifically to ways to avoid adventitious methylation. Key to achieving this goal was an understanding of the source of the unexpected methylation. Tetrahydro- $\beta$-carboline is made by acid-catalyzed reaction of formaldehyde with 3-(2-aminoethyl)indole (5). (See Scheme 1, adapted from E. D. Cox and J. M. Cook ${ }^{4}$ to indicate routes for byproduct formation during catalytic hydrogenation). In the presence of a proton source, the equilibrium between tetrahydro- $\beta$-carboline (10) and intermediates 7 - 9 will be established. If water is also present, 5 and formaldehyde can also be generated. If these steps occur during a catalytic hydrogenation, $\boldsymbol{6}$ and $\mathbf{7}$ can be hydrogenated directly to the corresponding 3-[2-(methylamino)ethyl]indole. Further, formaldehyde can reductively methylate any of the amino functionality in $\mathbf{5}-\mathbf{1 0}$ to form a variety of mono- and dimethylated byproducts.

In the previous reactions with in situ-reduced catalyst, most of the solvent from the catalyst pre-reduction (with the water formed by catalyst reduction) was removed via an internal filter in the reactor. Because of the position of the filter inside our reactor, about $5 \%$ of that solvent (and coproduct water) remained behind, and was present during hydrogenation of 9 -ethyl- $\beta$-carboline. To test whether this was sufficient water to cause byproduct formation, 10 (tetrahydrocarboline; THC) was hydrogenated with rhodium/lithium aluminate. However, after pre-reduction, the catalyst was washed thoroughly with anhydrous solvent before introduction of 10 . Hydrogenation $\left(160^{\circ} \mathrm{C}\right.$; 1250 psig $\mathrm{H}_{2} ; 15$ hrs; 20 wt. \% catalyst) provided high (92\%) conversion with good selectivity: $79 \%$ perhydro- $\beta$-carboline (11), $18 \%$ partially reduced 10 , and only $1 \%$ $\mathrm{N}$-methylated 11 and $0.6 \%$ perhydro-5. With the same protocol under more forcing 
conditions (same as above, with 30 wt. \% catalyst for 24 hrs.), conversion was higher (98\%). While 11 still predominated (60 - 65\%), significant concentrations of monomethylated and dimethylated 10, as well as of perhydro-5 and N-methyl-perhydro5, were formed. To test whether high conversion and selectivity might be obtained in a shorter time with a higher catalyst loading, the last reaction was repeated for 15 hours. While conversion remained high $(99 \%$, with no partially hydrogenated intermediates detected), selectivity to 11 (71\%) was little improved; N-methyl-perhydro-2 and Nmethyl-11 were the major byproducts ( $21 \%$ and $8 \%$, respectively). This result clearly shows the role of the catalyst in promoting byproduct formation. Consequently, the lower catalyst loading (20 wt. \%, based on tetrahydrocarboline) was used. With a newly cleaned reactor, conversion was higher (>99\%, with no partially hydrogenated intermediates detected) and selectivity markedly lower (54\% 11, 30\% N-methylperhydro5, and $13 \% \mathrm{~N}$-methyl-11) than those observed prior to cleaning the reactor. To determine the catalyst loading required to balance conversion and selectivity, analogous reactions were done with 10 wt. \%, 5 wt. \% and 2.5 wt. \% catalyst loadings. Results of these reactions are collected in Table 5-1. With the lowest catalyst loading, quantitative conversion and high selectivity to 11 were obtained. $\mathrm{N}^{1}$-methylperhydro-3-(2aminoethyl)indole (12) (Figure 5-3), a primary amine-containing byproduct, was not detected. If the latter were to be present, it might interfere with subsequent dehydrogenation.

Table 5-1. Effect of catalyst loading on hydrogenation of tetrahydrocarboline (THC).

\begin{tabular}{ccccccc}
\hline \multirow{2}{*}{$\begin{array}{c}\text { Catalyst } \\
\text { Loading }^{\mathrm{a}}\end{array}$} & & & \multicolumn{4}{c}{ Selectivity $^{\mathrm{c}}$} \\
\cline { 5 - 7 } & Time & Conversion $^{\mathrm{b}}$ & $\mathbf{1 1}$ & $\begin{array}{c}\text { Partially } \\
\text { Hydro- } \\
\text { genated }^{\mathrm{d}}\end{array}$ & $\begin{array}{c}\text { N-Methyl- } \\
\text { perhydro-5 }\end{array}$ & $\begin{array}{c}\text { N-Methyl- } \\
\text { perhydro- } \\
\mathbf{1 1}\end{array}$ \\
\hline & & & & & 7 & 9 \\
10 & 12 & 100 & 75 & 8 & 2 & 7 \\
5 & 12 & 100 & 81 & 9 & $\mathrm{ND}^{\mathrm{e}}$ & 2 \\
2.5 & 10 & 67 & 50 & 15 & ND $^{\mathrm{e}}$ & 5 \\
2.5 & 13 & 87 & 73 & 9 & ND $^{\mathrm{e}}$ & 6 \\
2.5 & 16 & 94 & 76 & 10 & ND $^{\mathrm{e}}$ & 7 \\
2.5 & 19 & 99 & 92 & ND $^{\mathrm{e}}$ & & \\
\hline
\end{tabular}


Notes for Table 5-1:

a) Weight percent of $4 \%$ rhodium on lithium aluminate, based on tetrahydrocarboline. Reactions carried out at $160^{\circ} \mathrm{C}$ and 1250 psig hydrogen pressure.

b) Based on tetrahydrocarboline.

c) Area percent GC analysis, rounded to nearest whole percent. Peaks identified by EI and CI GC-MS.

d) Hexahydro- and octahydrocarboline.

e) Not detected.

Based on this promising result with tetrahydrocarboline, hydrogenation of $\mathrm{N}$ methylcarboline (NMC) was attempted. With $2.5 \mathrm{wt}$ \% \% catalyst and the same temperature and pressure, only $20 \%$ conversion of NMC to the tetrahydro derivative was obtained after 16 hours at room temperature. Repetition of this reaction with $5 \mathrm{wt}$ \% catalyst provided only $40 \%$ conversion to tetrahydro-NMC after 64 hours. 
<smiles>NCCc1c[nH]c2ccccc12</smiles>

5<smiles></smiles>

$\|$<smiles>c1ccc2c(c1)NCCN2</smiles>

9

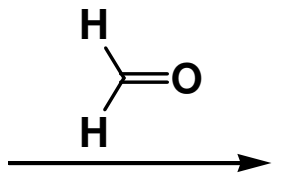<smiles>OCNCCc1c[nH]c2ccccc12</smiles>

6<smiles>OCCO</smiles><smiles>C1=NCCc2c1[nH]c1ccccc21</smiles>

7

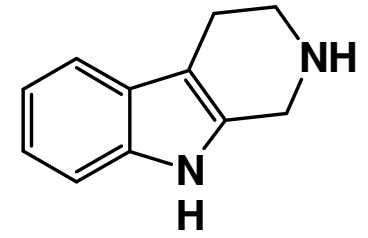

10

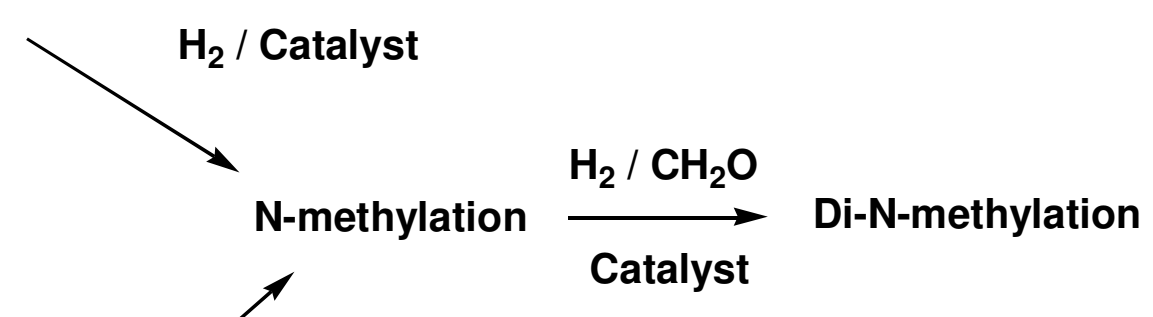

$\mathrm{H}_{2}$ / Catalyst

Scheme 5-1. Synthesis of tetrahydro- $\beta$-carboline and potential mechanisms for methylated byproduct formation during the hydrogenation of tetrahydro- $\beta$-carboline. 


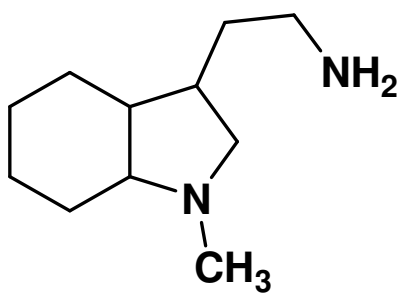

12

Figure 5-3. Structure of $\mathrm{N}^{1}$-methylperhydro-3-(2-aminoethyl)indole.

As a result of the severe inhibition of catalysis in attempted hydrogenations of $\mathrm{N}$-methylcarboline with rhodium/lithium aluminate, and in conjunction with the promising results for selective hydrogenation of tetrahydrocarboline (THC), attention was focused on the latter approach to provide sufficient material for dehydrogenation testing.

Repetition of the hydrogenation of THC with $2.5 \mathrm{wt} . \%$ rhodium/lithium aluminate $\left(160^{\circ} \mathrm{C}\right.$; $1250 \mathrm{psig}_{2} ; 16$ hours) at a higher concentration (1.75 grams of substrate, vs.1 gram used previously) provided very similar conversion and selectivity (Table 5-2; Run A). While a slightly higher conversion was obtained in the second run (Table 5-2; Run B), the selectivity was comparable to those of the other reactions. In both cases, good selectivities to perhydrocarboline and partially hydrogenated THC intermediates were obtained. While moderate quantities of $\mathrm{N}$-methyl-perhydrocarboline were formed, this byproduct has only secondary and tertiary nitrogen sites, and would not be expected to interfere with dehydrogenation. Finally, perhydro-3(2-amino-ethyl)indole and its $\mathrm{N}$-methyl derivative 12, primary amine-containing byproducts, were not detected. If the latter were to be present, they might interfere with subsequent dehydrogenation.

The dehydrogenation of perhydrocarboline samples (containing small amounts of partially hydrogenated carboline) was attempted using both platinum- and palladiumbased catalysts. The temperature of the dehydrogenation reactions, using neat samples of perhydrocarboline, was slowly ramped from 100 to $200^{\circ} \mathrm{C}\left(1^{\circ} \mathrm{C} / \mathrm{min}\right.$.). No hydrogen evolution was observed until temperatures above $150^{\circ} \mathrm{C}$ were reached, and despite 
prolonged heating at $200^{\circ} \mathrm{C}$, less than 0.2 wt. \% hydrogen was evolved. GC/MS analysis of the reaction products showed high reaction selectivity, but verified the very low conversion of perhydrocarboline to partially hydrogenated carboline intermediates.

Table 5-2. Hydrogenation of tetrahydrocarboline $(\mathrm{THC})^{\mathrm{a}}$

\begin{tabular}{lcccc}
\hline Component & (MW) & Scouting Run $^{\text {b }}$ & Run A & Run B \\
\hline & & & & \\
Perhydrocarboline & $(180)$ & 76 & 72 & 75 \\
Partially hydrogenated THC & $(178)$ & 9 & 7 & 10 \\
& $(176)$ & 2 & 3 & 2 \\
THC & $(174)$ & 1 & 5 & 2 \\
N-Methylperhydrocarboline & $(172)$ & 6 & 8 & ND $^{c}$ \\
N-Methylperhydro-3-(2-aminoethyl)indole & $(194)$ & 6 & 6 & 11 \\
Perhydro-3-(2-aminoethyl)indole & $(182)$ & ND $^{c}$ & Trace? $^{\mathrm{d}}$ & Trace? $^{\mathrm{d}}$ \\
& $(168)$ & ND $^{\mathrm{c}}$ & Trace? $^{\mathrm{d}}$ & Trace? $^{\mathrm{d}}$ \\
\hline
\end{tabular}

Notes for Table 5-2:

a) Reaction composition (GC A\%) after $16 \mathrm{hrs} . / 160^{\circ} \mathrm{C} / 1250 \mathrm{psig} \mathrm{H}_{2}$.

b) Initial reaction with 1.0 gram of substrate.

c) Not detected.

d) Very small $(<0.1 \mathrm{~A} \%)$ peaks were present at similar retention times to those of these components. These peaks could not be conclusively identified by EI GC-MS.

Owing to their relative availability, the foregoing investigation focused on $\beta$-carboline and tetrahydro- $\beta$-carboline. An isomeric structure, $\beta$-carboline (13) or its tetrahydro derivative 14, may not be as susceptible to acid-mediated hydrolytic ring opening (Figure 5-4).

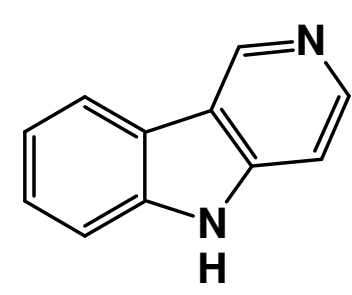

13

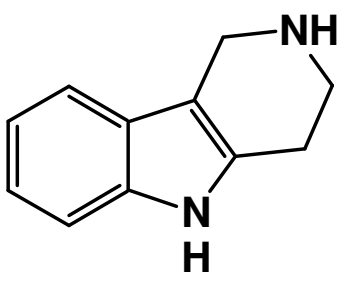

14

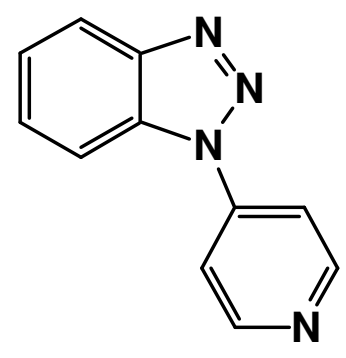

15

Figure 5-4. Alternative liquid carrier candidates with multiple nitrogen heteroatoms. 
Specifically, 14 has been made by variations of the Fischer Indole synthesis ${ }^{5,6}$ (e.g., by reaction of in situ-generated 4-piperidone with phenylhydrazine hydrochloride; Scheme $5-2){ }^{6}$ Further, preparation of $\mathbf{1 3}$ by a multistep route has been reported. ${ }^{7}$

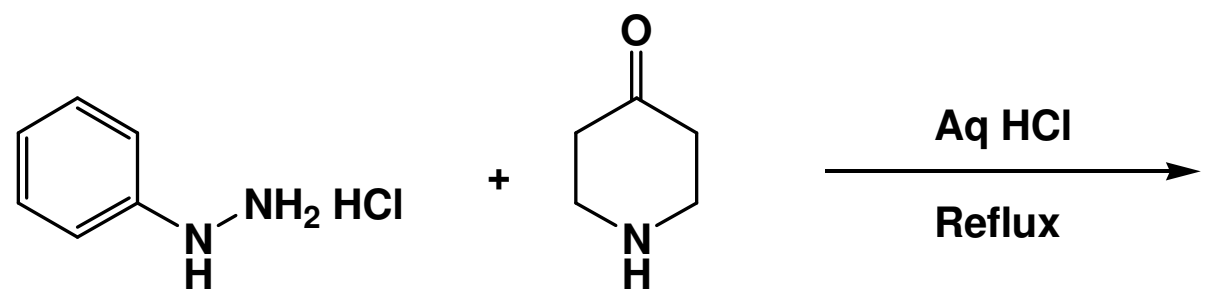

Scheme 5-2. Synthesis of 2,3,4,5-Tetrahydro-1H-pyrido[4,3-b]indole.

The last step in the sequence is cyclization of $\mathbf{1 5}$ in hot "syrupy" phosphoric acid. It should be noted that the multistep routes used to obtain 13 and 14 render them of academic interest rather than of practical utility.

\section{B. Azaindoles}

Thermodynamic calculations suggest that the overall average enthalpy for dehydrogenation of hydrogenated 7-azaindole and $\mathrm{N}$-alkylated derivatives is low and could enable the dehydrogenation of the saturated compounds under moderate conditions. We calculated the stepwise energies for two possible dehydrogenation pathways and found very even $\Delta \mathrm{E}$ values for one pathway (Scheme 5-3). 


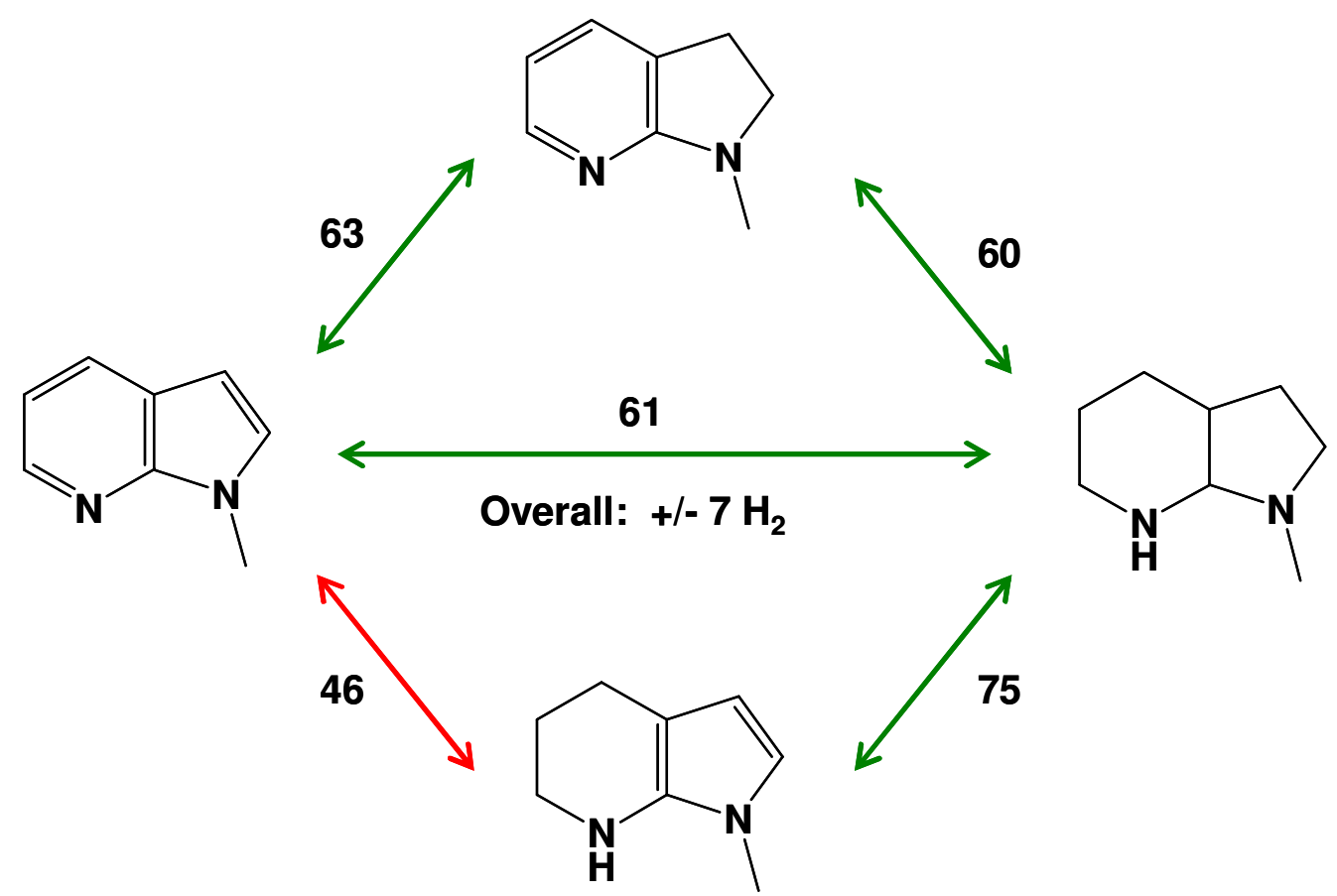

Scheme 5-3. Calculation of electronic energies for two possible dehydrogenation pathways for 7-aza-N-methylindole. Values are $\mathrm{kJ} / \mathrm{mole} \mathrm{H}_{2}$.

Our initial hydrogenation testing revealed that it was difficult to fully hydrogenate 7-azaindole. Using a $\mathrm{Ru} / \mathrm{Al}_{2} \mathrm{O}_{3}$ catalyst at $150^{\circ} \mathrm{C}$ and either 150 psig or 1000 psig hydrogen pressure resulted in the hydrogenation of only the carbons in the 2 and 3 positions of the five-membered ring (Scheme 4).

Higher conversion was achieved using a $\mathrm{Rh} / \mathrm{Al}_{2} \mathrm{O}_{3}$ catalyst and acetic acid solvent under 500 psig hydrogen at room temperature. An isomer of hexahydro-7-azaindole was recovered in which one double bond remains unsaturated. It is likely that the remaining unsaturation is the result of formation of a very stable imine (Scheme 5-4, lower left). Attempting additional hydrogenation of the hexahydro-7-azaindole with a $\mathrm{Pt} / \mathrm{Pd} / \mathrm{Al}_{2} \mathrm{O}_{3}$ catalyst at $100^{\circ} \mathrm{C}$ and 600 psig hydrogen for six hours resulted in only $10 \%$ conversion to perhydro-7-azaindole. 


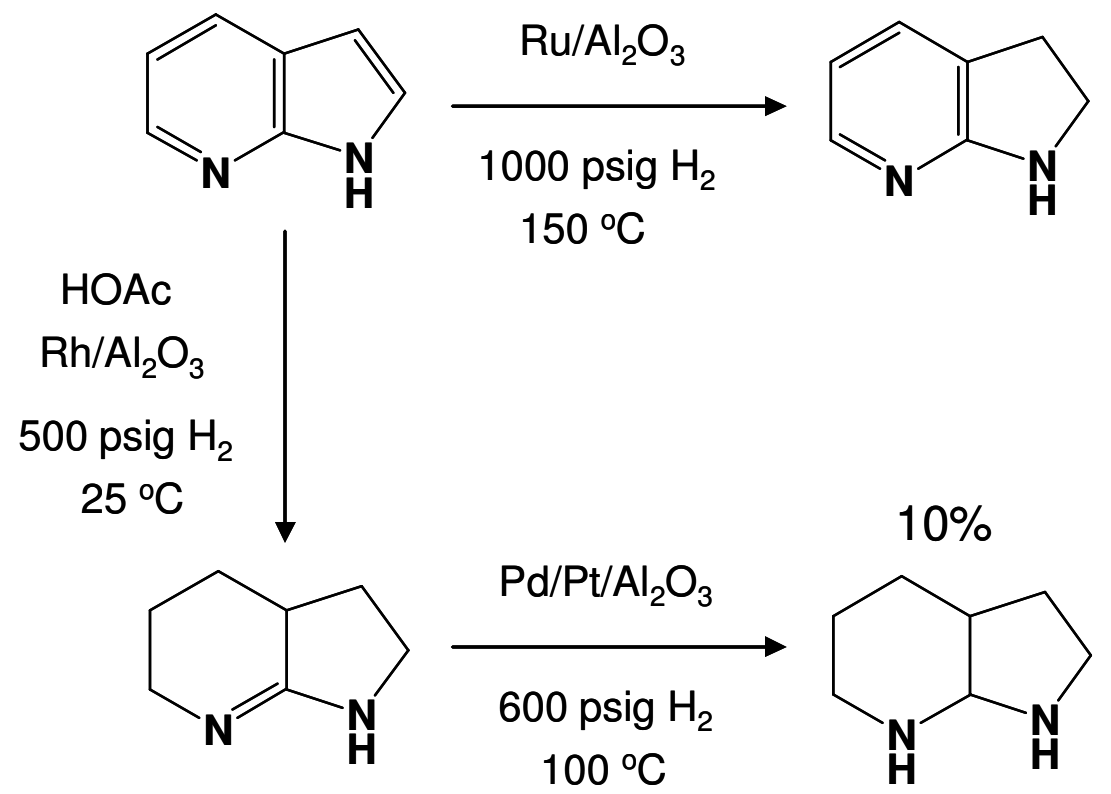

Scheme 5-4. Hydrogenation of 7-azaindole.

Investigation of alternate hydrogenation catalysts and conditions resulted in highly variable results. For example, hydrogenation of 7 -azaindole using $\mathrm{Rh} / \mathrm{LiAl}_{5} \mathrm{O}_{8}$ in $\mathrm{THF}$ at $160^{\circ} \mathrm{C}$ and $800 \mathrm{psig} \mathrm{H}_{2}$ resulted in the formation of oligomers. The $\mathrm{HCl}$ salt of 7-azaindole was prepared using anhydrous $\mathrm{HCl}$ in ether. Hydrogenation of the $\mathrm{HCl}$ salt of 7-azaindole using platinum oxide catalyst in ethanol resulted in byproducts formed from C-N bond cleavage.

As a result of the difficulty of obtaining perhydro-7-azaindole, we investigated the hydrogenation of alkylated derivatives of 7 -azaindole. In Chapter 4 we detail a number of indole-based liquid carrier candidates which demonstrated higher hydrogenation selectivity after the alkylation of the nitrogen atom in the five-membered ring. Alkylation of the nitrogen on the five-membered ring to produce 1-methyl-7-azaindole was achieved in high yield by deprotonation with $\mathrm{NaH}$ and methylation using iodomethane in tetrahydrofuran. Based upon ${ }^{1} \mathrm{H}$ NMR analysis, we observed that the reaction products were completely alkylated at the nitrogen atom in the five-membered ring. A small amount (ca. 7\%) of the products contained a second methyl group, presumably located at the nitrogen atom in the six-membered ring. 
A wide variety of hydrogenation catalysts and reaction conditions were explored to achieve the selective perhydrogenation of 1-methyl-7-azaindole (Scheme 5-5). Under both neutral and acidic conditions, the resulting imine product (structure confirmed by ${ }^{1} \mathrm{H}$ and $\left.{ }^{13} \mathrm{C} N M R\right)$ was not reduced, as might be expected from the very low $\Delta \mathrm{E}(-20 \mathrm{~kJ} / \mathrm{mol}$ $\mathrm{H}_{2}$ ) for this reaction.

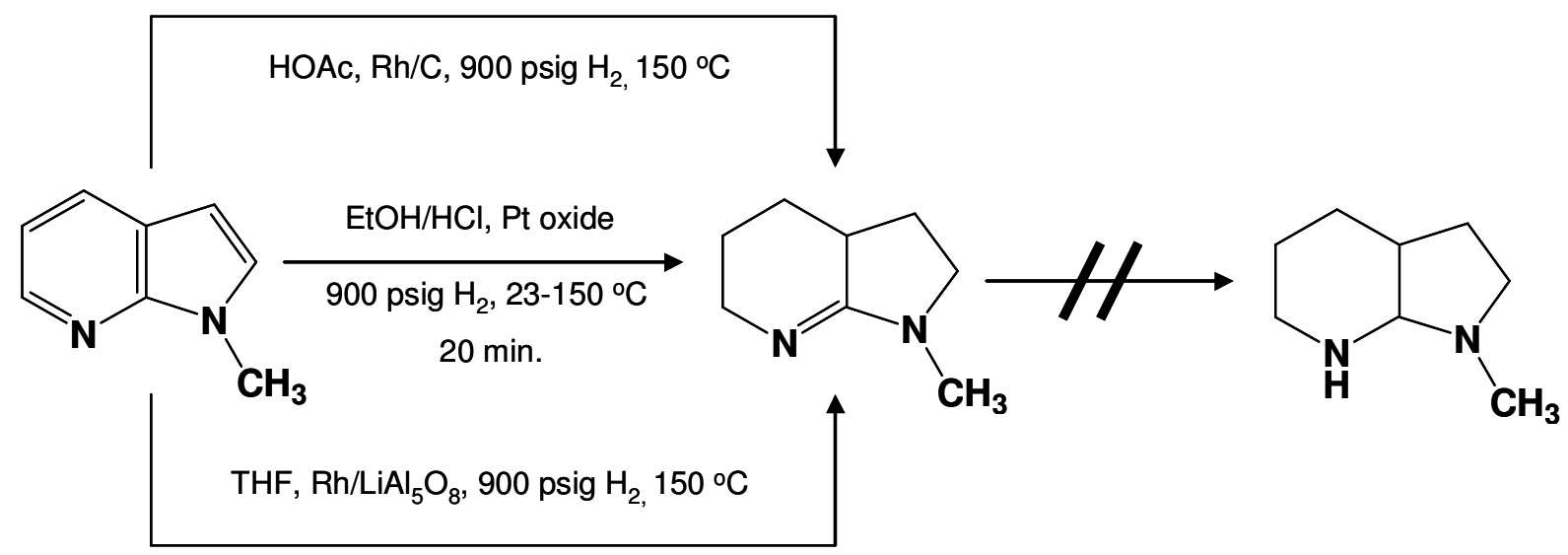

Scheme 5-5. Hydrogenation of 1-methyl-7-azaindole.

These results are similar to the attempted hydrogenation of 2,2'-bipyrimidine (Chapter 6), in which a very stable diimine is formed as the product of hydrogenation. From our assay of reaction conditions, it did appear that the hydrogenation under acidic conditions was much more rapid than the hydrogenation in THF.

The isolated hexahydro-1-methyl-7-azaindole from the hydrogenation reactions was tested for dehydrogenation at $200^{\circ} \mathrm{C}$ using a $5 \% \mathrm{Pd} / \mathrm{C}$ dehydrogenation catalyst. Unfortunately, we found that no hydrogen was evolved and hexahydro-1-methyl-7azaindole was recovered from the attempted dehydrogenation.

It may be possible that preparing isomeric azaindoles with the nitrogens in the 4,5 or 6 positions of the six-membered ring would avoid formation of this stable imidoformamide 
intermediate. However, due to a lack of suitable synthetic routes to these isomers, no additional research on azaindole liquid carrier prototypes was performed.

\section{1-Methylbenzimidazole}

The benzimidazole class of potential hydrogen carriers was attractive for exploration due to the structural similarity to the successful indole carrier molecules (Chapter 4 ) and the perceived thermodynamic advantages of substitution of an additional nitrogen atom in the five-membered ring. One commercially available member of this class is 1 methylbenzimidazole (m.p. $59^{\circ} \mathrm{C}$, b.p. $154^{\circ} \mathrm{C} / 12$ torr). The hydrogenation of 1 methylbenzimidazole was performed neat, using $5 \%$ rhodium on carbon at $150^{\circ} \mathrm{C}$ and 850 psia $\mathrm{H}_{2}$ pressure. Within 30 minutes at the reaction temperature, the hydrogen pressure drop ceased, indicating a rapid completion of the hydrogenation reaction. Analysis of the hydrogenation product by GC-MS showed quantitative conversion to a single tetrahydro-1-methylbenzimidazole isomer. Structural information from ${ }^{1} \mathrm{H}$ and ${ }^{13} \mathrm{C}$ NMR spectroscopy indicated that the hydrogenation occurred exclusively at the sixmembered ring (Scheme 5-6).

Calculation of the electronic energies $(\Delta \mathrm{E})$ of hydrogenation for the saturation of 1-methylbenzimidazole in two steps indicates that the incomplete hydrogenation was due to a thermodynamic limitation for the hydrogenation of the tetrahydro-1methylbenzimidazole intermediate. The average $\Delta \mathrm{E}$ for the hydrogenation of the tetrahydro-1-methylbenzimidazole shown in Scheme 5-6 was ca. $40 \mathrm{~kJ} / \mathrm{mol} \mathrm{H}_{2}$. The extremely low $\Delta \mathrm{H}$ that can be estimated from the $\Delta \mathrm{E}$ clearly would not allow for hydrogenation under feasible conditions of temperature and pressure. Thus, the addition of a second nitrogen atom in the 3 position of the five-membered ring of an $\mathrm{N}$-alkylated indole results in a larger-than-desired lowering of the average $\Delta \mathrm{H}$ for hydrogenation of the five-membered ring. 


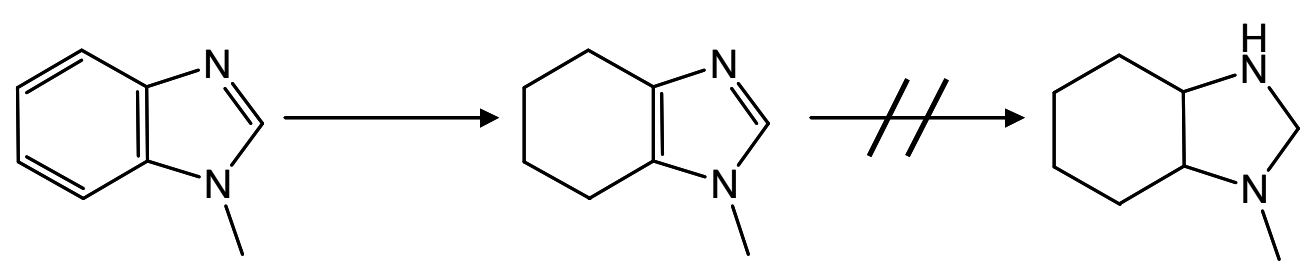

Scheme 5-6. Hydrogenation of 1-methylbenzimidazole.

Predictive computational modeling was used to identify a carrier prototype that may mediate the extremely low $\Delta \mathrm{H}$ of tetrahydro-1-methylbenzimidazole. Substitution of a pyridyl group at the 1 position of benzimidazole, rather than a methyl group, results in a carrier candidate that has a higher theoretical hydrogen capacity and more suitable thermodynamics for completely reversible hydrogenation. The calculations show that one of two potential reaction pathways has intermediate energies that are between 55 and $71 \mathrm{~kJ} / \mathrm{mol} \mathrm{H}$ (Scheme 5-7).

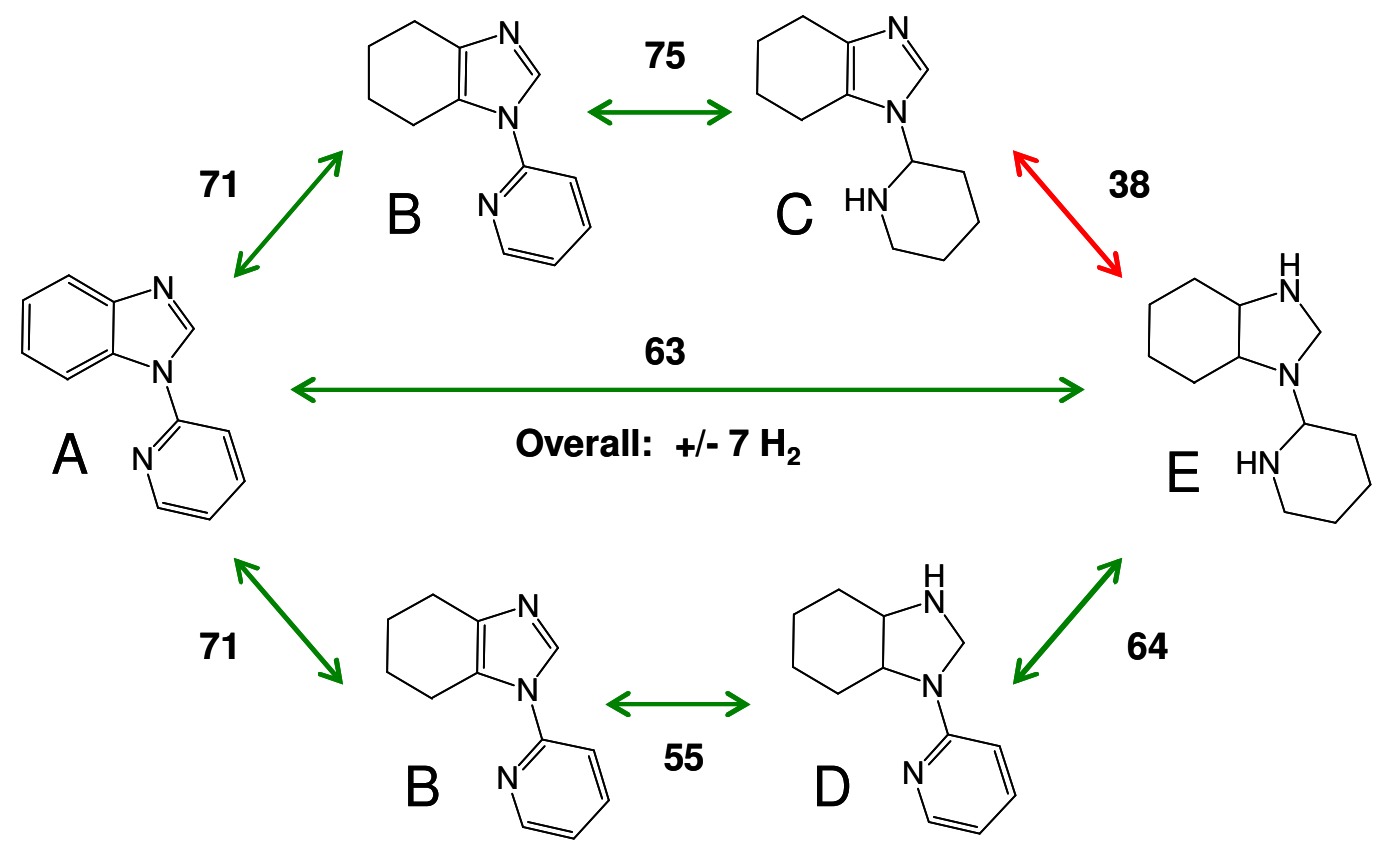

Scheme 5-7. Possible dehydrogenation pathways for 1-(pyrid-2-yl)benzimidazole. Calculated $\Delta \mathrm{E}$ values for dehydrogenation are in $\mathrm{kJ} / \mathrm{mole} \mathrm{H}_{2}$. 
While the upper pathway in Scheme 5-7 suffers from a step with an extremely low $\Delta \mathrm{H}$ $(\mathbf{C} \rightarrow \mathbf{E})$, the lower pathway suggests that the five-membered ring of the tetrahydro intermediate may be hydrogenated if the pyridyl group is unsaturated $(\mathbf{B} \rightarrow \mathbf{D})$. One possible contribution to the $17 \mathrm{~kJ} / \mathrm{mol} \mathrm{H}_{2}$ energy difference for the $\mathbf{C} \rightarrow \mathbf{E}$ and $\mathbf{B} \rightarrow \mathbf{D}$ transformations is that the aromatic 2-pyridyl group can act as an electron-withdrawing group, thus lowering the enthalpy of hydrogenation of the electron-rich five-membered ring. The saturated 2-piperidyl group in the upper pathway has the opposite influence, acting as an electron-donating group to the five-membered ring, contributing to a very low heat of hydrogenation for the $\mathbf{C} \rightarrow \mathbf{E}$ transformation.

Unfortunately, due to the lack of an identified method for the synthesis of 1-(pyrid-2$\mathrm{yl}$ )benzimidazole, no experimental verification of these computational predictions was forthcoming.

\section{Conclusions}

Despite promising thermodynamics for the reversible hydrogenation of the carbolineand azaindole-based liquid carrier candidates, we were unable to achieve the high selectivity and conversion required for a successful liquid carrier prototype. The carboline-based carriers demonstrated problematic reactivity during the hydrogenation, leading to unwanted methylated byproducts. Selectively $\mathrm{N}$-methylated $\beta$-carboline showed very slow hydrogenation rates under conditions suitable for high selectivity. Our attempts to dehydrogenate unalkylated perhydro- $\beta$-carboline did not result in appreciable hydrogen release.

Both 7-azaindole and 1-methyl-7-azaindole were hydrogenated with high selectivity to a hexahydro imine product which was resistant to complete saturation. The position of the nitrogen atom in the six-membered ring likely leads to the very stable imine intermediate. Attempted dehydrogenation of hexahydro-1-methyl-7-azaindole was unsuccessful. 
The 1-methylbenzimidazole carrier candidate was hydrogenated with high selectivity to a tetrahydro intermediate product which was resistant to complete saturation due to thermodynamic limitations. Substitution of the methyl group for substituents that act as electron-withdrawing groups might lead to more suitable thermodynamics for complete, reversible hydrogenation.

\section{Experimental Details}

Anhydrous tetrahydrofuran (under nitrogen), dimethyl carbonate, 1,4-diazabicyclo[2.2.2]octane, and tetrahydrocarboline were obtained from commercial sources and used as received. Rhodium on lithium aluminate catalyst (4 wt. \% rhodium loading) was obtained from Engelhard (product number C004117). ' $\mathrm{H}$ and ${ }^{13} \mathrm{C} N M R$ spectra were recorded as solutions in either deuterochloroform or perdeuteromethanol on a Bruker DMX-500 FT-NMR equipped with a $5 \mathrm{~mm}$ QNP probe. In all cases, the chemical shift was referenced to the solvent peak. Separations were effected on an Agilent 6890 Gas Chromatograph equipped with a fused silica RTX-35 column (30 $\mathrm{m} \times 0.25 \mathrm{~mm} ; 0.25 \mu \mathrm{m}$ film thickness). GC mass spectra were determined with a Finnigan SSQ7000 single quadrupole mass spectrometer; molecular weights and the number of nitrogen-bonded hydrogens were confirmed by chemical ionization (perdeuteroammonia).

Methylation of $\beta$-carboline. To a $100 \mathrm{~mL}$ three-necked round bottom flask equipped with a magnetic stirrer, condenser, nitrogen inlet and outlet, and rubber septum was added $2.5 \mathrm{~g}\left(1.489 \times 10^{-2}\right.$ mole $) \beta$-carboline and $0.167 \mathrm{~g}\left(1.489 \times 10^{-3} \mathrm{~mole}\right)$ 1,4-diazabicyclo[2.2.2]octane. The flask was purged with nitrogen for 15 min., after which $50 \mathrm{~mL}$ dimethyl carbonate was added via syringe. Upon heating to reflux with stirring, the suspended solids dissolved. After $9.5 \mathrm{hrs}$ under reflux, GC analysis showed complete disappearance of the starting material and appearance of a single product. Low-resolution GC-MS confirmed the anticipated molecular weight of 182 , which corresponds to introduction of one methyl group. Exclusive methylation of the indole nitrogen was confirmed by ${ }^{13} \mathrm{C}$ NMR (Figure 5-5). Owing to the water solubility of the 
product, the isolation procedure of reference 3 could not be used. Instead, volatiles were removed from the cooled reaction product with a rotary evaporator. The resulting solid was dried under nitrogen at $55^{\circ} \mathrm{C}$. GC analysis after $2-3$ days showed no traces of residual 1,4-diazabicyclo[2.2.2]octane in the product.

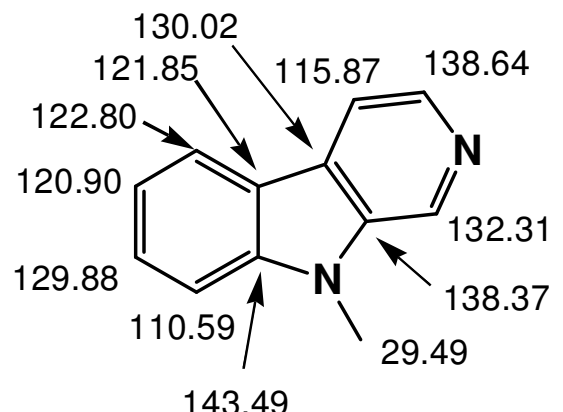

Figure $5-5 .{ }^{13} \mathrm{C}$ NMR chemical shifts of $\mathrm{N}$-methyl- $\beta$-carboline.

Catalyst reduction. A $100 \mathrm{~mL}$ Parr stainless steel reactor equipped with an internal cooling coil, a dip tube with a $0.5 \mu \mathrm{m}$ filter, and constant pressure gas delivery was charged with the desired quantity of $4 \%$ Rh/lithium aluminate (typically, $0.025-0.5 \mathrm{~g}$, depending on the amount of substrate to be hydrogenated and the desired catalyst loading) and $45 \mathrm{~mL}$ anhydrous THF. The reactor was closed, purged with nitrogen and hydrogen, and pressurized to ca. 500 psig with hydrogen. The mixture was heated to $190^{\circ} \mathrm{C}$ with stirring and pressurized to $800 \mathrm{psig}$. After 2 hours, the mixture was cooled to $50^{\circ} \mathrm{C}$, and the THF was removed under stirring via the internal filter. If the reduced catalyst was to be washed prior to hydrogenation of a substrate (either $\mathrm{N}$-methylcarboline or tetrahydrocarboline), hydrogen pressure was vented to ca. $20 \mathrm{psig}$ after removing the initial charge of THF. Fresh THF, $55 \mathrm{~mL}$, was introduced with a piston pump through the dip tube, and the mixture was stirred for $10-15$ minutes. After pressurizing to ca. 400 psig with hydrogen, THF was removed with stirring via the dip tube. To ensure complete removal of water (coproduct of catalyst reduction), this washing process was repeated at least three times. 
Hydrogenation of N-ethylcarboline (NEC) or tetrahydrocarboline (THC). In all cases, the catalyst was reduced prior to hydrogenation of the substrate. If desired (see discussion), the reduced catalyst was washed with fresh THF prior to hydrogenation of THC. A solution of the substrate in $65 \mathrm{~mL}$ anhydrous THF was prepared immediately prior to use. The concentration was chosen so that $50 \mathrm{~mL}$ of the solution contained the desired quantity of NEC or THC. The remainder of the solution was used to prime the piston pump and to maintain a residual amount in the pump reservoir (to prevent adventitious introduction of air into the hydrogenation). The solution (50 mL) was pumped into the reactor with moderate stirring (400 rpm). Subsequently, the reactor was purged twice with hydrogen, and then pressurized to 750 psig with hydrogen. The mixture was heated to $160^{\circ} \mathrm{C}$ with stirring (1000 rpm) and pressurized to $1250 \mathrm{psig}$. The reaction was maintained under these conditions for the desired time, 8 - $48 \mathrm{hrs}$. Progress of the reaction was monitored by GC analysis of samples withdrawn through the dip tube. Subsequent cooling to ambient, filtration through the dip tube and removal of solvent under vacuum provided the hydrogenated product.

Hydrogenation of N-methylcarboline (NMC). The procedure for THC was used, with the additional step of filtering the NMC solution through a $0.45 \mu \mathrm{m}$ filter prior to introduction into the reactor.

\section{$\underline{\text { References }}$}

1. See discussion of calculations, Chapter 1.

2. Cao, R.; Yu, F.; Wang, Z.; Peng, W. European Patent No. 1,634,881 A1, 2006, Example 49 (Xinjiang Huashidan Pharmaceutical Research Co.); Cao, R.; Chen, Q.; Hou, X.; Chen, H.; Guan, H.; Ma, Y.; Peng, W.; Xu, A. Bioorganic and Medicinal Chemistry, 2004, 12, 4613.

3. Shieh, W.-C.; Dell, S.; Bach, A.; Repic, O.; Blacklock, T.J. J. Org. Chem., 2003, 68, 1954.

4. Cox, E.D.; Cook, J.M. Chem. Rev., 1995, 95, 1797.

5. Cattanach, C.J.; Cohen, A.; Heath-Brown, B. J. Chem. Soc., C, 1968, 1235.

6. Spickett, R.G.W. J. Med. Chem., 1966, 9, 436.

7. Eftink, M.R.; Jia, J.; Hu, D.; Ghiron, C.A. J. Phys. Chem., 1995, 99, 5713. 


\section{Chapter 6: Hydrogen Carriers Incorporating Aromatic Ring Systems with 6-membered Rings}

\section{Introduction}

Among the range of aromatic molecules that are amenable to reversible catalytic hydrogenation for hydrogen storage, the molecules with nitrogen atoms that contain fused and/or linked six-membered rings are a promising subset. The principal potential advantages include the ability to add one hydrogen atom to every carbon and nitrogen atom in the molecule, yielding a theoretical hydrogen storage capacity of ca. 7.2 wt. \% hydrogen for every candidate in this class of carriers. This is superior to the classes of molecules containing 5-membered rings (i.e., indoles, carbazoles) where not all of the atoms in the ring structure can be saturated with hydrogen and the maximum theoretical capacity is less than $7 \mathrm{wt}$. \%. Many of the most promising candidates contain fused and/or linked six-membered rings, are commercially available, and have moderate melting points and low volatility.

While the reversible hydrogenation of nitrogen-containing aromatics (e.g., pyridine) has been disclosed in the chemical literature, there are fewer corresponding examples than found for conventional aromatics such as benzene and naphthalene. The product of nitrogen-containing aromatic hydrogenation is a secondary amine (e.g., piperidine). Potential complications exist for secondary amine products vs. saturated aromatics, including oxidation of the amines by contact with air and corrosion of metals.

In this chapter we report the results of our computational and experimental studies on several categories of molecules, including carriers with fully fused ring systems (naphthyridines, phenanthrolenes), 6-membered rings linked by single carbon-carbon bonds (dipyridyls, terpyridines), and molecules containing more than one nitrogen atom in a single ring (bipyrimidine, quinazoline). 


\section{Results and Discussion}

\section{A. Phenanthrolines}

Phenanthrolines are molecules which are formed from the ring fusion of three six-membered rings containing a total of two nitrogen atoms. Several representative phenanthroline isomers are shown in Figure 6-1.

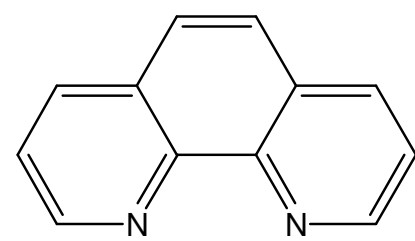

1,10-phenanthroline

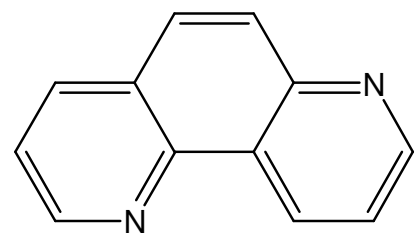

1,7-phenanthroline

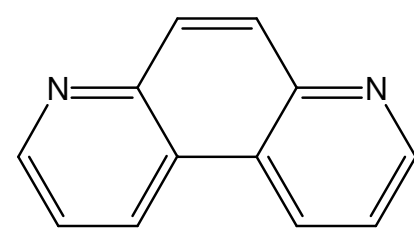

4,7-phenanthroline

Figure 6-1. The structures of three phenanthroline isomers.

These liquid carrier candidates were studied because they generally have reasonably good average and stepwise thermodynamics (e.g., 4,7-phenanthroline), low vapor pressures, and, in some cases, commercial availability.

\section{4,7-phenanthroline}

The molecule 4,7-phenanthroline was chosen for experimental study because our initial calculations of the overall average energy change upon hydrogenation $(\Delta \mathrm{E})$ showed promising thermodynamics for dehydrogenation. As discussed in Chapter 1, the calculations of energy changes due to hydrogenation of candidate liquid carrier molecules can eliminate candidates that do not have the appropriate overall thermodynamics for facile reversible hydrogenation. However, we have found that it is important to calculate the energies of transformation through likely intermediates rather than rely solely on the overall energy of hydrogenation. For some carrier candidates, dehydrogenation pathways can include intermediate steps with unfavorable energies 
(e.g., perhydro-4,7-phenanthroline). The overall calculated $\Delta \mathrm{E}$ of $-64 \mathrm{~kJ} / \mathrm{mol} \mathrm{H}_{2}$ for hydrogenation is favorable for low-temperature dehydrogenation (est. $\Delta \mathrm{H}$ of $-49 \mathrm{~kJ} / \mathrm{mol}$ $\mathrm{H}_{2}$ ). However, one potential pathway proceeds through intermediate steps with very different energies, while another potential pathway has more uniform energies. The pathways shown in Scheme 6-1 assume dehydrogenation pathways that liberate hydrogen from one aromatic ring per step. One pathway avoids a very high reaction energy until the final dehydrogenation step (Scheme 6-1, lower path). The most promising pathway for the dehydrogenation of perhydro-4,7-phenanthroline has three steps, with the highest energy step showing an average $\Delta \mathrm{E}$ of $81 \mathrm{kcal} / \mathrm{mol} \mathrm{H}_{2}$ for conversion of an octahydro intermediate to a dihydro intermediate (Scheme 6-1, upper path).

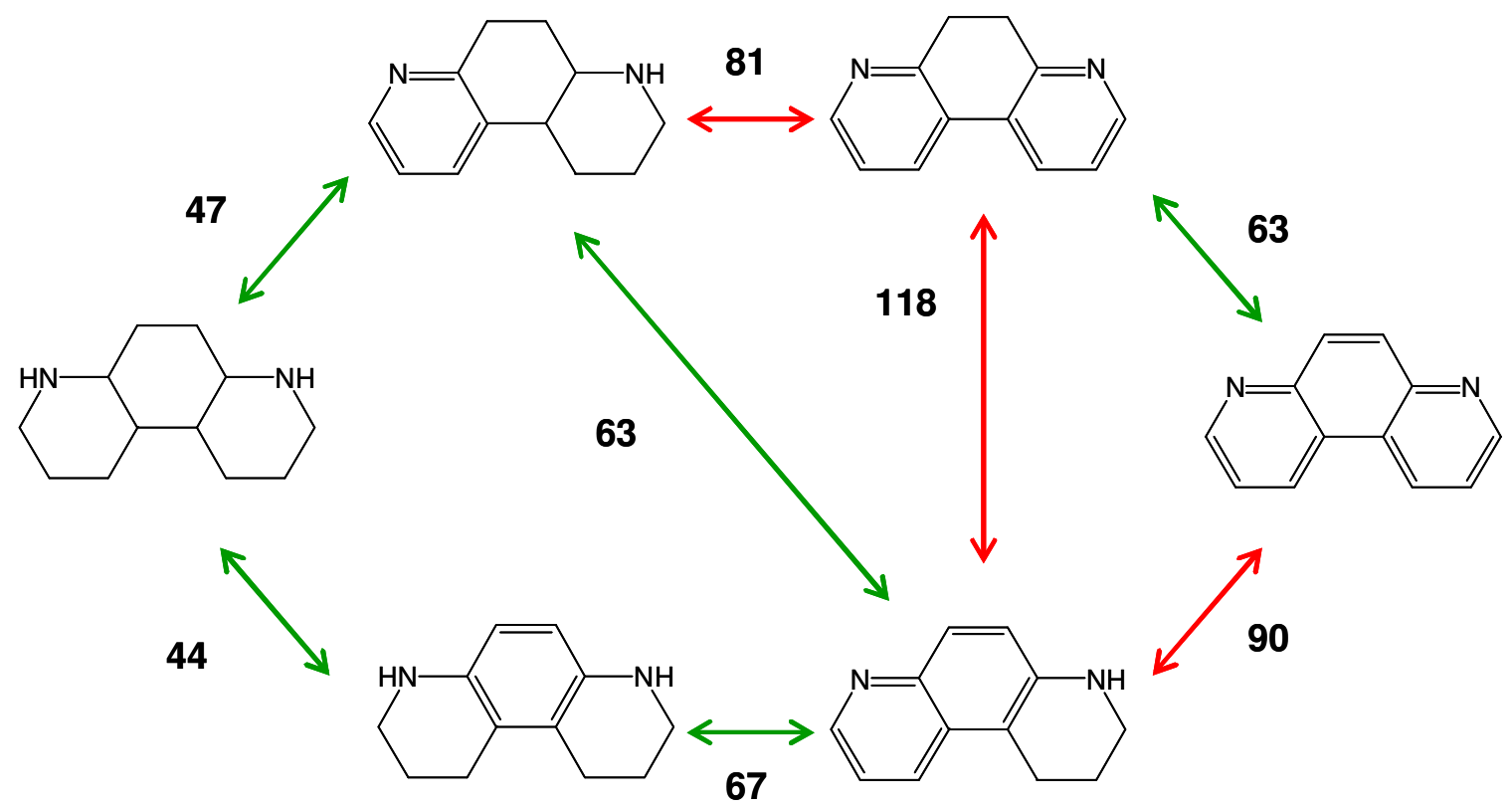

Scheme 6-1. Possible dehydrogenation pathways for perhydro-4,7-phenanthroline.

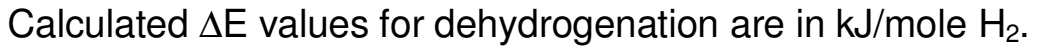

Our initial attempts to hydrogenate commercially available 4,7-phenanthroline resulted in low conversion to perhydro-4,7-phenanthroline. In the course of adjusting the catalytic reaction conditions to improve the conversion, we found that pre-activation of the catalyst was necessary for high conversion to perhydro-4,7-phenanthroline. 
Pre-activation of the catalyst involved heating a slurry of the catalyst in isopropanol under a high pressure of hydrogen. The catalyst was then handled only under an inert atmosphere and washed extensively with degassed THF to remove residual isopropanol (residual isopropanol can result in alkylation of the nitrogen atoms in the hydrogenated products). Perhydro-4,7-phenanthrolene was obtained in good yield and high purity by hydrogenation in $\mathrm{THF}$ solvent with pre-activated $5 \% \mathrm{Rh} / \mathrm{LiAl} \mathrm{O}_{8}$ at $160^{\circ} \mathrm{C}$ and $800 \mathrm{psig}$ hydrogen pressure.

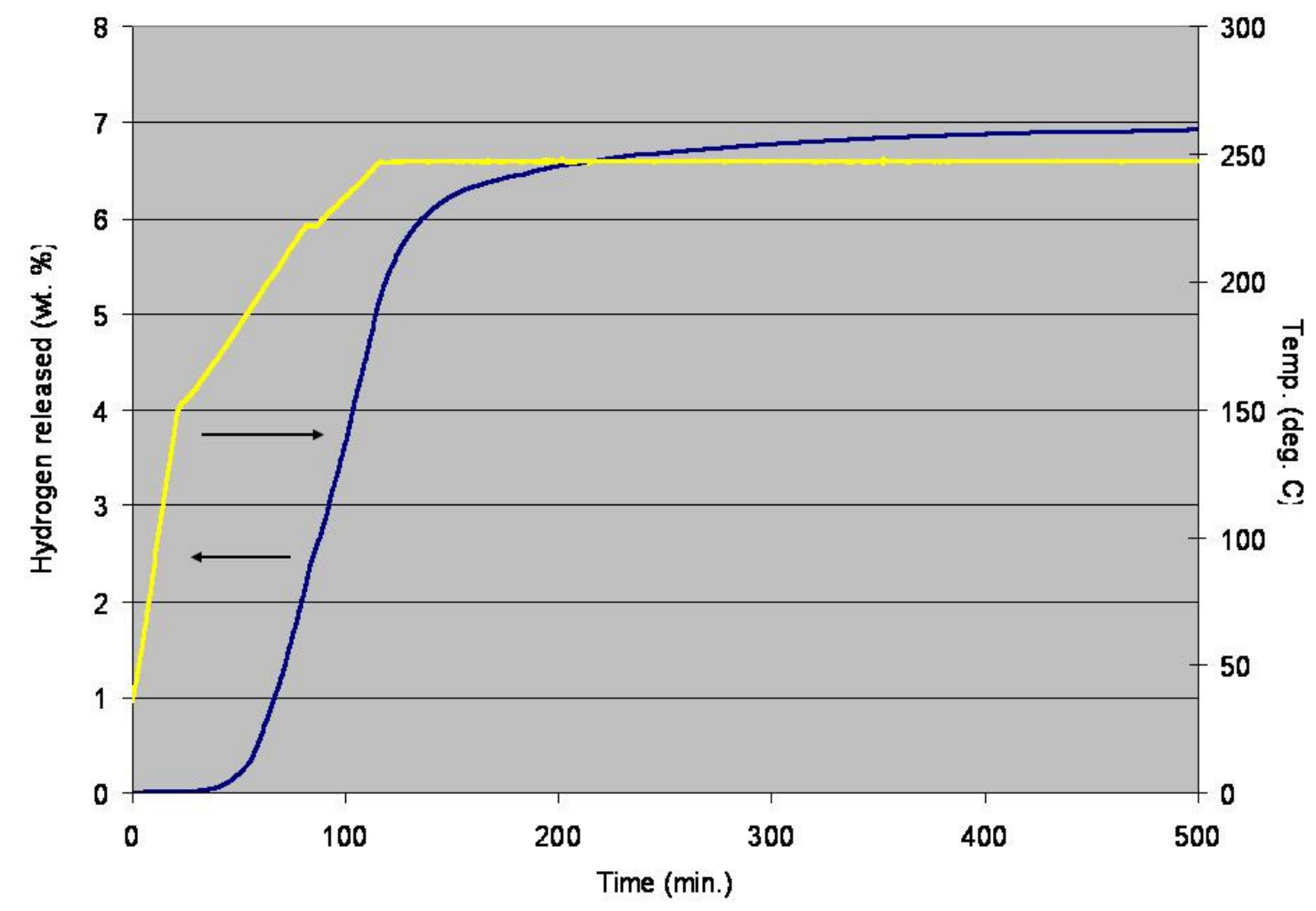

Figure 6-2. Dehydrogenation of perhydro-4,7-phenanthroline with $\mathrm{Pd} / \mathrm{C}$ catalyst.

Samples of perhydro-4,7-phenanthroline (MW 194) were dehydrogenated at $250^{\circ} \mathrm{C}$ under one atmosphere hydrogen using $5 \% \mathrm{Pd} / \mathrm{C}$ and $5 \% \mathrm{Pd} / \mathrm{LiAl}{ }_{5} \mathrm{O}_{8}$ catalysts. After 12 hours at $250^{\circ} \mathrm{C}$, the sample which was dehydrogenated using a $\mathrm{Pd} / \mathrm{C}$ catalyst demonstrated a reversible hydrogen storage capacity of $7.0 \mathrm{wt}$ \% (Figure 6-2, 
Table 6-1). The sample containing the lithium aluminate-supported palladium showed less activity (which was in agreement with preliminary catalyst screening studies on other isomers such as 1,10-phenanthroline).

Table 6-1. GC-MS analysis of products from dehydrogenation of perhydro-4,7-phenanthroline at $250^{\circ} \mathrm{C}$.

\begin{tabular}{|c|c|c|c|c|c|}
\hline Catalyst & $\begin{array}{c}\text { MW 180 } \\
(\%)\end{array}$ & $\begin{array}{c}\text { MW 182 } \\
(\%)\end{array}$ & $\begin{array}{c}\text { MW 184 } \\
(\%)\end{array}$ & $\begin{array}{c}\text { MW 188 } \\
(\%)\end{array}$ & $\begin{array}{c}\text { MW 194 } \\
(\%)\end{array}$ \\
\hline $5 \% \mathrm{Pd} / \mathrm{C}$ & 83 & 8 & 9 & 0 & 0 \\
\hline $5 \% \mathrm{Pd} / \mathrm{LiAl} \mathrm{O}_{8}$ & 43 & 10 & 36 & 5 & 5 \\
\hline
\end{tabular}

Proposed structures for the products detected by GC-MS in mixtures of dehydrogenated 4,7-phenanthroline are shown in Scheme 6-2.

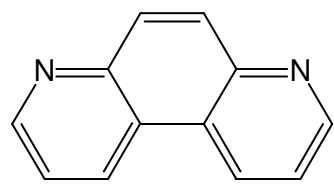

MW 180

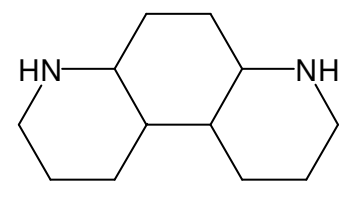

MW 194
$\Delta$
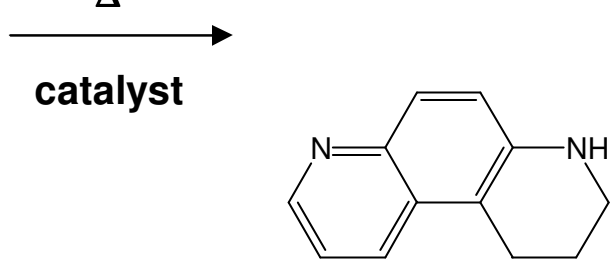

MW 184

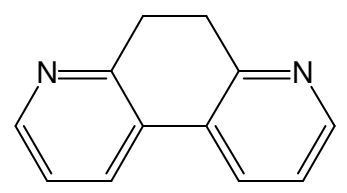

MW 182

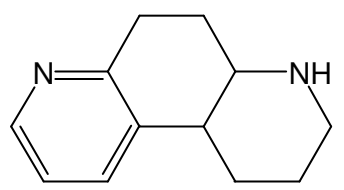

MW 188

Scheme 6-2. Products detected by GC-MS in mixtures of dehydrogenated 4,7-phenanthroline at $225^{\circ} \mathrm{C}$ and $250^{\circ} \mathrm{C}$. 
In a second set of experiments, perhydro-4,7-phenanthroline was dehydrogenated with several different catalysts at $225^{\circ} \mathrm{C}$ (Figure 6-3, Table 6-2); however, at temperatures below $225^{\circ} \mathrm{C}$, the rate of hydrogen evolution is slow. A slurry of perhydro-4,7phenanthroline and 8.75 wt.\% of $5 \% \mathrm{Pd} / \mathrm{C}$ showed a working hydrogen capacity at $225^{\circ} \mathrm{C}$ of $6.7 \mathrm{wt} \%$. Once hydrogen evolution effectively ceased in this experiment, an additional $150 \mathrm{mg}$ of the $5 \% \mathrm{Pd} / \mathrm{C}$ catalyst was added to the reaction mixture, but no additional hydrogen was evolved, indicating that catalyst deactivation is not a factor in the decreased working capacity at $225^{\circ} \mathrm{C}$. It appears that thermodynamic limitations are responsible for the lower-than-theoretical yield of hydrogen. When the catalyst loading was increased to 11.25 wt. \%, the observed working capacity was slightly less than observed when using lower catalyst loadings. This could be the result of decreased stirring efficiency due to the increased viscosity of the slurry.

Scheme 6-1 shows several dehydrogenation reaction pathways that fully hydrogenated 4,7-phenanthroline, and related intermediates could follow during dehydrogenation. The calculations reveal that a large thermodynamic barrier $\left(\Delta \mathrm{E}\right.$ of $\left.90 \mathrm{~kJ} / \mathrm{mol} \mathrm{H}_{2}\right)$ exists for one of the tetrahydro-4,7-phenanthroline intermediates to dehydrogenate to the fully unsaturated 4,7-phenanthroline. Scheme 6-1 also shows that the dehydrogenation of this tetrahydro-4,7-phenanthroline intermediate to dihydro-4,7-phenanthroline is highly unfavorable. There may be other catalytic reactions, such as isomerizations, which are thermodynamically more favored. Furthermore, since the dehydrogenation experiments are conducted under 1 atmosphere hydrogen pressure, the potential for some equilibrium between intermediates exists, and even if the equilibrium constant favors the dehydrogenated state, a small population of one intermediate may feed an alternate pathway and allow for higher dehydrogenation conversion.

Table 6-2. GC-MS analysis of products from dehydrogenation of perhydro-4,7phenanthroline using $5 \%$ palladium on carbon at $225^{\circ} \mathrm{C}$.

\begin{tabular}{|c|c|c|c|}
\hline Catalyst & MW 180 (\%) & MW 182 (\%) & MW 184 (\%) \\
\hline PD/C & 46 & 9 & 45 \\
\hline
\end{tabular}


In an experiment designed to gain some insight on influencing the dehydrogenation pathway followed by perhydro-4,7-phenanthroline and improving the hydrogen yield, 2.5 wt. \% Pt-Ir supported on alumina was added to the dehydrogenation reaction, along with $8.75 \mathrm{wt}$ \% $\mathrm{Pd} / \mathrm{C}$. The Pt-lr catalyst was chosen to serve as a possible isomerization and/or hydrogenation catalyst. The results of this experiment, provided in Figure 6-3, show that no enhancement was gained by the addition of a second isomerizationhydrogenation catalyst to the existing dehydrogenation catalyst.

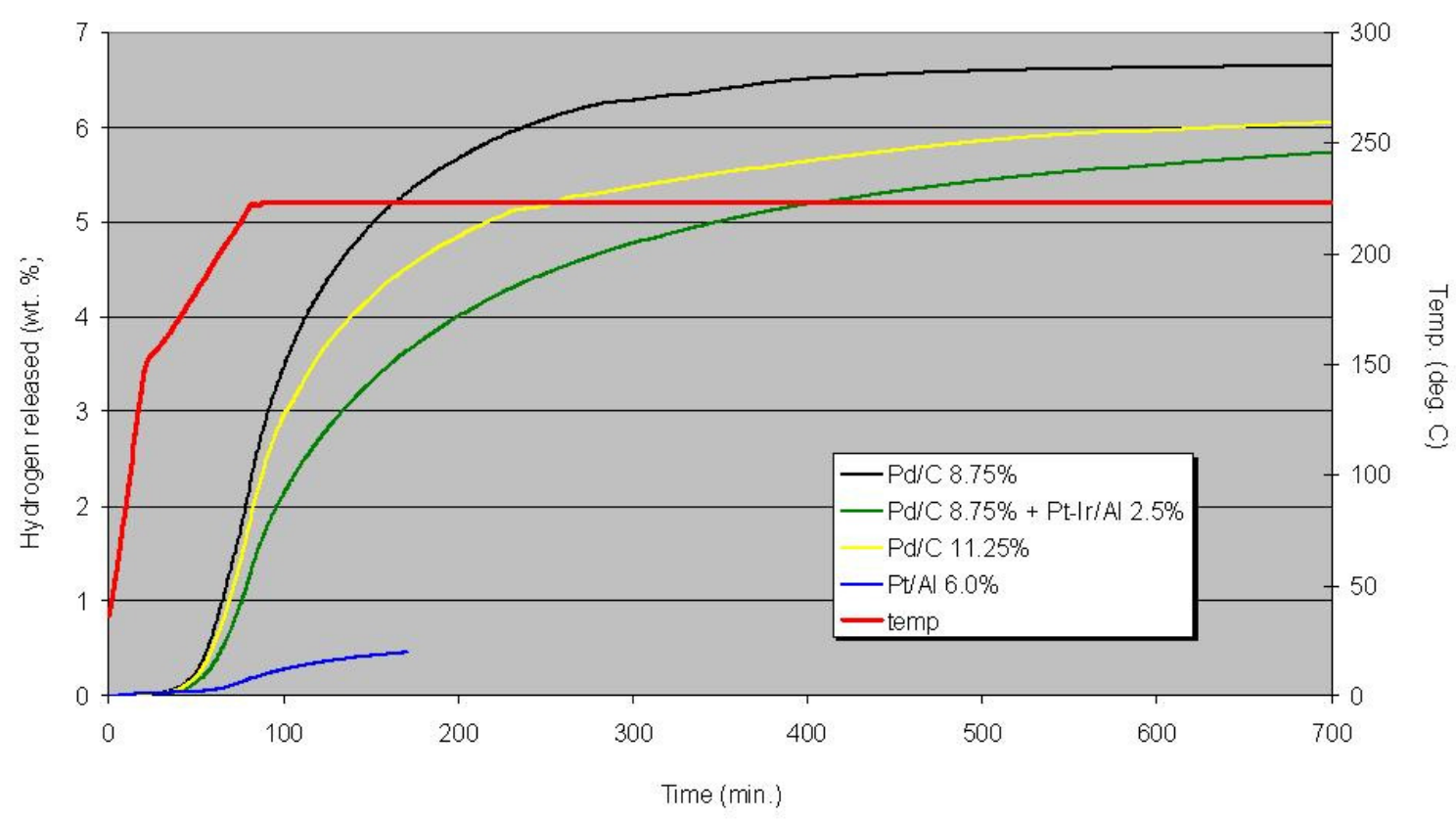

Figure 6-3. Dehydrogenation of perhydro-4,7-phenanthroline at $225^{\circ} \mathrm{C}$.

In our experience with indole/carbazole-related liquid carrier candidates (Chapters 3 and 4), the activity of pure platinum catalysts and platinum-based catalysts is generally superior to catalysts containing palladium. However, we found that palladium-based catalysts are more effective than platinum for the dehydrogenation of perhydro-4,7phenanthroline. This finding shows that general trends in dehydrogenation catalyst activity cannot necessary be expected for classes of liquid carrier prototypes with significantly different molecular structures. Thus, as a general rule, any newly discovered liquid carrier prototypes which demonstrate promising performance in 
preliminary testing must be tested with a broad range of dehydrogenation catalysts before their overall utility can be determined with a high degree of confidence.

\section{1,10-phenanthroline}

Another isomer of phenanthroline that is commercially available is 1,10-phenanthroline. Our initial hydrogenation experiments utilized the same conditions as used for the 4,7phenanthroline isomer (preactivated $5 \% \mathrm{Rh} / \mathrm{LiAl}_{5} \mathrm{O}_{8}, 160^{\circ} \mathrm{C}, 800$ psig hydrogen) and resulted in a quantitative yield and very high selectivity to a hydrogenated product. Unfortunately, this product was not the desired perhydrogenated compound, but octahydro-1,10-phenanthroline. The proposed structure of the octahydro-1,10phenanthroline isomer shown in Scheme 6-3 was assigned using IR and NMR data. The calculated stepwise thermodynamics of hydrogenation (Scheme 6-4) shows several potential pathways that should allow for complete hydrogenation. There are no steps within these pathways with $\Delta \mathrm{E}$ values that would be too low for high equilibrium conversion under the conditions employed for hydrogenation. Therefore, we speculate that the termination of hydrogenation at the stage of an octahydro intermediate is the result of catalyst inhibition by the octahydro product, possibly due to strong coordination of the chelating secondary amine groups on the octahydro-1,10-phenanthroline. The molecular structure of 1,10-phenanthroline has the two nitrogen atoms substituted in ideal positions on the rings to chelate metal atoms (1,10-phenanthroline is used frequently as a strong-binding, chelating ligand in inorganic coordination complexes). Additional efforts directed at obtaining high yields of perhydro-1,10-phenanthroline were unsuccessful.
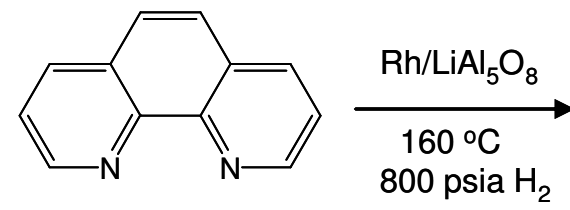

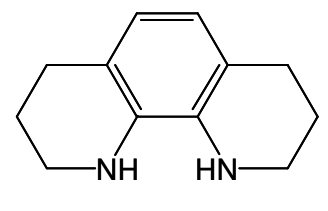

$100 \%$

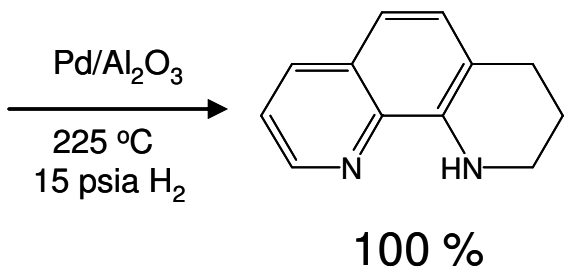

$100 \%$

Scheme 6-3. Hydrogenation/dehydrogenation of 1,10-phenanthroline. 
The stepwise energies shown in Scheme 6-4 suggest that the octahydro-1,10phenanthroline intermediate with unsaturation present in the middle ring should liberate two moles of hydrogen at a moderate temperature (avg. $\Delta \mathrm{E}$ of $63 \mathrm{~kJ} / \mathrm{mol}$ ), but not proceed to the fully unsaturated 1,10-phenanthroline (avg. $\Delta \mathrm{E}$ of $92 \mathrm{~kJ} / \mathrm{mol}$ ). Unlike 4,7phenanthroline, there does not appear to be a feasible pathway for generating an alternate isomer of octahydro-1,10-phenanthroline from the tetrahydro-1,10phenanthroline isomer, where the outer ring is saturated (Scheme 6-4, upper right), as the $\Delta \mathrm{E}$ for this transformation is too low for high equilibrium conversion (avg. $\Delta \mathrm{E}$ of 46 $\mathrm{kJ} / \mathrm{mol})$.

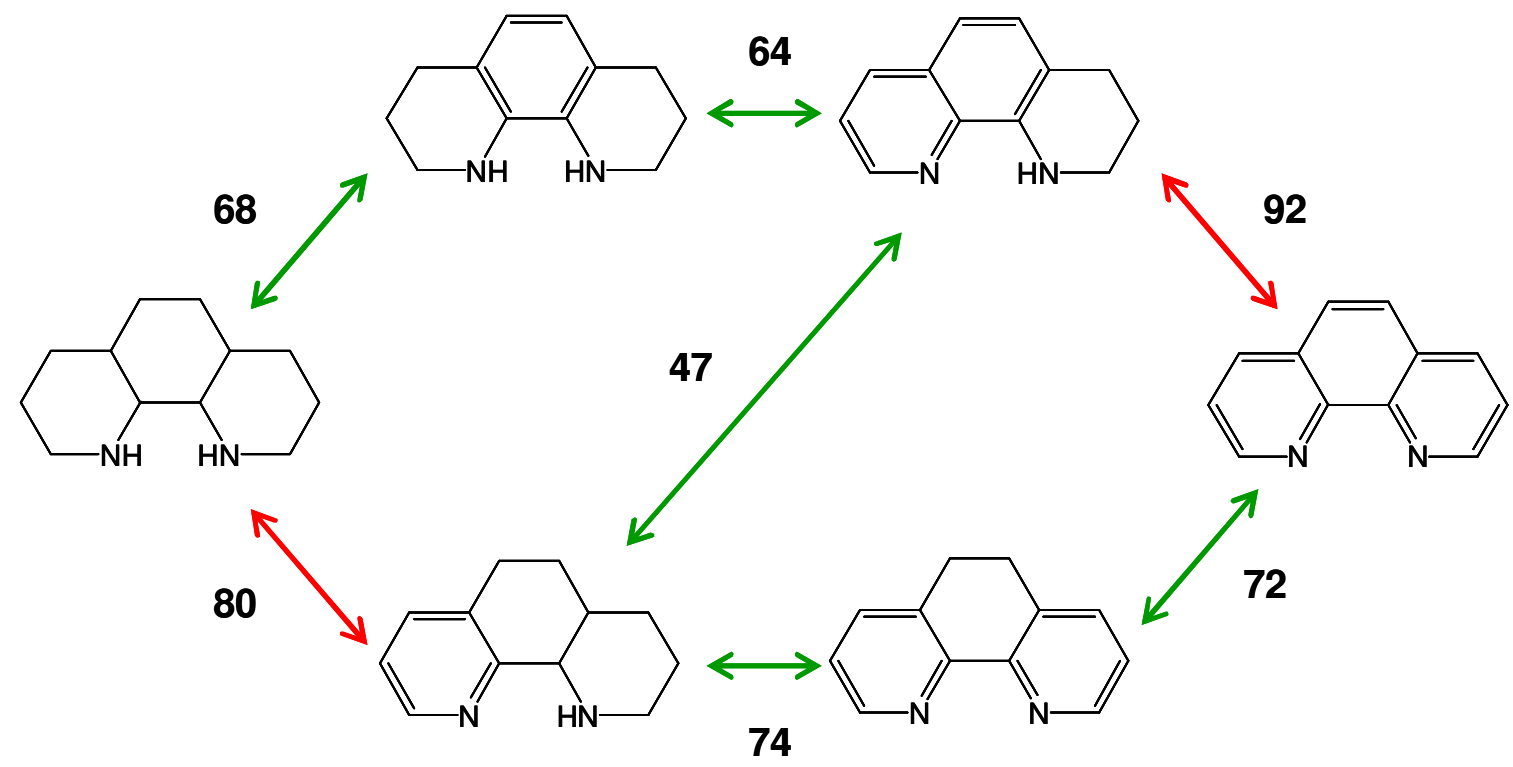

Scheme 6-4. Dehydrogenation pathways for perhydro-1,10-phenanthroline. Calculated $\Delta \mathrm{E}$ values for dehydrogenation are in $\mathrm{kJ} /$ mole $\mathrm{H}_{2}$.

Dehydrogenation of octahydro-1,10-phenanthroline with palladium on alumina did yield a tetrahydro-1,10-phenanthroline (Scheme 6-3). In agreement with our expectations based upon the calculated energies in Scheme 6-4, no additional dehydrogenation to 1,10-phenanthroline was apparent at $225^{\circ} \mathrm{C}$. In a similar trend observed for 
4,7-phenanthroline, palladium was far more effective than platinum for the dehydrogenation of octahydro-1,10-phenanthroline (Figure 6-4).

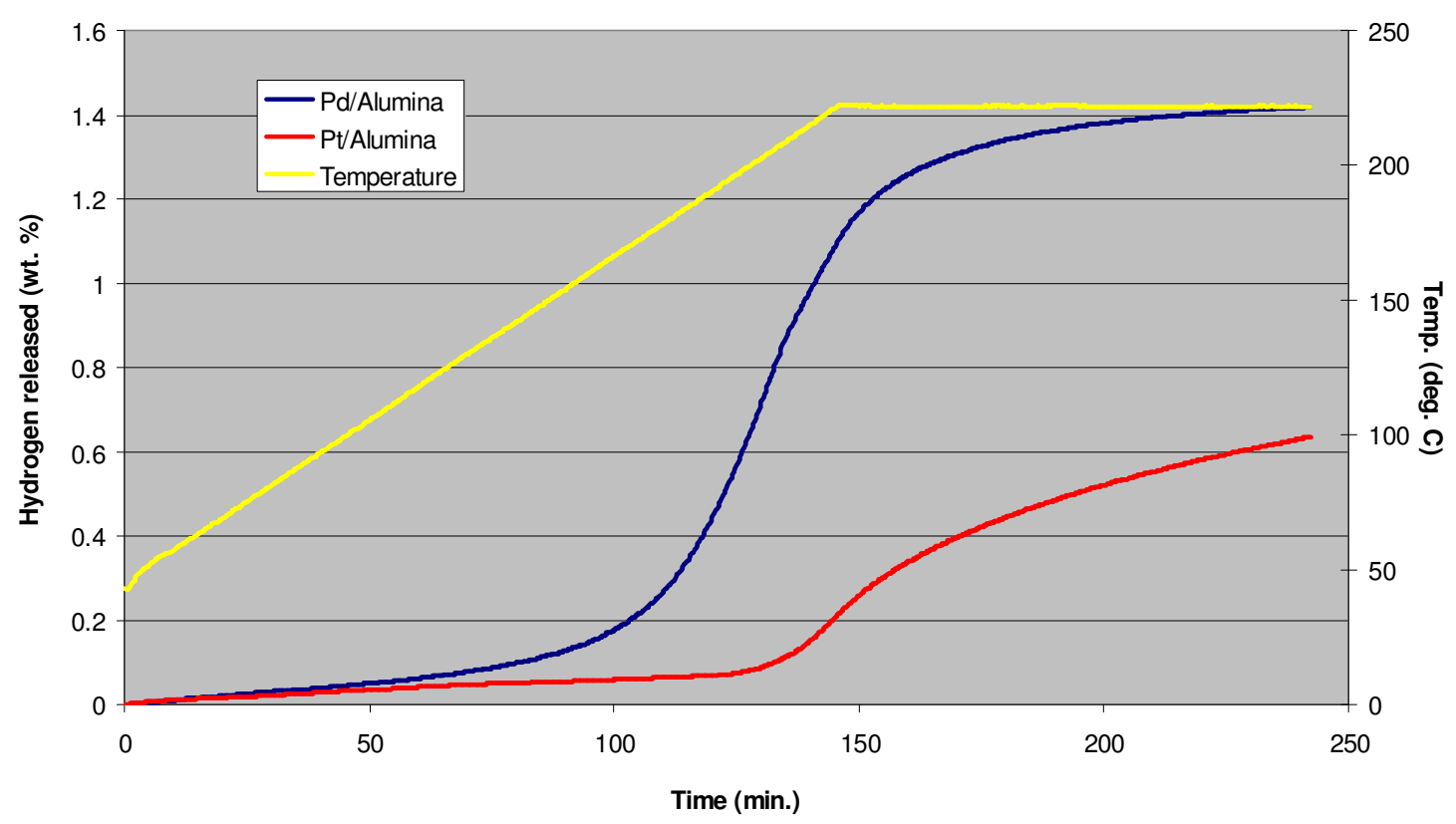

Figure 6-4. Dehydrogenation of octahydro-1,10-phenanthroline.

\section{1,7-phenanthroline}

The liquid carrier prototype 1,7-phenanthroline is commercially available in limited quantities. We employed the same conditions used for the hydrogenation of 4,7-phenanthroline (pre-activated $5 \% \mathrm{Rh} / \mathrm{LiAl}_{5} \mathrm{O}_{8}, 160^{\circ} \mathrm{C}, 800$ psig hydrogen), and this resulted in a yield and very high selectivity to perhydro-1,7-phenanthroline. The use of moderate temperatures during the hydrogenation reaction is important because the same hydrogenation catalyst at $225^{\circ} \mathrm{C}$ resulted in complete conversion of 1,7-phenanthroline to the tricyclic compound decahydropyrido[3,2,1-ij]quinoline, a molecule containing only one nitrogen atom (Scheme 6-5). This structural rearrangement and loss of nitrogen obviously involves hydrogenolysis and reformation of C-N bonds. 


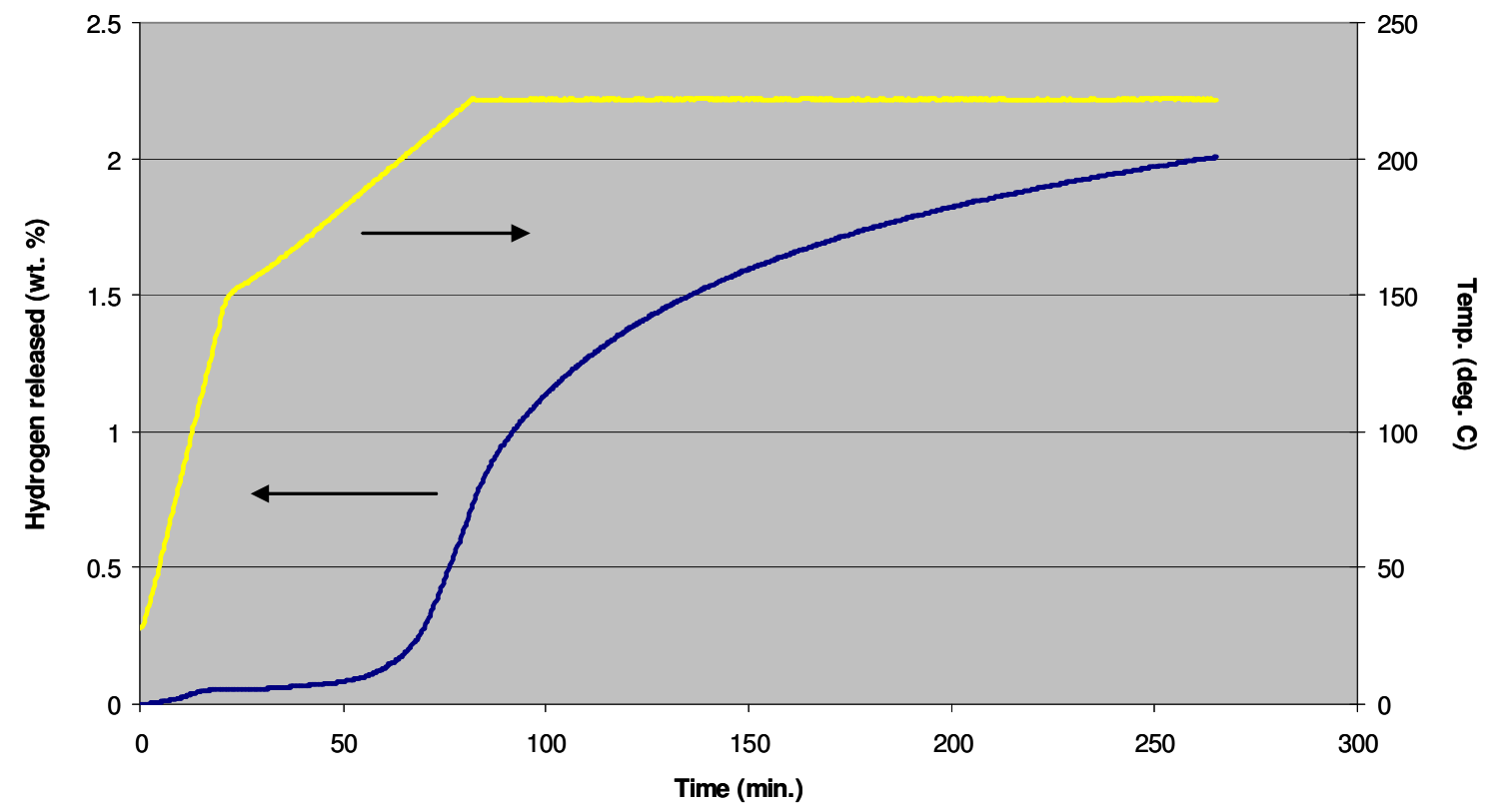

Figure 6-5. Dehydrogenation of perhydro-1,7-phenanthroline.

The dehydrogenation testing of perhydro-1,7-phenanthroline was performed using palladium on carbon and temperature ramping to $225^{\circ} \mathrm{C}$ under $1 \mathrm{~atm}$. hydrogen (Figure 6-5). Only a fraction of the theoretical hydrogen capacity of perhydro-1,7phenanthroline was realized after 3 hours of reaction at $225^{\circ} \mathrm{C}$. GC-MS analysis of the dehydrogenated products showed two unexpected peaks with MW 173 and MW 179, one and seven mass units lower than fully dehydrogenated 1,7-phenanthroline. The MW 173 material was separated from the crude product mixture by column chromatography and analyzed by ${ }^{1} \mathrm{H}$ and ${ }^{13} \mathrm{C}$ NMR. From these data, it was determined that the products formed with masses 173 and 179 are the highly condensed, cyclic structures shown in Scheme 6-5. The fully saturated compound with a MW of 179 is the same product that is obtained from the hydrogenation experiment conducted at $225^{\circ} \mathrm{C}$. 


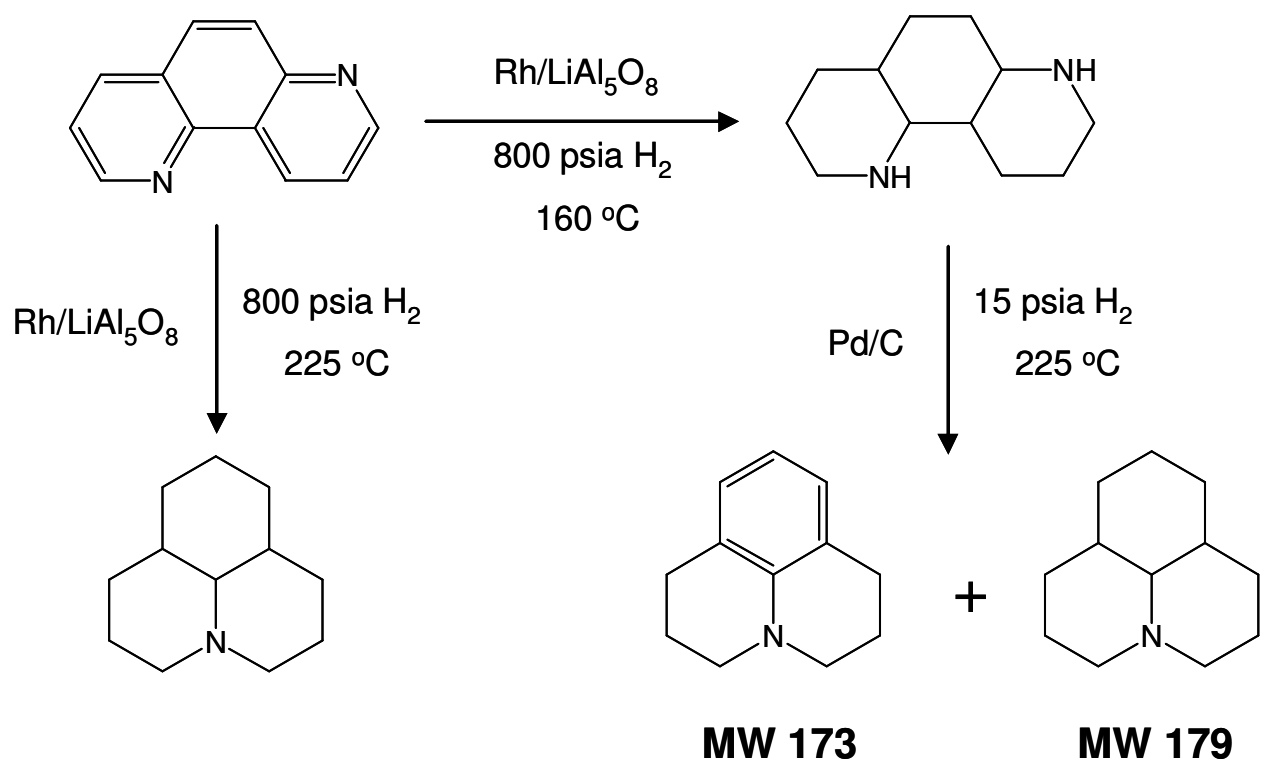

Scheme 6-5. Hydrogenation/dehydrogenation of 1,7-phenanthroline.

The dehydrogenation reaction yielded a product mixture that was evenly distributed between the MW 179 and MW 173 products at a conversion of 90\%. The very poor selectivity of the perhydro-1,7-phenanthroline dehydrogenation might be explained in part by the molecular structure. The arrangement of nitrogen atoms in 1,7-phenanthroline allows for easy cyclization once the loss of ammonia occurs, since a six-membered ring is formed. Since the remaining saturated ring system in decahydropyrido[3,2,1-ij]quinoline cannot become fully aromatic upon dehydrogenation due to the tertiary nitrogen atom, dehydrogenation beyond mass 173 would not be expected at these temperatures.

\section{Other phenanthrolines}

Having evaluated the commercially available 4,7-, 1,10-, and 1,7-phenanthroline liquid carrier prototypes, we attempted to investigate other isomers that were available through published organic synthesis procedures. Through a two-step reaction sequence, a set of 2,7-, 2,8-, and 2,9-phenanthrolines is accessible. The first step involves the 
preparation of bis-pyridyl ethylene precursors through catalytic coupling of bromopyridines with vinyl-substituted pyridines, as illustrated in Scheme 6-6.<smiles>C=Cc1ccncc1</smiles>

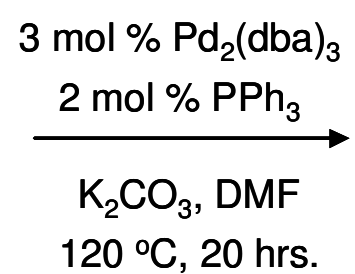<smiles>C(=C/c1cccnc1)\c1ccncc1</smiles>
$87 \%$

Scheme 6-6. Synthesis of bis-pyridyl ethylene by palladium-catalyzed coupling.

Synthesis of substantial quantities of the bis-pyridyl ethylene precursors was afforded by the high-yield, palladium-catalyzed coupling reactions. However, the cyclization of these precursors by UV irradiation using a $450 \mathrm{~W}$ medium discharge mercury lamp had to be conducted under conditions of high dilution to prevent intermolecular coupling (Scheme 6-7). This resulted in severe limitations on the amount of product that could be generated in a single reaction.
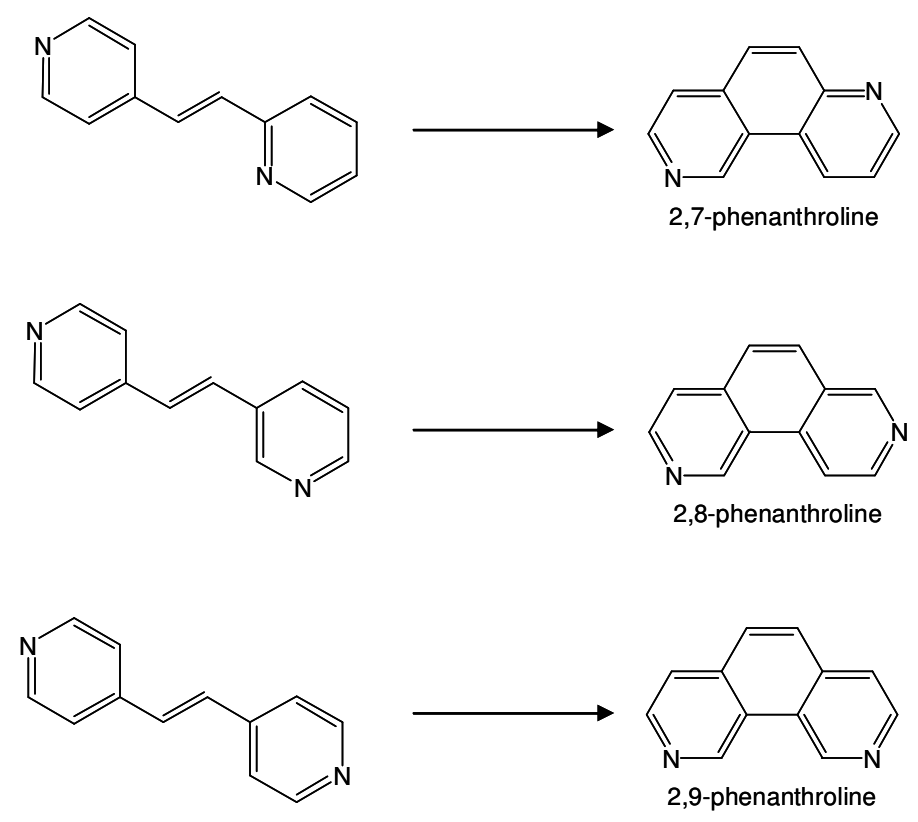

Scheme 6-7. Cyclization of bis-pyridyl ethylene precursors. 
Despite the very small amounts of material available for testing, preliminary hydrogenation experiments were conducted to assess the relative selectivity of the 2,7-, 2,8-, and 2,9-phenanthrolines. Under hydrogenation conditions similar to those used for 1,10-phenanthroline, the hydrogenation of 2,7-phenanthroline yielded a similar octahydro- product (Scheme 6-8, top). However, unlike octahydro-1,10-phenanthroline, the secondary amine groups in octahydro-2,7-phenanthroline are not positioned in the molecule in a manner that should lead to strong chelation of a metal catalyst. Other factors that may influence the selectivity, such as steric crowding of the middle aromatic ring, may be responsible for the failure to fully hydrogenate 2,7-phenanthroline. The hydrogenation of 2,8-phenanthroline was conducted using a rhodium catalyst and conditions similar to those used for 2,7-phenanthroline (Scheme 6-8, middle). The hydrogenation reaction resulted in a mixture of tetrahydro- and octahydro-2,8phenanthroline products.
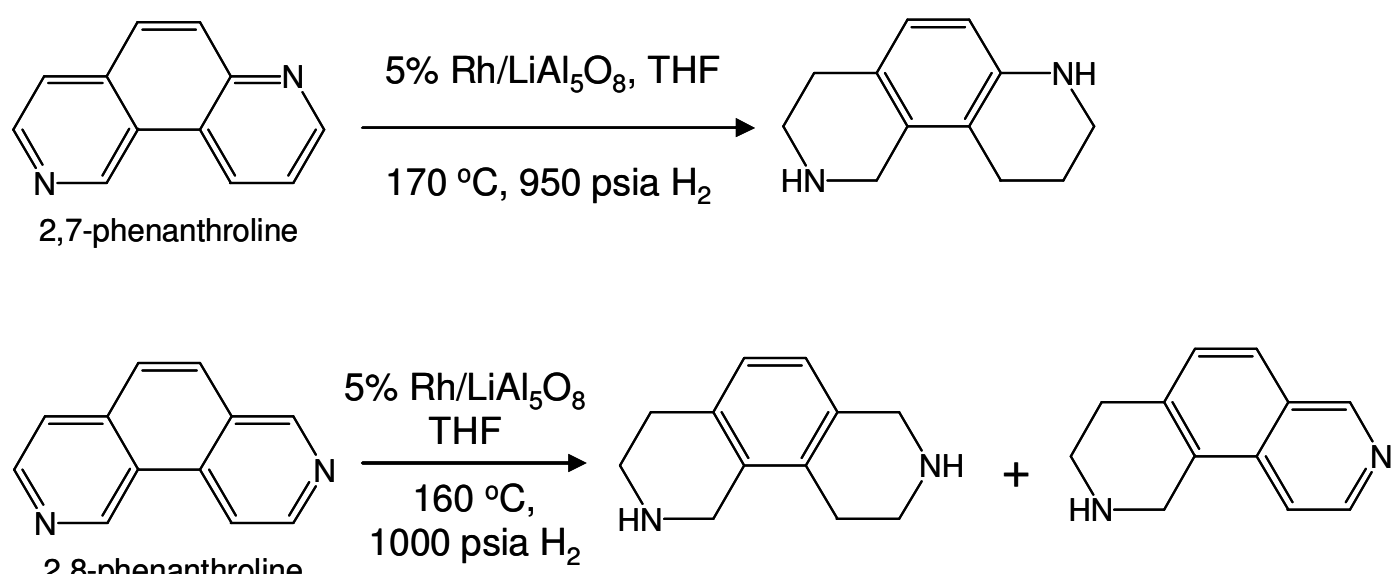

2,8-phenanthroline

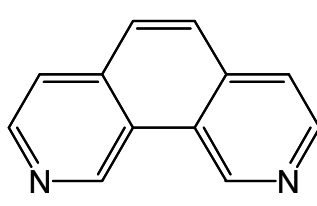

2,9-phenanthroline

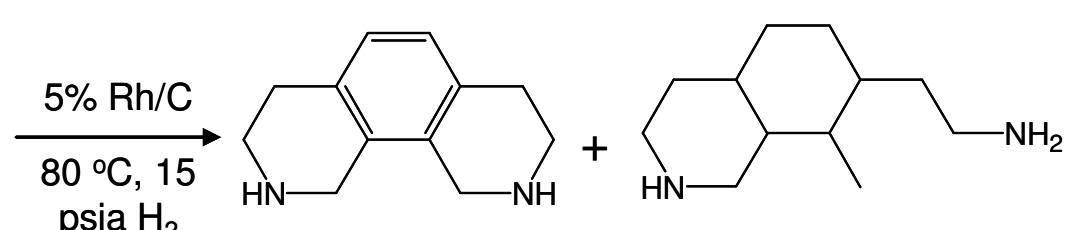
$\mathrm{HCl}$ salt

Scheme 6-8. Hydrogenation reactions of 2,7-, 2,8-, and 2,9-phenanthroline. 
Unlike the 2,7- and 2,8- isomers, 2,9-phenanthroline could not be hydrogenated with any appreciable conversion using supported rhodium hydrogenation catalysts. Therefore, we investigated alternate hydrogenation strategies in an effort to produce perhydro-2,9phenanthroline. One generally useful strategy for nitrogen-substituted aromatics is the hydrogenation of a protonated derivative of the aromatic. Therefore, we prepared the $\mathrm{HCl}$ (hydrochloric acid) salt of 2,9-phenanthroline by reaction of 2,9-phenanthroline with anhydrous $\mathrm{HCl}$ (Scheme 6-8, bottom). The $\mathrm{HCl}$ salt of 2,9-phenanthroline was hydrogenated under very mild conditions $\left(80^{\circ} \mathrm{C}, 15\right.$ psia hydrogen pressure) using a rhodium-on-carbon catalyst. Despite the mild conditions, the reaction products included ring-opened species that were formed via C-N hydrogenolysis. In recognition of the poor hydrogenation conversions and selectivities for 2,7-, 2,8-, and 2,9-phenanthrolines and the difficulty of preparing large quantities of these compounds by photolysis, no additional studies of these liquid carrier prototypes were performed.

In summary, a wide range of phenanthroline isomers was obtained and evaluated for catalytic hydrogenation and dehydrogenation. We found wide variations in the catalytic selectivity between isomers under essentially identical hydrogenation conditions. While 1,7- and 4,7-phenanthroline were successfully hydrogenated, other isomers (e.g., 1,10-, 2,7-, and 2,8-phenanthroline) only yielded partially hydrogenated products. The most successful prototype carrier was 4,7-phenanthroline, which yielded a reversible hydrogen storage capacity of ca. 7 wt. \% hydrogen, albeit only at high temperatures.

\section{B. Dipyridyls}

Dipyridyls are molecules containing two 6-membered rings connected by a single carbon-carbon bond. Each ring contains one nitrogen atom, and a small number of regioisomers are possible with variations of the position of the nitrogen atoms relative to the carbon-carbon bond joining the rings. With commercial availability, moderate hydrogenation enthalpies, and high hydrogen capacity (>7 wt. \%), dipyridyls were considered to be good candidates for hydrogen carriers. 


\section{2,4'-dipyridyl}

Our initial attention was focused on the hydrogenation of 2,4'-dipyridyl (Scheme 6-9). The catalysts evaluated for the hydrogenation of 2,4'-dipyridyl were $5 \%$ rhodium/carbon and $4 \%$ rhodium/lithium aluminate. While high conversions $(\geq 98 \%)$ were obtained with both catalysts under moderate hydrogenation conditions $\left(180-190^{\circ} \mathrm{C} ; 1250\right.$ psig hydrogen; 12 hrs.), the observed selectivities to the fully reduced product were moderate (47-67\%). A mixture of partially hydrogenated intermediates constituted the balance of the product mixture. Extension of the rhodium/carbon-catalyzed reaction to 24 hours provided high conversion ( $\geq 98 \%$ ) and selectivity (90\% 2,4'-bipiperidine; $10 \%$ halfreduced intermediates). GC-MS analyses verified that no ring cleavage products such as pyridine or piperidine were formed. Hydrogenation with $4 \%$ rhodium/lithium aluminate under the longer time conditions $\left(190^{\circ} \mathrm{C}\right.$; 1250 psig; $24 \mathrm{hrs}$.) provided the fully hydrogenated product in high conversion (>99\%) and selectivity ( $\geq 97 \%)$.
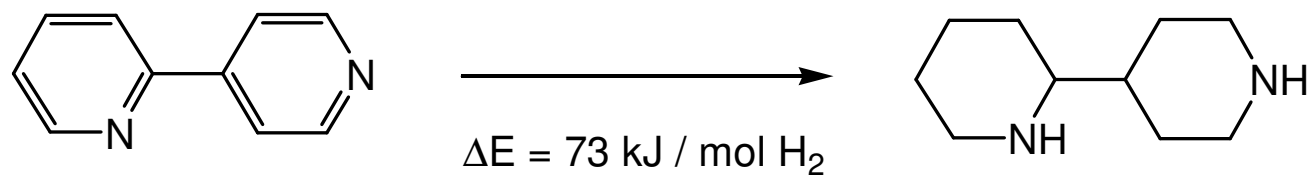

Scheme 6-9. Hydrogenation of 2,4'-dipyridyl.

Dehydrogenation of perhydro-2,4'-dipyridyl with either palladium (Pd/carbon and $\mathrm{Pd}$ /lithium aluminate) or platinum (Pt/alumina) catalysts ( 6 wt. \% catalyst; $200-225^{\circ} \mathrm{C}$ ) yielded low working capacities (Figure 6-6). No 2,4'-bypyridyl was detected in the products of the dehydrogenation reaction by GC-MS analysis. Fragmentation products such as pyridine or piperidine were also absent. Analysis by chemical ionization GC-MS revealed that the three major products had a molecular weight of 319 , very similar fragmentation patterns, and one exchangeable hydrogen. While the observed molecular weight corresponds to dimerization of 2,4'-bypiperidine with loss of ammonia, the 
pathway by which such a transformation would occur and the constitution of the dimers are unclear.

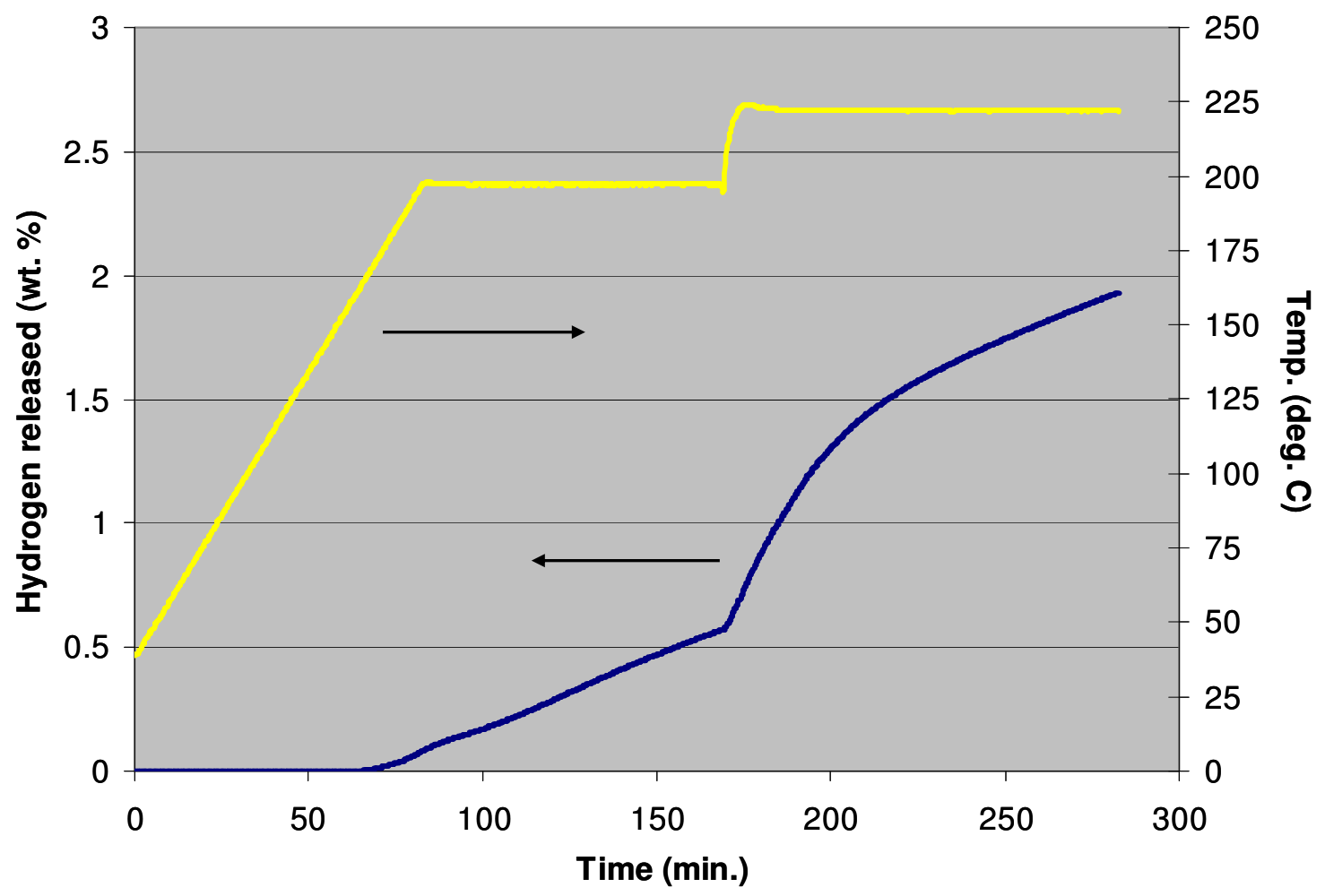

Figure 6-6. Dehydrogenation of 2,4'-dipyridyl with $\mathrm{Pt} / \mathrm{Al}_{2} \mathrm{O}_{3}$ catalyst.

\section{4,4'-dipyridyl}

An alternate dipyridyl chosen for evaluation was the commercially available 4,4'- isomer. Hydrogenation of 4,4'-dipyridyl proceeded with high conversion and selectivity (>95\%) under the same conditions used for 2,4'-dipyridyl. However, in contrast to the 2,4'-isomer, dehydrogenation with $\mathrm{Pd}$ /lithium aluminate proceeded slowly; only small amounts $(<10 \%)$ of the half-saturated intermediate and 4,4'-dipyridyl were formed at $200^{\circ} \mathrm{C}$ (Scheme 6-10). 


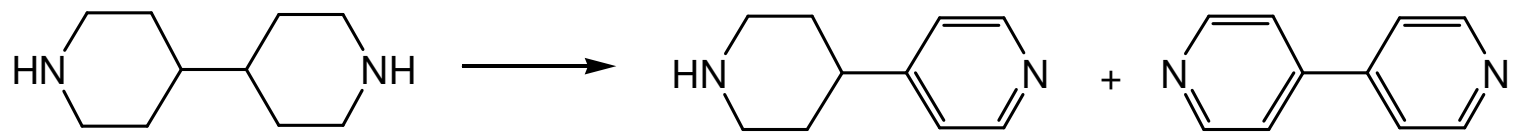

Scheme 6-10. Dehydrogenation of perhydro-4,4'-dipyridyl.

After 6 hours at $225^{\circ} \mathrm{C}$, a mixture (ca $1: 1: 1.6$ ) of saturated:half-saturated:aromatic components was formed. From the hydrogen flow data, only $56 \%$ of the theoretical hydrogen capacity was evolved (Figure 6-7).

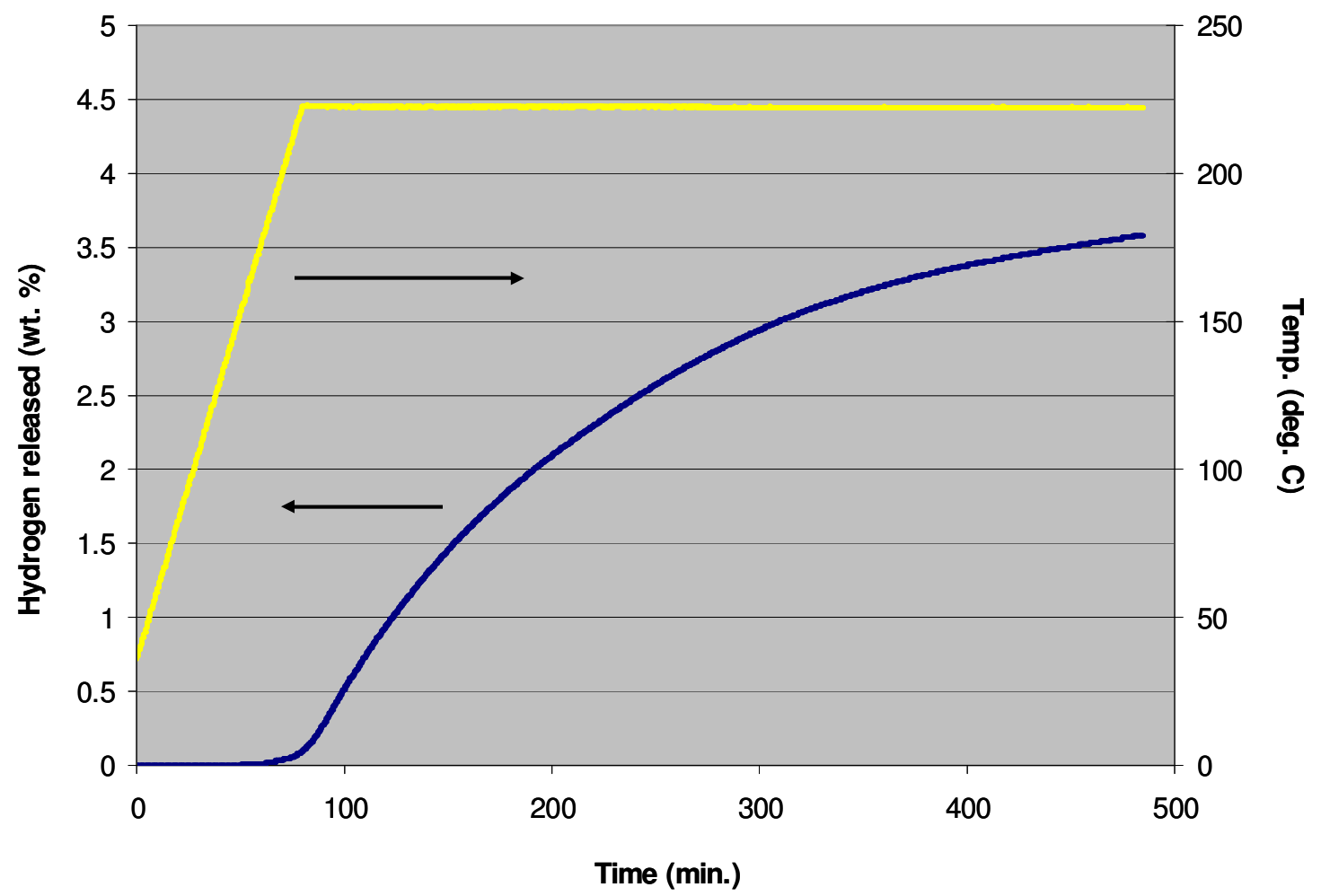

Figure 6-7. Dehydrogenation of perhydro-4,4'-bypyridyl. 
To determine whether the $\geq 7 \%$ theoretical hydrogen capacity could be obtained with 4,4'-dipyridyl, and whether that could be done selectively, dehydrogenation at higher temperatures (e.g., $250^{\circ} \mathrm{C}$ ) was evaluated. Hydrogen evolution in this experiment began at $180^{\circ} \mathrm{C}$ and subsided after 6 hours at $250^{\circ} \mathrm{C} ; 70 \%$ of the theoretical hydrogen capacity was evolved. GC-MS analysis of the dehydrogenation products showed high (98\%) conversion of perhydro-4,4'-bypyridyl. However, while 4,4'-bipyridyl was the major product $(66 \%)$, significant quantities of low- and high-eluting byproducts were also present. $\mathrm{ND}_{3} \mathrm{Cl}$ GC-MS and MALDI MS showed that several isomeric dimers of 4,4'-bipyridyl had been formed, as had small amounts of the corresponding trimers.

In summary, 2,4'- and 4,4'-dipyridyl were readily hydrogenated to the corresponding perhydrogenated compounds. Unfortunately, the preliminary dehydrogenation testing that was conducted suggests that while hydrogen can be generated via partial dehydrogenation, the selectivity of these reactions is not suitable for a successful liquid carrier. The perhydro-2,4'-dipyridyl dehydrogenation resulted in a mixture of high molecular weight species, and it is therefore probable that ammonia and/or other gaseous amines were formed during the decomposition. Perhydro-4,4'-dipyridyl dehydrogenation was somewhat more selective, but still resulted in the formation of high molecular weight species (dimers and trimers).

\section{2,2'-bipyrimidine}

Bipyrimidine has a similar molecular structure to the dipyridyl liquid carriers. The difference is that an additional nitrogen atom is substituted in the ring structures, giving a total of two nitrogen atoms per ring. Additional nitrogen incorporation in the rings should lower the average heat of hydrogenation relative to the dipyridyls. One isomer, 2,2'-bipyrimidine, was commercially available and was selected for testing. The hydrogenation was conducted using rhodium on lithium aluminate in THF solvent at $120^{\circ} \mathrm{C}$ and 850 psia hydrogen pressure. Under these conditions, we observed a quantitative conversion to octahydro-2,2'-bipyrimidine. This partially hydrogenated compound is a conjugated di-imine (Scheme 6-11, middle). Our thermodynamic 
calculations indicated that the heat of hydrogenation of the two imines was too low for hydrogenation to yield the perhydrogenated compound.

It has been shown in the literature that the heat of hydrogenation of nitrogen heterocycles can be increased by protonation of the heterocycle. Therefore, we performed the hydrogenation of 2,2'-bipyrimidine using a rhodium on carbon catalyst in a mixed aqueous $\mathrm{HCl} / \mathrm{THF}$ solvent system. Unfortunately, the hydrogenation again yielded only octahydro-2,2'-bipyrimidine. Due to the lack of an available route to perhydro-2,2'-bipyrimidine via hydrogenation, no dehydrogenation testing of this molecule was attempted.

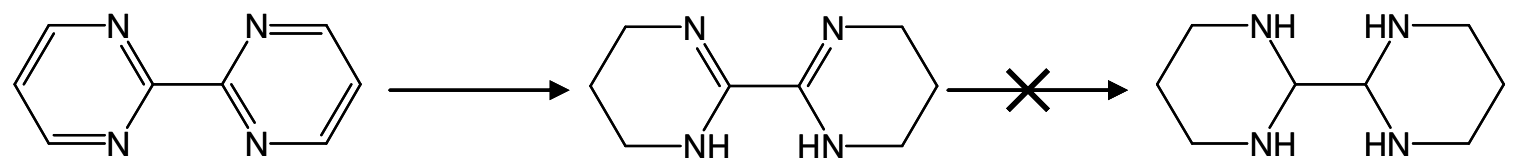

Scheme 6-11. Hydrogenation of 2,2'-bipyrimidine.

\section{Quinazoline}

Quinazoline is an analog of naphthalene containing two nitrogen atoms in one of the two rings. This commercially available compound has a melting point of $46-48^{\circ} \mathrm{C}$ and a boiling point of $243^{\circ} \mathrm{C}$ at 760 torr. The hydrogenation of quinazoline in THF solvent using a rhodium on bohemite catalyst at $100^{\circ} \mathrm{C}$ and 800 psia hydrogen pressure resulted in partial hydrogenation to tetrahydroquinazoline and ca. 10\% byproducts of ringopening hydrogenolysis. The tetrahydroquinazoline is easily oxidized, and handling this material in air during the post-reaction workup resulted in most of the tetrahydroquinazoline becoming oxidized to the cyclic diamide compound quinazoline2,4-dione (Scheme 6-12). The diamide was characterized by IR spectroscopy, showing characteristic $v(C O)$ stretches at 1600 and $1640 \mathrm{~cm}^{-1}$. The production of quinazoline2,4-dione was eliminated by handling the hydrogenated materials under inert atmosphere conditions. Employing alternate hydrogenation catalysts such as ruthenium 
on lithium aluminate also resulted in a mixture of partial hydrogenation to tetrahydroquinazoline and bond cleavage reactions. In an attempt to lower the hydrogenation temperature to inhibit the hydrogenolysis, we hydrogenated quinazoline in aqueous $\mathrm{HCl}$ at $60^{\circ} \mathrm{C}$ using a rhodium-on-carbon catalyst. This resulted in a higher level of hydrogenation; however, the major product was a thermodynamically stable imine that could not be further saturated.

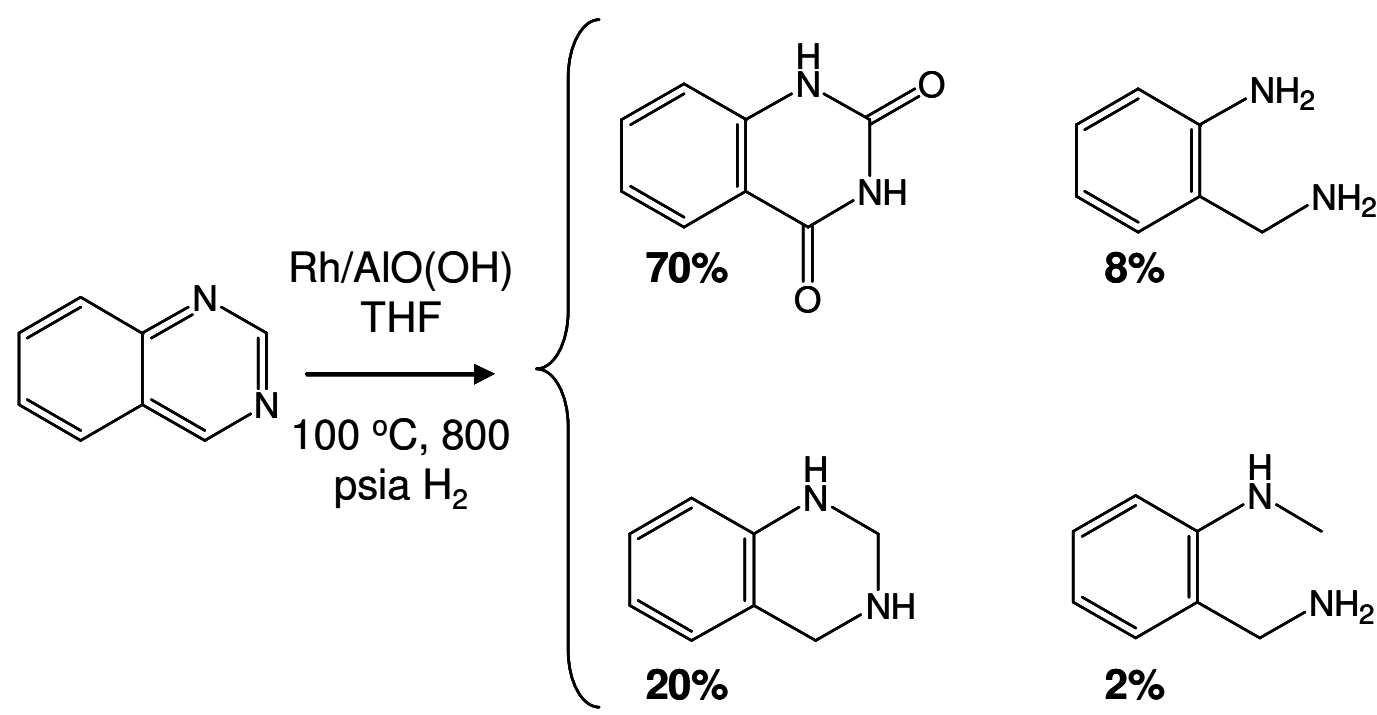

Scheme 6-12. Products of quinazoline hydrogenation after exposure to air during postreaction purification.

\section{E. Terpyridines}

Terpyridines are a higher molecular weight analog of the dipyridyl liquid carrier prototypes (section $B$ ). Unlike dipyridyls, a broad range of isomeric structures is possible due to the combination of various regioisomers (i.e., "linear" vs. "bent") and positions of nitrogen atom substitution in the rings (Figure 6-8). As part of our development of new liquid carriers, we investigated the hydrogenation and dehydrogenation of terpyridines. The isomer $2,2^{\prime}: 6$ ',2"-terpyridine is the only commercially available product. However, because of the proximity of the nitrogen 
atoms, this isomer is expected to act as a strong chelating agent, and this might deactivate hydrogenation and/or dehydrogenation catalysts. To circumvent this potential problem, we instead targeted 4,2':6',4"-terpyridine as a liquid carrier prototype.

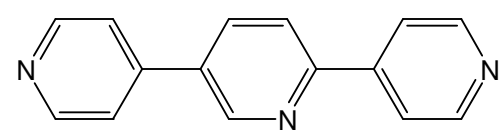

4,2':5',4"-terpyridine<smiles>c1cc(-c2ccncc2)nc(-c2ccncc2)c1</smiles>

4,2':6',4"-terpyridine<smiles>c1cc(-c2cncc(-c3ccncc3)c2)ccn1</smiles>

4,3':5',4"-terpyridine

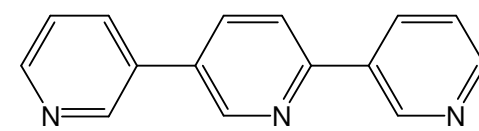

3,2':5',3"-terpyridine<smiles>c1cncc(-c2cccc(-c3cccnc3)n2)c1</smiles>

3,2':6',3"-terpyridine<smiles>c1cncc(-c2cncc(-c3cccnc3)c2)c1</smiles>

3,3':5',3"-terpyridine

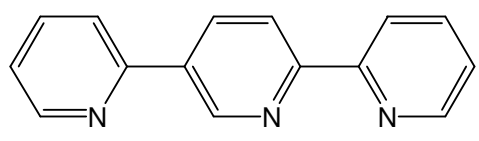

2,2':5',2"-terpyridine<smiles>c1ccc(-c2cccc(-c3ccccn3)n2)nc1</smiles>

2,2':6',2"-terpyridine<smiles>c1ccc(-c2cncc(-c3ccccn3)c2)nc1</smiles>

2,3':5',2"-terpyridine

Figure 6-8. Structures of selected terpyridine isomers.

A convenient method for synthesizing 4,2':6', 4"-terpyridine consists of palladiumcatalyzed cross-coupling reaction of 4-trimethylstannylpyridine with 2,6-dibromopyridine (Scheme 6-13).<smiles>CC(C)(C)C(C)(C)[Si](C)(C)C</smiles>

Scheme 6-13. Synthesis of $4,2^{\prime}: 6^{\prime}, 4^{\prime \prime}$-terpyridine. 
Hydrogenation of $4,2^{\prime}: 6^{\prime}, 4^{\prime \prime}$-terpyridine in THF solvent using pre-activated $5 \%$ rhodium on lithium aluminate resulted in quantitative conversion to a dodecahydro-4,2':6',4"terpyridine isomer, where the two outer rings are hydrogenated (Scheme 6-14). The structure of this partially hydrogenated product was determined by GC-MS and ${ }^{1} \mathrm{H}$ NMR analyses.<smiles>CO[C@@H](C1CCNCC1c1cccc(C2CCNCC2)n1)C(C)(F)F</smiles>

Scheme 6-14. Hydrogenation of 4,2':6',4"-terpyridine.

Since the hydrogenation of 4,2':6',4'-terpyridine failed to yield the desired perhydrogenated compound, alternative hydrogenation conditions were explored. The tri-hydrochloride salt of 4,2':6',4"-terpyridine was prepared by dissolving the free amine in anhydrous ether and adding 3.5 equivalents of a $2 \mathrm{M}$ solution of hydrogen chloride in dry ether. Hydrogenation of the protonated 4,2':6',4'-terpyridine in absolute ethanol with platinum oxide gave only undetermined low- and high-molecular-weight products (Scheme 6-15). Hydrogenation of protonated 4,2':6',4"-terpyridine with pre-activated rhodium on lithium aluminate in THF under the conditions shown in Scheme 6-14 yielded decahydro-4,2':6',4"-terpyridine, along with small amounts of byproducts.<smiles>Clc1cccc(-c2ccncc2)n1</smiles>

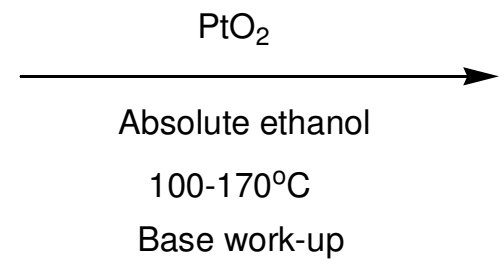

High and low molecular weight products

Scheme 6-15. Hydrogenation of 4,2':6',4"-terpyridine $3 \mathrm{HCl}$. 
Because of the poor results achieved from the hydrogenation of 4,2':6',4"-terpyridine and the tri-hydrochloride salt of 4,2':6',4"-terpyridine, no dehydrogenation testing on the partially hydrogenated products or other experiments was conducted using terpyridines.

\section{F. Naphthyridines}

Naphthyridines (also known as diazanaphthalenes or pyridopyridines) are another interesting class of liquid carrier protoypes that have theoretical reversible hydrogen capacities greater than $7 \mathrm{wt}$ \% and the potential for low melting points, depending on the symmetry of the molecules. The average heat of hydrogenation for this class of molecules is approximately $55-60 \mathrm{~kJ} / \mathrm{mol} \mathrm{H}$, based upon the calculated $\Delta \mathrm{E}$ for hydrogenation of a number of isomers (Table 6-3).

Table 6-3. Physical data and calculated $\Delta \mathrm{E}$ of hydrogenation for naphthyridine isomers.

\begin{tabular}{|c|c|c|c|c|c|}
\hline Structure & & & & & \\
\hline Name & $\begin{array}{c}1,5- \\
\text { naphthyridine }\end{array}$ & $\begin{array}{c}1,6- \\
\text { naphthyridine }\end{array}$ & $\begin{array}{c}1,7- \\
\text { naphthyridine }\end{array}$ & $\begin{array}{c}2,6- \\
\text { naphthyridine }\end{array}$ & $\begin{array}{c}2,7- \\
\text { naphthyridine }\end{array}$ \\
\hline $\begin{array}{c}\text { Calc. average } \Delta \mathrm{E} \text { of } \\
\text { hydrogenation } \\
\left(\text { trans, } \mathrm{kJ} / \mathrm{mol} \mathrm{H}_{2}\right)\end{array}$ & 72 & 72 & 73 & 73 & \\
\hline $\begin{array}{c}\text { Calc. average } \Delta \mathrm{E} \text { of } \\
\text { hydrogenation (cis, } \\
\mathrm{kJ} / \mathrm{mol} \mathrm{H}_{2} \text { ) }\end{array}$ & 68 & & 70 & 72 & \\
\hline Melting point $\left({ }^{\circ} \mathrm{C}\right)$ & $60-62$ & $25-30$ & $64-65$ & $118-199$ & 96 \\
\hline $\begin{array}{c}\text { Boiling point }\left({ }^{\circ} \mathrm{C} /\right. \\
\text { Torr })\end{array}$ & $80 / 0.5$ & $150 / 54$ & & & \\
\hline
\end{tabular}

While all of the possible naphthyridine isomers have been described at some level of detail in the chemical research literature, only the 1,5- and 1,6- isomers were commercially available in gram quantities. Data on several perhydronaphthyridines have also been reported in the literature (Table 6-4). 
Table 6-4. Physical data for perhydronaphthyridine isomers.

\begin{tabular}{|c|c|c|c|c|c|}
\hline Structure & & $\begin{array}{c}38-39(\mathrm{cis}) \\
72-73\end{array}$ & & \\
\hline Name & $\begin{array}{c}\text { decahydro-1,5- } \\
\text { naphthyridine }\end{array}$ & $\begin{array}{c}\text { decahydro-1,6- } \\
\text { naphthyridine }\end{array}$ & $\begin{array}{c}\text { decahydro-1,7- } \\
\text { naphthyridine }\end{array}$ & $\begin{array}{c}\text { decahydro-2,6- } \\
\text { naphthyridine }\end{array}$ & $\begin{array}{c}\text { decahydro-2,7- } \\
\text { naphthyridine }\end{array}$ \\
\hline Melting point (') & $\begin{array}{c}176-177 \\
(\text { trans })\end{array}$ & $74 / 1$ & $76 / 1.3$ & & \\
\hline $\begin{array}{c}\text { Boiling point (' } \\
\text { Torr) }\end{array}$ & $66 / 0.3(\mathrm{cis})$ & 74 & & \\
\hline
\end{tabular}

\section{1,5-naphthyridine}

1,5-naphthyridine was prepared by the Skraup method which consists of reacting 2-aminopyridine with glycerol in water and sulfuric acid using the sodium salt of nitrobenzene sulfonic acid as an oxidizing reagent for aromatizing the second ring of the dihydro-1,5-naphthyridine reaction intermediate (Scheme 6-16). Synthesizing the fully aromatic product required the use of a large excess of the oxidizing agent and this limited the scale-up of the reaction.<smiles>Nc1cccnc1</smiles><smiles>OCC(O)CO</smiles><smiles>O=[N+]([O-])[O-]</smiles><smiles>c1cnc2cccnc2c1</smiles>

Scheme 6-16. Synthesis of 1,5-naphthyridine via the Skraup method.

Since our ultimately desired product is perhydro-1,5-naphthyridine, there is no need to aromatize the dihydro-1,5-naphthyridine intermediate. Therefore, we decided to perform the reaction in Scheme 6-16 without an oxidizing reagent. Surprisingly, the reaction still yielded 1,5-naphthyridine. This indicates that aromaticity is the thermodynamic driving 
force and that under the reaction conditions dihydro-1,5-naphthyridine is easily dehydrogenated to the fully aromatic product (Scheme 6-17). The elimination of the oxidizing reagent allowed the reaction to be easily performed on a larger scale and made the product recovery easier. The crude 1,5-naphthyridine product was purified by sublimation.

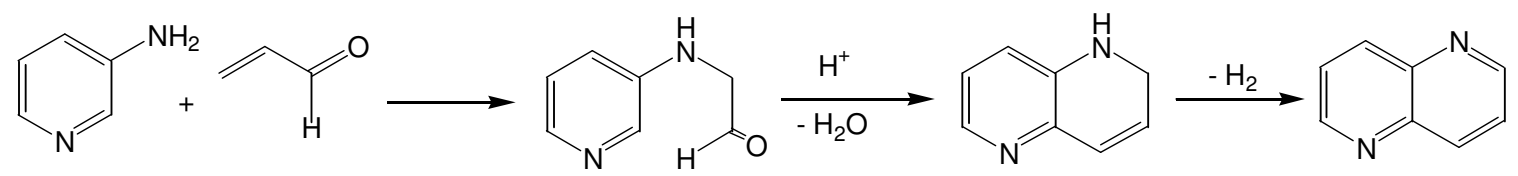

Scheme 6-17. Synthesis of 1,5-naphthyridine.

Hydrogenation of 1,5-naphthyridine as the free amine or as a hydrochloride salt with various solvents and catalysts was investigated. Hydrogenation with rhodium or ruthenium on lithium aluminate in THF, dioxane or ethanol solvents yielded only tetrahydro-1,5-naphthyridine at temperatures lower than $100^{\circ} \mathrm{C}$. At higher temperatures, perhydro-1,5-naphthyridine along with higher molecular weight products were obtained suggesting that oligomerization is occurring during the hydrogenation. Hydrogenation of the hydrochloride salt of 1,5 -naphthyridine at $50^{\circ} \mathrm{C}$ also showed oligomerization products. Ultimately, synthesis of perhydro-1,5-naphthyridine was accomplished using rhodium on alumina in acetic acid at room temperature over a period of three days (Scheme 6-18). Perhydro-1,5-naphthyridine was purified by sublimation at $110^{\circ} \mathrm{C}$ and 1 torr to provide a white solid.

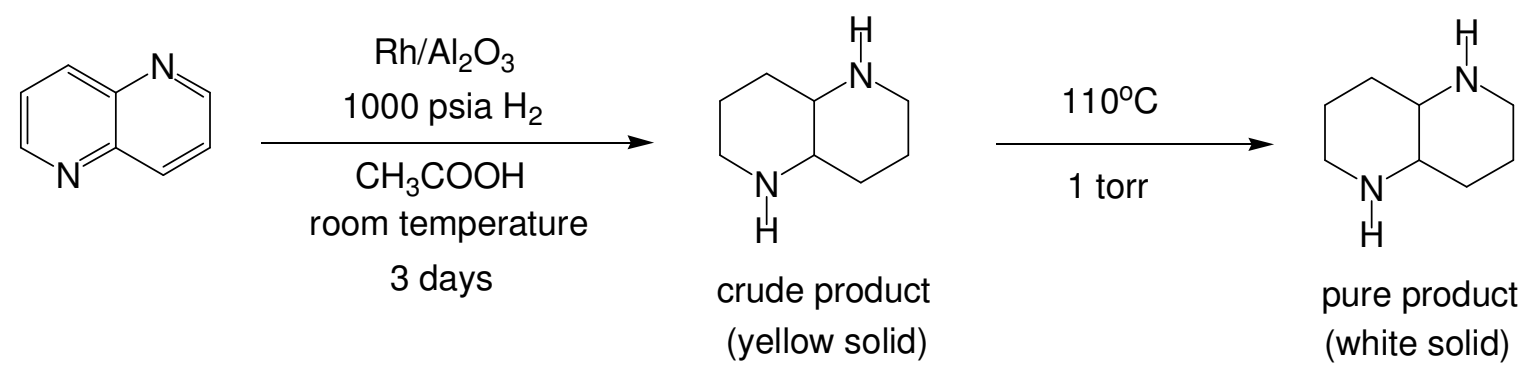

Scheme 6-18. Synthesis and purification of perhydro-1,5-naphthyridine. 
Because of the limited amount of purified perhydro-1,5-naphthyridine available, dehydrogenation was initially investigated using a mixture of perhydro-1,5-naphthyridine with perhydro-N-ethylcarbazole. Dehydrogenation was performed using a $5 \% \mathrm{Pd} / \mathrm{C}$ catalyst, ramping the temperature from room temperature to $200^{\circ} \mathrm{C}$ and holding at $200^{\circ} \mathrm{C}$ for 5 hours. GC-MS analysis of the dehydrogenated reaction mixture showed the expected dehydrogenation products of perhydro-N-ethylcarbazole, along with tetrahydro1,5-naphthyridine, perhydro-1,5-naphthyridine, and two peaks with molecular weights lower than that of 1,5-naphthyridine. Careful analysis of the fragmentation pattern of these peaks revealed that these products are derived from perhydro-1,5-naphthyridine, suggesting that the molecule is fragmenting in the dehydrogenation process (Scheme 6-19).

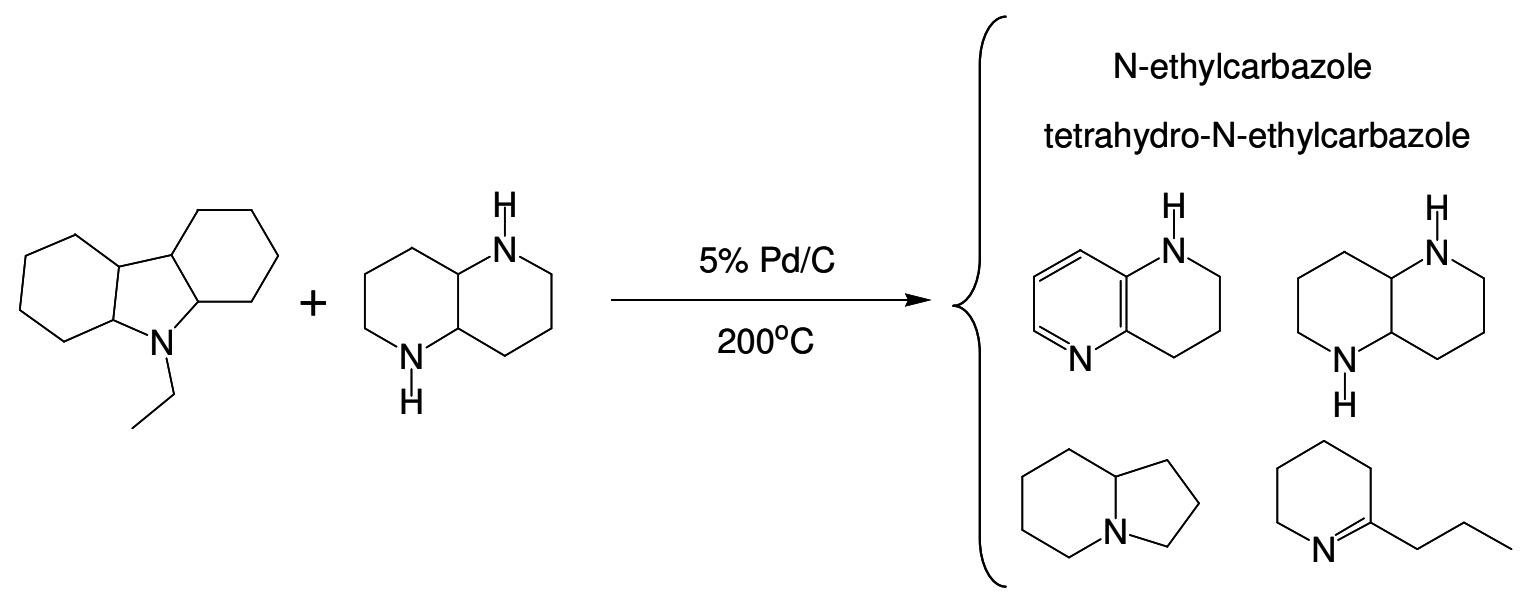

Scheme 6-19. Dehydrogenation of a mixture of perhydro-1,5-naphthyridine and perhydro-N-ethylcarbazole.

\section{1,6-naphthyridine}

The asymmetric isomer 1,6-naphthyridine is commercially available in limited quantities and has a melting point of $25^{\circ} \mathrm{C}$. In contrast to the difficulties encountered during the hydrogenation of 1,5-naphthyridine under neutral conditions, hydrogenation of 1,6-naphthyridine with pre-activated $5 \%$ rhodium on alumina at $100^{\circ} \mathrm{C}$ yielded a clean and quantitative conversion to tetrahydro-1,6-naphthyridine, and additional conversion at a higher temperature $\left(170^{\circ} \mathrm{C}, 6\right.$ hours $)$ yielded complete conversion to perhydro-1,6- 
naphthyridine, with only small amounts of byproducts. With a rhodium on lithium aluminate hydrogenation catalyst at the very mild reaction temperature of $40^{\circ} \mathrm{C}$, two tetrahydro-1,6-naphthyridine isomers were exclusively produced. Upon increasing the reaction temperature to $165^{\circ} \mathrm{C}$, perhydro-1,6-naphthyridine as a mixture of cis/trans isomers was recovered with $100 \%$ conversion and, depending on reaction conditions, in $>98 \%$ selectivity (Scheme 6-20).<smiles>c1cnc2ccncc2c1</smiles>

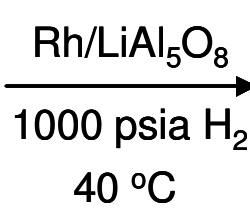<smiles>c1cnc2c(c1)CNCC2</smiles><smiles>c1cc2c(cn1)CCCN2</smiles><smiles>[R]OC(=O)OCC(C)C</smiles><smiles>C1CNC2CCNCC2C1</smiles>
$165^{\circ} \mathrm{C}$

Scheme 6-20. Hydrogenation of 1,6-naphthyridine.

The as-produced perhydro-1,6-naphthyridine was dehydrogenated using a $5 \%$ palladium-on-carbon catalyst by ramping the temperature to $225^{\circ} \mathrm{C}$ under one atmosphere of hydrogen and holding at $225^{\circ} \mathrm{C}$ for 30 minutes (Figure 6-9). GC-MS analysis of the product showed $100 \%$ conversion of perhydro-1,6-naphthyridine to tetrahydro-1,6-naphthyridine, corresponding to a 4.8 wt.\% hydrogen release which matches the amount of hydrogen measured during the dehydrogenation experiment. 


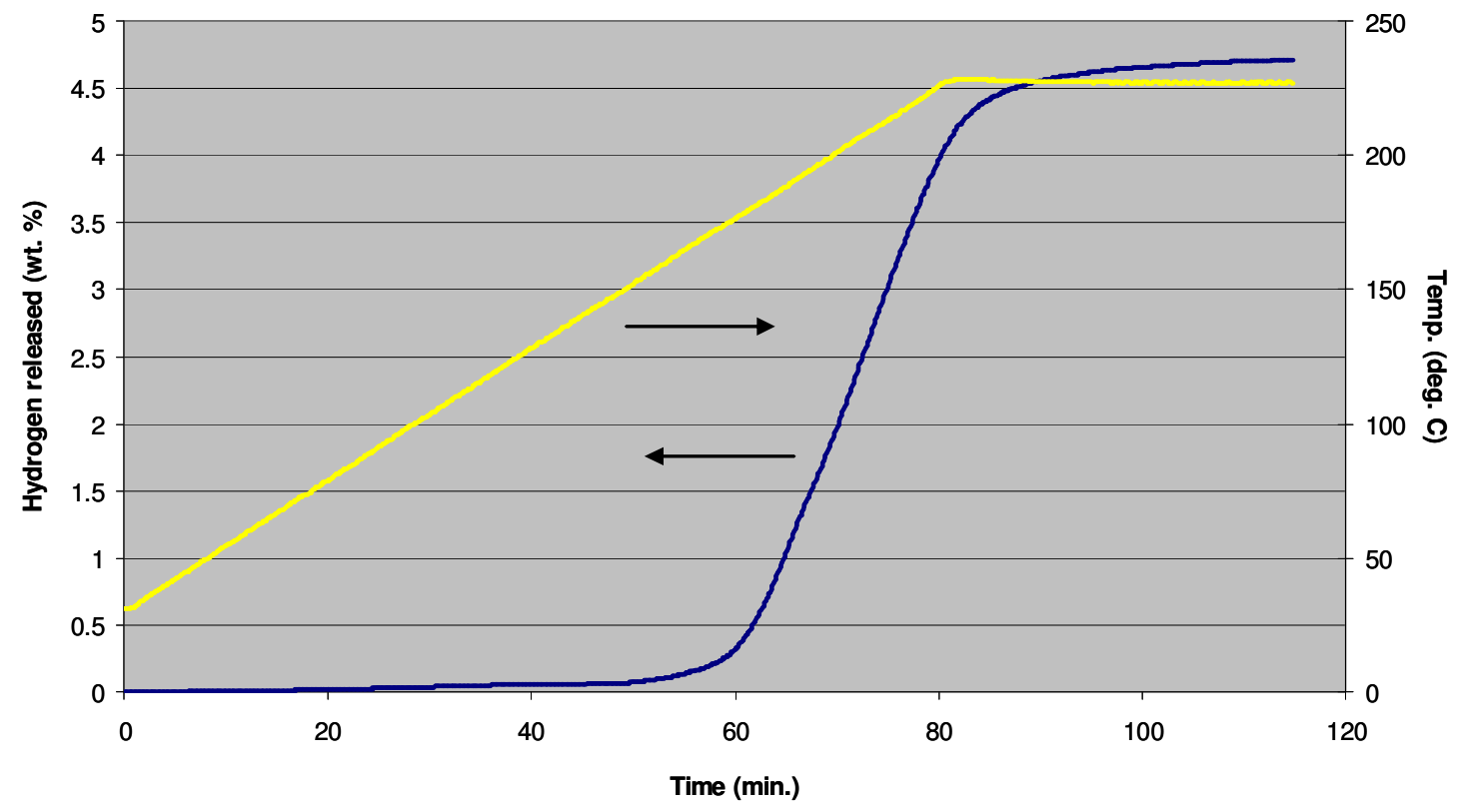

Figure 6-9. Dehydrogenation of perhydro-1,6-naphthyridine.

The tetrahydro-1,6-naphthyridine product isolated from the dehydrogenation experiment shown in Figure 6-9 was subjected to a second dehydrogenation experiment using a new aliquat of the 5\% palladium-on-carbon catalyst. As shown in Figure 6-10, temperatures of $>260^{\circ} \mathrm{C}$ were required to release additional hydrogen from the partially dehydrogenated carrier. The catalyst/liquid mixture was stirred at $270-280^{\circ} \mathrm{C}$ until the hydrogen flow effectively ceased. The dehydrogenated mixture was characterized by GC-MS analysis, and it was determined that the mixture contained $63 \%$ 1,6-naphthyridine, $27 \%$ tetrahydro-1,6-naphthyridine and $10 \%$ of a byproduct resulting from hydrogenolysis. Overall, the total amount of hydrogen released during the two dehydrogenation experiments was $5.8 \mathrm{wt}$. $\% \mathrm{H}_{2}$, and dehydrogenation selectivity was approximately $90 \%$. 


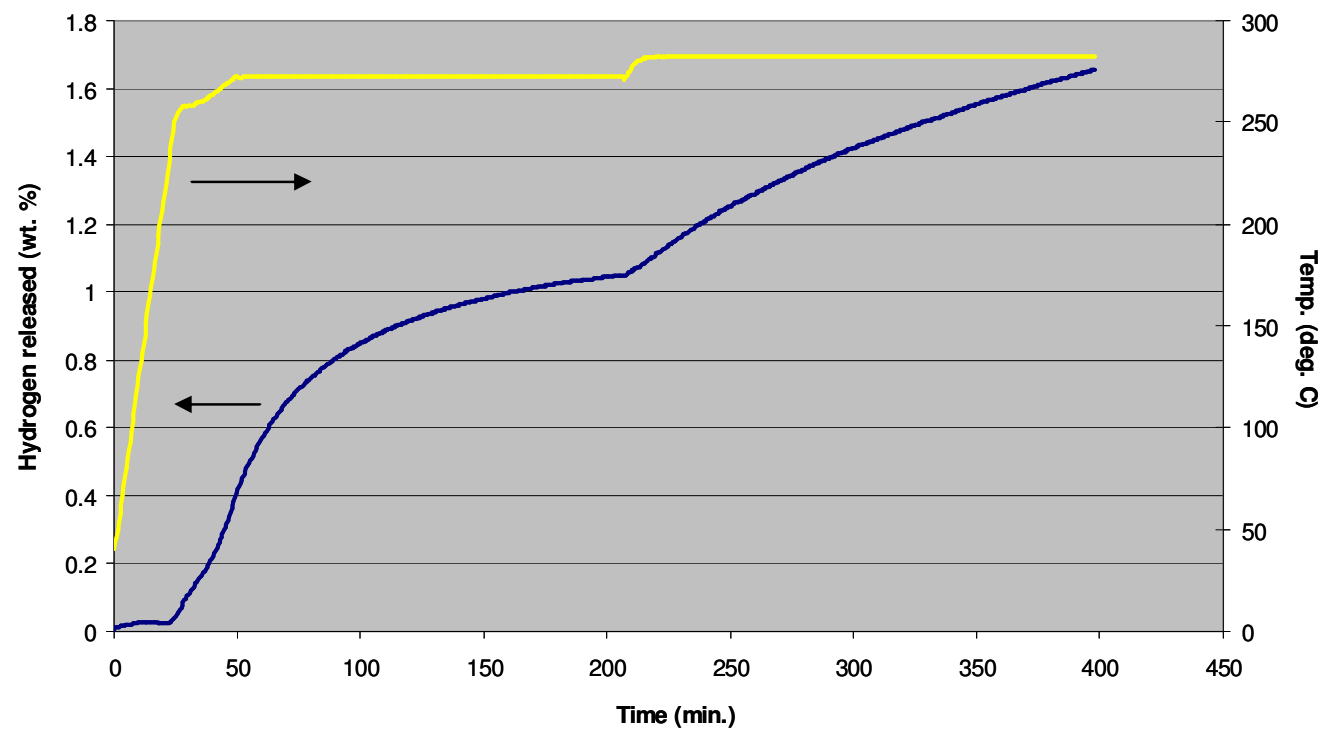

Figure 6-10. Dehydrogenation of tetrahydro-1,6-naphthyridine.

The identity of the tetrahydro-1,6-naphthyridine isomer and the high temperatures required for dehydrogenation of tetrahydro-1,6-naphthyridine are explained by the calculated hydrogenation energetics shown in Scheme 6-21.

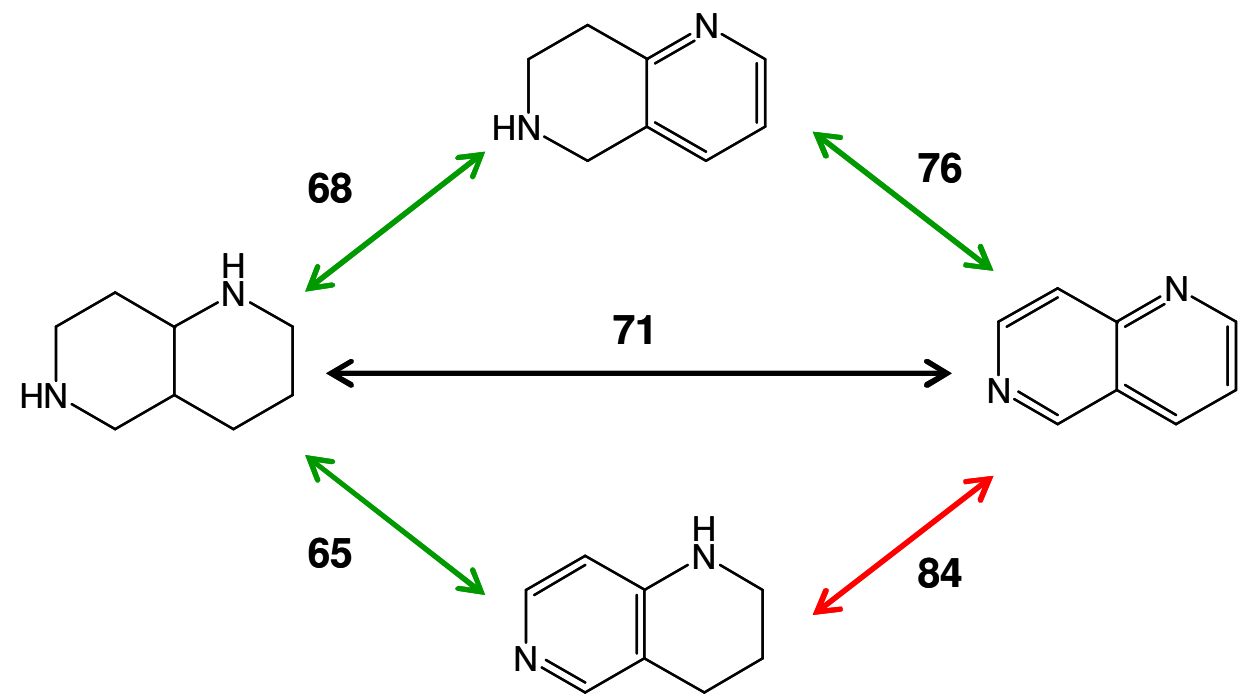

Scheme 6-21. Calculation of the electronic energy change, $\Delta \mathrm{E}$, for the dehydrogenation of perhydro-1,6-naphthyridine $\left(\mathrm{kJ} /\right.$ mole $\left._{2}\right)$. 
According to the calculated energies for the dehydrogenation of perhydro-1,6naphthyridine to form tetrahydro-1,6-naphthyridine, one isomer is favored by ca. $3.4 \mathrm{~kJ} / \mathrm{mol} \mathrm{H}_{2}$ (Scheme 6-21, lower pathway). The calculated $\Delta \mathrm{E}$ for the dehydrogenation of this tetrahydro-1,6-naphthyridine isomer is $84 \mathrm{~kJ} / \mathrm{mol} \mathrm{H}_{2}$, which is consistent with the very high temperatures necessary to achieve additional conversion to 1,6-naphthyridine. Thus, while the overall average $\Delta \mathrm{E}$ of $71 \mathrm{~kJ} / \mathrm{mol} \mathrm{H}_{2}$ for the dehydrogenation of perhydro-1,6-naphthyridine is appealing, the inequity of the average $\Delta \mathrm{E}$ for the two steps of the reaction pathway that was revealed by the experimental testing renders this carrier candidate less interesting as a practical liquid carrier.

\section{Other naphthyridine isomers}

The calculated stepwise thermodynamics for the dehydrogenation of the closely related 1,7-naphthyridine isomer shows that the average $\Delta E$ for the two steps are less uneven and perhaps would result in a viable pathway for complete dehydrogenation at moderate temperatures (Scheme 6-22, upper pathway). Similar calculations were performed for all of the remaining possible naphthyridine isomers (Scheme 6-23).

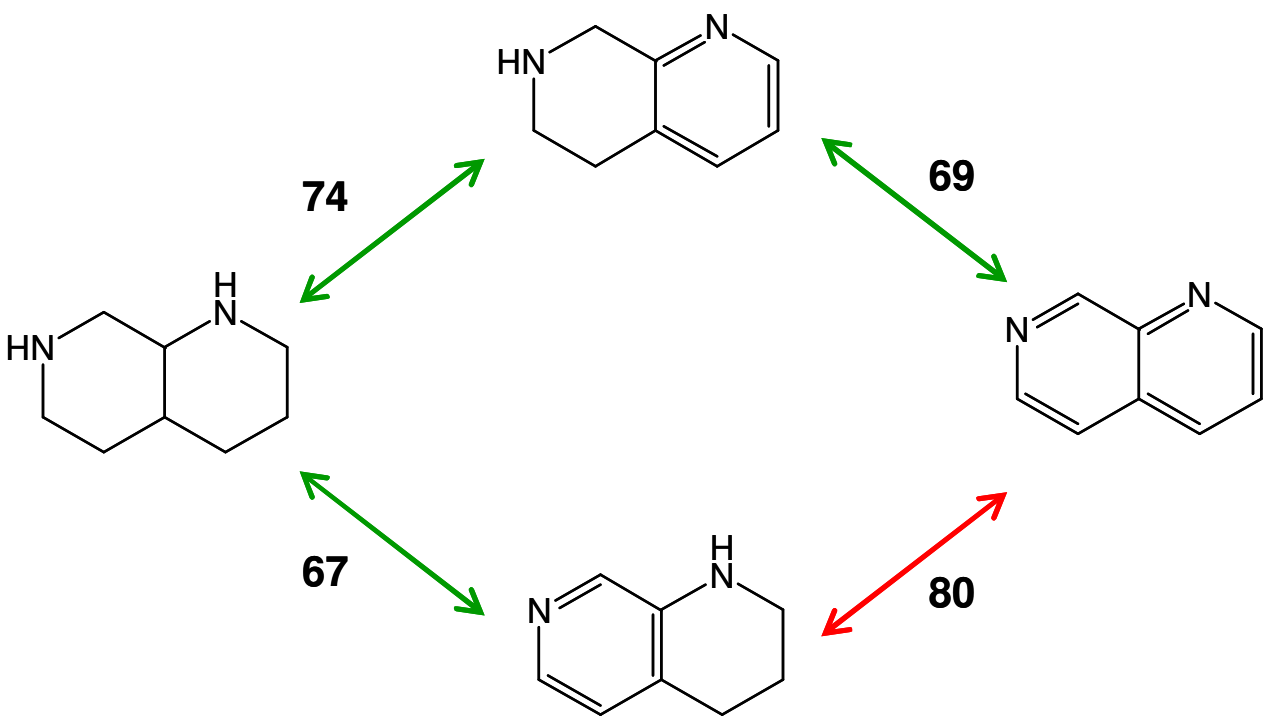

Scheme 6-22. Calculation of the electronic energy change, $\Delta \mathrm{E}$, for the dehydrogenation of perhydro-1,7-naphthyridine $\left(\mathrm{kJ} /\right.$ mole $\left._{2}\right)$. 
From these calculations, we determined that the isomers containing $C_{2}$ symmetry, such as 2,6-naphthyridine and 2,7-naphthyridine, afford reasonably good overall energetics (average $\Delta \mathrm{E}$ of $72 \mathrm{~kJ} / \mathrm{mole}_{2}$ ) and probable dehydrogenation pathways that do not contain any significant energetic barriers. Unfortunately, the lack of either commercial availability or simple synthetic procedures to prepare any of these naphthyridine isomers resulted in a decision not to pursue experimental testing for these carrier candidates.

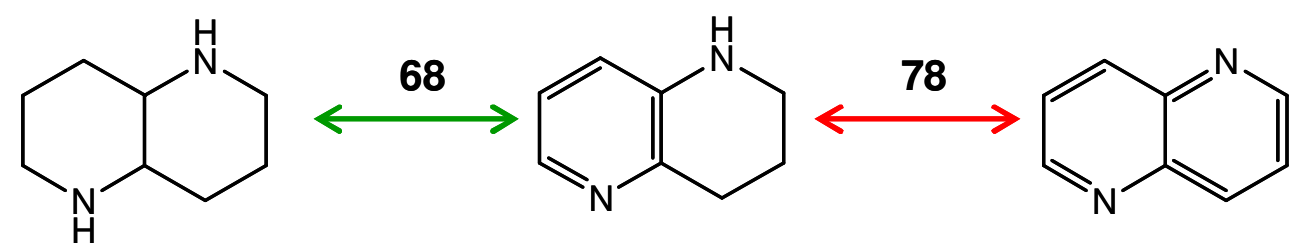

1,5-naphthyridine<smiles></smiles>

2,6-naphthyridine<smiles></smiles>

2,7-naphthyridine<smiles>C1CNC2NCCCC2C1</smiles><smiles>CC(=O)c1cc2c(nc1C)NCCC2</smiles><smiles>c1cnc2cccnc2c1</smiles>

1,8-naphthyridine

Scheme 6-23. Calculation of the electronic energy change, $\Delta \mathrm{E}$, for the dehydrogenation of various naphthyridine isomers $\left(\mathrm{kJ} / \mathrm{mole}_{2}\right)$. 


\section{Experimental Details}

See Appendix B for general experimental details.

\section{Preparation of $4,2^{\prime}: 6^{\prime}, 2^{\prime \prime}$-terpyridine}

A mixture of 4-trimethylstannylpyridine $(1 \mathrm{~g}, 4.1 \mathrm{mmol}), 2,6$-dibromopyridine

$(0.5 \mathrm{~g}, 2.1 \mathrm{mmol})$ and tetrakis(triphenylphosphine)palladium (40.5 mg, $0.26 \mathrm{mmol})$ in toluene $(60 \mathrm{~mL})$ was heated to $120^{\circ} \mathrm{C}$ with stirring for 8 hours. The reaction mixture was cooled to room temperature and extracted with water/ethyl acetate. The organic layer was dried with magnesium sulfate, filtered, and the solvent evaporated.

Chromatography on silica gel with ethyl acetate:hexanes (1:1) gave 0.4 grams of product.

\section{Hydrogenation of $4,2^{\prime}: 6^{\prime}, 2^{\prime \prime}$-terpyridine}

A 30 cc stainless steel pressure reactor was loaded with $0.5 \mathrm{~g}$ of $4,2^{\prime}: 6^{\prime}, 2^{\prime \prime}$-terpyridine in THF (10 mL) and $0.1 \mathrm{~g}$ of $5 \%$ pre-activated rhodium on lithium aluminate. After the headspace was purged with hydrogen, the hydrogen pressure was increased to $800 \mathrm{psia}$. The reactor was heated to $170^{\circ} \mathrm{C}$, and the hydrogen pressure increased to 950 psia. After 6 hours under these conditions with stirring, the reactor was cooled to $25^{\circ} \mathrm{C}$, and the contents were filtered to remove catalyst. GC-MS analysis showed complete conversion to a partially hydrogenated product (confirmed by ${ }^{1} \mathrm{H} N \mathrm{NR}$ ). The tri-hydrochloride salt was prepared by dissolving the free amine in anhydrous ether and adding 3.5 equivalents of a $2 \mathrm{M}$ solution of hydrogen chloride in dry ether. The white precipitate was isolated by evaporating the solvent and drying under vacuum. Hydrogenation of this material with platinum oxide in absolute ethanol at $170^{\circ} \mathrm{C}$ and $800 \mathrm{psi}$ of hydrogen gave high-molecular-weight products. Hydrogenation of the salt with pre-activated rhodium on lithium aluminate at $200^{\circ} \mathrm{C}$ and $900 \mathrm{psi}$ of hydrogen in THF gave partially hydrogenated product along with some bond cleavage byproducts. 


\section{Preparation of 1,5-naphthyridine}

The product 1,5-naphthyridine was prepared by a modification of the Skraup synthesis (without an oxidizing agent). To a solution of 3-aminopyridine (15 g, $0.16 \mathrm{~mol}$ ) dissolved in water $(90 \mathrm{~mL})$ and glycerol $(50 \mathrm{~mL})$ was slowly added concentrated sulfuric acid $(160 \mathrm{~mL})$ while the reaction mixture was cooled with an ice-water bath to $0^{\circ} \mathrm{C}$. At the end of the addition, the mixture was heated to $145^{\circ} \mathrm{C}$ with an oil bath for one hour. At longer reaction times, byproducts were formed. After cooling to room temperature, the reaction mixture was poured onto a concentrated solution of sodium hydroxide to $\mathrm{pH} \sim 14$, and then extracted with chloroform. The organic phase was washed with water, dried over magnesium sulfate, filtered, and the solvent was evaporated.

Chromatography on silica gel with ethyl acetate gave 7 grams of brown solid. Eleven grams of the brown solid (combined products of multiple reactions) was sublimed at $93^{\circ} \mathrm{C}$ under $1 \mathrm{~mm} \mathrm{Hg}$ pressure to give 10.4 grams of a white solid which, upon standing at room temperature for several days in air, showed a slight coloration.

\section{Hydrogenation of 1,5-naphthyridine}

Hydrogenation of 1,5-naphthyridine as the free amine or as a hydrochloride salt using various solvents and catalysts was investigated. Hydrogenation with rhodium or ruthenium on lithium aluminate catalyst and tetrahydrofuran, dioxane, or ethanol solvents gave only tetrahydro-1,5-naphthyridine as a product at temperatures lower than $100^{\circ} \mathrm{C}$. At higher reaction temperatures, perhydro-1,5-naphthyridine along with higher molecular weight products was obtained, suggesting that oligomerization is occurring. Hydrogenation of the hydrochloride salt of 1,5 -naphthyridine at $50^{\circ} \mathrm{C}$ showed the presence of oligomers in the products. Hydrogenation of 1,5-naphthyridine with high conversion and selectivity was ultimately accomplished using a rhodium-on-alumina catalyst in acetic acid solvent at room temperature over a period of three days.

Perhydro-1,5-naphthyridine was purified by sublimation at $110^{\circ} \mathrm{C}$ and 1 torr pressure to provide a white solid product. 


\section{Dehydrogenation of 1,5-naphthyridine}

Because of the limited amount of material available for testing, dehydrogenation of 1,5-naphthyridine was carried out as a mixture with perhydro- $\mathrm{N}$-ethylcarbazole with $5 \% \mathrm{Pd} / \mathrm{C}$. The dehydrogenation was performed by ramping the temperature from room temperature to $200^{\circ} \mathrm{C}$ and holding at $200^{\circ} \mathrm{C}$ for 5 hours under 1 atm. hydrogen pressure. GC-MS analysis of the dehydrogenation mixture showed the typical dehydrogenation products of perhydro-N-ethylcarbazole, along with the partially dehydrogenated 1,5-naphthyridine (one unsaturated ring), the starting perhydro-1,5-naphthyridine, and two peaks with molecular weights lower than that of 1,5-naphthyridine. Careful analysis of the fragmentation pattern of these peaks revealed that these products are derived from perhydro-1,5-naphthyridine, suggesting that the molecule is fragmenting in the dehydrogenation process.

\section{Dehydrogenation of hydrogenated 1,6-naphthyridine}

A $0.6 \mathrm{~g}$ sample of a 50:50 mixture containing tetrahydro-1,6-naphthyridine and perhydro1,6-naphthyridine was added to $3.5 \mathrm{~g}$ of perhydro- $\mathrm{N}$-ethylcarbazole and $250 \mathrm{mg}$ of $5 \% \mathrm{Pd} / \mathrm{C}$ catalyst. The sample mixture was then heated to $200^{\circ} \mathrm{C}$ for $5.5 \mathrm{hrs}$. while it was stirred at $500 \mathrm{rpm}$. After the hydrogen evolution flow rate dropped below $1 \mathrm{cc} / \mathrm{min}$., the catalyst was filtered off and the mixture was analyzed by GC-MS. The reaction yielded $100 \%$ conversion to tetrahydro-1,6-naphthyridine, and no fully dehydrogenated 1,6-naphthyridine was detected. 


\section{Chapter 7: Oxygen-containing Hydrogen Carriers}

\section{Introduction}

Throughout this report, we have detailed in several chapters the synthesis and testing of carriers incorporating nitrogen heteroatoms (N-heterocycles). In an effort to broaden the field of potential organic liquid hydrogen carriers, we investigated a number of aromatic molecules containing oxygen heteroatoms. There is a wide variety of organic functional groups that contain oxygen, including hydroxyl, ether, ketone, aldehyde, and carboxylic acid groups. Unlike nitrogen atoms, oxygen atoms cannot be substituted for carbon in six-membered aromatic ring systems (i.e., there is no oxygen-containing analog of pyridine).

However, there are oxygen-containing functional groups that can be reversibly hydrogenated. A well-known example is the reversible hydrogenation of acetone to isopropanol. The dehydrogenation of isopropanol can be accomplished using ruthenium catalysts under relatively mild conditions. ${ }^{1}$ It is believed that the slightly lower boiling point of acetone vs. isopropanol assists the conversion as the dehydrogenated product (acetone) can be distilled, pushing the reaction equilibrium towards the products (acetone $+\mathrm{H}_{2}$ ).

Our work was focused on two classes of oxygen-containing carriers. First, we investigated aromatic systems where the presence of oxygen in an ether group reduces the $\Delta \mathrm{H}_{\mathrm{D}}{ }^{0}$ of the aromatic systems (e.g., dibenzofuran). Here, the oxygen atom does not add hydrogen during the hydrogenation, but instead modifies the thermodynamics of the aromatic rings (similar to the nitrogen atom in carbazole). The second class of liquid carrier candidates is aromatic molecules containing secondary alcohol groups that can be dehydrogenated to ketones, yielding hydrogen. 


\section{Results and Discussion}

\section{A. Dibenzofuran}

Dibenzofuran, a relatively inexpensive commercial product, has a melting point similar to that of $\mathrm{N}$-ethylcarbazole, but has a higher hydrogen weight capacity (6.6 wt. \% versus $5.8 \mathrm{wt}$. \%). As discussed in detail in Chapter 1 , the presence of the oxygen atom in the five-membered ring reduces the $\alpha-\mathrm{CH}$ bond strength and lowers the average heat of hydrogenation for dibenzofuran relative to fluorene (Figure 7-1).

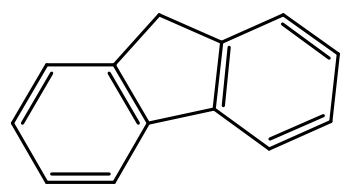

fluorene<smiles>O=C1c2ccccc2-c2ccccc21</smiles>

fluorenone<smiles>c1ccc2c(c1)oc1ccccc12</smiles>

dibenzofuran<smiles>c1ccc2c(c1)[nH]c1ccccc12</smiles>

carbazole

60.5

56.7

Figure 7-1. Average enthalpies $\left(\mathrm{kJ} / \mathrm{mol} \mathrm{H}_{2}\right)$ of dehydrogenation $\left(\Delta \mathrm{H}_{\mathrm{D}}{ }^{\circ}\right)$ at $300 \mathrm{~K}$ from ab initio DFT calculations. The enthalpy value for fluorenone represents only the dehydrogenation of the aromatic rings.

Our initial attempt at catalytic hydrogenation of dibenzofuran using a ruthenium on lithium aluminate catalyst at $170^{\circ} \mathrm{C}$ resulted in products of hydrogenolysis of the carbonoxygen bonds (e.g., dicyclohexyl). Dibenzofuran was hydrogenated with higher selectivity using $5 \%$ ruthenium on lithium aluminate at $100^{\circ} \mathrm{C}$ and 900 psia hydrogen pressure. GC-MS analysis showed complete conversion of dibenzofuran to perhydrodibenzofuran and dodecahydrobiphenylene (Scheme 7-1). The GC-MS analysis also revealed that the perhydrodibenzofuran was present in the hydrogenated mixture as two isomers in a ratio of 60:1. The dodecahydrobiphenylene was removed from the crude product mixture by vacuum distillation to yield a sample of perhydrodibenzofuran for dehydrogenation testing. 

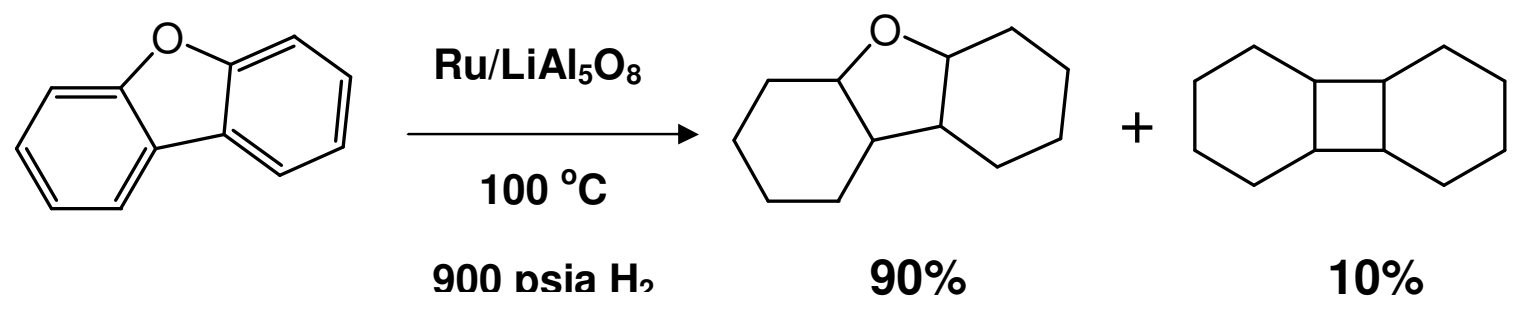

Scheme 7-1. Hydrogenation of dibenzofuran.

Our initial dehydrogenation testing of perhydrodibenzofuran utilized of a set of catalysts previously used for the dehydrogenation of perhydro-N-ethylcarbazole (Figure 7-2). The testing was designed to allow for the comparison of activity of each catalyst for dibenzofuran vs. N-ethylcarbazole to see if the order of catalyst activity was the same for the two carrier molecules. In general, the catalysts that were most effective at dehydrogenation of perhydro-N-ethylcarbazole did not work well with perhydrodibenzofuran. Of the 5 catalysts screened, palladium on carbon and palladium on alumina showed the best rates. The one platinum-based catalyst that was tested showed a high initial dehydrogenation rate, but the activity decreased rapidly after ca. $15 \%$ conversion of perhydrodibenzofuran. As expected, the dehydrogenation conversions of dibenzofuran (avg. $\Delta \mathrm{H}_{\mathrm{D}}=57 \mathrm{~kJ} / \mathrm{mol} \mathrm{H}_{2}$ ) with the various catalysts at $200^{\circ} \mathrm{C}$ were low when compared with perhydro- $\mathrm{N}$-ethylcarbazole (avg. $\Delta \mathrm{H}_{\mathrm{D}}=51 \mathrm{~kJ} / \mathrm{mol} \mathrm{H}_{2}$ ). 


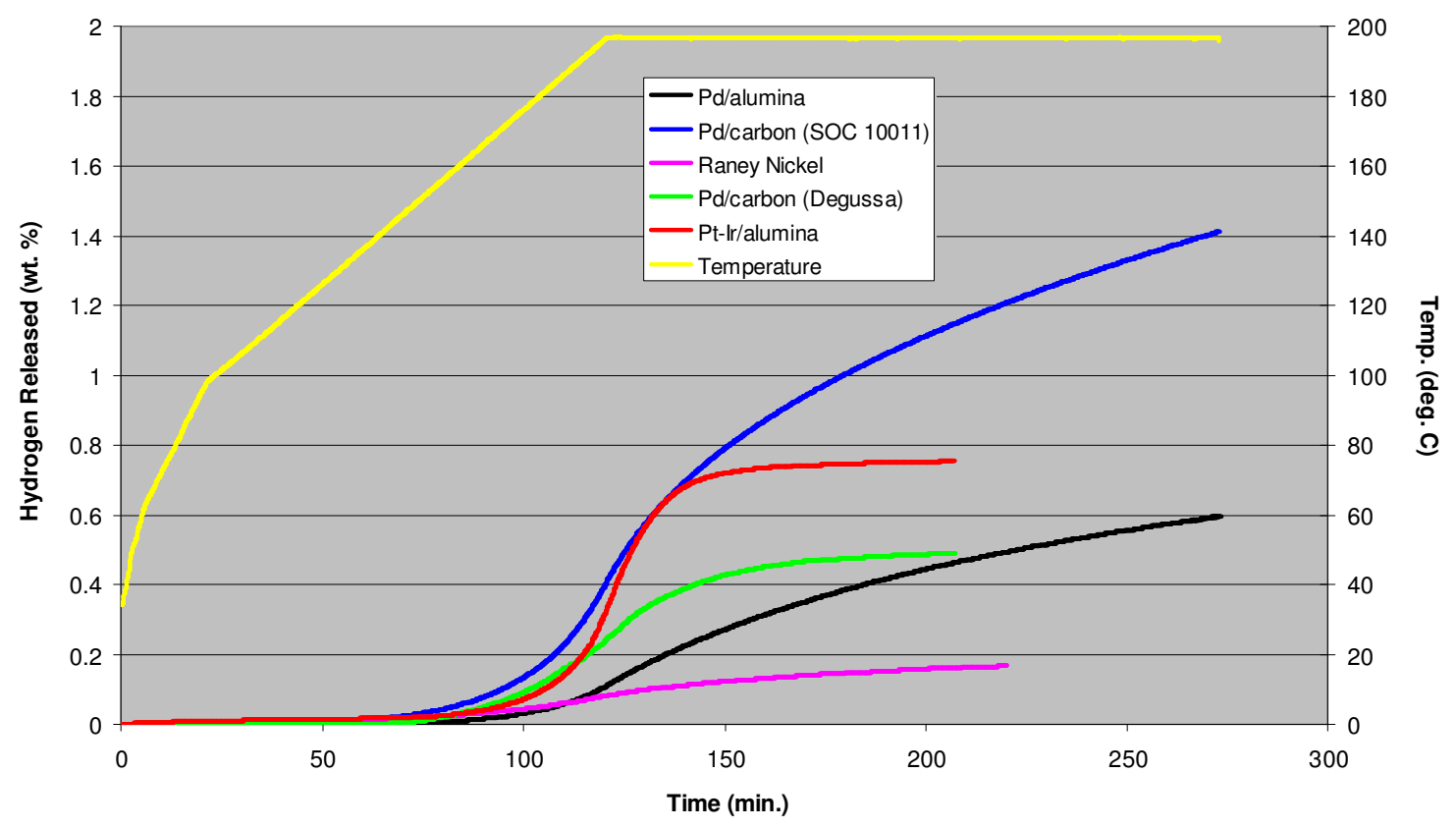

Figure 7-2. Catalyst rate comparison for the dehydrogenation of perhydrodibenzofuran.

Analysis of the perhydrodibenzofuran dehydrogenation products by GC-MS showed the fully dehydrogenated product, partially dehydrogenated products (loss of two, three and four molecules of hydrogen) and, interestingly, multiple isomers of perhydrodibenzofuran, even though the starting material was composed of only one isomer. No bond cleavage or hydrogenolysis products were observed after dehydrogenation at a maximum temperature of $200^{\circ} \mathrm{C}$. 


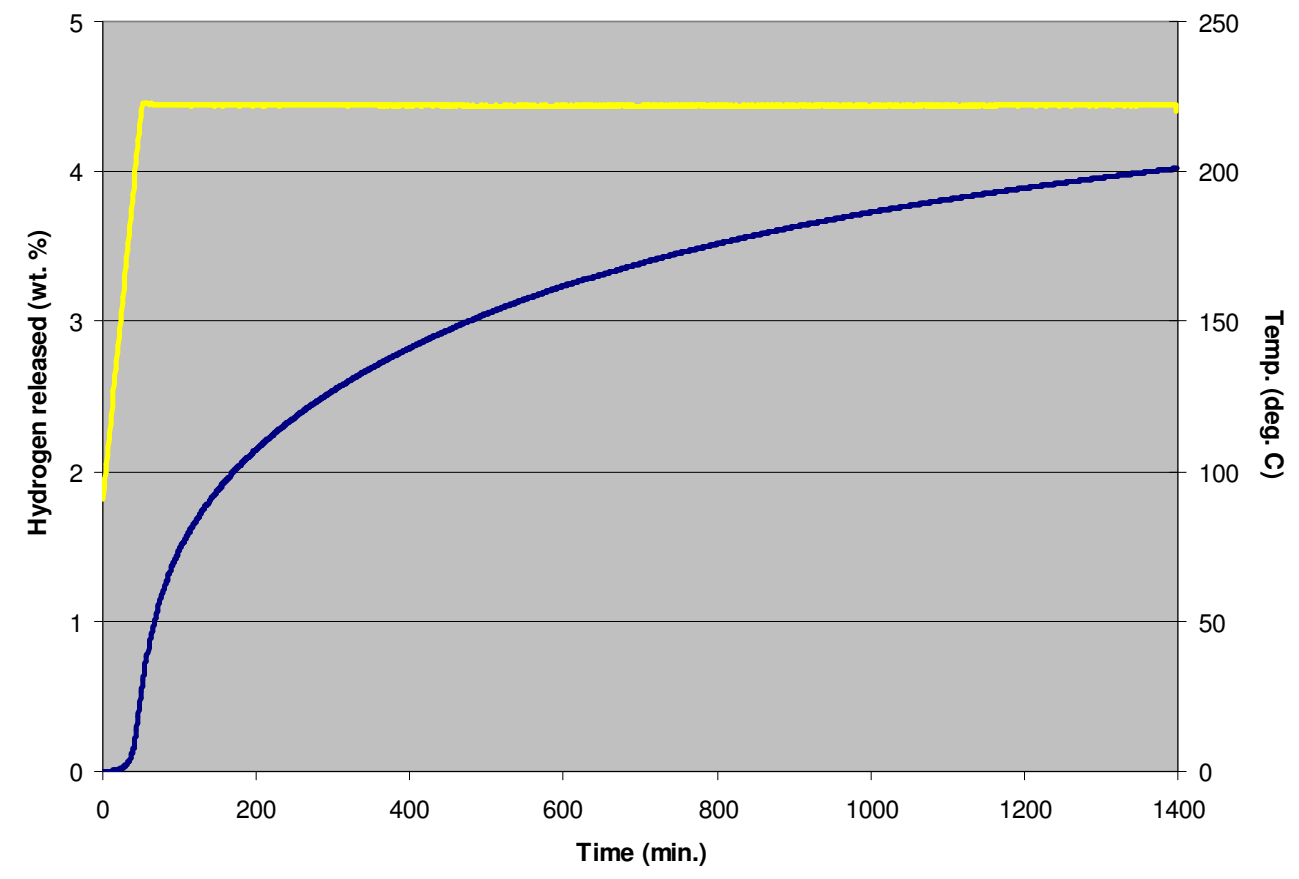

Figure 7-3. Dehydrogenation of perhydrodibenzofuran with palladium on carbon.

Since the dehydrogenation rates were slow at $200^{\circ} \mathrm{C}$, we investigated higher dehydrogenation temperatures. Dehydrogenation of perhydrodibenzofuran with $4 \%$ palladium on carbon under $1 \mathrm{~atm}$. of hydrogen yielded $4 \mathrm{wt}$. \% of hydrogen after ca. 1400 minutes at $225^{\circ} \mathrm{C}$ (Figure 7-3). GC-MS analysis of the products showed a mixture consisting of dibenzofuran (39\%), octahydrodibenzofuran (8\%), hexahydrodibenzofuran (2\%), tetrahydrodibenzofuran (13\%) and perhydrodibenzofuran $(38 \%)$ as a mixture of four isomers. No bond cleavage or hydrogenolysis products were observed after dehydrogenation at a maximum temperature of $225^{\circ} \mathrm{C}$.

\section{B. N-methylphenoxazine}

Phenoxazine is a commercially available chemical compound that contains a single oxygen heteroatom (ether group) and a nitrogen heteroatom (secondary amine group). The average energy of hydrogenation for the $\mathrm{N}$-methyl derivative was calculated to be 
$63 \mathrm{~kJ} / \mathrm{mol} \mathrm{H}_{2}$, and the loss of hydrogen in two steps of 3 equivalents of $\mathrm{H}_{2}$ yields calculated intermediate energies that are favorably consistent (Scheme 7-2).<smiles>CCCCc1ccc2c(c1)OC1CCCCC1N(C)N2c1ccc2c(c1)N(C)c1ccccc1O2</smiles>

Scheme 7-2. Stepwise dehydrogenation of perhydro-N-methylphenoxazine.

The N-methylation of phenoxazine was performed with a standard alkylation procedure using iodomethane (Scheme 7-3). The crude N-methylphenoxazine was hydrogenated with high selectivity and yield to perhydro-N-methylphenoxazine using ruthenium on lithium aluminate at $120^{\circ} \mathrm{C}$.<smiles>c1ccc2c(c1)Nc1ccccc1O2</smiles><smiles>COc1ccc2c(c1)N(C)c1ccccc1O2</smiles>

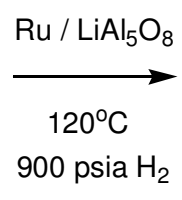<smiles>CN1C2CCCCC2OC2CCCCC21</smiles>

Scheme 7-3. Alkylation and hydrogenation of phenoxazine.

Dehydrogenation of perhydro-N-methylphenoxazine using $5 \%$ palladium on carbon resulted in the generation of hydrogen and yielded some fully dehydrogenated $\mathrm{N}$-methylphenoxazine and hexahydro-N-methylphenoxazine. Unfortunately, the dehydrogenation also yielded byproducts resulting from C-O bond hydrogenolysis, the primary byproduct being diphenylmethylamine (Scheme 7-4). 
<smiles></smiles>

Scheme 7-4. Dehydrogenation of perhydro-N-methylphenoxazine.

\section{C. $9(10 \mathrm{H})$-acridanone}

The molecule $9(10 \mathrm{H})$-acridanone has a structure similar to phenoxazine. The substitution of the ether oxygen atom for a carbonyl group offers an additional site for reversible hydrogenation, increasing the theoretical hydrogen capacity relative to phenoxazine. Since $9(10 \mathrm{H})$-acridanone has a very high melting point $\left(>300^{\circ} \mathrm{C}\right)$, we explored the alkylation of the nitrogen atom to lower the melting point. $\mathrm{N}$ ethylacridanone (m.p. $150^{\circ} \mathrm{C}$ ) was synthesized using one of our standard alkylation procedures (Scheme 7-5).<smiles>CCn1c2ccccc2c(=O)c2ccc(O)cc21</smiles>

Scheme 7-5. Synthesis of N-ethylacridanone.

Unfortunately, attempts to selectively hydrogenate $\mathrm{N}$-ethylacridanone typically resulted in a mixture of products including $\mathrm{N}$-ethylacridine (produced from hydrogenolysis of the C-O bond). Despite the production of some of the desired perhydroacridinol, the low hydrogenation selectivity (Scheme 7-6) resulted in our termination of any additional research on this carrier candidate. 


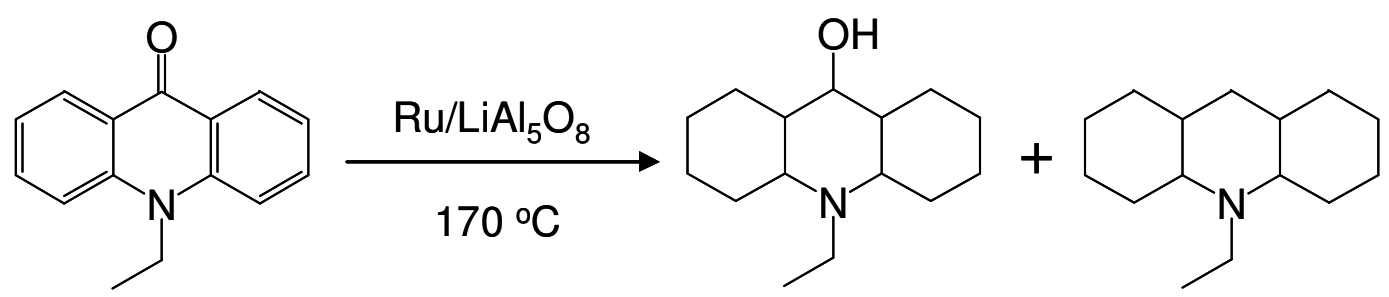

Scheme 7-6. Hydrogenation of N-ethylacridanone.

\section{2-ethylanthraquinone}

The compound 2-ethylanthraquinone is readily reduced to the diol and oxidized to the quinone in the commercial production of hydrogen peroxide. Attempts to hydrogenate 2-ethylanthraquinone under our standard hydrogenation conditions $\left(\mathrm{Ru} / \mathrm{LiAl}_{5} \mathrm{O}_{8}, 800 \mathrm{psia}\right.$ $\mathrm{H}_{2}, 115-170^{\circ} \mathrm{C}$ ) resulted in significant amounts of $\mathrm{C}-\mathrm{O}$ bond cleavage byproducts. However, using $5 \% \mathrm{Pd} / \mathrm{C}$ at $70^{\circ} \mathrm{C}$, we were able to partially hydrogenate 2-ethylanthraquinone with significantly less bond cleavage, but at the expense of high conversion (Scheme 7-7).<smiles>CCc1ccc2c(c1)C(=O)c1ccccc1C2=O</smiles>

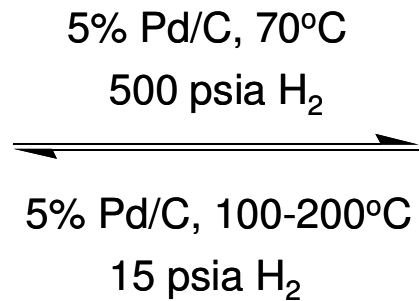<smiles>CCc1ccc2c(O)c3c(c(O)c2c1)CCCC3</smiles>

Scheme 7-7. Reversible hydrogenation of 2-ethylanthraquinone.

The partially hydrogenated 2-ethylanthraquinone diol was handled under an inert atmosphere, since it readily oxidizes to the quinone in air. The low hydrogenation conversion could not be improved beyond tetrahydroanthrahydroquinodiol without hydrogenolysis. Nevertheless, we wanted to determine if we could find conditions that would convert the diol back to the quinone by dehydrogenation. 
We mixed tetrahydroanthrahydroquinodiol with $5 \% \mathrm{Pd} / \mathrm{C}$ and very slowly heated the reaction mixture to $200^{\circ} \mathrm{C}$ under one atmosphere of hydrogen. The amount of evolved hydrogen was very low, and GC-MS analysis showed only $5 \%$ conversion to 2-ethylanthraquinone. Due to the high temperature necessary for this conversion, some hydrogenolysis via dehydration occurred. Since we were unable to completely avoid hydrogenolysis during hydrogenation or dehydrogenation, we discontinued studying this carrier candidate.

\section{E. Xanthrone}

Xanthrone is structurally similar to anthraquinone, but contains only one carbonyl group. In an effort to compare the hydrogenolysis reactivity with other carbonyl-containing substrates, we investigated the hydrogenation of xanthrone. As was the case with the other carbonyl-containing substrates, xanthrone could not be hydrogenated with high selectivity. With xanthrone we found two operative bond cleavage reactions:

1) $\mathrm{C}=\mathrm{O}$ cleavage and 2) $\mathrm{C}-\mathrm{O}$ cleavage of the ether group (Scheme 7-8).

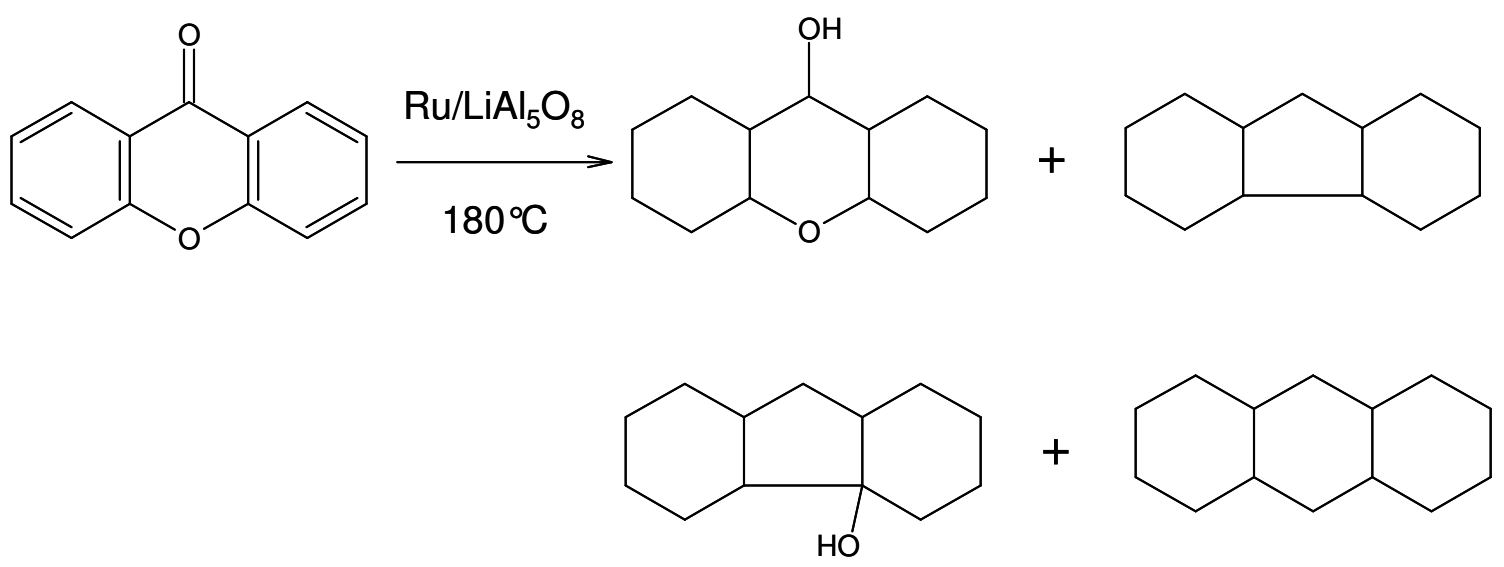

Scheme 7-8. Hydrogenation of xanthrone.

Dehydration of perhydroxanthrol or partially hydrogenated alcohol intermediates appears to be the usual route of oxygen atom loss. The hydrogenation conditions that were investigated ranged from a temperature of $23^{\circ} \mathrm{C}$, where no hydrogenation takes place, to 
temperatures as high as $180^{\circ} \mathrm{C}$. Raney nickel, ruthenium on alumina, and the less active hydrogenation catalyst palladium on carbon were tested for the hydrogenation of neat xanthrone and xanthrone dissolved in ethanol. The best conversion to fully saturated product was obtained with $\mathrm{Ru} / \mathrm{LiAl}_{5} \mathrm{O}_{8}$ at $180^{\circ} \mathrm{C}$. However, the selectivity was only approximately $50 \%$, yielding a number of byproducts from $\mathrm{C}-\mathrm{O}$ bond cleavage.

\section{F. Benzophthalimide}

Benzophthalimide is a commercially available substrate that has a moderate hydrogen capacity if the amide groups are reversibly hydrogenated. Under our standard hydrogenation conditions, we were able to obtain a high yield of hexahydrobenzophthalimide (Scheme 7-9).

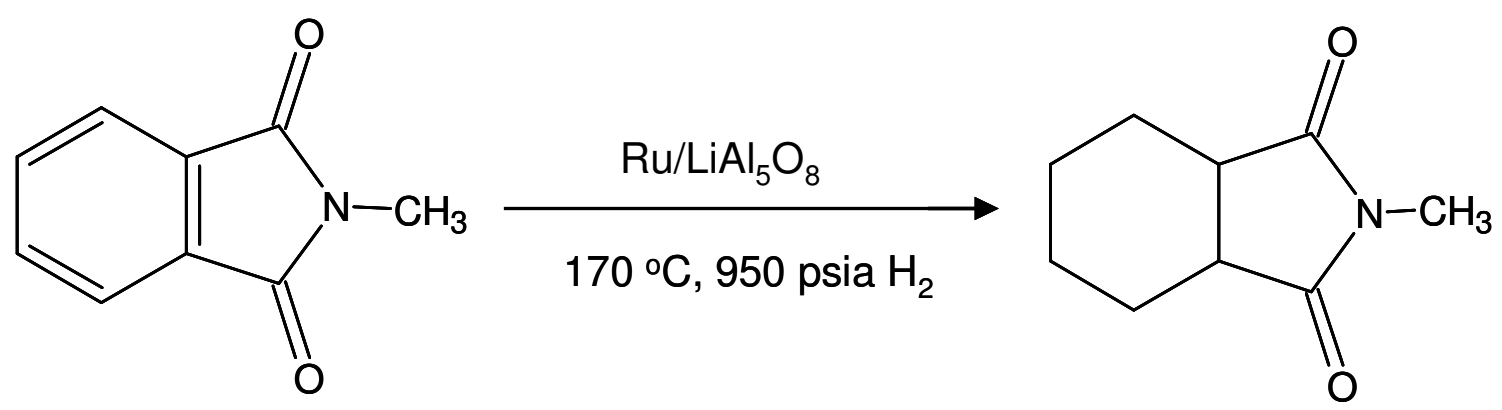

Scheme 7-9. Hydrogenation of benzophthalimide.

The six-membered aromatic ring readily hydrogenates, leaving the carbonyl groups unreduced. While amides are generally difficult to hydrogenate, we thought the phthalimide carbonyls may be less electron rich and perhaps susceptible to hydrogenation. Because of the low hydrogen capacity of hexahydrobenzophthalimide, we did not pursue dehydrogenation testing of this compound. 


\section{G. Phthalic anhydride}

Phthalic anhydride is structurally similar to benzophthalimide. Under hydrogenation testing conditions identical to those employed for benzophthalimide, we found that the anhydride was prone to $\mathrm{C}=\mathrm{O}$ bond cleavage, yielding a lactone product (Scheme 7-10).

Reducing the temperature during the hydrogenation reaction resulted in reduced $\mathrm{C}=\mathrm{O}$ bond cleavage, but also lower conversion to hydrogenated products.

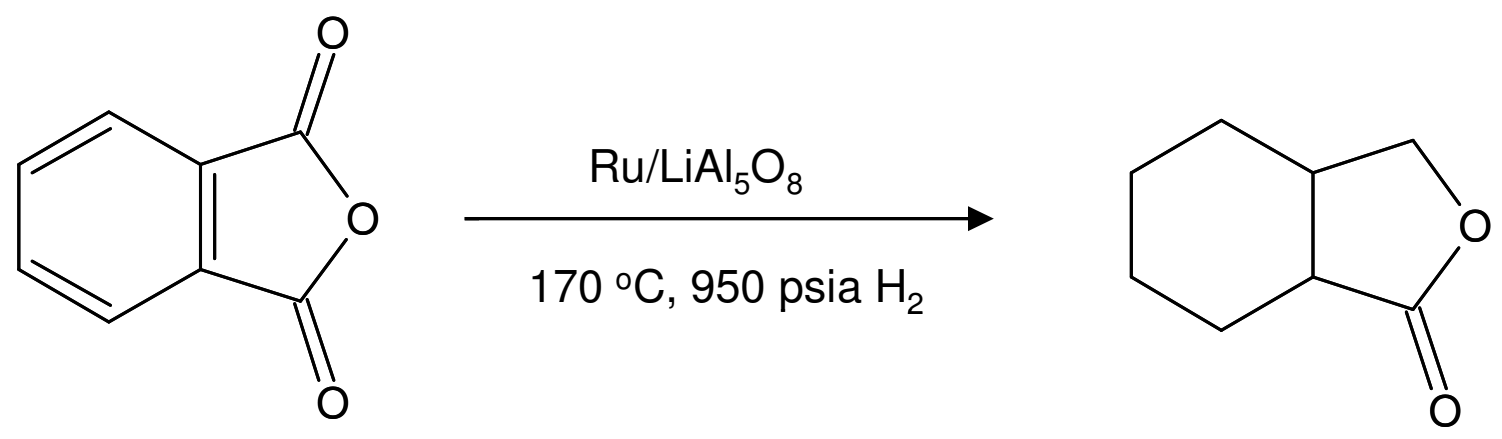

Scheme 7-10. Hydrogenation of phthalic anhydride.

\section{H. Benzo-1,3-dioxofuran}

The molecule benzo-1,3-dioxofuran (Figure 7-4) has a moderate theoretical hydrogen capacity due to the weight of the $\mathrm{O}-\mathrm{CH}_{2}-\mathrm{O}$ moiety. However, we anticipated that the heat of hydrogenation may be low due to the presence of two heteroatoms and the fused five-membered ring. This motivated a brief study of this potential liquid carrier.

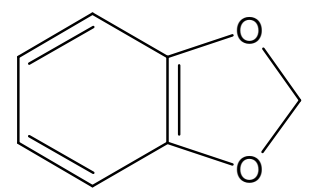

Figure 7-4. Structure of benzo-1,3-dioxofuran. 
Hydrogenation testing using our standard conditions demonstrated that this molecule was resistant to hydrogenation. It is possible that the heat of hydrogenation is too low to allow significant conversion under our usual conditions of temperature and hydrogen pressure. We thus attempted to hydrogenate this carrier under acidic conditions used for other liquid carrier candidates with a low $\Delta \mathrm{H}$ (e.g., Rh black, aqueous $\mathrm{HCl}, 100$ psig $\mathrm{H}_{2}, 80-150^{\circ} \mathrm{C}$ ), but observed no conversion to hydrogenated products.

\section{Conclusions}

The oxygen atom-containing liquid carrier candidates tested were generally unsuccessful due to issues with $\mathrm{C}-\mathrm{O}$ bond hydrogenolysis. Moderate hydrogenation conditions often resulted in incomplete conversion, while more aggressive conditions inevitably resulted in poor selectivity.

One oxygen-containing heterocycle was successful and should merit additional consideration as a liquid carrier candidate. Dibenzofuran was hydrogenated with high yield and selectivity using catalysts and conditions amenable to large-scale industrial hydrogenation. The dehydrogenation of perhydrodibenzofuran was conducted at moderate temperatures (e.g., $200-225^{\circ} \mathrm{C}$ ) and resulted in good selectivity and low-tomoderate conversions. It is likely that some performance improvement could be realized by additional research. Optimization of the catalytic hydrogenation could involve exploring methods for producing specific destabilized isomers of perhydrodibenzofuran. Additional dehydrogenation catalyst testing might yield increased rates while maintaining high selectivity.

\section{Experimental Details}

See Appendix B for general experimental details. 


\section{Reversible Hydrogenation of Dibenzofuran}

A $100 \mathrm{cc}$ stainless steel pressure reactor was loaded with $50 \mathrm{~g}$ dibenzofuran and $5.0 \mathrm{~g}$ of $5 \%$ ruthenium on lithium aluminate. After the headspace was purged with hydrogen, the hydrogen pressure was increased to $900 \mathrm{psia}$. The reactor was heated to $100^{\circ} \mathrm{C}$. After 4 hours, the reactor was cooled to $25^{\circ} \mathrm{C}$, and the contents filtered to remove catalyst. GC-MS analysis showed complete conversion of dibenzofuran to perhydrodibenzofuran $(86 \%)$ and dodecahydrobiphenylene (14\%). The GC/MS analysis also revealed that the perhydrodibenzofuran was present in the hydrogenated mixture as two different isomers that were resolved on the GC column in the ratio of 60:1. The reaction mixture was distilled at $89^{\circ} \mathrm{C}$ at a pressure of $8 \times 10^{-2}$ torr to yield a distillate that was $>99 \%$ perhydrodibenzofuran. The perhydrodibenzofuran was degassed by evacuation $\left(1 \times 10^{-3}\right.$ torr) at $20^{\circ} \mathrm{C}$ for 20 minutes. Under inert atmosphere, $4.0 \mathrm{~g}$ perhydrodibenzofuran and $0.1 \mathrm{~g}$ of $4 \%$ palladium on carbon (dehydrogenation catalyst) were placed in a $20 \mathrm{cc}$ stirred tank reactor and the reactor sealed. The reactor was connected to a manifold containing a vacuum source, hydrogen source, and a flow measurement system consisting of calibrated 10 and $100 \mathrm{sccm}$ flow meters in series. After evacuation of residual air from the manifold lines, hydrogen was purged through the reactor to displace the argon from the reactor headspace. The reactor was heated to $225^{\circ} \mathrm{C}$ at a rate of $3.75^{\circ} \mathrm{C} / \mathrm{min}$. with stirring $(500 \mathrm{rpm})$ under 15 psia hydrogen. After 1400 minutes at $220^{\circ} \mathrm{C}, 4 \mathrm{wt}$ \% of hydrogen was desorbed. After the reactor was cooled to $25^{\circ} \mathrm{C}$ and filtering was conducted to remove the catalyst, GC-MS analysis of the dehydrogenated liquid showed the presence of dibenzofuran (39\%), octahydrodibenzofuran $(8 \%)$, hexahydrodibenzofuran ( $2 \%)$, tetrahydrodibenzofuran (13\%) and perhydro-dibenzofuran (38\% as a mixture of four isomers).

\section{References}

1. Karvembu, R.; Priyarega, S. React. Kinet. React. Lett. 88, 333 (2006). 


\section{Chapter 8. Amine/Nitrile Couples as Potential Carriers}

\section{Introduction}

The catalytic hydrogenation of alkyl and aromatic nitriles to primary amines is well known. However, there is only scant literature relating to the reverse process, the selective dehydrogenation of the amines. This reaction could in principle allow a greater than 1:1 H:C hydrogen storage capacity for the liquid carrier. The thermodynamic barrier for catalytically releasing hydrogen from amines is greater than cyclohexane to benzene $\left(\Delta \mathrm{H}_{\mathrm{D}}{ }^{\circ}>67 \mathrm{~kJ} / \mathrm{mole} \mathrm{H}_{2}\right.$, see Table $1 \mathrm{~d}$, Appendix A). Nevertheless, we investigated primary amine/nitrile pair molecules for hydrogen storage with the promise of higher capacities, while recognizing the need for generally high dehydrogenation temperatures.

There are reports of a dehydrogenation of low MW aliphatic primary amines to alkylnitriles in gas phase reactions at relatively high temperatures $\left(\sim 400^{\circ} \mathrm{C}\right)$ over acid-base functional catalysts (e.g., $\mathrm{ZrO}_{2}$ ) with olefins, and secondary amines are substantial byproducts. ${ }^{1}$ At dilute gas-phase conditions $n$-Butylamine is dehydrogenated at $400^{\circ} \mathrm{C}$ over the $\left(\mathrm{H}_{3} \mathrm{O}\right)_{2}\left[\left(\mathrm{Mo}_{6} \mathrm{Cl}_{8}\right) \mathrm{Cl}_{3}\right]^{\cdot} 6 \mathrm{H}_{2} \mathrm{O}$ cluster catalyst to propionitrile, with the imine $\mathrm{PrCH}=\mathrm{NBu}$ as a major byproduct. ${ }^{2}$ There is a very brief report of the conversion of a solution of benzylamine in toluene to benzonitrile at $110^{\circ} \mathrm{C}$ under a flow of $\mathrm{N}_{2}$ in the presence of a homogeneous catalyst $\left[\mathrm{RhH}\left(\mathrm{P}^{\mathrm{i}} \mathrm{P}_{\mathrm{r}}\right)_{3}\right]$. Our goal in the present work is to find bulk (i.e., undiluted) liquid phase primary amine systems that could be selectively dehydrogenated to nitriles. We know of no examples of this process. The reverse process, the rehydrogenation of a nitrile to the amine, was considered to be well known art and was thus not investigated.

\section{$\underline{\text { Results and Discussion }}$}

Aromatic and heteroaromatic nitrile couples were evaluated for use as potential hydrogen carriers (Scheme 8-1). These candidates possess several potential advantages over other classes of carriers, including a very high capacity (>7 wt. \%) and ready availability. 
<smiles>N#Cc1cccc(C#N)c1</smiles>

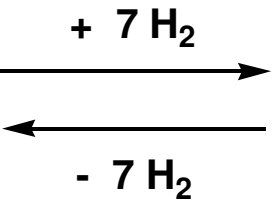<smiles>NCC1CCCC(CN)C1</smiles>

\section{$\Delta \mathrm{E}=85 \mathrm{~kJ} / \mathrm{mol} \mathrm{H}$; $9.87 \%$ capacity}<smiles>N#Cc1ccncc1</smiles>

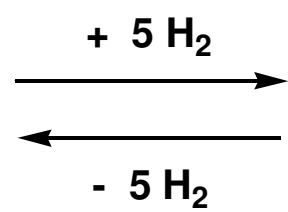<smiles>NCC1CCNCC1</smiles>

$\Delta \mathrm{E}=82 \mathrm{~kJ} / \mathrm{mol} \mathrm{H}_{2}$; $8.77 \%$ capacity

Scheme 8-1. (Hetero)aromatic nitriles as potential hydrogen carriers (average electronic energy [Appendix $\mathrm{A}] \Delta \mathrm{E}$ shown in $\mathrm{kJ} / \mathrm{mol} \mathrm{H}_{2}$ ).

Dehydrogenation of benzylamine and m-xylenediamine with nickel catalysts has been shown to proceed with deamination to form dibenzylamine and oligomeric xylylamines, respectively. ${ }^{4}$ Further, hydrogenation of aromatic and heteroaromatic nitriles to the corresponding aminomethyl compounds is known to be accompanied by deamination to the corresponding methyl (hetero)aromatic. ${ }^{5,6}$ This observation probably derives from formation and subsequent hydrogenolysis of the aromatic methylamine (Equation 1). The hydrogenolytic lability of benzylamines has been exploited extensively in their use as protecting groups for amines in many syntheses of complicated organic molecules. ${ }^{7}$

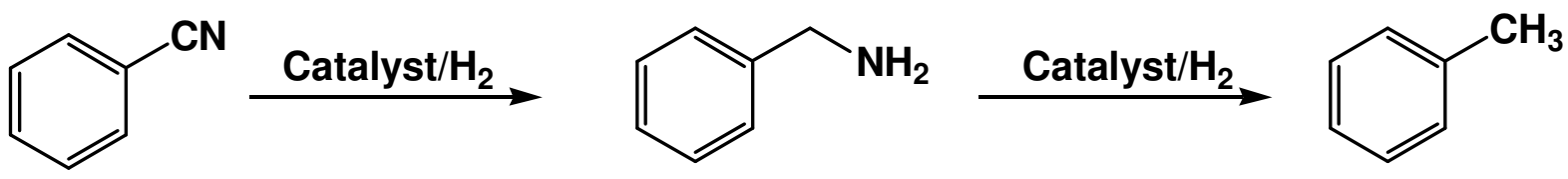

In an effort to control condensation reactions during dehydrogenation, our first step was to evaluate lithium-modified catalysts for dehydrogenation of bis(aminomethyl)cyclohexane (BAC) to 1,3-dicyano-benzene (Equation 2). 

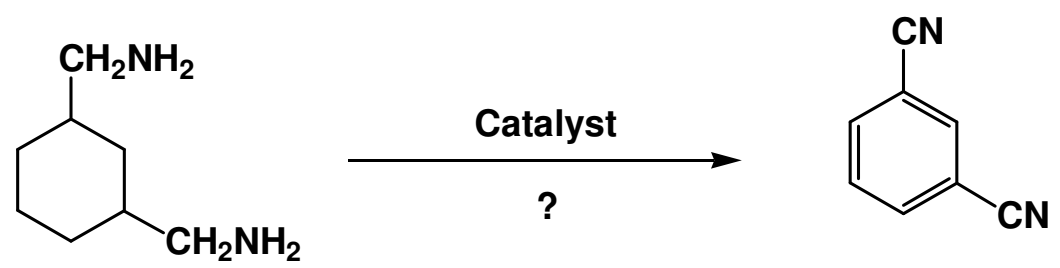

This reaction was chosen to try to provide a realistic test of the catalysts' ability to control condensation and hydrogenolysis reactions without the added complexities (particularly the high electrophilicity of the nitrile group, which further facilitates condensation reactions) that would be introduced by consideration of heteroaromatic systems. The two initial sets of catalysts that were tested were metals on a lithium-containing support (e.g., Ru, Pd, Pt on lithium aluminate) and $\mathrm{Pt}$ on alumina catalysts that were modified by addition of varying amounts of lithium. In the first dehydrogenation, $\mathrm{BAC}$ was treated with $\mathrm{Pd} /$ lithium aluminate at $200^{\circ} \mathrm{C}$. Although conversion of BAC was high (>95\%), only $30 \%$ of the theoretical hydrogen capacity was evolved. Analysis by Chemical lonization Mass Spectrometry (CI MS; perdeuteroammonia as the ionizing agent) showed that no nitrile-containing products resulted from the attempted dehydrogenation. Instead, two heterocyclic amines derived from BAC by ring closure of an intermediate imine and subsequent hydrogenolysis and dehydrogenation were formed (Scheme 2). MALDI MS showed that extensive oligomerization had also occurred; results are summarized in Scheme 8-2.

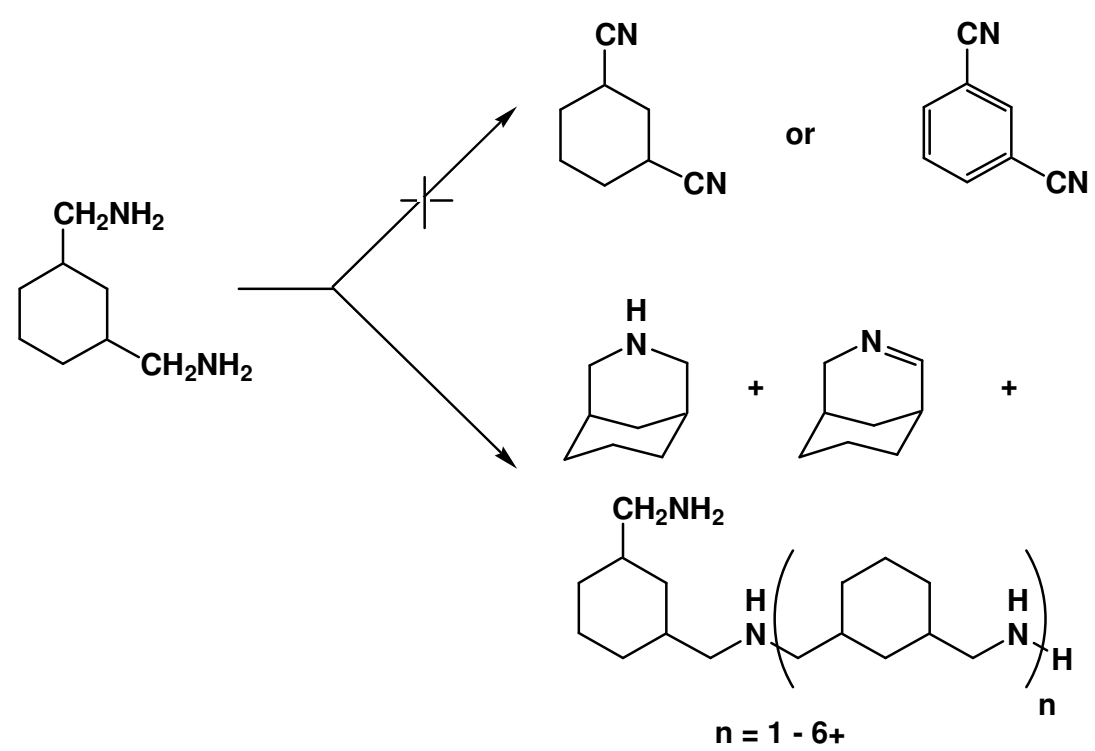

Scheme 8-2. Dehydrogenation of bis(aminomethyl)cyclohexane. 
Very similar results were obtained with all of the catalyst candidates. These included Pt/alumina (with no added lithium) and Pt/alumina with three concentrations of added Li (0.005 wt. \%, 0.05 wt. \%, and 0.5 wt. \%; all as lithium hydroxide, and all added by the incipient wetness technique). Despite varying the concentration of post-added lithium over three decades, no significant effect was observed on the selectivity of dehydrogenation. To determine whether lithium incorporated into the support might be more effective than post-added lithium, Pt/lithium aluminate was tested. Further, since ruthenium gives high selectivities to primary amines in the hydrogenations of dinitriles, ${ }^{8} \mathrm{Ru} /$ /ithium aluminate was also evaluated to determine whether it might provide improved selectivity for the reverse reaction. No significant improvement over the above results was found with either of the latter catalyst candidates.

To avoid the formation of byproducts via intramolecular cyclization, dehydrogenation of metaxylenediamine (MXDA) was evaluated (Equation 3). With this substrate, intramolecular cyclization is not possible.
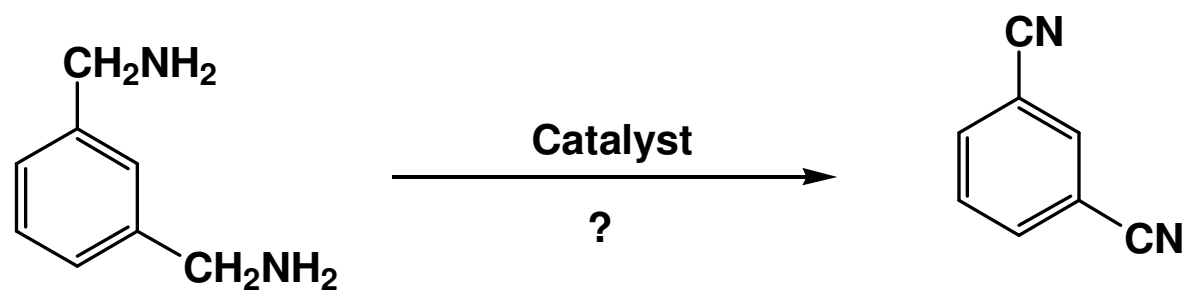

Under typical dehydrogenation conditions $\left(150^{\circ} \mathrm{C}\right)$ with palladium/lithium aluminate, no nitrilecontaining products were found. Instead, deamination and/or oligomerization intervened (Scheme 8-3). 

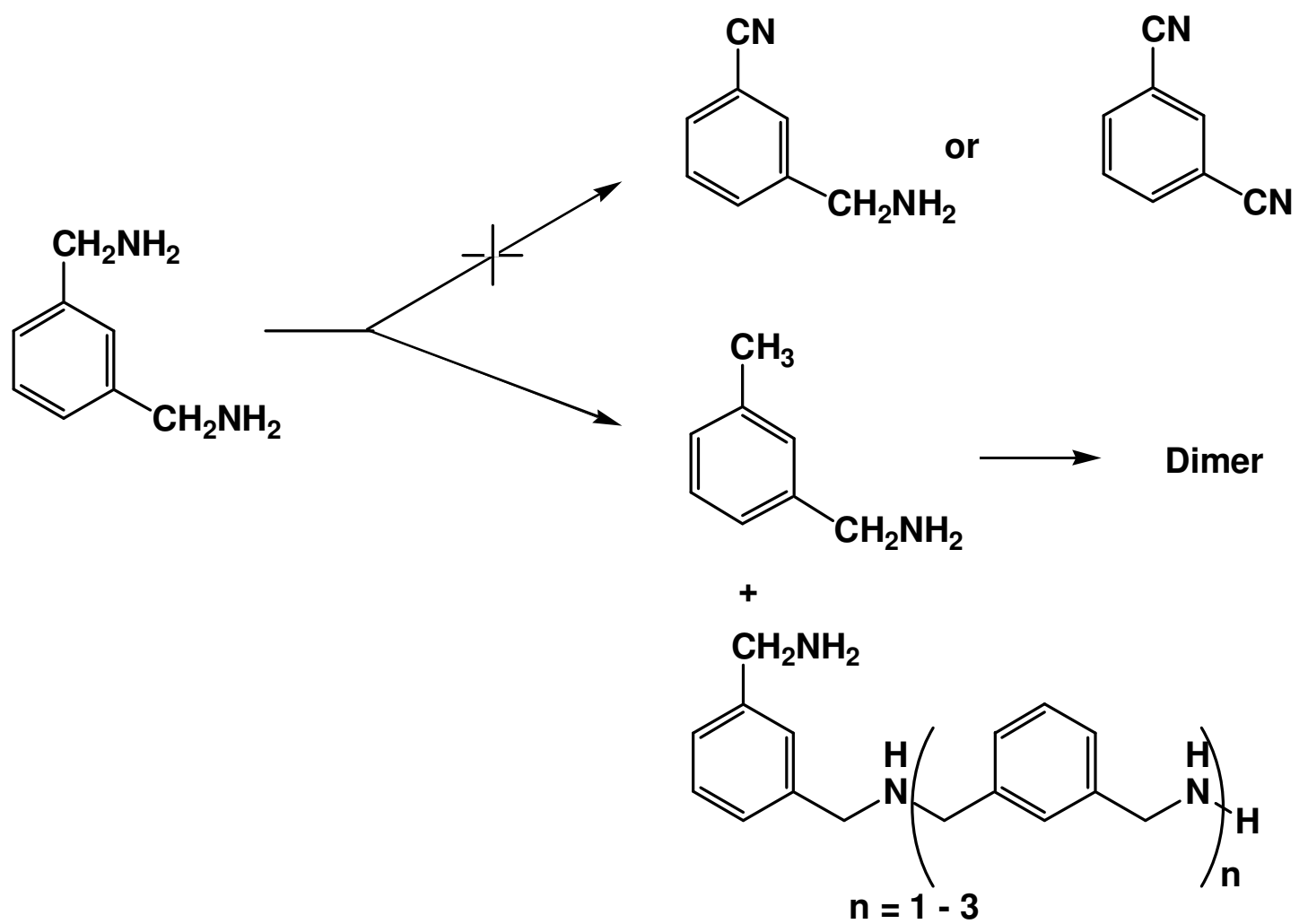

Scheme 8-3. Dehydrogenation of m-xylenediamine.

In addition to supported catalysts, sponge metal catalysts are also useful for selective hydrogenations. Specifically, chromium-promoted sponge cobalt is used for selective hydrogenation of nitriles. ${ }^{9}$ To evaluate whether this might also function as a selective dehydrogenation catalyst, a representative type was tested for dehydrogenation of MXDA. Using Raney ${ }^{\circledR} 2724$, a commercial chromium-promoted sponge cobalt catalyst, a somewhat higher reaction temperature $\left(200^{\circ} \mathrm{C}\right)$ was required to achieve a reasonable conversion $(65 \%)$ of MXDA. However, no difference in selectivity between the latter reaction and the previous dehydrogenation with palladium/lithium aluminate was found.

Regardless of the substrate which was dehydrogenated, BAC or MXDA, no nitrile-containing products were formed. In both instances, competing pathways such as deamination, cyclization or cyclodimerization, and oligomerization intervened. These results clearly indicated that if an 
aminomethyl-containing substrate were to be successful as a hydrogen carrier, among other potential issues it should have only one aminomethyl group.

At this point, molecular modeling calculations were employed to better understand the underlying energetics for the dehydrogenation of amines to nitriles, with the expectation of finding substrate molecules for which this transformation was more likely to occur. Specifically, we sought to determine how the nature of the $\mathrm{R}$ group in the primary amine $\left(\mathrm{R}-\mathrm{CH}_{2} \mathrm{NH}_{2}\right)$ affects the energetics of amine dehydrogenation to a nitrile. As a first test case, the energetics for the stepwise dehydrogenation of n-propylamine and 3-amino-1-propene to the corresponding nitriles and intermediate imines were examined by comparison of the calculated energies. The results are shown in Scheme 8-4. The vinyl imine intermediate, 1, is stabilized vis-a-vis the saturated analog, $\mathbf{2}$, which renders it less energetically accessible from the amine and also facilitates a dehydrogenation to the nitrile.
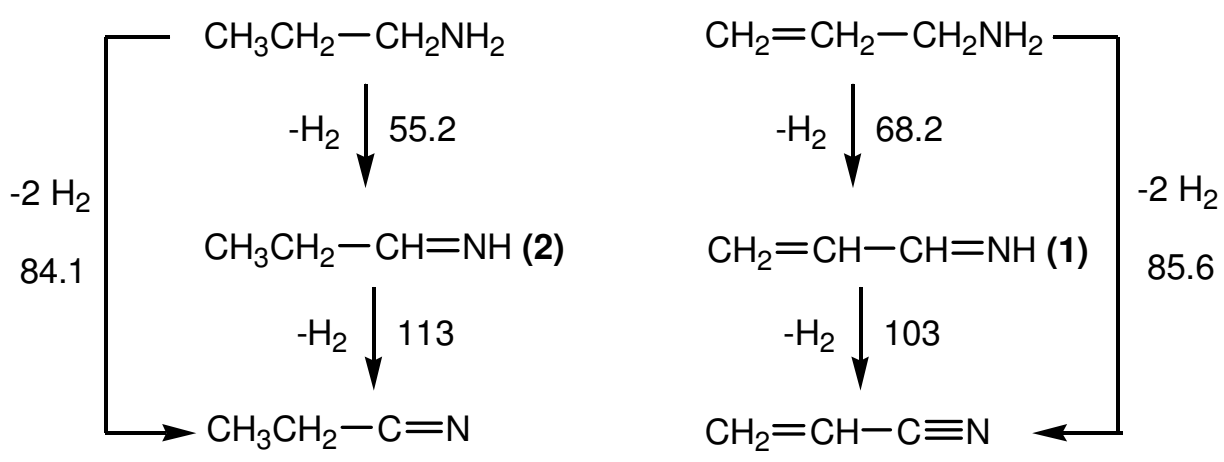

Scheme 8-4. Calculated electronic energies for the stepwise and overall conversion of n-propylamine and 3-amino-1-propene to the corresponding nitriles and the imine intermediates. Electronic energies (B3LYP functional with $6-311 \mathrm{G}^{* *}$ basis set) are all expressed in $\mathrm{kJ} / \mathrm{mole}_{2}$.

Considering the apparent advantage of an unsaturated functional group that is potentially highly conjugated with an imine, we chose to investigate the 3-methylamino/3-cyanoindole couple (Scheme 8-5). A more rapid dehydrogenation of the imine to the nitrile might be expected to diminish side reactions such as polymerization. Such reactions can occur spontaneously (as in 
the trimerization of 1-piperidine into its hexahydro-1,3,5 derivative ${ }^{12}$ ) or via nucleophilic attack on the imine carbon atom by species such as the precursor alkyl amine.

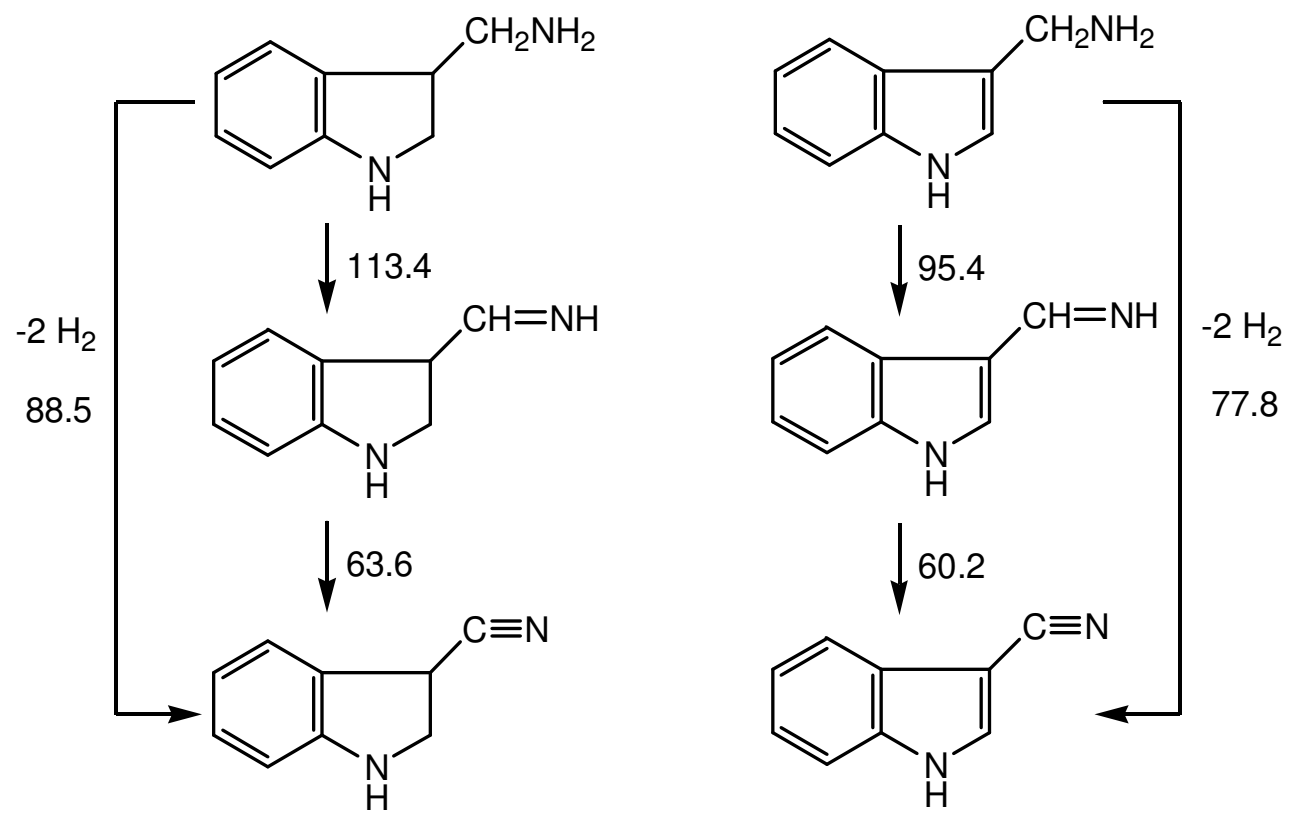

Scheme 8-5. Calculated electronic energies $\left(\mathrm{kJ} / \mathrm{mole}_{2}\right)$ for the stepwise and overall conversion of 3-aminomethyl indole and 3-aminomethyl indolene to the corresponding nitrile and imine intermediates.

From a comparison of the two aminomethyl group dehydrogenation sequences in Scheme 8-5, it is apparent that the indolyl functional group favorably lowers both reaction steps. The lowering of the first step most likely results from a decrease of the $\mathrm{C}-\mathrm{H}$ bond dissociation energy (BDE) of the amine (see Chapter 1 discussion on $\mathrm{C}-\mathrm{H}$ BDEs), with a stabilization of the imine intermediate accounting for a more facile dehydrogenation to the nitrile in the second step.

To experimentally test this hypothesis, preparation of 3-aminomethylindole (Figure 8-1) by lithium aluminum hydride reduction of 3-cyanoindole was attempted following a literature procedure. $^{10}$ Although conversion was complete, selectivity was poor $(<60 \%$; the same as that obtained in the literature), and isolation of the product was complicated by its air and base sensitivity. 


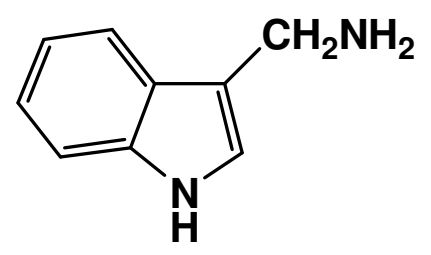

Figure 8-1. The molecular structure of 3-aminomethylindole (3).

As an alternative, 1-methyl-3-aminomethylindole (4) was proposed as a more tractable substrate (Figure 8-2).

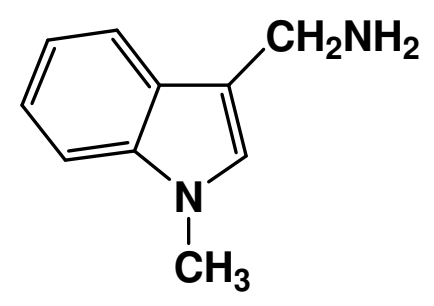

Figure 8-2. The molecular structure of 1-methyl-3-aminomethylindole (4).

Inclusion of the methyl group should improve base stability of the ring system and block a site for condensation with reactive intermediates during dehydrogenation. Preparation of the immediate precursor, N-methyl-3-cyanoindole (Figure 8-3), has been reported in high yield $(98 \%) .^{11}$

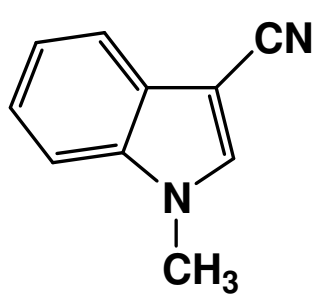

Figure 8-3. The molecular structure of N-methyl-3-cyanoindole (5). 
Not only was this procedure reproducible, but it could be simplified because the cosolvent, dimethylformamide (DMF), could be excluded without detriment. Exclusion of DMF simplified product isolation and avoided a material that would have poisoned the catalyst for subsequent hydrogenation of the nitrile to the amine. Conversion of $\mathbf{5}$ to $\mathbf{4}$ was readily accomplished with Raney ${ }^{\circledR} 2724$ in high conversion and selectivity (both $>98 \%$ ). While 4 is air sensitive and rapidly discolored on standing, it could be stored in a vacuum oven under nitrogen at ambient temperature for at least six days with minimal $(5 \%)$ decomposition. Upon dehydrogenation with palladium/lithium aluminate at $150^{\circ} \mathrm{C}$, small amounts of $\mathrm{N}$-methyl-3-methylindole $(<10 \%)$, 3 -methylindole $(<5 \%)$, and a dimer were formed. When the reaction was performed at $175^{\circ} \mathrm{C}$ to increase the conversion, $\mathrm{N}$-methyl-3-methylindole was the major product observed by GC analysis (85\%); lesser quantities of 3-methylindole (7\%) and $\mathrm{N}$-methyl-3-cyanoindole (5) $(6 \%)$ were also formed. Further, MALDI MS showed the presence of dimeric and trimeric species (e.g., see Scheme 8-6). This is the first time that an aminomethyl compound as a single phase has been dehydrogenated under hydrogen to the corresponding nitrile. However, owing to the low selectivity and multiplicity of side reactions such as hydrogenolysis of $\mathbf{4}$ to $\mathrm{N}$-methyl-3methylindole and 3-methylindole accompanied by condensation of $\mathbf{4}$ with the corresponding imine intermediate and subsequent loss of ammonia to yield $\mathbf{6}$, no further work was performed.

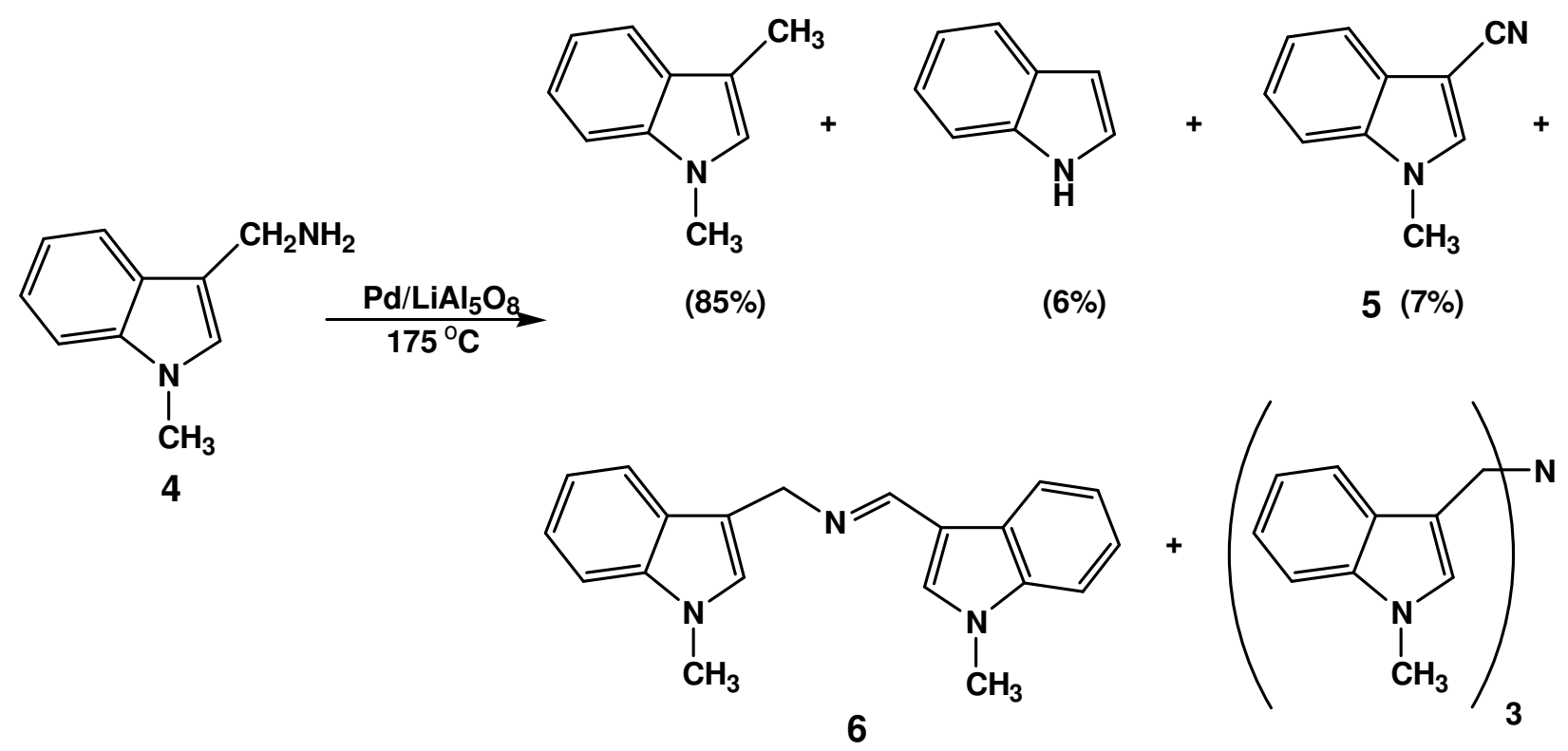

Scheme 8-6. Dehydrogenation of N-methyl-3-aminomethylndole. 


\section{Experimental Details}

\section{General Details}

Anhydrous tetrahydrofuran (under nitrogen), dimethyl carbonate, 1,4-diazabicyclo[2.2.2]octane, and 3-cyanoindole were obtained from commercial sources and used as received. Ruthenium on lithium aluminate catalyst ( $5 \mathrm{wt}$. \% rhodium loading) was obtained from Engelhard (product number SOC02152), as were $5 \%$ palladium on carbon and $5 \%$ palladium on lithium aluminate. Raney ${ }^{\circledR} 2724$ was obtained from W. R. Grace Company. ${ }^{1} \mathrm{H}$ and ${ }^{13} \mathrm{C}$ NMR spectra were recorded in solution in either deuterochloroform or perdeuteromethanol on a Bruker DMX-500 FT-NMR equipped with a $5 \mathrm{~mm}$ QNP probe. In all cases, the chemical shift was referenced to the solvent peak. Separations were effected on an Agilent 6890 Gas Chromatograph equipped with a fused silica RTX-35 column ( $30 \mathrm{~m} \times 0.25 \mathrm{~mm} ; 0.25 \mu \mathrm{m}$ film thickness). GC mass spectra were determined with a Finnigan SSQ7000 single quadrupole mass spectrometer; molecular weights and the number of nitrogen-bonded hydrogens were confirmed by chemical ionization (perdeuteroammonia). MALDI mass spectra were determined on a Bruker Biflex III using a dihydroxybenzoic acid matrix and no mass suppression.

\section{Platinum on alumina catalysts}

Platinum on alumina ( 5 wt. $\%$ platinum loading) was prepared by the incipient wetness method. ${ }^{13}$ Lithium (as lithium hydroxide; $0.005 \mathrm{wt} . \%, 0.05 \mathrm{wt} . \%$, and $0.5 \mathrm{wt} . \%$ ) was subsequently introduced onto portions of this catalyst, also by the incipient wetness method.

\section{Dehydrogenation procedures}

A $40 \mathrm{~mL}$ stainless steel reactor equipped with a Parr magnetic stirrer was charged with 4.0 grams of the desired substrate and $250 \mathrm{mg}$ of the dehydrogenation catalyst in a glove box. The reactor was assembled in the glove box, the hand valve was closed to mitigate exposure to air, and the reactor was removed from the glove box. After the reactor was tightened in a vise, it was connected to a gas manifold. Prior to opening the hand valve of the reactor, the connecting line was evacuated with a vacuum pump. Subsequently, the line was pressurized 
with 10 - 20 psig of hydrogen; this cycle was repeated twice and then the hand valve was opened. The reactor was pressurized to 200 psig with hydrogen and then vented; this cycle was repeated twice. After pressurization to 200 psig for the third time, the reactor was vented to 60 psig with hydrogen, isolated from the gas manifold, and valving redirected to allow the hydrogen to flow through a Brooks Model 5860 flow meter and controller to purge it. When all of the hydrogen was vented through the flowmeter, heating and stirring (500 rpm) were started. Hydrogen evolution was then monitored via the Brooks flowmeter. The temperature of dehydrogenation was chosen to maximize hydrogen evolution; reactions were allowed to run until hydrogen evolution was complete. Dehydrogenations of BAC were carried out at $200^{\circ} \mathrm{C}$ for 15 hours; those of MXDA were performed at $125-225^{\circ} \mathrm{C}$ for times ranging from $1.75-15$ hours, and those of $\mathrm{N}$-methyl-3-aminomethylindole were done at $150-175^{\circ} \mathrm{C}$ for $1-15$ hours.

\section{Methylation of 3-cyanoindole}

The procedure of reference 9 was followed, with omission of DMF. GC analysis of the reaction after $7 \mathrm{hrs}$. showed the presence of $\mathrm{N}$-methyl-3-cyanoindole (70\%) and unchanged 3-cyanoindole (30\%). After heating at $95^{\circ} \mathrm{C}$ for an additional 6 hours, only N-methyl-3cyanoindole was observed. Isolation via the procedure of reference 9 gave N-methyl-3cyanoindole $(1.04 \mathrm{~g}, 95 \%$ yield).

\section{Hydrogenation of N-methyl-3-cyanoindole}

A $100 \mathrm{~mL}$ Parr stainless steel reactor equipped with an internal cooling coil, a dip tube with a $0.5 \mu \mathrm{m}$ filter, and constant pressure gas delivery was charged with $0.05 \mathrm{~g}$ Raney ${ }^{\circledR} 2724$ (weighed with a pycnometer), $1 \mathrm{~g} \mathrm{~N}$-methyl-3-cyanoindole, and $65 \mathrm{~mL}$ tetrahydrofuran. The reactor was closed, purged with nitrogen and hydrogen, pressurized to 600 psig with hydrogen, and heated to $100^{\circ} \mathrm{C}$. Upon attaining $100^{\circ} \mathrm{C}$, the hydrogen pressure was increased to 1000 psig. The reaction was maintained at $100^{\circ} \mathrm{C} / 1000$ psig for $8 \mathrm{hrs}$. Cooling and filtration through the dip tube provided a solution of $\mathrm{N}$-methyl-3-aminomethylindole in tetrahydrofuran. Evaporation of the solvent under vacuum provided pure $\mathrm{N}$-methyl-3-aminomethylindole (0.99 g, 96\% yield). 


\section{References}

1. Xu, B.-Q.; Yamaguchi, T.; Tanabe, K. Applied Catalysis 1991, 75, 75.

2. Kamiguchi, S.; Nakanura, A.; Suzuki, A.; Kodomari, M.,; Nomura, M.; Iwasama, Y.;Chikara, T. J. of Catalysis 2005, 230, 204.

3. Yoshida, T.; Okano, T.; Otsuka, S. JCS Chem. Comm. 1979, 870.

4. Tashiro, M.; Mitsuno, S; Ito, M. Yuki Gosei Kagaku Kyokaishi, 1967, 25, 405.

5. Greenfield, H. Ind. Eng. Chem., Prod. Res. Dev. 1976, 15, 156.

6. Takagawa. M., U.S. Pat. No. 5,371,293 (Mitsubishi Gas Chemical Co., Inc.), 1994.

7. Protecting Groups, $3^{\text {rd }}$ Ed., P. J. Kocienski, Thieme, New York, 2005, pp. 570 - 585.

8. Catalytic Hydrogenation in Organic Syntheses, P. N. Rylander, Academic Press, New York, 1979, p. 142.

9. Johnson, T. A., U.S. Pat. No. 5,869,653 (Air Products and Chemicals), 1999.

10. Ho, K.-K.; Baldwin, J.J.; Bohnstedt, A.C.; Kultgen, S.G.; McDonald, E.; Gao, T.; Murphy, J.R.; Rankovic, Z.; Horlick, R.; Appell, K.C. U.S. Pat. No. 7,084,154 (Pharmacopeia Drug Discovery), 2006; procedure MM.

11. Shieh, W.-C.; Dell, S.; Bach, A.; Repic, O.; Blacklock, T. J. J. Org. Chem. 2003, 68, 1954.

12. DeKimpe, N.; Stevens, C. J. Org. Chem. 1993, 58, 2904.

13. Ding, H.; Armor, J.N.; Emig, L.A.; Ramprasad. D.; Vedage, G.A.; Wilhelm, F.C. U.S. Pat Np. 6,184,416 (Air Products and Chemicals), 2001, Example 7. 


\section{Chapter 9: Autothermal Hydrogen Storage and Delivery Employing Liquid Carriers}

\section{Introduction}

The widespread use of hydrogen as an energy carrier depends critically on devising technologies for the effective storage and delivery of hydrogen to a power source. Merchant hydrogen used in industrial processes is conveyed as a compressed gas or a cryogenic liquid, which are the current means for hydrogen storage in fuel cell and hydrogen internal combustion engine (ICE) prototype vehicles. There is continuing intense research on devising ways of storing hydrogen at higher effective densities by reversible containment in various carrier media. These carrier media comprise metal alloy hydrides, complex hydrides, metal amides and physical adsorbents such as high surface carbons and metal-organic framework materials. Of growing interest has been the storage of hydrogen via catalytic hydrogenation and dehydrogenation of liquid-phase organic molecule pairs (e.g., toluene/methylcyclohexane, naphthalene/decalin, and N-ethylcarbazole/perhydro-N-ethylcarbazole; the latter discussed in detail in Chapter 3).

The containment of hydrogen by any carrier media is accompanied by a generation of heat, while a corresponding input of thermal energy $\left(\Delta \mathrm{H}_{\mathrm{D}}\right.$, the heat of desorption), at the appropriate temperature, is necessary for hydrogen release. The $\Delta H_{D}$ is essentially the energy cost for storing the hydrogen and overcoming the entropy loss $\left(-\Delta S_{D}\right)$ in "condensing" or "fixing" the gas. These quantities are related by the familiar thermodynamic relationships in Equation 1, where $\Delta G_{D}$ is the Gibbs free energy for desorption, $R$ is the gas constant, and $K_{D}$ is the equilibrium constant for the reaction in Equation 2.

$$
\begin{aligned}
& \Delta \mathrm{G}_{\mathrm{D}}=-\mathrm{RT} \ln \mathrm{K}_{\mathrm{D}}=\Delta \mathrm{H}_{\mathrm{D}}-\mathrm{T} \Delta \mathrm{S}_{\mathrm{D}} \\
& \text { Hydrogenated Carrier } \stackrel{\mathrm{K}_{\mathrm{D}}}{\rightleftarrows} \text { Carrier }+\mathrm{H}_{2}
\end{aligned}
$$

For hydrogen carriers that operate via physical adsorption, the $\mathrm{H}_{2}$ molecule remains intact and retains some mobility in the adsorbed state. In the adsorbed state, $\Delta S_{D}$ is of the order of

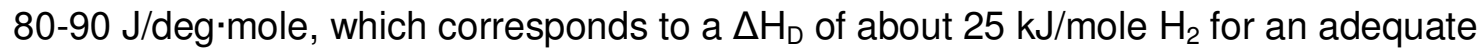
reversibility at near-ambient conditions. With hydrogen storage materials such as metal hydrides and organic liquid carriers, the hydrogen molecule dissociates on absorption and is 
relatively "fixed" in the captured state (i.e., as $\mathrm{C}-\mathrm{H}$ bonds in the organic molecules). In this case, $\Delta \mathrm{S}_{\mathrm{D}}$ is larger and approaches the value of the standard entropy $\left(\mathrm{S}^{\circ}\right)$ for free hydrogen, which is $125 \mathrm{~J} / \mathrm{deg} \cdot \mathrm{mole}$. Consequently, for a given Gibbs free energy change, the organic carriers are associated with a higher $\Delta \mathrm{H}_{\mathrm{D}}$ and higher desorption temperatures, e.g., $>300^{\circ} \mathrm{C}$, for dehydrogenation of cyclohexane to benzene (Appendix $A$ ).

In our search for the "ideal" organic liquid carrier, we were challenged with finding hydrogenated aromatic/aromatic molecule pairs with minimal heats of dehydrogenation, $\Delta H_{D}$, that approach $40 \mathrm{~kJ} / \mathrm{mole} \mathrm{H}_{2}$. This minimal heat of dehydrogenation and a $\Delta \mathrm{S}_{\mathrm{D}}$ of $125 \mathrm{~J} / \mathrm{deg} \cdot \mathrm{mole}$ would, in theory, allow a very moderate desorption temperature, $T_{D}$, of ca. $80^{\circ} \mathrm{C}$. The lower limit for $\Delta H_{D}$ is set by the need to have an adequate equilibrium at practical conditions for a re-hydrogenation of the carrier (reference 19 in Chapter 1). A hydrogen desorption temperature of $80^{\circ} \mathrm{C}$, or preferably even lower temperature, was considered necessary for providing the needed $\Delta H_{D}$ with the rejected waste heat from a PEM fuel cell (cf. Figure 1-3 in Chapter 1). Unfortunately the goal of finding organic liquid carriers that can practically deliver hydrogen at such mild temperatures has, at least in the course of this project, remained elusive. Traditional organic liquid carriers such as decalin require temperatures of $250-300^{\circ} \mathrm{C}$ or higher for high dehydrogenation conversion. One of the best overall liquid carrier candidates from our present work, perhydro-N-ethylcarbazole, has a $\Delta \mathrm{H}_{\mathrm{D}}$ of $50 \mathrm{~kJ} /$ mole $\mathrm{H}_{2}$ and demonstrates good rates of hydrogen desorption in the presence of supported Pt or Pd catalysts at $\sim 200^{\circ} \mathrm{C}$ (Chapter 3 ). The required $\Delta \mathrm{H}_{\mathrm{D}}$ at this approximate temperature could conceivably be drawn from the exhaust of a hydrogen ICE or from the rejected waste heat of a phosphoric acid or $\mathrm{PBI} / \mathrm{H}_{3} \mathrm{PO}_{4}$ fuel cell which functions in the range of $160-200^{\circ} \mathrm{C}$. For a stationary fuel cell system, electrical heating or heat from combustion of a secondary fuel could be employed to supply the $\Delta H_{D}$. Nevertheless, we were challenged with finding ways to supply the necessary heat for delivering hydrogen from an organic carrier to a PEM fuel cell, currently the most widely used option of powering hydrogen-fueled vehicles.

The necessary $\Delta \mathrm{H}_{\mathrm{D}}$ at a temperature of $\sim 200^{\circ} \mathrm{C}$ could certainly be obtained from the combustion of a portion of the generated hydrogen, as has been proposed for the methylcyclohexane/toluene $+3 \mathrm{H}_{2}$ carrier $^{1,2}$. For the perhydro- $\mathrm{N}$-ethylcarbazole liquid carrier with $\Delta H_{D}$ of $52 \mathrm{~kJ} /$ mole $_{2}$, the use of at least $20 \%$ of the LHV of hydrogen would be required. This hydrogen consumption could be much higher in practice, considering heat transfer limitations. 
Another option for supplying heat entails the combustion of a portion of the dehydrogenated carrier. This concept was investigated by Pitault et al. ${ }^{3}$ using two intimate, thermally linked reactors. The first reactor is for the catalytic dehydrogenation of a methylcyclohexane carrier, and the second reactor is for a catalyzed combustion of a portion of the toluene reaction byproduct. This approach has the evident disadvantages of using a relatively expensive fuel and the inevitable release of $\mathrm{CO}_{2}$ and possible emissions of $\mathrm{NO}_{\mathrm{x}}$.

More recent approaches towards a thermally efficient hydrogen storage involve the use of multiple carriers which provide hydrogen by a combination of inherently endothermic and exothermic chemical reactions. Gelsey ${ }^{4}$ describes an apparatus where, for example, the heat required for releasing hydrogen from a metal hydride is provided by the exothermic catalytic hydrolysis of sodium borohydride to hydrogen and sodium borate. Wechsler et al. ${ }^{5}$ have investigated the concept of providing a bifunctional carrier (e.g., N-heterocycle: $\mathrm{BH}_{3}$ adducts) and mixtures of carriers such as of indoline and amino boranes (e.g., $\mathrm{H}_{3} \mathrm{~N}: \mathrm{BH}_{3}$ ) where ideally the $\mathrm{N}$-heterocyclic molecules undergo an endothermic dehydrogenation with the necessary heat and additional hydrogen coming from a hydrolysis of the borane. Recent patent applications ${ }^{6,7}$ claim an essentially autothermal production of hydrogen by a combination of endothermic and exothermic $\mathrm{H}_{2}$-releasing chemical reactions.

A recognized limitation of these approaches is that the exothermic $\mathrm{H}_{2}$-releasing carriers cannot for thermodynamic reasons ${ }^{8}$ be directly regenerated with hydrogen. Their regeneration necessarily requires multiple reaction steps, potentially involving electrochemical transformations, resulting in a significant barrier to implementation and added cost of operation. Given these less than satisfactory options, we investigated the possibility of developing a hydrogen-regenerable organic liquid carrier which could both store hydrogen and provide the heat energy for liberating the gas.

\section{Autothermal carrier design and $\mathrm{H}_{2}$ delivery processes}

Envisaged as an autothermal carrier is a composition which can provide hydrogen by means of a catalytic dehydrogenation reaction and additionally furnish thermal energy from a selective catalytic oxidation reaction using air or oxygen as the oxidant. In an appropriate reactor, this oxidation provides the heat energy at the required temperature for the dehydrogenation 
reaction. The dehydrogenated and partially oxidized "spent" carrier is regenerated in a catalytic hydrogenation process.

One envisaged cyclic process for delivering hydrogen and heat from the carrier and its total regeneration at a remote site with hydrogen is shown in Figure 9-1. Onboard or at a stationary site, the carrier is catalytically dehydrogenated. After separation of the hydrogen, the resultant liquid stream is oxidized with air, thus providing at steady state of operation the needed heat for the dehydrogenation step. Off-board, and preferably at a large industrial site, the carrier is regenerated in a catalytic reduction with hydrogen.

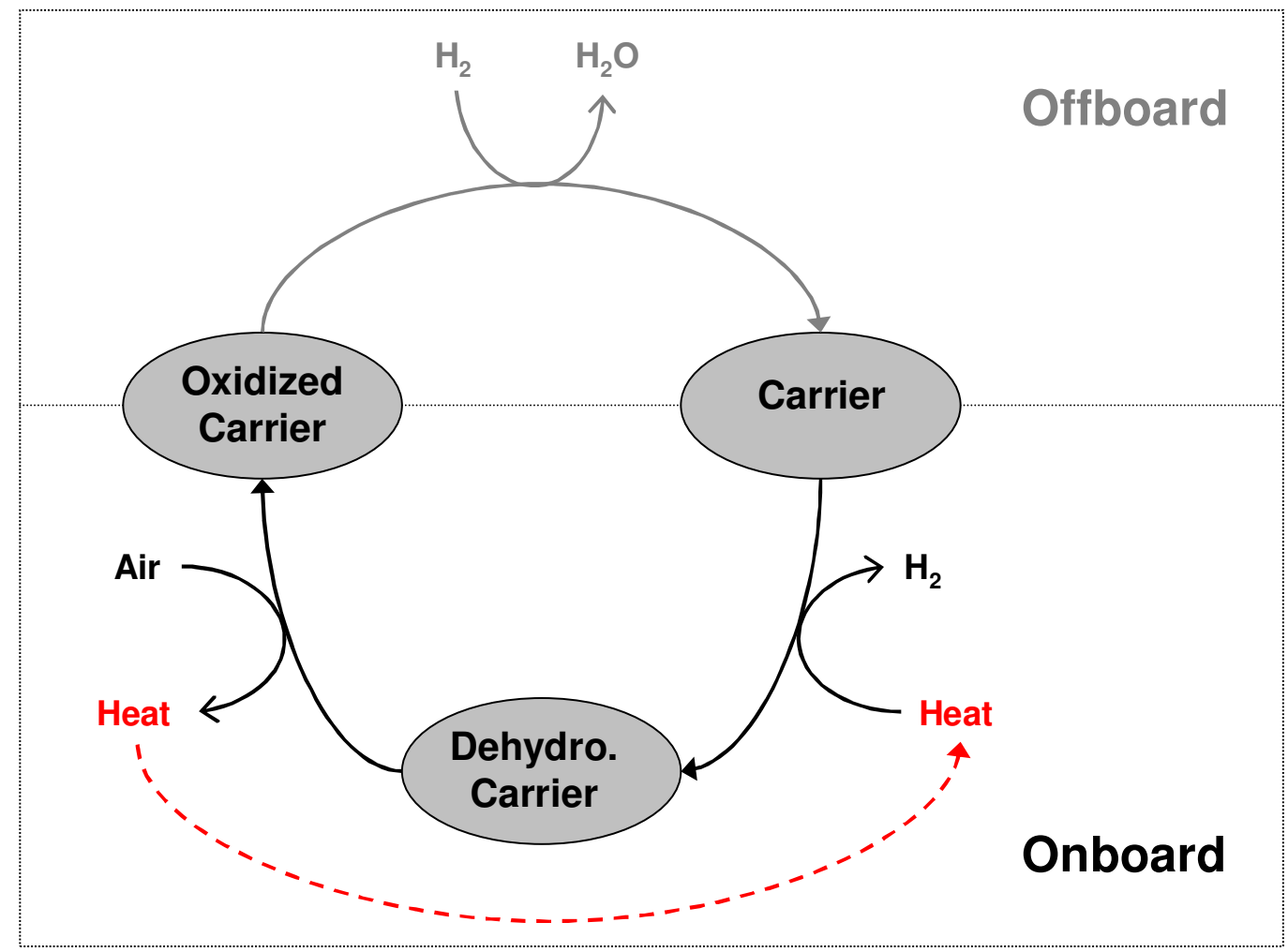

Figure 9-1. General schematic of one autothermal carrier cycle.

The autothermal cycle shown in Figure 9-1 features sequential dehydrogenation and oxidation of the liquid carrier, wherein the carrier is substantially dehydrogenated prior to the heatgenerating, selective-oxidation step. In principle, this cycle could be run in reverse with the oxidation process as the first step. This would have the advantage of providing the initially 
needed heat for the subsequent dehydrogenation, but it greatly raises the challenge of providing carriers that could undergo the required extremely selective oxidation chemistry. Yet another potential autothermal cycle directs a split perhydrogenated carrier feed to undergo two simultaneous reactions where the selective oxidation of the perhydrogenated carrier proceeds to only supply heat to the endothermic dehydrogenation (Figure 9-2). This may have system engineering design advantages, but it leads to a significant lowering of the carrier's available $\mathrm{H}_{2}$ storage capacity ${ }^{9}$.

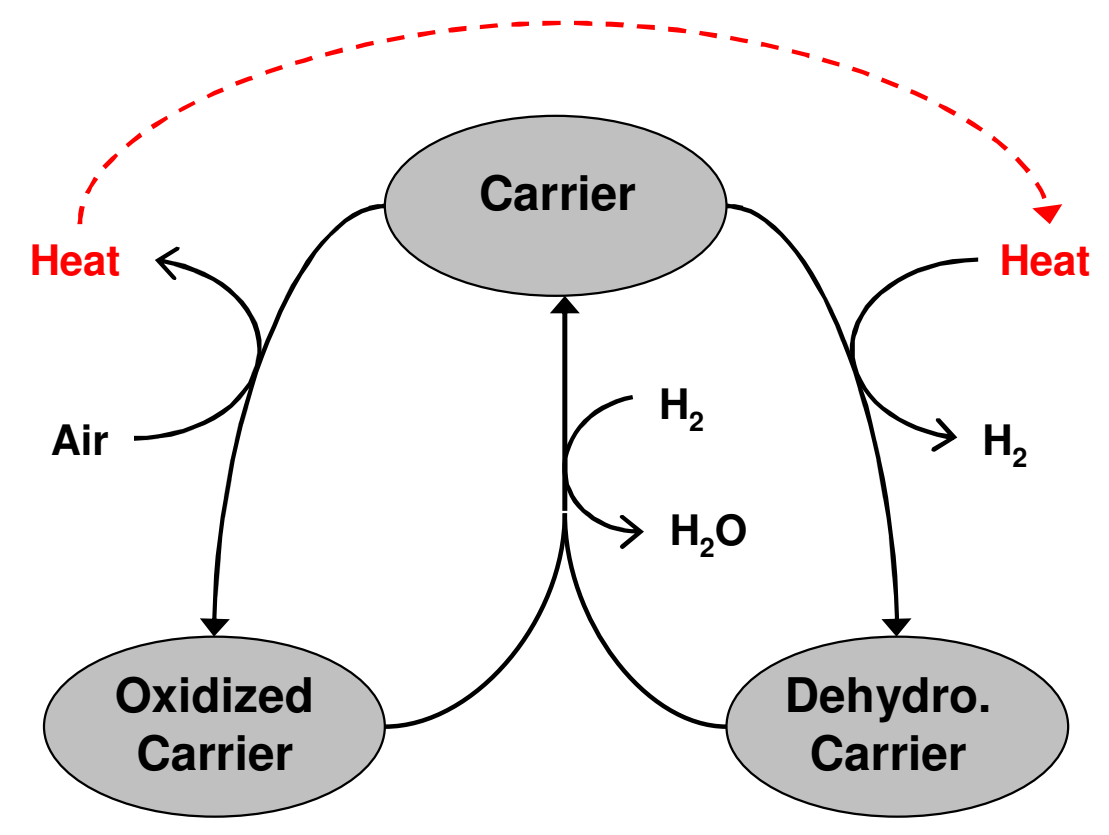

Figure 9-2. Alternative autothermal carrier cycle schematic.

The schematic shown in Figure 9-1, which is overall the most realistic autothermal carrier cycle for providing hydrogen onboard a vehicle or at a stationary site, is discussed below with reference to Scheme 9-1. 


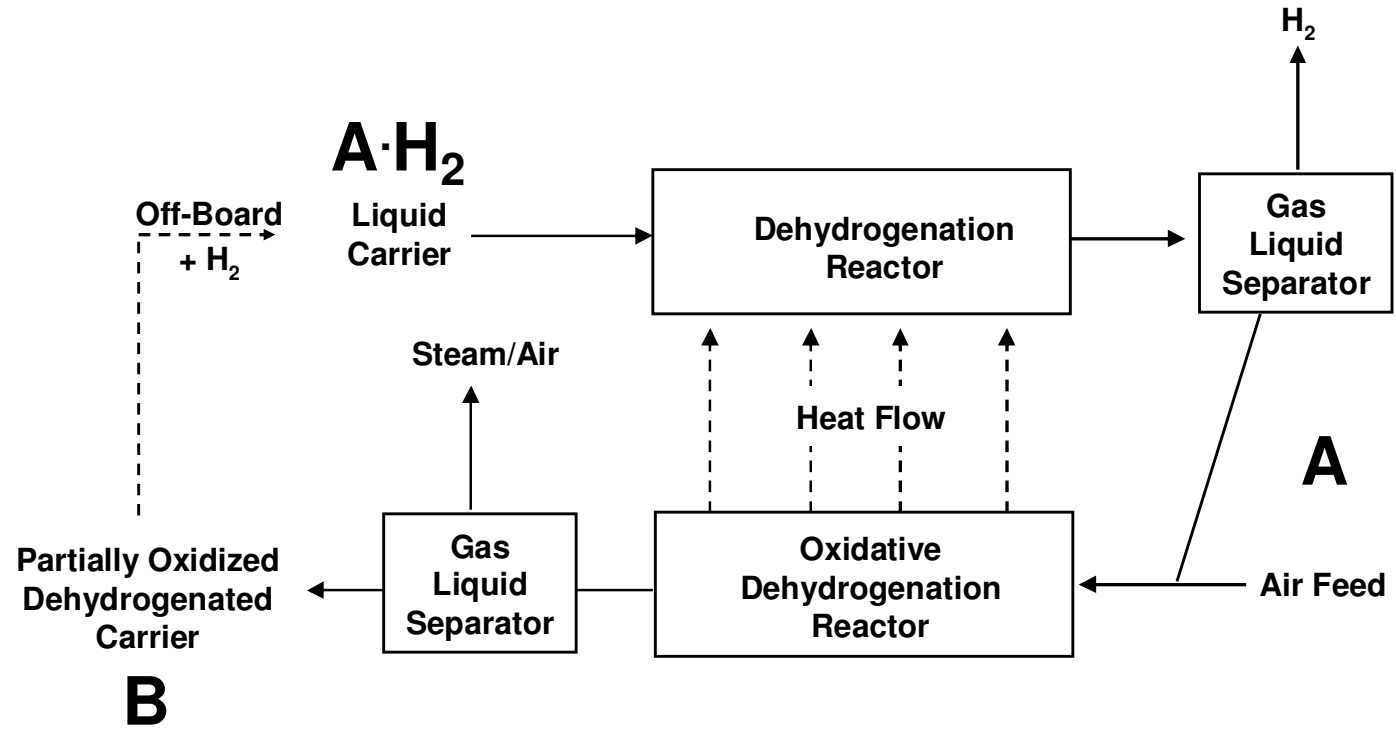

Scheme 9-1. Autothermal dehydrogenation reactor system schematic.

The "loaded" liquid carrier, $\mathbf{A} \cdot \mathbf{H}_{2}$, is passed into the dehydrogenation reactor, where it is brought to reaction temperature by heat that is transferred from the oxidative dehydrogenation reactor. The effluent liquid and hydrogen gas mixture is resolved in the gas/liquid separator, providing the required $\mathrm{H}_{2}$ fuel. The "spent" carrier $\mathbf{A}$ is mixed with an air feed and led to the second reactor, where it is partially oxidized to the extent that is necessary for providing the required heat for $\mathrm{H}_{2}$ production. The steam byproduct and oxygen-depleted air are vented in an exhaust stream, while $\mathbf{B}$, the partially oxidized dehydrogenated carrier, is collected for regeneration at an external site with hydrogen.

In contrast to our original concept (Figure 1-3, Chapter 1), the hydrogen is produced without any thermal input from the power source. The power source's waste heat can now be used for other vehicle requirements (heating, air conditioning, etc.).

In these processes, the carrier's two distinct roles in providing hydrogen as (a) a source of $\mathrm{H}_{2}$ via a catalytic dehydrogenation process and (b) as a substrate for an exothermic selective oxidation may be realized with the carrier as a mixture of two active components. However, in order to minimize carrier mass, it would be advantageous that the autothermal carrier consist of a single bifunctional molecule. 
The following are experimental data on two such bifunctional molecules as carriers in potential hydrogen storage and delivery processes.

\section{Experimental Results}

\section{A. 1-(Carbazolyl)-2-hydroxypropane carrier (1)}

This carbazole derivative was used to illustrate the concept of a dual-function autothermal liquid carrier. Given our considerable experience with the reversible hydrogenation of carbazole derivatives (Chapter 3), we attempted a selective oxidation of $\mathbf{1}$. Reaction of $\mathbf{1}$ with a flow of dry air in the presence of a $\mathrm{Ru} / \mathrm{Al}_{2} \mathrm{O}_{3}$ catalyst at $190^{\circ} \mathrm{C}$ led to a $90 \%$ conversion of the secondary alcohol functional group to the corresponding ketone, $\mathbf{2}$, as shown in Scheme 9-2.

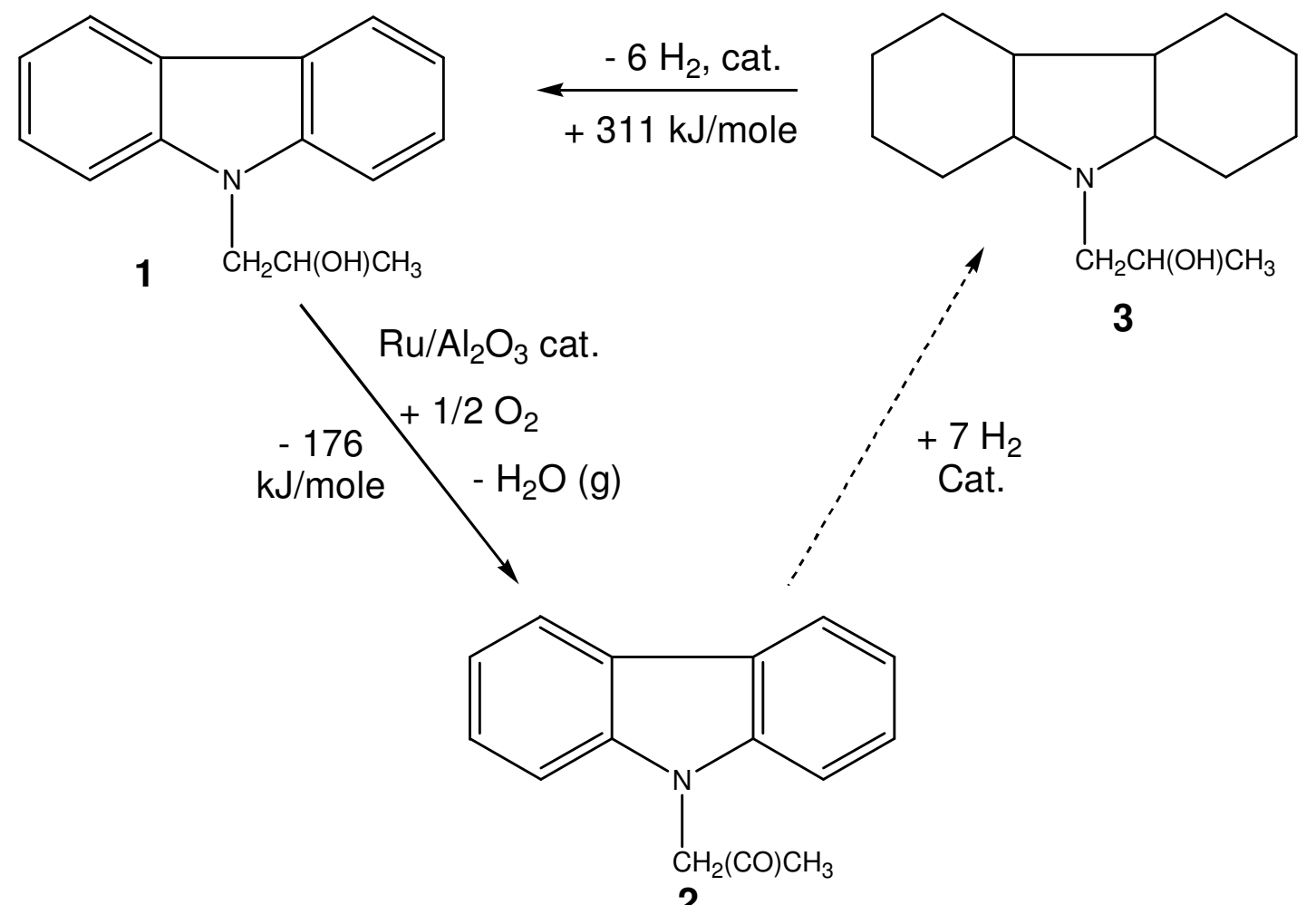

Scheme 9-2. Illustration of the concept of a dual-function carrier. The hydrogen-loaded carrier, 3 , undergoes an endothermic dehydrogenation to 1 , a heat-releasing selective oxidation to 2 , and a reduction of $\mathbf{3}$ with hydrogen offboard the vehicle, completing the cycle. 
We determined the quantity of byproduct water produced in the reaction by capturing the water to verify that $\mathbf{2}$ is formed from a selective oxidation reaction rather than simply a catalytic dehydrogenation process (which would yield hydrogen instead of water). Using the calculated reaction heats shown in Scheme 9-2, the oxidation step potentially supplies only $56 \%$ of the needed $\Delta \mathrm{H}_{\mathrm{D}}$. However, we estimate that selective oxidation of the primary alcohol moiety in 1-carbazoyl-2-hydroxypropane to the corresponding carboxylic acid at $70 \%$ conversion would be sufficient for supplying the entire heat of dehydrogenation.

\section{B. The perhydrofluorene/fluorene/fluorenone carrier system}

Using this carrier system, it was possible to experimentally demonstrate the individual steps of a cyclic hydrogen storage and delivery process using an autothermal carrier as illustrated in Scheme 9-3.

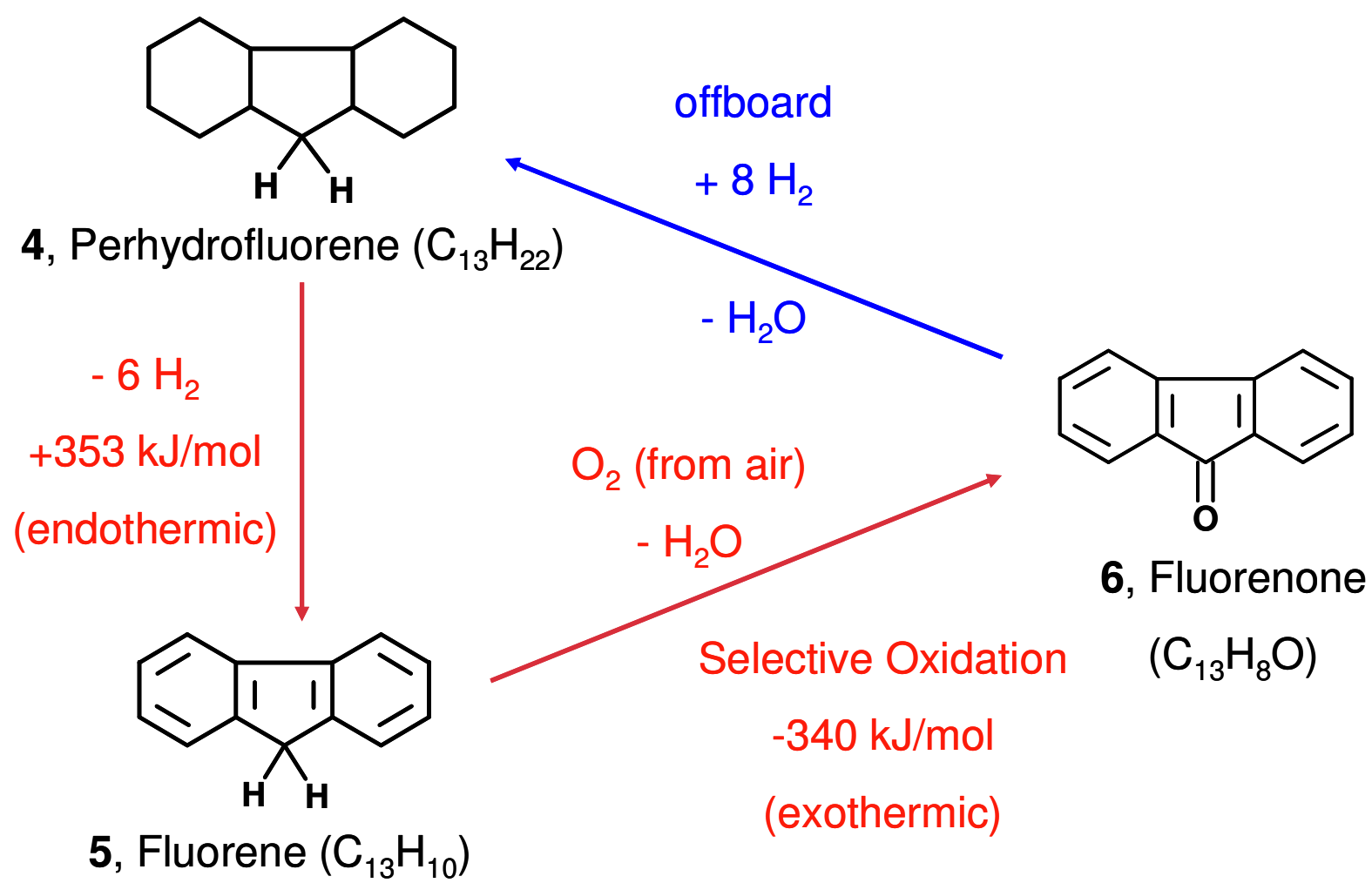

Scheme 9-3. The perhydrofluorene/fluorene/fluorenone, dehydrogenation, selective oxidation and re-hydrogenation cycle. 
The sequence comprises a catalytic dehydrogenation of perhydrofluorene (4) to fluorene (5) with a selective oxidation of fluorene to fluorenone (6) that provides close to the required $\Delta \mathrm{H}_{D}$ for the dehydrogenation step. Off-board the vehicle or at a remote site, the 9-fluorenone "spent" carrier is re-hydrogenated to perhydrofluorene, with the only significant fuel for the cyclic process being hydrogen.

(1) Dehydrogenation of cis,cis-perhydrofluorene to fluorene

The dehydrogenation of cis,cis-perhydrofluorene as a neat liquid (b.p. $253^{\circ} \mathrm{C}$ ) was conducted at temperatures of $200-235^{\circ} \mathrm{C}$ in the presence of a $5 \% \mathrm{Pt}^{\prime} / \mathrm{Al}_{2} \mathrm{O}_{3}$ catalyst under $1 \mathrm{~atm}$. hydrogen pressure. The hydrogen generation time/temperature profile for the reaction as determined from the hydrogen flow rate is shown in Figure 9-3.

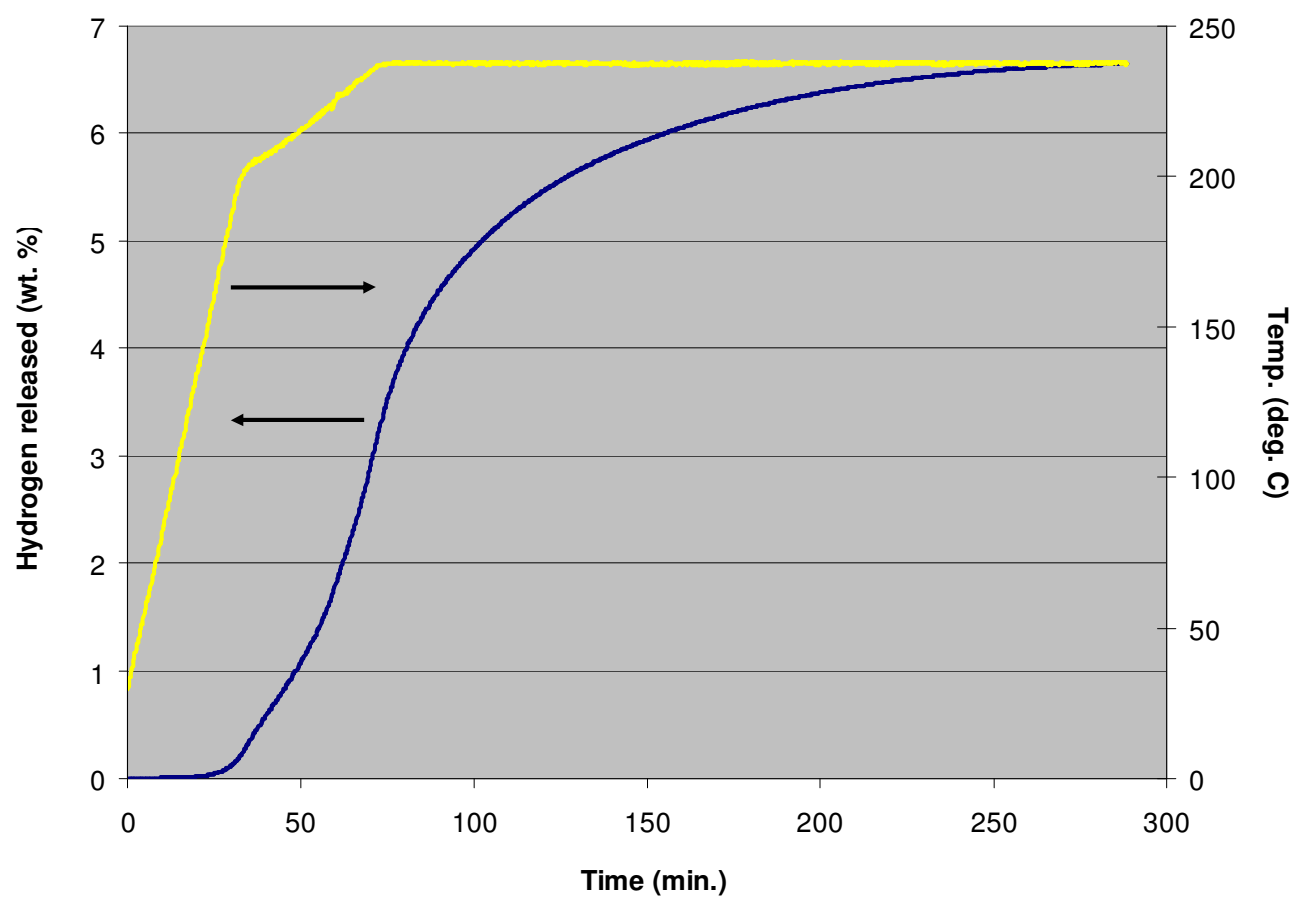

Figure 9-3. Dehydrogenation of cis,cis-perhydrofluorene. 
An effective 6.65 wt\% hydrogen capacity for perhydrofluorene was determined from the $\mathrm{H}_{2}$ flow measurements. This was confirmed by GC analyses indicating a 99\% conversion of perhydrofluorene to fluorene with no reaction byproducts evident in the dehydrogenated liquid carrier.

(2) Aerobic oxidation of fluorene to 9-fluorenone

The second step of an autothermal process, devising a selective oxidation of fluorene to 9-fluorenone in the liquid phase with air at higher temperatures than the $\mathrm{H}_{2}$-generation process, proved to be quite challenging. In view of the recent interest in using supported gold catalysts for selective oxidation ${ }^{10-12}$, a number of such compositions were evaluated for the selective oxidation of fluorene. Of particular interest was the $\mathrm{Au}-\mathrm{Pd} / \mathrm{SiO}_{2} / \mathrm{TiO}_{2}$ catalyst ${ }^{12}$ which had proved effective for a selective oxidation of activated hydrocarbon groups (alkyl substituents on aromatics) and seemed appropriate to investigate for an oxidation of the activated methylene group of fluorene.

(I) Fluorene/catalyst oxidation reactions in the condensed phase

An unmixed slurry consisting of a mixture of fluorene $\left(0.6 \mathrm{~g}\right.$, m.p. $111-114^{\circ} \mathrm{C}$, b.p. $\left.298^{\circ} \mathrm{C}\right)$ and the $\mathrm{Au}-\mathrm{Pd} / \mathrm{SiO}_{2} / \mathrm{TiO}_{2}$ catalyst $(0.3 \mathrm{~g})$ contained in a 0.5 " diameter quartz tube was heated to $225^{\circ} \mathrm{C}$ and contacted with a flowing air stream for a reaction time which ranged from 1-4 hours. Products were assayed both by GC-MS and gel permeation chromatography (GPC). Under these conditions, fluorene (5) was oxidized to a viscous oily liquid containing 9-fluorenone (6), the fluorene dimers 7 and 8, some hydroxyl group modified dimers (9), as well as oligomers (Scheme 9-4). 
<smiles>[CH]C1c2ccccc2-c2ccccc21</smiles>

$\frac{\text { air, } 225^{\circ} \mathrm{C}}{\mathrm{Au}-\mathrm{Pd} / \mathrm{SiO}_{2} / \mathrm{TiO}_{2}}$ 5<smiles>c1ccc(-c2c(-c3ccccc3)c3ccccc3c3ccccc23)cc1</smiles>

8<smiles></smiles>

$\mathrm{OH}$<smiles>Oc1ccc2c(c1)-c1ccccc1C2C1c2ccccc2-c2ccccc21</smiles>

+ oligomers

Scheme 9-4. Products from the oxidation of a 2:1 fluorene to $\mathrm{Au}-\mathrm{Pd} / \mathrm{SiO}_{2} / \mathrm{TiO}_{2}$ catalyst mixture with air at $225^{\circ} \mathrm{C}$.

From the GC-MS analyses, a 2.5:1 ratio of fluorene dimers (compounds 7 and 8 ) to 9-fluorenone (6) was observed after the first hour of reaction time. This ratio ultimately diminished to 0.2:1, with 9-fluorenone being the predominant product (Figure 9-4).

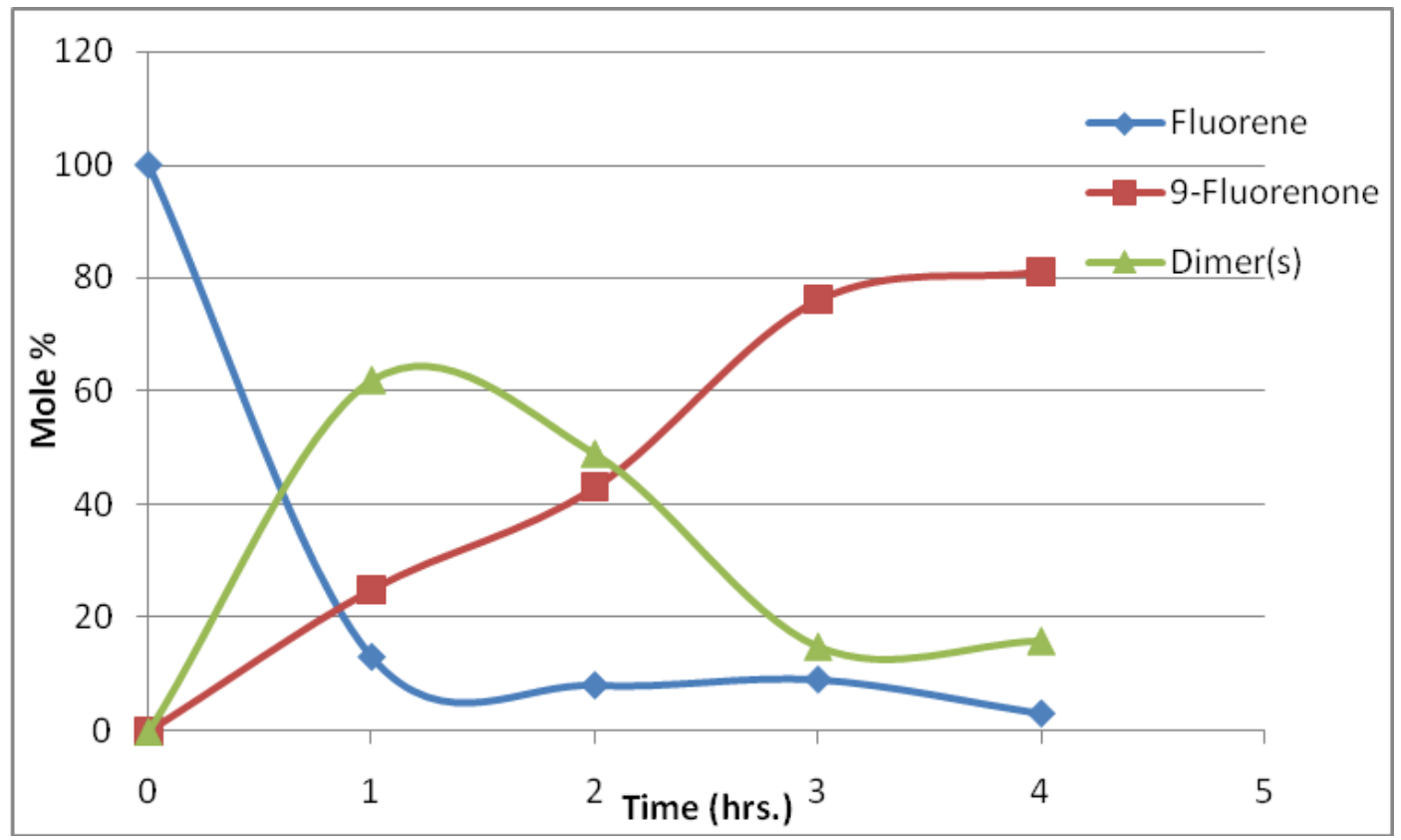

Figure 9-4. GC-MS analyses of the product mixture composition from the aerobic oxidation at $225^{\circ} \mathrm{C}$ of a $2: 1$ fluorene $\mathrm{Au}-\mathrm{Pd} / \mathrm{SiO}_{2} / \mathrm{TiO}_{2}$ catalyst mixture. 
Using GPC analyses, it was unfortunately not possible to separate fluorene and 9fluorenone. However, the data clearly point to the formation, even initially (after $1 \mathrm{hr}$ ), of fluorene dimer and trimer species and the relative amount of dimer diminishing as the reaction progresses (Figure 9-5). At the same reaction conditions in the total absence of oxygen, fluorene dimers are the only observed products by GC-MS analysis.

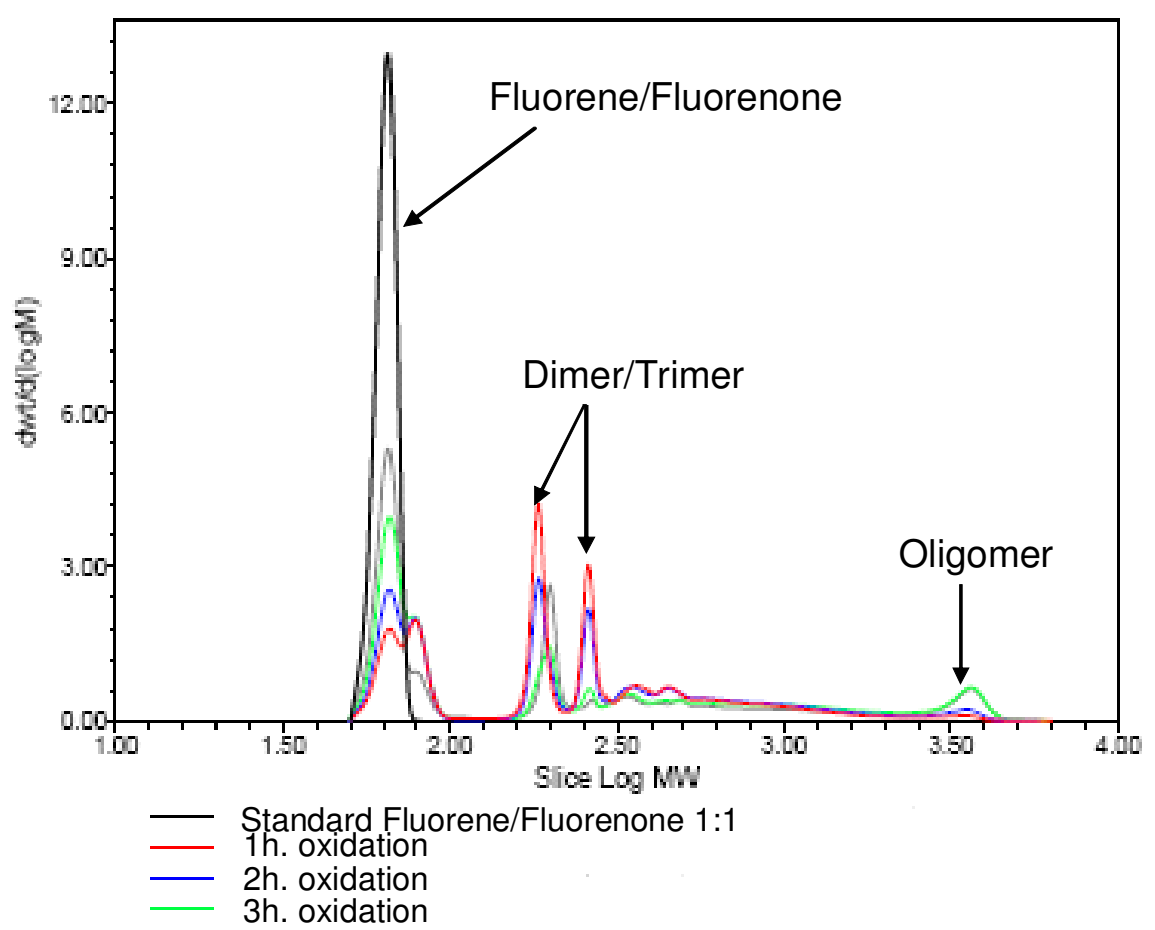

Figure 9-5. Gel permeation chromatography (GPC) data for the aerobic oxidation of a 2:1 fluorene to $\mathrm{Au}-\mathrm{Pd} / \mathrm{SiO}_{2} / \mathrm{TiO}_{2}$ catalyst mixture at $225^{\circ} \mathrm{C}$. The "oligomer" peak corresponds to approximately a trimer of fluorene/fluorenone.

These data are suggestive of a reaction mechanism where fluorene, presumably through the intermediacy of a fluorenyl radical, undergoes a dimerization/dehydrogenation process and in parallel is oxidized to 9-fluorenone. The dimers (7-9) are then oxidized to trimer and possibly higher oligomeric species (Scheme 9-5). 


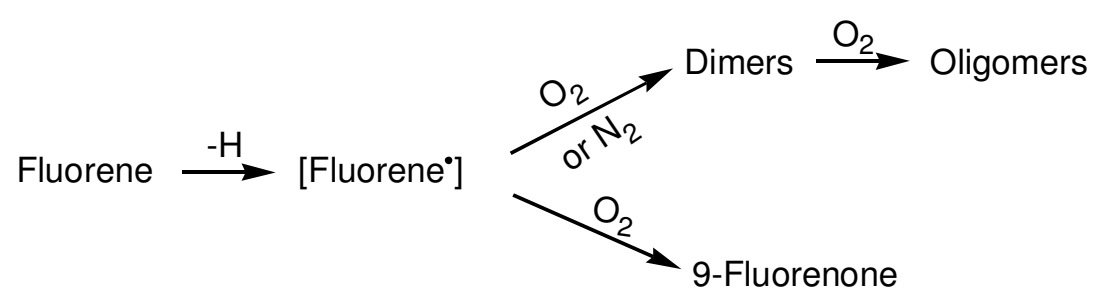

Scheme 9-5. Parallel mechanisms for fluorene reactivity.

(II) Gas phase selective oxidation of fluorene to 9-fluorenone

A high selectivity for the oxidation of fluorene to 9-fluorenone is necessary for providing the maximum exotherm and for facile regeneration of the oxidation product with hydrogen. Thus, in order to minimize the undesired formation of fluorene dimers, we attempted the oxidation of fluorene in the gas phase. The apparatus used for the gas phase oxidation studies (Figure 9-6) consisted of a $3 \mathrm{~mm}$ diameter quartz tube contained horizontally within two tubular furnaces in series (zone 1 followed by zone 2).

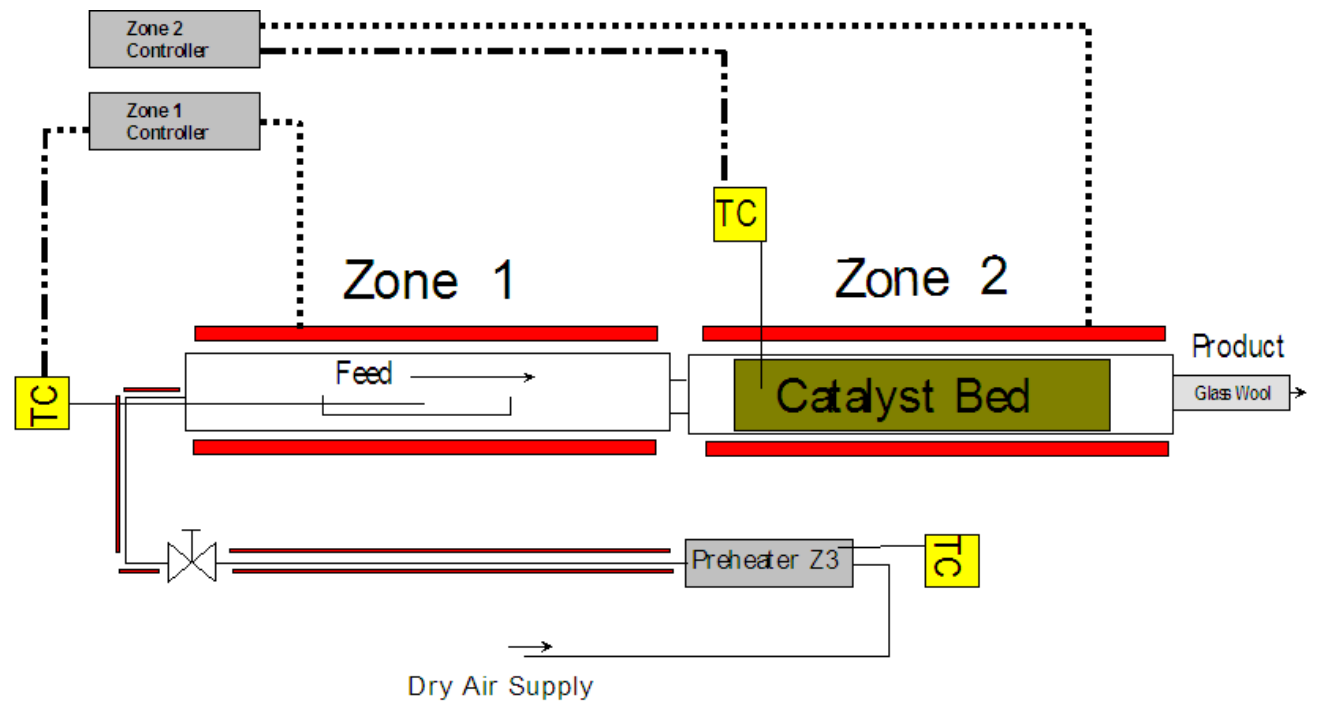

Figure 9-6. Schematic of reactor apparatus for vapor phase selective oxidation reactions. 
Approximately $2 \mathrm{~g}$ of fluorene was loaded into a quartz boat which was inserted into the section of the tube contained in heating zone 1 . The powdered or pelleted catalyst was loaded into zone 2 of the tube and held in place by quartz wool plugs. A rotameter was used to gauge an adjustable air flow over the fluorene sample and through the catalyst bed. The reaction product(s) which deposited downstream in the cooler section of the quartz tube (containing glass wool) were collected and analyzed by GC-MS.

\section{a. Vapor phase aerobic oxidation of fluorene with $\mathrm{Au}-\mathrm{Pd} / \mathrm{SiO}_{2} / \underline{\mathrm{TiO}}_{2}$}

In the vapor phase oxidation apparatus, $1.5 \mathrm{~g}$ fluorene were loaded into zone 1 , and $2.6 \mathrm{~g}$ of $\mathrm{Au}-\mathrm{Pd} / \mathrm{SiO}_{2} / \mathrm{TiO}_{2}$ catalyst were inserted in zone 2 of the quartz tube. Air was passed through the apparatus at $14 \mathrm{sccm}$ and the catalyst was heated to $300^{\circ} \mathrm{C}$. When a stable $300^{\circ} \mathrm{C}$ temperature was reached in zone 2 , the fluorene in zone 1 was heated to $200^{\circ} \mathrm{C}$. Under these conditions the vapor pressure of fluorene is ca. 120 torr, and the estimated fluorene-to-oxygen ratio is ca. 0.8:1. After 1.5 hours of reaction time, the product collected in the cold downstream glass wool plug showed only unreacted fluorene and fluorene dimer. There were no traces of 9-fluorenone.

\section{b. Vapor-phase aerobic oxidation of fluorene to 9-fluorenone using} Cs-promoted iron-vanadium oxide catalysts

Our subsequent experiments toward the gas-phase oxidation of fluorene built upon the substantial existing literature on the gas-phase oxidation of fluorene to 9-fluorenone using vanadium oxide-based catalysts ${ }^{13-14}$. For example, studies by Baerns et $\mathrm{al}^{14(\mathrm{a})}$ have shown that substantially modifying $\mathrm{V}_{2} \mathrm{O}_{5}$ with $\mathrm{Fe}_{2} \mathrm{O}_{3}$ and doping the mixed oxides with small levels of cesium sulfate led to $\mathrm{Cs}$-promoted iron-vanadium oxide catalysts that were remarkably selective for fluorene oxidation. A $99 \%$ selectivity for the oxidation of fluorene to fluorenone was reported for a $\mathrm{V} / \mathrm{Fe} / \mathrm{Cs}$ catalyst composition with an atomic ratio of 1:1.4:0.06, respectively. Unfortunately, the literature reports (e.g., reference 5a) provide only meager details for synthesizing the catalyst. We deployed a procedure essentially consisting of reacting a solution of $\mathrm{NH}_{4} \mathrm{VO}_{3}, \mathrm{Fe}\left(\mathrm{NO}_{3}\right)_{3}$ and $\mathrm{CsNO}_{3}$ with ammonium bicarbonate, which led to precipitation of a solid which was dried, calcined at $400^{\circ} \mathrm{C}$, mixed with $\mathrm{Al}_{2} \mathrm{O}_{3} \cdot \mathrm{H}_{2} \mathrm{O}$, tableted, and calcined a second time. Additional details about the catalyst synthesis are provided in the experimental section. 
In the vapor-phase oxidation apparatus, $2 \mathrm{~g}$ of fluorene were loaded in the quartz boat (zone 1), and $10 \mathrm{~g}$ of the cesium-promoted iron-vanadium catalyst in the form of $1 / 8$ " by $1 / 8$ " (cylindrical) tablets, physically admixed with $1 / 32$ " diameter inert silica beads (Fuji Silysia $\varphi-50$ ), was placed in zone 2 of the quartz tube. The total catalyst volume was $15 \mathrm{cc}$. With a flow of air at $240 \mathrm{sccm}$ through zones 1 and 2 , the catalyst in zone 2 was heated to $350^{\circ} \mathrm{C}$. Upon reaching this temperature, the fluorene in zone 1 was heated to $150^{\circ} \mathrm{C}$. After 1.5 hours of reaction time, the product which condensed in the cooler downstream sections of the quartz tube was collected. GC-MS analyses showed a reaction product that was $>99 \%$ 9-fluorenone with no observable starting material and, in particular, no fluorene dimer.

The vapor-phase oxidation experiment was then repeated with a lower air flow of $60 \mathrm{sccm}$, higher fluorene sample temperature of $200^{\circ} \mathrm{C}$, and the same catalyst bed temperature of $350^{\circ} \mathrm{C}$. After 2 hours on stream, the reactor tube was cooled and the light yellow deposit of reaction product which had formed downstream of the catalyst bed was collected and analyzed by GC-MS. Similar to the earlier experiment, 9-fluorenone was the only product ( 99\%) observed by GC-MS analysis. The catalyst used in this reaction was extracted with acetone. Analysis of the resulting solution showed that 9-fluorenone was the only substance extracted from the catalyst. GC-MS analysis of the remaining solid in zone 1 indicated the presence of only fluorene and fluorene dimer (ca. 21 area \%), which had formed from a direct uncatalyzed oxidation of fluorene at $200^{\circ} \mathrm{C}$.

This work confirmed that it was possible to effect a very selective conversion of gas-phase fluorene to 9-fluorenone. No attempts were made to further characterize and optimize the reaction, since a great deal of information about this reaction can be found in the literature ${ }^{5}$.

\section{(3) Hydrogenation of 9-fluorenone to cis, cis-perhydrofluorene}

Our initial attempts to hydrogenate 9-fluorenone using a $5 \% \mathrm{Ru} / \mathrm{Al}_{2} \mathrm{O}_{3}$ catalyst resulted in an incomplete conversion under our standard hydrogenation conditions (1000 psia $\mathrm{H}_{2}$ pressure, $190^{\circ} \mathrm{C}$ ). While the aromatic rings were saturated, very little C-O bond hydrogenolysis was observed, resulting in production of 9-fluorenol (hydroxyl group on the methylene carbon). Replacement of ruthenium with a $5 \% \mathrm{Rh} /$ carbon catalyst also resulted in an incomplete conversion under our standard hydrogenation conditions. The major product was perhydrofluorene, present as multiple isomers, but some 9-fluorenol was present according 
to the GC-MS analysis. By switching the catalyst to a mixture of palladium on carbon and rhodium on carbon and performing the hydrogenation at $170^{\circ} \mathrm{C}$ and $1000 \mathrm{psia} \mathrm{H}_{2}$ pressure, complete hydrogenolysis of the $\mathrm{C}-\mathrm{O}$ bond and a quantitative yield of perhydrofluorene (as multiple isomers) was afforded. It appears that under these hydrogenation conditions, rhodium-based catalysts are active for catalytic isomerization to give a mixture of isomeric perhydrofluorene products. However, since the cis-syn-cis isomer of perhydrofluorene is most desirable for the dehydrogenation step of the autothermal cycle, we continued to develop a hydrogenation process that would demonstrate a good yield of cis-syn-cisperhydrofluorene from 9-fluorenone.

Our attention turned to a 2-step sequential hydrogenation process, where one catalyst is used to cleave the $\mathrm{C}-\mathrm{O}$ bond through hydrogenolysis and a second catalyst is used to saturate the aromatic rings. Since the "determination" of the isomer distribution most likely occurs during the ring saturation, we looked to a catalyst that previously had demonstrated low activity for catalytic isomerization. In our previous use of ruthenium-based catalysts for $\mathrm{N}$-ethylcarbazole hydrogenation, we were able to obtain mixtures rich in more energetic isomers at low hydrogenation temperatures (Chapter 3 ).

A solution of 9-fluorenone in THF solvent was treated with hydrogen (110 psia) for 16 hours in the presence of an $\mathrm{Au}-\mathrm{Pd} / \mathrm{SiO}_{2} / \mathrm{TiO}_{2}$ catalyst. Removal of the catalyst afforded a solution consisting of a mixture of fluorene (53.5\%) and hexahydrofluorene (46.5\%), with no remaining 9-fluorenone. This mixture of products was then subjected to additional hydrogenation in the presence of a $5 \% \mathrm{Ru} / \mathrm{Al}_{2} \mathrm{O}_{3}$ catalyst under 1000 psia $\mathrm{H}_{2}$ at $190^{\circ} \mathrm{C}$ for 4 hours. The cis-syn-cis isomer of perhydrofluorene was the only product detected by GC-MS analysis. The assignment of the cis-syn-cis isomer was confirmed by INADEQUATE ${ }^{13} \mathrm{C}$ NMR. Full details are provided in the Experimental Section.

\section{(4) Vapor-phase partial, selective oxidation of cis-syn-cis-perhydrofluorene}

Within our concept for autothermal $\mathrm{H}_{2}$ storage and delivery, as illustrated in Figures 9-1 and 9-2, an initial production of hydrogen requires the dehydrogenation reactor to have a sufficient heat supply at reaction temperature. In principle, this could be realized by introducing the perhydrogenated ("fully loaded") carrier, $\mathbf{A} \cdot \mathbf{H}_{2}$, and air into the oxidation reactor, where it is converted to $\mathbf{B}$, the partially oxidized dehydrogenated carrier, with production of a large amount of heat. This heat would be the $\Delta \mathrm{H}$ (exotherm) for 
$\mathbf{A} \cdot \mathbf{H}_{2}+1 / 2 \mathrm{O}_{2} \rightarrow \mathbf{A}+\mathrm{H}_{2} \mathrm{O}$ in combination with the exotherm for $\mathbf{A}+\mathrm{O}_{2} \rightarrow \mathbf{B}+\mathrm{H}_{2} \mathrm{O}$. We illustrate this concept here by demonstrating a direct partial oxidation of cis-syn-cisperhydrofluorene.

A sample of $2 \mathrm{~g}$ cis-syn-cis-perhydrofluorene (Aldrich, 97\%) was loaded into a quartz boat in zone 1 of the gas-phase reactor apparatus (Figure 9-6). A $10 \mathrm{~g}$ aliquot of cesium-promoted iron-vanadium catalyst in the form of $1 / 8$ " by $1 / 8$ " (cylindrical) tablets, physically admixed with 1/32" diameter inert silica beads, was placed in zone 2 of the quartz tube. Air was purged through the system at a rate of $60 \mathrm{sccm}$. Upon heating the catalyst to $350^{\circ} \mathrm{C}$, the sample in zone 1 was heated to $150^{\circ} \mathrm{C}$ and held at this temperature for 30 minutes before being heated to $200^{\circ} \mathrm{C}$ for an additional 1.5 hours. The material which deposited downstream of the heated catalyst was dissolved in acetone and analyzed by GC-MS. The results indicated a $39 \%$ conversion of perhydrofluorene to a product mixture containing $20 \%$ fluorene and 14\% 9-fluorenone, with the balance consisting mainly of partially hydrogenated fluorene. This experiment demonstrates that perhydrofluorene can be oxidized in a fairly selective catalytic reaction to produce products that can readily be re-hydrogenated to perhydrofluorene. Even this partial conversion of perhydrofluorene to a mixture of $20 \%$ fluorene and $14 \% 9$-fluorenone (neglecting the other minor by-products) is expected to generate ca. $1260 \mathrm{~kJ} /$ mole of converted perhydrofluorene which, neglecting system thermal losses, should be sufficient heat for releasing all of the hydrogen from 3.6 moles of perhydrofluorene.

\section{Discussion}

Our autothermal $\mathrm{H}_{2}$ storage and delivery concept was illustrated experimentally using two compositions where carbazole and fluorene are the hydrogen carriers, and the secondary alcohol and methylene groups, respectively, are the selectively oxidizable functions that can be regenerated by catalytic hydrogenation. Schematically, and in general terms, the autothermal carrier may be viewed as a molecule where two functions are present and connected by covalent bond(s), as shown in Figure 9-7. 


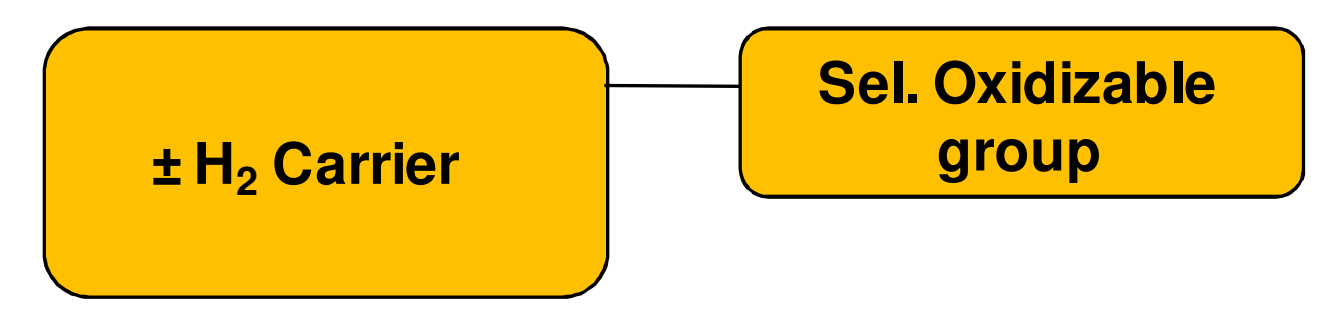

Figure 9-7. Schematic of a dual-function autothermal hydrogen carrier molecule.

For maximum $\mathrm{H}_{2}$ storage and release capacity, the appended selectively oxidizable functional group should provide a very large amount of heat per unit formula weight. There are a considerable number of possibilities for selectively oxidizable and, potentially, hydrogenregenerable functional groups. Activated hydrocarbon functional groups, such as alkyl groups attached to aromatic rings, can be oxidized to alcohols, aldehydes, ketones and carboxylic acids. Primary and secondary alcohols can be oxidized to aldehydes and ketones, respectively. A catalytic oxidation of $1^{\circ}, 2^{\circ}$ and $3^{\circ}$ amines can result in the production of, respectively, nitriles, imines and formyl products. Another example is the possibility of sulfides undergoing a selective oxidative conversion to sulfoxides and sulfones.

The calorific value (kcal/gram FW) of functional groups in exemplary molecules for given selective oxidation reactions was estimated from the respective thermochemistry and is shown in the last column of Table 9-1. Of highest thermal value are the activated hydrocarbon-tocarboxylic acid, aldehyde and ketone transformations (entries 1a, c, d in Table 9-1), oxidation of benzyl alcohols to acids ( $2 b$ in Table 9-1), conversion of $1^{\circ}$ amines to nitriles (entry 4 in Table 9-1), and oxidation of N-methyl tertiary amines to formamides (entry 6 in Table 9-1).

For ultimate utility in an autothermal cycle, the oxidation reactions have to be selective with respect to being reversible in a catalytic reaction with hydrogen. Thus, any carbon-carbon breaking reactions or formation of $\mathrm{CO}_{2}$ would be precluded. Provided here is a review (with imbedded references) of the literature on the catalytic selective oxidation of the functional groups in the representative molecules of Table 9-1, and on the potential hydrogenation catalysis for their regeneration. 


\section{Review: Selective Oxidation and Regeneration of Functional Groups}

1. Activated hydrocarbon groups. Activated hydrocarbon groups are defined as linear or branched saturated hydrocarbon groups that are attached to an aromatic molecule. Examples include the methyl group in toluene, the ethyl group in ethylbenzene, the isopropyl group in cumene, the methylene group in diphenylmethane, and the methylene group in fluorene. The carbon atom of the hydrocarbon group that is immediately adjacent to the aromatic moiety (e.g., a benzyl carbon) is considered to be "activated" towards chemical reactivity, including oxidation, vis-a-vis the carbon atom of a saturated hydrocarbon molecule. Oxidation of activated hydrocarbon functional groups, such as the side chain groups on aromatic molecules (e.g., toluene as in 1a, 1b, 1c in Table 9-1) can be accomplished, using air as the oxidant, with nanoporous molecular sieves ${ }^{15}$ and by cobalt tetraphenylporphyrin catalysts ${ }^{16}$. A catalytic air oxidation of diphenylmethane to benzophenone using air as the oxidant over alumina-supported $\mathrm{NaF}$ and $\mathrm{KF}$ catalysts has been reported. ${ }^{17} \mathrm{~V}$. R. Choudhary et al. have reported the selective oxidation of diphenylmethane to benzophenone and ethylbenzene to acetophenone with oxygen over an Mg-Al hydrotalcite catalyst. ${ }^{18}$ Arylaldehydes (e.g., $\mathrm{PhCHO}$ ) and arylcarboxylic acids can be hydrogenated (Reactions $2 a, b$ and $c$ in Table 9-1) to the corresponding benzyl alcohols. The benzyl alcohols in turn can undergo hydrogenolysis (reactions with $\mathrm{C}-\mathrm{O}$ bond rupture by $\mathrm{H}_{2}$ ) to regenerate the benzylic hydrocarbon. Useful catalysts for the hydrogenolysis reactions are palladium catalysts which have a lesser tendency to hydrogenate the aromatic ring. ${ }^{19}$ As relating to Reaction 1c in Table 9-1, polyoxometallate catalysts have been employed for an oxidative dehydrogenation of cyclic saturated hydrocarbons (e.g., decalin), to yield partially hydrogenated cyclic compounds. ${ }^{20}$ The partially hydrogenated cyclic compounds may then be fully reduced with hydrogen over supported platinum, platinum oxide or rhodium and ruthenium catalysts to regenerate the carrier. 


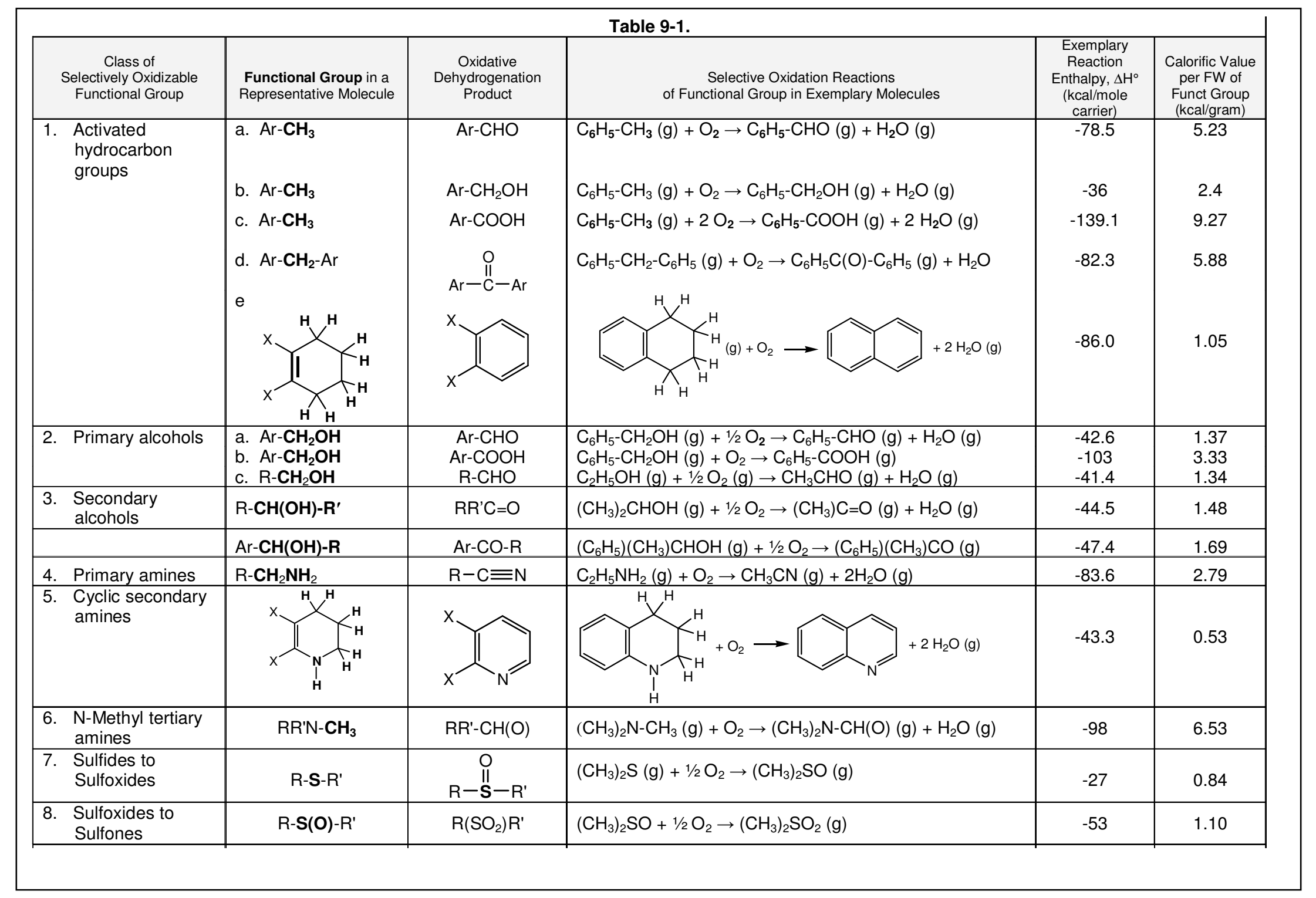


2. Primary alcohols. The selective oxidation of alcohols, with air or oxygen, has been disclosed in a large number of publications. ${ }^{21}$ The most investigated primary alcohol substrates are those that are part of a conjugated system, i.e., benzyl alcohol (Reactions 2a, 2b in Table 9-1). The selective oxidation of primary alcohols to aldehydes and carboxylic acids can be accomplished using a variety of catalysts ranging from supported $\mathrm{Pt}$ group metals (usually with $\mathrm{Bi}$ or $\mathrm{Pb}$ promoters), redox metal oxides such as $\mathrm{RuO}_{2} \cdot \mathrm{xH}_{2} \mathrm{O}, \mathrm{Ru}$-supported hydrotalcites, and redox molecular sieves. Oxidation of non-conjugated primary alcohols (e.g., ethanol) are illustrated by Reaction $2 \mathrm{c}$ in Table 9-1. Platinum group metals may be used in the presence of some water and base (Mallott et al., p. 3052). With nanoparticle gold catalysts on magnesium aluminate, a selective conversion of aqueous ethanol to acetic acid has been disclosed..$^{22}$ Highly dispersed gold on nanocrystalline cerium oxide permits a selective oxidation of aliphatic primary alcohols in the presence of base and water to carboxylic acids (e.g., n-hexanol to hexanoic acid). ${ }^{23}$ In the substantial absence of solvent, carboxylic acid esters may also be formed:

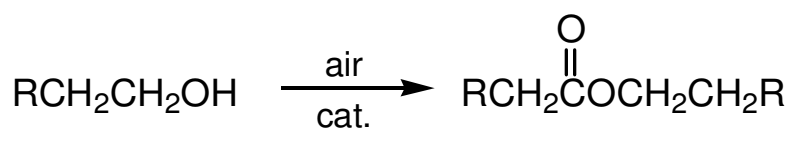

Carboxylic acid esters are potentially useful as selective oxidation products since they can also be hydrogenated back to the alcohol. The selective hydrogenation of aldehydes, carboxylic acids and carboxylic acid esters to the corresponding primary alcohols can also be performed.

Aldehydes can be hydrogenated to the corresponding alcohols using Raney $\mathrm{Ni}, \mathrm{Pt}$ oxide, and copper-chromium catalysts. ${ }^{24}$ The hydrogenation of carboxylic acids to alcohols usually entails more forcing conditions such as higher temperatures and elevated hydrogen pressures. Typical catalysts disclosed in the literature are copper chromite, $\mathrm{Ru} / \mathrm{C}$, and rhenium or rhodium oxides (Rylander, pgs. 64-65; Nishimura, pgs. 387-391). Carboxylic acid esters are typically hydrogenated over copper-chromium oxide catalysts at ca. $250^{\circ} \mathrm{C}$ and high hydrogen pressures. However, the hydrogenation of esters under 
relatively mild conditions is possible, as demonstrated by the conversion of methyl acetate at $60^{\circ} \mathrm{C}$ and 6 atm. $\mathrm{H}_{2}$ pressure to methanol, ethanol and ethyl acetate, using potassium-doped ruthenium on carbon catalysts. ${ }^{25}$

3. Secondary alcohols. The selective aerobic oxidation of secondary alcohols to ketones (Reaction 3, Table 9-1) can be accomplished by a variety of oxide-supported ruthenium and platinum catalysts, even in the absence of solvent (Mallott, pgs. 3052-3053). A selective catalytic hydrogenation of ketones to regenerate the alcohol can likewise be accomplished, for example, by using Raney nickel with base promoters, supported Ru and Rh catalysts, or, specifically for the hydrogenation of aliphatic ketones, Pd on oxide supports (Rylander, pgs. 8284; Nishimura, pgs. 185-193).

4. Primary amines and cyclic secondary amines. Processes for the catalytic oxidative dehydrogenation of amines to imines or nitriles (Reaction 4 in Table 9-1) with air or oxygen can be employed. The aerobic oxidation of benzylamine with $\mathrm{O}_{2}(1 \mathrm{~atm}$.) in the presence of a copper catalyst $\left(\mathrm{CuCl}_{2}\right)$ and molecular sieve $3 \mathrm{~A}$ (as a drying agent) to yield a mixture of benzonitrile and $\mathrm{N}$-benzylidenebenzylamine has been described ${ }^{26}$ :

$$
\mathrm{Ph}-\mathrm{CH}_{2}-\mathrm{NH}_{2} \stackrel{\mathrm{CuCl}_{2}}{\longrightarrow} \mathrm{PhC} \equiv \mathrm{N}+\mathrm{PhCH}=\mathrm{N}-\mathrm{CH}_{2} \mathrm{Ph}
$$

The benzylamine dehydrogenation reactions are zero-order in oxygen, which implies that air (or perhaps oxygen-depleted air from a fuel cell) should be a suitable oxidant.

The dehydrogenation of aliphatic amines appears to be more selective. For example, $\mathrm{N}$-octylamine can be converted to the corresponding $\mathrm{N}$-heptylnitrile in $96 \%$ isolated yield at $80^{\circ} \mathrm{C}$ and $1 \mathrm{~atm} . \mathrm{O}_{2}$ pressure. Even more efficient aerobic dehydrogenations of primary amines to nitriles and imines have been described. ${ }^{27}$ Using ruthenium on alumina catalysts, it was possible to fully convert an array of amines to the corresponding nitriles with good selectivity using $\mathrm{O}_{2}\left(1 \mathrm{~atm}\right.$.) at $130^{\circ} \mathrm{C}$. The selectivities are greatest for the oxidative dehydrogenation of long-chain amines to nitriles and reactions involving an oxidative dehydrogenation of cyclic secondary amines where an aromatic amine is the product. Examples are the conversions of indolene to indole and 1,2,3,4-tetrahydroquinoline to quinoline (Reaction 5 in Table 9-1), which both proceed with greater than $99 \%$ selectivity. 
5. N-Methyl tertiary amines. The methyl group of a tertiary $\mathrm{N}$-methyl amine can be selectively oxidized with air or oxygen to a $-\mathrm{N}(\mathrm{CO}) \mathrm{H}$ (N-formyl) group (Reaction 6 in Table 9-1) by a variety of catalytic systems. The conversion of trimethylamine to dimethylformamide by reaction with oxygen in the presence of a $15 \% \mathrm{Pd} /$ charcoal catalyst has been reported. ${ }^{28}$ An improved process resulted from the use of cupric chloride or other metal halides as catalysts. ${ }^{29}$ A variety of complex metal oxide systems ranging from xerogels, aerogels, and metal-substituted heteropolyacids can be used for the preparation of substituted formamides, $\mathrm{R}^{\prime} \mathrm{RN}(\mathrm{CO}) \mathrm{H}$, from tertiary $\mathrm{N}$-methylamines. ${ }^{30}$ The hydrogenation of acid amides, $\mathrm{RCONH}_{2}$, to amines may be performed with copper-chromium oxide and copper-barium-chromium oxide catalysts under relatively forcing conditions (Nishimura, pgs. 406-411). Specifically, a hydrogenation of formamides to methylamines in the presence of methyl carbonyl catalysts has been reported. ${ }^{31}$

6. Oxidation of sulfides to sulfoxides and sulfones. There are available catalytic methodologies for a selective oxidation of organic sulfides, $\mathrm{R}_{2} \mathrm{~S}$, (alternatively known as thioethers) to sulfoxides, $\mathrm{R}_{2} \mathrm{~S}=\mathrm{O}$, and sulfones, $\mathrm{R}_{2} \mathrm{SO}_{2}$, (Reactions 7, 8 in Table 9-1]. ${ }^{32}$ For example, a selective oxidation of sulfides to sulfoxides under aerobic conditions may be accomplished using the binary catalytic system, $\mathrm{BiBr}_{3}-\mathrm{Bi}\left(\mathrm{NO}_{3}\right)_{3} \cdot{ }^{33}$ The reduction of sulfoxides and sulfones to thioethers is usually accomplished with chemical reductants such as $\mathrm{NaBH}_{4} / \mathrm{I}_{2} \cdot{ }^{34}$ However, reductions of $\mathrm{R}_{2} \mathrm{SO}$ or $\mathrm{R}_{2} \mathrm{SO}_{2}$ functional groups to $\mathrm{R}_{2} \mathrm{~S}$ should be possible using $\mathrm{H}_{2}$ and Raney Ni catalysts. An indication of this is that some thiol products are formed in the hydrogenation of unsaturated organic sulfones. ${ }^{35}$

\section{Conclusions}

Autothermal hydrogen storage cycles offer the potential for hydrogen storage systems that deliver hydrogen to a power source without the need for external heat integration or burning a portion of the hydrogen generated at the site of hydrogen use. The thermodynamic requirements of reducing the oxidized carrier back to the perhydrogenated state are met at the regeneration site, where 'excess' hydrogen (i.e., more hydrogen than is reversibly stored) is used to regenerate both functions of the carrier (hydrogen delivery and heat generation). This is desirable from an economic perspective, since the price of hydrogen is lower at large, centrally located industrial sites.

We have established each step of a (nearly) autothermal cycle using cis,cis-perhydrofluorene as the autothermal liquid carrier prototype. All three stages of the autothermal cycle (dehydrogenation, 
selective oxidation, rehydrogenation) involve catalytic reactions that have been demonstrated at $>98 \%$ selectivity for the fluorene-based intermediates. Selective oxidation of perhydrofluorene was established by preliminary experiments and offers the potential for supply of the excess heat needed for system start-up.

\section{Experimental Details}

\section{Experimental methodology - analytical}

The GC-MS instrument consists of a Hewlett Packard model 6890 gas chromatograph (RTx-35 column, $30 \mathrm{~m}$ length, $0.25 \mathrm{~mm}$ inner diameter, $0.25 \mu \mathrm{m}$ packing thickness) and an Agilent model 5973 mass spectrometer. The GC-MS analytical methods used typically employed temperature ramping to $310^{\circ} \mathrm{C}$. Gel Permeation Chromatography (GPC) analysis was performed using an Alliance 2695 instrument with High Resolution, Low Mass Gel Permeation (maximum 2,000 Da) on three HR 0.5 Columns. Detection utilized a Waters Corporation Model 2414 Differential Refractometer. Matrix Assisted Laser Desorption lonization (MALDI) mass spectroscopy was performed on a Bruker Biflex III instrument with no mass suppression. For ${ }^{13} \mathrm{C}$ NMR spectroscopy, a Bruker Advance $300 \mathrm{MHz}$ instrument was employed.

\section{Preparation of $\mathrm{Au}-\mathrm{Pd} / \mathrm{SiO}_{2} / \mathrm{TiO}_{2}$ catalyst}

The oxidation catalyst was prepared using the following procedure. Titanium(IV) isopropoxide (Aldrich, $99.999 \%)$ in the amount of $2.14 \mathrm{~g}(7.5 \mathrm{mmol})$ was dissolved in $50 \mathrm{ml}$ 2-propanol, and this solution was added to $12 \mathrm{~g}$ of powdered Cariact Q-10 silica (Fuji Silysia). The suspension was stirred for one hour at room temperature before the solvent was removed in vacuo at $50^{\circ} \mathrm{C}$. The resulting solid was dried in air at $110^{\circ} \mathrm{C}$ for 3 hours, followed by calcination in air at $600^{\circ} \mathrm{C}$ for 4 hours. In a round bottom flask was added $1.89 \mathrm{~g}(5.6 \mathrm{mmol}) \mathrm{HAuCl}_{4}$ (Strem, 99.9\%) and $300 \mathrm{ml}$ deionized water. The solution was heated to $65^{\circ} \mathrm{C}$ with stirring, and the $\mathrm{pH}$ adjusted to ca. 10 using $1 \mathrm{M} \mathrm{NaOH}$. To the resulting solution was added $0.86 \mathrm{~g}$ (2.9 mmol) $\mathrm{Pd}\left(\mathrm{NH}_{3}\right)_{4}\left(\mathrm{NO}_{3}\right)_{2}$ (Alfa Aesar) dissolved in $25 \mathrm{ml}$ deionized water and $11.5 \mathrm{~g}$ of the $\mathrm{SiO}_{2} / \mathrm{TiO}_{2}$ support. The slurry was then stirred for one hour at $65^{\circ} \mathrm{C}$ and cooled to room temperature. The catalyst was then filtered and washed three times with $350 \mathrm{ml}$ deionized water until a $\mathrm{AgNO}_{3}$ test showed no chloride ion was present in the deionized water wash. The filter cake was then dried at $110^{\circ} \mathrm{C}$ overnight followed by a 3.5 hour calcination in air at $400^{\circ} \mathrm{C}$. 


\section{Preparation of $\mathrm{V} / \mathrm{Fe} / \mathrm{Cs}$ catalyst}

Ammonium vanadate, $\mathrm{NH}_{4} \mathrm{VO}_{3}(11.7 \mathrm{~g}, 0.10$ mole) was added to 1 liter of a $0.51 \mathrm{M}$ solution of ammonium bicarbonate, $\mathrm{NH}_{4} \mathrm{HCO}_{3}$ with stirring until it had completely dissolved. To this was added drop wise a solution of $\mathrm{Fe}\left(\mathrm{NO}_{3}\right)_{3} \cdot 9 \mathrm{H}_{2} \mathrm{O}\left(56.6 \mathrm{~g}, 0.14\right.$ mole) and cesium nitrate, $\mathrm{CsNO}_{3},(1.17 \mathrm{~g}$, $0.006 \mathrm{~mole})$ in $500 \mathrm{ml}$ of water. When addition was complete, the solution $(\mathrm{pH}=8.5)$ and precipitate mixture was heated to $95^{\circ} \mathrm{C}$, where it was held for $1 / 2$ hour with stirring and then cooled. The mixture was then filtered and the precipitate washed only once with 1.5 liters of water (mother liquor $\mathrm{pH}=8.5$, wash $\mathrm{H}_{2} \mathrm{O} \mathrm{pH}=8.2$ ). The remaining solid was dried at $110^{\circ} \mathrm{C}$ overnight and calcined in air for 2 hours at $400^{\circ} \mathrm{C}$.

Elemental analyses (by X-ray fluorescence): V (19.49\%), Fe (44.95\%), Cs (0.544\%) corresponding to $\mathrm{V}: F e: C s=1: 2.1: 0.01$. The sample was amorphous by XRD. A 1:1 W,W mixture of the powder and Boehmite (fine $\mathrm{Al}_{2} \mathrm{O}_{3} \cdot \mathrm{H}_{2} \mathrm{O}$ powder) was pressed into 1/8" x 1/8" (cylindrical) tablets, which were then calcined at $450^{\circ} \mathrm{C}$ for 2 hours, thus providing the required catalyst.

\section{Catalytic hydrogenation of 9-fluorenone to cis,cis-perhydrofluorene}

A sample of $0.5 \mathrm{~g}(2.8 \mathrm{mmol}) 9$-fluorenone (Aldrich, $98 \%), 0.15 \mathrm{~g} \mathrm{Au}-\mathrm{Pd} / \mathrm{SiO}_{2} / \mathrm{TiO}_{2}$ catalyst and $5 \mathrm{ml}$ tetrahydrofuran (Fisher, HPLC Grade) was placed in a $20 \mathrm{cc}$ stirred tank stainless steel autoclave reactor and the reactor was then sealed. The reactor was connected to a manifold containing a vacuum source, high-pressure hydrogen source, and a high-pressure ballast. After evacuation of residual air from the manifold lines, hydrogen was purged through the reactor to displace the air from the reactor headspace. The mixture was hydrogenated at $110 \mathrm{psia}_{2}$ pressure and $70^{\circ} \mathrm{C}$ for 16 hours. After cooling and venting the reactor to atmospheric pressure, its contents were filtered to remove the catalyst. GC-MS analysis of the liquid product showed complete conversion of 9-fluorenone to two products: fluorene $(53.5 \%)$ and hexahydrofluorene $(46.5 \%)$. To this solution of fluorene and hexahydrofluorene was added $0.15 \mathrm{~g}$ of a ruthenium supported on alumina catalyst (Engelhard, $5 \% \mathrm{Ru}$ ), and the slurry was returned to the reactor. The mixture was hydrogenated at $1000 \mathrm{psia}$ $\mathrm{H}_{2}$ pressure and $190^{\circ} \mathrm{C}$ for 4 hours. After cooling and venting the reactor to atmospheric conditions, the catalyst was removed by filtration. GC-MS analysis of the product solution showed $>99 \%$ conversion and $>99 \%$ selectivity to perhydrofluorene. The cis,cis isomer of perhydrofluorene was obtained with $96 \%$ selectivity based on the GC retention time vs. commercial perhydrofluorene (Aldrich, 97\%). INADEQUATE ${ }^{13} \mathrm{C}$ NMR taken of the commercial perhydrofluorene confirmed the 
cis,cis stereochemistry of the four methine carbons in the central ring: $22.1 \mathrm{ppm}(\mathrm{s}), 24.3 \mathrm{ppm}(\mathrm{s})$, 24.4 ppm (s), 29.2 ppm (s), 35.3 ppm (s), 37.3 ppm (s), 43 ppm (s), as reported [R. Meusinger J. Prakt. Chem. 339, 128 (1997)].

\section{$\underline{\text { References and Notes }}$}

1. Grünenfelder, N.F.; Schucan, T.H. Int. J. Hydrogen Energy 1989, 14, 579.

2. Kesten, A.S., U.S. Pat, No, 4,567,033.

3. Fabien, H.; Lanure, V.; Pitault, I.; French Pat. No. 2,896,494.

4. Gelsey, J., U.S. Pat. No. 7,108,933. See also: Toshiyuki, E.; Matsumoto, T.; Yoshikiro, K.; JP 2007076992-A (Nippan Oil Co. Ltd.).

5. Wechsler, D.; Cui, Y.; Dean, D.; Davis, B. Jessop, P.G. J. Am. Chem. Soc. 2008, 130, 17203.

6. Thorn, D.L.; Tumas, W.; Ott, K.C.; and Burrell, K.; U.S. Pat. Appl. 2007/0183967A1.

7. Takahira, J.; Yutoka, J.; Tokohita, M. JP 2008189538-A (Nissan Motor Co., Ltd.).

8. Releasing $\mathrm{H}_{2}$ in an exothermic reaction (corresponding to a negative $\Delta H$ and a positive $\Delta S$ in Equation 1) necessarily makes the reverse process thermodynamically inaccessible $[\Delta \mathrm{G}$ (hydrogenation) >>0].

9. In the selective direct oxidation of the perhydrogenated carrier, $\mathrm{A} \cdot \mathrm{nH}_{2}$, to $\mathrm{B}$, the partially oxidized dehydrogenated carrier, the contributing chemical reactions and their corresponding enthalpies are as follows:

$$
\begin{array}{ll}
\mathrm{A} \cdot \mathrm{nH}_{2} \rightarrow \mathrm{A}+\mathrm{nH}_{2} & \Delta \mathrm{H}_{\mathrm{D}} \text { (endothermic) } \\
\mathrm{A}+\mathrm{O}_{2} \rightarrow \mathrm{B} & \Delta \mathrm{H}_{\mathrm{OX}} \text { (exothermic) } \\
\mathrm{nH}_{2}+\mathrm{n} / 2 \mathrm{O}_{2} \rightarrow \mathrm{nH}_{2} \mathrm{O} & \mathrm{nLHV}\left(\mathrm{H}_{2}\right) \text { exothermic }
\end{array}
$$

$A \cdot n \mathrm{H}_{2}+3 / 2 \mathrm{O}_{2} \rightarrow \mathrm{B}+\mathrm{nH}_{2} \mathrm{O}$

This oxidation of $A \cdot \mathrm{nH}_{2}$ to $\mathrm{B}$ minimally provides the necessary heat for a dehydrogenation of $\left[\Delta \mathrm{H}_{\mathrm{D}}+\Delta \mathrm{H}_{\mathrm{Ox}}+\mathrm{nLHV}\left(\mathrm{H}_{2}\right)\right] / \Delta \mathrm{H}_{\mathrm{D}}$ moles of carrier $\mathrm{A} \cdot \mathrm{nH}_{2}$ to provide $\mathrm{n}$ moles of $\mathrm{H}_{2}$. For an oxidation of perhydrofluorene to 9-fluorenone this ratio corresponds [from the data in Scheme 3 and for LHV $\left(\mathrm{H}_{2}\right)$ of $243 \mathrm{~kJ} / \mathrm{mole}$ ] to $\sim 4$ moles of perhydrofluorene undergoing dehydrogenation for one mole that is directly oxidized to 9-fluorenone, the common product. In this autothermal cycle the available $\mathrm{H}_{2}$ capacity is effectively reduced about $20 \%$. 
10. "Catalysis by Gold"; Bond, G.C.; Louis, C. and Thompson, D. T.; Catalytic Science Series Vol. 6, Imperial College Press, 2006.

11. Horuta, M. Catalysis Today, 1997, 36153.

12. Augustine, S.; Smith, D.W.; Hanes, R.M. and Evans, M.D.; PCT Pat. Appl. WO98/18553 (1998) (Millenium Petrochemicals Ltd.).

13. Hayashi, T., PCT Pat. Appl. WO2005033055 (2005, Nippon Shokubai Co. Ltd.).

14. (a) Majunke, F.; Borchert, H.; Baerns, M. Proceedings of the $10^{\text {th }}$ International Congress on Catalysis, 19-24 July 1992, Budapest, Hungary. In "New Frontiers in Catalysis", 1993, Elsevier Science Publ. , p. 707-717.

(b) Mleczko, L.; Panneck, V.; Baerns, M. Chem. Eng. Technol. 1994, 17, 169.

(c) Takahashi, T.; Yasuhisa, E.; Eur. Pat. Appl. EP0779264A1 (Nippon Shokubai Co. Ltd.)

15. Raja, R. et al. Catalysis Letters 2006, 110, 179.

16. C.-C. Guo et al. Applied Catalysis A - General 282, 55 (2005).

17. J. H. Clark et al. in Journal of Chemical Research Synopses 3, 102 (1994).

18. V. R. Choudhary et al. Journal of Catalysis 227, 257 (2004).

19. "Catalytic Hydrogenation in Organic Synthesis" by P. Rylander, Academic Press 1979, pages 271-275.

20. A. I. Nekhaev et al. Neftekhimiya 42, 455 (2002).

21. For a recent review see: T. Mallott et al. Chem. Rev. 104, 3037 (2004).

22. C. H. Christensen et al. Angew. Chem. Int. Ed. 45, 4648 (2006).

23. A. Abod et al. Angew. Chem. Int. Ed. 444066 (2005).

24. S. Nishimura, "Handbook of Heterogenous Catalytic Hydrogenation for Organic Synthesis", John Wiley \& Sons 2001, pgs. 170-178.

25. G. Pez, R. Grey, U.S. Pat. No. 4,359,404.

26. Y. Maeda, T. Nishimura, and S. Uemura Bull. Chem. Soc. Jpn. 76, 2399 (2003).

27. K. Yamaguchi and N. Mizuno Angew Chem. Int. Ed. 42, 1480 (2003).

28. Rosenblatt, U.S. Pat. No. 3,483,216.

29. Sauer, U.S. Pat. No. $4,042,621$.

30. D. Coulson et al. WO 01/53252 A2. 
31. R. M. Laine et al. C1 Molecule Chemistry 1, 1 (1984).

32. A. V. Mashkina Catalysis Reviews-Science and Engineering 32, 105 (1990).

33. K. Komatsu Chemistry Letters 12, 1229 (1997).

34. K. Baback Synthesis 3, 335 (2003).

35. L. Bateman J. Chem. Soc. 2888 (1958). 


\section{Appendix A: Computational Methods and Thermodynamic Data}

As explained in Chapter 1, the most discerning factor in selecting/devising hydrogen-reversible liquid carriers is the enthalpy or heat of dehydrogenation, $\Delta \mathrm{H}_{\mathrm{D}}$. Since enthalpy varies only slightly with temperature even a knowledge of this quantity at standard conditions $\left(\Delta H_{D}{ }^{\circ}\right)$ can be useful. $A$ difficulty is that enthalpy data is available for only a relatively small number of hydrogenated pi-conjugated/piconjugated molecule pairs. Fundamentally the energy of molecules can be totally described using quantum mechanics-based computational methods ${ }^{1}$. Practically this is done at various levels of theory depending on the size of the problem and available computational hardware and time. For an initial scoping of $\Delta \mathrm{H}_{\mathrm{D}}$ for a series of molecules we used the PM3 (Parametric Method 3) and the more precise hybrid density functional theory (DFT) for developing as needed a fuller thermodynamic profile of the molecule; the calculation of $\Delta \mathrm{H}_{\mathrm{D}}$ but also as needed, $\Delta \mathrm{S}_{\mathrm{D}}$, at a desired range of temperatures.

\section{THERMODYNAMIC CALCULATIONS USING THE PM3 ALGORITHM}

The PM3 method was implemented using the commercial software program package Spartan 02 and Spartan 04 by Wavefunction Inc., Irvine, CA. In performing the calculations, all structures were first fully optimized in their molecular geometry by an energy minimization procedure. The configuration of the hydrogenated species was carefully chosen so that the adjacent hydrogen atoms are present alternatively at opposite sides of the aromatic planes; the ultimate criteria being a selection of the isomer of lowest energy. It is known that PM3 incorrectly yields the heat of formation for the $\mathrm{H}_{2}$ molecule. However, by replacing it with the experimental value of the heat of formation for $\mathrm{H}_{2}$ at its standard state we obtain the value of heat of dehydrogenation at standard conditions, $\Delta \mathrm{H}_{\mathrm{D}}{ }^{\circ}$, for hydrogenation that is in fair agreement with the available experimental data. For example, for dehydrogenation of cyclohexane (gas) to benzene (gas), the calculated value of $\Delta \mathrm{H}_{\mathrm{D}}{ }^{\circ}$ is $76.0 \mathrm{~kJ} / \mathrm{mol} \mathrm{H}_{2}$ (exp. $68.7 \mathrm{~kJ} / \mathrm{mol} \mathrm{H}_{2}$ ); for the dehydrogenation of trans-decalin (gas) to naphthalene (gas), the calculated value is $71.3 \mathrm{~kJ} / \mathrm{mol} \mathrm{H}_{2}$ (exp. $-66.8 \mathrm{~kJ} / \mathrm{mol} \mathrm{H}_{2}$ ). While a similar level of accuracy was also found for other all carbon-hydrogen only aromatic compounds the technique appears to be somewhat less satisfactory when applied to molecules with nitrogen or other hetero atoms for which the $a b$ initio DFT method is much more reliable (see below). Nevertheless, PM3 works satisfactorily for providing the correct trend of the standard heat of formation, $\Delta \mathrm{H}_{\mathrm{f}}^{\circ}$ values over a range of similar molecules. And it is the only method available for very large systems (>about seven, five or six-membered rings) where the computational cost for the more sophisticated and higher precision ab initio methods would be 
impractical. Therefore we have only used the PM3 algorithm for calculating $\Delta \mathrm{H}_{\mathrm{f}}^{\circ}$ of hydrogenation for the series of polyaromatic hydrocarbons (Chapter 1 , Figures 4 and 5) which comprise relatively larger molecules (greater than about seven five- or six-membered rings) for which the computational cost of the $a b$ initio DFT methodology would be prohibitive.

\section{THERMODYNAMIC CALCULATIONS CARRIED OUT USING THE AB INITIO DENSITY FUNCTIONAL THEORY COMPUTATIONAL APPROACH}

In the ab initio DFT computational approach, the molecular geometry is as before carefully selected to ensure that the lowest energy conformer has been chosen. The final geometry optimization is carried out using (unless otherwise specified) the B3LYP functional with a 6-311G" basis set (see Chapter 5 and 10, respectively in Reference 1 for "Computational Chemistry"). This geometry calculation also provides the electronic energy, E, of the molecule. The molecule's normal vibrational frequencies are estimated using the harmonic oscillator approximation, derived from the second derivative of the energy. The frequencies are a measure of the vibrational energy of the molecule from which using standard methods of statistical mechanics, treating the molecule as an ideal gas, the total vibrational enthalpy (Hv) and entropy (Sv) are determined as a function of temperature. Rotational and translational contributions to the enthalpy $\left(H_{R}, H_{T}\right)$ and entropy $\left(S_{R}, S_{T}\right)$ and the external energy of the molecule (RT where $R$ is the gas constant) are also included. In summary, the enthalpy change, $\Delta \mathrm{H}$ for the hydrogenation of substrate $\mathrm{A}$ to $\mathrm{A}-\mathrm{H}_{2 n}$ (Equation 1 ) is given by Equation 1 .

$$
\Delta \mathrm{H}=\Delta \mathrm{E}+\mathrm{sHv}\left(\mathrm{A}-\mathrm{H}_{2 n}\right)-\mathrm{sHv}(\mathrm{A})-\left[\mathrm{Hv}\left(\mathrm{H}_{2}\right)+\mathrm{H}_{\mathrm{R}}\left(\mathrm{H}_{2}\right)+\mathrm{H}_{\mathrm{T}}\left(\mathrm{H}_{2}\right)+\mathrm{RT}\right]
$$

The harmonic oscillator approximation is known to result in an over-estimation of the vibrational frequencies and thus the vibrational enthalpy, $H_{v}$. This situation is commonly remedied (see Computational Chemistry Chapter 11 ) by modifying the calculated $H_{v}$ 's for the organic molecules by the scaling factor $\mathrm{s}$, which for a hydrogenation of aromatic molecules we have empirically determined as 0.8. This is a considerably larger scaling factor that is normally employed but is justified by the excellent agreement between calculated and experimental $\Delta H_{D}{ }^{\circ}$ (where $\Delta H_{D}=-\Delta H$ of Eqn. 1) values for a variety of hydrogenated/dehydrogenated molecule pairs for which experimental data is available (Table 1). The entropy change for hydrogenation, $\Delta S$ is given by Equation 2, where $\Delta S_{D}=-\Delta S$.

$$
\Delta S=S\left(A-H_{2 n}\right)-S\left(H_{v}\right)-\left[S_{v}\left(H_{2}\right)+S_{R}\left(H_{2}\right)+S_{T}\left(H_{2}\right)\right]
$$


It is noteworthy that for the hydrogenation of pi-unsaturated compounds the translational entropy for hydrogen, $\mathrm{S}_{\mathrm{T}}\left(\mathrm{H}_{2}\right)$, largely predominates and as assumed earlier, $\Delta \mathrm{S}_{\mathrm{H}_{2}}$ at standard condition for many systems is close to $-125 \mathrm{~J} / \mathrm{deg}$. mol.

$$
\begin{aligned}
& \Delta G=-R T \operatorname{lnK}=\Delta H-T \Delta S \\
& K=\left[A-H_{2 n}\right] /[A] P_{2}{ }^{n}
\end{aligned}
$$

The ab initio-estimated values of $\Delta \mathrm{H}$ and $\Delta \mathrm{S}$ along with Equations 3 and 4 (where the terms in square brackets refer to concentrations and $\mathrm{P}$ designates the $\mathrm{H}_{2}$ partial pressure) were used to predict the temperature at which $95.24 \%$ of the hydrogenated carrier, $\mathrm{A}-\mathrm{H}_{2 n}$, would be converted back to the pi-unsaturated substrate, $\mathrm{A}$, at $1 \mathrm{~atm}$. of $\mathrm{H}_{2}$ (i.e., where $\mathrm{K}$ of Equation 4 at reaction equilibrium at this temperature and $\mathrm{H}_{2}$ pressure is equal to $0.05 \mathrm{~atm}^{-\mathrm{n}}$ ].

Tables 1a-d show the standard heat of hydrogenation, $\Delta \mathrm{H}_{\mathrm{D}}{ }^{\circ}$, data for a wide range of potential hydrogen carriers as well as expected temperatures for a $95 \%$ dehydrogenation at equilibrium of the fully saturated (perhydrogenated) carrier molecules. Here, the carrier is identified as the aromatic molecule of the dehydrogenated/hydrogenated molecule pair. Unless otherwise indicated for the hydrogenated polycyclic hydrocarbons, an all-trans configuration for the $\mathrm{C}-\mathrm{H}$ bonds was employed. For the $\mathrm{N}$-containing hydrogenated polycyclic molecules the $\mathrm{N}$ atom "in" and "out" isomer of minimum energy was used as the starting structure. Included is a prediction of the corresponding temperatures calculated (using Equations 3 and 4 ) from experimental $\Delta H$ and $\Delta S$ data (where available from, for example, the NIST Standard Database No. 69 [March 2003], and the data collection in the HSC5 "Chemistry for Windows" software program package ${ }^{2}$ ).

Table 1a. Thermodynamic and reaction data for extended polycyclic aromatic hydrocarbons: calculated (calc.) and experimental (exp.) standard heats of dehydrogenation $\left(\Delta \mathrm{H}_{D}{ }^{\circ}\right.$ in $\mathrm{kJ} / \mathrm{mole}$ $\left.\mathrm{H}_{2}\right)$, estimated temperatures at $95 \%$ equilibrium conversion based on calculated $\left(\mathrm{T}_{95 \%} \mathrm{calc}\right.$.) and experimentally derived ( $T_{95 \%}$ exp) reaction enthalpy, and entropy data for the perhydrogenated to dehydrogenated carrier $+\mathrm{H}_{2}$ conversion. It is assumed that in this process one $\mathrm{H}_{2}$ molecule is generated per $\mathrm{C}=\mathrm{C}, \mathrm{C}=\mathrm{N}$ and/or $\mathrm{C}=\mathrm{O}$ double bond that is formed.

\begin{tabular}{|c|c|c|c|c|}
\hline $\begin{array}{c}\text { Dehydrogenated Carrier } \\
\text { Structure }\end{array}$ & $\begin{array}{c}\Delta \mathrm{H}_{\mathrm{D}}{ }^{\circ}(\mathbf{3 0 0} \mathrm{K}, \\
\text { calc.) }\end{array}$ & $\begin{array}{c}\Delta \mathrm{H}_{\mathrm{D}}{ }^{\circ}(298 \mathrm{~K}, \\
\text { exp.) }\end{array}$ & $\begin{array}{c}\mathrm{T}_{95 \%}{ }^{\circ} \mathrm{C} \\
\text { (calc.) }\end{array}$ & $\begin{array}{c}\mathrm{T}_{95 \%}{ }^{\circ} \mathrm{C} \\
\text { (exp.) }\end{array}$ \\
\hline & 65.3 & 68.7 & 319 & 318 \\
\hline
\end{tabular}


Table 1a (continued)

\begin{tabular}{|c|c|c|c|c|}
\hline $\begin{array}{c}\text { Dehydrogenated Carrier } \\
\text { Structure }\end{array}$ & $\begin{array}{c}\Delta \mathrm{H}_{\mathrm{D}^{\circ}(300 \mathrm{~K},} \\
\text { calc. })\end{array}$ & $\begin{array}{c}\Delta H_{D^{\circ}}{ }^{\circ}(298 \mathrm{~K}, \\
\text { exp.) }\end{array}$ & $\begin{array}{l}\mathrm{T}_{95 \%}{ }^{\circ} \mathrm{C} \\
\text { (calc.) }\end{array}$ & $\begin{array}{l}\mathrm{T}_{95 \%}{ }^{\circ} \mathrm{C} \\
\text { (exp.) }\end{array}$ \\
\hline cis & $63.2^{\mathrm{a}}$ & 64.0 & 244 & 262 \\
\hline trans & $66.1^{\mathrm{b}}$ & 66.6 & 273 & 280 \\
\hline & 61.0 & & 226 & \\
\hline & 54.4 & & 169 & \\
\hline & $58.1^{\mathrm{C}}$ & & 206 & \\
\hline & 59.0 & & & \\
\hline & 54.0 & & & \\
\hline
\end{tabular}


Table 1a (continued)

\begin{tabular}{|c|c|c|c|c|}
\hline $\begin{array}{c}\text { Dehydrogenated Carrier } \\
\text { Structure }\end{array}$ & $\begin{array}{c}\Delta \mathrm{H}_{\mathrm{D}}{ }^{\circ}(\mathbf{3 0 0} \mathrm{K}, \\
\text { calc.) }\end{array}$ & $\begin{array}{c}\Delta \mathrm{H}_{\mathrm{D}}{ }^{\circ}(\mathbf{2 9 8} \mathrm{K}, \\
\text { exp.) }\end{array}$ & $\begin{array}{c}\mathrm{T}_{95 \%}{ }^{\circ} \mathrm{C} \\
\text { (calc.) }\end{array}$ & $\begin{array}{c}\mathrm{T}_{95 \%}{ }^{\circ} \mathrm{C} \\
\text { (exp.) }\end{array}$ \\
\hline & 218 & & \\
\hline
\end{tabular}


Table 1a (continued)

\begin{tabular}{|c|c|c|c|c|}
\hline $\begin{array}{c}\text { Dehydrogenated Carrier } \\
\text { Structure }\end{array}$ & $\begin{array}{c}\Delta \mathrm{H}_{\mathrm{D}}{ }^{\circ}(\mathbf{3 0 0} \mathrm{K}, \\
\text { calc.) }\end{array}$ & $\begin{array}{c}\Delta \mathrm{H}_{\mathrm{D}}{ }^{\circ}(298 \mathrm{~K}, \\
\text { exp.) }\end{array}$ & $\begin{array}{c}\mathrm{T}_{95 \%}{ }^{\circ} \mathrm{C} \\
\text { (calc.) }\end{array}$ & $\begin{array}{c}\mathrm{T}_{95 \%}{ }^{\circ} \mathrm{C} \\
\text { (exp.) }\end{array}$ \\
\hline & 61.9 & 237 & \\
\hline $\begin{array}{c}\mathrm{CH}_{3} \mathrm{COCH} \mathrm{H}_{3}+\mathrm{H}_{2} \rightarrow \\
\mathrm{CH}_{3} \mathrm{CH}(\mathrm{OH}) \mathrm{CH}_{3}\end{array}$ & 54.2 & 51.6 & & \\
\hline
\end{tabular}

${ }^{a}$ Heat of dehydrogenation to form cis-decalin.

${ }^{\mathrm{b}}$ Heat of dehydrogenation to form the trans-decalin.

Includes $\Delta \mathrm{H}_{D}{ }^{\circ}$ for $>\mathrm{CHCOH} \rightarrow>=\mathrm{O}+\mathrm{H}_{2}$.

Table 1b. Thermodynamics and reaction data for extended pi-conjugated substrates with nitrogen heteroatoms.

\begin{tabular}{|c|c|c|c|c|}
\hline $\begin{array}{c}\text { Dehydrogenated Carrier } \\
\text { Structure }\end{array}$ & $\begin{array}{c}\Delta \mathrm{H}_{\mathrm{D}}{ }^{\circ}(\mathbf{3 0 0} \mathrm{K}, \\
\text { (calc.) }\end{array}$ & $\begin{array}{c}\Delta \mathrm{H}_{\mathrm{D}}{ }^{\circ} \mathbf{( 2 9 8 \mathrm { K } ,} \\
\text { exp.) }\end{array}$ & $\begin{array}{c}\mathrm{T}_{95 \%}{ }^{\circ}{ }^{\circ} \mathbf{C} \\
\text { (calc.) }\end{array}$ & $\begin{array}{c}\mathbf{T}_{95 \%}{ }^{\circ} \mathbf{C} \\
\text { (exp.) }\end{array}$ \\
\hline & 55.2 & 55.9 & 248 & 274 \\
\hline & 63.6 & 62.6 & 268 & 262 \\
\hline
\end{tabular}


Table 1b (continued)

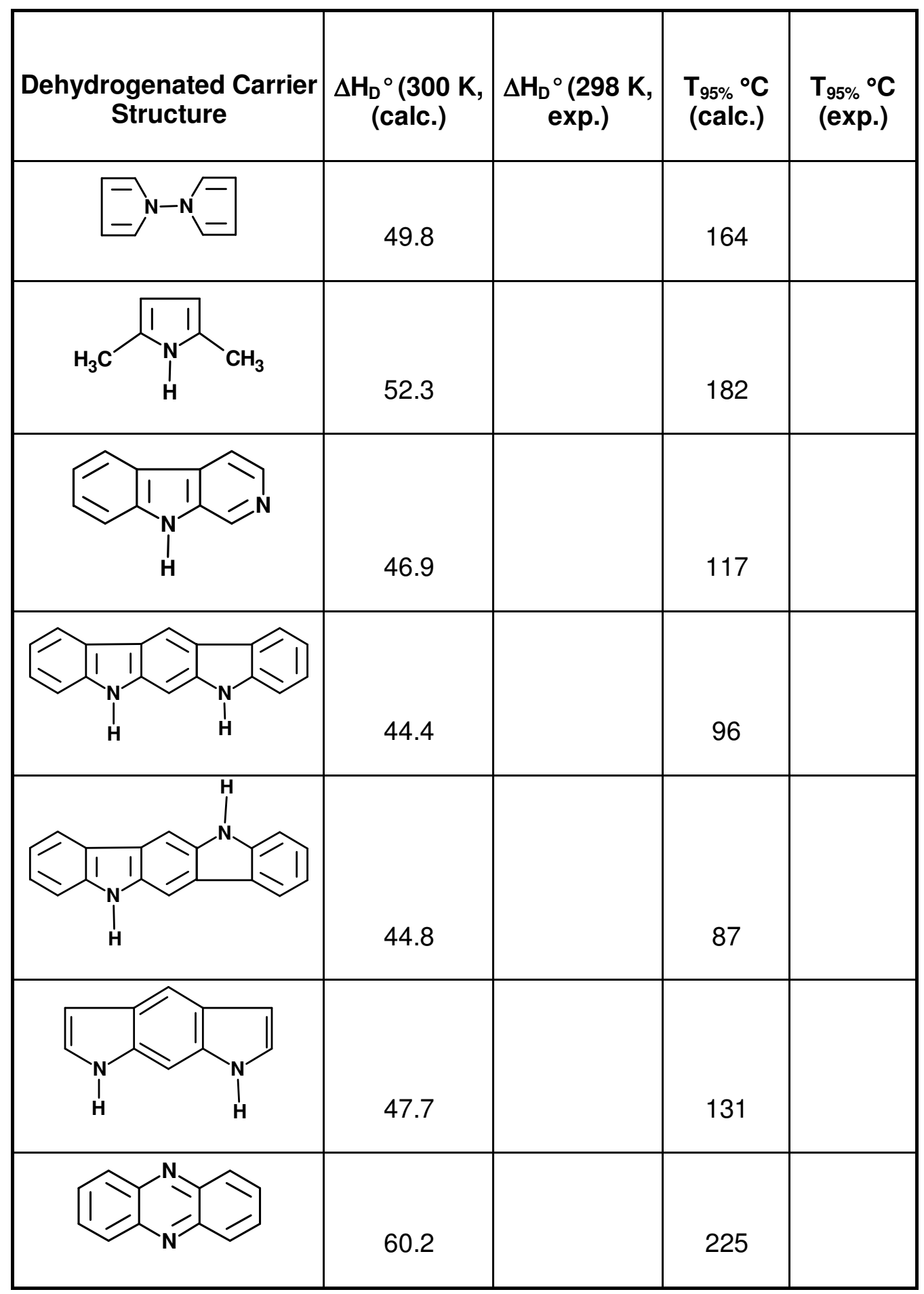


Table 1b (continued)

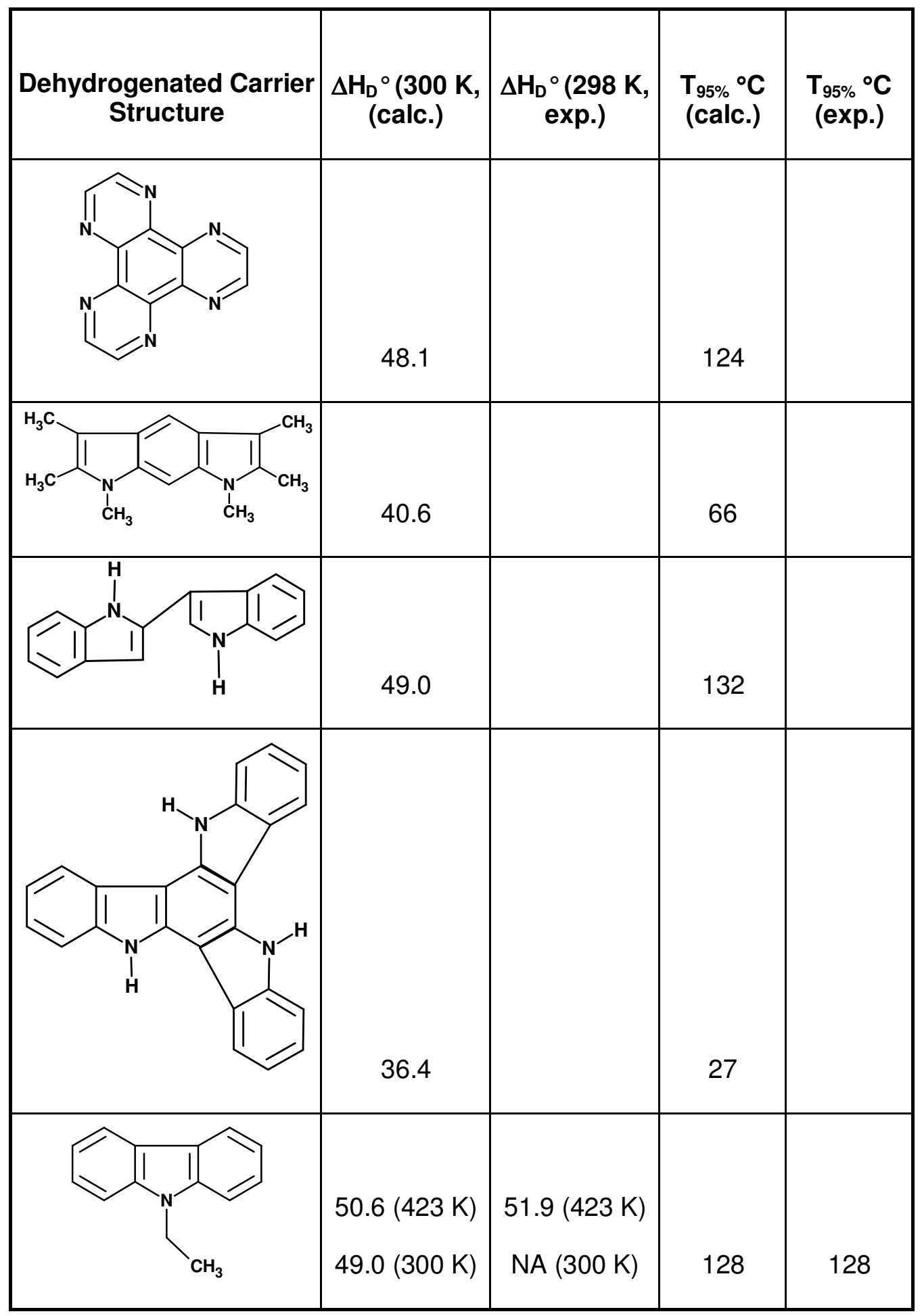


Table 1b (continued)

\begin{tabular}{|c|c|c|c|c|}
\hline $\begin{array}{c}\text { Dehydrogenated Carrier } \\
\text { Structure }\end{array}$ & $\begin{array}{l}\Delta \mathrm{H}_{\mathrm{D}}^{\circ}(300 \mathrm{~K}, \\
\quad \text { (calc.) }\end{array}$ & $\begin{array}{l}\Delta H_{D}{ }^{\circ}(298 \mathrm{~K}, \\
\text { exp.) }\end{array}$ & $\begin{array}{c}\mathrm{T}_{95 \%}{ }^{\circ} \mathrm{C} \\
\text { (calc.) }\end{array}$ & $\begin{array}{l}\mathrm{T}_{95 \%}{ }^{\circ} \mathrm{C} \\
(\exp .)\end{array}$ \\
\hline & 51.9 & & 164 & \\
\hline $\mathrm{H}$ & 59.4 & & 220 & \\
\hline & 61.9 & & 239 & \\
\hline & 52.3 & & 168 & \\
\hline & 51.0 & & 139 & \\
\hline & 57.7 & & 201 & \\
\hline & 63.1 & & 245 & \\
\hline
\end{tabular}


Table 1b (continued)

\begin{tabular}{|l|l|l|l|l|}
$\begin{array}{r}\text { Dehydrogenated Carrier } \\
\text { Structure }\end{array}$ & $\begin{array}{c}\Delta \mathrm{H}_{\mathrm{D}}{ }^{\circ}(300 \mathrm{~K}, \\
\text { (calc.) }\end{array}$ & $\begin{array}{c}\Delta \mathrm{H}_{\mathrm{D}}{ }^{\circ}(\mathbf{2 9 8} \mathrm{K}, \\
\text { exp.) }\end{array}$ & $\begin{array}{c}\mathrm{T}_{95 \%}{ }^{\circ} \mathrm{C} \\
\text { (calc.) }\end{array}$ & $\begin{array}{c}\mathbf{T}_{95 \%}{ }^{\circ} \mathrm{C} \\
\text { (exp.) }\end{array}$ \\
\hline & & & & \\
\hline
\end{tabular}


Table 1b (continued)

\begin{tabular}{|l|l|l|l|l|}
\hline & & & \\
\hline
\end{tabular}


Table 1b (continued)

\begin{tabular}{|c|c|c|c|c|}
\hline $\begin{array}{c}\text { Dehydrogenated Carrier } \\
\text { Structure }\end{array}$ & $\begin{array}{c}\Delta H_{D}^{\circ}(300 \mathrm{~K}, \\
\text { (calc.) }\end{array}$ & $\begin{array}{c}\Delta \mathrm{H}_{\mathrm{D}}^{\circ}(298 \mathrm{~K}, \\
\text { exp.) }\end{array}$ & $\begin{array}{l}\mathrm{T}_{95 \%}{ }^{\circ} \mathrm{C} \\
\text { (calc.) }\end{array}$ & $\begin{array}{l}\mathrm{T}_{95 \%}{ }^{\circ} \mathrm{C} \\
\text { (exp.) }\end{array}$ \\
\hline $\mathrm{CH}_{3} \mathrm{CN}+\mathrm{H}_{2} \rightarrow \mathrm{CH}_{3} \mathrm{CH}=\mathrm{NH}$ & 49.0 & 42.7 & & \\
\hline $\begin{array}{c}\mathrm{CH}_{3} \mathrm{CH}=\mathrm{NH}+\mathrm{H}_{2} \rightarrow \\
\mathrm{CH}_{3} \mathrm{CH}_{2} \mathrm{NH}_{2}\end{array}$ & 82.8 & 95.4 & & \\
\hline
\end{tabular}

${ }^{\mathrm{c}}$ Includes $\Delta \mathrm{HD}^{\circ}$ for $>\mathrm{CHOH} \rightarrow>\mathrm{C}=\mathrm{O}+\mathrm{H}_{2}$

Table 1c. Thermodynamics and reaction data for extended pi-conjugated substrates with heteroatoms other than nitrogen and comparative data for diphenylsilanes.

\begin{tabular}{|c|c|c|c|c|}
\hline $\begin{array}{c}\text { Dehydrogenated Carrier } \\
\text { Structure }\end{array}$ & $\begin{array}{l}\Delta \mathrm{H}_{\mathrm{D}}^{\circ}(300 \mathrm{~K}) \\
\text { (calc.) }\end{array}$ & $\begin{array}{c}\Delta H_{D}{ }^{\circ}(298 \mathrm{~K}, \\
\text { exp. })\end{array}$ & $\begin{array}{l}\mathrm{T}_{95 \%}{ }^{\circ} \mathrm{C} \\
\text { (calc.) }\end{array}$ & $\begin{array}{l}\mathrm{T}_{95 \%}{ }^{\circ} \mathrm{C} \\
\text { (exp.) }\end{array}$ \\
\hline & $\begin{array}{c}\text { (See note a) } \\
42.7\end{array}$ & & 52 & \\
\hline & $\begin{array}{c}56.5 \\
52.3(\text { cis-syn- } \\
\text { cis })\end{array}$ & & 197 & \\
\hline & 68.6 & & 285 & \\
\hline & 65.3 & & 275 & \\
\hline
\end{tabular}


Table 1c (continued)

\begin{tabular}{|l|l|l|l|l|}
\hline $\begin{array}{c}\text { Dehydrogenated Carrier } \\
\text { Structure }\end{array}$ & $\begin{array}{c}\Delta \mathrm{H}_{\mathrm{D}}{ }^{\circ}(300 \mathrm{~K}, \\
\text { (calc.) }\end{array}$ & $\begin{array}{c}\Delta \mathrm{H}_{\mathrm{D}}{ }^{\circ}(298 \mathrm{~K}, \\
\text { exp.) }\end{array}$ & $\begin{array}{c}\mathrm{T}_{95 \%}{ }^{\circ} \mathrm{C} \\
\text { (calc.) }\end{array}$ & $\begin{array}{c}\mathrm{T}_{95 \%}{ }^{\circ} \mathrm{C} \\
\text { (exp.) }\end{array}$ \\
\hline
\end{tabular}

Note: (a) Full geometry optimization with MP2/6-311** yielded an atomization energy of $-43.5 \mathrm{~kJ} / \mathrm{mole}_{2}$ vs. $-73.2 \mathrm{~kJ} / \mathrm{mole}_{2}$ calculated with B3LYP/6-311G**. An MP2 calculation would give a too small enthalpy of dehydrogenation to be reasonable.

Table 1d. Thermodynamics and reaction data for Pi-conjugated organic polymers and oligomers and comparative data for phenylene oligomers.

\begin{tabular}{|c|c|c|c|c|}
\hline $\begin{array}{c}\text { Dehydrogenated Carrier } \\
\text { Structure }\end{array}$ & $\begin{array}{c}\Delta \mathrm{H}_{\mathrm{D}}{ }^{\circ}(300 \mathrm{~K}) \\
\text { (calc.) }\end{array}$ & $\begin{array}{c}\Delta \mathrm{H}_{\mathrm{D}}{ }^{\circ}(298 \mathrm{~K}, \\
\text { exp.) }\end{array}$ & $\begin{array}{c}\mathrm{T}_{95 \%}{ }^{\circ} \mathrm{C} \\
\text { (calc.) }\end{array}$ & $\begin{array}{c}\mathrm{T}_{95 \%}{ }^{\circ} \mathrm{C} \\
\text { (exp.) }\end{array}$ \\
\hline
\end{tabular}


Table 1d (continued)

\begin{tabular}{|c|c|c|c|c|}
\hline $\begin{array}{c}\text { Dehydrogenated Carrier } \\
\text { Structure }\end{array}$ & $\begin{array}{c}\Delta \mathrm{H}_{\mathrm{D}}{ }^{\circ}(\mathbf{3 0 0} \mathrm{K}, \\
\text { (calc.) }\end{array}$ & $\begin{array}{c}\Delta \mathrm{H}_{\mathrm{D}}{ }^{\circ} \text { (298 K, } \\
\text { exp.) }\end{array}$ & $\begin{array}{c}\mathrm{T}_{95 \%}{ }^{\circ} \mathrm{C} \\
\text { (calc.) }\end{array}$ & $\begin{array}{c}\mathrm{T}_{95 \%}{ }^{\circ} \mathrm{C} \\
\text { (exp.) }\end{array}$ \\
\hline
\end{tabular}

\section{Calculation of standard heats of formation}

In classical (phenomenological) chemical thermodynamics, the enthalpy and entropy quantities are referred to a defined standard state of a substance (e.g., for carbon as graphite at $298.16 \mathrm{~K}$ at $1 \mathrm{~atm}$.). It is not possible to a priori calculate, by quantum and statistical mechanics methods, with a very high degree of precision the standard state of a substance. In order to be able to estimate standard enthalpy and entropy values some experimental data has to be invoked. Thus, the enthalpy of formation of a molecule at $0{ }^{\circ} \mathrm{K}, \Delta \mathrm{H}$, is the sum of the enthalpies of formation of the isolated atoms which are known experimental quantities. From this is subtracted the atomization energy of the molecule, which can be calculated using quantum and statistical mechanics. This procedure as detailed by Pople et al. in Reference 3, including a correction for ambient conditions, was used to calculate $\mathrm{H}_{\mathrm{f}}$. The resulting standard heats of formation, $\mathrm{H}_{\mathrm{f}}$, for the hydrocarbon and $\mathrm{N}$-heterocyclic molecules shown in Figures 8 and 9 of Chapter 1 are provided here in Tables 2 and 3, respectively. 
Table 2. Calculated standard heats of formation, $\mathrm{H}_{\mathrm{f}}^{\circ}$, and dehydrogenation enthalpies, $\Delta \mathrm{H}^{\circ}$, $\left(\mathrm{kJ} / \mathrm{mole}_{2}\right)$ for the hydrocarbon molecules shown in Figure 8 of Chapter 1.

\begin{tabular}{|c|c|c|c|c|c|}
\hline Molecule & $\mathbf{H}_{\mathbf{f}}{ }^{\circ}$ (dehydr) & $\mathbf{H}_{\mathrm{f}}{ }^{\circ}$ (hydr 1) & $\mathbf{H}_{\mathrm{f}}{ }^{\circ}$ (hydr 2) & $\Delta \mathbf{H 1}$ & $\Delta \mathbf{H 2}$ \\
\hline 1 & 25.48056 & & & & \\
\hline 1 & & -40.41744 & & 65.898 & \\
\hline 2 & 28.685504 & & & & \\
\hline 2 & & -37.73968 & & 66.42518 & \\
\hline 3 & 34.091232 & & -34.89456 & & 63.58006 \\
\hline 3 & & -32.400896 & & 66.49213 & \\
\hline 4 & 31.141512 & & & & \\
\hline 4 & & -31.283768 & & 62.42528 & \\
\hline 5 & 33.0666152 & & & & \\
\hline 5 & & -21.685672 & & & \\
\hline 5 & & & -27.48888 & & \\
\hline
\end{tabular}

Column 1: "Molecule" identifies the molecule pair along the row of Figure 8 [i.e., benzene (1), naphthalene (2), anthracene (3), etc.].

Column 2: The $\mathrm{H}_{\mathrm{f}}{ }^{\circ}$ for the aromatic (dehydrogenated) molecule of the pair.

Column 3: The $\mathrm{H}_{\mathrm{f}}{ }^{\circ}$ for the hydrogenated molecule in its most stable (all trans) form.

Column 4: $\Delta \mathrm{H} 1$ is the $\Delta \mathrm{H}_{\mathrm{D}}{ }^{\circ}$ for the dehydrogenation of the most stable (all trans) isomer.

Column 5: $\Delta \mathrm{H}_{2}$ is the $\Delta \mathrm{H}_{\mathrm{D}}{ }^{\circ}$ for the dehydrogenation of the least stable isomer of structure as represented in Figure 8 (Chapter 4). 
Table 3. Calculated standard heats of formation, $\mathrm{H}_{\mathrm{f}}{ }^{\circ}$, and dehydrogenation enthalpies, $\Delta \mathrm{H}^{\circ},\left(\mathrm{kJ} / \mathrm{mole}_{2}\right)$ for the $\mathrm{N}$-heterocyclic molecules in Figure 9 of Chapter 1.

\begin{tabular}{|c|c|c|c|c|c|}
\hline Molecule & $\mathrm{H}_{\mathrm{f}}{ }^{\circ}$ (dehydr) & $\mathrm{H}_{\mathrm{f}}{ }^{\circ}$ (hydr 1) & $\mathrm{H}_{\mathrm{f}}{ }^{\circ}$ (hydr 2) & $\Delta \mathrm{H} 1$ & $\Delta \mathrm{H} 2$ \\
\hline 1 & 37.90704 & & & & \\
\hline 1 & & -26.19184 & & 64.09888 & \\
\hline 2 & 46.090944 & & & & \\
\hline 2 & & -10.81564 & & 56.90658 & \\
\hline 2 & & & -3.26352 & & 49.35446 \\
\hline 3 & 39.354704 & & & & \\
\hline 3 & & -24.242096 & & 63.5968 & \\
\hline 4 & 43.442472 & & & & \\
\hline 4 & & -12.372088 & & 55.81456 & \\
\hline 5 & 34.463608 & & & & \\
\hline 5 & & -16.90336 & & 51.36697 & \\
\hline 6 & 43.099384 & & & & \\
\hline 6 & & -4.24676 & & 47.34614 & \\
\hline 6 & & & -3.142184 & & 46.24157 \\
\hline
\end{tabular}

Column 1: "Molecule" refers to the molecule pair along the row of Figure 9 [i.e., pyridine (1), naphthyridine (2), etc.]. Descriptions for columns 2 to 5 are as for Table 2. 


\section{Calculation of bond dissociation energies}

The calculation of homolytic $\mathrm{C}-\mathrm{H}$ bond dissociation energies provided valuable insight into molecular characteristics which significantly influence the dissociation enthalpies, $\Delta H_{D}$, of hydrogenated hydrogen carrier molecules. It was particularly valuable for discerning the effect of $\mathrm{N}$ and $\mathrm{O}$ heteroatoms on $\Delta \mathrm{H}_{\mathrm{D}}$.

The bond dissociation energy BDE (strictly, enthalpy, $\mathrm{H}$ ) for the process of homolytic $\mathrm{C}-\mathrm{H}$ bond dissociation $\left(\mathrm{RC}-\mathrm{H} \rightarrow \mathrm{RC}^{*}+\mathrm{H}^{*}\right)$ is given by Equation 5 .

$$
B D E=H\left(R C^{*}\right)+H\left(H^{*}\right)-H(R C-H)
$$

This $B D E$ is equivalent to the enthalpy change, $\Delta \mathrm{H}$, for the reaction and is the sum of the electronic energy, $\mathrm{E}$, and the internal and translation energy of the molecule, which for computational efficacy is considered to be an ideal gas $(\Delta \mathrm{H}=\mathrm{E}+$ zero point energy $+\mathrm{RT}$ vibrational energy correction + rotational + translational + "ideal gas contribution").

The Spartan 06 suite of programs (Wavefunction Inc., Irvine, CA) was used for the calculations. Limited test calculations for estimating the BDE of selected organic amine were performed:

1. Triethylamine $\left[\left(\mathrm{H}-\mathrm{CH}_{2} \mathrm{CH}_{2}\right) \mathrm{N}\left(\mathrm{CH}_{2} \mathrm{CH}_{3}\right)_{2}\right]$

$\operatorname{BDE}(\mathrm{C}-\mathrm{H})=369 \mathrm{~kJ} / \mathrm{mole}$

Experimental values: $379 \pm 1.7 \mathrm{~kJ} / \mathrm{mole}^{4} ; 382 \pm 4 \mathrm{~kJ} / \mathrm{mole}^{5}$

2. Pyrrolidine:<smiles></smiles>

$\operatorname{BDE}(\mathrm{C}-\mathrm{H})=370 \mathrm{~kJ} / \mathrm{mole}$

Experimental: $377 \pm 10 \mathrm{~kJ} / \mathrm{mole}^{6}$ 


\section{Check of BDE calculation using isodesmic approach}

As a check, particularly for the BDE for the carbon adjacent to nitrogen in perhydro $\mathrm{N}$-ethyl carbazole, the following approach of employing an isodesmic (same number of bonds in reactants and products) was employed.

Employing the C-H BDE for methanol:

$$
\begin{aligned}
& \mathrm{HOCH}_{2}-\mathrm{H}+\mathrm{R}^{\cdot}=\mathrm{HOCH}_{2}{ }^{\circ}+\mathrm{R}-\mathrm{H} \ldots \ldots(1) \\
& \mathrm{HOCH}_{2}{ }^{\circ}+\mathrm{H}^{\cdot}=\mathrm{HO}_{2}-\mathrm{H} \ldots \ldots \ldots \ldots \text { (2) }
\end{aligned}
$$

$$
\mathrm{R}^{*}+\mathrm{H}^{\cdot}=\mathrm{R}-\mathrm{H}
$$

where $\mathrm{R}^{*}$ is the radical remaining from a dissociation of the $\mathrm{C}-\mathrm{H}$ bond of the subject molecule.

Using the value of $401.7 \mathrm{~kJ} / \mathrm{mole}$ for the $\mathrm{BDE}$ of $\mathrm{CH}_{3} \mathrm{OH},{ }^{6}$ the $\mathrm{BDE}$ for the $\mathrm{C}-\mathrm{H}$ bond at the carbon atom adjacent to $\mathrm{N}$ in $\mathrm{N}$-ethylcarbazole is estimated to be $352 \mathrm{~kJ} / \mathrm{mole}$. This is slightly more than the $341 \mathrm{~kJ} / \mathrm{mole}$ as calculated by the direct method but is still significantly lower than the BDE of C-H bonds for a wide range of strained and "normal" hydrocarbons ${ }^{7}$. This is ascribed to the effect of the neighboring nitrogen atom.

\section{References}

1. "Computational Chemistry - A Practical Guide for Applying Techniques to Real World Problems", D. Young, Wiley-Interscience, NY, 2007.

2. Outokumpu Research, Finland.

3. Curtiss, L.A.; Raghavachani, K.; Redfern, P.C., Pople, J.A. J. Chem. Phys. 1997, 106, 1063.

4. Dombrowski, G.W.; Dinnocenzo, J.P.; Farid, S.; Goodman, J.L.; Gould, I.R. J. Org. Chem. 1999, $64,427$.

5. Laleve, J.; Allonas, X.; and Fouassier, J.-P., J. Am. Chem. Soc. 2002, 124, 9613.

6. Wayner, D.D.M.; Clark, K.B.; Rouk, A.; Yu, D.; Armstrong, D.A. J. Am. Chem. Soc. 1997, 119, 8925.

7. Feng, Y.; Liu, L.; Wang, J.-T.; Zhao, S.W.; Guo, Q.-X. J. Org. Chem. 2004, 69, 3129. 


\section{Appendix B: General Experimental Details}

\section{General synthetic and analytical procedures}

Commercial reagents were used without purification unless stated otherwise.

Gas Chromatography/ Mass Spectrometry (GC/MS) analyses were performed with an HP 5890 Series GC coupled to a 5972 Series Mass Selective Detector using a HP-5 column (30 meters) or with an HP 6890 Series GC coupled with a 5973 Inert Mass Selective Detector (Agilent Technologies) with a HP-35 column (30 meters). Proton $\left({ }^{1} \mathrm{H}\right)$ and carbon $\left({ }^{13} \mathrm{C}\right)$ nuclear magnetic resonance (NMR) spectra were collected on 300,400 or $500 \mathrm{MHz}$ Bruker instruments with an internal standard.

\section{Hydrogenation catalyst pre-activation}

In a typical procedure, the pre-catalyst was loaded into a stirred tank reactor along with tetrahydrofuran or isopropanol and heated to $160^{\circ} \mathrm{C}$ under $400 \mathrm{psig}$ hydrogen pressure. After 4 hours under these conditions, the reactor was cooled to room temperature and vented to atmospheric pressure. The reactor was then brought into an argon-filled glove box where the catalyst was filtered, washed with dry tetrahydrofuran, and dried under vacuum.

\section{General hydrogenation procedure}

In an argon-filled glove box, the substrate was dissolved in tetrahydrofuran or other hydrogenation reaction solvent and added to a $50-300 \mathrm{cc}$ stirred tank reactor. The hydrogenation catalyst (e.g., $\mathrm{Ru} / \mathrm{LiAl} \mathrm{O}_{8}, \mathrm{Rh} / \mathrm{Al}_{2} \mathrm{O}_{3}$ or $\mathrm{Rh} / \mathrm{C}$ ) was weighed and the hydrogenation catalyst added to the reactor (up to 2 mole \% catalyst - on a metal weight basis). The reactor was then sealed inside of the glovebox and attached to a hydrogenation manifold. The manifold has a source of high pressure hydrogen and a pressure gauge. The headspace of the reactor was purged with hydrogen by charging the reactor to high pressure and venting to atmospheric pressure. The reactor was then pressurized to $800-900$ psig such that when heating, the reactor pressure does not exceed 1000 psig hydrogen. The reactor was then heated to $160^{\circ} \mathrm{C}$ under a $7{ }^{\circ} \mathrm{C} / \mathrm{min}$. 
heating rate up to $180^{\circ} \mathrm{C}$ with stirring at $500-600 \mathrm{rpm}$. The reaction pressure is monitored and once the pressure drop ceases, the reactor is cooled to room temperature and vented to atmospheric pressure. The contents of the reactor are then filtered and analyzed by GC-MS to determine the hydrogenation conversion and selectivity.

\section{General dehydrogenation procedure}

At least 4 grams of hydrogenated substrate was degassed under vacuum and brought into an inert atmosphere glove box. The substrate and $<10$ wt. \% catalyst (or approximately $1 \mathrm{~mol} \%$ metal) were loaded into a $20 \mathrm{cc}$ stirred tank reactor. The reactor was sealed inside of the glovebox and connected to a dehydrogenation manifold (Figure 1). The dehydrogenation testing manifold contains five cells that can hold $20 \mathrm{cc}$ reactors. The manifold can supply vacuum, helium, or hydrogen to the reactors. Each reactor can be vented to atmosphere through a series of two hydrogen flow meters (10 sccm and $100 \mathrm{sccm}$ ). The head space of the reactor is purged with hydrogen and pressurized to one atmosphere hydrogen. The temperature of the reactor is ramped at a controlled rate to $225^{\circ} \mathrm{C}$ while stirring at $500-800 \mathrm{rpm}$. The effluent hydrogen gas is tabulated by the mass flow meters. When hydrogen evolution ends, the reactor is cooled to room temperature. The contents are then filtered and analyzed by GC-MS.

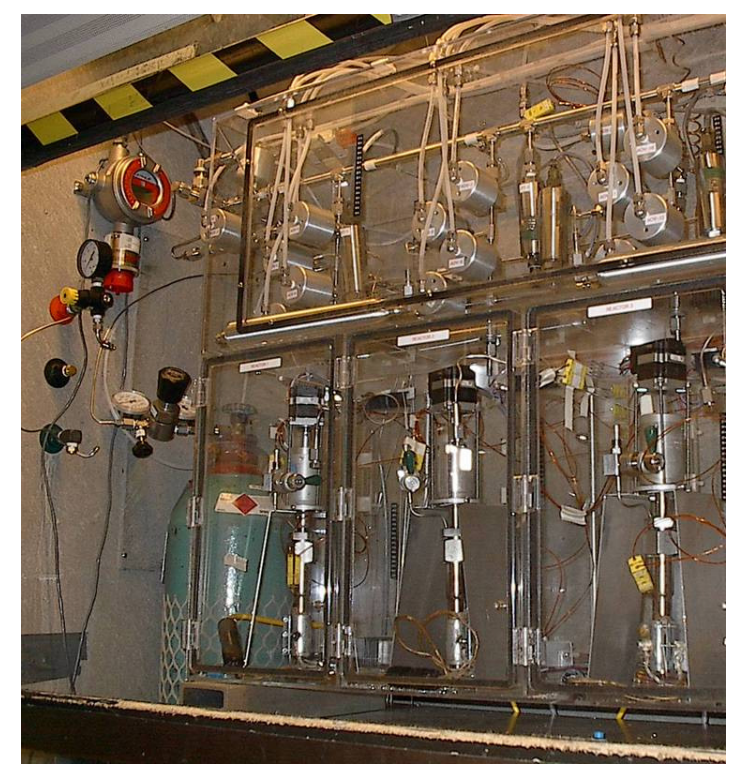

Figure 1. Picture of dehydrogenation testing equipment. 


\section{Dehydrogenation catalyst testing}

For comparison of dehydrogenation catalyst activity, two $20 \mathrm{cc}$ stirred tank reactors were used in the same positions in the dehydrogenation testing manifold for every catalyst screening reaction. The relative performance of the two reactors was tested by setting up identical dehydrogenation runs (i.e., same batch of perhydro-N-ethylcarbazole, same batch of catalyst) and comparing the data (Figure 2).

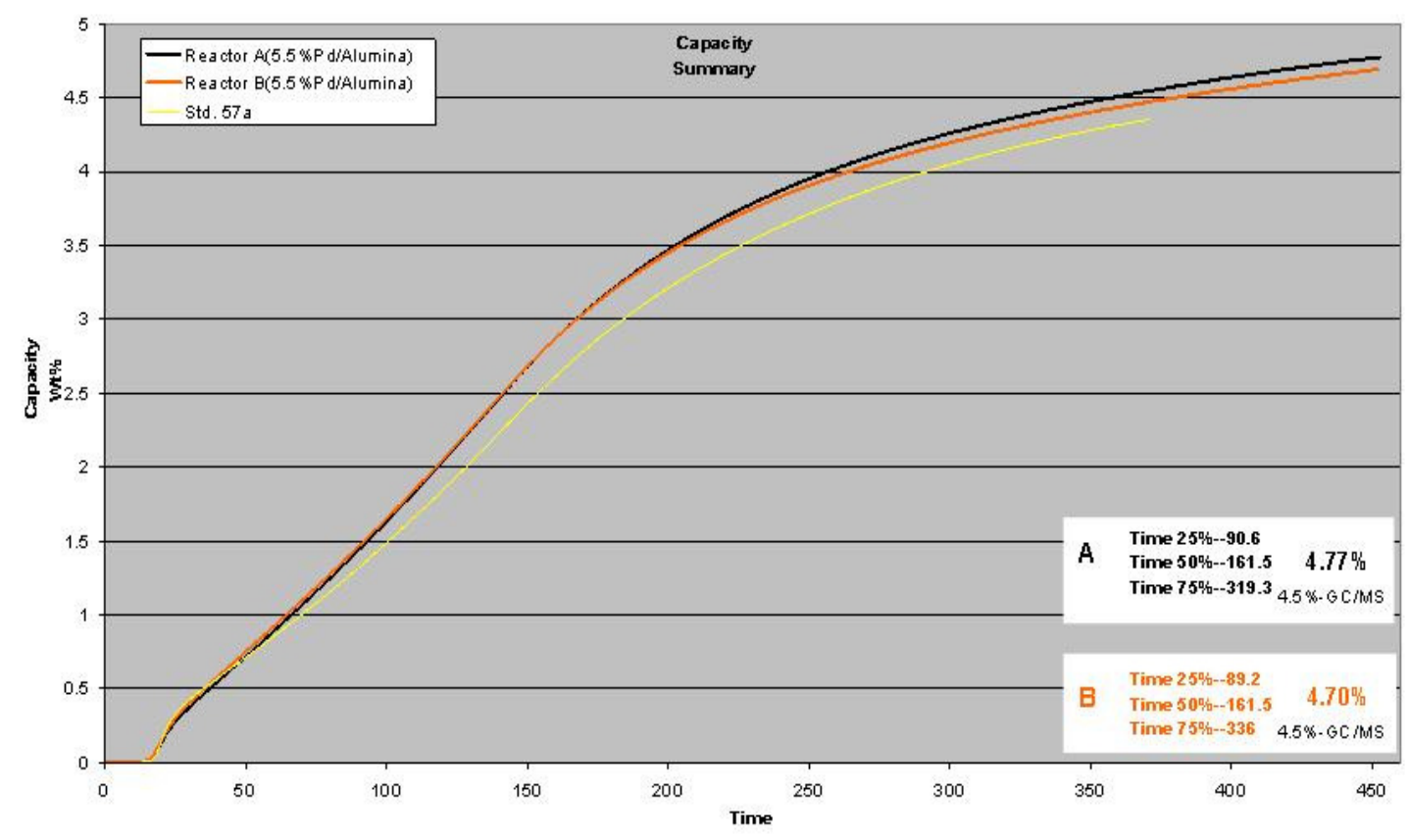

Figure 2. Dehydrogenation reactor testing data.

The performance of these reactors was periodically checked by performing a dehydrogenation with a reference catalyst ( $5 \%$ palladium on alumina, Engelhard catalyst 99021). Efforts were undertaken to use the same batch of perhydro-N-ethylcarbazole for screening a set of dehydrogenation catalysts. When changing batches of perhydro$\mathrm{N}$-ethylcarbazole, a set of dehydrogenation experiments with the reference catalyst were used to check for significant differences in the dehydrogenation rate and selectivity. 
The chioce of standard reactor stirring rate was carefully established so that the rate of hydrogen evolution would not be limited by mass-transfer, but instead reflect the rate of the catalytic dehydrogenation reaction. Our testing involved performing the dehydrogenation of perhydro-N-ethylcarbazole with the same catalyst in the same reactors, but increasing the stirring rate from $250 \mathrm{rpm}$ (Figure 3) to $500 \mathrm{rpm}$ (Figure 4), and finally to $750 \mathrm{rpm}$.

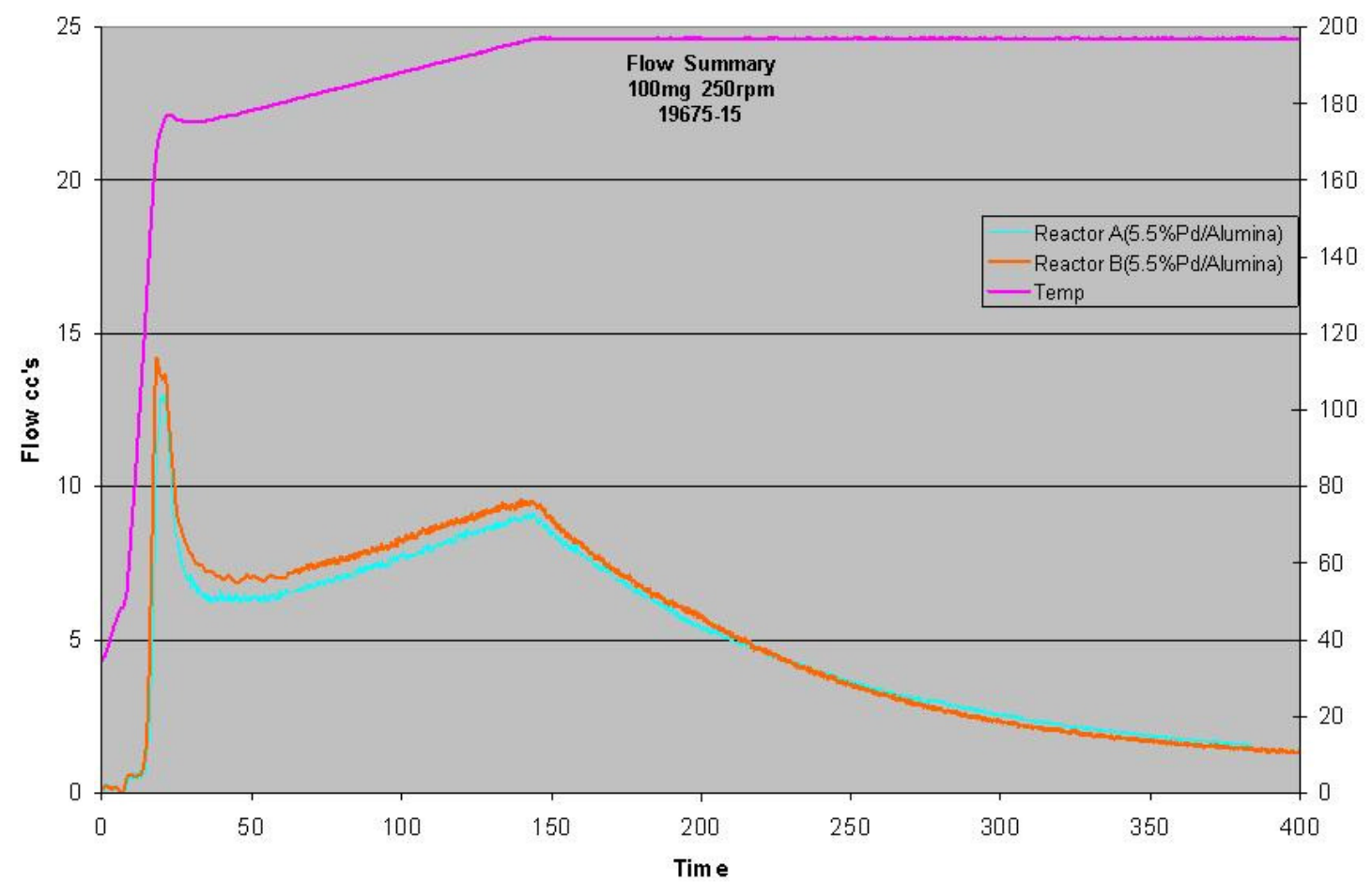

Figure 3. Hydrogen flow data for perhydro-N-ethylcarbazole dehydrogenation conducted with a 250 rpm stirring rate.

We found that there was a faster dehydrogenation rate for the reaction conducted with a $500 \mathrm{rpm}$ stirring rate relative to the reaction conducted with a $250 \mathrm{rpm}$ stirring rate. However, there was a negligible difference between reactions conducted with $500 \mathrm{rpm}$ and 750 rpm stirring rates. Therefore, we performed all dehydrogenation catalyst testing using a stirring rate of at least $500 \mathrm{rpm}$. 


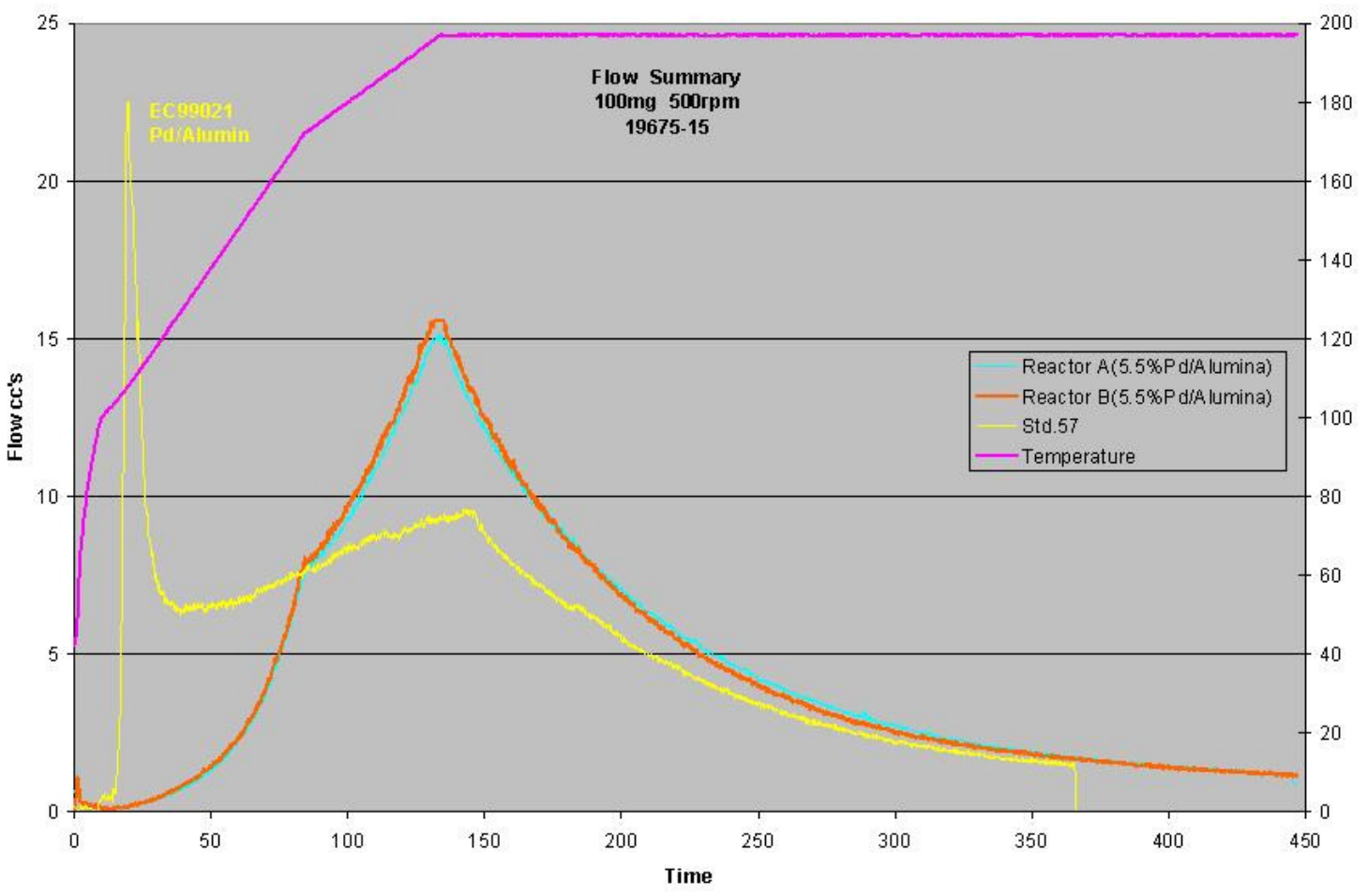

Figure 3. Hydrogen flow data for perhydro-N-ethylcarbazole dehydrogenation conducted with a 500 rpm stirring rate. 


\section{Appendix C: List of Abbreviations}

The following is a table of abbreviations (listed in alphabetical order) found in the Executive Summary, Chapters 1-9, and Appendices A and B of the final report.

\begin{tabular}{|c|c|}
\hline amu & Atomic Mass Unit \\
\hline b.p. & Boiling Point \\
\hline BDE & Bond Dissociation Energy \\
\hline CC & Cubic Centimeter \\
\hline $\mathrm{Cl}$ & Chemical lonization \\
\hline $\mathrm{dba}$ & Dibenzylideneacetone \\
\hline DEP & Direct Exposure Probe \\
\hline DFT & Density Functional Theory \\
\hline DMAC & Dimethylacetamide \\
\hline DMF & Dimethylformamide \\
\hline $\mathrm{DOE}$ & Department of Energy \\
\hline dppp & Diphenylphosphinopropane \\
\hline EH\&S & Environment, Health and Safety \\
\hline El & Electron Ionization \\
\hline FW & Formula Weight \\
\hline g & Gram \\
\hline GC-MS & Gas Chromatography-Mass Spectroscopy \\
\hline GPC & Gel Permeation Chromatography \\
\hline HPLC & High Performance Liquid Chromatography \\
\hline ICE & Internal Combustion Engine \\
\hline INADEQUATE & Incredible Natural Abundance Double Quantum Transfer Experiment \\
\hline IR & Infrared \\
\hline $\mathrm{kJ}$ & Kilojoule \\
\hline LHV & Lower Heating Value \\
\hline LPAH & Large Polyaromatic Hydrocarbon \\
\hline M & Molar \\
\hline m.p. & Melting Point \\
\hline MALDI & Matrix-Assisted Laser Desorption lonization \\
\hline $\mathrm{mL}$ & Milliliter \\
\hline MW & Molecular Weight \\
\hline NMP & N-methylpyrrolidone \\
\hline NMR & Nuclear Magnetic Resonance \\
\hline $\mathrm{PAH}$ & Polyaromatic Hydrocarbon \\
\hline $\mathrm{PBI}$ & Polybenzimidazole \\
\hline PEM & Proton Exchange Membrane \\
\hline PM3 & Parametric Method 3 \\
\hline ppm & Parts Per Million \\
\hline psi & Pounds per Square Inch \\
\hline psia & Pounds per Square Inch, Absolute \\
\hline psig & Pounds per Square Inch, Gauge \\
\hline
\end{tabular}




\section{List of Abbreviations (continued)}

\begin{tabular}{|l|l|}
\hline RF & Radio Frequency \\
\hline rpm & Revolutions Per Minute \\
\hline sccm & Standard Cubic Centimeters per Minute \\
\hline TGA & Thermogravimetric Analysis \\
\hline THF & Tetrahydrofuran \\
\hline UV & Ultraviolet \\
\hline
\end{tabular}

